Supporting Information

\title{
Copper-Catalyzed Cross-Coupling of Benzylic C-H Bonds and Azoles with Controlled $N$-Site Selectivity
}

\author{
Si-Jie Chen ${ }^{\dagger}$, Dung L. Golden ${ }^{\dagger}$, Shane W. Krska ${ }^{\ddagger}$ and Shannon S. Stahl ${ }^{\dagger *}$ \\ 'Department of Chemistry, University of Wisconsin-Madison, 1101 University Avenue, Madison, Wisconsin 53706, \\ United States \\ * High-Throughput Experimentation and Lead Discovery Capabilities, Merck \& Co., Inc., Kenilworth, New Jersey \\ 07033, United States \\ *Correspondence to: stahl@chem.wisc.edu
}

\section{Table of Contents}

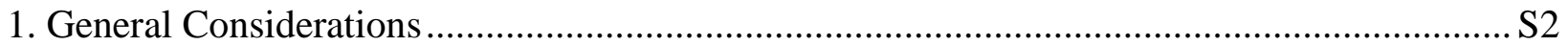

2. Experimental Procedures for Cross-Coupling Reactions ................................................. S2

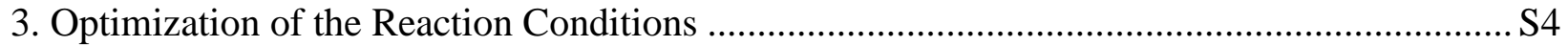

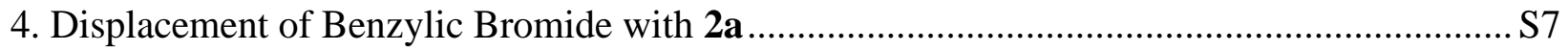

5. Additional Screening Data with Benzylic C-H Substrates and Azoles................................. S8

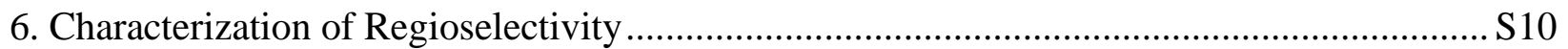

7. Crystallization and Crystallographic Data for $\left[\left(\mathrm{C}_{6} \mathrm{H}_{5}\right) \mathrm{N}\left(\mathrm{CH}_{3}\right)_{3}\right]_{2}\left[\mathrm{CuBr}_{4}\right]$ and $\mathbf{2 a} \ldots \ldots \ldots \ldots \ldots . . . . . . . .11$

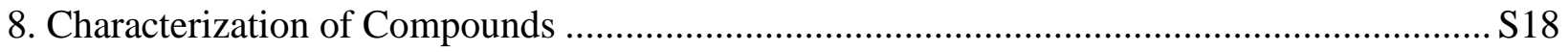

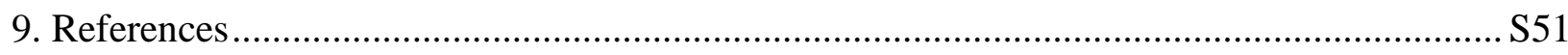

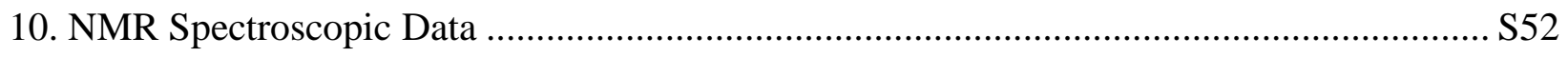




\section{General Considerations}

All reagents were purchased and used as received unless otherwise noted. $\mathrm{Cu}$ salts were purchased from Aldrich. Benzylic C-H substrates were purchased from Alfa Aesar, Ambeed, Ark Pharm, AstaTech, Chem-Impex, Combi-Blocks, Enamine, Matrix Chemicals, Millipore Sigma, Oakwood Chemicals and TCI America. Additives were purchased from Aldrich or TCI America. $N$-Fluorobenzenesulfonimide (NFSI) was purchased from Ark Pharm and Combi-Blocks. Diisopropyl phosphites were purchased from Aldrich, Oakwood Chemicals, Alfa Aesar and TCI America. All the reagents were used without further purification. Substrate 1q was prepared with the procedure reported in literature. ${ }^{1}$

${ }^{1} \mathrm{H},{ }^{13} \mathrm{C},{ }^{19} \mathrm{~F}$ and all the 2D NMR spectra were recorded on Bruker $400 \mathrm{MHz}$ or Bruker $500 \mathrm{MHz}$ spectrometers and chemical shifts are reported in parts per million (ppm). ${ }^{1} \mathrm{H}$ NMR spectra were referenced to tetramethylsilane at $0.00 \mathrm{ppm}$ and ${ }^{13} \mathrm{C}$ NMR spectra were referenced to $\mathrm{CDCl}_{3}$ at $77.16 \mathrm{ppm}$. Column chromatography was performed using a Biotage Isolera One ${ }^{\circledR}$ with reusable 25 g SNAP Ultra ${ }^{\circledR}$ cartridges, $25 \mathrm{~g}$ Sfärß cartridges or standard silica cartridges. Further purification of impure samples was conducted using preparative thin-layer chromatography with Analtech ${ }^{\circledR}$ Glass-Backed Silica G UNIPLATES ${ }^{\circledR}$. High-resolution mass spectra were obtained using a Thermo Q Exactive ${ }^{\mathrm{TM}}$ Plus (ESI or ASAP-MS) by the mass spectrometry facility at the University of Wisconsin. Melting points were determined using a DigiMelt MPA160 SRS melting point apparatus.

\section{Experimental Procedures for Cross-Coupling Reactions}

General Procedure (I) for Cross Coupling of $\mathbf{N}-\mathbf{H}$ Nucleophiles and Benzylic C-H Substrates (pressure tube, temperature $\geq \mathbf{5 0}^{\circ} \mathrm{C}$ )

Copper(I) chloride (2.0 mg, $0.020 \mathrm{mmol}, 10 \mathrm{~mol} \%)$, tetrabutylammonium chloride (2.8 mg, $0.020 \mathrm{mmol}$, $10 \mathrm{~mol} \%$ or $16.8 \mathrm{mg}, 0.060 \mathrm{mmol}, 30 \mathrm{~mol} \%$ ), NFSI ( $94.6 \mathrm{mg}, 0.30 \mathrm{mmol}, 1.5$ equiv), $\mathrm{N}-\mathrm{H}$ nucleophile ( $0.50 \mathrm{mmol}, 2.5$ equiv) and benzylic substrate (if solid, $0.20 \mathrm{mmol}, 1.0$ equiv) were added to a glass pressure tube under air. The pressure tube was then moved to a glove box under $\mathrm{N}_{2}$ atmosphere. Solvent $(1.0 \mathrm{~mL})$, benzylic substrate (if liquid, $0.20 \mathrm{mmol}, 1.0$ equiv) and diisopropyl phosphite $(16.3 \mu \mathrm{L}, 0.10$ mmol, 0.5 equiv) were added to the tube. The tube was sealed in the glove box and taken out to a hot plate. The sealed tube was heated at $50{ }^{\circ} \mathrm{C}$ with stirring for $16 \mathrm{~h}$. When the reaction finished, the mixture was cooled down to room temperature. Then the mixture was evaporated under vacuum and the crude mixture was purified by column chromatography (silica gel, eluted by pentane:ethyl acetate $=20: 1$ to $4: 1$ ).

Trimethylsilyl triflate $\left(3.6 \mu \mathrm{L}, 0.020 \mathrm{mmol}, 10 \mathrm{~mol}^{\circ}\right)$ or $\mathrm{BF}_{3} \cdot \mathrm{OEt}_{2}(2.5 \mu \mathrm{L}, 0.020 \mathrm{mmol}, 10 \mathrm{~mol} \%)$ was added in the glove box when they were used as an additive instead of tetrabutylammonium chloride. 


\section{General Procedure (II) for Cross Coupling of N-H nucleophiles and Benzylic C-H Substrates (glass}

vial, temperature $\leq 40^{\circ} \mathrm{C}$ )

Copper(I) chloride (2.0 mg, $0.020 \mathrm{mmol}, 10 \mathrm{~mol} \%$ ), tetrabutylammonium chloride $(5.6 \mathrm{mg}, 0.020 \mathrm{mmol}$, $10 \mathrm{~mol} \%$ or $16.8 \mathrm{mg}, 0.060 \mathrm{mmol}, 30 \mathrm{~mol} \%$ ), NFSI (94.6 mg, $0.30 \mathrm{mmol}, 1.5$ equiv), $\mathrm{N}-\mathrm{H}$ nucleophile ( $0.50 \mathrm{mmol}, 2.5$ equiv) and benzylic substrate (if solid, $0.20 \mathrm{mmol}, 1.0$ equiv) were added under air to a 4 $\mathrm{ml}$ borosilicate glass vial containing a magnetic stir bar. Then the vial was capped with a pierceable Teflon cap. A needle was used to pierce through the cap to facilitate exchange of the vial headspace with the atmosphere. Then the vial was moved into a glove box, through three vacuum-nitrogen-backfill cycles. The needle was removed, and the vial was taken out of the glove box (now sealed under an inert gas). Solvent (1.0 mL), benzylic substrate (if liquid, $0.20 \mathrm{mmol}, 1.0$ equiv), and diisopropyl phosphite (16.3 $\mu \mathrm{L}$, $0.10 \mathrm{mmol}, 0.5$ equiv) were added into the vial by injection through the cap. The sealed vial was heated at $30{ }^{\circ} \mathrm{C}$ and stirred for $16 \mathrm{~h}$. When the reaction finished, the mixture was cooled down to room temperature. Then the mixture was evaporated under vacuum and the crude mixture was purified by column chromatography (silica gel, eluted by pentane:ethyl acetate $=20: 1$ to $4: 1$ ). 


\section{Optimization of the Reaction Conditions}

Optimization of reaction conditions were conducted according to general procedure (I) or (II), with variations specified in each table. Reaction yields were monitored by ${ }^{1} \mathrm{H}$ NMR spectroscopy with 0.2 mmol mesitylene as the internal standard.

Table S1a. Assessment of Ancillary Ligands

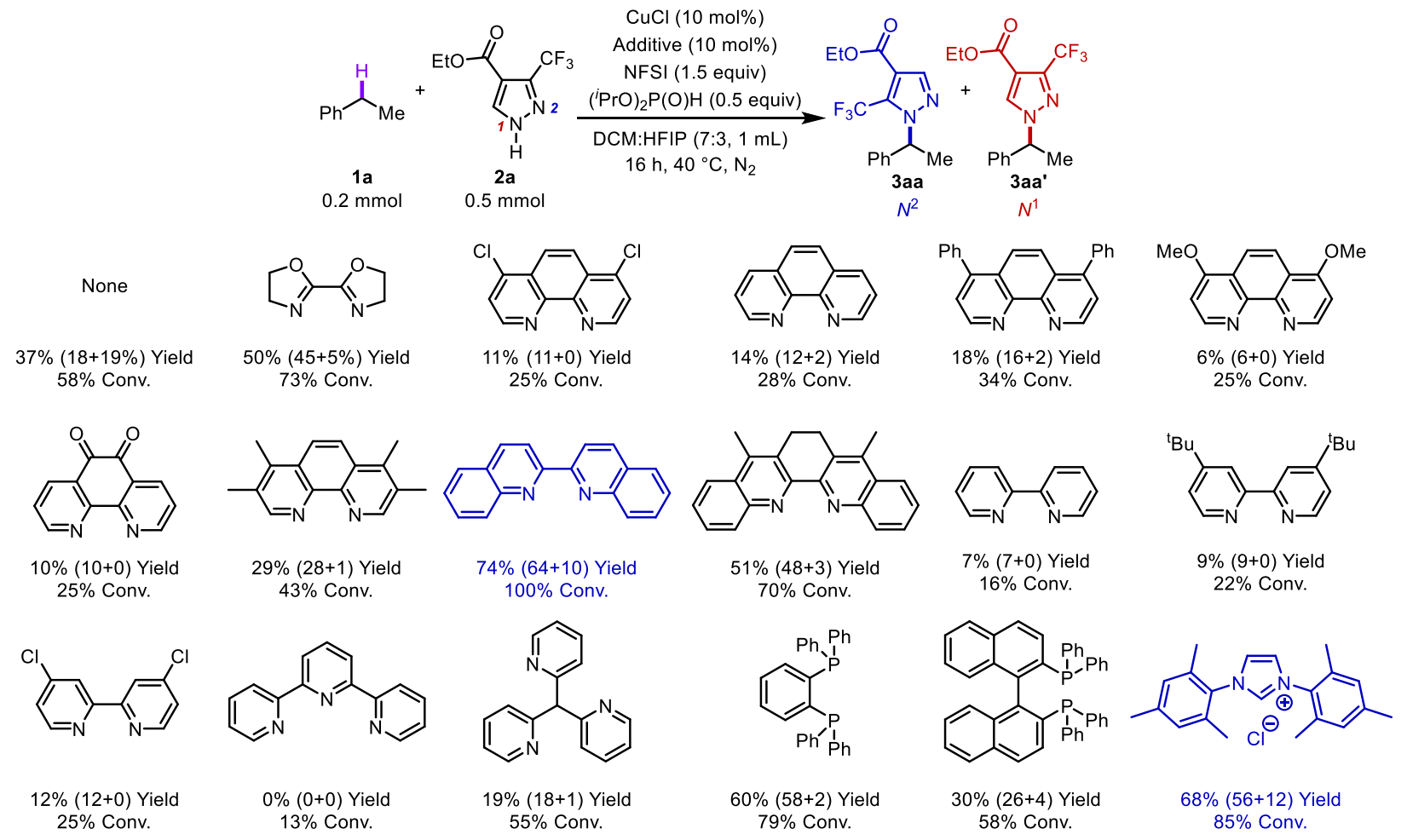

Table S2b. Ligand Comparison with Selected Nucleophiles

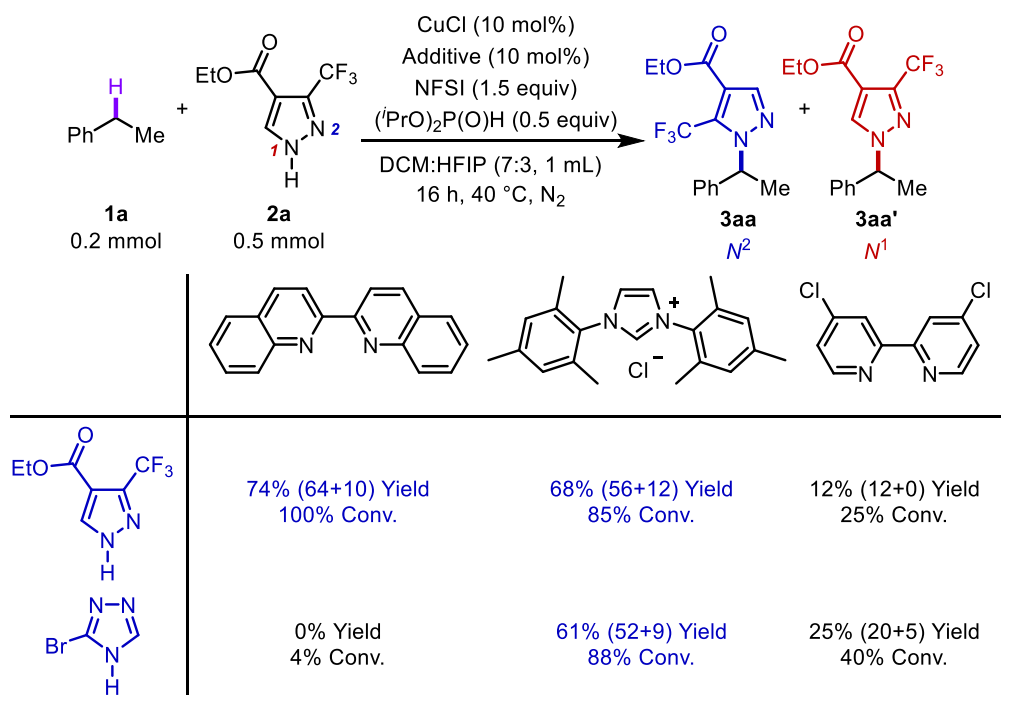


Table S2. Effect of Different Additives in Controlling the Regioselectivity ${ }^{a}$

\begin{tabular}{|c|c|c|c|c|c|}
\hline & $\begin{array}{c}\mathrm{Ph}_{\mathrm{Me}} \\
1 \mathrm{a} \\
0.2 \mathrm{mmol}\end{array}$ & cto- & $\begin{array}{l}\mathrm{CuBr}_{2}(10 \mathrm{~mol} \%) \\
\text { Additive }(10 \mathrm{~mol} \%) \\
\text { NFSI }(1.5 \text { equiv }) \\
\stackrel{\text { (PrO })_{2} \mathrm{P}(\mathrm{O}) \mathrm{H}(0.5 \text { equiv })}{\longrightarrow} \\
\underset{\mathrm{DCM}: \mathrm{HFIP}(7: 3,1 \mathrm{~mL})}{16 \mathrm{~h}, 50^{\circ} \mathrm{C}, \mathrm{N}_{2}}\end{array}$ & 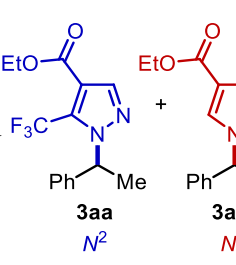 & \\
\hline Entry & Additive & Conv. of 1a (\%) & Yield of $\boldsymbol{N}-2(\%)$ & Yield of $\mathbf{N - 1}(\%)$ & $N-2: N-1$ \\
\hline 1 & $\mathrm{IMes}^{+} \mathrm{Cl}^{-}$ & 100 & 63 & 7 & $9.0: 1$ \\
\hline 2 & $\mathrm{TBA}^{+} \mathrm{Br}$ & 100 & 72 & 4 & $18: 1$ \\
\hline 3 & $\mathrm{TBA}^{+} \mathrm{NO}_{3}^{-}$ & 91 & 54 & 2 & $13: 1$ \\
\hline 4 & $\mathrm{TBA}^{+} \mathrm{Cl}^{-}$ & 100 & 70 & 6 & $11.7: 1$ \\
\hline 5 & (none) & 89 & 57 & 5 & 9.3:1 \\
\hline 6 & $\mathrm{TBA}^{+} \mathrm{PF}_{6}^{-}$ & 91 & 57 & 11 & $5.8: 1$ \\
\hline 7 & $\mathrm{TBA}^{+} \mathrm{HSO}_{4}^{-}$ & 100 & 57 & 10 & $5.7: 1$ \\
\hline 8 & $\mathrm{TBA}^{+} \mathrm{BH}_{2}^{-}$ & 88 & 52 & 24 & $2.2: 1$ \\
\hline 9 & $\mathrm{TBA}^{+} \mathrm{OTf}^{-}$ & 96 & 45 & 23 & $2.1: 1$ \\
\hline 10 & $\mathrm{TBA}^{+} \mathrm{ClO}_{4}^{-}$ & 100 & 63 & 34 & $1.7: 1$ \\
\hline 11 & TBDMSCI & 94 & 44 & 27 & $1.6: 1$ \\
\hline 12 & TMSCN & 91 & 42 & 28 & $1.5: 1$ \\
\hline 13 & TIPSCI & 91 & 36 & 29 & $1.2: 1$ \\
\hline 14 & $\mathrm{TBA}^{+} \mathrm{BF}_{4}^{-}$ & 100 & 26 & 34 & $1: 1.3$ \\
\hline 15 & $\mathrm{TEA}^{+} \mathrm{BF}_{4}^{-}$ & 97 & 35 & 43 & $1: 1.2$ \\
\hline 16 & $(\mathrm{Ph})_{3} \mathrm{SiCl}$ & 97 & 34 & 37 & $1: 1.1$ \\
\hline 17 & TMSCI & 94 & 30 & 47 & $1: 1.8$ \\
\hline 18 & $\mathrm{BF}_{3} \bullet \mathrm{OEt}_{2}$ & 91 & 6 & 60 & $1: 10$ \\
\hline 19 & TMSOTf & 42 & 2 & 38 & $1: 19$ \\
\hline $20^{b}$ & TMSOTf & 100 & 1 & 66 & $1: 66$ \\
\hline
\end{tabular}

Note: The screenings of salts in this study indicate the imidazolium cation identified in previous screening results does not play an important role in reactivity or $N^{1} / N^{2}$ regioselectivity and can be replaced by tetrabutylammonium cation. When the additive contains a Lewis acid, such as TMSCl or $\mathrm{BF}_{3} \cdot \mathrm{OEt}_{2}$, the regioselectiviy switiches to favor $N^{1}$. 
Table S3. Optimization of the Reaction Conditions with Various Solvents, Cu Salts and Temperature $^{\mathrm{a}}$

\begin{tabular}{|c|c|c|c|c|c|c|c|c|c|}
\hline & & $\begin{array}{c}\mathrm{Ph}_{\mathrm{Me}} \\
0.2 \mathrm{mmol}\end{array}$ & $\begin{array}{r}2 \\
0.5 \mathrm{n}\end{array}$ & & $\begin{array}{r}\text { TC } \\
\mathrm{N} \\
\text { ('PrO) } \\
\mathrm{DCM} \\
1\end{array}$ & $\begin{array}{l}\text { ( }(10 \text { mol\%) } \\
\mathrm{BBr}(\mathrm{X} \text { equiv) } \\
\mathrm{SI}(\mathrm{Y} \text { equiv) } \\
\text { (O) } \mathrm{H}(0.5 \text { equiv) } \\
\mathrm{FIP}(7: 3,1.0 \mathrm{ml}) \\
\text { h, } 50^{\circ} \mathrm{C}, \mathrm{N}_{2}\end{array}$ & 3aa & & \\
\hline Entry & {$[\mathrm{Cu}]$} & Solvent & $\mathrm{X}$ & $\mathrm{Y}$ & Z & Conv. of $1(\%)$ & Yield of $\mathbf{N - 2}(\%)$ & Yield of $N-1(\%)$ & $N-2: N-1$ \\
\hline 1 & $\mathrm{CuCl}$ & DCE & 0.1 & 1.5 & 2.5 & 94 & 60 & 3 & $20: 1$ \\
\hline 2 & $\mathrm{CuCl}$ & $\mathrm{PhH}$ & 0.1 & 1.5 & 2.5 & 58 & 12 & 0 & - \\
\hline 3 & $\mathrm{CuCl}$ & $\mathrm{PhCl}$ & 0.1 & 1.5 & 2.5 & 91 & 48 & 0 & - \\
\hline 4 & $\mathrm{CuCl}$ & $\mathrm{PhCF}_{3}$ & 0.1 & 1.5 & 2.5 & 64 & 24 & 1 & $24: 1$ \\
\hline 5 & $\mathrm{CuCl}$ & $\mathrm{PhNO}_{2}$ & 0.1 & 1.5 & 2.5 & 64 & 12 & 1 & $12: 1$ \\
\hline 6 & $\mathrm{CuCl}$ & $\mathrm{MeNO}_{2}$ & 0.1 & 1.5 & 2.5 & 97 & 21 & 34 & $1: 1.6$ \\
\hline 7 & $\mathrm{CuCl}$ & DCM & 0.1 & 1.5 & 2.5 & 100 & 48 & 8 & $6: 1$ \\
\hline 8 & $\mathrm{CuCl}$ & HFIP & 0.1 & 1.5 & 2.5 & 91 & 2 & 39 & $1: 19.5$ \\
\hline 10 & $\mathrm{CuCl}$ & DCM:HFIP=8:2 & 0.1 & 1.5 & 2.5 & 100 & 63 & 9 & $7: 1$ \\
\hline 11 & $\mathrm{CuCl}$ & DCM:HFIP=6:4 & 0.1 & 1.5 & 2.5 & 100 & 45 & 16 & $2.8: 1$ \\
\hline 12 & $\mathrm{CuCl}$ & DCM:HFIP=7:3 & 0.1 & 1.5 & 2.5 & 100 & 63 & 8 & $7.9: 1$ \\
\hline 13 & CuOAc & DCM:HFIP=7:3 & 0.1 & 1.5 & 2.5 & 94 & 66 & 6 & $11: 1$ \\
\hline 14 & Cul & DCM:HFIP=7:3 & 0.1 & 1.5 & 2.5 & 100 & 57 & 11 & $5.2: 1$ \\
\hline 15 & $\mathrm{CuBr}$ & $\mathrm{DCM}: \mathrm{HFIP}=7: 3$ & 0.1 & 1.5 & 2.5 & 100 & 57 & 11 & $5.2: 1$ \\
\hline 16 & $\mathrm{CuCl}_{2}$ & DCM:HFIP=7:3 & 0.1 & 1.5 & 2.5 & 100 & 60 & 10 & $6: 1$ \\
\hline 17 & $\mathrm{CuBr}_{2}$ & DCM:HFIP=7:3 & 0.1 & 1.5 & 2.5 & 100 & 63 & 11 & $5.7: 1$ \\
\hline 18 & $\mathrm{CuBr}_{2}$ & DCM:HFIP=7:3 & 0.1 & 1.0 & 2.5 & 94 & 60 & 8 & 7.5:1 \\
\hline 19 & $\mathrm{CuBr}_{2}$ & DCM:HFIP=7:3 & 0.1 & 2.0 & 2.5 & 100 & 63 & 8 & 7.9:1 \\
\hline 20 & $\mathrm{CuBr}_{2}$ & DCM:HFIP=7:3 & 0.1 & 1.5 & 2.5 & 100 & 69 & 6 & $11.5: 1$ \\
\hline 21 & $\mathrm{CuBr}_{2}$ & DCM:HFIP=7:3 & 0.3 & 1.5 & 2.5 & 97 & 72 & 2 & $36: 1$ \\
\hline 22 & $\mathrm{CuCl}$ & DCM:HFIP=7:3 & $0.3^{a}$ & 1.5 & 2.5 & 100 & 76 & 4 & 19:1 \\
\hline 23 & $\mathrm{CuCl}$ & DCM:HFIP=7:3 & $0.1^{\mathrm{a}}$ & 1.5 & 2.5 & 100 & 75 & 4 & 19:1 \\
\hline
\end{tabular}

a Reactions were conducted with TBACl instead of TBABr. DCE, 1,2-dichloroethane; DCM, dichloromethane; HFIP, hexafluoroisopropanol; Conv., conversion.

Table S4. Investigation of various reductants with ethylbenzene as the substrate

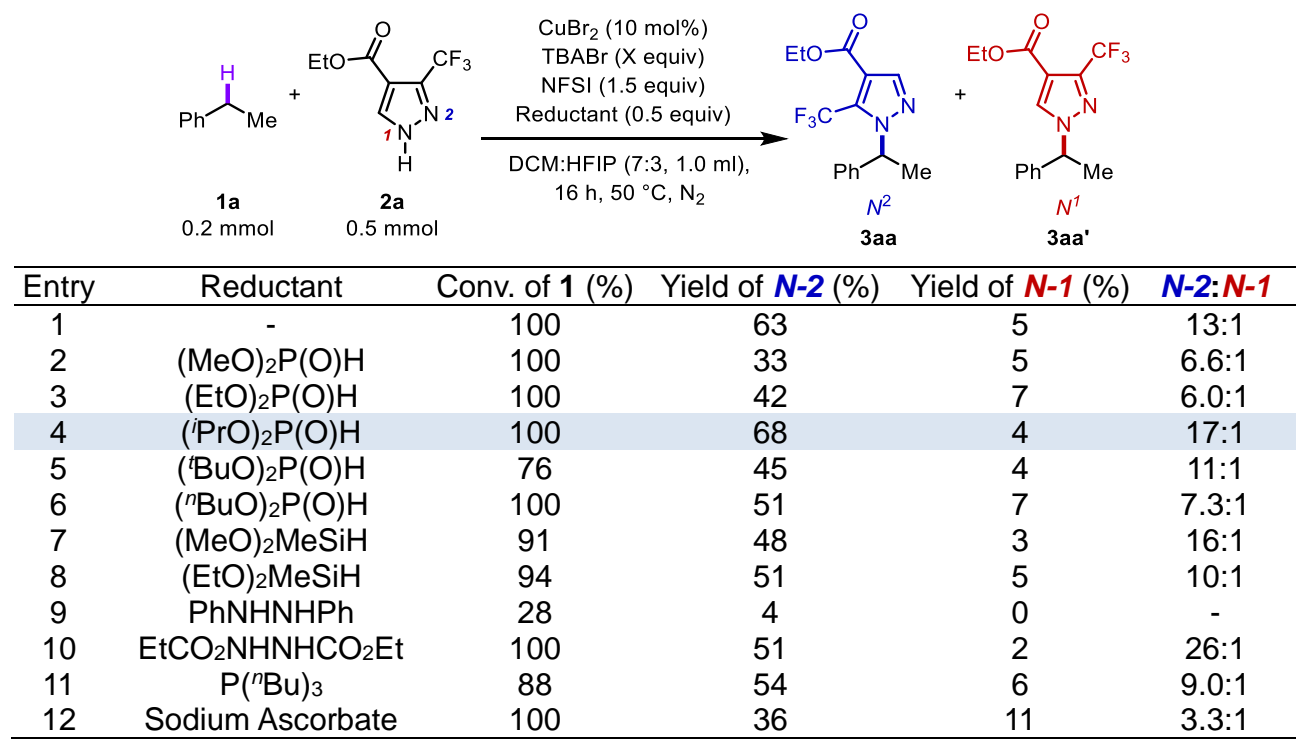




\section{Displacement of Benzylic Bromide with 2a}
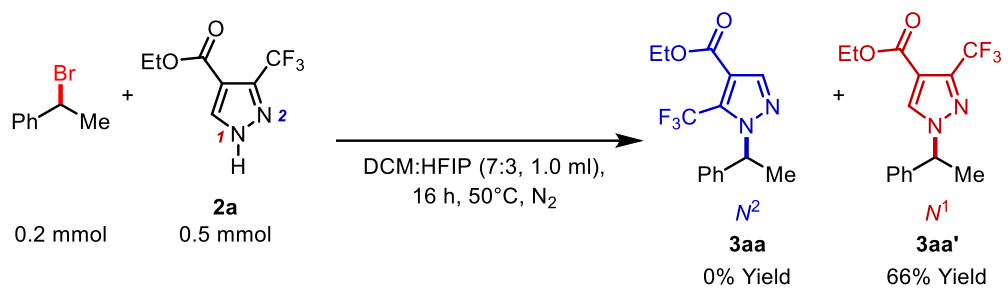

2a (104 $\mathrm{mg}, 0.50 \mathrm{mmol}, 2.5$ equiv) was added to a glass pressure tube under air. The pressure tube was then moved to a glove box under $\mathrm{N}_{2}$ atmosphere. DCM:HFIP (7:3, 1.0 mL) and (1-Bromoethyl)benzene ( $27.3 \mu \mathrm{L}, 0.20 \mathrm{mmol}, 1.0$ equiv) and were added to the tube. The tube was sealed in the glove box and taken out to a hot plate. The sealed tube was heated at $50{ }^{\circ} \mathrm{C}$ with stirring for $16 \mathrm{~h}$. When the reaction finished, the mixture was cooled down to room temperature. Then an aliquot of the mixture was taken, diluted with $\mathrm{CDCl}_{3}$. Reaction yield was monitored by ${ }^{1} \mathrm{H}$ NMR spectroscopy with $0.2 \mathrm{mmol}$ mesitylene as the internal standard. 


\section{Additional Screening Data with Benzylic C-H Substrates and Azoles}

Not all substrates tested afforded good reactivity in the cross-coupling reactions, and a survey of suboptimal results are provided in Figure S1. Ethylbenzene derivatives with electron-deficient parasubstituents are less reactive toward $\mathrm{C}-\mathrm{H}$ activation, and lower conversions and yields are observed (Figure S1A and B). Pyridine-containing substrates afford the desirable products but often with low yields (Figure S1C). More electron-rich azoles appear to inhibit the $\mathrm{C}-\mathrm{N}$ cross coupling reactivity, possibly reflecting coordination/inhibition of the copper catalyst or side reactivity with NFSI (Figure S1D). The presence of large substituents near the benzylic $\mathrm{C}-\mathrm{H}$ site also inhibits the conversion of the starting material (Figure S1E). Reaction yields were monitored by ${ }^{1} \mathrm{H}$ NMR spectroscopy with $0.2 \mathrm{mmol}$ mesitylene as the internal standard.
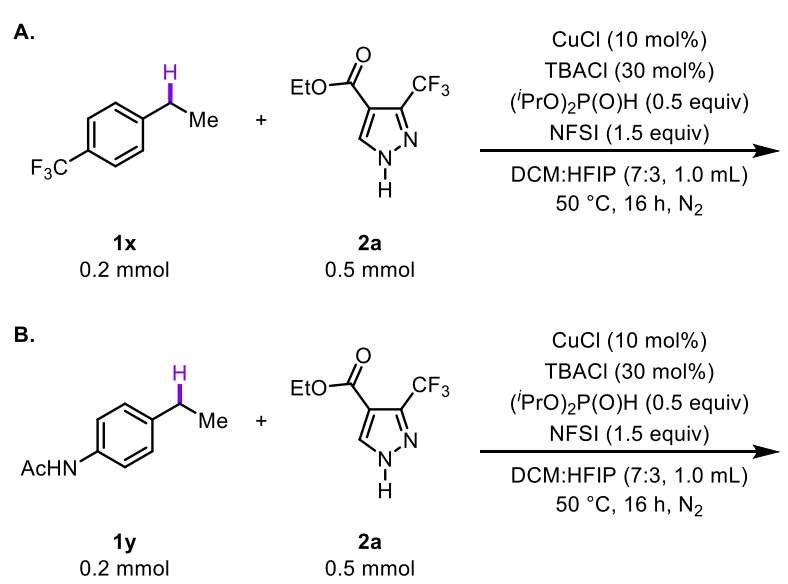

c.
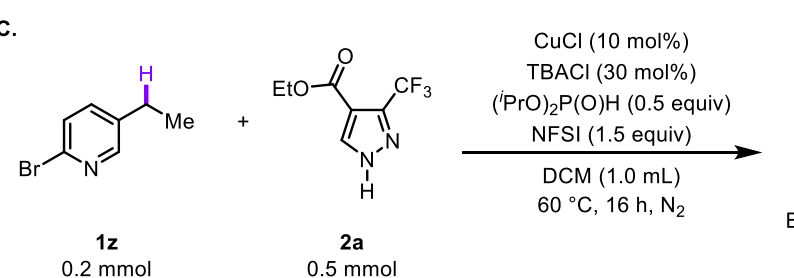

D.
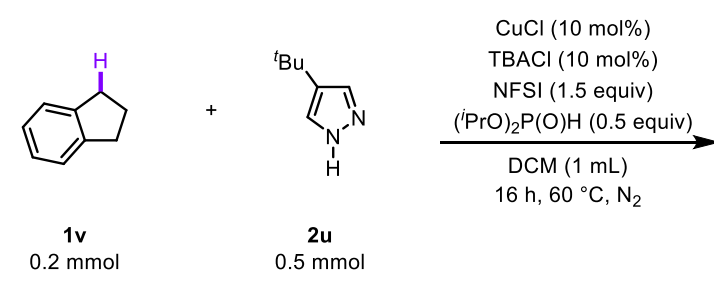

E.
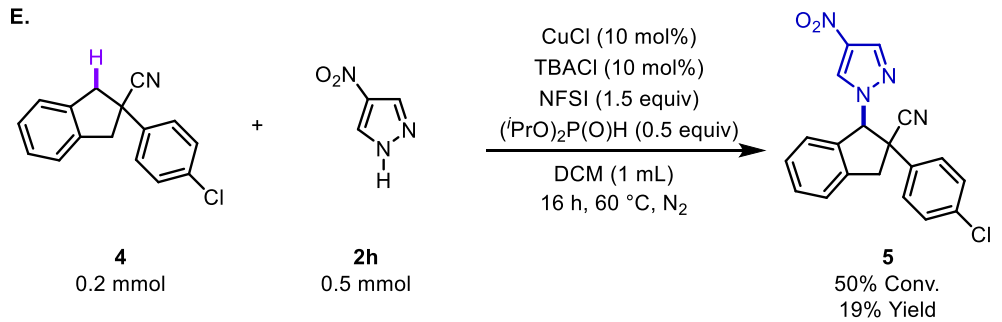

Figure S1. Screening data with additional benzylic C-H substrates and azoles. 
A selection of cross-coupling reactions of benzylic $\mathrm{C}-\mathrm{H}$ substrates and azoles were further analyzed to provide insights into the mass balance of this method.

- While TBABr has shown high selectivity for $N-2$ regioisomer in coupling reactions with $\mathbf{2 a}$, when electron-rich substrates like $\mathbf{1 h}$ were employed, formation of brominated products on the arene were observed. Replacing $\mathrm{TBABr}$ with $\mathrm{TBACl}$ avoided this side reactivity (Figure $\mathrm{S} 2 \mathrm{~A}$ ).

- Minor side products were observed where the byproduct benzenesulfonimide from NFSI was introduced to the benzylic sites, especially when cyclic substrates were engaged (Figure S2B and $\mathrm{S} 2 \mathrm{C})$.

A.

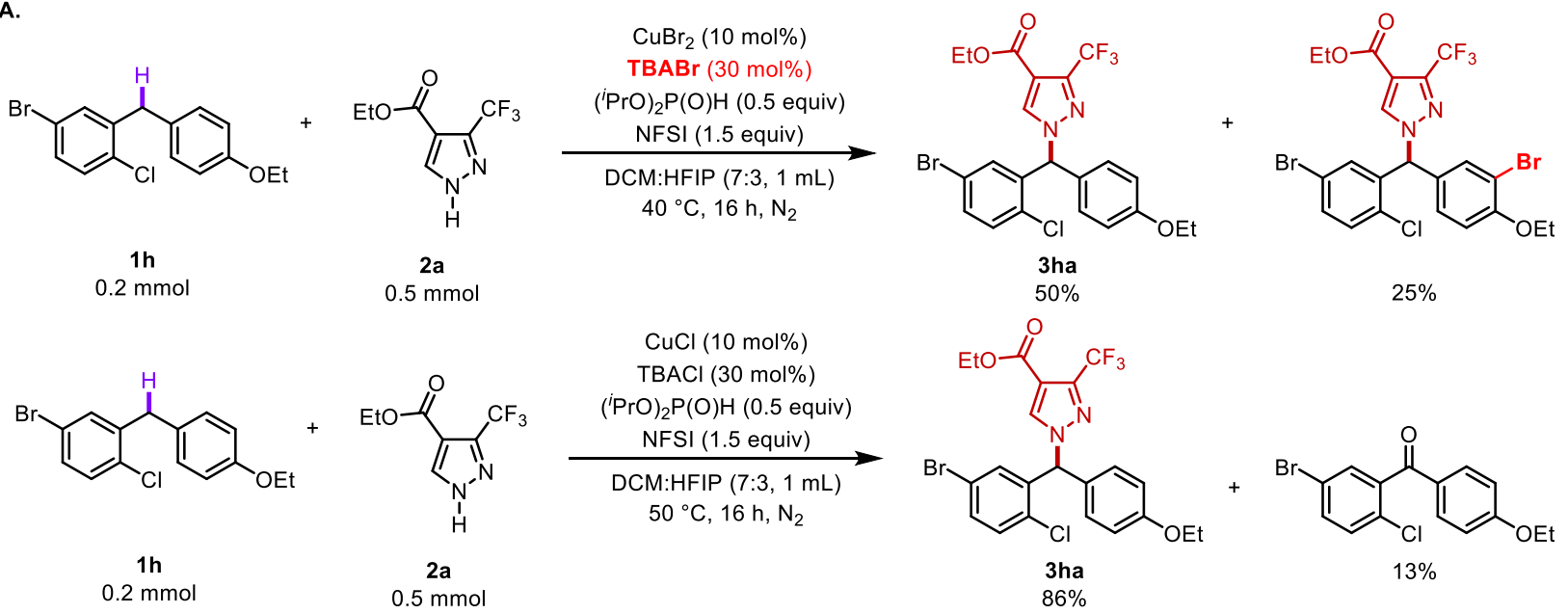

B.
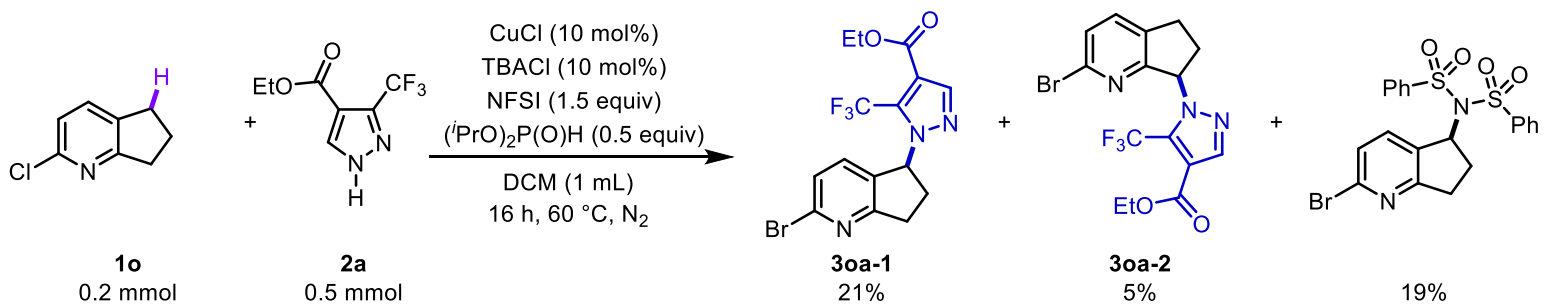

C.

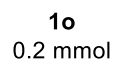

$$
\stackrel{2 a}{0.5 \mathrm{mmol}}
$$

$\mathrm{CuCl}(10 \mathrm{~mol} \%)$
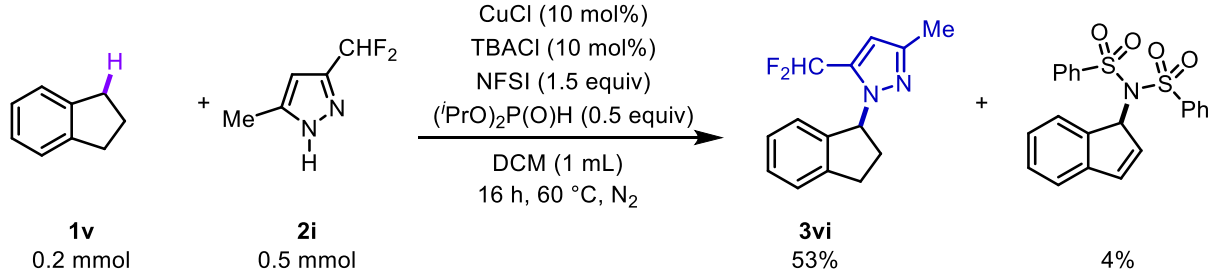

Figure S2. Further analyses of reaction outcomes of cross couplings of benzylic C-H bonds and azoles 


\section{Characterization of Regioselectivity}

\section{A. Benzylic $\mathbf{C}-\mathbf{H}$ cross coupling products with 2a}

All the $N-1$ and $N-2$ regioisomers were assigned based on 2D NMR spectra (HSQC and HMBC), along with additional characteristics in the ${ }^{13} \mathrm{C}$ and ${ }^{19} \mathrm{~F}$ NMR spectra.
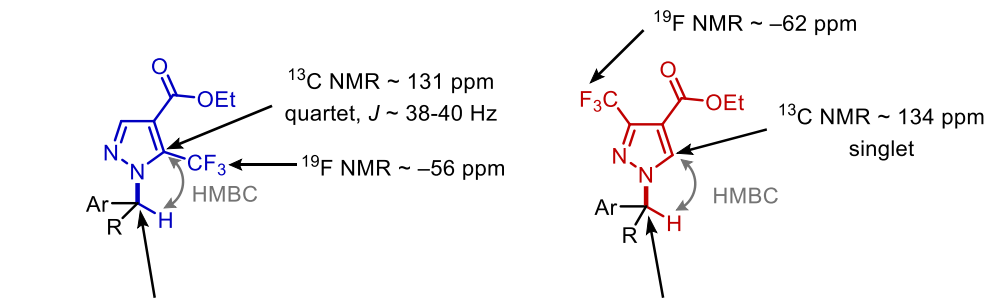

${ }^{13} \mathrm{C}$ NMR: quartet, $J \sim 3-4 \mathrm{~Hz}$

${ }^{13} \mathrm{C}$ NMR: singlet

$N^{2}$ isomer

$N^{1}$ isomer

Figure S3. Evidence for regioisomer assignment of benzylic C-H cross coupling products with 2a

\section{B. Benzylic $\mathbf{C}-\mathbf{H}$ cross coupling products with other ambidentate azoles}

Regioisomers with other azoles were assigned based on 2D NMR spectra (HSQC and HMBC), literature values and/or the number of peaks in ${ }^{13} \mathrm{C}$ NMR spectra.
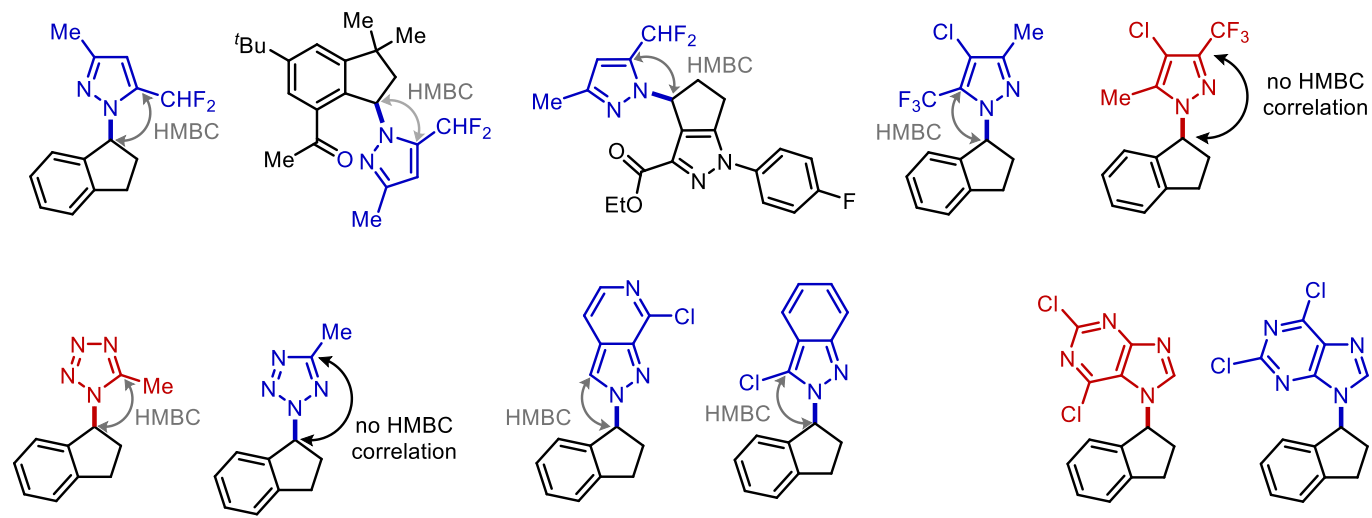

HMBC analysis

Reported chemical shift in literature, Reported chemical shift and where structures were confirmed by reactivity in literature ${ }^{2}$ X-ray diffraction of single crystals ${ }^{3}$
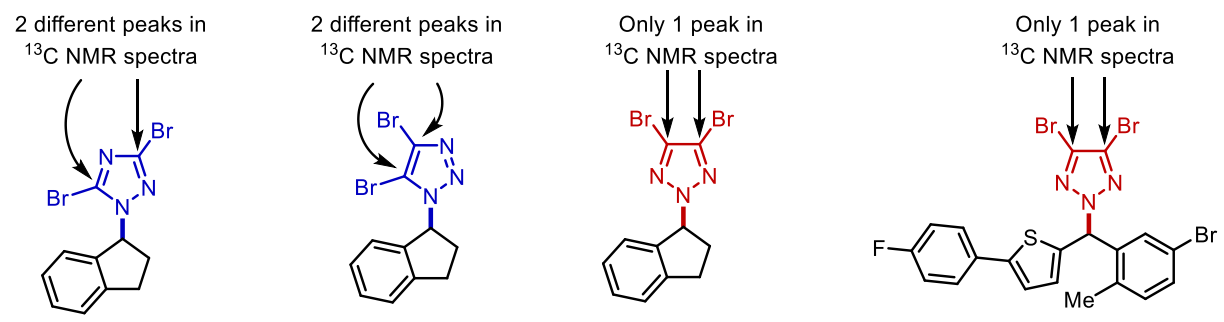

Figure S4. Proof of regioisomer assignment for benzylic $\mathrm{C}-\mathrm{H}$ cross coupling products with other ambidentate azoles 


\section{Crystallization and Crystallographic Data for $\left[\left(\mathrm{C}_{6} \mathrm{H}_{5}\right) \mathrm{N}\left(\mathrm{CH}_{3}\right)_{3}\right]_{2}[\mathrm{CuBr} 4]$ and $2 \mathrm{a}$}

\section{Preparation}

Copper(I) bromide (28.7 mg, $0.2 \mathrm{mmol}, 1.0$ equiv) and trimethylphenylammonium bromide (129.7 $\mathrm{mg}$, $0.6 \mathrm{mmol}, 3.0$ equiv) were weighed into a $4 \mathrm{~mL}$ borosilicate glass vial in a glove box under nitrogen atmosphere, followed by the addition of $1 \mathrm{~mL}$ 1,1,1,3,3,3-hexafluoroisopropanol. Nfluorobenzenesulfonimide $(31.5 \mathrm{mg}, 0.1 \mathrm{mmol}, 0.5$ equiv) was then added into the solution. The vial was then sealed with a Teflon cap and the reaction mixture was heated at $50^{\circ} \mathrm{C}$ for $30 \mathrm{~min}$. After that, 2a ( $166.5 \mathrm{mg}, 0.4 \mathrm{mmol}, 4.0$ equiv) was added into the solution. The reaction mixture was then removed from the glovebox and heated at $50^{\circ} \mathrm{C}$ on a hot plate for $16 \mathrm{~h}$ before cooling down to room temperature. The $4 \mathrm{~mL}$ glass vial was uncapped and placed in a $15 \mathrm{~mL}$ borosilicate glass vial containing pentane. The $15 \mathrm{~mL}$ glass vial was then sealed with a $15 \mathrm{~mL}$ Teflon cap to allow vapor diffusion. Two kinds of crystals were found in the glass vial: blue crystal $\left[\left(\mathrm{C}_{6} \mathrm{H}_{5}\right) \mathrm{N}\left(\mathrm{CH}_{3}\right)_{3}\right]_{2}\left[\mathrm{CuBr}_{4}\right]$ and colorless crystal $2 \mathbf{a}$.

\section{Crystallographic Data for $\left[\left(\mathrm{C}_{6} \mathrm{H}_{5}\right) \mathrm{N}\left(\mathrm{CH}_{3}\right)_{3}\right]_{2}\left[\mathrm{CuBr}_{4}\right]$} Data Collection

A blue crystal with approximate dimensions $0.14 \times 0.01 \times 0.005 \mathrm{~mm}^{3}$ was selected under oil under ambient conditions and attached to the tip of a MiTeGen MicroMount $\odot$. The crystal was mounted in a stream of cold nitrogen at 100(1) $\mathrm{K}$ and centered in the X-ray beam by using a video camera.

The crystal evaluation and data collection were performed on a Bruker Quazar SMART APEXII diffractometer with $\mathrm{Mo} \mathrm{K}_{\alpha}(\lambda=0.71073 \AA)$ radiation and a diffractometer to crystal distance of $4.96 \mathrm{~cm}^{4}$ The initial cell constants were obtained from three series of $\omega$ scans at different starting angles. Each series consisted of 12 frames collected at intervals of $0.5^{\circ}$ in a $6^{\circ}$ range about $\omega$ with the exposure time of 30 seconds per frame. The reflections were successfully indexed by an automated indexing routine built in the APEXII program suite. The final cell constants were calculated from a set of 5662 strong reflections from the actual data collection.

The data were collected by using a full sphere data collection routine to survey reciprocal space to the extent of a full sphere to a resolution of $0.80 \AA$ A. A total of 28817 data were harvested by collecting 4 sets of frames with $0.6^{\circ}$ scans in $\omega$ and $\varphi$ with exposure times of $180 \mathrm{sec}$ per frame. These highly redundant datasets were corrected for Lorentz and polarization effects. The absorption correction was based on fitting a function to the empirical transmission surface as sampled by multiple equivalent measurements. ${ }^{5}$ 


\section{Structure Solution and Refinement}

The systematic absences in the diffraction data were consistent for the space groups $P n$ and $P 2 / n$. The $E$ statistics strongly suggested the centrosymmetric space group $P 2 / n$ that yielded chemically reasonable and computationally stable results of refinement. . $^{6-11}$

A successful solution by direct methods provided most non-hydrogen atoms from the $E$-map. The remaining non-hydrogen atoms were located in an alternating series of least-squares cycles and difference Fourier maps. All non-hydrogen atoms were refined with anisotropic displacement coefficients. All hydrogen atoms were included in the structure factor calculation at idealized positions and were allowed to ride on the neighboring atoms with relative isotropic displacement coefficients.

The structure crystallizes as an inorganic salt with the formula $\left[\left(\mathrm{C}_{6} \mathrm{H}_{5}\right) \mathrm{N}\left(\mathrm{CH}_{3}\right)_{3}\right]_{2}\left[\mathrm{CuBr}_{4}\right]$. Atom $\mathrm{Br} 4$ in the $\left[\mathrm{CuBr}_{4}\right]^{2-}$ anion is disordered over two positions, with a major component occupancy of $58.5(16) \%$.

The structure also contains two trimethylaniline cations. One of these cations resides on a crystallographic general position and is fully occupied. The second cation is disordered over two different crystallographic sites. Both of these sites are crystallographic special positions corresponding to two-fold axes. Thus, this cation presents itself in the symmetry-independent unit as being $50 \%$ occupied at two different crystallographic sites and is equally disordered across the crystallographic two-fold axis at each site. This cation was refined with 1,2 and 1,3 distance restraints, as well as with atomic displacement parameter restraints and constraints.

The final least-squares refinement of 305 parameters against 4749 data resulted in residuals $R$ (based on $F^{2}$ for $I \geq 2 \sigma$ ) and $w R$ (based on $F^{2}$ for all data) of 0.0453 and 0.0962 , respectively. The final difference Fourier map was featureless.

\section{Summary}

Crystal Data for $\mathrm{C}_{18} \mathrm{H}_{28} \mathrm{Br}_{4} \mathrm{CuN}_{2}(M=655.60 \mathrm{~g} / \mathrm{mol})$ : monoclinic, space group $P 2 / n$ (no. 13), $a=$ 9.247(4) $\AA, b=8.397(3) \AA, c=29.837(10) \AA, \beta=93.608(11)^{\circ}, V=2312.2(14) \AA^{3}, Z=4, T=99.99 \mathrm{~K}$, $\mu(\mathrm{Mo} \mathrm{K} \alpha)=7.862 \mathrm{~mm}^{-1}$, Dcalc $=1.883 \mathrm{~g} / \mathrm{cm}^{3}, 28817$ reflections measured $\left(2.736^{\circ} \leq 2 \Theta \leq 52.85^{\circ}\right), 4749$ unique $\left(R_{\text {int }}=0.0705, \mathrm{R}_{\text {sigma }}=0.0545\right)$ which were used in all calculations. The final $R_{1}$ was 0.0453 (I > $2 \sigma(\mathrm{I}))$ and $w R_{2}$ was 0.0962 (all data). 


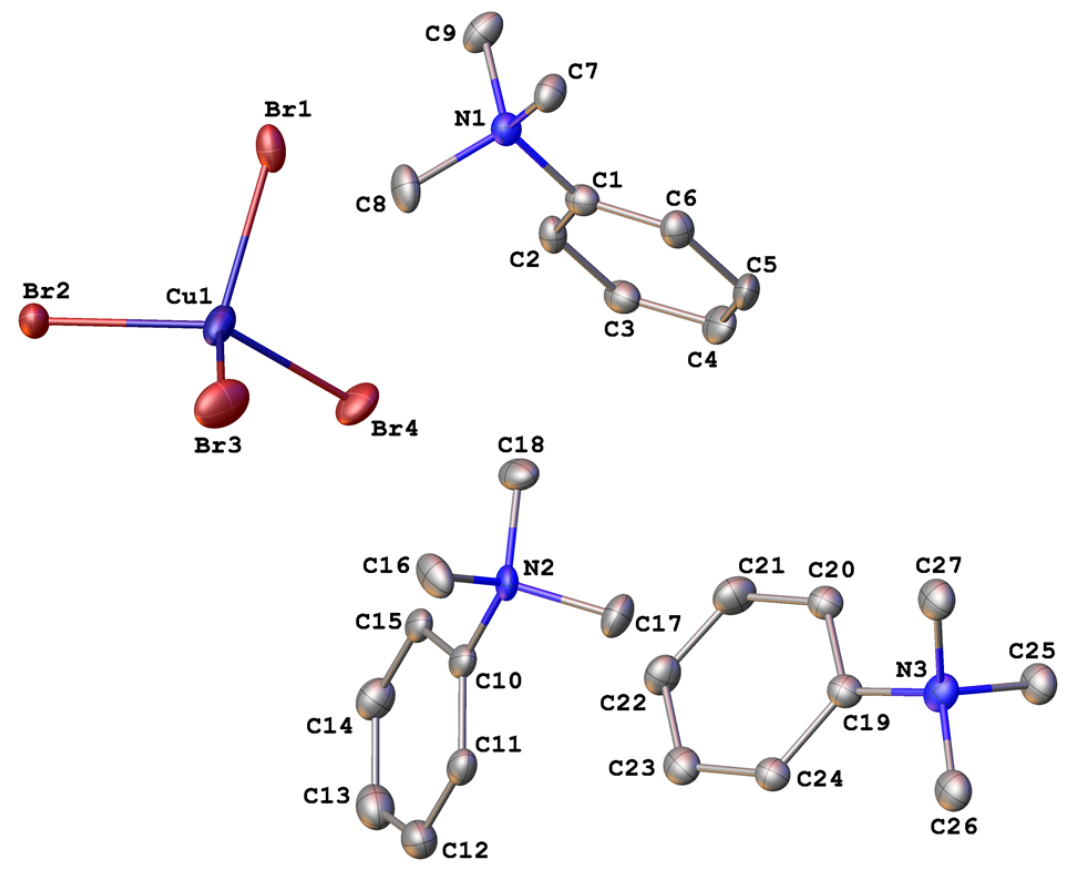

Figure S5. A molecular drawing of $\left[\left(\mathrm{C}_{6} \mathrm{H}_{5}\right) \mathrm{N}\left(\mathrm{CH}_{3}\right)_{3}\right]_{2}\left[\mathrm{CuBr}_{4}\right]$. The $\mathrm{N}, \mathrm{N}, \mathrm{N}$-trimethylanilinium cations containing atoms $\mathrm{N} 2$ and N3 are 50\% occupied. All atoms are shown with 50\% probability ellipsoids. All $\mathrm{H}$ atoms and minor disorder components are omitted. 
Table S5. Crystal data and structure refinement for $\left[\left(\mathrm{C}_{6} \mathrm{H}_{5}\right) \mathbf{N}\left(\mathrm{CH}_{3}\right)_{3}\right]_{2}\left[\mathrm{CuBr}_{4}\right]$.

Empirical formula

Formula weight

Temperature/K

Crystal system

Space group

$\mathrm{a} / \AA ̊$

$\mathrm{b} / \AA$

$\mathrm{c} / \AA$

$\alpha /{ }^{\circ}$

$\beta /{ }^{\circ}$

$\gamma /{ }^{\circ}$

Volume $/ \AA^{3}$

Z

$\rho_{\text {calc }} \mathrm{g} / \mathrm{cm}^{3}$

$\mu / \mathrm{mm}^{-1}$

$\mathrm{F}(000)$

Crystal size $/ \mathrm{mm}^{3}$

Radiation

$2 \Theta$ range for data collection/ ${ }^{\circ}$

Index ranges

Reflections collected

Independent reflections

Data/restraints/parameters

Goodness-of-fit on $\mathrm{F}^{2}$

Final $\mathrm{R}$ indexes $[\mathrm{I}>=2 \sigma(\mathrm{I})]$

Final $R$ indexes [all data]

Largest diff. peak/hole / e $\AA^{-3}$
$\left[\left(\mathrm{C}_{6} \mathrm{H}_{5}\right) \mathrm{N}\left(\mathrm{CH}_{3}\right)_{3}\right]_{2}\left[\mathrm{CuBr}_{4}\right]$

655.60

99.99

monoclinic

$P 2 / n$

$9.247(4)$

$8.397(3)$

29.837(10)

90

93.608(11)

90

2312.2(14)

4

1.883

7.862

1276.0

$0.14 \times 0.01 \times 0.005$

Mo Ka $(\lambda=0.71073)$

2.736 to 52.85

$-11 \leq \mathrm{h} \leq 11,-10 \leq \mathrm{k} \leq 9,-37 \leq 1 \leq 35$

28817

$4749\left[R_{\text {int }}=0.0705, R_{\text {sigma }}=0.0545\right]$

4749/95/305

1.067

$\mathrm{R}_{1}=0.0453, \mathrm{wR}_{2}=0.0879$

$\mathrm{R}_{1}=0.0727, \mathrm{wR}_{2}=0.0962$

$0.70 /-1.02$ 


\section{Crystallographic Data for 2a \\ Data Collection}

A colorless crystal with approximate dimensions $0.50 \times 0.40 \times 0.40 \mathrm{~mm}^{3}$ was selected under oil under ambient conditions and attached to the tip of a MiTeGen MicroMount $\odot$. The crystal was mounted in a stream of cold nitrogen at $100(1) \mathrm{K}$ and centered in the X-ray beam by using a video camera.

The crystal evaluation and data collection were performed on a Bruker SMART APEXII diffractometer with $\mathrm{Cu} \mathrm{K} \alpha=1.54178 \AA)$ radiation and the diffractometer to crystal distance of $4.03 \mathrm{~cm}{ }^{4}$

The initial cell constants were obtained from three series of $\omega$ scans at different starting angles. Each series consisted of 50 frames collected at intervals of $0.5^{\circ}$ in a $25^{\circ}$ range about $\omega$ with an exposure time of 10 seconds per frame. The reflections were successfully indexed by an automated indexing routine built in the APEX3 program. The final cell constants were calculated from a set of 2234 strong reflections from the actual data collection.

The data were collected by using a full sphere data collection routine to survey reciprocal space to the extent of a full sphere to a resolution of $0.81 \AA$. A total of 3882 data were harvested by collecting 9 sets of frames with $0.6^{\circ}$ scans in $\omega$ and $\varphi$ with an exposure time 5-20 sec per frame. These highly redundant datasets were corrected for Lorentz and polarization effects. The absorption correction was based on fitting a function to the empirical transmission surface as sampled by multiple equivalent measurements. ${ }^{5}$

\section{Structure Solution and Refinement}

The systematic absences in the diffraction data were consistent for the space groups $P 2{ }_{1} / m$ and $P 2_{1}$. The $E$-statistics strongly suggested the centrosymmetric space group $P 2_{1} / m$ that yielded chemically reasonable and computationally stable results of refinement. . $^{6-11}$

A successful solution by direct methods provided most non-hydrogen atoms from the $E$-map. The remaining non-hydrogen atoms were located in an alternating series of least-squares cycles and difference Fourier maps. All non-hydrogen atoms were refined with anisotropic displacement coefficients.

The molecule resides on a crystallographic mirror plane.

The final least-squares refinement of 99 parameters against 912 data resulted in residuals $R$ (based on $F^{2}$ for $I \geq 2 \sigma$ ) and $w R$ (based on $F^{2}$ for all data) of 0.0305 and 0.0814 , respectively. The final difference Fourier map was featureless.

\section{Summary}

Crystal Data for $\mathrm{C}_{7} \mathrm{H}_{7} \mathrm{~F}_{3} \mathrm{~N}_{2} \mathrm{O}_{2}(M=208.15 \mathrm{~g} / \mathrm{mol})$ : monoclinic, space group $P 2_{1} / m$ (no. 11), $a=$

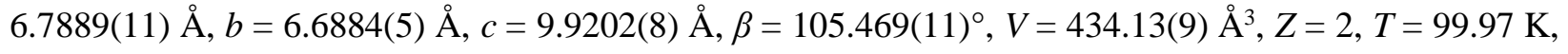
$\mu(\mathrm{Cu} \mathrm{K} \alpha)=1.420 \mathrm{~mm}^{-1}$, Dcalc $=1.592 \mathrm{~g} / \mathrm{cm}^{3}, 3882$ reflections measured $\left(9.25^{\circ} \leq 2 \Theta \leq 144.162^{\circ}\right), 912$ unique $\left(R_{\text {int }}=0.0278, \mathrm{R}_{\text {sigma }}=0.0202\right)$ which were used in all calculations. The final $R_{1}$ was 0.0305 (I > $2 \sigma(\mathrm{I}))$ and $w R_{2}$ was 0.0814 (all data). 


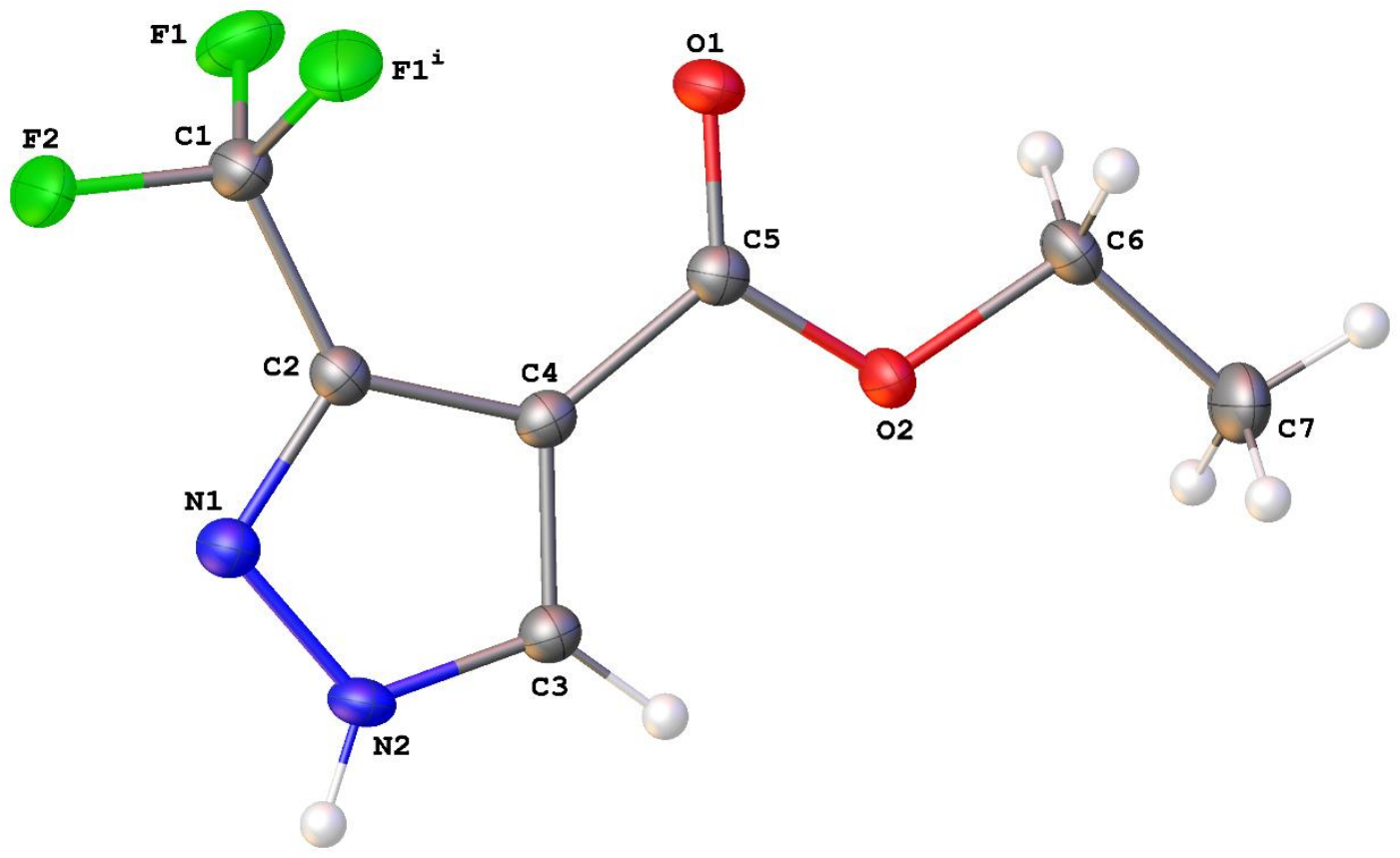

Figure S6. A molecular drawing of 2a shown with $50 \%$ probability ellipsoids for non-hydrogen atoms. [Symmetry code: (i) $x, 3 / 2-y, z$.] 
Table S6. Crystal data and structure refinement for 2a.

Empirical formula

Formula weight

Temperature/K

Crystal system

Space group

$\mathrm{a} / \AA$

$\mathrm{b} / \AA$

$\mathrm{c} / \AA$

$\alpha /{ }^{\circ}$

$\beta /{ }^{\circ}$

$\gamma /{ }^{\circ}$

Volume $/ \AA^{3}$

Z

$\rho_{\text {calc }} \mathrm{g} / \mathrm{cm}^{3}$

$\mu / \mathrm{mm}^{-1}$

$\mathrm{F}(000)$

Crystal size $/ \mathrm{mm}^{3}$

Radiation
$\mathrm{C}_{7} \mathrm{H}_{7} \mathrm{~F}_{3} \mathrm{~N}_{2} \mathrm{O}_{2}$

208.15

99.97

monoclinic

$P 2_{1} / m$

$6.7889(11)$

$6.6884(5)$

$9.9202(8)$

90

105.469(11)

90

434.13(9)

2

1.592

1.420

212.0

$0.5 \times 0.4 \times 0.4$

$\mathrm{Cu} \mathrm{K \alpha}(\lambda=1.54178)$

$2 \Theta$ range for data collection $/{ }^{\circ} 9.25$ to 144.162

Index ranges $\quad-7 \leq \mathrm{h} \leq 8,-8 \leq \mathrm{k} \leq 8,-12 \leq 1 \leq 12$

Reflections collected $\quad 3882$

Independent reflections $\quad 912\left[\mathrm{R}_{\text {int }}=0.0278, \mathrm{R}_{\text {sigma }}=0.0202\right]$

Data/restraints/parameters $\quad 912 / 0 / 99$

Goodness-of-fit on $\mathrm{F}^{2} \quad 1.073$

Final $R$ indexes $[I>=2 \sigma(I)] \quad R_{1}=0.0305, w_{2}=0.0788$

Final $\mathrm{R}$ indexes [all data] $\quad \mathrm{R}_{1}=0.0343, \mathrm{wR}_{2}=0.0814$

Largest diff. peak/hole / e $\AA^{-3}$ 0.28/-0.27 


\section{Characterization of Compounds}<smiles>CCOC(=O)c1cnn(C(C)c2ccccc2)c1C(F)(F)F</smiles>

Ethyl 1-(1-phenylethyl)-5-(trifluoromethyl)-1H-pyrazole-4-carboxylate, 3aa

Reaction run using ethylbenzene 1a $(24.5 \mu \mathrm{L}, 0.2 \mathrm{mmol}, 1.0$ equiv), ethyl 3-(trifluoromethyl)-1Hpyrazole-4-carboxylate $\mathbf{2 a}(104.1 \mathrm{mg}, 0.50 \mathrm{mmol}, 2.5$ equiv), and tetrabutylammonium chloride (5.6 $\mathrm{mg}$, $0.02 \mathrm{mmol}, 0.1$ equiv) following the general procedure I (pressure tube). Yield $=47.3 \mathrm{mg}(76 \%)$ of paleyellow liquid. TLC (pentane:EtOAc, 9:1 v/v): $R_{\mathrm{f}}=0.71$.

${ }^{1} \mathrm{H}$ NMR $\left(\mathrm{CDCl}_{3}, 500 \mathrm{MHz}\right): 7.99(\mathrm{~s}, 1 \mathrm{H}), 7.40-7.19(\mathrm{~m}, 5 \mathrm{H}), 5.79$ (q, $\left.J=6.9 \mathrm{~Hz}, 1 \mathrm{H}\right), 4.31$ (q, $J=7.1$

$\mathrm{Hz}, 2 \mathrm{H}), 1.94(\mathrm{~d}, J=6.9 \mathrm{~Hz}, 3 \mathrm{H}), 1.33(\mathrm{t}, J=7.1 \mathrm{~Hz}, 3 \mathrm{H}) \mathrm{ppm}$.

${ }^{13} \mathrm{C} \mathrm{NMR}\left(\mathrm{CDCl}_{3}, 126 \mathrm{MHz}\right): 161.2,141.5,140.5,131.4(\mathrm{q}, J=40.1 \mathrm{~Hz}), 128.7,128.1,126.3,119.7(\mathrm{q}, J$ $=271.2 \mathrm{~Hz}), 115.8(\mathrm{q}, J=1.5 \mathrm{~Hz}), 61.1,61.0(\mathrm{q}, J=3.6 \mathrm{~Hz}), 22.1,14.1 \mathrm{ppm}$.

${ }^{19} \mathrm{~F} \mathrm{NMR}\left(\mathrm{CDCl}_{3}, 377 \mathrm{MHz}\right):-55.9 \mathrm{ppm}$.

HRMS Calculated for $\left[\mathrm{C}_{15} \mathrm{H}_{15} \mathrm{~F}_{3} \mathrm{~N}_{2} \mathrm{O}_{2}+\mathrm{H}\right]^{+}: 313.1158$, Found: 313.1153 .<smiles>CCOC(=O)c1cn(C(C)c2ccccc2)nc1C(F)(F)F</smiles>

Ethyl 1-(1-phenylethyl)-3-(trifluoromethyl)-1H-pyrazole-4-carboxylate, 3aa'

Reaction run using ethylbenzene 1a $(24.5 \mu \mathrm{L}, 0.2 \mathrm{mmol}, 1.0$ equiv), ethyl 3-(trifluoromethyl)-1Hpyrazole-4-carboxylate $2 \mathbf{a}$ (104.1 mg, $0.50 \mathrm{mmol}, 2.5$ equiv), and TMSOTf (3.6 $\mu \mathrm{L}, 0.02 \mathrm{mmol}, 0.1 \mathrm{equiv}$ ) following the general procedure I (pressure tube). Yield $=39.6 \mathrm{mg}(63 \%)$ of clear colorless liquid. TLC (pentane:EtOAc, 9:1 v/v): $\mathrm{R}_{\mathrm{f}}=0.38$.

${ }^{1} \mathrm{H}$ NMR $\left(\mathrm{CDCl}_{3}, 500 \mathrm{MHz}\right): 7.91(\mathrm{~s}, 1 \mathrm{H}), 7.42-7.32(\mathrm{~m}, 3 \mathrm{H}), 7.28-7.26(\mathrm{~m}, 2 \mathrm{H}), 5.56(\mathrm{q}, J=7.1 \mathrm{~Hz}$, $1 \mathrm{H}), 4.29(\mathrm{q}, J=7.1 \mathrm{~Hz}, 2 \mathrm{H}), 1.92(\mathrm{~d}, J=7.1 \mathrm{~Hz}, 3 \mathrm{H}), 1.32(\mathrm{t}, J=7.1 \mathrm{~Hz}, 3 \mathrm{H}) \mathrm{ppm}$.

${ }^{13} \mathrm{C} \mathrm{NMR}\left(\mathrm{CDCl}_{3}, 126 \mathrm{MHz}\right): 161.0,141.2(\mathrm{q}, J=38.3 \mathrm{~Hz}), 139.4,134.1,129.1,128.8,126.7,120.5(\mathrm{q}, J$ $=269.6 \mathrm{~Hz}), 113.2,62.6,61.0,21.1,14.1 \mathrm{ppm}$.

${ }^{19} \mathrm{~F} \mathrm{NMR}\left(\mathrm{CDCl}_{3}, 377 \mathrm{MHz}\right):-61.9 \mathrm{ppm}$.

HRMS Calculated for $\left[\mathrm{C}_{15} \mathrm{H}_{15} \mathrm{~F}_{3} \mathrm{~N}_{2} \mathrm{O}_{2}+\mathrm{H}\right]^{+}: 313.1158$, Found: 313.1157. 
<smiles>CCOC(=O)c1cn(C(C)c2ccc(OC)cc2)nc1C(F)(F)F</smiles>

Ethyl 1-(1-(4-methoxyphenyl)ethyl)-3-(trifluoromethyl)-1H-pyrazole-4-carboxylate, 3ba'

Reaction run using 4-ethylanisole $\mathbf{1 b}(29.3 \mu \mathrm{L}, 0.2 \mathrm{mmol}, 1.0$ equiv), ethyl 3-(trifluoromethyl)-1Hpyrazole-4-carboxylate $\mathbf{2 a}(104.1 \mathrm{mg}, 0.50 \mathrm{mmol}, 2.5$ equiv), and trimethylsilyl triflate $(3.6 \mu \mathrm{L}, 0.02$ mmol, 0.1 equiv) at $60^{\circ} \mathrm{C}$ following the general procedure I (pressure tube). Yield $=54.7 \mathrm{mg}(80 \%)$ of clear colorless liquid. TLC (pentane:EtOAc, 9:1 v/v): $\mathrm{R}_{\mathrm{f}}=0.42$.

${ }^{1} \mathrm{H} \mathrm{NMR}\left(\mathrm{CDCl}_{3}, 500 \mathrm{MHz}\right): 7.84(\mathrm{~s}, 1 \mathrm{H}), 7.26-7.20(\mathrm{~m}, 2 \mathrm{H}), 6.94-6.86(\mathrm{~m}, 2 \mathrm{H}), 5.51$ (q, J = 7.0 Hz,

$1 \mathrm{H}), 4.28(\mathrm{q}, J=7.1 \mathrm{~Hz}, 2 \mathrm{H}), 3.81(\mathrm{~s}, 3 \mathrm{H}), 1.89(\mathrm{~d}, J=7.0 \mathrm{~Hz}, 3 \mathrm{H}), 1.32(\mathrm{t}, J=7.1 \mathrm{~Hz}, 3 \mathrm{H}) \mathrm{ppm}$.

${ }^{13} \mathrm{C} \mathrm{NMR}\left(\mathrm{CDCl}_{3}, 126 \mathrm{MHz}\right): 161.0,159.8,141.1(\mathrm{q}, J=38.4 \mathrm{~Hz}), 133.8,131.1,128.1,120.5(\mathrm{q}, J=$ $269.6 \mathrm{~Hz}), 114.4,113.0,62.1,60.8,55.3,21.1,14.1 \mathrm{ppm}$.

${ }^{19} \mathrm{~F} \mathrm{NMR}\left(\mathrm{CDCl}_{3}, 377 \mathrm{MHz}\right)$ : $-61.9 \mathrm{ppm}$.

HRMS Calculated for $\left[\mathrm{C}_{16} \mathrm{H}_{17} \mathrm{~F}_{3} \mathrm{~N}_{2} \mathrm{O}_{3}+\mathrm{Na}\right]^{+}:$365.1083, Found: 365.1089 .<smiles>CCOC(=O)c1cnn(C(C)c2ccc(Br)cc2)c1C(F)(F)F</smiles>

Ethyl 1-(1-(4-bromophenyl)ethyl)-5-(trifluoromethyl)-1H-pyrazole-4-carboxylate, 3ca

Reaction run using 4-bromoethylbenzene $1 \mathrm{c}(27.5 \mu \mathrm{L}, 0.2 \mathrm{mmol}, 1.0$ equiv), ethyl 3-(trifluoromethyl)1H-pyrazole-4-carboxylate $\mathbf{2 a}(104.1 \mathrm{mg}, 0.50 \mathrm{mmol}, 2.5$ equiv), and tetrabutylammonium chloride (5.6 $\mathrm{mg}, 0.02 \mathrm{mmol}, 0.1$ equiv) following the general procedure I (pressure tube). Yield $=57.6 \mathrm{mg}(74 \%)$ of clear colorless liquid. TLC (pentane:EtOAc, 9:1 v/v): $\mathrm{R}_{\mathrm{f}}=0.61$.

${ }^{1} \mathrm{H}$ NMR $\left(\mathrm{CDCl}_{3}, 500 \mathrm{MHz}\right): 7.98(\mathrm{~s}, 1 \mathrm{H}), 7.52-7.39$ (m, 2H), $7.24-7.05$ (m, 2H), 5.73 (q, J = 6.9 Hz, $1 \mathrm{H}), 4.31(\mathrm{q}, J=7.1 \mathrm{~Hz}, 2 \mathrm{H}), 1.92(\mathrm{~d}, J=6.9 \mathrm{~Hz}, 3 \mathrm{H}), 1.34(\mathrm{t}, J=7.1 \mathrm{~Hz}, 3 \mathrm{H}) \mathrm{ppm}$.

${ }^{13} \mathrm{C} \mathrm{NMR}\left(\mathrm{CDCl}_{3}, 126 \mathrm{MHz}\right): 161.1,141.7,139.5,131.9,131.4(\mathrm{q}, J=39.9 \mathrm{~Hz}), 128.2,122.3,119.6(\mathrm{q}, J$ $=271.3 \mathrm{~Hz}), 115.9(\mathrm{q}, J=1.6 \mathrm{~Hz}), 61.2,60.5(\mathrm{q}, J=3.4 \mathrm{~Hz}), 22.1,14.1 \mathrm{ppm}$.

${ }^{19} \mathrm{~F} \mathrm{NMR}\left(\mathrm{CDCl}_{3}, 377 \mathrm{MHz}\right):-55.9 \mathrm{ppm}$.

HRMS Calculated for $\left[\mathrm{C}_{15} \mathrm{H}_{14} \mathrm{BrF}_{3} \mathrm{~N}_{2} \mathrm{O}_{2}+\mathrm{H}\right]^{+}:$391.0264, Found: 391.0257. 


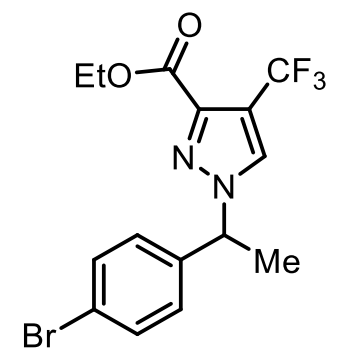

Ethyl 1-(1-(4-bromophenyl)ethyl)-5-(trifluoromethyl)-1H-pyrazole-4-carboxylate, 3ca'

Reaction run using 4-bromoethylbenzene 1c $(27.5 \mu \mathrm{L}, 0.2 \mathrm{mmol}, 1.0$ equiv), ethyl 3-(trifluoromethyl)1H-pyrazole-4-carboxylate 2a (104.1 mg, $0.50 \mathrm{mmol}, 2.5$ equiv), and trimethylsilyl triflate $(3.6 \mu \mathrm{L}, 0.02$ mmol, 0.1 equiv) at $60^{\circ} \mathrm{C}$ following the general procedure $\mathrm{I}$ (pressure tube). Yield $=78.2 \mathrm{mg}(35 \%)$ of pale-yellow semisolid. TLC (pentane:EtOAc, 9:1 v/v): $\mathrm{R}_{\mathrm{f}}=0.31 . \mathrm{Mp}: 89-90{ }^{\circ} \mathrm{C}$

${ }^{1} \mathrm{H}$ NMR $\left(\mathrm{CDCl}_{3}, 500 \mathrm{MHz}\right): 7.92(\mathrm{~s}, 1 \mathrm{H}), 7.71-7.41(\mathrm{~m}, 2 \mathrm{H}), 7.18-7.11(\mathrm{~m}, 2 \mathrm{H}), 5.51(\mathrm{q}, J=7.1 \mathrm{~Hz}$, $1 \mathrm{H}), 4.30(\mathrm{q}, J=7.1 \mathrm{~Hz}, 2 \mathrm{H}), 1.90(\mathrm{~d}, J=7.1 \mathrm{~Hz}, 3 \mathrm{H}), 1.33(\mathrm{t}, J=7.1 \mathrm{~Hz}, 3 \mathrm{H}) \mathrm{ppm}$.

${ }^{13} \mathrm{C} \mathrm{NMR}\left(\mathrm{CDCl}_{3}, 126 \mathrm{MHz}\right): 160.9,141.4(\mathrm{q}, J=38.6 \mathrm{~Hz}), 138.5,134.0,132.3,128.3,122.8,120.4(\mathrm{q}, J$ $=269.7 \mathrm{~Hz}), 113.4(\mathrm{~d}, J=1.2 \mathrm{~Hz}), 62.0,61.0,21.0,14.1 \mathrm{ppm}$.

${ }^{19} \mathrm{~F} \mathrm{NMR}\left(\mathrm{CDCl}_{3}, 377 \mathrm{MHz}\right)$ : $-61.94 \mathrm{ppm}$.

HRMS Calculated for $\left[\mathrm{C}_{15} \mathrm{H}_{14} \mathrm{BrF}_{3} \mathrm{~N}_{2} \mathrm{O}_{2}+\mathrm{Na}\right]^{+}$: 413.0083, Found: 413.0083.<smiles>CCOC(=O)c1cnn(C(C)c2ccc(OC(C)=O)cc2)c1C(F)(F)F</smiles>

Ethyl 1-(1-(4-acetoxyphenyl)ethyl)-5-(trifluoromethyl)-1H-pyrazole-4-carboxylate, 3da

Reaction run using 4-ethylphenyl acetate $1 \mathbf{d}(32.0 \mu \mathrm{L}, 0.2 \mathrm{mmol}, 1.0$ equiv), ethyl 3-(trifluoromethyl)1H-pyrazole-4-carboxylate $\mathbf{2 a}(104.1 \mathrm{mg}, 0.50 \mathrm{mmol}, 2.5$ equiv), and tetrabutylammonium chloride (5.6 $\mathrm{mg}, 0.02 \mathrm{mmol}, 0.1$ equiv) following the general procedure I (pressure tube). Yield $=51.5 \mathrm{mg}(70 \%)$ of brown solid. TLC (pentane:EtOAc, 9:1 v/v): $\mathrm{R}_{\mathrm{f}}=0.33 . \mathrm{Mp}: 88-90{ }^{\circ} \mathrm{C}$

${ }^{1} \mathrm{H}$ NMR $\left(\mathrm{CDCl}_{3}, 500 \mathrm{MHz}\right): 7.98(\mathrm{~s}, 1 \mathrm{H}), 7.34-7.28(\mathrm{~m}, 2 \mathrm{H}), 7.10-7.01$ (m, 2H), 5.77 (q, J = 6.9 Hz, $1 \mathrm{H}), 4.31(\mathrm{q}, J=7.1 \mathrm{~Hz}, 2 \mathrm{H}), 2.28(\mathrm{~s}, 3 \mathrm{H}), 1.93(\mathrm{~d}, J=6.9 \mathrm{~Hz}, 3 \mathrm{H}), 1.34(\mathrm{t}, J=7.1 \mathrm{~Hz}, 3 \mathrm{H}) \mathrm{ppm}$.

${ }^{13} \mathrm{C}$ NMR $\left(\mathrm{CDCl}_{3}, 126 \mathrm{MHz}\right): 169.3,161.1,150.4,141.6,137.9,131.3(\mathrm{q}, J=40.0 \mathrm{~Hz}), 127.7,121.8$, $119.6(\mathrm{q}, J=271.2 \mathrm{~Hz}), 115.8(\mathrm{q}, J=1.6 \mathrm{~Hz}), 61.1,60.5(\mathrm{q}, J=3.4 \mathrm{~Hz}), 22.2,21.1,14.1 \mathrm{ppm}$.

${ }^{19} \mathrm{~F} \mathrm{NMR}\left(\mathrm{CDCl}_{3}, 377 \mathrm{MHz}\right):-55.8 \mathrm{ppm}$.

HRMS Calculated for $\left[\mathrm{C}_{17} \mathrm{H}_{17} \mathrm{~F}_{3} \mathrm{~N}_{2} \mathrm{O}_{4}+\mathrm{H}\right]^{+}: 371.1213$, Found: 371.1218 . 
<smiles>CCOC(=O)c1cnn(C(c2ccccc2)C(C)C)c1C(F)(F)F</smiles>

Ethyl 1-(2-methyl-1-phenylpropyl)-5-(trifluoromethyl)-1H-pyrazole-4-carboxylat, 3ea Reaction run using isobutylbenzene $1 \mathrm{e}(31.4 \mu \mathrm{L}, 0.2 \mathrm{mmol}, 1.0$ equiv), ethyl 3-(trifluoromethyl)-1Hpyrazole-4-carboxylate $2 \mathrm{a}(104.1 \mathrm{mg}, 0.50 \mathrm{mmol}, 2.5$ equiv), and tetrabutylammonium chloride (5.6 $\mathrm{mg}$, $0.02 \mathrm{mmol}, 0.1$ equiv) following the general procedure I (pressure tube). Yield $=45.8 \mathrm{mg}(67 \%)$ of yellow liquid. TLC (pentane:EtOAc, 9:1 v/v): $\mathrm{R}_{\mathrm{f}}=0.47$.

${ }^{1} \mathrm{H}$ NMR $\left(\mathrm{CDCl}_{3}, 400 \mathrm{MHz}\right): 7.98$ (s, 1H), 7.57 - 7.46 (m, 2H), $7.41-7.26(\mathrm{~m}, 3 \mathrm{H}), 4.96$ (d, J = 10.6 Hz,

$1 \mathrm{H}), 4.29$ (q, $J=7.1 \mathrm{~Hz}, 2 \mathrm{H}), 2.94(\mathrm{dh}, J=12.6,6.4,5.8 \mathrm{~Hz}, 1 \mathrm{H}), 1.33(\mathrm{t}, J=7.1 \mathrm{~Hz}, 4 \mathrm{H}), 0.82(\mathrm{~d}, J$ $=2.3 \mathrm{~Hz}, 3 \mathrm{H}), 0.80(\mathrm{~d}, J=2.0 \mathrm{~Hz}, 3 \mathrm{H}) \mathrm{ppm}$.

${ }^{13} \mathrm{C} \mathrm{NMR}\left(\mathrm{CDCl}_{3}, 126 \mathrm{MHz}\right): 161.3,141.7,138.1,131.9(\mathrm{q}, J=39.6 \mathrm{~Hz}), 128.5,128.4,128.2,119.7$ (q, $J$ $=271.3 \mathrm{~Hz}), 115.1(\mathrm{q}, J=1.5 \mathrm{~Hz}), 72.7(\mathrm{q}, J=3.0 \mathrm{~Hz}), 61.1,33.6,19.9,19.7,14.1 \mathrm{ppm}$.

${ }^{19} \mathrm{~F} \mathrm{NMR}\left(\mathrm{CDCl}_{3}, 377 \mathrm{MHz}\right):-54.9 \mathrm{ppm}$.

HRMS Calculated for $\left[\mathrm{C}_{17} \mathrm{H}_{19} \mathrm{~F}_{3} \mathrm{~N}_{2} \mathrm{O}_{2}+\mathrm{H}\right]^{+}: 341.1471$, Found: 341.1470 .

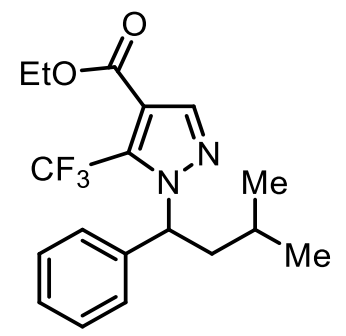

Ethyl 1-(3-methyl-1-phenylbutyl)-5-(trifluoromethyl)-1H-pyrazole-4-carboxylate, 3fa

Reaction run using isopentylbenzene 1f $(34.7 \mu \mathrm{L}, 0.2 \mathrm{mmol}, 1.0$ equiv), ethyl 3-(trifluoromethyl)-1Hpyrazole-4-carboxylate $2 \mathbf{a}(104.1 \mathrm{mg}, 0.50 \mathrm{mmol}, 2.5$ equiv), and tetrabutylammonium chloride (5.6 $\mathrm{mg}$, $0.02 \mathrm{mmol}, 0.1$ equiv) following the general procedure I (pressure tube). Yield $=42.1 \mathrm{mg}(59 \%)$ of paleyellow semisolid. TLC (pentane:EtOAc, 9:1 v/v): $\mathrm{R}_{\mathrm{f}}=0.67$.

${ }^{1} \mathrm{H}$ NMR $\left(\mathrm{CDCl}_{3}, 500 \mathrm{MHz}\right): 7.99(\mathrm{~s}, 1 \mathrm{H}), 7.38-7.25(\mathrm{~m}, 5 \mathrm{H}), 5.63(\mathrm{dd}, J=9.1,6.0 \mathrm{~Hz}, 1 \mathrm{H}), 4.30(\mathrm{q}, J$ $=7.1 \mathrm{~Hz}, 2 \mathrm{H}), 2.50(\mathrm{ddd}, J=14.5,9.1,5.8 \mathrm{~Hz}, 1 \mathrm{H}), 2.00(\mathrm{ddd}, J=14.0,7.9,6.0 \mathrm{~Hz}, 1 \mathrm{H}), 1.42-1.36$ $(\mathrm{m}, 1 \mathrm{H}), 1.33(\mathrm{t}, J=7.1 \mathrm{~Hz}, 3 \mathrm{H}), 0.95(\mathrm{~d}, J=6.6 \mathrm{~Hz}, 3 \mathrm{H}), 0.92(\mathrm{~d}, J=6.6 \mathrm{~Hz}, 3 \mathrm{H}) \mathrm{ppm}$.

${ }^{13} \mathrm{C} \mathrm{NMR}\left(\mathrm{CDCl}_{3}, 126 \mathrm{MHz}\right): 141.5,139.7,131.72(\mathrm{q}, J=39.7 \mathrm{~Hz}), 128.7,128.2,127.0,119.7(\mathrm{q}, J=$ $271.3 \mathrm{~Hz}), 115.6(\mathrm{q}, J=1.5 \mathrm{~Hz}), 63.9(\mathrm{q}, J=3.1 \mathrm{~Hz}), 61.1,45.1,29.7,24.9,22.5,21.9,14.1 \mathrm{ppm}$.

${ }^{19} \mathrm{~F} \mathrm{NMR}\left(\mathrm{CDCl}_{3}, 377 \mathrm{MHz}\right)$ : $-55.6 \mathrm{ppm}$.

HRMS Calculated for $\left[\mathrm{C}_{18} \mathrm{H}_{21} \mathrm{~F}_{3} \mathrm{~N}_{2} \mathrm{O}_{2}+\mathrm{Na}\right]^{+}:$377.1447, Found: 377.1444. 


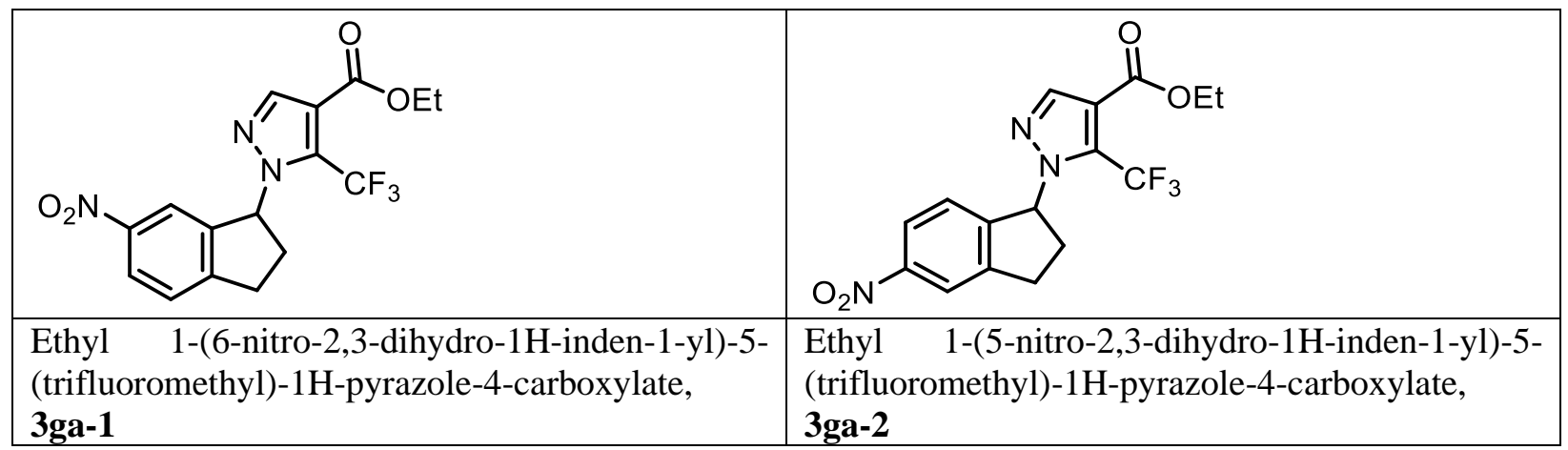

Reaction run using 5-nitroindane $1 \mathrm{~g}$ (32.7 mg, $0.2 \mathrm{mmol}, 1.0$ equiv), ethyl 3-(trifluoromethyl)-1Hpyrazole-4-carboxylate $\mathbf{2 a}$ ( $104.1 \mathrm{mg}, 0.50 \mathrm{mmol}, 2.5$ equiv), copper(II) bromide ( $4.5 \mathrm{mg}, 0.02 \mathrm{mmol}, 0.1$ equiv.), and tetrabutylammonium bromide (19.3 $\mathrm{mg}, 0.06 \mathrm{mmol}, 0.3$ equiv) following the general procedure I (pressure tube) and two regioisomers were isolated.

3ga-1: Yield $=14.5 \mathrm{mg}(20 \%)$ of pale-yellow semisolid. TLC (pentane:EtOAc, 4:1 v/v): $\mathbf{R}_{\mathrm{f}}=0.47$.

${ }^{1} \mathrm{H}$ NMR $\left(\mathrm{CDCl}_{3}, 500 \mathrm{MHz}\right): \delta 8.20(\mathrm{dd}, J=8.4,2.2 \mathrm{~Hz}, 1 \mathrm{H}), 7.90(\mathrm{~s}, 1 \mathrm{H}), 7.89(\mathrm{~d}, J=2.1 \mathrm{~Hz}, 1 \mathrm{H}), 7.49$ $(\mathrm{d}, J=8.4 \mathrm{~Hz}, 1 \mathrm{H}), 6.16(\mathrm{dd}, J=7.9,6.0 \mathrm{~Hz}, 1 \mathrm{H}), 4.35(\mathrm{q}, J=7.1 \mathrm{~Hz}, 2 \mathrm{H}), 3.40(\mathrm{ddd}, J=17.0,8.9$, $5.2 \mathrm{~Hz}, 1 \mathrm{H}), 3.11(\mathrm{ddd}, J=16.9,8.8,6.2 \mathrm{~Hz}, 1 \mathrm{H}), 2.80(\mathrm{dtd}, J=13.6,8.4,5.2 \mathrm{~Hz}, 1 \mathrm{H}), 2.61$ (ddt, $J=$ $12.7,9.0,6.1 \mathrm{~Hz}, 1 \mathrm{H}), 1.37(\mathrm{t}, J=7.1 \mathrm{~Hz}, 3 \mathrm{H}) \mathrm{ppm}$

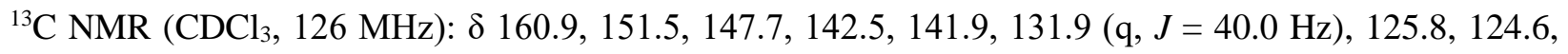
120.0, $119.7(\mathrm{q}, J=271.3 \mathrm{~Hz}), 115.9(\mathrm{q}, J=1.3 \mathrm{~Hz}), 65.4(\mathrm{q}, J=3.5 \mathrm{~Hz}), 61.3,33.7,31.0,14.1 \mathrm{ppm}$.

${ }^{19} \mathrm{~F}$ NMR (377 MHz, $\left.\mathrm{CDCl}_{3}\right)$ : $-55.5 \mathrm{ppm}$.

HRMS Calculated for $\left[\mathrm{C}_{16} \mathrm{H}_{14} \mathrm{~F}_{3} \mathrm{~N}_{3} \mathrm{O}_{4}+\mathrm{NH}_{4}\right]^{+}: 387.1275$, Found: 387.1271.

3ga-2: Yield $=9.8 \mathrm{mg}(13 \%)$ of pale-yellow liquid. TLC (pentane:EtOAc, $4: 1 \mathrm{v} / \mathrm{v}$ ): $\mathrm{R}_{\mathrm{f}}=0.59$.

${ }^{1} \mathrm{H} \mathrm{NMR}\left(\mathrm{CDCl}_{3}, 500 \mathrm{MHz}\right): \delta 8.19(\mathrm{~d}, J=2.1 \mathrm{~Hz}, 1 \mathrm{H}), 8.09(\mathrm{dd}, J=8.4,2.1 \mathrm{~Hz}, 1 \mathrm{H}), 7.90(\mathrm{~s}, 1 \mathrm{H}), 7.17$ $(\mathrm{d}, J=8.3 \mathrm{~Hz}, 1 \mathrm{H}), 6.15(\mathrm{dd}, J=8.2,6.0 \mathrm{~Hz}, 1 \mathrm{H}), 4.35(\mathrm{q}, J=7.1 \mathrm{~Hz}, 2 \mathrm{H}), 3.40(\mathrm{ddd}, J=16.3,9.0$, $5.0 \mathrm{~Hz}, 1 \mathrm{H}), 3.12(\mathrm{dt}, J=15.9,7.6 \mathrm{~Hz}, 1 \mathrm{H}), 2.81(\mathrm{dtd}, J=13.4,8.4,5.0 \mathrm{~Hz}, 1 \mathrm{H}), 2.64$ (ddt, $J=13.1$, 8.9, $6.3 \mathrm{~Hz}, 1 \mathrm{H}), 1.37(\mathrm{t}, J=7.1 \mathrm{~Hz}, 3 \mathrm{H}) \mathrm{ppm}$.

${ }^{13} \mathrm{C} \mathrm{NMR}\left(\mathrm{CDCl}_{3}, 126 \mathrm{MHz}\right): \delta 160.9,148.8,147.0,145.7,142.4,131.9$ (q, $\left.J=40.0 \mathrm{~Hz}\right), 125.1,122.8$, $120.5,119.7(\mathrm{q}, J=271.5 \mathrm{~Hz}), 115.9(\mathrm{q}, J=1.3 \mathrm{~Hz}), 65.4(\mathrm{q}, J=3.5 \mathrm{~Hz}), 61.3,33.6,30.7,14.1 \mathrm{ppm}$. ${ }^{19} \mathrm{~F}$ NMR (377 MHz, $\mathrm{CDCl}_{3}$ ): -55.6 ppm.

HRMS Calculated for $\left[\mathrm{C}_{16} \mathrm{H}_{14} \mathrm{~F}_{3} \mathrm{~N}_{3} \mathrm{O}_{4}+\mathrm{NH}_{4}\right]^{+}: 387.1275$, Found: 387.1271.<smiles>CCOC(=O)c1cn(C(c2ccc(OCC)cc2)c2cc(Br)ccc2Cl)nc1C(F)(F)F</smiles> 
Ethyl 1-((5-bromo-2-chlorophenyl)(4-ethoxyphenyl)methyl)-3-(trifluoromethyl)-1H-pyrazole-4carboxylate, 3ha'

Reaction run using 4-(5-bromo-2-chlorobenzyl)phenyl ethyl ether $\mathbf{1 h}$ (65.1 $\mathrm{mg}, 0.2 \mathrm{mmol}, 1.0$ equiv), ethyl 3-(trifluoromethyl)-1H-pyrazole-4-carboxylate 2a (104.1 mg, $0.50 \mathrm{mmol}, 2.5$ equiv), and tetrabutylammonium chloride $(5.6 \mathrm{mg}, 0.02 \mathrm{mmol}, 0.1$ equiv) following the general procedure I (pressure tube). Yield $=91.5 \mathrm{mg}(86 \%)$ of yellow solid. TLC (pentane:EtOAc, 9:1 v/v): $\mathrm{R}_{\mathrm{f}}=0.44 . \mathrm{Mp}: 84-87{ }^{\circ} \mathrm{C}$ ${ }^{1} \mathrm{H} \mathrm{NMR}\left(\mathrm{CDCl}_{3}, 500 \mathrm{MHz}\right): 7.74(\mathrm{~s}, 1 \mathrm{H}), 7.44(\mathrm{dd}, J=8.5,2.3 \mathrm{~Hz}, 1 \mathrm{H}), 7.29(\mathrm{~d}, J=8.5 \mathrm{~Hz}, 1 \mathrm{H}), 7.07-$

$7.01(\mathrm{~m}, 2 \mathrm{H}), 6.98(\mathrm{~s}, 1 \mathrm{H}), 6.95-6.88(\mathrm{~m}, 2 \mathrm{H}), 6.81(\mathrm{~d}, J=2.3 \mathrm{~Hz}, 1 \mathrm{H}), 4.31(\mathrm{q}, J=7.1 \mathrm{~Hz}, 2 \mathrm{H})$, $4.05(\mathrm{q}, J=7.0 \mathrm{~Hz}, 2 \mathrm{H}), 1.43(\mathrm{t}, J=7.0 \mathrm{~Hz}, 3 \mathrm{H}), 1.34(\mathrm{t}, J=7.1 \mathrm{~Hz}, 3 \mathrm{H}) \mathrm{ppm}$.

${ }^{13} \mathrm{C} \mathrm{NMR}\left(\mathrm{CDCl}_{3}, 126 \mathrm{MHz}\right): 160.9,159.7,142.3(\mathrm{q}, J=38.5 \mathrm{~Hz}), 138.0,136.1,132.9,132.5,131.6$, $131.3,129.9,126.7,121.1,120.2(\mathrm{q}, J=269.9 \mathrm{~Hz}), 115.2,113.2,67.1,63.6,61.1,14.8,14.0 \mathrm{ppm}$.

${ }^{19} \mathrm{~F} \mathrm{NMR}\left(\mathrm{CDCl}_{3}, 377 \mathrm{MHz}\right)$ : $-62.0 \mathrm{ppm}$.

HRMS Calculated for $\left[\mathrm{C}_{22} \mathrm{H}_{19} \mathrm{BrClF}_{3} \mathrm{~N}_{2} \mathrm{O}_{3}+\mathrm{Na}\right]^{+}:$553.0112, Found: 553.0112.<smiles>CCOC(=O)c1cn(C(c2ccc(F)cc2)c2ccc(F)cc2)nc1C(F)(F)F</smiles>

Ethyl 1-(bis(4-fluorophenyl)methyl)-3-(trifluoromethyl)-1H-pyrazole-4-carboxylate, 3ia'

Reaction run using 4,4'-difluorodiphenylmethane $\mathbf{1 i}$ (40.8 $\mathrm{mg}, 0.2 \mathrm{mmol}, 1.0$ equiv), ethyl 3(trifluoromethyl)-1H-pyrazole-4-carboxylate $2 \mathrm{a}$ (104.1 $\mathrm{mg}, 0.50 \mathrm{mmol}, 2.5$ equiv), copper(II) bromide (4.5 mg, $0.02 \mathrm{mmol}, 0.1$ equiv.), and tetrabutylammonium bromide (19.3 $\mathrm{mg}, 0.06 \mathrm{mmol}, 0.3 \mathrm{equiv}$ ) following the general procedure $\mathrm{I}$ (pressure tube). Yield $=78.3 \mathrm{mg}(95 \%)$ of yellow solid. TLC (pentane:EtOAc, 9:1 v/v): $\mathrm{R}_{\mathrm{f}}=0.30 . \mathrm{Mp}: 96-97^{\circ} \mathrm{C}$

${ }^{1} \mathrm{H} \mathrm{NMR}\left(\mathrm{CDCl}_{3}, 500 \mathrm{MHz}\right): 7.83-7.78(\mathrm{~m}, 1 \mathrm{H}), 7.12-7.03(\mathrm{~m}, 8 \mathrm{H}), 6.78(\mathrm{~s}, 1 \mathrm{H}), 4.30$ (q, J = $7.1 \mathrm{~Hz}$, $2 \mathrm{H}), 1.33(\mathrm{t}, J=7.1 \mathrm{~Hz}, 3 \mathrm{H}) \mathrm{ppm}$.

${ }^{13} \mathrm{C} \mathrm{NMR}\left(\mathrm{CDCl}_{3}, 126 \mathrm{MHz}\right): 162.8(\mathrm{~d}, J=249.1 \mathrm{~Hz}), 161.8,160.8,142.0(\mathrm{q}, J=38.6 \mathrm{~Hz}), 135.4,133.3$ $(\mathrm{d}, J=3.3 \mathrm{~Hz}), 129.9$ (d, $J=8.3 \mathrm{~Hz}), 120.3(\mathrm{q}, J=269.8 \mathrm{~Hz}), 116.2(\mathrm{~d}, J=21.8 \mathrm{~Hz}), 113.5,69.3$, 61.1, $14.0 \mathrm{ppm}$.

${ }^{19} \mathrm{~F}$ NMR $\left(\mathrm{CDCl}_{3}, 377 \mathrm{MHz}\right):-61.97,-112.26 \mathrm{ppm}$.

HRMS Calculated for $\left[\mathrm{C}_{20} \mathrm{H}_{15} \mathrm{~F}_{5} \mathrm{~N}_{2} \mathrm{O}_{2}+\mathrm{Na}\right]^{+}$: 433.0946, Found: 433.0951.<smiles>CCOC(=O)c1cnn(C2CCOc3ccccc32)c1C(F)(F)F</smiles> 
Ethyl 1-(chroman-4-yl)-5-(trifluoromethyl)-1H-pyrazole-4-carboxylate, 3ja

Reaction run using chroman $\mathbf{1 j}(25.3 \mu \mathrm{L}, 0.2 \mathrm{mmol}, 1.0$ equiv), ethyl 3-(trifluoromethyl)-1H-pyrazole-4carboxylate $2 \mathrm{a}(104.1 \mathrm{mg}, 0.50 \mathrm{mmol}, 2.5$ equiv), and tetrabutylammonium chloride (16.8 $\mathrm{mg}, 0.06 \mathrm{mmol}$, 0.3 equiv) following the general procedure I (pressure tube). Yield $=29.4 \mathrm{mg}(43 \%)$ of yellow semisolid. TLC (pentane:EtOAc, 15:1 v/v): $\mathbf{R}_{\mathrm{f}}=0.51 .3 \mathbf{j} \mathbf{a}^{\prime}(9.7 \mathrm{mg}, 14 \%)$ was also isolated in this reaction.

${ }^{1} \mathrm{H} \mathrm{NMR}\left(\mathrm{CDCl}_{3}, 500 \mathrm{MHz}\right): 7.91(\mathrm{~s}, 1 \mathrm{H}), 7.22(\mathrm{ddd}, J=8.5,6.9,1.5 \mathrm{~Hz}, 1 \mathrm{H}), 6.92(\mathrm{dd}, J=8.3,1.0 \mathrm{~Hz}$, $1 \mathrm{H}), 6.85(\mathrm{td}, J=7.6,1.1 \mathrm{~Hz}, 1 \mathrm{H}), 6.75(\mathrm{dd}, J=7.9,0.9 \mathrm{~Hz}, 1 \mathrm{H}), 5.82(\mathrm{t}, J=5.7 \mathrm{~Hz}, 1 \mathrm{H}), 4.51(\mathrm{dt}, J$ $=11.6,5.7 \mathrm{~Hz}, 1 \mathrm{H}), 4.34(\mathrm{q}, J=7.2 \mathrm{~Hz}, 2 \mathrm{H}), 4.28(\mathrm{dt}, J=10.8,5.0 \mathrm{~Hz}, 1 \mathrm{H}), 2.45(\mathrm{q}, J=5.6 \mathrm{~Hz}, 2 \mathrm{H})$, $1.36(\mathrm{t}, J=7.2 \mathrm{~Hz}, 3 \mathrm{H}) \mathrm{ppm}$.

${ }^{13} \mathrm{C} \mathrm{NMR}\left(\mathrm{CDCl}_{3}, 126 \mathrm{MHz}\right): 161.1,155.2,141.9,132.0(\mathrm{q}, J=39.9 \mathrm{~Hz}), 130.0,128.6,120.9,119.8(\mathrm{q}, J$ $=271.2 \mathrm{~Hz}), 119.1,117.6,115.7(\mathrm{~d}, J=1.6 \mathrm{~Hz}), 62.8,61.3,55.4(\mathrm{q}, J=3.6 \mathrm{~Hz}), 30.1,14.1 \mathrm{ppm}$.

${ }^{19} \mathrm{~F} \mathrm{NMR}\left(\mathrm{CDCl}_{3}, 377 \mathrm{MHz}\right):-55.7 \mathrm{ppm}$.

HRMS Calculated for $\left[\mathrm{C}_{16} \mathrm{H}_{15} \mathrm{~F}_{3} \mathrm{~N}_{2} \mathrm{O}_{3}+\mathrm{Na}\right]^{+}:$363.0927, Found: 363.0918.<smiles>CCOC(=O)c1nn(C2CCOc3ccccc32)cc1C(F)(F)F</smiles>

Ethyl 1-(chroman-4-yl)-4-(trifluoromethyl)-1H-pyrazole-3-carboxylate, 3ja'

Reaction run using chroman $\mathbf{1 j}(25.3 \mu \mathrm{L}, 0.2 \mathrm{mmol}, 1.0$ equiv), ethyl 3-(trifluoromethyl)-1H-pyrazole-4carboxylate $2 \mathrm{a}(104.1 \mathrm{mg}, 0.50 \mathrm{mmol}, 2.5$ equiv), and trimethylsilyl triflate ( $3.6 \mu \mathrm{L}, 0.02 \mathrm{mmol}, 0.1 \mathrm{equiv})$ at $60^{\circ} \mathrm{C}$ following the general procedure I (pressure tube). Yield $=25.4 \mathrm{mg}(37 \%)$ of white solid. TLC (pentane:EtOAc, 9:1 v/v): $\mathrm{R}_{\mathrm{f}}=0.94 . \mathrm{Mp}: 86-88^{\circ} \mathrm{C}$

${ }^{1} \mathrm{H} \mathrm{NMR}\left(\mathrm{CDCl}_{3}, 500 \mathrm{MHz}\right): 7.71(\mathrm{~s}, 1 \mathrm{H}), 7.33(\mathrm{ddd}, J=8.6,7.3,1.7 \mathrm{~Hz}, 1 \mathrm{H}), 7.08(\mathrm{dd}, J=8.1,1.8 \mathrm{~Hz}$, $1 \mathrm{H}), 6.97(\mathrm{~m}, 2 \mathrm{H}), 5.58(\mathrm{t}, J=4.3 \mathrm{~Hz}, 1 \mathrm{H}), 4.34-4.23(\mathrm{~m}, 3 \mathrm{H}), 3.92(\mathrm{td}, J=11.5,2.5 \mathrm{~Hz}, 1 \mathrm{H}), 2.53$ (dtd, $J=14.6,3.9,2.5 \mathrm{~Hz}, 1 \mathrm{H}), 2.43(\mathrm{ddt}, J=14.7,11.4,4.3 \mathrm{~Hz}, 1 \mathrm{H}), 1.31(\mathrm{t}, J=7.1 \mathrm{~Hz}, 3 \mathrm{H}) \mathrm{ppm}$.

${ }^{13} \mathrm{C} \mathrm{NMR}\left(\mathrm{CDCl}_{3}, 126 \mathrm{MHz}\right): 160.9,155.5,142.0(\mathrm{q}, J=38.4 \mathrm{~Hz}), 135.3,131.2,130.5,121.4,120.4(\mathrm{q}, J$ $=269.8 \mathrm{~Hz}), 118.0,116.4,112.9,61.5,61.0,56.7,28.9,14.0 \mathrm{ppm}$.

${ }^{19} \mathrm{~F} \mathrm{NMR}\left(\mathrm{CDCl}_{3}, 377 \mathrm{MHz}\right):-62.0 \mathrm{ppm}$.

HRMS Calculated for $\left[\mathrm{C}_{16} \mathrm{H}_{15} \mathrm{~F}_{3} \mathrm{~N}_{2} \mathrm{O}_{3}+\mathrm{Na}\right]^{+}:$363.0927, Found: 363.0922 .<smiles>CCOC(=O)c1cnn(C(C)c2ccc(C=O)s2)c1C(F)(F)F</smiles>

Ethyl 1-(1-(5-formylthiophen-2-yl)ethyl)-5-(trifluoromethyl)-1H-pyrazole-4-carboxylate, 3ka Reaction run using 5-ethyl-2-thiophenecarboxaldehyde $\mathbf{1 k}(25.1 \mu \mathrm{L}, 0.2 \mathrm{mmol}, 1.0$ equiv), ethyl 3- 
(trifluoromethyl)-1H-pyrazole-4-carboxylate $\quad 2 \mathrm{a} \quad\left(\begin{array}{llllll}104.1 & \mathrm{mg}, & 0.50 & \mathrm{mmol}, & 2.5 & \text { equiv}), \\ \text { and }\end{array}\right.$ tetrabutylammonium chloride $(16.8 \mathrm{mg}, 0.06 \mathrm{mmol}, 0.3$ equiv) following the general procedure I (pressure tube). Yield $=37.7 \mathrm{mg}(54 \%)$ of pale-yellow liquid. TLC (pentane:EtOAc, 4:1 v/v): $R_{\mathrm{f}}=0.52$.

3ka' (13\%) was observed in ${ }^{1} \mathrm{H}$ NMR analysis of the reaction crude, with $0.2 \mathrm{mmol}$ mesitylene as the external standard.

${ }^{1} \mathrm{H}$ NMR $\left(\mathrm{CDCl}_{3}, 500 \mathrm{MHz}\right): \delta 9.86(\mathrm{~s}, 1 \mathrm{H}), 8.00(\mathrm{~s}, 1 \mathrm{H}), 7.61(\mathrm{~d}, J=3.9 \mathrm{~Hz}, 1 \mathrm{H}), 7.05(\mathrm{~d}, J=3.9 \mathrm{~Hz}$, $1 \mathrm{H}), 6.05(\mathrm{q}, J=6.8 \mathrm{~Hz}, 1 \mathrm{H}), 4.33(\mathrm{q}, J=7.1 \mathrm{~Hz}, 2 \mathrm{H}), 2.03(\mathrm{~d}, J=6.8 \mathrm{~Hz}, 3 \mathrm{H}), 1.35(\mathrm{t}, J=7.1 \mathrm{~Hz}$, $3 \mathrm{H}) \mathrm{ppm}$.

${ }^{13} \mathrm{C} \mathrm{NMR}\left(\mathrm{CDCl}_{3}, 126 \mathrm{MHz}\right): \delta 182.8,160.8,153.1,143.8,142.2,135.7,131.2(\mathrm{q}, J=40.2 \mathrm{~Hz}), 126.5$, $116.1(\mathrm{q}, J=1.3 \mathrm{~Hz}), 119.5(\mathrm{q}, J=271.4 \mathrm{~Hz}), 61.3,56.8(\mathrm{q}, J=3.7 \mathrm{~Hz}), 22.9,14.1 \mathrm{ppm}$.

${ }^{19} \mathrm{~F}$ NMR (377 MHz, $\left.\mathrm{CDCl}_{3}\right)$ : $-55.9 \mathrm{ppm}$.

HRMS Calculated for $\left[\mathrm{C}_{14} \mathrm{H}_{13} \mathrm{BF}_{3} \mathrm{~N}_{2} \mathrm{O}_{3} \mathrm{~S}+\mathrm{Na}\right]^{+}: 369.0491$, Found: 369.0485 .<smiles>CCOC(=O)c1nn(C(C)c2ccc(C=O)s2)cc1C(F)(F)F</smiles>

Ethyl 1-(1-(5-formylthiophen-2-yl)ethyl)-4-(trifluoromethyl)-1H-pyrazole-3-carboxylate, 3ka'

Reaction run using 5-ethyl-2-thiophenecarboxaldehyde $\mathbf{1 k}(25.1 \mu \mathrm{L}, 0.2 \mathrm{mmol}, 1.0$ equiv), ethyl 3(trifluoromethyl)-1H-pyrazole-4-carboxylate $2 \mathrm{a}$ (104.1 $\mathrm{mg}, 0.50 \mathrm{mmol}, 2.5$ equiv), and trimethylsilyl triflate $\left(3.6 \mu \mathrm{L}, 0.02 \mathrm{mmol}, 0.1\right.$ equiv) at $60^{\circ} \mathrm{C}$ following the general procedure $\mathrm{I}$ (pressure tube). Yield $=$ $34.6 \mathrm{mg}(50 \%)$ of yellow semisolid. TLC (pentane:EtOAc, 9:1 v/v): $\mathrm{R}_{\mathrm{f}}=0.91$.

${ }^{1} \mathrm{H} \mathrm{NMR}\left(\mathrm{CDCl}_{3}, 500 \mathrm{MHz}\right): 9.88(\mathrm{~s}, 1 \mathrm{H}), 8.04(\mathrm{~s}, 1 \mathrm{H}), 7.66(\mathrm{~d}, J=3.8 \mathrm{~Hz}, 1 \mathrm{H}), 7.11(\mathrm{~d}, J=3.8 \mathrm{~Hz}, 1 \mathrm{H})$,

$5.83(\mathrm{q}, J=7.0 \mathrm{~Hz}, 1 \mathrm{H}), 4.31(\mathrm{q}, J=7.1 \mathrm{~Hz}, 2 \mathrm{H}), 2.02(\mathrm{~d}, J=7.1 \mathrm{~Hz}, 3 \mathrm{H}), 1.34(\mathrm{t}, J=7.1 \mathrm{~Hz}, 4 \mathrm{H})$ ppm.

${ }^{13} \mathrm{C} \mathrm{NMR}\left(\mathrm{CDCl}_{3}, 126 \mathrm{MHz}\right): 182.8,160.6,152.2,143.9,141.8(\mathrm{q}, J=38.7 \mathrm{~Hz}), 136.1,133.7,126.94$, $120.2(\mathrm{q}, J=269.8 \mathrm{~Hz}), 113.8,61.1,58.3,21.8,14.0 \mathrm{ppm}$.

${ }^{19} \mathrm{~F} \mathrm{NMR}\left(\mathrm{CDCl}_{3}, 377 \mathrm{MHz}\right):-62.0 \mathrm{ppm}$.

HRMS Calculated for $\left[\mathrm{C}_{14} \mathrm{H}_{13} \mathrm{~F}_{3} \mathrm{~N}_{2} \mathrm{O}_{3} \mathrm{~S}+\mathrm{Na}\right]^{+}:$369.0491, Found: 369.0487.<smiles>CCCCC(c1cc(Br)sc1Br)n1ncc(C(=O)OCC)c1C(F)(F)F</smiles> 
Ethyl 1-(1-(2,5-dibromothiophen-3-yl)hexyl)-5-(trifluoromethyl)-1H-pyrazole-4-carboxylate, 3la Reaction run using 2,5-dibromo-3-hexylthiophene 11 (42 $\mu \mathrm{L}, 0.2 \mathrm{mmol}, 1.0$ equiv), ethyl 3(trifluoromethyl)-1H-pyrazole-4-carboxylate $\quad \mathbf{2 a} \quad\left(\begin{array}{llllll}104.1 & \mathrm{mg}, & 0.50 & \mathrm{mmol}, & 2.5 & \text { equiv, and }\end{array}\right.$ tetrabutylammonium chloride $(16.8 \mathrm{mg}, 0.06 \mathrm{mmol}, 0.3$ equiv) following the general procedure I (pressure tube). Yield $=68.7 \mathrm{mg}(65 \%)$ of pale-yellow liquid. TLC (pentane:EtOAc, 9:1 v/v): $\mathrm{R}_{\mathrm{f}}=0.59$.

${ }^{1} \mathrm{H}$ NMR $\left(\mathrm{CDCl}_{3}, 500 \mathrm{MHz}\right): 8.00(\mathrm{~s}, 1 \mathrm{H}), 7.14(\mathrm{~s}, 1 \mathrm{H}), 5.69(\mathrm{dd}, J=8.7,6.3 \mathrm{~Hz}, 1 \mathrm{H}), 4.32$ (q, J = 7.1 Hz, 2H), 2.35 (dtd, $J=14.2,9.2,5.3 \mathrm{~Hz}, 1 \mathrm{H}), 2.03$ (ddt, $J=13.5,9.8,6.1 \mathrm{~Hz}, 1 \mathrm{H}), 1.35$ (t, $J=7.1 \mathrm{~Hz}$, $3 \mathrm{H}), 1.32-1.25(\mathrm{~m}, 4 \mathrm{H}), 1.26-1.19(\mathrm{~m}, 1 \mathrm{H}), 1.16-1.08(\mathrm{~m}, 1 \mathrm{H}), 0.88-0.83(\mathrm{~m}, 3 \mathrm{H}) \mathrm{ppm}$.

${ }^{13} \mathrm{C} \mathrm{NMR}\left(\mathrm{CDCl}_{3}, 126 \mathrm{MHz}\right): 161.1,142.0,139.9,132.0$ (q, $\left.J=40.1 \mathrm{~Hz}\right), 129.8,119.5$ (q, $\left.J=271.6 \mathrm{~Hz}\right)$, 115.6, 111.7, 110.0, 61.2, 60.1 (q, $J=3.4 \mathrm{~Hz}), 36.0,31.0,25.5,22.3,14.1,13.9$ ppm.

${ }^{19} \mathrm{~F} \mathrm{NMR}\left(\mathrm{CDCl}_{3}, 377 \mathrm{MHz}\right):-55.7 \mathrm{ppm}$.

HRMS Calculated for $\left[\mathrm{C}_{17} \mathrm{H}_{19} \mathrm{BrF}_{3} \mathrm{~N}_{2} \mathrm{O}_{2} \mathrm{~S}+\mathrm{H}\right]^{+}:$530.9559, Found: 530.9554. 


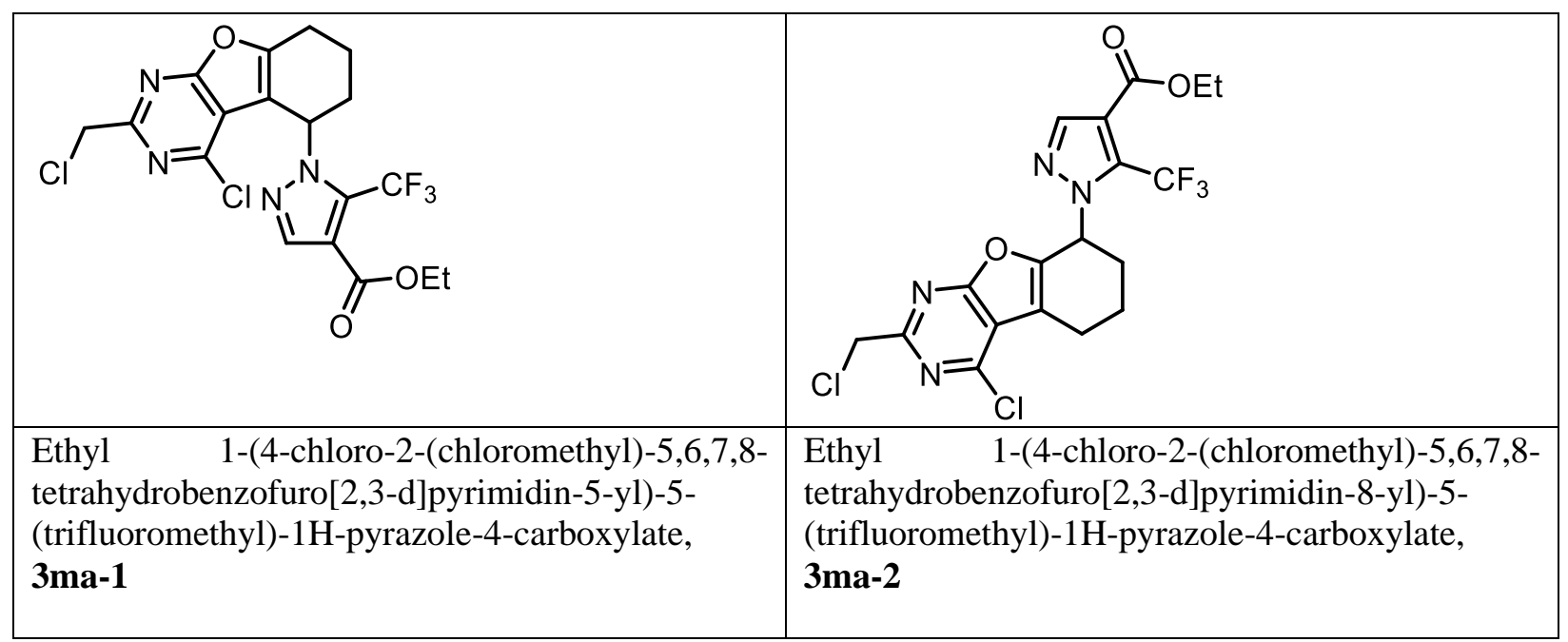

Reaction run using 3-chloro-5-(chloromethyl)-8-oxa-4,6-diazatricyclo[7.4.0.0\{2,7\}]trideca-1(9),2,4,6tetraene $\mathbf{1 m}$ (51.4 mg, $0.2 \mathrm{mmol}, 1.0$ equiv), ethyl 3-(trifluoromethyl)-1H-pyrazole-4-carboxylate 2a (104.1 mg, $0.50 \mathrm{mmol}, 2.5$ equiv), and tetrabutylammonium chloride (16.8 $\mathrm{mg}, 0.06 \mathrm{mmol}, 0.3 \mathrm{equiv}$ ) following the general procedure II (glass vial) at $40{ }^{\circ} \mathrm{C}$ and two regioisomers were isolated.

3ma-1: Yield $=72.3 \mathrm{mg}(45 \%)$ of yellow semisolid. TLC (pentane:EtOAc, 9:1 v/v): $\mathrm{R}_{\mathrm{f}}=0.32$.

${ }^{1} \mathrm{H} \mathrm{NMR}\left(\mathrm{CDCl}_{3}, 500 \mathrm{MHz}\right): 7.89(\mathrm{~s}, 1 \mathrm{H}), 5.87(\mathrm{t}, J=5.6 \mathrm{~Hz}, 1 \mathrm{H}), 4.73(\mathrm{~s}, 2 \mathrm{H}), 4.35$ (q, $\left.J=7.1 \mathrm{~Hz}, 2 \mathrm{H}\right)$, $3.11(\mathrm{dtd}, J=17.0,5.5,1.6 \mathrm{~Hz}, 1 \mathrm{H}), 2.92(\mathrm{dddd}, J=17.0,7.3,5.5,1.7 \mathrm{~Hz}, 1 \mathrm{H}), 2.43(\mathrm{q}, J=5.9 \mathrm{~Hz}$, 2H), $2.30-2.18(\mathrm{~m}, 1 \mathrm{H}), 2.00(\mathrm{dh}, J=17.2,5.6 \mathrm{~Hz}, 1 \mathrm{H}), 1.37(\mathrm{t}, J=7.1 \mathrm{~Hz}, 3 \mathrm{H}) \mathrm{ppm}$.

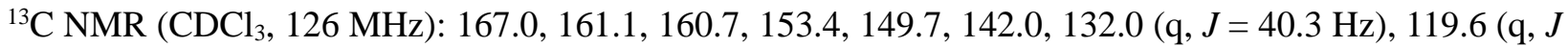
$=271.4 \mathrm{~Hz}), 117.2,116.3(\mathrm{q}, J=1.6 \mathrm{~Hz}), 116.1,61.3,54.5(\mathrm{q}, J=3.9 \mathrm{~Hz}), 46.2,30.8,21.0,19.4,14.1$ ppm.

${ }^{19} \mathrm{~F}$ NMR $\left(\mathrm{CDCl}_{3}, 377 \mathrm{MHz}\right):-55.8 \mathrm{ppm}$.

HRMS Calculated for $\left[\mathrm{C}_{18} \mathrm{H}_{15} \mathrm{Cl}_{2} \mathrm{~F}_{3} \mathrm{~N}_{4} \mathrm{O}_{3}+\mathrm{H}\right]^{+}:$463.0546, Found: 463.0543.

3ma-2: Yield $=8.1 \mathrm{mg}(9 \%)$ of yellow semisolid. TLC (pentane:EtOAc, 4:1 v/v): $\mathrm{R}_{\mathrm{f}}=0.39$.

${ }^{1} \mathrm{H} \mathrm{NMR}\left(\mathrm{CDCl}_{3}, 500 \mathrm{MHz}\right): 7.84(\mathrm{~s}, 1 \mathrm{H}), 6.07(\mathrm{t}, J=4.5 \mathrm{~Hz}, 2 \mathrm{H}), 4.73(\mathrm{~s}, 2 \mathrm{H}), 4.34(\mathrm{q}, J=7.1 \mathrm{~Hz}, 2 \mathrm{H})$, $3.05(\mathrm{dt}, J=18.1,5.1 \mathrm{~Hz}, 1 \mathrm{H}), 2.86(\mathrm{dt}, J=17.5,7.2 \mathrm{~Hz}, 1 \mathrm{H}), 2.34(\mathrm{ddt}, J=16.8,8.7,2.8 \mathrm{~Hz}, 1 \mathrm{H})$, $2.26-2.09(\mathrm{~m}, 2 \mathrm{H}), 2.08-1.95(\mathrm{~m}, 2 \mathrm{H}), 1.36(\mathrm{t}, J=7.1 \mathrm{~Hz}, 3 \mathrm{H}) \mathrm{ppm}$.

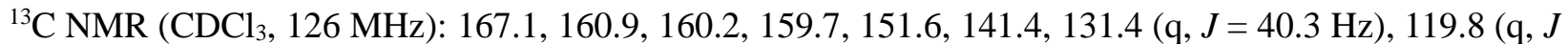
$=271.6 \mathrm{~Hz}), 116.7,115.5,109.3,61.3,54.2(\mathrm{q}, J=3.8 \mathrm{~Hz}), 46.3,31.2,23.1,18.2,14.1 \mathrm{ppm}$.

${ }^{19} \mathrm{~F} \mathrm{NMR}\left(\mathrm{CDCl}_{3}, 377 \mathrm{MHz}\right):-56.0 \mathrm{ppm}$.

HRMS Calculated for $\left[\mathrm{C}_{18} \mathrm{H}_{15} \mathrm{Cl}_{2} \mathrm{~F}_{3} \mathrm{~N}_{4} \mathrm{O}_{3}+\mathrm{H}\right]^{+}:$463.0546, Found: 463.0545. 


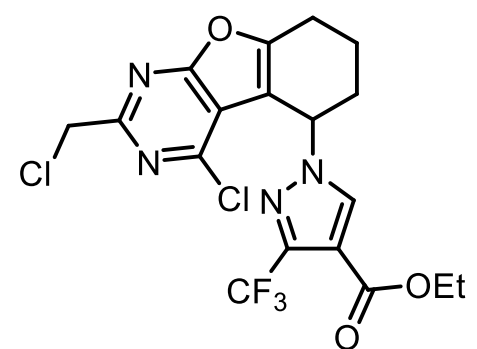

Ethyl 1-(4-chloro-2-(chloromethyl)-5,6,7,8-tetrahydrobenzofuro[2,3-d]pyrimidin-5-yl)-3-(trifluoromethyl)-1H-pyrazole-4-carboxylate, 3ma'-1

Reaction run using 3-chloro-5-(chloromethyl)-8-oxa-4,6-diazatricyclo[7.4.0.0\{2,7\}]trideca-1(9),2,4,6tetraene $1 \mathrm{~m}$ (51.4 mg, $0.2 \mathrm{mmol}, 1.0$ equiv), ethyl 3-(trifluoromethyl)-1H-pyrazole-4-carboxylate 2a (104.1 mg, $0.50 \mathrm{mmol}, 2.5$ equiv), and $\mathrm{BF}_{3} \cdot \mathrm{Et}_{2} \mathrm{O}\left(2.5 \mu \mathrm{L}, 0.02 \mathrm{mmol}, 0.1\right.$ equiv) at $60^{\circ} \mathrm{C}$ following the general procedure I (pressure tube) and two regioisomers were isolated.

3ma'-1: Yield $=52.6 \mathrm{mg}(58 \%)$ of colorless semisolid. TLC (pentane:Et $\left.\mathrm{E}_{2} \mathrm{O}, 1: 1 \mathrm{v} / \mathrm{v}\right): \mathrm{R}_{\mathrm{f}}=0.18$.

${ }^{1} \mathrm{H} \mathrm{NMR}\left(\mathrm{CDCl}_{3}, 400 \mathrm{MHz}\right): 7.93(\mathrm{~s}, 1 \mathrm{H}), 5.65(\mathrm{t}, J=5.0 \mathrm{~Hz}, 1 \mathrm{H}), 4.76(\mathrm{~s}, 2 \mathrm{H}), 4.31(\mathrm{q}, J=7.2 \mathrm{~Hz}, 2 \mathrm{H})$,

$3.12(\mathrm{dt}, J=17.4,5.3 \mathrm{~Hz}, 1 \mathrm{H}), 2.97-2.82(\mathrm{~m}, 1 \mathrm{H}), 2.57(\mathrm{dq}, J=14.3,4.7 \mathrm{~Hz}, 1 \mathrm{H}), 2.48-2.31(\mathrm{~m}$, $1 \mathrm{H}), 2.04(\mathrm{p}, J=5.2 \mathrm{~Hz}, 2 \mathrm{H}), 1.34(\mathrm{t}, J=7.1 \mathrm{~Hz}, 3 \mathrm{H}) \mathrm{ppm}$.

${ }^{13} \mathrm{C} \mathrm{NMR}\left(\mathrm{CDCl}_{3}, 126 \mathrm{MHz}\right): 167.3,161.7,160.6,153.9,148.6,142.2(\mathrm{q}, J=38.8 \mathrm{~Hz}), 135.1,120.2$ (q, $J$ $=269.8 \mathrm{~Hz}), 118.4,115.9,113.6,61.1,55.4,46.2,29.9,21.1,18.8,14.1 \mathrm{ppm}$.

${ }^{19} \mathrm{~F} \mathrm{NMR}\left(\mathrm{CDCl}_{3}, 377 \mathrm{MHz}\right)$ : -62.1 ppm.

HRMS Calculated for $\left[\mathrm{C}_{18} \mathrm{H}_{15} \mathrm{Cl}_{2} \mathrm{~F}_{3} \mathrm{~N}_{4} \mathrm{O}_{3}+\mathrm{H}\right]^{+}:$463.0546, Found: 463.0539 .

3ma-1: Yield $=12.9 \mathrm{mg}(14 \%)$ of yellow semisolid. TLC (pentane:EtOAc, 9:1 v/v): $\mathrm{R}_{\mathrm{f}}=0.32$. 


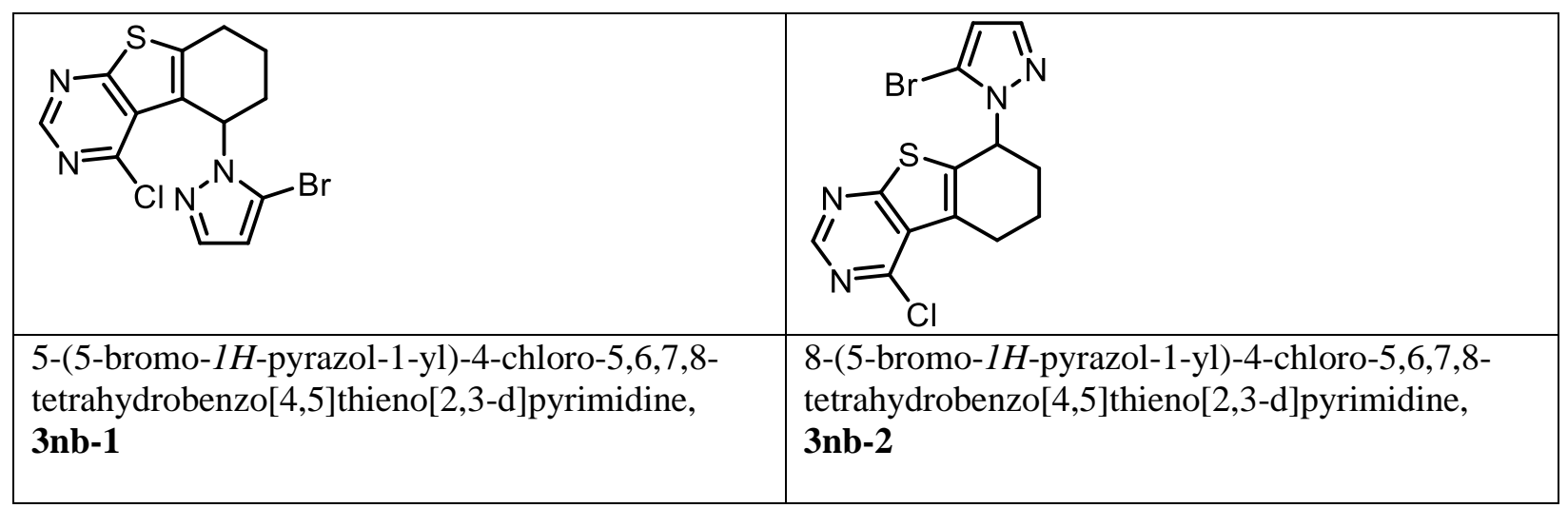

Reaction run using 4-chloro-5,6,7,8-tetrahydro[1]benzothieno[2,3-d]pyrimidine 1n (44.8 mg, $0.2 \mathrm{mmol}$, 1.0 equiv), 3-bromopyrazle $2 \mathbf{b}$ ( $73.5 \mathrm{mg}, 0.50 \mathrm{mmol}, 2.5$ equiv), copper(II) bromide ( $4.5 \mathrm{mg}, 0.02 \mathrm{mmol}$, 0.1 equiv.), and tetrabutylammonium bromide (19.3 $\mathrm{mg}, 0.06 \mathrm{mmol}, 0.3$ equiv) following the general procedure I (pressure tube) and two regioisomers were isolated.

3nb-1: Yield $=28.1 \mathrm{mg}(38 \%)$ of white solid. (pentane:EtOAc, $2: 1 \mathrm{v} / \mathrm{v}): \mathrm{R}_{\mathrm{f}}=0.48$.

${ }^{1} \mathrm{H} \mathrm{NMR}\left(\mathrm{CDCl}_{3}, 500 \mathrm{MHz}\right): \delta 8.72(\mathrm{~s}, 1 \mathrm{H}), 7.39(\mathrm{~d}, J=2.0 \mathrm{~Hz}, 1 \mathrm{H}), 6.39(\mathrm{~d}, J=1.9 \mathrm{~Hz}, 1 \mathrm{H}), 6.18(\mathrm{t}, J=$ $2.4 \mathrm{~Hz}, 1 \mathrm{H}), 3.19$ (ddd, $J=17.8,5.4,2.1 \mathrm{~Hz}, 1 \mathrm{H}), 3.02-2.81(\mathrm{~m}, 1 \mathrm{H}), 2.33$ (ddt, $J=13.8,5.0,2.7$ $\mathrm{Hz}, 1 \mathrm{H}), 2.15$ (tdd, $J=13.7,4.5,2.9 \mathrm{~Hz}, 1 \mathrm{H}), 2.04$ (tddd, $J=13.6,11.3,5.3,2.5 \mathrm{~Hz}, 1 \mathrm{H}), 1.96-1.86$ $(\mathrm{m}, 1 \mathrm{H}) \mathrm{ppm}$.

${ }^{13} \mathrm{C} \mathrm{NMR}\left(\mathrm{CDCl}_{3}, 126 \mathrm{MHz}\right): 168.8,152.8,152.0,145.6,139.8,127.9,122.7,112.0,109.2,52.7,29.9$, 26.1, $17.0 \mathrm{ppm}$.

HRMS Calculated for $\left[\mathrm{C}_{13} \mathrm{H}_{10} \mathrm{BrClN}{ }_{4} \mathrm{~S}+\mathrm{H}\right]^{+}:$368.9571, Found: 368.9570.

3nb-2: Yield $=18.8 \mathrm{mg}(25 \%)$ of white solid. (pentane:EtOAc, $2: 1 \mathrm{v} / \mathrm{v}$ ): $\mathrm{R}_{\mathrm{f}}=0.06$.

${ }^{1} \mathrm{H} \mathrm{NMR}\left(\mathrm{CDCl}_{3}, 500 \mathrm{MHz}\right): 8.75(\mathrm{~s}, 1 \mathrm{H}), 7.56(\mathrm{~d}, J=1.8 \mathrm{~Hz}, 1 \mathrm{H}), 6.40(\mathrm{~d}, J=1.9 \mathrm{~Hz}, 1 \mathrm{H}), 5.86(\mathrm{dd}, J=$ 7.7, $5.4 \mathrm{~Hz}, 1 \mathrm{H}), 3.37-3.14(\mathrm{~m}, 2 \mathrm{H}), 2.52-2.40(\mathrm{~m}, 1 \mathrm{H}), 2.42-2.27(\mathrm{~m}, 2 \mathrm{H}), 2.07-1.95(\mathrm{~m}, 1 \mathrm{H})$ ppm.

${ }^{13} \mathrm{C} \mathrm{NMR}\left(\mathrm{CDCl}_{3}, 126 \mathrm{MHz}\right): 169.6,154.5,152.5,141.3,137.7,131.0,128.3,112.8,109.1,55.6,29.9$, 26.1, $20.5 \mathrm{ppm}$.

HRMS Calculated for $\left[\mathrm{C}_{13} \mathrm{H}_{10} \mathrm{BrClN}_{4} \mathrm{~S}+\mathrm{H}\right]^{+}$: 368.9571, Found: 368.9571.

\begin{tabular}{|c|c|}
\hline $\mathrm{H}_{\mathrm{OE}}$ & II \\
\hline $\begin{array}{l}\text { Ethyl 1-(2-chloro-6,7-dihydro-5H- } \\
\text { cyclopenta[b]pyridin-5-yl)-5-(trifluoromethyl)- } \\
\text { 1H-pyrazole-4-carboxylate, 3oa-1 }\end{array}$ & $\begin{array}{l}\text { Ethyl 1-(2-chloro-6,7-dihydro-5H- } \\
\text { cyclopenta[b]pyridin-7-yl)-5-(trifluoromethyl)- } \\
\text { 1H-pyrazole-4-carboxylate, 3oa-2 }\end{array}$ \\
\hline
\end{tabular}


Reaction run using 2-chloro-6,7-dihydro-5H-cyclopenta[b]pyridine 10 (30.7 mg, $0.2 \mathrm{mmol}, 1.0$ equiv), ethyl 3-(trifluoromethyl)-1H-pyrazole-4-carboxylate 2a (104.1 mg, $0.50 \mathrm{mmol}, 2.5$ equiv), and tetrabutylammonium chloride $(5.6 \mathrm{mg}, 0.02 \mathrm{mmol}, 0.1$ equiv) following the general procedure I (pressure tube) at $60^{\circ} \mathrm{C}$ and two regioisomers were isolated.

3oa-1: Yield $=14.0 \mathrm{mg}(20 \%)$ of light-yellow liquid. TLC (pentane:EtOAc, $4: 1 \mathrm{v} / \mathrm{v}$ ): $\mathbf{R}_{\mathrm{f}}=0.27$.

${ }^{1} \mathrm{H} \mathrm{NMR}\left(\mathrm{CDCl}_{3}, 400 \mathrm{MHz}\right): \delta 7.89(\mathrm{~s}, 1 \mathrm{H}), 7.34(\mathrm{~d}, J=8.1 \mathrm{~Hz}, 1 \mathrm{H}), 7.17(\mathrm{~d}, J=8.1 \mathrm{~Hz}, 1 \mathrm{H}), 6.09(\mathrm{dd}, J$ $=8.4,5.2 \mathrm{~Hz}, 1 \mathrm{H}), 4.34(\mathrm{q}, J=7.1 \mathrm{~Hz}, 2 \mathrm{H}), 3.41(\mathrm{ddd}, J=17.1,9.3,5.5 \mathrm{~Hz}, 1 \mathrm{H}), 3.11(\mathrm{ddd}, J=17.3$, 8.9, $6.1 \mathrm{~Hz}, 1 \mathrm{H}), 2.77$ (dtd, $J=14.0,8.7,5.5 \mathrm{~Hz}, 1 \mathrm{H}), 2.57$ (ddt, $J=14.3,9.3,5.7 \mathrm{~Hz}, 1 \mathrm{H}), 1.36(\mathrm{t}, J$ $=7.1 \mathrm{~Hz}, 3 \mathrm{H}) \mathrm{ppm}$.

${ }^{13} \mathrm{C} \mathrm{NMR}\left(\mathrm{CDCl}_{3}, 126 \mathrm{MHz}\right): \delta 165.6,160.9,152.5,142.3,135.1,132.3,131.7(\mathrm{q}, J=39.9 \mathrm{~Hz}), 122.4$, $119.7(\mathrm{q}, J=271.2 \mathrm{~Hz}), 115.9,63.7(\mathrm{q}, J=3.5 \mathrm{~Hz}), 61.4,32.4,31.6,14.2 \mathrm{ppm}$.

${ }^{19} \mathrm{~F}$ NMR (377 MHz, $\mathrm{CDCl}_{3}$ ): $-55.6 \mathrm{ppm}$.

HRMS Calculated for $\left[\mathrm{C}_{15} \mathrm{H}_{13} \mathrm{ClF}_{3} \mathrm{~N}_{3} \mathrm{O}_{2}+\mathrm{H}\right]^{+}: 360.0721$, Found: 360.0717.

3oa-2: Yield $=4.0 \mathrm{mg}(6 \%)$ of light-yellow liquid. TLC (pentane:EtOAc, 9:1 v/v): $\mathrm{R}_{\mathrm{f}}=0.16$.

${ }^{1} \mathrm{H}$ NMR (CDCl + DCM, $\left.400 \mathrm{MHz}\right): \delta 7.89(\mathrm{~s}, 1 \mathrm{H}), 7.62(\mathrm{~d}, J=8.0 \mathrm{~Hz}, 1 \mathrm{H}), 7.26(\mathrm{~d}, J=8.3 \mathrm{~Hz}, 3 \mathrm{H})$, $6.05(\mathrm{dd}, J=8.6,4.9 \mathrm{~Hz}, 1 \mathrm{H}), 4.33(\mathrm{qd}, J=7.1,1.7 \mathrm{~Hz}, 2 \mathrm{H}), 3.24(\mathrm{ddd}, J=15.4,9.0,5.7 \mathrm{~Hz}, 1 \mathrm{H})$, 2.99 (ddd, $J=16.3,8.8,5.2 \mathrm{~Hz}, 1 \mathrm{H}$ ), 2.77 (dtd, $J=14.3,8.7,5.8 \mathrm{~Hz}, 1 \mathrm{H}$ ), 2.48 (ddt, $J=14.1,9.6,5.1$ $\mathrm{Hz}, 1 \mathrm{H}), 1.35$ (t, $J=7.1 \mathrm{~Hz}, 3 \mathrm{H})$. ppm.

${ }^{13} \mathrm{C} \mathrm{NMR}\left(\mathrm{CDCl}_{3}, 126 \mathrm{MHz}\right): \delta 161.1,160.1,150.7,142.2,136.4,135.7,131.7(\mathrm{q}, J=39.9 \mathrm{~Hz}), 124.3$, $119.7(\mathrm{q}, J=271.2 \mathrm{~Hz}), 115.9,65.52(\mathrm{q}, J=3.9 \mathrm{~Hz}), 61.1,31.7,27.9,14.1 \mathrm{ppm}$.

${ }^{19} \mathrm{~F}$ NMR (377 MHz, $\left.\mathrm{CDCl}_{3}\right)$ : $-55.7 \mathrm{ppm}$.

HRMS Calculated for $\left[\mathrm{C}_{15} \mathrm{H}_{13} \mathrm{ClF}_{3} \mathrm{~N}_{3} \mathrm{O}_{2}+\mathrm{Na}\right]^{+}:$382.0541, Found: 382.0538 .

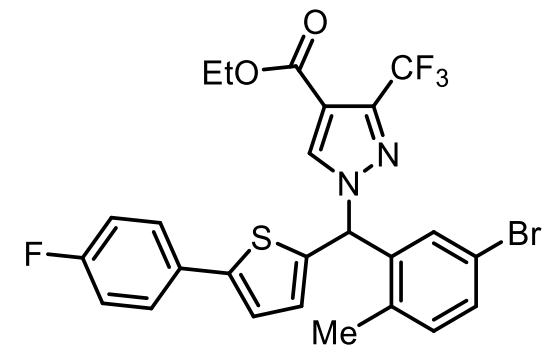

Ethyl 1-((5-bromo-2-methylphenyl)(5-(4-fluorophenyl)thiophen-2-yl)methyl)-3-(trifluoromethyl)-1Hpyrazole-4-carboxylate, 3pa'

Reaction run using 2-(5-bromo-2-methylbenzyl)-5-(4-fluorophenyl)thiophene 1p (68.6 mg, $0.2 \mathrm{mmol}, 1.0$ equiv), ethyl 3-(trifluoromethyl)-1H-pyrazole-4-carboxylate 2a (104.1 mg, $0.50 \mathrm{mmol}, 2.5$ equiv), and tetrabutylammonium chloride $(5.6 \mathrm{mg}, 0.02 \mathrm{mmol}, 0.1$ equiv) following the general procedure II (glass vial) at $30{ }^{\circ} \mathrm{C}$. Yield $=101.6 \mathrm{mg}(90 \%)$ of yellow solid. TLC (pentane:EtOAc, 9:1 v/v): $\mathrm{R}_{\mathrm{f}}=0.56 . \mathrm{Mp}$ : $115-118^{\circ} \mathrm{C}$.

${ }^{1} \mathrm{H}$ NMR $\left(\mathrm{CDCl}_{3}, 500 \mathrm{MHz}\right): 7.94(\mathrm{~s}, 1 \mathrm{H}), 7.57-7.49(\mathrm{~m}, 2 \mathrm{H}), 7.43(\mathrm{dd}, J=8.1,2.1 \mathrm{~Hz}, 1 \mathrm{H}), 7.15(\mathrm{~d}, J$ 
$=3.7 \mathrm{~Hz}, 1 \mathrm{H}), 7.11(\mathrm{~d}, J=8.2 \mathrm{~Hz}, 1 \mathrm{H}), 7.08(\mathrm{t}, J=8.6 \mathrm{~Hz}, 2 \mathrm{H}), 7.02(\mathrm{~s}, 1 \mathrm{H}), 6.85(\mathrm{~d}, J=2.0 \mathrm{~Hz}, 1 \mathrm{H})$, $6.80(\mathrm{dd}, J=3.8,0.9 \mathrm{~Hz}, 1 \mathrm{H}), 4.33(\mathrm{q}, J=7.1 \mathrm{~Hz}, 2 \mathrm{H}), 2.23(\mathrm{~s}, 3 \mathrm{H}), 1.35(\mathrm{t}, J=7.1 \mathrm{~Hz}, 3 \mathrm{H}) \mathrm{ppm}$.

${ }^{13} \mathrm{C} \mathrm{NMR}\left(\mathrm{CDCl}_{3}, 126 \mathrm{MHz}\right): 162.7$ (d, $\left.J=248.6 \mathrm{~Hz}\right), 160.8,146.0,142.3$ (q, $\left.J=38.6 \mathrm{~Hz}\right), 138.2,137.4$, 135.5, 134.87, 132.8, 132.1, 130.0, 129.7 (d, $J=3.4 \mathrm{~Hz}), 128.7,127.7(\mathrm{~d}, J=8.2 \mathrm{~Hz}), 123.1,120.4$, $120.2(\mathrm{q}, J=270.0 \mathrm{~Hz}), 116.1(\mathrm{~d}, J=21.9 \mathrm{~Hz}), 113.6,63.1,61.2,18.8,14.1 \mathrm{ppm}$.

${ }^{19} \mathrm{~F} \mathrm{NMR}\left(\mathrm{CDCl}_{3}, 377 \mathrm{MHz}\right):-62.0,-113.2 \mathrm{ppm}$.

HRMS Calculated for $\left[\mathrm{C}_{25} \mathrm{H}_{19} \mathrm{BrF}_{4} \mathrm{~N}_{2} \mathrm{O}_{2} \mathrm{~S}+\mathrm{NH}_{4}\right]^{+}$: 584.0625, Found: 584.0617.

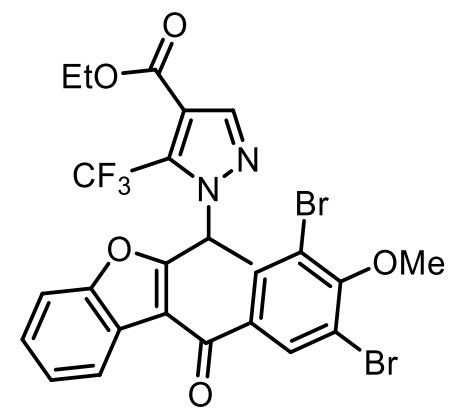

Ethyl 1-(1-(3-(3,5-dibromo-4-methoxybenzoyl)benzofuran-2-yl)ethyl)-5-(trifluoromethyl)-1H-pyrazole4-carboxylate, $\mathbf{3 q a}$

Reaction run using benzbromarone methyl ether $\mathbf{1 q}(87.6 \mathrm{mg}, 0.2 \mathrm{mmol}, 1.0$ equiv), ethyl 3(trifluoromethyl)-1H-pyrazole-4-carboxylate $\quad \mathbf{2 a} \quad(104.1 \quad \mathrm{mg}, \quad 0.50 \quad \mathrm{mmol}, \quad 2.5$ equiv), and tetrabutylammonium chloride $(5.6 \mathrm{mg}, 0.02 \mathrm{mmol}, 0.1$ equiv) following the general procedure I (pressure tube). Yield $=99.0 \mathrm{mg}(77 \%)$ of pale-yellow semisolid. TLC (pentane:EtOAc, 9:1 v/v): $R_{\mathrm{f}}=0.42$.

${ }^{1} \mathrm{H}$ NMR $\left(\mathrm{CDCl}_{3}, 500 \mathrm{MHz}\right): \delta 7.99$ (s, 1H), 7.94 (s, 2H), 7.60 (dt, $\left.J=8.5,0.9 \mathrm{~Hz}, 1 \mathrm{H}\right), 7.39$ (ddd, $J=8.4$,

$7.0,1.5 \mathrm{~Hz}, 1 \mathrm{H}), 7.27(\mathrm{td}, J=7.5,7.1,0.8 \mathrm{~Hz}, 2 \mathrm{H}), 7.23(\mathrm{dd}, J=8.0,0.7 \mathrm{~Hz}, 1 \mathrm{H}), 6.37(\mathrm{q}, J=6.9 \mathrm{~Hz}$, $1 \mathrm{H}), 4.31(\mathrm{qd}, J=7.2,1.1 \mathrm{~Hz}, 2 \mathrm{H}), 3.98(\mathrm{~s}, 3 \mathrm{H}), 2.08(\mathrm{~d}, J=6.9 \mathrm{~Hz}, 3 \mathrm{H}), 1.34(\mathrm{t}, J=7.1 \mathrm{~Hz}, 3 \mathrm{H})$ ppm.

${ }^{13} \mathrm{C} \mathrm{NMR}\left(\mathrm{CDCl}_{3}, 126 \mathrm{MHz}\right): \delta 187.4,160.9,158.8,158.3,154.0,141.9,136.1,133.7,131.4(\mathrm{q}, J=40.0$

$\mathrm{Hz}), 126.0,125.2,124.4,121.4,119.4(\mathrm{q}, J=271.3 \mathrm{~Hz}), 118.7,117.1,116.3(\mathrm{q}, J=1.3 \mathrm{~Hz}), 112.2$,

$61.2,60.9,54.1(\mathrm{q}, J=3.6 \mathrm{~Hz}), 19.5,14.1 \mathrm{ppm}$.

${ }^{19} \mathrm{~F} \mathrm{NMR}\left(377 \mathrm{MHz}, \mathrm{CDCl}_{3}\right)$ : $-56.4 \mathrm{ppm}$.

HRMS Calculated for $\left[\mathrm{C}_{25} \mathrm{H}_{19} \mathrm{Br}_{2} \mathrm{~F}_{3} \mathrm{~N}_{2} \mathrm{O}_{5}+\mathrm{H}\right]^{+}:$642.9686, Found: 642.9687.<smiles>CCOC(=O)c1cn(C2c3oc4ccccc4c3C(=O)c3cc(Br)c(OC)c(Br)c32)nc1C(F)(F)F</smiles> 
Ethyl 1-(1-(3-(3,5-dibromo-4-methoxybenzoyl)benzofuran-2-yl)ethyl)-3-(trifluoromethyl)-1H-pyrazole4-carboxylate, 3qa'

Reaction run using benzbromarone methyl ether $\mathbf{1 q}(87.6 \mathrm{mg}, 0.2 \mathrm{mmol}, 1.0$ equiv), ethyl 3(trifluoromethyl)-1H-pyrazole-4-carboxylate $2 \mathrm{a}\left(104.1 \mathrm{mg}, 0.50 \mathrm{mmol}, 2.5\right.$ equiv), and $\mathrm{BF}_{3} \bullet \mathrm{Et}_{2} \mathrm{O}(2.5 \mu \mathrm{L}$, $0.02 \mathrm{mmol}, 0.1$ equiv) following the general procedure I (pressure tube) at $60{ }^{\circ} \mathrm{C}$. Yield $=58.0 \mathrm{mg}(45 \%)$ of pale-yellow semisolid. TLC (pentane:EtOAc, 4:1 v/v): $\mathrm{R}_{\mathrm{f}}=0.56 . \mathrm{Mp}: 152-155{ }^{\circ} \mathrm{C}$.

${ }^{1} \mathrm{H} \mathrm{NMR}\left(\mathrm{CDCl}_{3}, 500 \mathrm{MHz}\right): \delta 8.31(\mathrm{~s}, 1 \mathrm{H}), 8.01(\mathrm{~s}, 2 \mathrm{H}), 7.59(\mathrm{dt}, J=8.4,0.9 \mathrm{~Hz}, 1 \mathrm{H}), 7.42$ (ddd, $J=8.5$, $4.7,3.8 \mathrm{~Hz}, 1 \mathrm{H}), 7.30(\mathrm{t}, J=1.0 \mathrm{~Hz}, 1 \mathrm{H}), 7.29(\mathrm{~d}, J=0.8 \mathrm{~Hz}, 1 \mathrm{H}), 6.06(\mathrm{q}, J=7.1 \mathrm{~Hz}, 1 \mathrm{H}), 4.32(\mathrm{q}, J$ $=7.1 \mathrm{~Hz}, 2 \mathrm{H}), 4.00(\mathrm{~s}, 3 \mathrm{H}), 2.06(\mathrm{~d}, J=7.1 \mathrm{~Hz}, 3 \mathrm{H}), 1.35(\mathrm{t}, J=7.1 \mathrm{~Hz}, 3 \mathrm{H}) \mathrm{ppm}$.

${ }^{13} \mathrm{C} \mathrm{NMR}\left(\mathrm{CDCl}_{3}, 126 \mathrm{MHz}\right): \delta 187.3,160.8,158.5,158.2,154.0,141.7$ (q, $\left.J=38.7 \mathrm{~Hz}\right), 135.9,134.8$, $133.9,126.3,125.2,124.6,121.6,120.3(\mathrm{q}, J=269.9 \mathrm{~Hz}), 118.8,117.7,113.6,112.2,61.0,60.9,55.0$, $18.8,14.1 \mathrm{ppm}$.

${ }^{19} \mathrm{~F}$ NMR (377 MHz, $\left.\mathrm{CDCl}_{3}\right):-62.0 \mathrm{ppm}$.

HRMS Calculated for $\left[\mathrm{C}_{25} \mathrm{H}_{19} \mathrm{Br}_{2} \mathrm{~F}_{3} \mathrm{~N}_{2} \mathrm{O}_{5}+\mathrm{H}\right]^{+}:$642.9686, Found: 642.9687. 
<smiles>CC(=O)c1cc(C(C)C)cc2c1C(n1nccc1Br)CC2(C)C</smiles>

1-(3-(4-bromo-1H-pyrazol-1-yl)-6-(tert-butyl)-1,1-dimethyl-2,3-dihydro-1H-inden-4-yl)ethan-1-one, 3rb Reaction run using celestolide $\mathbf{1 r}(48.9 \mathrm{mg}, 0.2 \mathrm{mmol}, 1.0$ equiv), 3-bromopyrazole $2 \mathbf{b}$ (73.5 $\mathrm{mg}, 0.50$ mmol, 2.5 equiv), and tetrabutylammonium chloride $(5.6 \mathrm{mg}, 0.02 \mathrm{mmol}, 0.1$ equiv) following the general procedure I (pressure tube). Yield $=54.2 \mathrm{mg}(70 \%)$ of white solid. TLC (pentane:EtOAc, 9:1 v/v): $\mathrm{R}_{\mathrm{f}}=0.46 . \mathrm{Mp}: 88-90^{\circ} \mathrm{C}$

${ }^{1} \mathrm{H}$ NMR $\left(\mathrm{CDCl}_{3}, 500 \mathrm{MHz}\right): 7.63(\mathrm{~s}, 1 \mathrm{H}), 7.42(\mathrm{~d}, J=1.8 \mathrm{~Hz}, 1 \mathrm{H}), 7.36(\mathrm{~d}, J=1.9 \mathrm{~Hz}, 1 \mathrm{H}), 6.40(\mathrm{dd}, J=$

8.2, $6.5 \mathrm{~Hz}, 1 \mathrm{H}), 6.26(\mathrm{~d}, 1 \mathrm{H}), 2.53(\mathrm{dd}, J=13.1,8.3 \mathrm{~Hz}, 1 \mathrm{H}), 2.35(\mathrm{~s}, 3 \mathrm{H}), 2.09$ (dd, $J=13.1,6.5 \mathrm{~Hz}$, $1 \mathrm{H}), 1.42(\mathrm{~s}, 3 \mathrm{H}), 1.37$ (s, 9H), $1.32(\mathrm{~s}, 3 \mathrm{H}) \mathrm{ppm}$.

${ }^{13} \mathrm{C} \mathrm{NMR}\left(\mathrm{CDCl}_{3}, 126 \mathrm{MHz}\right): 199.9,154.2,152.4,139.6,135.4,135.1,125.1,123.0,113.8,107.6,61.6$, 49.3, 42.4, 34.9, 31.4, 30.3, 29.3, 27.6.ppm.

HRMS Calculated for $\left[\mathrm{C}_{20} \mathrm{H}_{25} \mathrm{BrN}_{2} \mathrm{O}+\mathrm{H}\right]^{+}: 389.1223$, Found: 389.1220 .<smiles>CCOC(=O)c1cnn(C(c2ccc(C(C)C(=O)OC)cc2)C(C)C)c1C(F)(F)F</smiles>

Ethyl 1-(1-(4-(1-methoxy-1-oxopropan-2-yl)phenyl)-2-methylpropyl)-5-(trifluoromethyl)-1H-pyrazole-4carboxylate, 3sa

Reaction run using ibuprofen methyl ester $1 \mathrm{~s}(41.6 \mu \mathrm{L}, 0.2 \mathrm{mmol}, 1.0$ equiv), ethyl 3-(trifluoromethyl)1H-pyrazole-4-carboxylate $\mathbf{2 a}(104.1 \mathrm{mg}, 0.50 \mathrm{mmol}, 2.5$ equiv), and tetrabutylammonium chloride (5.6 $\mathrm{mg}, 0.02 \mathrm{mmol}, 0.1$ equiv) following the general procedure II (glass vial) at $40{ }^{\circ} \mathrm{C}$. . Yield $=55.7 \mathrm{mg}$ $(65 \%)$ of white solid. TLC (pentane:EtOAc, $9: 1 \mathrm{v} / \mathrm{v}$ ): $\mathrm{R}_{\mathrm{f}}=0.45 . \mathrm{Mp}: 83-86^{\circ} \mathrm{C}$.

${ }^{1} \mathrm{H}$ NMR $\left(\mathrm{CDCl}_{3}, 500 \mathrm{MHz}\right): 7.97(\mathrm{~s}, 1 \mathrm{H}), 7.47(\mathrm{~d}, J=8.3 \mathrm{~Hz}, 2 \mathrm{H}), 7.25(\mathrm{~d}, J=8.3 \mathrm{~Hz}, 2 \mathrm{H}), 4.94(\mathrm{~d}, J=$ $10.6 \mathrm{~Hz}, 1 \mathrm{H}), 4.29(\mathrm{q}, J=7.1 \mathrm{~Hz}, 2 \mathrm{H}), 3.70(\mathrm{q}, J=7.2 \mathrm{~Hz}, 1 \mathrm{H}), 3.65(\mathrm{~s}, 3 \mathrm{H}), 2.97-2.86(\mathrm{~m}, 1 \mathrm{H})$, $1.47(\mathrm{~d}, J=7.2 \mathrm{~Hz}, 3 \mathrm{H}), 1.33(\mathrm{t}, J=7.1 \mathrm{~Hz}, 3 \mathrm{H}), 0.81(\mathrm{~d}, J=6.8 \mathrm{~Hz}, 3 \mathrm{H}), 0.79(\mathrm{~d}, J=6.4 \mathrm{~Hz}, 3 \mathrm{H})$ ppm.

${ }^{13} \mathrm{C} \mathrm{NMR}\left(\mathrm{CDCl}_{3}, 126 \mathrm{MHz}\right): 174.8(\mathrm{~d}, J=1.3 \mathrm{~Hz}), 161.3,141.7,140.6(\mathrm{~d}, J=1.0 \mathrm{~Hz}), 136.9,131.9(\mathrm{q}, J$ $=39.8 \mathrm{~Hz}), 128.5,127.6,119.7(\mathrm{q}, J=271.3 \mathrm{~Hz}), 115.1(\mathrm{q}, J=1.5 \mathrm{~Hz}), 72.3(\mathrm{q}, J=2.9 \mathrm{~Hz}), 61.1$, 52.1, 45.1, 33.6, 20.0, 19.7, 18.5, $14.1 \mathrm{ppm}$.

${ }^{19} \mathrm{~F} \mathrm{NMR}\left(\mathrm{CDCl}_{3}, 377 \mathrm{MHz}\right)$ : $-54.9 \mathrm{ppm}$.

HRMS Calculated for $\left[\mathrm{C}_{21} \mathrm{H}_{25} \mathrm{~F}_{3} \mathrm{~N}_{2} \mathrm{O}_{4}+\mathrm{NH}_{4}\right]^{+}$: 444.2105, Found: 444.2101. 


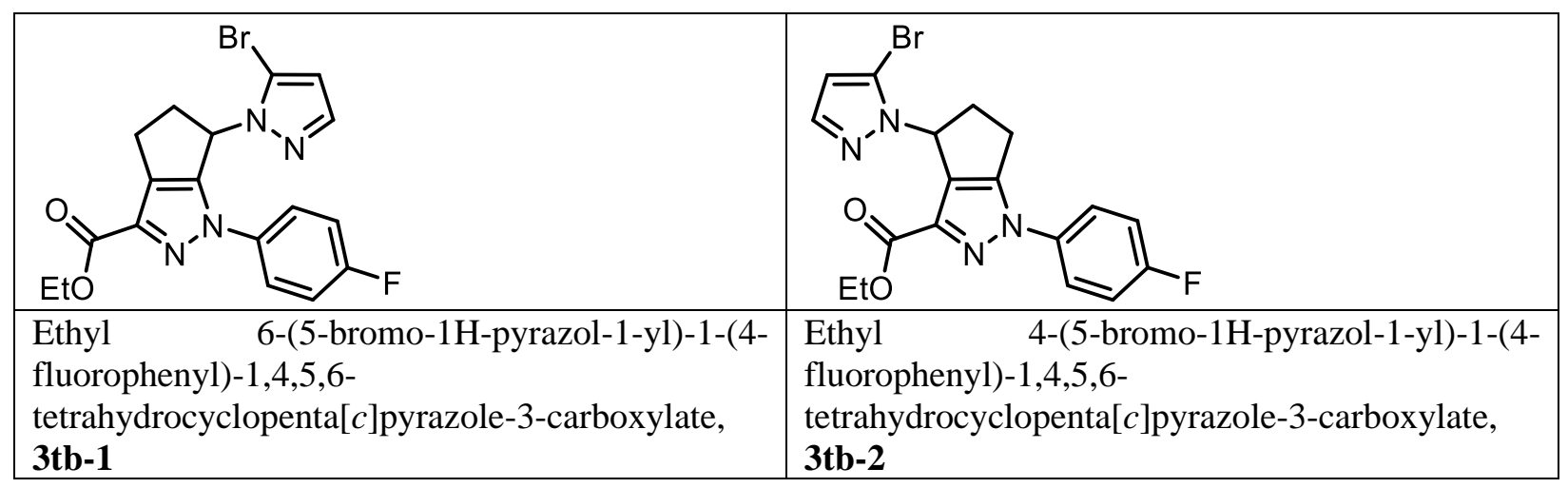

Reaction run ethyl 1-(4-fluorophenyl)-1,4,5,6-tetrahydrocyclopenta[c]pyrazole-3-carboxylate $1 \mathbf{1 t}$ (54.5 mg, $0.2 \mathrm{mmol}, 1.0$ equiv), ethyl 3-(trifluoromethyl)-1H-pyrazole-4-carboxylate 2a (104.1 mg, $0.50 \mathrm{mmol}, 2.5$ equiv), and tetrabutylammonium chloride $(5.6 \mathrm{mg}, 0.02 \mathrm{mmol}, 0.1$ equiv) following the general procedure $\mathrm{I}$ (pressure tube) and two regioisomers were isolated.

3tb-1: Yield $=36.0 \mathrm{mg}(43 \%)$ of light yellow amorphous solid. TLC (pentane:EtOAc, 4:1 v/v): $\mathrm{R}_{\mathrm{f}}=0.17$. ${ }^{1} \mathrm{H} \mathrm{NMR}\left(\mathrm{CDCl}_{3}, 500 \mathrm{MHz}\right): 7.99-7.61(\mathrm{~m}, 2 \mathrm{H}), 7.50(\mathrm{~d}, J=1.9 \mathrm{~Hz}, 1 \mathrm{H}), 7.22-7.10(\mathrm{~m}, 2 \mathrm{H}), 6.30(\mathrm{~d}$, $J=1.9 \mathrm{~Hz}, 1 \mathrm{H}), 6.09(\mathrm{dd}, J=8.4,3.8 \mathrm{~Hz}, 1 \mathrm{H}), 4.23(\mathrm{qt}, J=7.4,3.8 \mathrm{~Hz}, 2 \mathrm{H}), 3.38$ (dddd, $J=15.4$, 8.9, 5.1, $1.3 \mathrm{~Hz}, 1 \mathrm{H}), 3.22$ (dtd, $J=13.6,8.6,5.1 \mathrm{~Hz}, 1 \mathrm{H}), 3.03(\mathrm{ddd}, J=15.4,8.9,4.4 \mathrm{~Hz}, 1 \mathrm{H}), 2.80$ (ddt, $J=13.2,8.6,4.2 \mathrm{~Hz}, 1 \mathrm{H}), 1.18(\mathrm{t}, J=7.1 \mathrm{~Hz}, 3 \mathrm{H}) \mathrm{ppm}$.

${ }^{13} \mathrm{C} \mathrm{NMR}\left(\mathrm{CDCl}_{3}, 126 \mathrm{MHz}\right): 161.5(\mathrm{~d}, J=247.6 \mathrm{~Hz}), 161.4,151.3,140.6,138.3,135.7(\mathrm{~d}, J=3.1 \mathrm{~Hz})$, $129.2,122.2(\mathrm{~d}, J=8.6 \mathrm{~Hz}), 116.3(\mathrm{~d}, J=23.0 \mathrm{~Hz}), 112.4,108.0,60.9,55.8,39.5,25.1,14.2 \mathrm{ppm}$.

${ }^{19} \mathrm{~F} \mathrm{NMR}\left(\mathrm{CDCl}_{3}, 377 \mathrm{MHz}\right)$ : $-114.2 \mathrm{ppm}$.

HRMS Calculated for $\left[\mathrm{C}_{18} \mathrm{H}_{16} \mathrm{BrFN}_{4} \mathrm{O}_{2}+\mathrm{H}\right]^{+}:$419.0513, Found: 419.0508.

3tb-2: Yield $=5.1 \mathrm{mg}(6 \%)$ of light-yellow semisolid. TLC (pentane:EtOAc, 4:1 v/v): $\mathrm{R}_{\mathrm{f}}=0.25$.

${ }^{1} \mathrm{H}$ NMR $\left(\mathrm{CDCl}_{3}, 500 \mathrm{MHz}\right): 7.45(\mathrm{~d}, J=1.9 \mathrm{~Hz}, 1 \mathrm{H}), 7.21-7.14(\mathrm{~m}, 2 \mathrm{H}), 6.97(\mathrm{t}, J=8.6 \mathrm{~Hz}, 2 \mathrm{H}), 6.24$ $(\mathrm{d}, J=1.9 \mathrm{~Hz}, 1 \mathrm{H}), 6.07(\mathrm{q}, J=4.4 \mathrm{~Hz}, 1 \mathrm{H}), 4.42(\mathrm{qd}, J=7.1,1.3 \mathrm{~Hz}, 2 \mathrm{H}), 3.21(\mathrm{qd}, J=7.9,3.8 \mathrm{~Hz}$, $2 \mathrm{H}), 3.02-2.89(\mathrm{~m}, 1 \mathrm{H}), 2.86-2.74(\mathrm{~m}, 1 \mathrm{H}), 1.40(\mathrm{t}, J=7.1 \mathrm{~Hz}, 3 \mathrm{H}) \mathrm{ppm}$.

${ }^{13} \mathrm{C} \mathrm{NMR}\left(\mathrm{CDCl}_{3}, 126 \mathrm{MHz}\right): 162.2,161.8(\mathrm{~d}, J=248.1 \mathrm{~Hz}), 146.6,141.4,138.4,135.1,134.7,123.5(\mathrm{~d}$, $J=8.7 \mathrm{~Hz}), 116.0(\mathrm{~d}, J=23.1 \mathrm{~Hz}), 112.2,108.9,61.0,56.9,40.1,22.4,14.4 \mathrm{ppm}$.

${ }^{19} \mathrm{~F}$ NMR $\left(\mathrm{CDCl}_{3}, 377 \mathrm{MHz}\right)$ : $-113.6 \mathrm{ppm}$.

HRMS Calculated for $\left[\mathrm{C}_{18} \mathrm{H}_{16} \mathrm{BrFN}_{4} \mathrm{O}_{2}+\mathrm{H}\right]^{+}: 419.0513$, Found: 419.0509. 


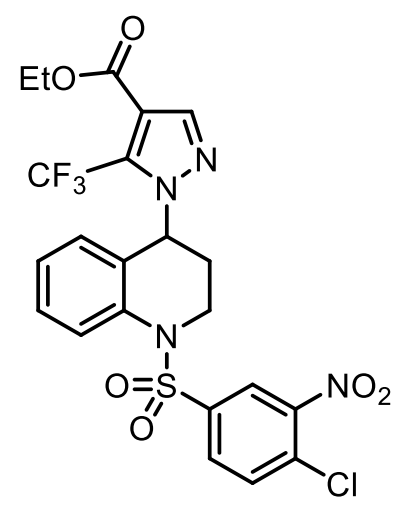

Ethyl 1-(1-((4-chloro-3-nitrophenyl)sulfonyl)-1,2,3,4-tetrahydroquinolin-4-yl)-5-(trifluoromethyl)-1Hpyrazole-4-carboxylate, 3ua

Reaction run using 1-(4-chloro-3-nitrobenzenesulfonyl)-1,2,3,4-tetrahydroquinoline 1u (70.6 mg, 0.2 mmol, 1.0 equiv), ethyl 3-(trifluoromethyl)-1H-pyrazole-4-carboxylate 2a (104.1 mg, $0.50 \mathrm{mmol}, 2.5$ equiv), and tetrabutylammonium chloride $(5.6 \mathrm{mg}, 0.02 \mathrm{mmol}, 0.1$ equiv) following the general procedure $\mathrm{I}$ (pressure tube). Yield $=47.0 \mathrm{mg}(42 \%)$ of yellow semisolid. TLC (pentane:EtOAc, 4:1 v/v): $\mathrm{R}_{\mathrm{f}}=0.40$. ${ }^{1} \mathrm{H} \mathrm{NMR}\left(\mathrm{CDCl}_{3}, 500 \mathrm{MHz}\right): 8.08(\mathrm{~d}, J=2.2 \mathrm{~Hz}, 1 \mathrm{H}), 7.90(\mathrm{dd}, J=8.4,1.1 \mathrm{~Hz}, 1 \mathrm{H}), 7.78(\mathrm{dd}, J=8.5$,

$2.2 \mathrm{~Hz}, 1 \mathrm{H}), 7.71(\mathrm{~s}, 1 \mathrm{H}), 7.63(\mathrm{~d}, J=8.5 \mathrm{~Hz}, 1 \mathrm{H}), 7.35(\mathrm{ddd}, J=8.5,7.2,1.1 \mathrm{~Hz}, 2 \mathrm{H}), 7.14(\mathrm{td}, J=$ 7.6, $1.2 \mathrm{~Hz}, 1 \mathrm{H}), 6.68(\mathrm{~d}, J=7.4 \mathrm{~Hz}, 1 \mathrm{H}), 5.59$ (t, $J=7.2 \mathrm{~Hz}, 1 \mathrm{H}), 4.33(\mathrm{q}, J=7.1 \mathrm{~Hz}, 3 \mathrm{H}), 4.32-$ $4.25(\mathrm{~m}, 1 \mathrm{H}), 3.95$ (ddd, $J=13.9,9.3,3.9 \mathrm{~Hz}, 1 \mathrm{H}), 2.22-2.09(\mathrm{~m}, 2 \mathrm{H}), 1.35(\mathrm{t}, J=7.1 \mathrm{~Hz}, 3 \mathrm{H}) \mathrm{ppm}$. ${ }^{13} \mathrm{C} \mathrm{NMR}\left(\mathrm{CDCl}_{3}, 126 \mathrm{MHz}\right) \delta 160.7,148.0,142.1,139.3,136.2,133.0,132.1$ (q, $\left.J=39.8 \mathrm{~Hz}\right), 132.1$, $131.2,129.3,128.5,127.1,126.5,124.7,124.7,119.5(\mathrm{q}, J=271.4 \mathrm{~Hz}), 115.7,61.4,56.9(\mathrm{q}, J=3.2$ $\mathrm{Hz}), 44.7,28.5,14.1 \mathrm{ppm}$.

${ }^{19} \mathrm{~F}$ NMR $\left(\mathrm{CDCl}_{3}, 377 \mathrm{MHz}\right):-55.93 \mathrm{ppm}$.

HRMS Calculated for $\left[\mathrm{C}_{22} \mathrm{H}_{18} \mathrm{ClF}_{3} \mathrm{~N}_{4} \mathrm{O}_{6} \mathrm{~S}+\mathrm{Na}\right]^{+}:$581.0480, Found: 581.0478 .

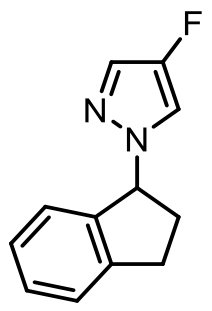

1-(2,3-dihydro-1 $H$-inden-1-yl)-4-fluoro- $1 H$-pyrazole, 3vc

Reaction run using indane $1 \mathbf{v}(24.5 \mu \mathrm{L}, 0.2 \mathrm{mmol}, 1.0$ equiv), 4-fluoropyrazole $2 \mathrm{c}$ (43.0 $\mathrm{mg}, 0.50 \mathrm{mmol}$, 2.5 equiv), and tetrabutylammonium chloride $(5.6 \mathrm{mg}, 0.02 \mathrm{mmol}, 0.1$ equiv) following the general procedure I (pressure tube) at $60{ }^{\circ} \mathrm{C}$. Yield $=33.4 \mathrm{mg}(82 \%)$ of yellow liquid. TLC (pentane:EtOAc, 9:1 $\mathrm{v} / \mathrm{v}): \mathrm{R}_{\mathrm{f}}=0.50$.

${ }^{1} \mathrm{H}$ NMR $\left(\mathrm{CDCl}_{3}, 400 \mathrm{MHz}\right): 7.36(\mathrm{~d}, J=4.4 \mathrm{~Hz}, 1 \mathrm{H}), 7.34-7.28(\mathrm{~m}, 2 \mathrm{H}), 7.22(\mathrm{td}, J=7.0,6.3,2.3 \mathrm{~Hz}$, $1 \mathrm{H}), 7.16(\mathrm{~d}, J=7.5 \mathrm{~Hz}, 1 \mathrm{H}), 7.07(\mathrm{~d}, J=4.7 \mathrm{~Hz}, 1 \mathrm{H}), 5.76$ (t, $J=7.0 \mathrm{~Hz}, 1 \mathrm{H}), 3.12$ (ddd, $J=16.0$, 8.7, $5.2 \mathrm{~Hz}, 1 \mathrm{H}), 2.96(\mathrm{ddd}, J=15.8,8.4,6.6 \mathrm{~Hz}, 1 \mathrm{H}), 2.68(\mathrm{dtd}, J=13.5,8.2,5.2 \mathrm{~Hz}, 1 \mathrm{H}$ ), 2.30 (ddt, $J=13.4,8.7,6.2 \mathrm{~Hz}, 1 \mathrm{H}) \mathrm{ppm}$. 
${ }^{13} \mathrm{C} \mathrm{NMR}\left(\mathrm{CDCl}_{3}, 101 \mathrm{MHz}\right): 149.6(\mathrm{~d}, \mathrm{~J}=246.0 \mathrm{~Hz}), 143.8,140.6,129.0,127.1,126.1$ (d, J = $\left.13.5 \mathrm{~Hz}\right)$, 125.2, 124.8, $113.6(\mathrm{~d}, \mathrm{~J}=27.8 \mathrm{~Hz}), 68.0,33.7,30.4 \mathrm{ppm}$.

${ }^{19} \mathrm{~F}$ NMR $\left(\mathrm{CDCl}_{3}, 377 \mathrm{MHz}\right):-176.5 \mathrm{ppm}$.

HRMS Calculated for $\left[\mathrm{C}_{12} \mathrm{H}_{11} \mathrm{FN}_{2}+\mathrm{H}\right]^{+}:$203.0979, Found: 203.0978.

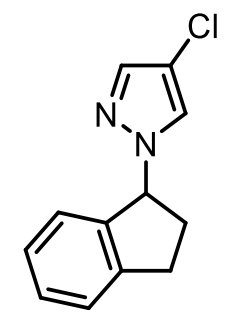

4-chloro-1-(2,3-dihydro-1H-inden-1-yl)-1H-pyrazole, 3vd

Reaction run using indane $1 \mathbf{v}(24.5 \mu \mathrm{L}, 0.2 \mathrm{mmol}, 1.0$ equiv), 4-chloropyrazole $2 \mathbf{2 d}(51.3 \mathrm{mg}, 0.50 \mathrm{mmol}$, 2.5 equiv), and tetrabutylammonium chloride (5.6 $\mathrm{mg}, 0.02 \mathrm{mmol}, 0.1$ equiv) following the general procedure I (pressure tube) at $60^{\circ} \mathrm{C}$. Yield $=28.8 \mathrm{mg}(66 \%)$ of colorless liquid. TLC (pentane:EtOAc, 9:1 $\mathrm{v} / \mathrm{v}): \mathrm{R}_{\mathrm{f}}=0.57$.

${ }^{1} \mathrm{H}$ NMR $\left(\mathrm{CDCl}_{3}, 500 \mathrm{MHz}\right): 7.45$ (s, 1H), 7.32 (m, 2H), 7.23 (ddd, $\left.J=8.0,6.5,2.1 \mathrm{~Hz}, 1 \mathrm{H}\right), 7.17$ (m, $2 \mathrm{H}), 5.81(\mathrm{dd}, J=7.9,5.6 \mathrm{~Hz}, 1 \mathrm{H}), 3.13(\mathrm{ddd}, J=14.4,8.6,5.6 \mathrm{~Hz}, 1 \mathrm{H}), 2.96(\mathrm{ddd}, J=15.7,8.2,6.4$ $\mathrm{Hz}, 1 \mathrm{H}), 2.68$ (dtd, $J=13.6,8.2,5.6 \mathrm{~Hz}, 1 \mathrm{H}), 2.33$ (ddt, $J=11.9,8.6,5.9 \mathrm{~Hz}, 1 \mathrm{H}) \mathrm{ppm}$.

${ }^{13} \mathrm{C} \mathrm{NMR}\left(\mathrm{CDCl}_{3}, 126 \mathrm{MHz}\right): 144.0,140.4,137.7,129.0,127.1,125.6,125.2,124.9,109.8,67.7,33.7$, $30.4 \mathrm{ppm}$.

HRMS Calculated for $\left[\mathrm{C}_{12} \mathrm{H}_{11} \mathrm{ClN}_{2} \mathrm{~N}_{2}+\mathrm{H}\right]^{+}: 219.0684$, Found: 219.0684.

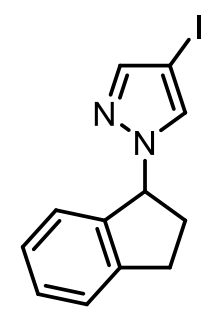

1-(2,3-dihydro- $1 H$-inden-1-yl)-4-iodo- $1 H$-pyrazole, 3ve

Reaction run using indane $1 \mathbf{v}(24.5 \mu \mathrm{L}, 0.2 \mathrm{mmol}, 1.0$ equiv), 4-iodopyrazole $2 \mathbf{e}$ (97.0 mg, $0.50 \mathrm{mmol}, 2.5$ equiv), and tetrabutylammonium chloride $(5.6 \mathrm{mg}, 0.02 \mathrm{mmol}, 0.1$ equiv) following the general procedure $\mathrm{I}$ (pressure tube) at $60^{\circ} \mathrm{C}$. Yield $=37.5 \mathrm{mg}(60 \%)$ of light-yellow liquid. TLC (pentane:EtOAc, 9:1 v/v): $\mathrm{R}_{\mathrm{f}}=0.60$.

${ }^{1} \mathrm{H}$ NMR $\left(\mathrm{CDCl}_{3}, 400 \mathrm{MHz}\right): 7.54(\mathrm{~s}, 1 \mathrm{H}), 7.37-7.28(\mathrm{~m}, 2 \mathrm{H}), 7.27-7.19(\mathrm{~m}, 2 \mathrm{H}), 7.17$ (d, J = 7.6 Hz, $1 \mathrm{H}), 5.87(\mathrm{dd}, J=7.9,5.5 \mathrm{~Hz}, 1 \mathrm{H}), 3.13(\mathrm{ddd}, J=16.0,8.6,5.6 \mathrm{~Hz}, 1 \mathrm{H}), 2.96(\mathrm{ddd}, J=15.7,8.4,6.1$ $\mathrm{Hz}, 1 \mathrm{H}), 2.68(\mathrm{dtd}, J=13.7,8.2,5.6 \mathrm{~Hz}, 1 \mathrm{H}), 2.33$ (ddt, $J=14.1,8.6,5.8 \mathrm{~Hz}, 1 \mathrm{H}) \mathrm{ppm}$.

${ }^{13} \mathrm{C} \mathrm{NMR}\left(\mathrm{CDCl}_{3}, 101 \mathrm{MHz}\right): 144.4,144.0,140.4,132.1,129.1,127.2,125.2,125.0,67.5,56.1,33.9$, $30.4 \mathrm{ppm}$.

HRMS Calculated for $\left[\mathrm{C}_{12} \mathrm{H}_{11} \mathrm{IN}_{2}+\mathrm{H}\right]^{+}: 311.0040$, Found: 311.0036 . 


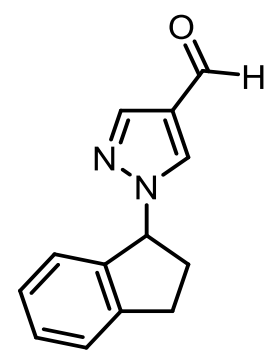

1-(2,3-dihydro- $1 H$-inden-1-yl)-1H-pyrazole-4-carbaldehyde, $3 \mathbf{v f}$

Reaction run using indane $1 \mathbf{v}(24.5 \mu \mathrm{L}, 0.2 \mathrm{mmol}, 1.0$ equiv), pyrazole-4-carboxaldehyde $\mathbf{2 f}$ ( $48.0 \mathrm{mg}$, $0.50 \mathrm{mmol}, 2.5$ equiv), and tetrabutylammonium chloride $(5.6 \mathrm{mg}, 0.02 \mathrm{mmol}, 0.1$ equiv) following the general procedure I (pressure tube) at $60^{\circ} \mathrm{C}$. Yield $=30.2 \mathrm{mg}(71 \%)$ of light-yellow liquid. TLC (pentane:EtOAc, 9:1 v/v): $\mathrm{R}_{\mathrm{f}}=0.17$.

${ }^{1} \mathrm{H}$ NMR $\left(\mathrm{CDCl}_{3}, 400 \mathrm{MHz}\right): 9.80(\mathrm{~s}, 1 \mathrm{H}), 8.01$ (s, 1H), 7.73 (s, 1H), 7.39 - $7.34(\mathrm{~m}, 2 \mathrm{H}), 7.30$ - 7.23 (m,

$1 \mathrm{H}), 7.21(\mathrm{~d}, J=7.6 \mathrm{~Hz}, 1 \mathrm{H}), 5.90(\mathrm{dd}, J=7.9,4.9 \mathrm{~Hz}, 1 \mathrm{H}), 3.16(\mathrm{ddd}, J=15.2,8.5,6.2 \mathrm{~Hz}, 1 \mathrm{H})$,

3.00 (ddd, $J=16.1,8.5,5.4 \mathrm{~Hz}, 1 \mathrm{H}), 2.73$ (dtd, $J=14.2,8.2,6.2 \mathrm{~Hz}, 1 \mathrm{H}), 2.41$ (ddt, $J=13.7,8.5,5.2$

$\mathrm{Hz}, 1 \mathrm{H}) \mathrm{ppm}$.

${ }^{13} \mathrm{C} \mathrm{NMR}\left(\mathrm{CDCl}_{3}, 101 \mathrm{MHz}\right): 184.1,144.3,141.0,139.6,131.1,129.4,127.3,125.4,125.0,124.2,67.5$, 33.7, $30.4 \mathrm{ppm}$.

HRMS Calculated for $\left[\mathrm{C}_{13} \mathrm{H}_{12} \mathrm{~N}_{2} \mathrm{O}+\mathrm{H}\right]^{+}:$213.1022, Found: 213.1021.

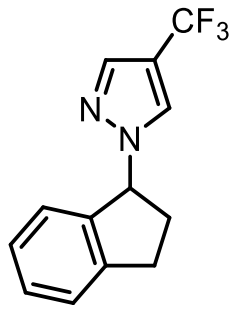

1-(2,3-dihydro-1H-inden-1-yl)-4-(trifluoromethyl)-1H-pyrazole, 3vg

Reaction run using indane $1 \mathbf{v}(24.5 \mu \mathrm{L}, 0.2 \mathrm{mmol}, 1.0$ equiv), 4-trifluoromethylpyrazole $\mathbf{2 g}(68.0 \mathrm{mg}$, $0.50 \mathrm{mmol}, 2.5$ equiv), and tetrabutylammonium chloride $(5.6 \mathrm{mg}, 0.02 \mathrm{mmol}, 0.1$ equiv) following the general procedure $\mathrm{I}$ (pressure tube) at $60^{\circ} \mathrm{C}$. Yield $=36.0 \mathrm{mg}(71 \%)$ of colorless liquid. TLC (pentane:EtOAc, 9:1 v/v): $\mathrm{R}_{\mathrm{f}}=0.61$.

${ }^{1} \mathrm{H} \mathrm{NMR}\left(\mathrm{CDCl}_{3}, 500 \mathrm{MHz}\right): 7.74(\mathrm{~s}, 1 \mathrm{H}), 7.48(\mathrm{~s}, 1 \mathrm{H}), 7.39-7.31(\mathrm{~m}, 2 \mathrm{H}), 7.30-7.21(\mathrm{~m}, 1 \mathrm{H}), 7.19$ (d, $J=7.5 \mathrm{~Hz}, 1 \mathrm{H}), 5.89$ (dd, $J=7.9,5.3 \mathrm{~Hz}, 1 \mathrm{H}), 3.15$ (ddd, $J=15.9,8.6,5.7 \mathrm{~Hz}, 1 \mathrm{H}), 2.99$ (ddd, $J=$ $15.9,8.5,5.9 \mathrm{~Hz}, 1 \mathrm{H}), 2.72(\mathrm{dtd}, J=13.9,8.2,5.8 \mathrm{~Hz}, 1 \mathrm{H}), 2.38(\mathrm{ddt}, J=13.9,8.6,5.6 \mathrm{~Hz}, 1 \mathrm{H}) \mathrm{ppm}$. ${ }^{13} \mathrm{C} \mathrm{NMR}\left(\mathrm{CDCl}_{3}, 126 \mathrm{MHz}\right): 144.1,139.9,137.1$ (q, $\left.J=2.8 \mathrm{~Hz}\right), 129.3,127.3,126.9$ (q, $\left.J=3.6 \mathrm{~Hz}\right)$, $125.3,124.9,122.7(\mathrm{q}, J=265.9 \mathrm{~Hz}), 113.5(\mathrm{q}, J=38.1 \mathrm{~Hz}), 67.5,33.8,30.4 \mathrm{ppm}$.

${ }^{19} \mathrm{~F} \mathrm{NMR}\left(\mathrm{CDCl}_{3}, 377 \mathrm{MHz}\right)$ : $-56.3 \mathrm{ppm}$.

HRMS Calculated for $\left[\mathrm{C}_{13} \mathrm{H}_{11} \mathrm{~F}_{3} \mathrm{~N}_{2}+\mathrm{H}\right]^{+}:$: 253.0947, Found: 253.0944. 


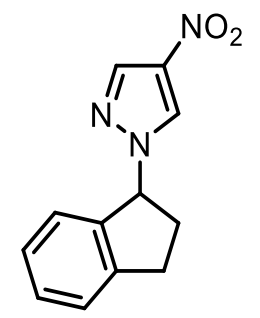

1-(2,3-dihydro-1 $H$-inden-1-yl)-4-nitro-1 $H$-pyrazole, $3 \mathbf{v h}$

Reaction run using indane $1 \mathbf{v}(24.5 \mu \mathrm{L}, 0.2 \mathrm{mmol}, 1.0$ equiv), 4-nitropyrazole $\mathbf{2 h}(56.5 \mathrm{mg}, 0.50 \mathrm{mmol}$, 2.5 equiv), and tetrabutylammonium chloride $(5.6 \mathrm{mg}, 0.02 \mathrm{mmol}, 0.1$ equiv) following the general procedure I (pressure tube) at $60^{\circ} \mathrm{C}$. Yield $=38.2 \mathrm{mg}(83 \%)$ of dark yellow liquid. TLC (pentane:EtOAc, $9: 1 \mathrm{v} / \mathrm{v}): \mathrm{R}_{\mathrm{f}}=0.40$.

${ }^{1} \mathrm{H}$ NMR $\left(\mathrm{CDCl}_{3}, 400 \mathrm{MHz}\right): 8.11(\mathrm{~s}, 1 \mathrm{H}), 7.89(\mathrm{~s}, 1 \mathrm{H}), 7.38(\mathrm{~d}, J=3.9 \mathrm{~Hz}, 2 \mathrm{H}), 7.29$ (dt, $J=8.5,4.2 \mathrm{~Hz}$, $1 \mathrm{H}), 7.23(\mathrm{~d}, J=7.6 \mathrm{~Hz}, 1 \mathrm{H}), 5.87$ (dd, $J=7.9,4.6 \mathrm{~Hz}, 1 \mathrm{H}), 3.17$ (ddd, $J=15.4,8.5,6.5 \mathrm{~Hz}, 1 \mathrm{H})$, $3.02(\mathrm{ddd}, J=16.2,8.6,5.1 \mathrm{~Hz}, 1 \mathrm{H}), 2.74(\mathrm{dtd}, J=14.5,8.2,6.5 \mathrm{~Hz}, 1 \mathrm{H}), 2.41$ (ddt, $J=13.5,8.4,4.9$ $\mathrm{Hz}, 1 \mathrm{H}) \mathrm{ppm}$.

${ }^{13} \mathrm{C} \mathrm{NMR}\left(\mathrm{CDCl}_{3}, 101 \mathrm{MHz}\right)$ : 144.4, 138.8, 136.0, 129.8, 127.5, 127.0, 125.5, 125.0, 68.3, 33.6, $30.3 \mathrm{ppm}$. HRMS Calculated for $\left[\mathrm{C}_{12} \mathrm{H}_{11} \mathrm{~N}_{3} \mathrm{O}_{2}+\mathrm{Na}\right]^{+}:$252.0743, Found: 252.0740 .

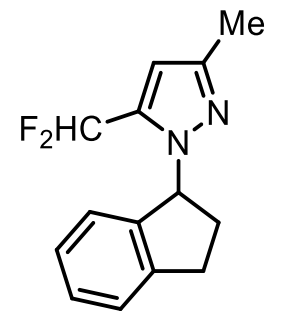

5-(difluoromethyl)-1-(2,3-dihydro-1H-inden-1-yl)-3-methyl-1 $H$-pyrazole, 3vi

Reaction run using indane $\mathbf{1 v}(24.5 \mu \mathrm{L}, 0.2 \mathrm{mmol}, 1.0$ equiv), 3-(difluoromethyl)-5-methyl- $1 H$-pyrazole $2 \mathbf{i}(66.0 \mathrm{mg}, 0.50 \mathrm{mmol}, 2.5$ equiv), and tetrabutylammonium chloride (5.6 mg, $0.02 \mathrm{mmol}, 0.1$ equiv) following the general procedure I (pressure tube) in DCM. Yield $=25.7 \mathrm{mg}(52 \%)$ of while solid. TLC (pentane:EtOAc, 9:1 v/v): $\mathrm{R}_{\mathrm{f}}=0.47 . \mathrm{Mp}: 75-77^{\circ} \mathrm{C}$.

${ }^{1} \mathrm{H} \mathrm{NMR}\left(\mathrm{CDCl}_{3}, 500 \mathrm{MHz}\right): 7.29(\mathrm{~d}, J=7.5 \mathrm{~Hz}, 1 \mathrm{H}), 7.25(\mathrm{t}, J=7.3 \mathrm{~Hz}, 1 \mathrm{H}), 7.16(\mathrm{t}, J=7.3 \mathrm{~Hz}, 1 \mathrm{H})$, $6.99(\mathrm{~d}, J=7.6 \mathrm{~Hz}, 1 \mathrm{H}), 6.65(\mathrm{t}, J=54.1 \mathrm{~Hz}, 1 \mathrm{H}), 6.28(\mathrm{~s}, 1 \mathrm{H}), 5.93(\mathrm{t}, J=7.9 \mathrm{~Hz}, 1 \mathrm{H}), 3.23(\mathrm{ddd}, J$ $=15.9,9.1,3.5 \mathrm{~Hz}, 1 \mathrm{H}), 2.97(\mathrm{dt}, J=16.2,8.3 \mathrm{~Hz}, 1 \mathrm{H}), 2.70-2.62(\mathrm{~m}, 1 \mathrm{H}), 2.57(\mathrm{dq}, J=13.1,8.3$ $\mathrm{Hz}, 1 \mathrm{H}), 2.22$ (s, 3H) ppm.

${ }^{13} \mathrm{C} \mathrm{NMR}\left(\mathrm{CDCl}_{3}, 126 \mathrm{MHz}\right): 148.3,143.3,141.3,136.1(\mathrm{t}, J=26.0 \mathrm{~Hz}), 128.4,126.8,125.1,124.1$, $108.8(\mathrm{t}, J=235.5 \mathrm{~Hz}), 106.4,65.2,32.9,30.5,13.6 \mathrm{ppm}$.

${ }^{19} \mathrm{~F} \mathrm{NMR}\left(\mathrm{CDCl}_{3}, 377 \mathrm{MHz}\right):-109.1(\mathrm{~d}, J=307.2 \mathrm{~Hz}),-111.7(\mathrm{~d}, J=307.2 \mathrm{~Hz}) \mathrm{ppm}$.

HRMS Calculated for $\left[\mathrm{C}_{14} \mathrm{H}_{14} \mathrm{~F}_{2} \mathrm{~N}_{2}+\mathrm{H}\right]^{+}: 249.1198$, Found: 249.1193. 


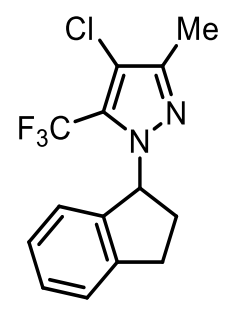

4-chloro-1-(2,3-dihydro-1H-inden-1-yl)-3-methyl-5-(trifluoromethyl)-1 $H$-pyrazole, 3vj

Reaction run using indane $1 \mathbf{v}(24.5 \mu \mathrm{L}, 0.2 \mathrm{mmol}, 1.0$ equiv), 4-Chloro-3-trifluoromethyl-5-(methyl) pyrazole $2 \mathbf{j}$ ( $92.3 \mathrm{mg}, 0.50 \mathrm{mmol}, 2.5$ equiv), and tetrabutylammonium chloride (5.6 $\mathrm{mg}, 0.02 \mathrm{mmol}, 0.1$ equiv) following the general procedure I (pressure tube) in DCM. Yield $=32.7 \mathrm{mg}(55 \%)$ of colorless liquid. TLC (pentane: $\mathrm{Et}_{2} \mathrm{O}, 20: 1 \mathrm{v} / \mathrm{v}$ ): $\mathrm{R}_{\mathrm{f}}=0.56$.

${ }^{1} \mathrm{H}$ NMR $\left(\mathrm{CDCl}_{3}, 500 \mathrm{MHz}\right): 7.30(\mathrm{~d}, J=7.5 \mathrm{~Hz}, 1 \mathrm{H}), 7.30-7.25(\mathrm{~m}, 1 \mathrm{H}), 7.18(\mathrm{t}, J=7.3 \mathrm{~Hz}, 1 \mathrm{H}), 7.00$ $(\mathrm{d}, J=7.6 \mathrm{~Hz}, 1 \mathrm{H}), 5.89$ (t, $J=7.4 \mathrm{~Hz}, 1 \mathrm{H}), 3.26$ (ddd, $J=15.7,9.0,4.3 \mathrm{~Hz}, 1 \mathrm{H}), 2.97$ (dt, $J=15.8$, $7.9 \mathrm{~Hz}, 1 \mathrm{H}), 2.62(\mathrm{dtt}, J=12.4,8.5,4.2 \mathrm{~Hz}, 1 \mathrm{H}), 2.54(\mathrm{ddd}, J=13.1,6.7,1.7 \mathrm{~Hz}, 1 \mathrm{H}), 2.17$ (s, 3H) ppm.

${ }^{13} \mathrm{C}$ NMR ( $\left.\mathrm{CDCl}_{3}, 126 \mathrm{MHz}\right): 146.9,143.5,140.6,128.6,127.8$ (q, $\left.J=37.6 \mathrm{~Hz}\right), 126.9,125.1,124.1$, $120.0(\mathrm{q}, J=270.2 \mathrm{~Hz}), 110.4,65.7(\mathrm{q}, J=2.3 \mathrm{~Hz}), 32.8,30.6,11.3 \mathrm{ppm}$.

${ }^{19} \mathrm{~F} \mathrm{NMR}\left(\mathrm{CDCl}_{3}, 377 \mathrm{MHz}\right):-57.3 \mathrm{ppm}$.

HRMS Calculated for $\left[\mathrm{C}_{14} \mathrm{H}_{12} \mathrm{ClF}_{3} \mathrm{~N}_{2}+\mathrm{Na}\right]^{+}:$323.0533, Found: 323.0529.

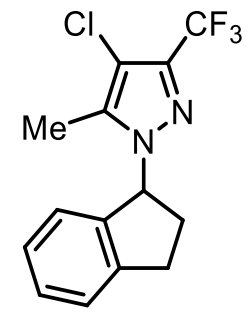

4-chloro-1-(2,3-dihydro-1H-inden-1-yl)-5-methyl-3-(trifluoromethyl)-1 $H$-pyrazole, 3vj'

Reaction run using indane $\mathbf{1 v}(24.5 \mu \mathrm{L}, 0.2 \mathrm{mmol}, 1.0$ equiv), 4-Chloro-3-trifluoromethyl-5-(methyl) pyrazole $2 \mathbf{j}$ ( $92.3 \mathrm{mg}, 0.50 \mathrm{mmol}, 2.5$ equiv), and $\mathrm{BF}_{3} \bullet \mathrm{Et}_{2} \mathrm{O}(2.5 \mu \mathrm{L}, 0.02 \mathrm{mmol}, 0.1$ equiv) following the general procedure $\mathrm{I}$ (pressure tube) at $60^{\circ} \mathrm{C}$. Yield $=27.9 \mathrm{mg}(46 \%)$ of colorless liquid. TLC (pentane:EtOAc, 9:1 v/v): $\mathrm{R}_{\mathrm{f}}=0.63$.

${ }^{1} \mathrm{H} \mathrm{NMR}\left(\mathrm{CDCl}_{3}, 500 \mathrm{MHz}\right): 7.33-7.26(\mathrm{~m}, 2 \mathrm{H}), 7.19(\mathrm{td}, J=7.2,1.6 \mathrm{~Hz}, 1 \mathrm{H}), 6.96(\mathrm{~d}, J=7.6 \mathrm{~Hz}, 1 \mathrm{H})$, 5.90 (t, $J=7.9 \mathrm{~Hz}, 1 \mathrm{H}), 3.23(\mathrm{ddd}, J=16.0,9.2,3.7 \mathrm{~Hz}, 1 \mathrm{H}), 3.01(\mathrm{dt}, J=16.2,8.2 \mathrm{~Hz}, 1 \mathrm{H}), 2.68$ (dtd, $J=13.5,8.5,3.7 \mathrm{~Hz}, 1 \mathrm{H}), 2.50(\mathrm{ddt}, J=13.4,9.3,7.8 \mathrm{~Hz}, 1 \mathrm{H}) \mathrm{ppm}$.

${ }^{13} \mathrm{C} \mathrm{NMR}\left(\mathrm{CDCl}_{3}, 126 \mathrm{MHz}\right): 143.4,140.1,137.7,137.6$ (q, $\left.J=37.3 \mathrm{~Hz}\right), 129.0,127.2,125.4,124.1$, 120.9 (q, $J=269.3 \mathrm{~Hz}), 107.5,66.6,32.1,30.7,9.6 \mathrm{ppm}$.

${ }^{19} \mathrm{~F} \mathrm{NMR}\left(\mathrm{CDCl}_{3}, 377 \mathrm{MHz}\right):-62.3 \mathrm{ppm}$.

HRMS Calculated for $\left[\mathrm{C}_{14} \mathrm{H}_{12} \mathrm{ClF}_{3} \mathrm{~N}_{2}+\mathrm{H}\right]^{+}:$301.0714, Found: 301.0710 . 


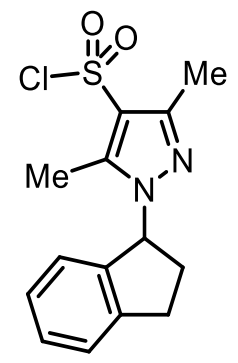

1-(2,3-dihydro-1H-inden-1-yl)-3,5-dimethyl-1 $H$-pyrazole-4-sulfonyl chloride, 3vk

Reaction run using indane $1 \mathbf{v}(24.5 \mu \mathrm{L}, 0.2 \mathrm{mmol}, 1.0$ equiv), 3,5-dimethyl- $1 H$-pyrazole-4-sulfonyl chloride $2 \mathbf{k}$ ( $97.3 \mathrm{mg}, 0.50 \mathrm{mmol}, 2.5$ equiv), and tetrabutylammonium chloride (5.6 $\mathrm{mg}, 0.02 \mathrm{mmol}, 0.1$ equiv) following the general procedure I (pressure tube) in DCM. Yield $=32.7 \mathrm{mg}(53 \%)$ of white solid. TLC (pentane:EtOAc, 9:1 v/v): $\mathrm{R}_{\mathrm{f}}=0.37 . \mathrm{Mp}: 69-71{ }^{\circ} \mathrm{C}$.

${ }^{1} \mathrm{H} \mathrm{NMR}\left(\mathrm{CDCl}_{3}, 500 \mathrm{MHz}\right): 7.33(\mathrm{~d}, J=7.5 \mathrm{~Hz}, 1 \mathrm{H}), 7.30(\mathrm{t}, J=7.4 \mathrm{~Hz}, 1 \mathrm{H}), 7.20(\mathrm{t}, J=7.3 \mathrm{~Hz}, 1 \mathrm{H})$,

$6.95(\mathrm{~d}, J=7.6 \mathrm{~Hz}, 1 \mathrm{H}), 5.83(\mathrm{t}, J=7.7 \mathrm{~Hz}, 1 \mathrm{H}), 3.28(\mathrm{ddd}, J=16.0,9.0,3.9 \mathrm{~Hz}, 1 \mathrm{H}), 3.02(\mathrm{dt}, J=$ 16.0, 8.1 Hz, 1H), $2.66(\mathrm{~m}, 1 \mathrm{H}), 2.63(\mathrm{~s}, 3 \mathrm{H}), 2.57$ (dq, $J=13.2,7.6 \mathrm{~Hz}, 1 \mathrm{H}), 2.42(\mathrm{~s}, 3 \mathrm{H}) \mathrm{ppm}$.

${ }^{13} \mathrm{C} \mathrm{NMR}\left(\mathrm{CDCl}_{3}, 126 \mathrm{MHz}\right): 148.4,143.4,142.9,139.9,128.9,127.1,125.4,123.8,121.6,64.2,32.1$, 30.7, 13.3, $10.9 \mathrm{ppm}$.

HRMS Calculated for $\left[\mathrm{C}_{14} \mathrm{H}_{15} \mathrm{ClN}_{2} \mathrm{O}_{2} \mathrm{~S}+\mathrm{H}\right]^{+}:$311.0616, Found: 311.0609 .

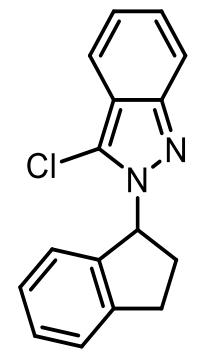

3-chloro-2-(2,3-dihydro-1H-inden-1-yl)-2H-indazole, 3vl

Reaction run using indane $\mathbf{1 v}(24.5 \mu \mathrm{L}, 0.2 \mathrm{mmol}, 1.0$ equiv), 3-chloroindazole $2 \mathrm{l}$ (38.2 $\mathrm{mg}, 0.50 \mathrm{mmol}$, 2.5 equiv), and tetrabutylammonium chloride $(5.6 \mathrm{mg}, 0.02 \mathrm{mmol}, 0.1$ equiv) following the general procedure I (pressure tube) at $60^{\circ} \mathrm{C}$. Yield $=17.2 \mathrm{mg}(32 \%)$ of colorless semisolid. TLC (pentane:EtOAc, $9: 1 \mathrm{v} / \mathrm{v}): \mathrm{R}_{\mathrm{f}}=0.71$.

${ }^{1} \mathrm{H}$ NMR $\left(\mathrm{CDCl}_{3}, 500 \mathrm{MHz}\right): 7.68(\mathrm{~d}, J=8.1 \mathrm{~Hz}, 1 \mathrm{H}), 7.35(\mathrm{~d}, J=7.5 \mathrm{~Hz}, 1 \mathrm{H}), 7.31(\mathrm{~d}, J=7.3 \mathrm{~Hz}, 1 \mathrm{H})$, $7.28(\mathrm{~d}, J=7.2 \mathrm{~Hz}, 1 \mathrm{H}), 7.18(\mathrm{t}, J=7.5 \mathrm{~Hz}, 1 \mathrm{H}), 7.14(\mathrm{t}, J=7.5 \mathrm{~Hz}, 1 \mathrm{H}), 7.07(\mathrm{~d}, J=8.5 \mathrm{~Hz}, 1 \mathrm{H})$, $6.99(\mathrm{~d}, J=7.6 \mathrm{~Hz}, 1 \mathrm{H}), 6.22(\mathrm{t}, J=7.9 \mathrm{~Hz}, 1 \mathrm{H}), 3.27(\mathrm{ddd}, J=15.9,9.1,3.4 \mathrm{~Hz}, 2 \mathrm{H}), 3.06(\mathrm{dt}, J=$ $16.2,8.3 \mathrm{~Hz}, 1 \mathrm{H}), 2.73(\mathrm{ddt}, J=16.8,8.4,4.2 \mathrm{~Hz}, 2 \mathrm{H}), 2.56(\mathrm{dq}, J=13.3,8.2 \mathrm{~Hz}, 1 \mathrm{H}) \mathrm{ppm}$.

${ }^{13} \mathrm{C} \mathrm{NMR}\left(\mathrm{CDCl}_{3}, 126 \mathrm{MHz}\right): 143.3,140.8,140.3,133.0,128.6,127.2,126.9,125.2,124.4,121.8,121.2$, $112.0,110.1,65.3,32.1,30.6 \mathrm{ppm}$.

HRMS Calculated for $\left[\mathrm{C}_{16} \mathrm{H}_{13} \mathrm{ClN}_{2}+\mathrm{H}\right]^{+}:$269.0840, Found: 269.0838 . 


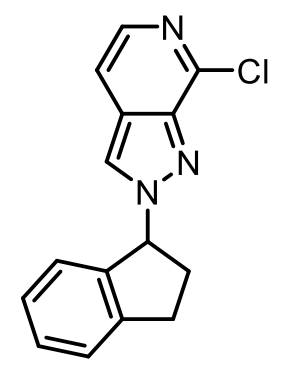

7-chloro-1-(2,3-dihydro-1H-inden-1-yl)-1H-pyrazolo[3,4-c]pyridine, 3vm

Reaction run using indane $\mathbf{1 v}(24.5 \mu \mathrm{L}, 0.2 \mathrm{mmol}, 1.0$ equiv), 7-Chloro-1H-pyrazolo[3,4-c]pyridine $\mathbf{2 m}$ (76.8 $\mathrm{mg}, 0.50 \mathrm{mmol}, 2.5$ equiv), and tetrabutylammonium chloride (5.6 mg, $0.02 \mathrm{mmol}, 0.1$ equiv) following the general procedure I (pressure tube) at $60^{\circ} \mathrm{C}$. Yield $=16.0 \mathrm{mg}(30 \%)$ of yellow semisolid. TLC (DCM:MeOH, 19:1 v/v): $\mathrm{R}_{\mathrm{f}}=0.60$.

${ }^{1} \mathrm{H}$ NMR $\left(\mathrm{CDCl}_{3}, 500 \mathrm{MHz}\right): 7.90(\mathrm{~d}, J=5.8 \mathrm{~Hz}, 1 \mathrm{H}), 7.70(\mathrm{~s}, 1 \mathrm{H}), 7.44-7.37(\mathrm{~m}, 2 \mathrm{H}), 7.36(\mathrm{~d}, J=6.0$

$\mathrm{Hz}, 1 \mathrm{H}), 7.31-7.22(\mathrm{~m}, 3 \mathrm{H}), 6.31(\mathrm{dd}, J=8.1,4.7 \mathrm{~Hz}, 1 \mathrm{H}), 3.21(\mathrm{ddd}, J=15.3,8.4,6.4 \mathrm{~Hz}, 1 \mathrm{H})$,

3.07 (ddd, $J=16.2,8.6,5.2 \mathrm{~Hz}, 1 \mathrm{H}$ ), 2.90 (dtd, $J=14.5,8.3,6.3 \mathrm{~Hz}, 1 \mathrm{H}$ ), 2.50 (ddt, $J=13.6,8.6,5.0$ $\mathrm{Hz}, 1 \mathrm{H}) \mathrm{ppm}$.

${ }^{13} \mathrm{C} \mathrm{NMR}\left(\mathrm{CDCl}_{3}, 126 \mathrm{MHz}\right): 144.5,142.9,142.9,139.5,137.2,129.7,127.5,125.6,125.5,125.3,122.3$, 113.6, 69.5, 34.7, $30.5 \mathrm{ppm}$.

HRMS Calculated for $\left[\mathrm{C}_{15} \mathrm{H}_{12} \mathrm{ClN}_{3}+\mathrm{Na}\right]^{+}:$292.0612, Found: 292.0607.

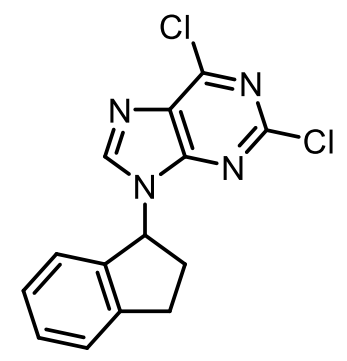

2,6-dichloro-9-(2,3-dihydro-1H-inden-1-yl)-9H-purine, 3vn

Reaction run using indane $1 \mathbf{v}(24.5 \mu \mathrm{L}, 0.2 \mathrm{mmol}, 1.0$ equiv), 2,6-dichloropurine $\mathbf{2 n}(94.5 \mathrm{mg}, 0.50 \mathrm{mmol}$, 2.5 equiv), and tetrabutylammonium chloride $(5.6 \mathrm{mg}, 0.02 \mathrm{mmol}, 0.1$ equiv) following the general procedure I (pressure tube) in DCM. Yield $=21.5 \mathrm{mg}(35 \%)$ of white solid. TLC (pentane:EtOAc, 4:1 $\mathrm{v} / \mathrm{v}): \mathrm{R}_{\mathrm{f}}=0.26 . \mathrm{Mp}: 140-143{ }^{\circ} \mathrm{C} . \mathbf{3 v n}$ ' $(13.9 \mathrm{mg}, 23 \%)$ was also isolated in this reaction.

${ }^{1} \mathrm{H}$ NMR $\left(\mathrm{CDCl}_{3}, 400 \mathrm{MHz}\right): 7.79(\mathrm{~s}, 1 \mathrm{H}), 7.46-7.35(\mathrm{~m}, 2 \mathrm{H}), 7.27$ (d, $\left.J=7.7 \mathrm{~Hz}, 1 \mathrm{H}\right), 7.16(\mathrm{~d}, J=7.6$

$\mathrm{Hz}, 1 \mathrm{H}), 6.25(\mathrm{dd}, J=7.8,5.5 \mathrm{~Hz}, 1 \mathrm{H}), 3.22(\mathrm{ddd}, J=14.7,8.5,5.9 \mathrm{~Hz}, 1 \mathrm{H}), 3.10$ (ddd, $J=16.0,8.2$, $6.4 \mathrm{~Hz}, 1 \mathrm{H}), 2.87(\mathrm{dtd}, J=13.9,8.1,5.8 \mathrm{~Hz}, 1 \mathrm{H}), 2.28(\mathrm{ddt}, J=14.0,8.5,5.7 \mathrm{~Hz}, 1 \mathrm{H}) \mathrm{ppm}$.

${ }^{13} \mathrm{C} \mathrm{NMR}\left(\mathrm{CDCl}_{3}, 101 \mathrm{MHz}\right)$ : 153.2, 153.0, 151.8, 144.3, 144.1, 138.7, 131.0, 129.8, 127.7, 125.6, 124.6, 59.9, 34.1, 30.5 ppm.

HRMS Calculated for $\left[\mathrm{C}_{14} \mathrm{H}_{10} \mathrm{Cl}_{2} \mathrm{~N}_{4}+\mathrm{H}\right]^{+}:$305.0355, Found: 305.0353 . 


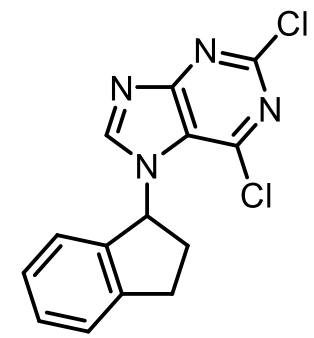

2,6-dichloro-7-(2,3-dihydro-1H-inden-1-yl)-7H-purine, 3vn'

Reaction run using indane $1 \mathbf{v}(24.5 \mu \mathrm{L}, 0.2 \mathrm{mmol}, 1.0$ equiv), 2,6-dichloropurine $2 \mathbf{n}(94.5 \mathrm{mg}, 0.50 \mathrm{mmol}$, 2.5 equiv), and $\mathrm{BF}_{3} \bullet \mathrm{Et}_{2} \mathrm{O}(2.5 \mu \mathrm{L}, 0.02 \mathrm{mmol}, 0.1$ equiv) following the general procedure $\mathrm{I}$ (pressure tube) at $60^{\circ} \mathrm{C}$. Yield $=38.5 \mathrm{mg}(63 \%)$ of white solid. TLC (pentane:EtOAc, $\left.4: 1 \mathrm{v} / \mathrm{v}\right): \mathrm{R}_{\mathrm{f}}=0.11 . \mathrm{Mp}: 167-$ $170{ }^{\circ} \mathrm{C}$.

${ }^{1} \mathrm{H}$ NMR $\left(\mathrm{CDCl}_{3}, 400 \mathrm{MHz}\right): 7.79(\mathrm{~s}, 1 \mathrm{H}), 7.54-7.41(\mathrm{~m}, 2 \mathrm{H}), 7.36(\mathrm{dt}, J=8.3,4.0 \mathrm{~Hz}, 1 \mathrm{H}), 7.31(\mathrm{~d}, J=$ $7.7 \mathrm{~Hz}, 1 \mathrm{H}), 6.47(\mathrm{dd}, J=7.6,4.2 \mathrm{~Hz}, 1 \mathrm{H}), 3.36-3.00(\mathrm{~m}, 2 \mathrm{H}), 2.87$ (dq, $J=15.1,7.7 \mathrm{~Hz}, 1 \mathrm{H}), 2.28$ (ddt, $J=13.1,8.4,4.6 \mathrm{~Hz}, 1 \mathrm{H}$ ) ppm.

${ }^{13} \mathrm{C} \mathrm{NMR}\left(\mathrm{CDCl}_{3}, 101 \mathrm{MHz}\right): 164.0,157.9,153.2,148.4,144.7,143.8,137.5,130.3,128.0,125.9,125.2$, $62.8,35.5,30.1 \mathrm{ppm}$.

HRMS Calculated for $\left[\mathrm{C}_{14} \mathrm{H}_{10} \mathrm{Cl}_{2} \mathrm{~N}_{4}+\mathrm{Na}\right]^{+}: 327.0175$, Found: 327.0173.

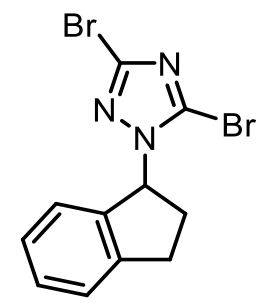

3,5-dibromo-1-(2,3-dihydro-1H-inden-1-yl)-1H-1,2,4-triazole, 3vo

Reaction run using indane $\mathbf{1 v}(24.5 \mu \mathrm{L}, 0.2 \mathrm{mmol}, 1.0$ equiv), 3,5-dibromo-1H-1,2,4-triazole $2 \mathrm{o}$ (113.4 $\mathrm{mg}, 0.50 \mathrm{mmol}, 2.5$ equiv), and tetrabutylammonium chloride (5.6 mg, $0.02 \mathrm{mmol}, 0.1$ equiv) following the general procedure I (pressure tube) at $60^{\circ} \mathrm{C}$. Yield $=53.1 \mathrm{mg}(77 \%)$ of white solid. TLC (pentane:EtOAc, 9:1 v/v): $\mathrm{R}_{\mathrm{f}}=0.52 . \mathrm{Mp}: 83-86^{\circ} \mathrm{C}$.

${ }^{1} \mathrm{H}$ NMR ( $\left.\mathrm{CDCl}_{3}+\mathrm{DCM}, 500 \mathrm{MHz}\right): 7.37-7.28(\mathrm{~m}, 2 \mathrm{H}), 7.24-7.16(\mathrm{~m}, 1 \mathrm{H}), 7.08(\mathrm{~d}, J=7.6 \mathrm{~Hz}, 1 \mathrm{H})$,

6.01 (dd, $J=8.2,6.0 \mathrm{~Hz}, 1 \mathrm{H}), 3.32$ (ddd, $J=15.9,8.9,4.9 \mathrm{~Hz}, 1 \mathrm{H}), 3.01$ (ddd, $J=15.6,8.5,6.6 \mathrm{~Hz}$,

$1 \mathrm{H}), 2.67(\mathrm{dtd}, J=13.4,8.4,5.0 \mathrm{~Hz}, 1 \mathrm{H}), 2.56(\mathrm{ddt}, J=13.2,8.9,6.3 \mathrm{~Hz}, 1 \mathrm{H}) \mathrm{ppm}$.

${ }^{13} \mathrm{C} \mathrm{NMR}\left(\mathrm{CDCl}_{3}, 126 \mathrm{MHz}\right)$ : 143.8, 140.4, 139.2, 129.3, 129.0, 127.1, 125.3, 124.1, 64.9, 31.9, $30.7 \mathrm{ppm}$. HRMS Calculated for $\left[\mathrm{C}_{11} \mathrm{H}_{9} \mathrm{Br}_{2} \mathrm{~N}_{3}+\mathrm{H}\right]^{+}:$341.9236, Found: 341.9233 . 


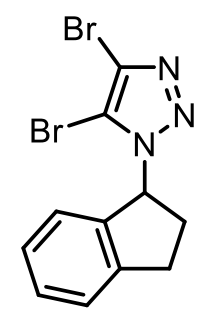

4,5-dibromo-1-(2,3-dihydro-1H-inden-1-yl)-1H-1,2,3-triazole, 3vp

Reaction run using indane $1 \mathbf{v}(24.5 \mu \mathrm{L}, 0.2 \mathrm{mmol}, 1.0$ equiv), 4,5-dibromo-1H-1,2,3-triazole $2 \mathbf{p}$ (113.4 $\mathrm{mg}, 0.50 \mathrm{mmol}, 2.5$ equiv), and tetrabutylammonium chloride (5.6 mg, $0.02 \mathrm{mmol}, 0.1$ equiv) following the general procedure I (pressure tube) in DCM. Yield $=25.4 \mathrm{mg}(37 \%)$ of colorless liquid. TLC (pentane:EtOAc, 9:1 v/v): $R_{\mathrm{f}}=0.47 .3 \mathrm{vp}$ ( $59 \mathrm{mg}, 8 \%$ ) was also isolated in this reaction.

${ }^{1} \mathrm{H} \mathrm{NMR}\left(\mathrm{CDCl}_{3}, 500 \mathrm{MHz}\right): 7.55-7.28(\mathrm{~m}, 2 \mathrm{H}), 7.21(\mathrm{t}, J=7.0 \mathrm{~Hz}, 1 \mathrm{H}), 7.11(\mathrm{~d}, J=7.7 \mathrm{~Hz}, 1 \mathrm{H}), 6.19$ (dd, $J=8.4,5.7 \mathrm{~Hz}, 1 \mathrm{H}), 3.38(\mathrm{ddd}, J=15.8,8.9,5.3 \mathrm{~Hz}, 1 \mathrm{H}), 3.07$ (ddd, $J=15.7,8.6,6.1 \mathrm{~Hz}, 1 \mathrm{H}$ ), 2.77 (dtd, $J=13.9,8.5,5.4 \mathrm{~Hz}, 1 \mathrm{H}), 2.63$ (ddt, $J=14.4,8.8,5.9 \mathrm{~Hz}, 1 \mathrm{H}) \mathrm{ppm}$.

${ }^{13} \mathrm{C} \mathrm{NMR}\left(\mathrm{CDCl}_{3}, 126 \mathrm{MHz}\right)$ : 144.0, 138.9, 129.4, 127.2, 125.3, 124.4, 123.4, 111.7, 66.1, 32.2, $30.9 \mathrm{ppm}$. HRMS Calculated for $\left[\mathrm{C}_{11} \mathrm{H}_{9} \mathrm{Br}_{2} \mathrm{~N}_{3}+\mathrm{Na}\right]^{+}: 363.9055$, Found: 363.9053 .

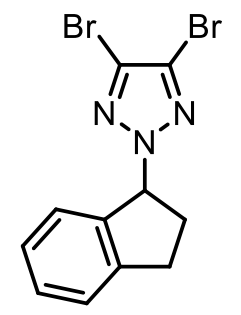

4,5-dibromo-2-(2,3-dihydro-1H-inden-1-yl)-2H-1,2,3-triazole, 3vp'

Reaction run using indane $\mathbf{1 v}(24.5 \mu \mathrm{L}, 0.2 \mathrm{mmol}, 1.0$ equiv), 4,5-dibromo-1H-1,2,3-triazole $\mathbf{2 p}$ (113.4 $\mathrm{mg}, 0.50 \mathrm{mmol}, 2.5$ equiv), and $\mathrm{BF}_{3} \bullet \mathrm{Et}_{2} \mathrm{O}(2.5 \mu \mathrm{L}, 0.02 \mathrm{mmol}, 0.1$ equiv) following the general procedure I (pressure tube) at $60^{\circ} \mathrm{C}$ in DCM. Yield $=5.9 \mathrm{mg}(8 \%)$ of colorless liquid. TLC (pentane:EtOAc, 9:1 v/v): $\mathrm{R}_{\mathrm{f}}=0.73$.

${ }^{1} \mathrm{H} \mathrm{NMR}\left(\mathrm{CDCl}_{3}, 500 \mathrm{MHz}\right): 7.34-7.30(\mathrm{~m}, 1 \mathrm{H}), 7.24-7.18(\mathrm{~m}, 1 \mathrm{H}), 6.10(\mathrm{dd}, J=7.5,5.9 \mathrm{~Hz}, 1 \mathrm{H})$, $3.48-3.22(\mathrm{~m}, 1 \mathrm{H}), 3.10-2.94(\mathrm{~m}, 1 \mathrm{H}), 2.90-2.57(\mathrm{~m}, 1 \mathrm{H})$. ppm.

${ }^{13} \mathrm{C} \mathrm{NMR}\left(\mathrm{CDCl}_{3}, 126 \mathrm{MHz}\right)$ : 144.0, 138.9, 129.4, 127.2, 125.3, 124.4, 123.4, 111.7, 66.1, 32.2, 30.9 ppm. HRMS Calculated for $\left[\mathrm{C}_{11} \mathrm{H}_{9} \mathrm{Br}_{2} \mathrm{~N}_{3}+\mathrm{Na}\right]^{+}: 363.9052$, Found: 363.9055 . 


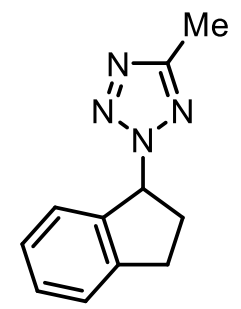

2-(2,3-dihydro-1H-inden-1-yl)-5-methyl-2H-tetrazole, 3vq

Reaction run using indane $1 \mathbf{v}(24.5 \mu \mathrm{L}, 0.2 \mathrm{mmol}, 1.0$ equiv), 5-methyl tetrazole $\mathbf{2 q}$ ( $42.0 \mathrm{mg}, 0.50 \mathrm{mmol}$, 2.5 equiv), and tetrabutylammonium chloride $(5.6 \mathrm{mg}, 0.02 \mathrm{mmol}, 0.1$ equiv) following the general procedure I (pressure tube) at $60^{\circ} \mathrm{C}$ in DCM. Yield $=20.0 \mathrm{mg}(50 \%)$ of colorless liquid. TLC (pentane: $\left.\mathrm{Et}_{2} \mathrm{O}, 20: 1 \mathrm{v} / \mathrm{v}\right): \mathrm{R}_{\mathrm{f}}=0.30 .3 \mathrm{vq}(3.6 \mathrm{mg}, 18 \%)$ was also isolated in this reaction.

${ }^{1} \mathrm{H}$ NMR $\left(\mathrm{CDCl}_{3}, 400 \mathrm{MHz}\right): 7.56$ - $7.29(\mathrm{~m}, 2 \mathrm{H}), 7.24-6.93(\mathrm{~m}, 2 \mathrm{H}), 6.36(\mathrm{dd}, J=8.1,5.2 \mathrm{~Hz}, 1 \mathrm{H})$,

3.37 (ddd, $J=15.3,8.6,6.1 \mathrm{~Hz}, 1 \mathrm{H}), 3.07$ (ddd, $J=15.9,8.4,5.8 \mathrm{~Hz}, 1 \mathrm{H}), 2.88-2.64(\mathrm{~m}, 2 \mathrm{H}), 2.50$ (s, 3H) ppm.

${ }^{13} \mathrm{C} \mathrm{NMR}\left(\mathrm{CDCl}_{3}, 101 \mathrm{MHz}\right): 163.1,144.0,139.2,129.4,127.1,125.2,124.8,68.0,32.1,30.8,11.0 \mathrm{ppm}$. HRMS Calculated for $\left[\mathrm{C}_{11} \mathrm{H}_{12} \mathrm{~N}_{4}+\mathrm{H}\right]^{+}:$201.1135, Found: 201.1132.<smiles>Cc1nnnn1C1CCc2ccccc21</smiles>

1-(2,3-dihydro-1H-inden-1-yl)-5-methyl-1H-tetrazole, 3vq'

Reaction run using indane $\mathbf{1 v}(24.5 \mu \mathrm{L}, 0.2 \mathrm{mmol}, 1.0$ equiv), 5-methyl tetrazole $\mathbf{2 q}$ (42.0 $\mathrm{mg}, 0.50 \mathrm{mmol}$, 2.5 equiv), and $\mathrm{BF}_{3} \bullet \mathrm{Et}_{2} \mathrm{O}(2.5 \mu \mathrm{L}, 0.02 \mathrm{mmol}, 0.1$ equiv) following the general procedure I (pressure tube) at $60^{\circ} \mathrm{C}$. Yield $=16.7 \mathrm{mg}(42 \%)$ of colorless liquid. TLC (pentane:Et $\left.t_{2} \mathrm{O}, 20: 1 \mathrm{v} / \mathrm{v}\right): \mathrm{R}_{\mathrm{f}}=0.30 .3 \mathrm{vq}(15.2$ $\mathrm{mg}, 37 \%)$ was also isolated in this reaction.

${ }^{1} \mathrm{H} \mathrm{NMR}\left(\mathrm{CDCl}_{3}, 600 \mathrm{MHz}\right): 7.37(\mathrm{~d}, J=7.6 \mathrm{~Hz}, 1 \mathrm{H}), 7.34(\mathrm{t}, J=7.3 \mathrm{~Hz}, 1 \mathrm{H}), 7.22(\mathrm{t}, J=7.3 \mathrm{~Hz}, 0 \mathrm{H})$, $6.99(\mathrm{~d}, J=7.7 \mathrm{~Hz}, 1 \mathrm{H}), 6.13(\mathrm{dd}, J=8.4,6.2 \mathrm{~Hz}, 1 \mathrm{H}), 3.33(\mathrm{ddd}, J=16.2,9.0,4.8 \mathrm{~Hz}, 1 \mathrm{H}), 3.09$ (ddd, $J=15.9,8.8,6.5 \mathrm{~Hz}, 1 \mathrm{H}), 2.81(\mathrm{dtd}, J=13.5,8.5,4.8 \mathrm{~Hz}, 1 \mathrm{H}), 2.49-2.40(\mathrm{~m}, 1 \mathrm{H}), 2.43$ (s, 3H) ppm.

${ }^{13} \mathrm{C} \mathrm{NMR}\left(\mathrm{CDCl}_{3}, 151 \mathrm{MHz}\right)$ : 150.9, 143.6, 138.5, 129.5, 127.4, 125.5, 124.1, 63.1, 32.6, 30.7, $9.5 \mathrm{ppm}$. HRMS Calculated for $\left[\mathrm{C}_{11} \mathrm{H}_{12} \mathrm{~N}_{4}+\mathrm{Na}\right]^{+}: 223.0954$, Found: 223.0952. 


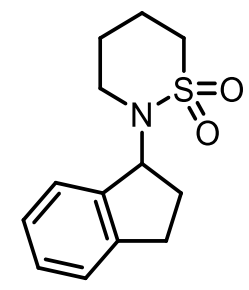

2-(2,3-dihydro-1H-inden-1-yl)-1,2-thiazinane 1,1-dioxide, 3vr

Reaction run using indane $\mathbf{1 v}(24.5 \mu \mathrm{L}, 0.2 \mathrm{mmol}, 1.0$ equiv), 1,4-propanesultam $2 \mathbf{r}(67.5 \mathrm{mg}, 0.50 \mathrm{mmol}$, 2.5 equiv), and tetrabutylammonium chloride $(5.6 \mathrm{mg}, 0.02 \mathrm{mmol}, 0.1$ equiv) following the general procedure I (pressure tube) in DCM. Yield $=23.5 \mathrm{mg}(47 \%)$ of colorless liquid. TLC (pentane:EtOAc, 9:1 v/v): $\mathrm{R}_{\mathrm{f}}=0.13$.

${ }^{1} \mathrm{H}$ NMR $\left(\mathrm{CDCl}_{3}, 500 \mathrm{MHz}\right): 7.40-7.30(\mathrm{~m}, 1 \mathrm{H}), 7.28-7.19(\mathrm{~m}, 3 \mathrm{H}), 5.67(\mathrm{t}, J=8.0 \mathrm{~Hz}, 1 \mathrm{H}), 3.24-$ $3.11(\mathrm{~m}, 2 \mathrm{H}), 3.07(\mathrm{ddd}, J=16.7,8.8,4.4 \mathrm{~Hz}, 1 \mathrm{H}), 3.03-2.90(\mathrm{~m}, 2 \mathrm{H}), 2.85(\mathrm{dt}, J=16.3,8.4 \mathrm{~Hz}$, $1 \mathrm{H}), 2.46-2.34(\mathrm{~m}, 1 \mathrm{H}), 2.31-2.15(\mathrm{~m}, 2 \mathrm{H}), 2.03(\mathrm{dq}, J=13.3,8.5 \mathrm{~Hz}, 1 \mathrm{H}), 1.64$ (ddtq, $J=22.3$, 13.6, 9.0, 4.1 Hz, 2H) ppm.

${ }^{13} \mathrm{C} \mathrm{NMR}\left(\mathrm{CDCl}_{3}, 126 \mathrm{MHz}\right): 143.6,140.3,128.1,126.8,124.9,124.6,59.7,51.1,43.8,30.4,29.3,24.9$, $24.2 \mathrm{ppm}$.

HRMS Calculated for $\left[\mathrm{C}_{13} \mathrm{H}_{17} \mathrm{NO}_{2} \mathrm{~S}+\mathrm{Na}\right]^{+}:$274.0872, Found: 274.0868 .

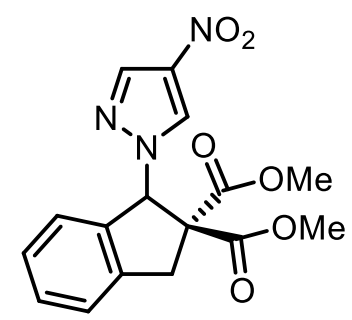

Dimethyl 1-(4-nitro-1H-pyrazol-1-yl)-1,3-dihydro-2H-indene-2,2-dicarboxylate, 3wh

Reaction run using 2,2-dimethyl 1,3-dihydroindene-2,2-dicarboxylate $1 \mathbf{w}$ (46.9 mg, $0.2 \mathrm{mmol}, 1.0$ equiv), 4-Nitropyrazole $\mathbf{2 h}$ (56.0 mg, $0.50 \mathrm{mmol}, 2.5$ equiv), and tetrabutylammonium chloride (5.6 mg, 0.02 mmol, 0.1 equiv) following the general procedure I (pressure tube). Yield $=44.7 \mathrm{mg}(65 \%)$ of yellow semisolid. TLC (pentane:EtOAc, 9:1 v/v): $\mathrm{R}_{\mathrm{f}}=0.10$.

${ }^{1} \mathrm{H} \mathrm{NMR}\left(\mathrm{CDCl}_{3}, 400 \mathrm{MHz}\right): 8.03(\mathrm{dd}, J=10.6,0.6 \mathrm{~Hz}, 1 \mathrm{H}), 7.42(\mathrm{td}, J=7.4,1.2 \mathrm{~Hz}, 1 \mathrm{H}), 7.37(\mathrm{~d}, J=$ $7.4 \mathrm{~Hz}, 1 \mathrm{H}), 7.32(\mathrm{t}, J=7.6 \mathrm{~Hz}, 1 \mathrm{H}), 7.22(\mathrm{~d}, J=7.6 \mathrm{~Hz}, 1 \mathrm{H}), 6.60(\mathrm{~s}, 1 \mathrm{H}), 4.09(\mathrm{~d}, J=16.8 \mathrm{~Hz}, 1 \mathrm{H})$, $3.79(\mathrm{~s}, 3 \mathrm{H}), 3.51(\mathrm{~s}, 3 \mathrm{H}), 3.47(\mathrm{~d}, J=16.9 \mathrm{~Hz}, 1 \mathrm{H}) \mathrm{ppm}$.

${ }^{13} \mathrm{C}$ NMR $\left(\mathrm{CDCl}_{3}, 101 \mathrm{MHz}\right): 170.1,167.6,140.6,136.3,136.1,135.9,130.5,129.4,128.3,125.23$, $125.18,71.3,65.0,53.6,53.3,38.8$. ppm.

HRMS Calculated for $\left[\mathrm{C}_{16} \mathrm{H}_{15} \mathrm{~N}_{3} \mathrm{O}_{6} \mathrm{~S}+\mathrm{Na}\right]^{+}: 368.0853$, Found: 368.0846. 


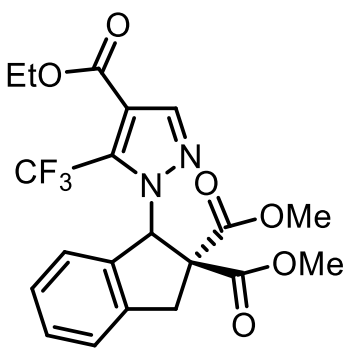

Dimethyl 1-(4-(ethoxycarbonyl)-5-(trifluoromethyl)-1H-pyrazol-1-yl)-1,3-dihydro-2H-indene-2,2dicarboxylate, 3wa

Reaction run using 2,2-dimethyl 1,3-dihydroindene-2,2-dicarboxylate $1 \mathbf{w}$ (46.9 mg, 0.2 mmol, 1.0 equiv), ethyl 3-(trifluoromethyl)-1H-pyrazole-4-carboxylate $2 \mathrm{a}\left(104.1 \mathrm{mg}, 0.50 \mathrm{mmol}, 2.5\right.$ equiv), and $\mathrm{BF}_{3} \bullet \mathrm{Et}_{2} \mathrm{O}$ ( $2.5 \mu \mathrm{L}, 0.02 \mathrm{mmol}, 0.1$ equiv) following the general procedure I (pressure tube) at $60^{\circ} \mathrm{C}$ in DCM. Yield $=45.1 \mathrm{mg}(51 \%)$ of yellow semisolid. TLC (pentane:EtOAc, 9:1 v/v): $\mathrm{R}_{\mathrm{f}}=0.20$.

${ }^{1} \mathrm{H} \mathrm{NMR}\left(\mathrm{CDCl}_{3}, 400 \mathrm{MHz}\right): 7.79$ (s, 1H), 7.41 - 7.29 (m, 2H), $7.25-7.16$ (m, 2H), $6.82(\mathrm{~s}, 1 \mathrm{H}), 4.39$ (d, $J=16.8 \mathrm{~Hz}, 1 \mathrm{H}), 4.31(\mathrm{q}, J=7.1 \mathrm{~Hz}, 2 \mathrm{H}), 3.76(\mathrm{~s}, 3 \mathrm{H}), 3.51(\mathrm{~d}, J=16.8 \mathrm{~Hz}, 1 \mathrm{H}), 3.46(\mathrm{~s}, 3 \mathrm{H}), 1.34$ $(\mathrm{t}, J=7.1 \mathrm{~Hz}, 3 \mathrm{H}) \mathrm{ppm}$.

${ }^{13} \mathrm{C} \mathrm{NMR}\left(\mathrm{CDCl}_{3}, 101 \mathrm{MHz}\right): 170.1,167.4,160.9,142.6,140.9,138.4,132.5$ (q, $\left.J=39.9 \mathrm{~Hz}\right), 129.9$, 127.8, 125.0, 124.6, $119.6(\mathrm{q}, J=271.8 \mathrm{~Hz}), 115.4,68.4(\mathrm{q}, J=4.3 \mathrm{~Hz}), 65.2,61.2,53.7,53.0,39.9$, $14.1 \mathrm{ppm}$.

${ }^{19} \mathrm{~F} \mathrm{NMR}\left(\mathrm{CDCl}_{3}, 377 \mathrm{MHz}\right):-56.6 \mathrm{ppm}$.

HRMS Calculated for $\left[\mathrm{C}_{20} \mathrm{H}_{19} \mathrm{~F}_{3} \mathrm{~N}_{2} \mathrm{O}_{6}+\mathrm{H}\right]^{+}:$441.1268, Found: 441.1262.

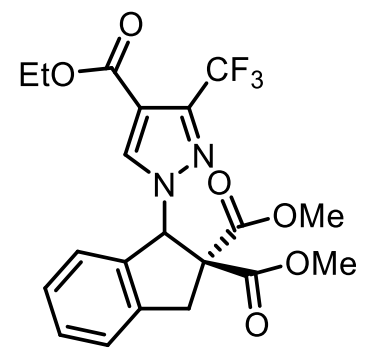

Dimethyl 1-(4-(ethoxycarbonyl)-3-(trifluoromethyl)-1H-pyrazol-1-yl)-1,3-dihydro-2H-indene-2,2dicarboxylate, 3wa'

Reaction run using 2,2-dimethyl 1,3-dihydroindene-2,2-dicarboxylate $1 \mathbf{w}$ (46.9 mg, $0.2 \mathrm{mmol}, 1.0$ equiv), ethyl 3-(trifluoromethyl)-1H-pyrazole-4-carboxylate $2 \mathbf{a}$ (104.1 mg, $0.50 \mathrm{mmol}, 2.5$ equiv), and $\mathrm{BF}_{3} \bullet \mathrm{Et}_{2} \mathrm{O}$ $\left(2.5 \mu \mathrm{L}, 0.02 \mathrm{mmol}, 0.1\right.$ equiv) following the general procedure $\mathrm{I}$ (pressure tube) at $60^{\circ} \mathrm{C}$. Yield $=40.6 \mathrm{mg}$ (46\%) of white semisolid. TLC (pentane:EtOAc, 9:1 v/v): $\mathrm{R}_{\mathrm{f}}=0.13$.

${ }^{1} \mathrm{H}$ NMR $\left(\mathrm{CDCl}_{3}, 400 \mathrm{MHz}\right): 7.79$ (s, 1H), 7.41 - 7.29 (m, 2H), 7.25 - 7.16 (m, 2H), $6.82(\mathrm{~s}, 1 \mathrm{H}), 4.39$ (d, $J=16.8 \mathrm{~Hz}, 1 \mathrm{H}), 4.31(\mathrm{q}, J=7.1 \mathrm{~Hz}, 2 \mathrm{H}), 3.76(\mathrm{~s}, 3 \mathrm{H}), 3.51(\mathrm{~d}, J=16.8 \mathrm{~Hz}, 1 \mathrm{H}), 3.46(\mathrm{~s}, 3 \mathrm{H}), 1.34$ (t, $J=7.1 \mathrm{~Hz}, 3 \mathrm{H}) \mathrm{ppm}$.

${ }^{13} \mathrm{C} \mathrm{NMR}\left(\mathrm{CDCl}_{3}, 101 \mathrm{MHz}\right): 170.1,167.4,160.9,142.6,140.9,138.4,132.5$ (q, $\left.J=39.9 \mathrm{~Hz}\right), 129.9$, 127.8, 125.0, 124.6, $119.6(\mathrm{q}, J=271.8 \mathrm{~Hz}), 115.4,68.4(\mathrm{q}, J=4.3 \mathrm{~Hz}), 65.2,61.2,53.7,53.0,39.9$, $14.1 \mathrm{ppm}$. 
${ }^{19} \mathrm{~F} \mathrm{NMR}\left(\mathrm{CDCl}_{3}, 377 \mathrm{MHz}\right):-56.6 \mathrm{ppm}$.

HRMS Calculated for $\left[\mathrm{C}_{20} \mathrm{H}_{19} \mathrm{~F}_{3} \mathrm{~N}_{2} \mathrm{O}_{6}+\mathrm{H}\right]^{+}: 441.1268$, Found: 441.1262 .<smiles>CC(=O)c1cc(C(C)(C)C)cc2c1C(n1cc(C=O)cn1)CC2(C)C</smiles>

1-(7-acetyl-5-(tert-butyl)-3,3-dimethyl-2,3-dihydro-1H-inden-1-yl)-1H-pyrazole-4-carbaldehyde, 3rf

Reaction run using celestolide $\mathbf{1 r}(48.9 \mathrm{mg}, 0.2 \mathrm{mmol}, 1.0$ equiv), pyrazole-4-carboxaldehyde $\mathbf{2 f}$ ( $48.0 \mathrm{mg}$, $0.50 \mathrm{mmol}, 2.5$ equiv), and tetrabutylammonium chloride $(5.6 \mathrm{mg}, 0.02 \mathrm{mmol}, 0.1$ equiv) following the general procedure I (pressure tube) at $60^{\circ} \mathrm{C}$. Yield $=61.0 \mathrm{mg}(90 \%)$ of colorless semisolid. TLC (pentane:EtOAc, $4: 1 \mathrm{v} / \mathrm{v}$ ): $\mathrm{R}_{\mathrm{f}}=0.29$.

${ }^{1} \mathrm{H} \mathrm{NMR}\left(\mathrm{CDCl}_{3}, 500 \mathrm{MHz}\right): 9.79$ (s, 1H), $7.93(\mathrm{~s}, 1 \mathrm{H}), 7.77$ (d, $\left.J=1.8 \mathrm{~Hz}, 1 \mathrm{H}\right), 7.74(\mathrm{~s}, 1 \mathrm{H}), 7.48(\mathrm{~d}, J=$

$1.8 \mathrm{~Hz}, 1 \mathrm{H}), 6.29(\mathrm{dd}, J=8.4,3.9 \mathrm{~Hz}, 1 \mathrm{H}), 2.54(\mathrm{dd}, J=13.8,8.4 \mathrm{~Hz}, 1 \mathrm{H}), 2.45(\mathrm{~s}, 3 \mathrm{H}), 2.37$ (dd, $J=$ $13.8,3.9 \mathrm{~Hz}, 1 \mathrm{H}), 1.40(\mathrm{~s}, 10 \mathrm{H}), 1.34(\mathrm{~s}, 3 \mathrm{H}), 1.30(\mathrm{~s}, 3 \mathrm{H}) \mathrm{ppm}$.

${ }^{13} \mathrm{C} \mathrm{NMR}\left(\mathrm{CDCl}_{3}, 126 \mathrm{MHz}\right): 199.2,184.3,155.4,153.8,140.8,134.4,132.7,126.2,124.0,123.1,65.0$, 49.0, 42.7, 35.1, 31.4, 30.3, 29.9, $27.8 \mathrm{ppm}$.

HRMS Calculated for $\left[\mathrm{C}_{21} \mathrm{H}_{26} \mathrm{~N}_{2} \mathrm{O}_{2}+\mathrm{Na}\right]^{+}: 361.1887$, Found: 361.1880 .<smiles>CC(=O)c1cc(C(C)(C)C)cc2c1C(n1nc(C)cc1C(F)F)CC2(C)C</smiles>

1-(6-(tert-butyl)-3-(5-(difluoromethyl)-3-methyl-1H-pyrazol-1-yl)-1,1-dimethyl-2,3-dihydro-1H-inden-4yl)ethan-1-one, 3ri

Reaction run using celestolide $1 \mathbf{r}(48.9 \mathrm{mg}, 0.2 \mathrm{mmol}, 1.0$ equiv), 3-(difluoromethyl)-5-methyl- $1 H$ pyrazole $2 \mathbf{i}(66.0 \mathrm{mg}, 0.50 \mathrm{mmol}, 2.5$ equiv), and tetrabutylammonium chloride (5.6 mg, $0.02 \mathrm{mmol}, 0.1$ equiv) following the general procedure $\mathrm{I}$ (pressure tube) at $60^{\circ} \mathrm{C}$ in $\mathrm{DCM}$. Yield $=38.7 \mathrm{mg}(52 \%)$ of white solid. TLC (pentane:EtOAc, 9:1 v/v): $\mathrm{R}_{\mathrm{f}}=0.56 . \mathrm{Mp}: 113-116^{\circ} \mathrm{C}$.

${ }^{1} \mathrm{H} \mathrm{NMR}\left(\mathrm{CDCl}_{3}, 400 \mathrm{MHz}\right): 7.54(\mathrm{~d}, J=1.8 \mathrm{~Hz}, 1 \mathrm{H}), 7.39(\mathrm{~d}, J=1.7 \mathrm{~Hz}, 1 \mathrm{H}), 7.09$ (dd, $J=58.3,53.1$

$\mathrm{Hz}, 1 \mathrm{H}), 6.19(\mathrm{~d}, J=2.2 \mathrm{~Hz}, 1 \mathrm{H}), 6.08(\mathrm{t}, J=7.9 \mathrm{~Hz}, 1 \mathrm{H}), 2.51(\mathrm{dd}, J=12.9,8.0 \mathrm{~Hz}, 1 \mathrm{H}), 2.30(\mathrm{~s}$, $3 \mathrm{H}), 2.21(\mathrm{dd}, J=12.9,7.9 \mathrm{~Hz}, 1 \mathrm{H}), 2.14(\mathrm{~s}, 3 \mathrm{H}), 1.47$ (s, 3H), $1.36(\mathrm{~s}, 9 \mathrm{H}), 1.27$ (s, 3H) ppm.

${ }^{13} \mathrm{C} \mathrm{NMR}\left(\mathrm{CDCl}_{3}, 101 \mathrm{MHz}\right): 200.5,153.8,152.3,147.4,137.5$ (dd, $\left.J=33.0,24.4 \mathrm{~Hz}\right), 135.7,135.4$, 
$124.4,122.8,109.8(\mathrm{dd}, J=235.3 \mathrm{~Hz}, J=235.4 \mathrm{~Hz}), 104.4(\mathrm{dd}, J=3.7,2.4 \mathrm{~Hz}), 61.9(\mathrm{~d}, J=2.0 \mathrm{~Hz})$, 50.0, 42.1, 34.9, 31.5, 30.3, 28.8, 27.8, $13.6 \mathrm{ppm}$.

${ }^{19} \mathrm{~F} \mathrm{NMR}\left(\mathrm{CDCl}_{3}, 377 \mathrm{MHz}\right)$ : $-106.96(\mathrm{~d}, J=307.0 \mathrm{~Hz}),-121.35(\mathrm{~d}, J=307.0 \mathrm{~Hz}) \mathrm{ppm}$.

HRMS Calculated for $\left[\mathrm{C}_{22} \mathrm{H}_{28} \mathrm{~F}_{2} \mathrm{~N}_{2} \mathrm{O}+\mathrm{Na}\right]^{+}:$397.2062, Found: 297.2057.<smiles>CCOC(=O)c1nn(-c2ccc(F)cc2)c2c1CCC2n1nc(C)cc1C(F)F</smiles>

Ethyl 6-(5-(difluoromethyl)-3-methyl-1H-pyrazol-1-yl)-1-(4-fluorophenyl)-1,4,5,6-tetrahydrocyclopenta[c]pyrazole-3-carboxylate, 3 ti

Reaction run using ethyl 1-(4-fluorophenyl)-1,4,5,6-tetrahydrocyclopenta[c]pyrazole-3-carboxylate 1t (54.5 mg, $0.2 \mathrm{mmol}, 1.0$ equiv), 3-(difluoromethyl)-5-methyl-1H-pyrazole $2 \mathbf{i}$ (66.0 mg, $0.50 \mathrm{mmol}, 2.5$ equiv), and tetrabutylammonium chloride $(5.6 \mathrm{mg}, 0.02 \mathrm{mmol}, 0.1$ equiv) following the general procedure I (pressure tube) at $50{ }^{\circ} \mathrm{C}$ in DCM. Yield $=33.6 \mathrm{mg}(42 \%)$ of pale yellow amorphous solid. TLC (DCM:MeOH, 50:1 v/v): $\mathrm{R}_{\mathrm{f}}=0.18$.

${ }^{1} \mathrm{H} \mathrm{NMR}\left(\mathrm{CDCl}_{3}, 500 \mathrm{MHz}\right): 7.72-7.65(\mathrm{~m}, 2 \mathrm{H}), 7.20-7.13(\mathrm{~m}, 2 \mathrm{H}), 6.99(\mathrm{dd}, J=56.5,53.2 \mathrm{~Hz}, 1 \mathrm{H})$, $6.25(\mathrm{~d}, J=1.9 \mathrm{~Hz}, 1 \mathrm{H}), 5.86(\mathrm{dd}, J=8.6,3.8 \mathrm{~Hz}, 1 \mathrm{H}), 4.36-4.14(\mathrm{~m}, 2 \mathrm{H}), 3.40$ (dddd, $J=15.1,8.7$, $5.1,1.3 \mathrm{~Hz}, 1 \mathrm{H}), 3.20(\mathrm{dtd}, J=13.6,8.4,5.0 \mathrm{~Hz}, 1 \mathrm{H}), 2.99$ (ddd, $J=15.2,8.6,4.5 \mathrm{~Hz}, 1 \mathrm{H}), 2.90$ (ddt, $J=13.2,8.7,4.2 \mathrm{~Hz}, 1 \mathrm{H}), 2.21(\mathrm{~s}, 3 \mathrm{H}), 1.19(\mathrm{t}, J=7.1 \mathrm{~Hz}, 3 \mathrm{H}) \mathrm{ppm}$.

${ }^{13} \mathrm{C} \mathrm{NMR}\left(\mathrm{CDCl}_{3}, 126 \mathrm{MHz}\right): 161.7,161.6(\mathrm{~d}, J=247.6 \mathrm{~Hz}), 151.5,148.1,138.2,136.1$ (dd, $J=30.2$, $25.0 \mathrm{~Hz}), 135.7(\mathrm{~d}, J=3.0 \mathrm{~Hz}), 129.6,122.3(\mathrm{~d}, J=8.4 \mathrm{~Hz}), 116.3(\mathrm{~d}, J=23.1 \mathrm{~Hz}), 109.2(\mathrm{t}, J=$ $235.7 \mathrm{~Hz}), 105.5(\mathrm{t}, J=3.8 \mathrm{~Hz}), 61.0,56.2(\mathrm{~d}, J=2.4 \mathrm{~Hz}), 39.8,25.2,14.1,13.7 \mathrm{ppm}$.

${ }^{19} \mathrm{~F} \mathrm{NMR}\left(\mathrm{CDCl}_{3}, 377 \mathrm{MHz}\right):-108.15(\mathrm{~d}, J=307.6 \mathrm{~Hz}),-114.20,-116.86(\mathrm{~d}, J=307.8 \mathrm{~Hz}) \mathrm{ppm}$.

HRMS Calculated for $\left[\mathrm{C}_{20} \mathrm{H}_{19} \mathrm{~F}_{3} \mathrm{~N}_{4} \mathrm{O}_{2}+\mathrm{H}\right]^{+}:$405.1533, Found: 405.1529.

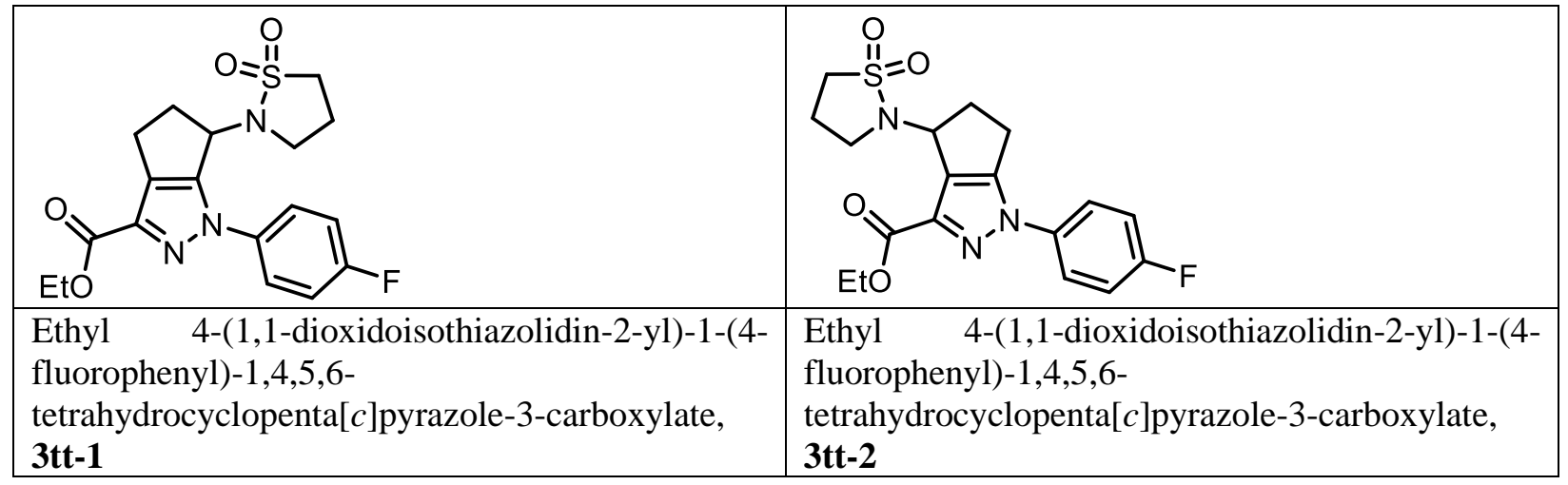

Reaction run using ethyl 1-(4-fluorophenyl)-1,4,5,6-tetrahydrocyclopenta[c]pyrazole-3-carboxylate 1t (54.5 mg, $0.2 \mathrm{mmol}, 1.0$ equiv), 1,3-propanesultam $2 \mathrm{t}(50.0 \mu \mathrm{L}, 0.50 \mathrm{mmol}, 2.5$ equiv), and 
tetrabutylammonium chloride $(5.6 \mathrm{mg}, 0.02 \mathrm{mmol}, 0.1$ equiv) following the general procedure I (pressure tube) at $40{ }^{\circ} \mathrm{C}$ in DCM, and two regioisomers were isolated.

3tt-1: Yield $=39.0 \mathrm{mg}(47 \%)$ of colorless semisolid. TLC (pentane:EtOAc, 1:2 v/v): $R_{\mathrm{f}}=0.33$.

${ }^{1} \mathrm{H}$ NMR $\left(\mathrm{CDCl}_{3}, 500 \mathrm{MHz}\right): 7.92-7.56(\mathrm{~m}, 2 \mathrm{H}), 7.19-6.96(\mathrm{~m}, 2 \mathrm{H}), 5.66-4.99(\mathrm{~m}, 1 \mathrm{H}), 4.42(\mathrm{qd}, J=$ 7.1, 3.0 Hz, 2H), $3.36-3.11(\mathrm{~m}, 5 \mathrm{H}), 3.03-2.89(\mathrm{~m}, 2 \mathrm{H}), 2.85$ (ddt, $J=13.6,5.2,2.8 \mathrm{~Hz}, 1 \mathrm{H}), 2.32$ (dddd, $J=13.3,9.0,6.4,2.6 \mathrm{~Hz}, 2 \mathrm{H}), 1.41(\mathrm{t}, J=7.1 \mathrm{~Hz}, 3 \mathrm{H})$. ppm.

${ }^{13} \mathrm{C} \mathrm{NMR}\left(\mathrm{CDCl}_{3}, 126 \mathrm{MHz}\right): 161.8,161.6(\mathrm{~d}, J=247.9 \mathrm{~Hz}), 151.4,139.0,135.6(\mathrm{~d}, J=3.0 \mathrm{~Hz}), 128.4$, $122.2(\mathrm{~d}, J=8.5 \mathrm{~Hz}), 116.4(\mathrm{~d}, J=23.0 \mathrm{~Hz}), 61.3,51.3,47.3,42.7,37.0,25.2,18.6,14.4 \mathrm{ppm}$.

${ }^{19} \mathrm{~F} \mathrm{NMR}\left(\mathrm{CDCl}_{3}, 377 \mathrm{MHz}\right):-113.9 \mathrm{ppm}$.

HRMS Calculated for $\left[\mathrm{C}_{18} \mathrm{H}_{20} \mathrm{FN}_{3} \mathrm{O}_{4} \mathrm{~S}+\mathrm{H}\right]^{+}:$394.1231, Found: 394.1226.

3tt-2: Yield $=4.0 \mathrm{mg}(5 \%)$ of pale yellow amorphous solid. TLC (pentane:EtOAc, $1: 1 \mathrm{v} / \mathrm{v}): \mathrm{R}_{\mathrm{f}}=0.33$.

${ }^{1} \mathrm{H}$ NMR $\left(\mathrm{CDCl}_{3}, 500 \mathrm{MHz}\right): 7.90-7.76(\mathrm{~m}, 2 \mathrm{H}), 7.21-7.11(\mathrm{~m}, 2 \mathrm{H}), 5.38(\mathrm{dd}, J=8.1,3.2 \mathrm{~Hz}, 1 \mathrm{H})$, $4.42(\mathrm{qd}, J=7.1,1.4 \mathrm{~Hz}, 2 \mathrm{H}), 3.18(\mathrm{dt}, J=9.1,7.3 \mathrm{~Hz}, 1 \mathrm{H}), 3.11$ (ddd, $J=12.7,8.2,6.8 \mathrm{~Hz}, 1 \mathrm{H})$, 3.01 (dddd, $J=24.5,13.6,9.0,7.1 \mathrm{~Hz}, 2 \mathrm{H}), 2.94-2.84(\mathrm{~m}, 2 \mathrm{H}), 2.81-2.69(\mathrm{~m}, 2 \mathrm{H}), 2.33-2.22$ (m, $1 \mathrm{H}), 2.18-2.07(\mathrm{~m}, 1 \mathrm{H}), 1.41(\mathrm{t}, J=7.1 \mathrm{~Hz}, 3 \mathrm{H}) \mathrm{ppm}$.

${ }^{13} \mathrm{C} \mathrm{NMR}\left(\mathrm{CDCl}_{3}, 126 \mathrm{MHz}\right): 162.1,161.7(\mathrm{~d}, J=247.8 \mathrm{~Hz}), 145.6,138.2,135.1(\mathrm{~d}, J=3.0 \mathrm{~Hz}), 134.6$, $122.4(\mathrm{~d}, J=8.4 \mathrm{~Hz}), 116.2(\mathrm{~d}, J=23.0 \mathrm{~Hz}), 61.1,51.1,46.8,40.7,36.0,22.8,18.4,14.1 \mathrm{ppm}$.

${ }^{19} \mathrm{~F} \mathrm{NMR}\left(\mathrm{CDCl}_{3}, 377 \mathrm{MHz}\right):-114.1 \mathrm{ppm}$.

HRMS Calculated for $\left[\mathrm{C}_{18} \mathrm{H}_{20} \mathrm{FN}_{3} \mathrm{O}_{4} \mathrm{~S}+\mathrm{H}\right]^{+}:$394.1231, Found: 394.1229.

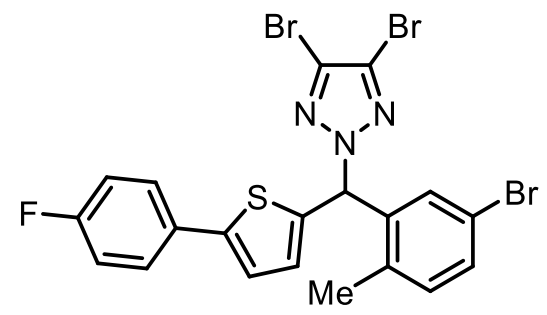

4,5-dibromo-2-((5-bromo-2-methylphenyl)(5-(4-fluorophenyl)thiophen-2-yl)methyl)-2H-1,2,3-triazole,

3pp'

Reaction run using 2-(5-bromo-2-methylbenzyl)-5-(4-fluorophenyl)thiophene 1p (68.6 mg, $0.2 \mathrm{mmol}, 1.0$ equiv), 4,5-Dibromo-1H-1,2,3-triazole $2 \mathbf{p}$ (113.4 mg, $0.50 \mathrm{mmol}, 2.5$ equiv), and tetrabutylammonium chloride $\left(5.6 \mathrm{mg}, 0.02 \mathrm{mmol}, 0.1\right.$ equiv) following the general procedure II (glass vial) procedure at $30{ }^{\circ} \mathrm{C}$. Yield $=81.8 \mathrm{mg}(70 \%)$ of colorless liquid. TLC (pentane:EtOAc, 9:1 v/v): $\mathrm{R}_{\mathrm{f}}=0.51$.

${ }^{1} \mathrm{H} \mathrm{NMR}\left(\mathrm{CDCl}_{3}, 500 \mathrm{MHz}\right): 7.58-7.46(\mathrm{~m}, 2 \mathrm{H}), 7.40(\mathrm{~d}, J=7.4 \mathrm{~Hz}, 2 \mathrm{H}), 7.20(\mathrm{~s}, 1 \mathrm{H}), 7.09$ (d, $J=3.2$

$\mathrm{Hz}, 2 \mathrm{H}), 7.08-6.94(\mathrm{~m}, 2 \mathrm{H}), 6.84(\mathrm{~d}, J=3.7 \mathrm{~Hz}, 1 \mathrm{H}), 2.29(\mathrm{~s}, 3 \mathrm{H}) \mathrm{ppm}$.

${ }^{13} \mathrm{C} \mathrm{NMR}\left(\mathrm{CDCl}_{3}, 101 \mathrm{MHz}\right): 162.6(\mathrm{~d}, J=248.1 \mathrm{~Hz}), 145.5,137.6,137.5,134.6,132.5,132.1,130.00$, $129.95,129.6,127.6(\mathrm{~d}, J=8.1 \mathrm{~Hz}), 125.6,122.8,120.3,116.0(\mathrm{~d}, J=21.9 \mathrm{~Hz}), 65.9,18.9$.

${ }^{19} \mathrm{~F}$ NMR $\left(\mathrm{CDCl}_{3}, 377 \mathrm{MHz}\right)$ : $-113.6 \mathrm{ppm}$.

HRMS Calculated for $\left[\mathrm{C}_{20} \mathrm{H}_{13} \mathrm{Br}_{3} \mathrm{FN}_{3} \mathrm{~S}+\mathrm{H}\right]^{+}:$583.8437, Found: 583.8444 . 


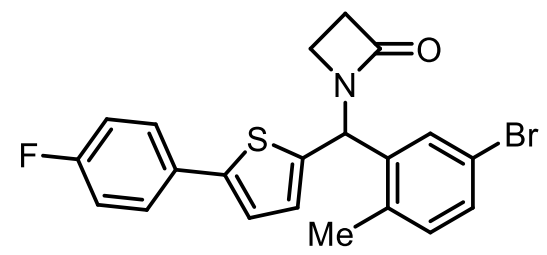

4,5-dibromo-2-((5-bromo-2-methylphenyl)(5-(4-fluorophenyl)thiophen-2-yl)methyl)-2H-1,2,3-triazole,

3 ps

Reaction run using 2-(5-bromo-2-methylbenzyl)-5-(4-fluorophenyl)thiophene 1p (68.6 mg, $0.2 \mathrm{mmol}, 1.0$ equiv), azetidin-2-one $2 \mathrm{~s}$ ( $35.0 \mathrm{mg}, 0.50 \mathrm{mmol}, 2.5$ equiv), and tetrabutylammonium chloride (5.6 $\mathrm{mg}$, $0.02 \mathrm{mmol}, 0.1$ equiv) following the general procedure II (glass vial) procedure at $30{ }^{\circ} \mathrm{C}$. Yield $=68.5 \mathrm{mg}$ (80\%) of light-yellow liquid. TLC (pentane:EtOAc, 4:1 v/v): $R_{\mathrm{f}}=0.10$.

${ }^{1} \mathrm{H} \mathrm{NMR}\left(\mathrm{CDCl}_{3}, 500 \mathrm{MHz}\right): 7.57-7.48(\mathrm{~m}, 2 \mathrm{H}), 7.41(\mathrm{~d}, J=2.1 \mathrm{~Hz}, 1 \mathrm{H}), 7.38(\mathrm{dd}, J=8.1,2.1 \mathrm{~Hz}, 1 \mathrm{H})$, $7.15-7.06(\mathrm{~m}, 2 \mathrm{H}), 7.06(\mathrm{t}, J=8.6 \mathrm{~Hz}, 2 \mathrm{H}), 6.72(\mathrm{~d}, J=3.8 \mathrm{~Hz}, 1 \mathrm{H}), 6.43(\mathrm{~s}, 1 \mathrm{H}), 3.38(\mathrm{q}, J=5.1$, $4.5 \mathrm{~Hz}, 1 \mathrm{H}), 3.24(\mathrm{dt}, J=5.7,4.0 \mathrm{~Hz}, 1 \mathrm{H}), 3.01(\mathrm{t}, J=4.2 \mathrm{~Hz}, 2 \mathrm{H}), 2.29$ (s, 3H) ppm.

${ }^{13} \mathrm{C} \mathrm{NMR}\left(\mathrm{CDCl}_{3}, 101 \mathrm{MHz}\right): 167.2,162.5(\mathrm{~d}, J=247.7 \mathrm{~Hz}), 143.7,140.7,139.2,135.1,132.7,131.3$, $130.2(\mathrm{~d}, J=3.5 \mathrm{~Hz}), 129.9,127.7,127.4(\mathrm{~d}, J=8.1 \mathrm{~Hz}), 122.9,119.8,115.9(\mathrm{~d}, J=21.8 \mathrm{~Hz}), 53.8$, 38.4, 36.1, $19.0 \mathrm{ppm}$.

${ }^{19} \mathrm{~F}$ NMR $\left(\mathrm{CDCl}_{3}, 471 \mathrm{MHz}\right):-114.0 \mathrm{ppm}$.

HRMS Calculated for $\left[\mathrm{C}_{21} \mathrm{H}_{17} \mathrm{BrFNOS}+\mathrm{H}\right]^{+}$: 452.0091, Found: 452.0089. 


\section{References}

1. Hu, H.; Chen, S.-J.; Mandal, M.; Pratik, S. M.; Buss, J. A.; Krska, S. W.; Cramer, C. J.; Stahl, S. S. Nat. Catal. 2020, 3, 358-367.

2. Sun, H.-L.; Yang, F.; Ye, W.-T.; Wang, J.-J.; Zhu, R. ACS Catal. 2020, 10, 4983-4989.

3. Liu, W.; Sengupta, S.; Peterson, J. L.; Akhmedov, N. G.; Shi, X. J. Org. Chem. 2007, 72, 50125015.

4. Bruker-AXS (2016). APEX3. Version 2016.5-0. Madison, Wisconsin, USA.

5. Krause, L.; Herbst-Irmer, R.; Sheldrick, G. M.; Stalke, D. J. Appl. Cryst. 2015, 48, 3-10.

6. Sheldrick, G. M. (2013b). XPREP. Version 2013/1. Georg-August-Universität Göttingen, Göttingen, Germany.

7. Sheldrick, G. M. (2013a). The SHELX homepage, http://shelx.uni-ac.gwdg.de/SHELX/.

8. Sheldrick, G. M. Acta Cryst. 2015, A71, 3-8.

9. Sheldrick, G. M. Acta Cryst. 2015, C71, 3-8.

10.Dolomanov, O. V.; Bourhis, L. J.; Gildea, R. J.; Howard, J. A. K.; Puschmann, H. J. Appl. Crystallogr. 2009, 42, 339-341.

11.Guzei, I. A. (2007-2013). Programs Gn. University of Wisconsin-Madison, Madison, Wisconsin, USA. 


\section{NMR Spectroscopic Data}

${ }^{1} \mathrm{H}$ NMR spectrum of 3aa in $\mathrm{CDCl}_{3}$ containing $0.03 \%$ (v/v) TMS (500 MHz).

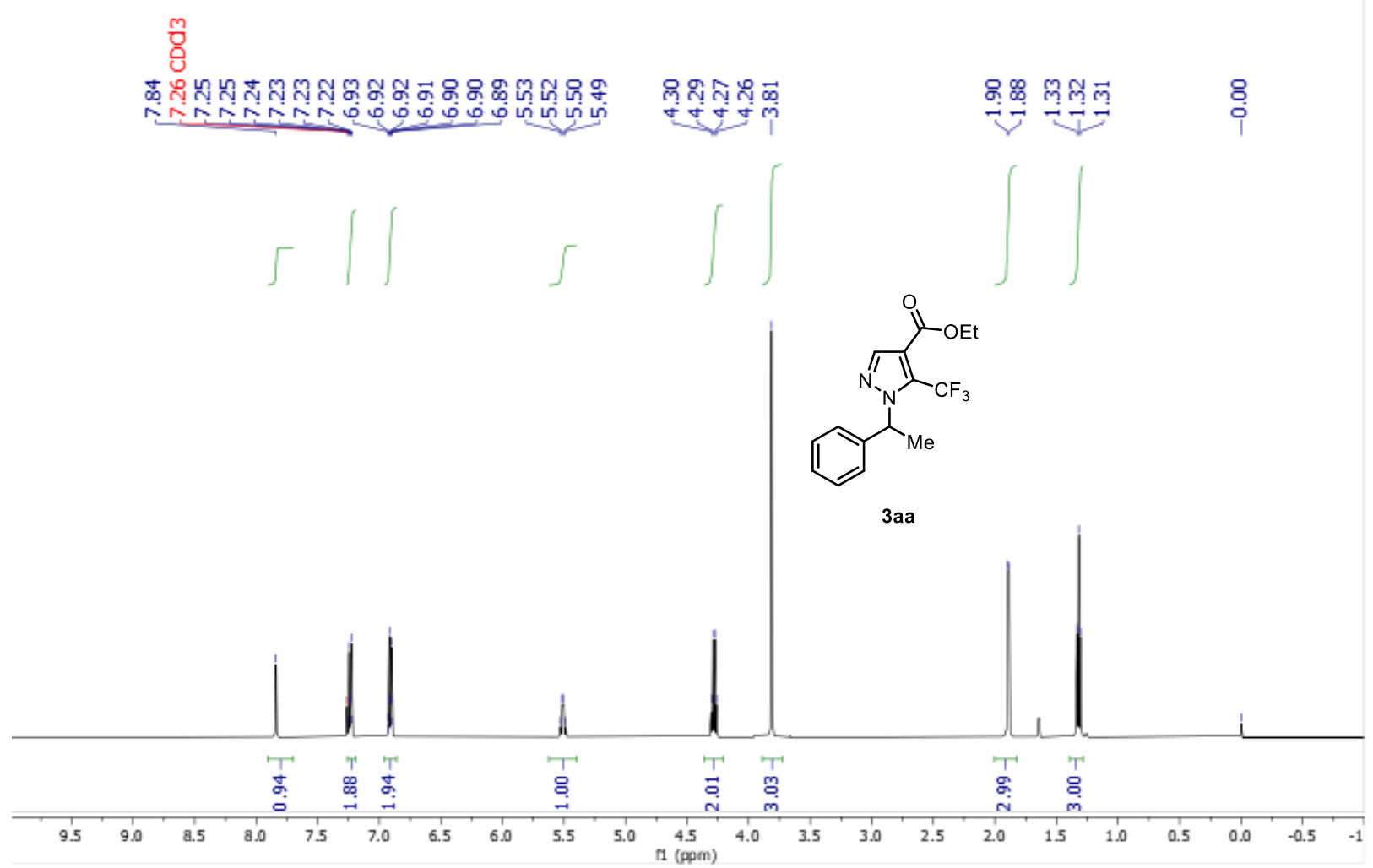

${ }^{13} \mathrm{C}$ NMR spectrum of 3aa in $\mathrm{CDCl}_{3}$ containing $0.03 \%(\mathrm{v} / \mathrm{v}) \mathrm{TMS}(126 \mathrm{MHz})$.

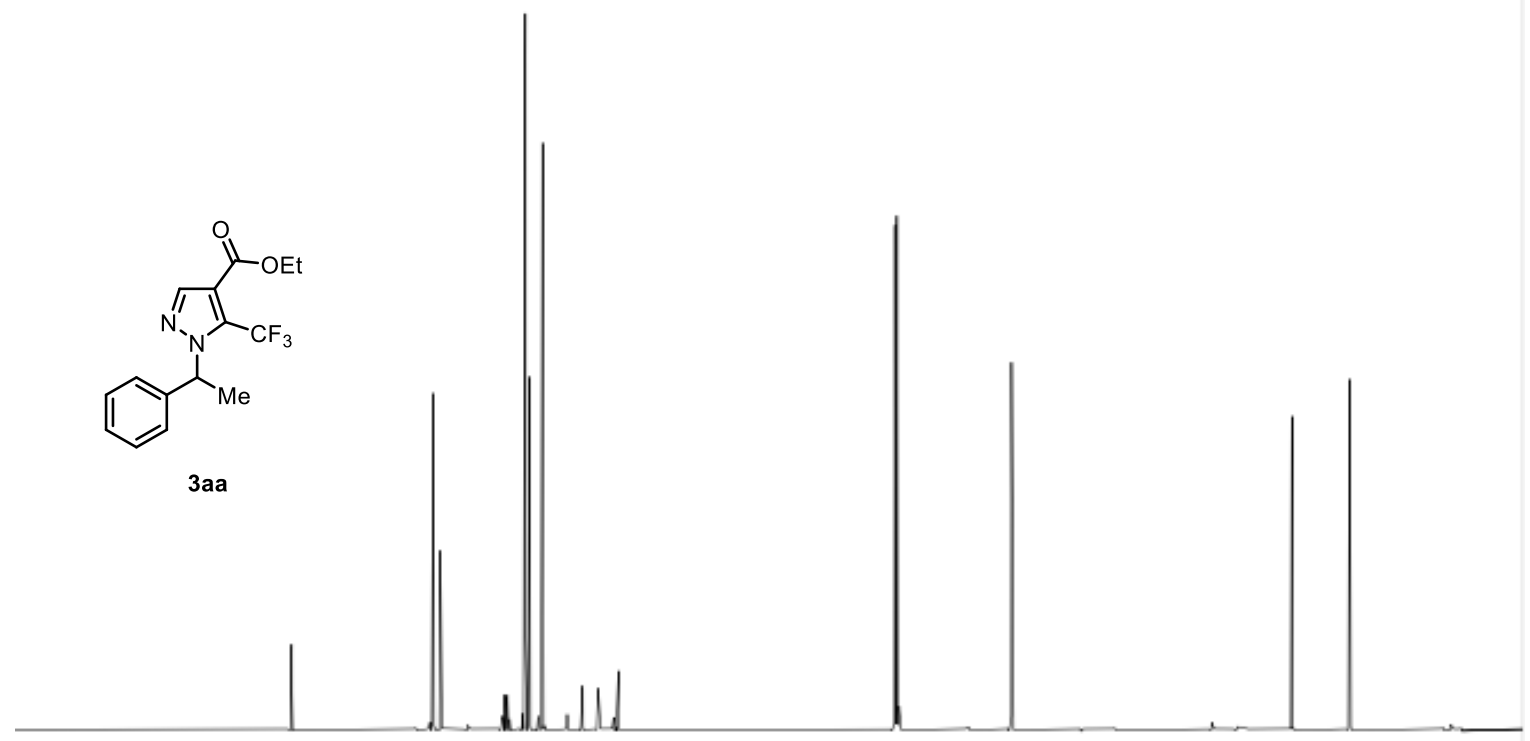

\begin{tabular}{llllllllllllllllllllll}
\hline 0 & 190 & 180 & 170 & 160 & 150 & 140 & 130 & 120 & 110 & 100 & 100 & 80 & 70 & 60 & 50 & 40 & 30 & 20 & 10 & 0 & -1
\end{tabular} 
${ }^{19} \mathrm{~F}$ NMR spectrum of $\mathbf{3 a a}$ in $\mathrm{CDCl}_{3}$ containing $0.03 \%$ (v/v) TMS (377 MHz).

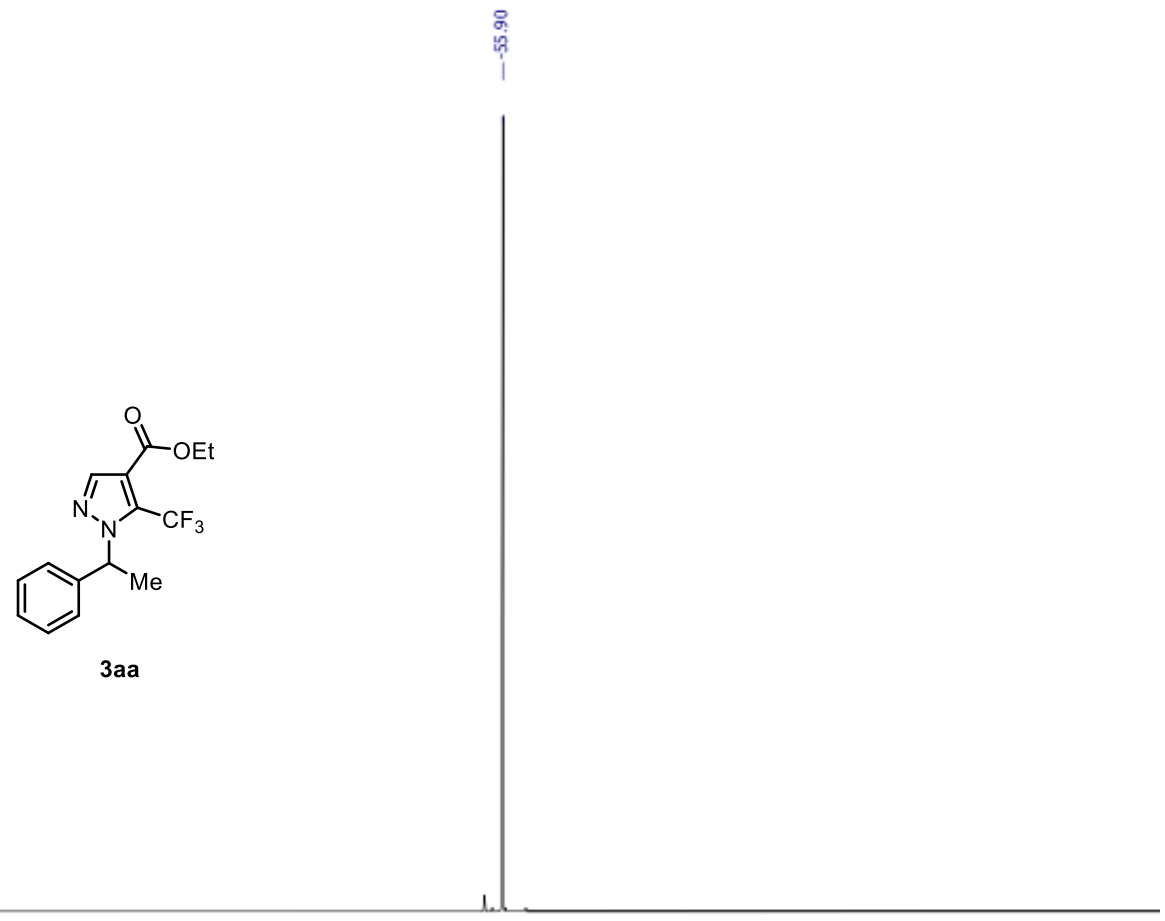

$\begin{array}{llllllllllllllllllllllllllllllllllllllllll}-41 & -42 & -43 & -44 & -45 & -46 & -47 & -48 & -49 & -50 & -51 & -52 & -53 & -54 & -55 & -56 & -57 & -58 & -59 & -60 & -61 & -62 & -63 & -64 & -65 & -66 & -67 & -68 & -69 & -7\end{array}$

HSQC NMR spectrum of 3aa in $\mathrm{CDCl}_{3}$ containing $0.03 \%$ (v/v) TMS (500, $126 \mathrm{MHz}$ ).

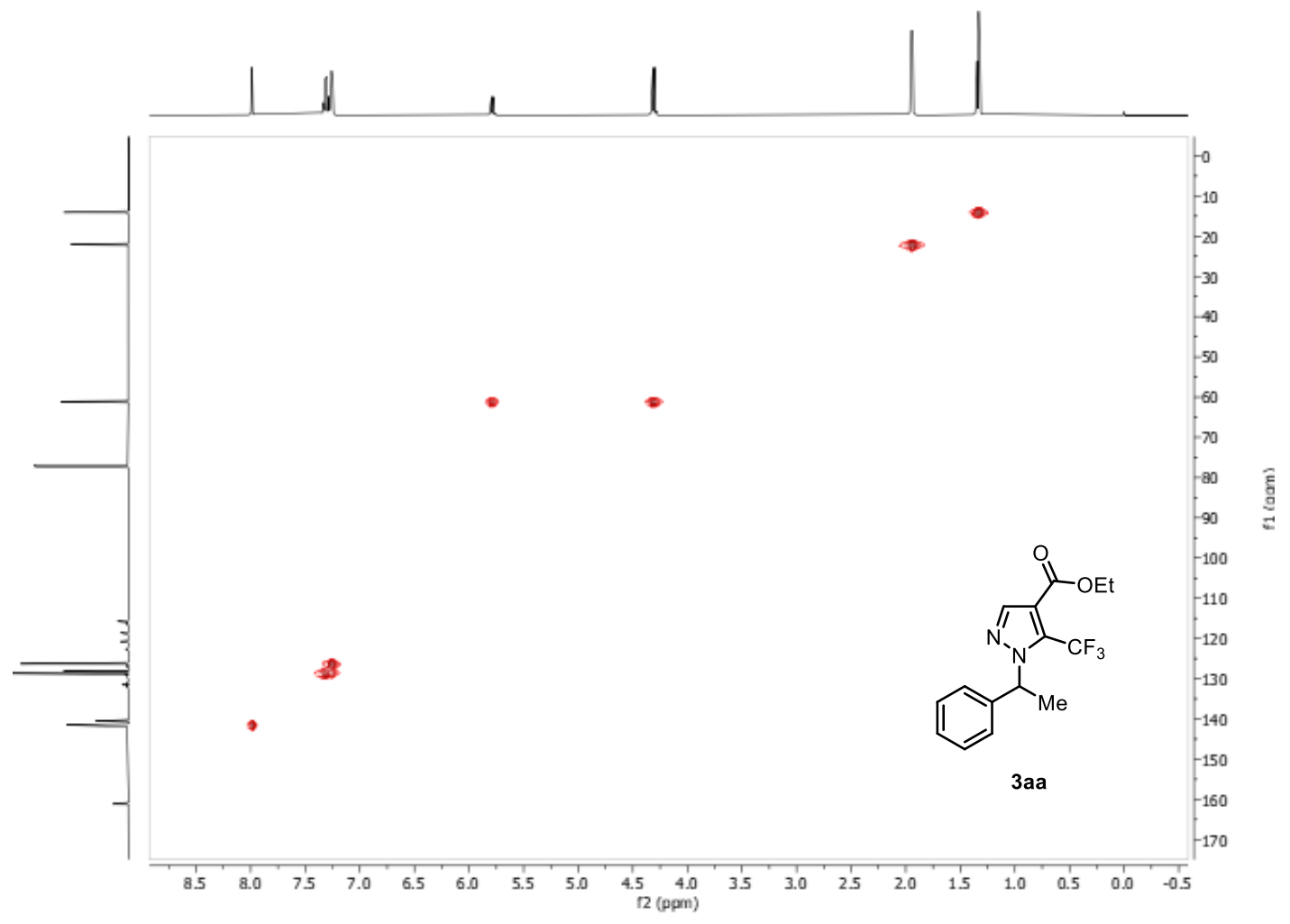


HMBC NMR spectrum of 3aa in $\mathrm{CDCl}_{3}$ containing $0.03 \%$ (v/v) TMS (500, $126 \mathrm{MHz}$ ).

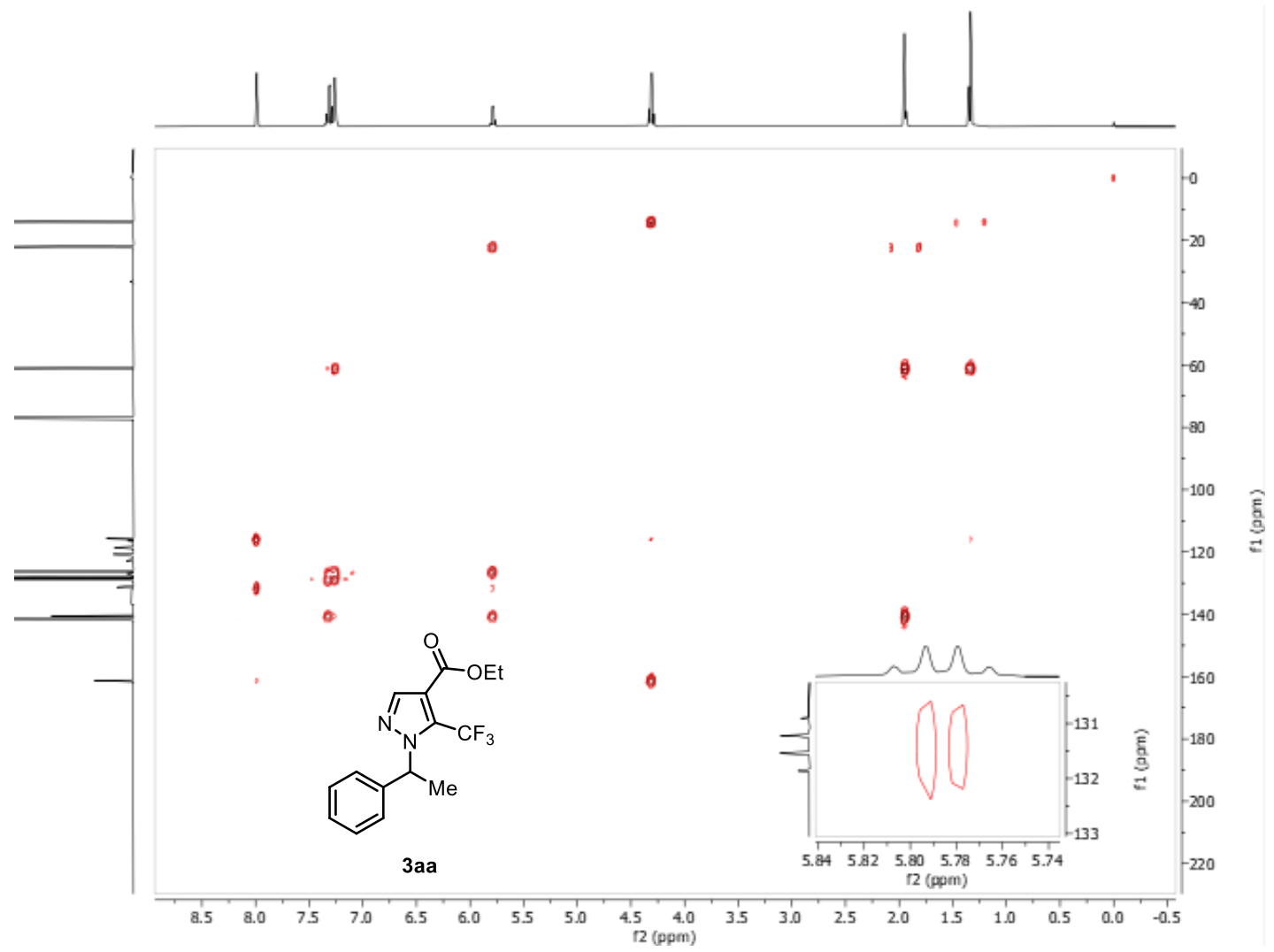

${ }^{1} \mathrm{H}$ NMR spectrum of 3aa' in $\mathrm{CDCl}_{3}$ containing $0.03 \%$ (v/v) TMS (500 MHz).

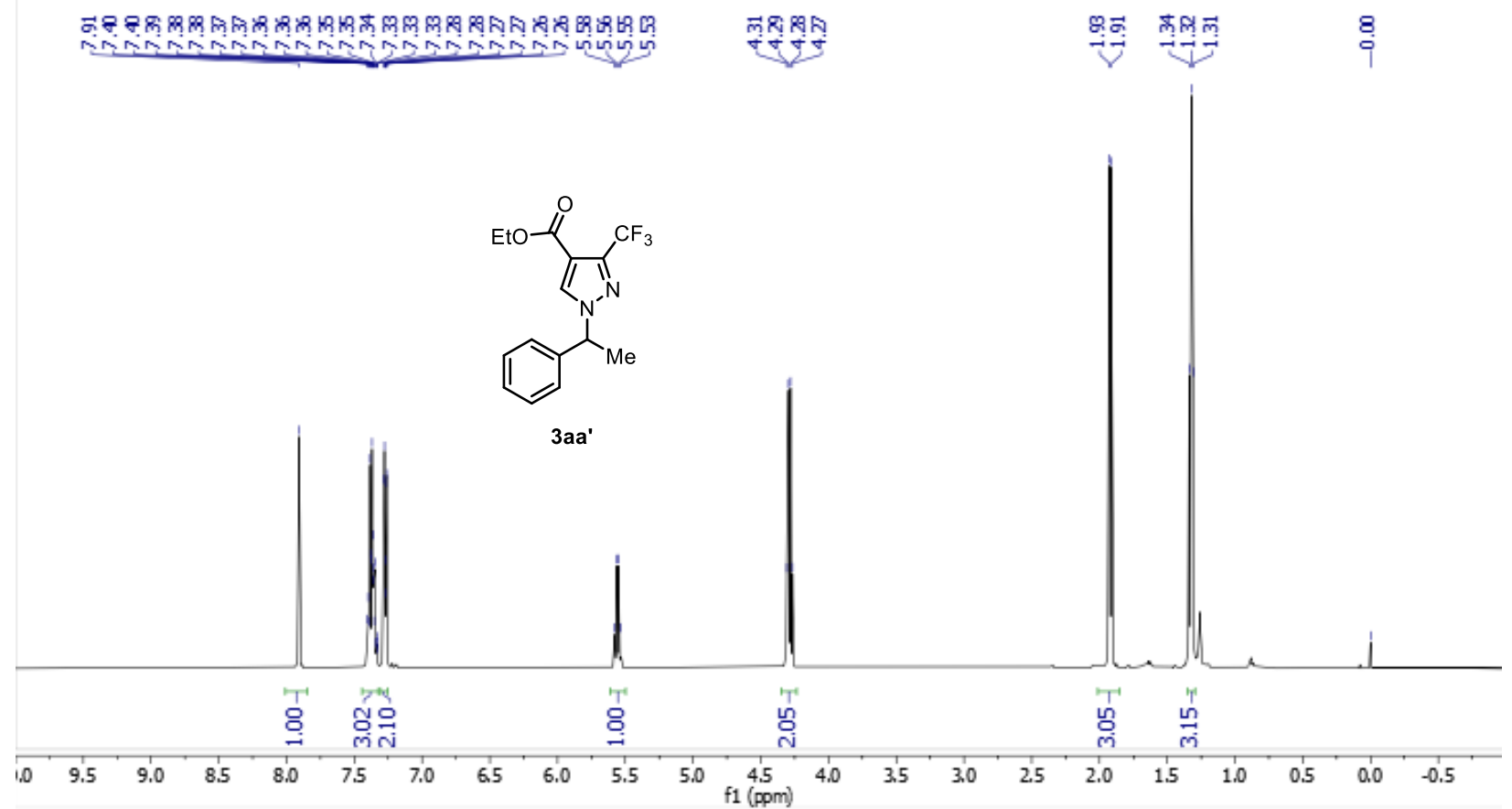


${ }^{13} \mathrm{C}$ NMR spectrum of 3aa' in $\mathrm{CDCl}_{3}$ containing $0.03 \%$ (v/v) TMS (126 MHz).

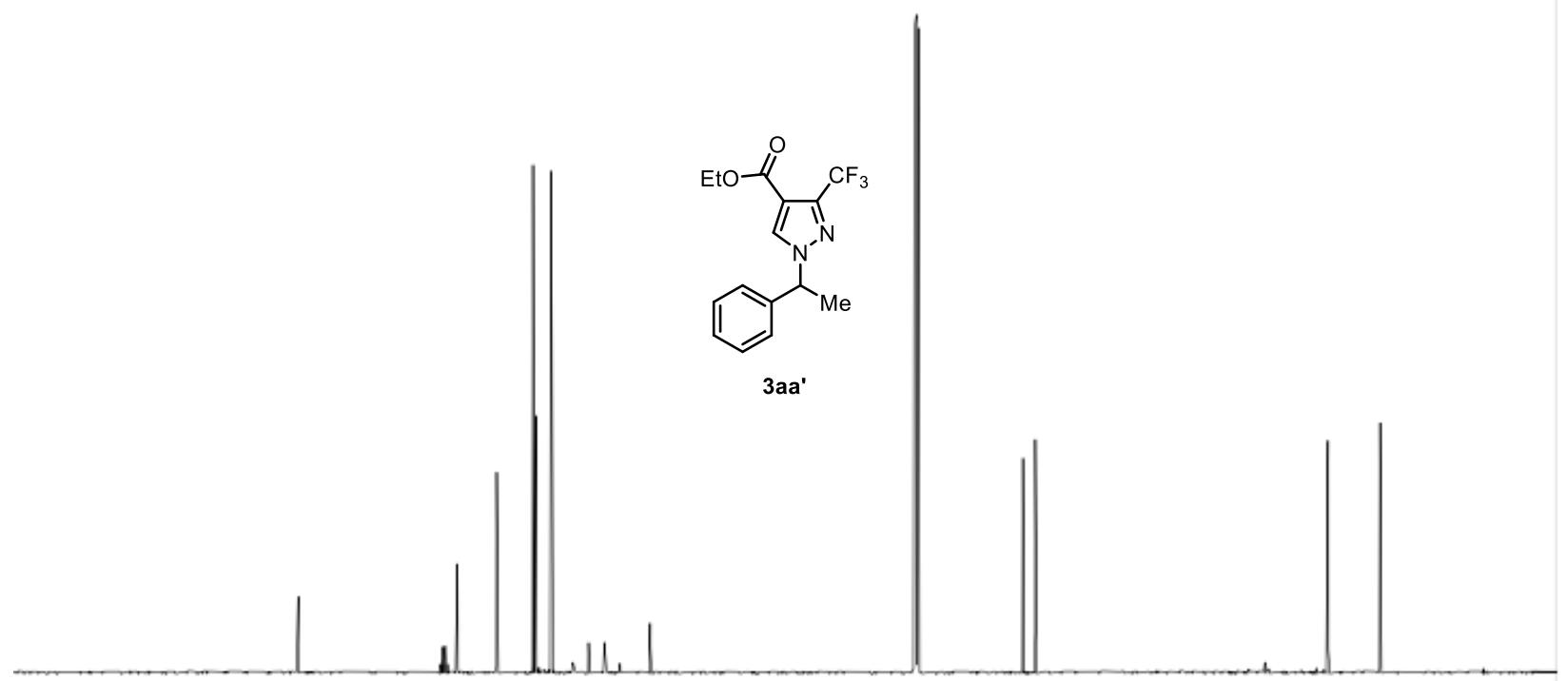

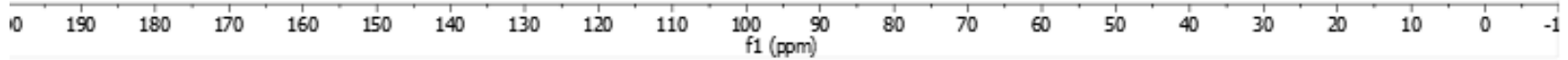

${ }^{19} \mathrm{~F}$ NMR spectrum of $3 \mathbf{a a}^{\prime}$ in $\mathrm{CDCl}_{3}$ containing $0.03 \%$ (v/v) TMS (377 MHz).

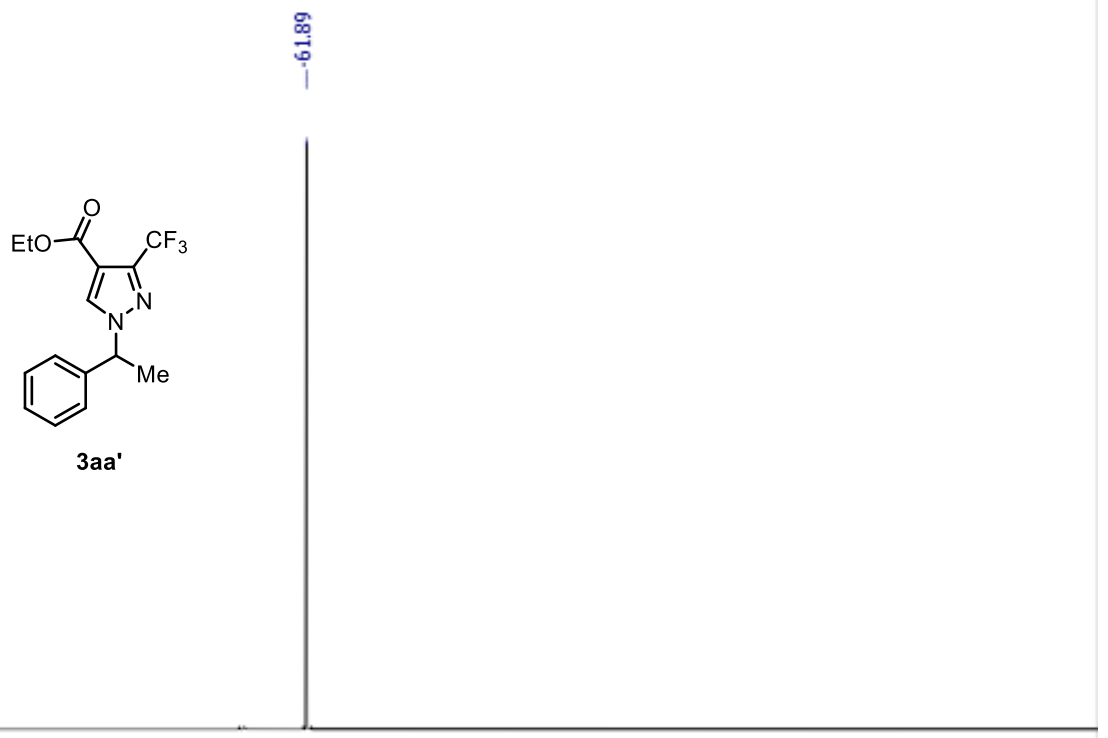

\begin{tabular}{lllllllllllllllllllllllllllllllllllll}
\hline 0 & 5 & 0 & -5 & -10 & -15 & -20 & -25 & -30 & -35 & -40 & -45 & -50 & -55 & -60 & -65 & -70 & -75 & -80 & -85 & -90 & -95 & -100 & -105 & -110 & -115 & -120 & -125 & -1
\end{tabular}

HSQC NMR spectrum of 3aa' in $\mathrm{CDCl}_{3}$ containing $0.03 \%$ (v/v) TMS $(500,126 \mathrm{MHz})$. 


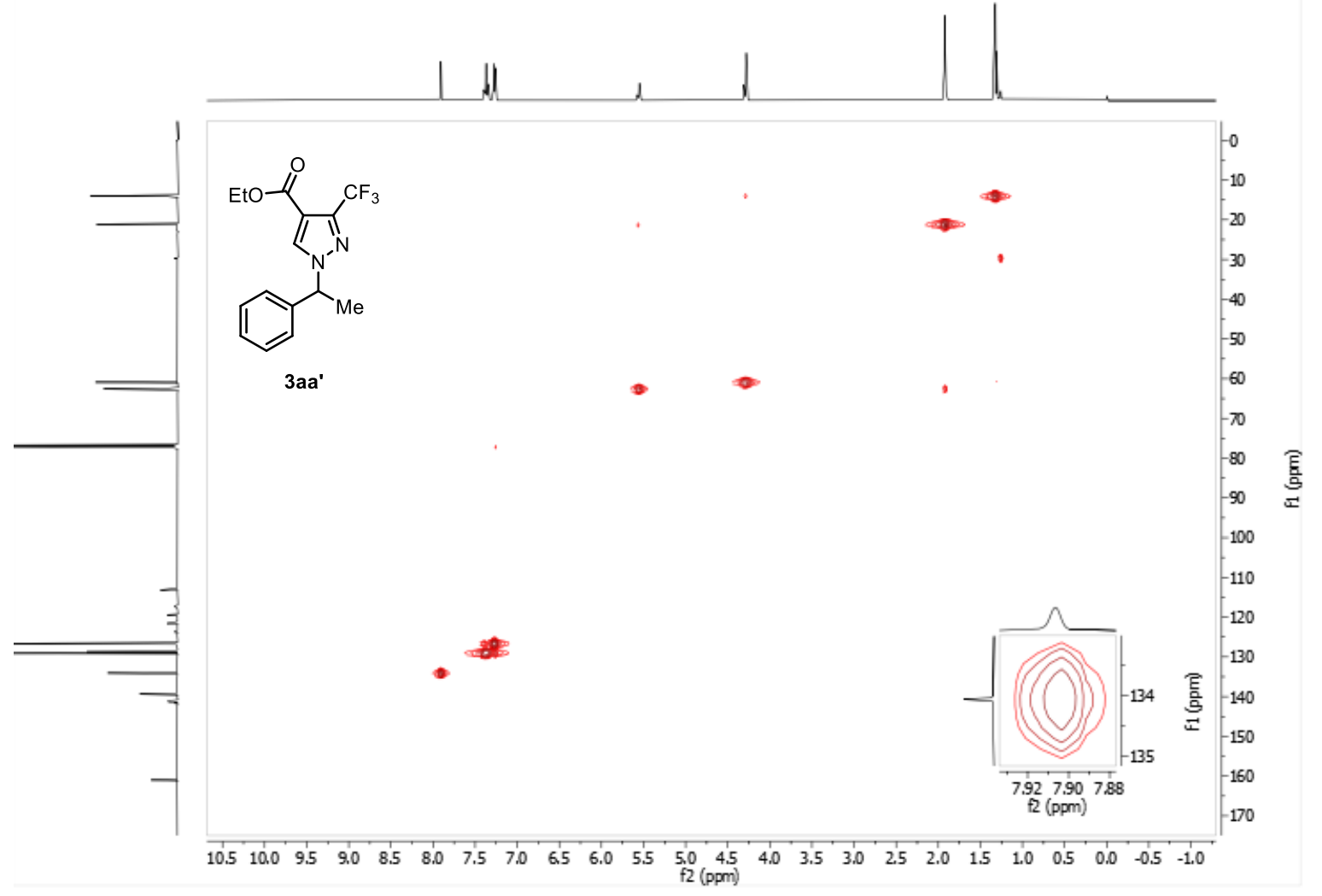

HMBC NMR spectrum of 3aa' in $\mathrm{CDCl}_{3}$ containing $0.03 \%$ (v/v) TMS (500, $126 \mathrm{MHz}$ ).

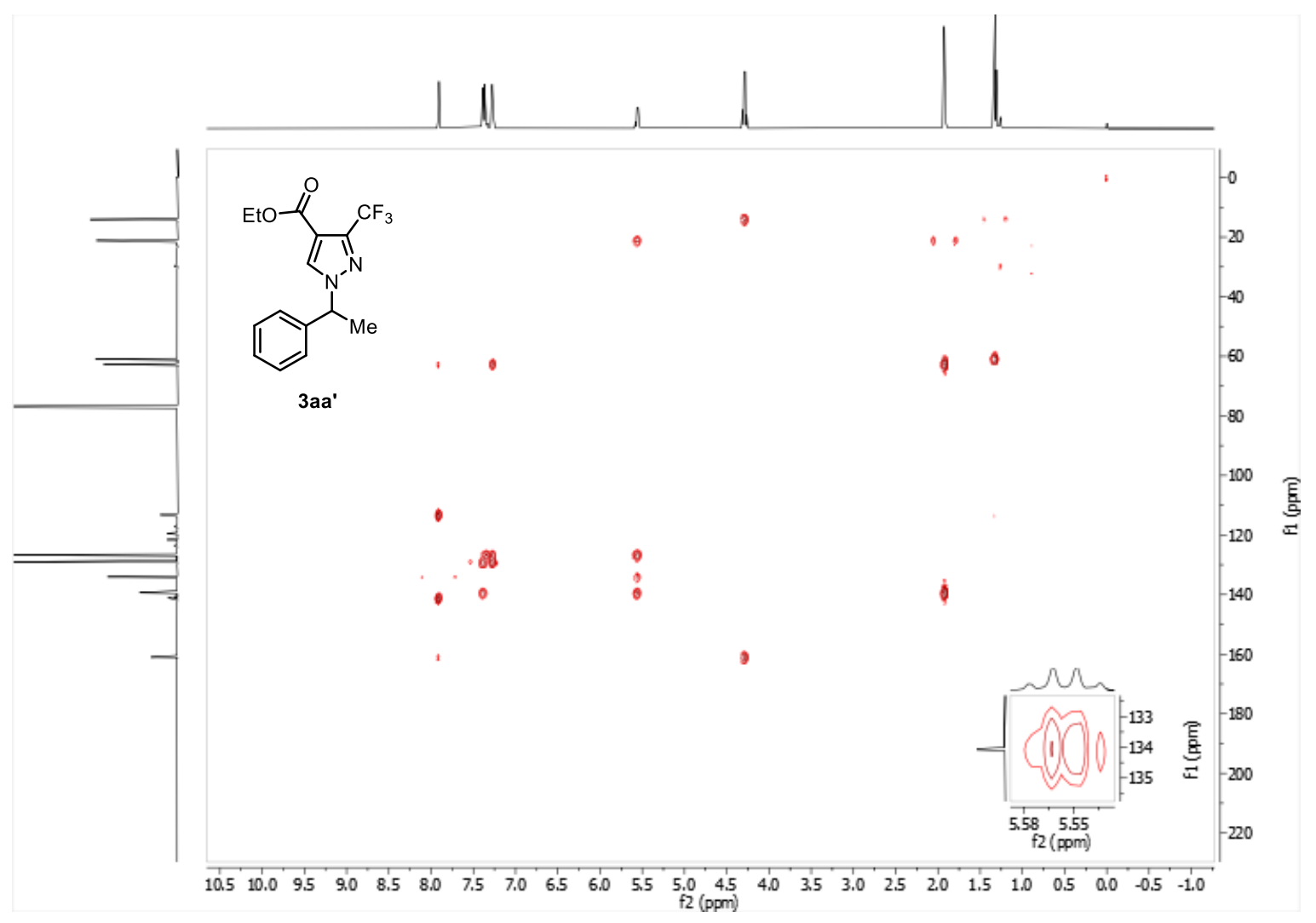


${ }^{1} \mathrm{H}$ NMR spectrum of $\mathbf{3 b a}$ in $\mathrm{CDCl}_{3}$ containing $0.03 \%(\mathrm{v} / \mathrm{v})$ TMS $(500 \mathrm{MHz})$.

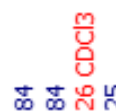

$\frac{m}{\mathrm{O}}$

๘

承

:
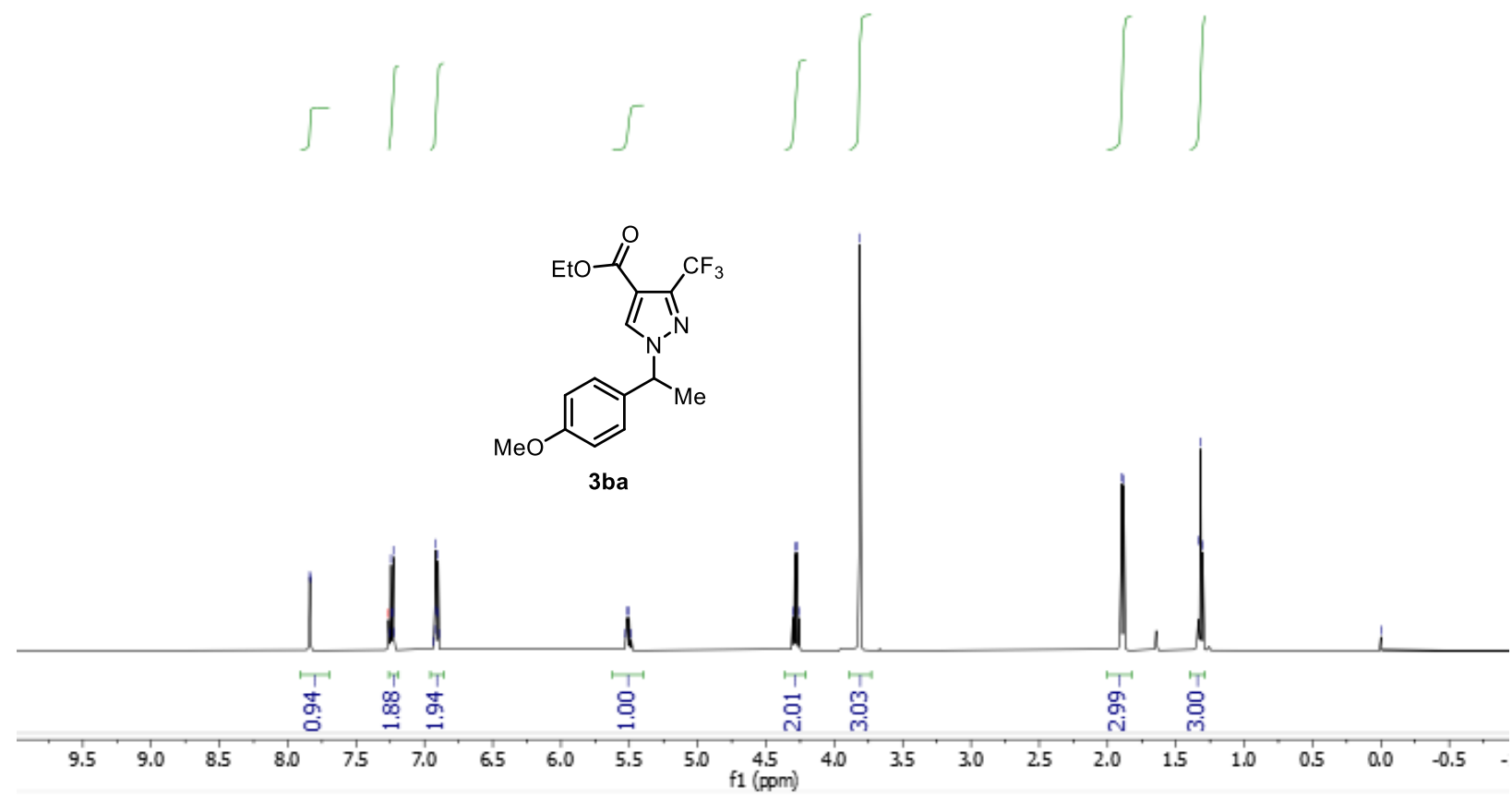

${ }^{13} \mathrm{C}$ NMR spectrum of $\mathbf{3 b a}$ in $\mathrm{CDCl}_{3}$ containing $0.03 \%$ (v/v) TMS (126 MHz).

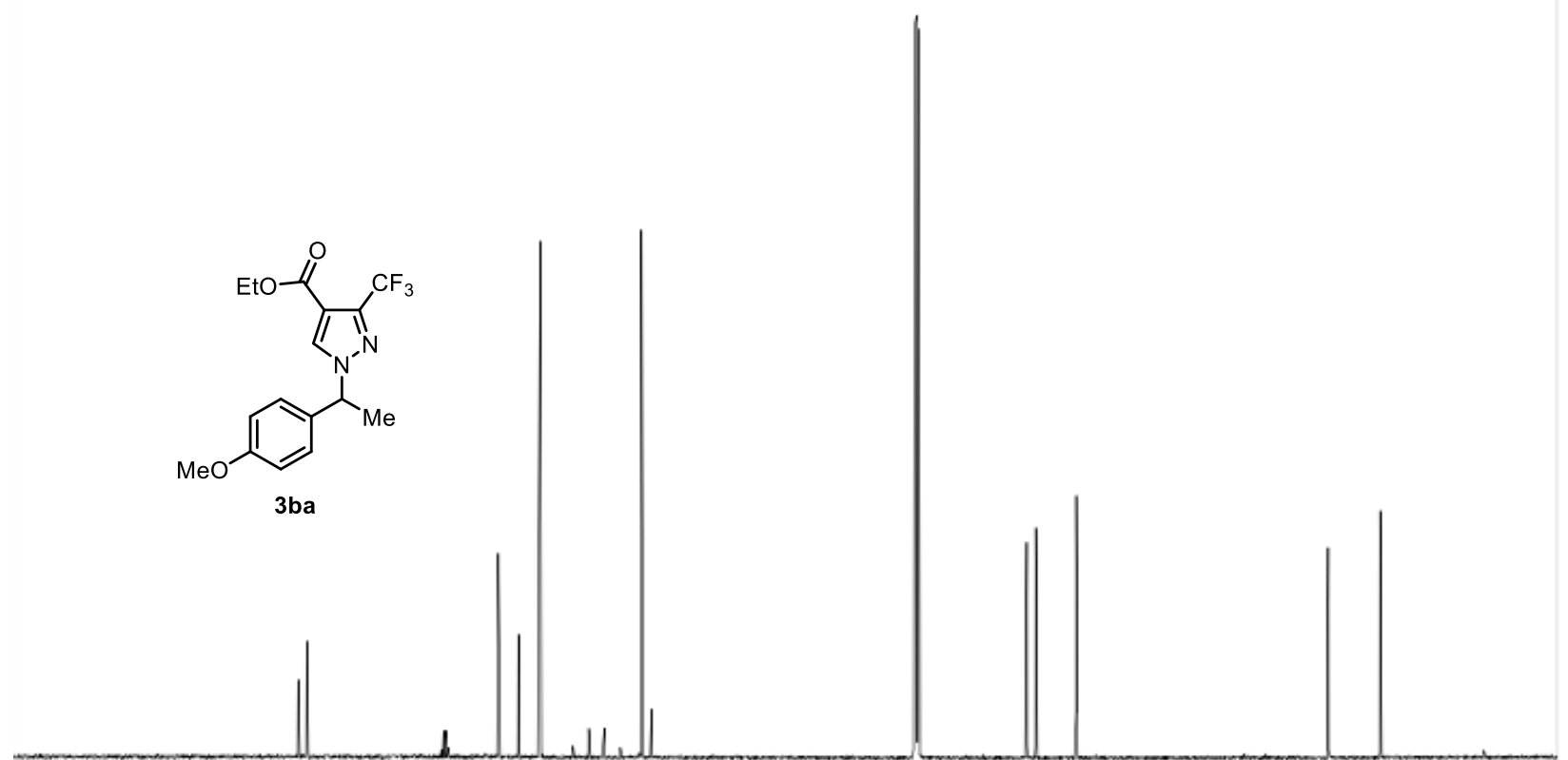

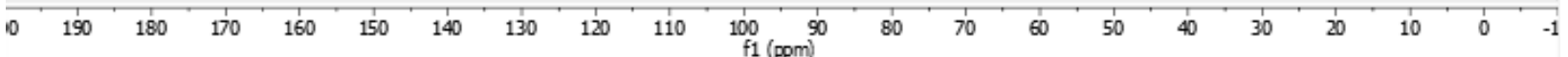


${ }^{19} \mathrm{~F}$ NMR spectrum of $\mathbf{3} \mathbf{b a}$ ' in $\mathrm{CDCl}_{3}$ containing $0.03 \%$ (v/v) TMS (377 MHz).

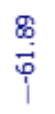
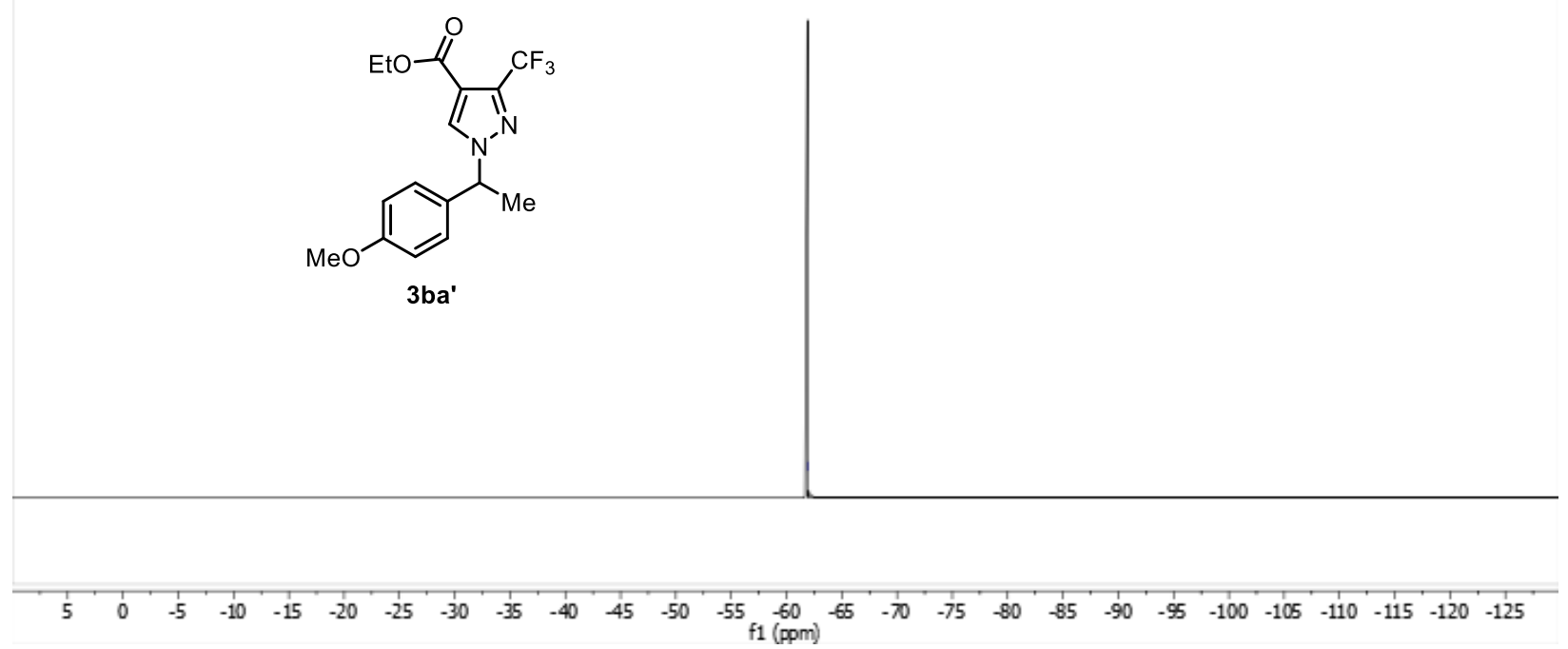

HSQC NMR spectrum of $3 \mathbf{b a}^{\prime}$ in $\mathrm{CDCl}_{3}$ containing $0.03 \%$ (v/v) TMS $(500,126 \mathrm{MHz})$.

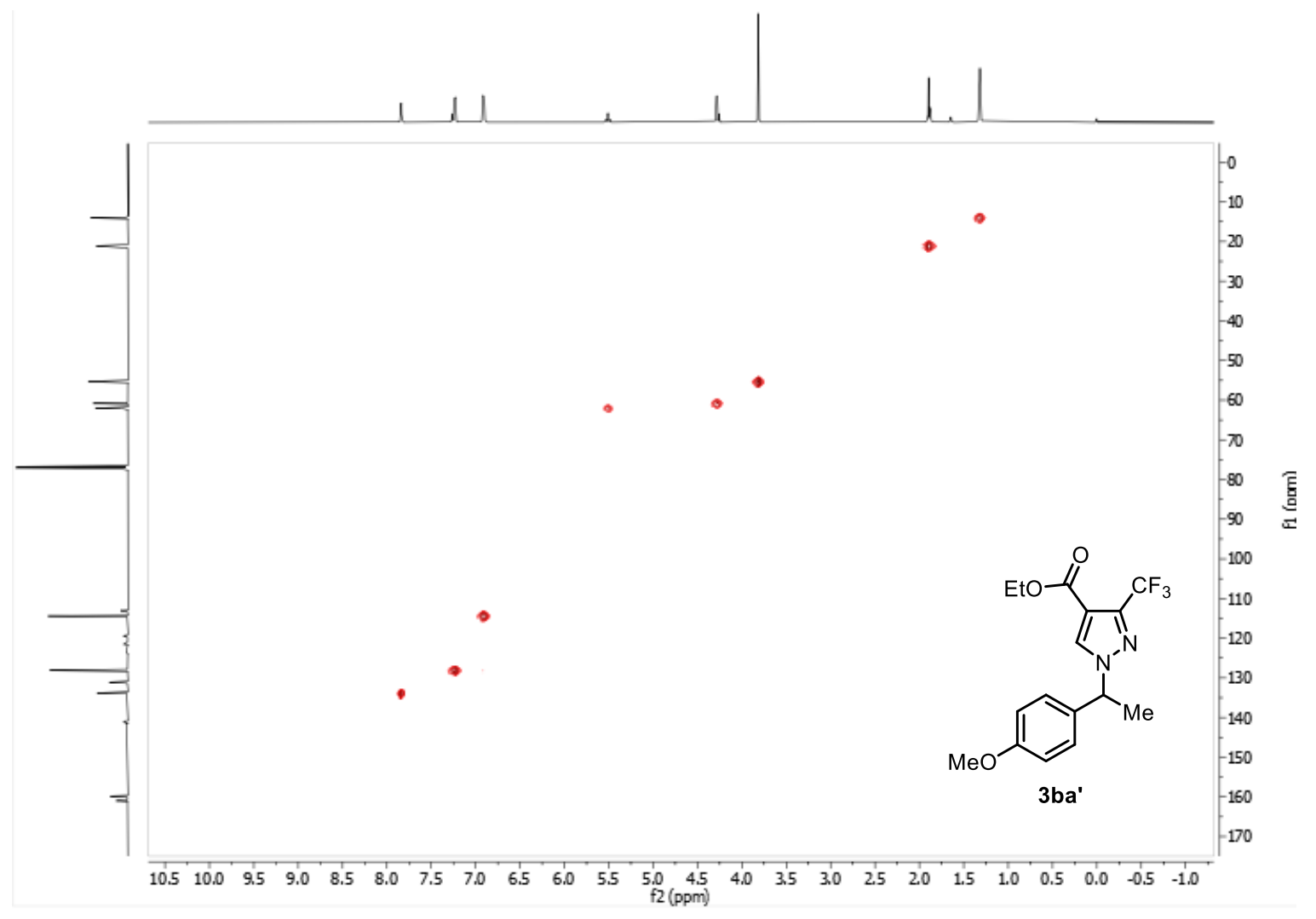


HMBC NMR spectrum of 3ba' in $\mathrm{CDCl}_{3}$ containing $0.03 \%$ (v/v) TMS (500, $\left.126 \mathrm{MHz}\right)$.

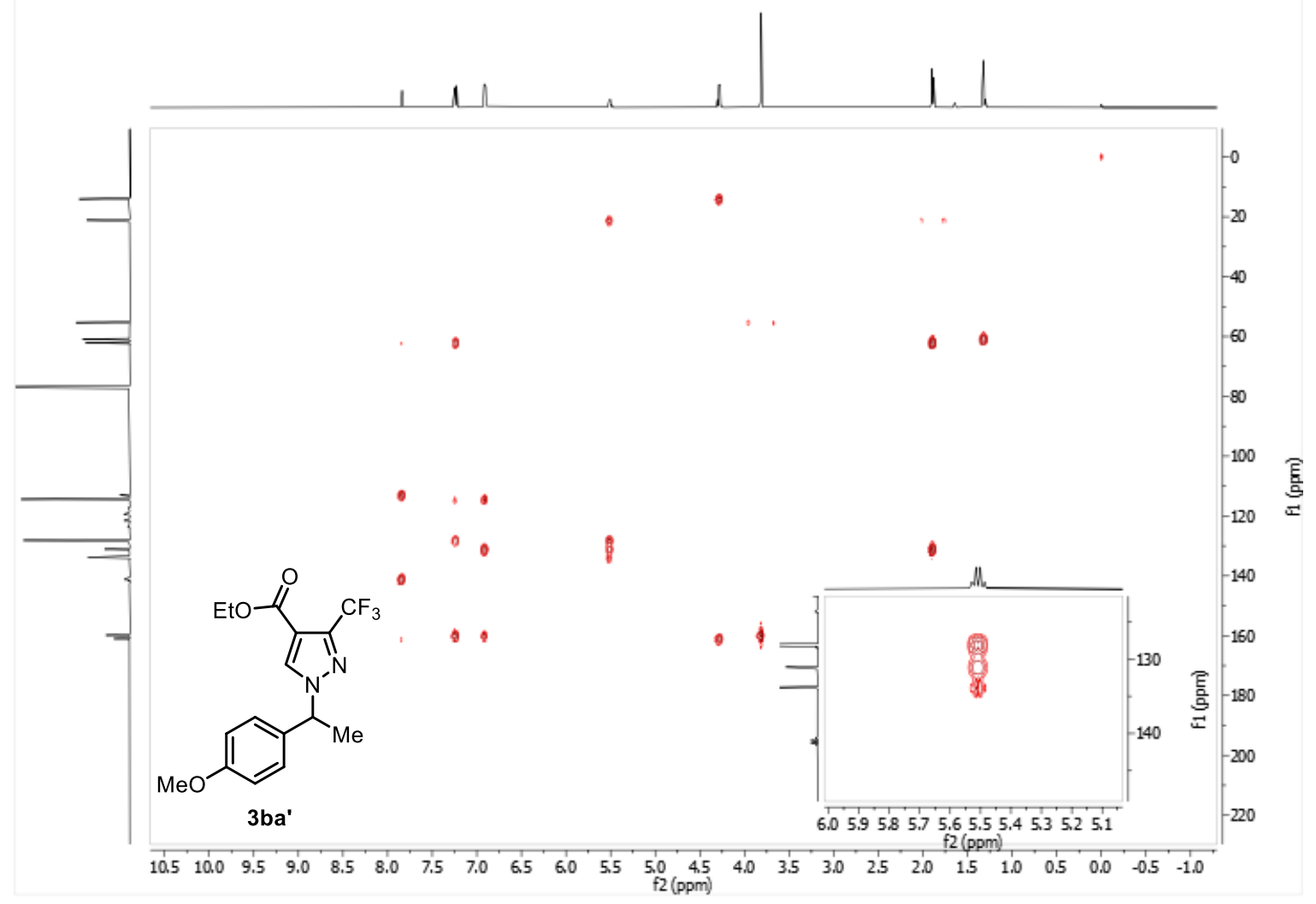

${ }^{1} \mathrm{H}$ NMR spectrum of $\mathbf{3 c a}$ in $\mathrm{CDCl}_{3}$ containing $0.03 \%$ (v/v) TMS (500 MHz).

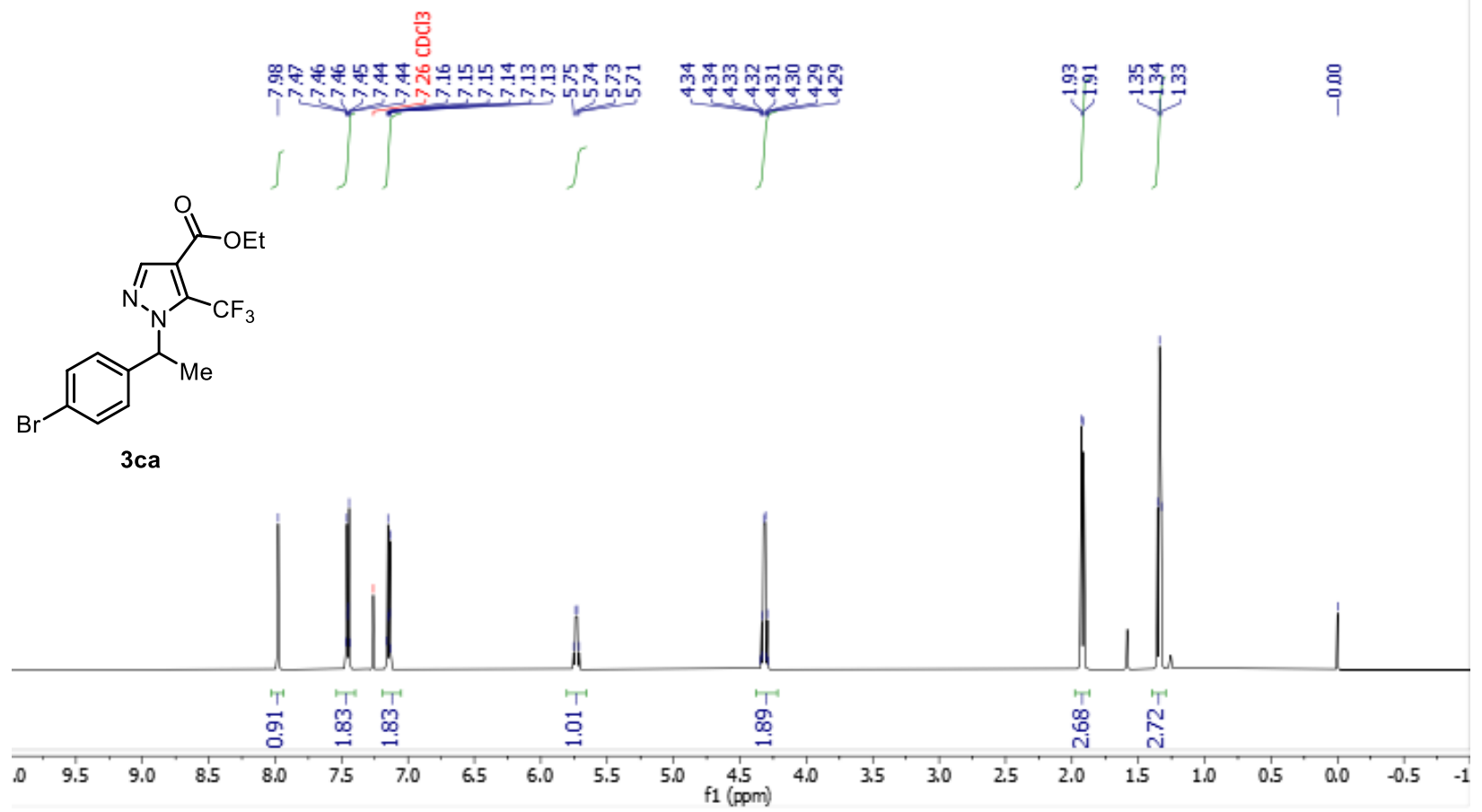


${ }^{13} \mathrm{C}$ NMR spectrum of $\mathbf{3 c a}$ in $\mathrm{CDCl}_{3}$ containing $0.03 \%$ (v/v) TMS (126 MHz).
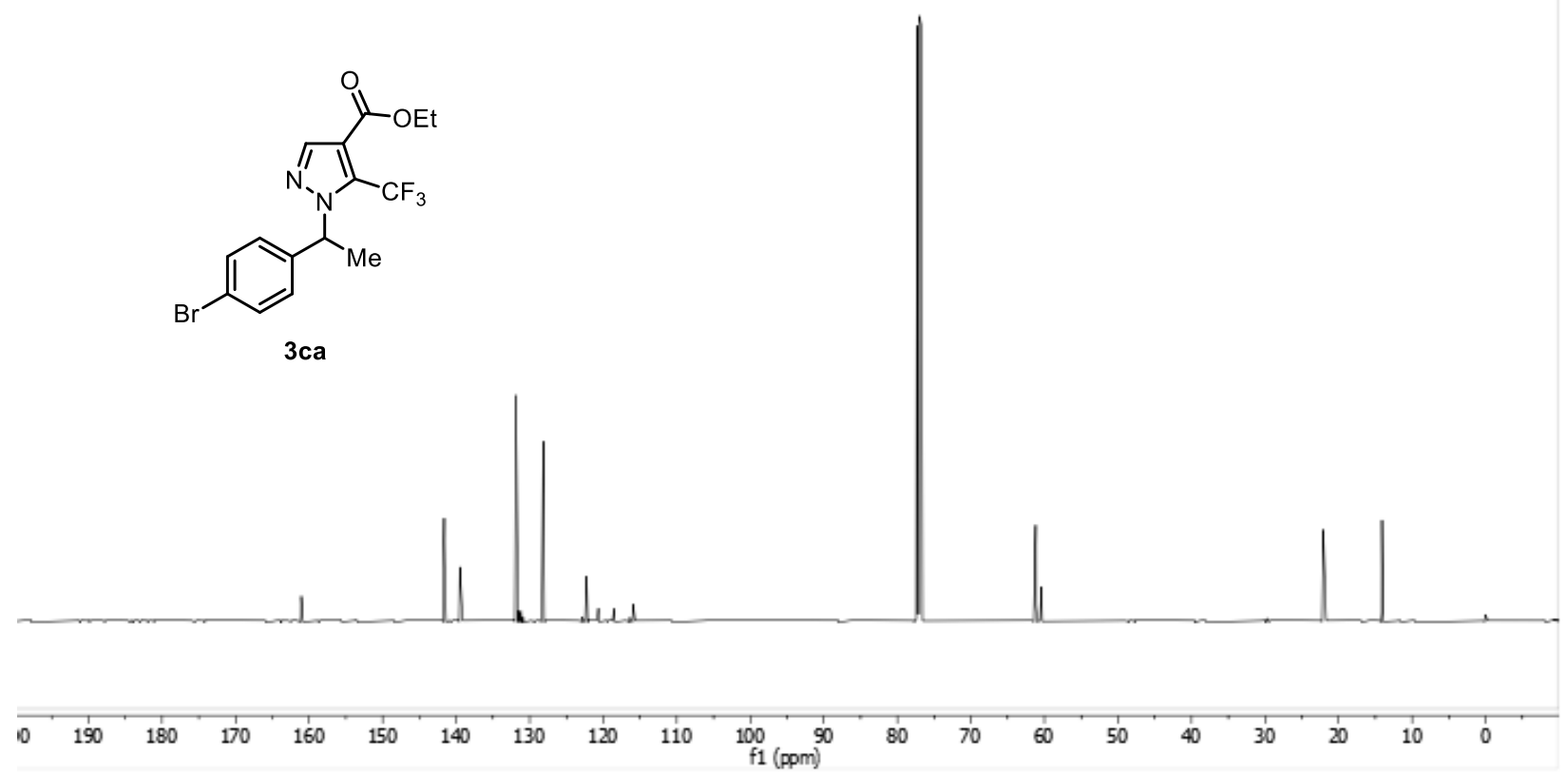

${ }^{19} \mathrm{~F}$ NMR spectrum of $3 \mathbf{c a}$ in $\mathrm{CDCl}_{3}$ containing $0.03 \%(\mathrm{v} / \mathrm{v})$ TMS $(377 \mathrm{MHz})$.

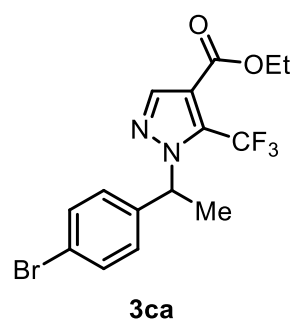


HSQC NMR spectrum of $3 \mathbf{c a}$ in $\mathrm{CDCl}_{3}$ containing $0.03 \%$ (v/v) TMS (500, $126 \mathrm{MHz}$ ).

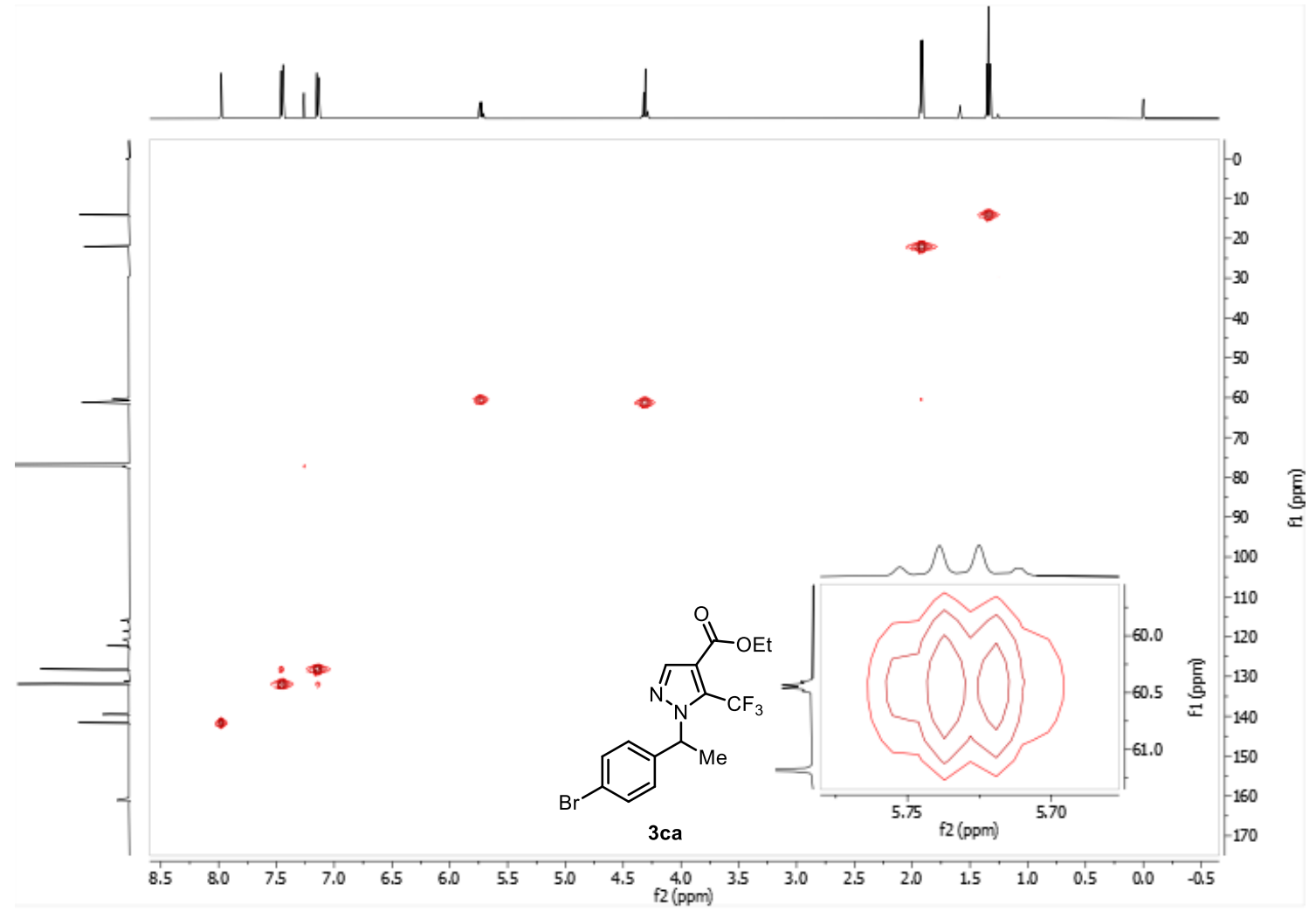

HMBC NMR spectrum of 3ca in $\mathrm{CDCl}_{3}$ containing $0.03 \%$ (v/v) TMS (500, $126 \mathrm{MHz}$ ).

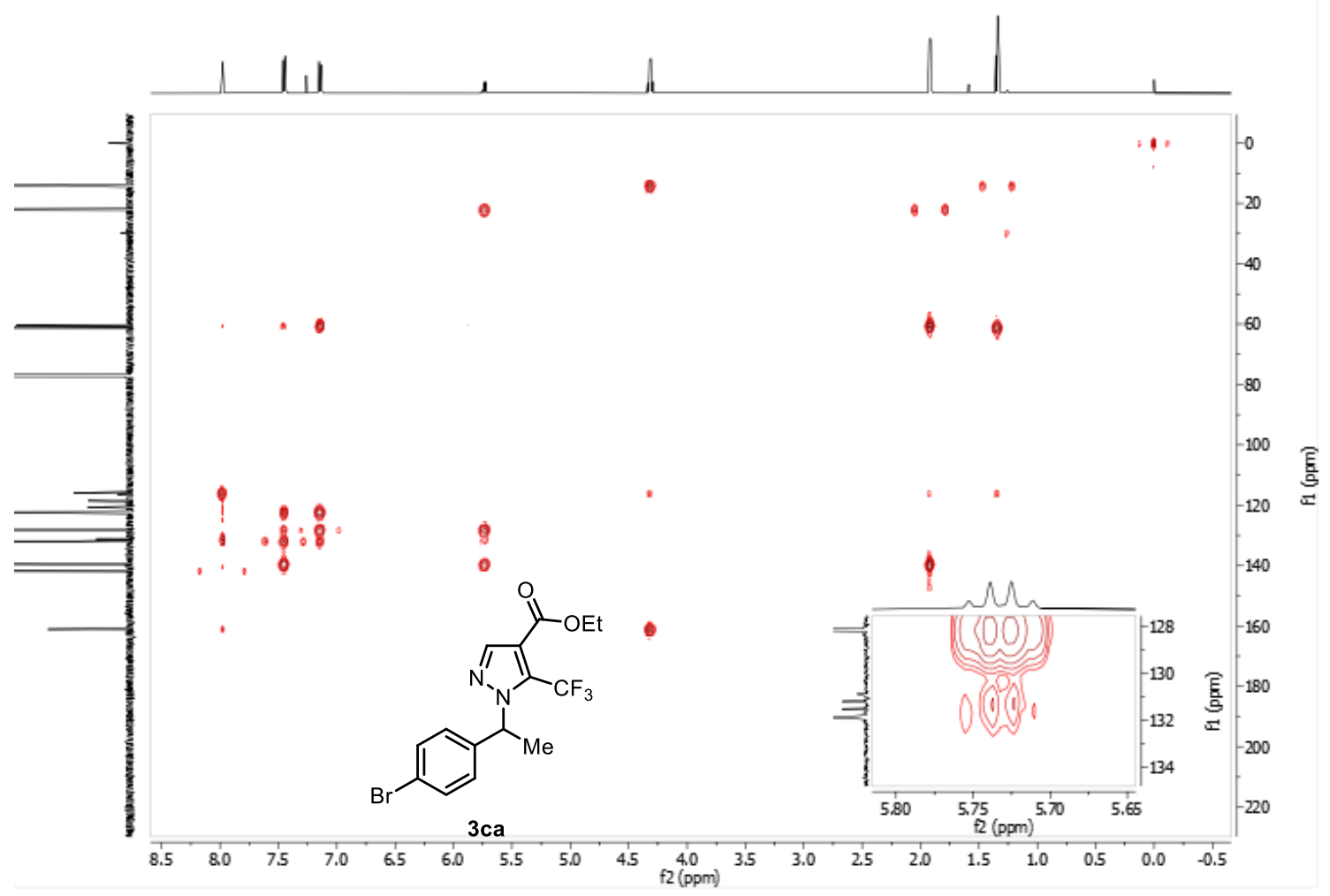


${ }^{1} \mathrm{H}$ NMR spectrum of $\mathbf{3} \mathbf{c a}^{\prime}$ in $\mathrm{CDCl}_{3}$ containing $0.03 \%$ (v/v) TMS (500 MHz).

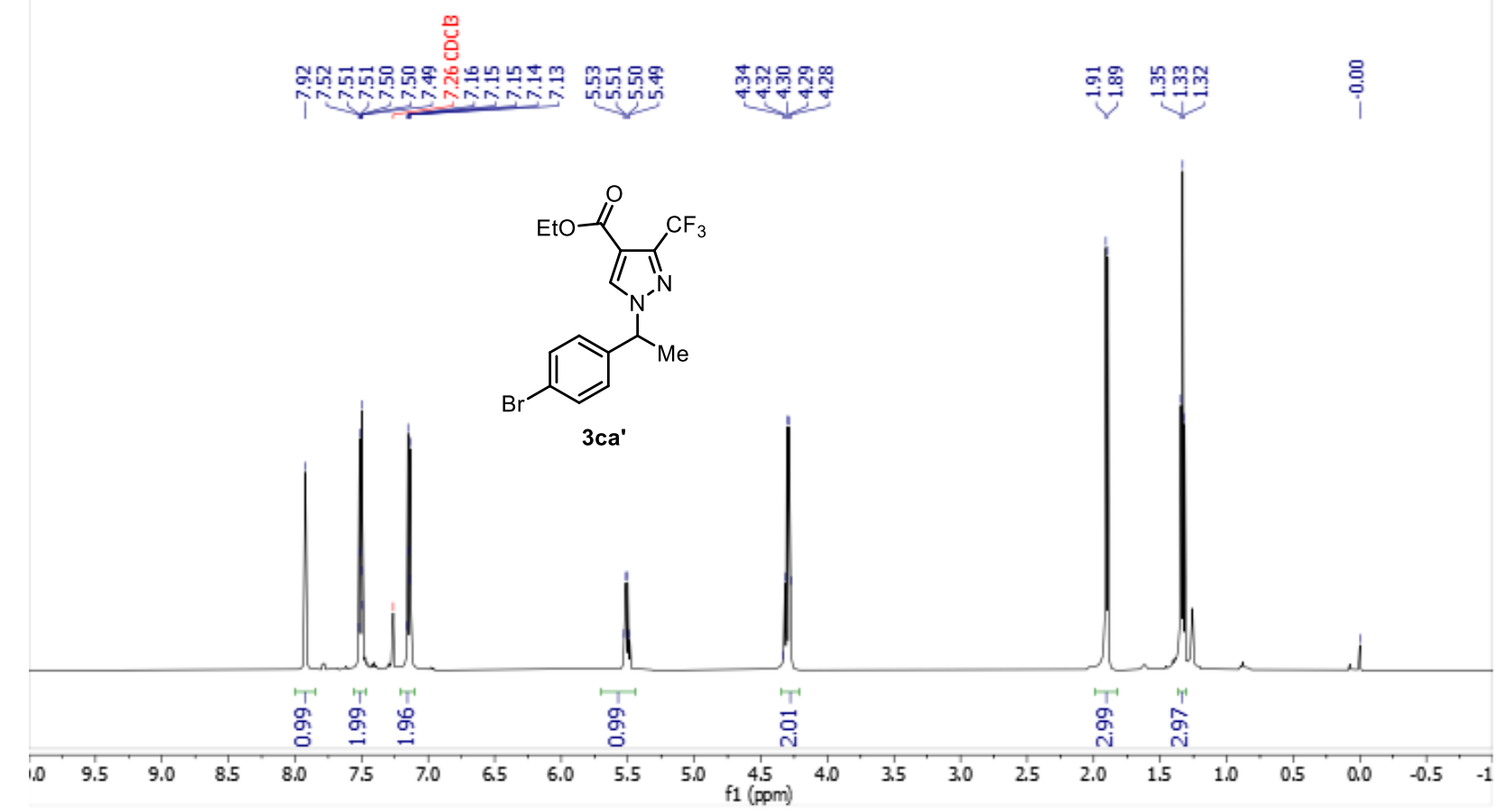

${ }^{13} \mathrm{C}$ NMR spectrum of $3 \mathbf{c a}$ ' in $\mathrm{CDCl}_{3}$ containing $0.03 \%$ (v/v) TMS (126 MHz).

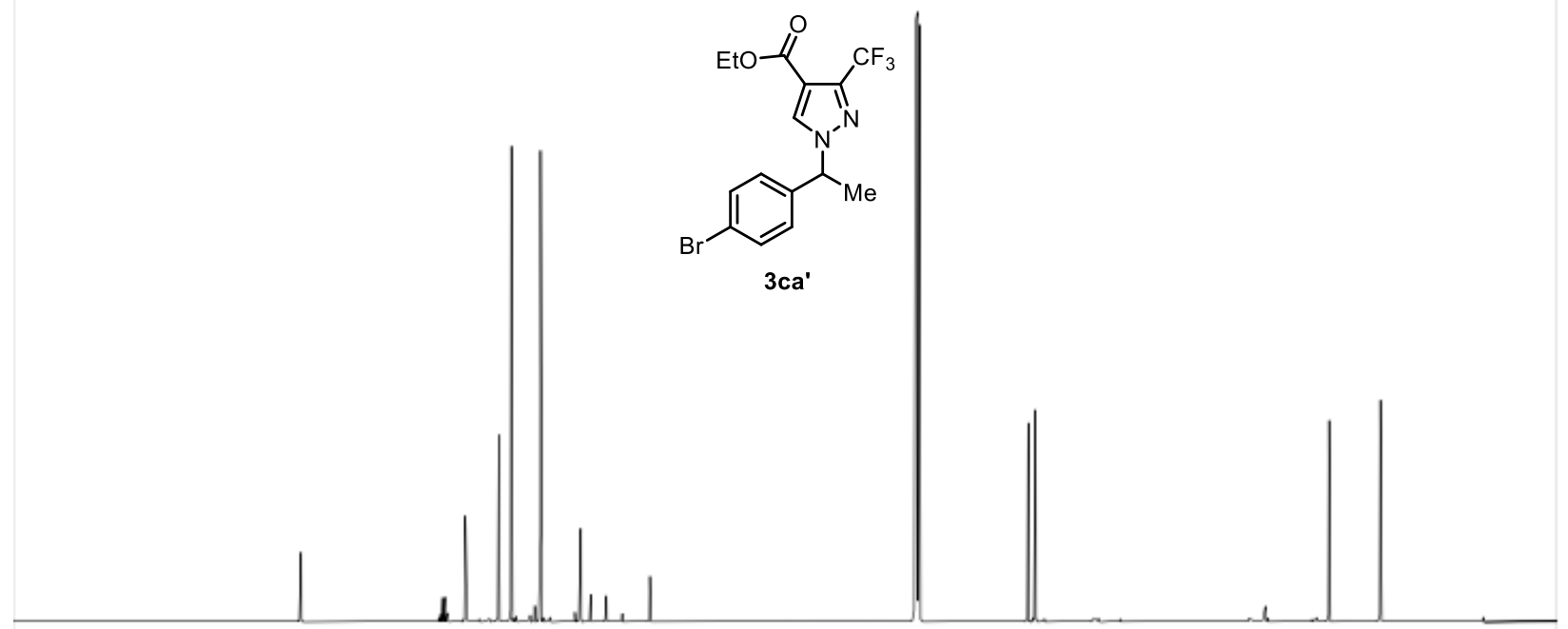

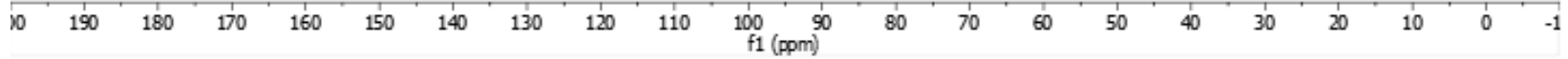


${ }^{19} \mathrm{~F}$ NMR spectrum of $\mathbf{3} \mathbf{c a}$ ' in $\mathrm{CDCl}_{3}$ containing $0.03 \%$ (v/v) TMS (377 MHz).
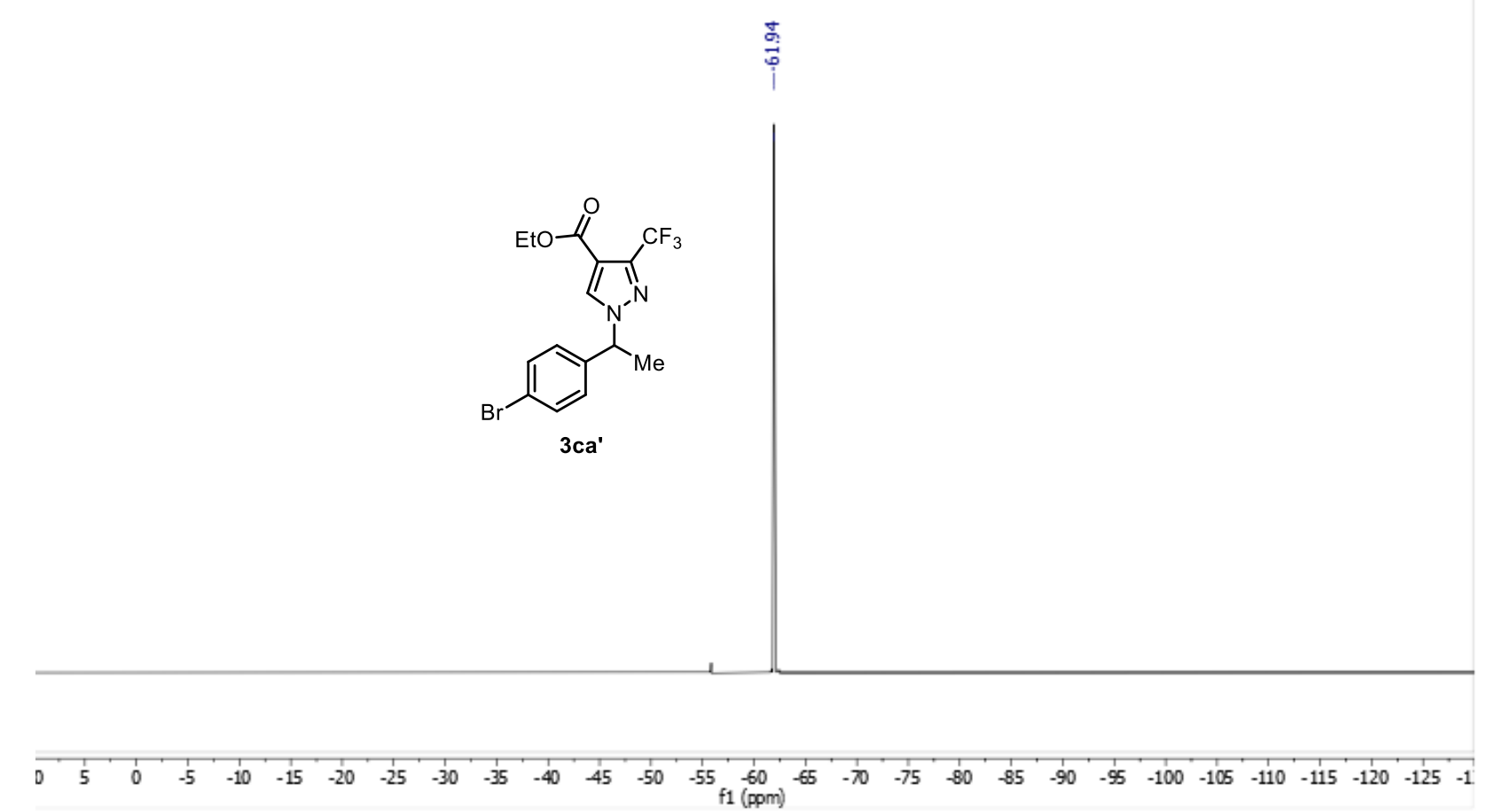

HSQC NMR spectrum of 3ca' in $\mathrm{CDCl}_{3}$ containing $0.03 \%$ (v/v) TMS (500, $126 \mathrm{MHz}$ ).

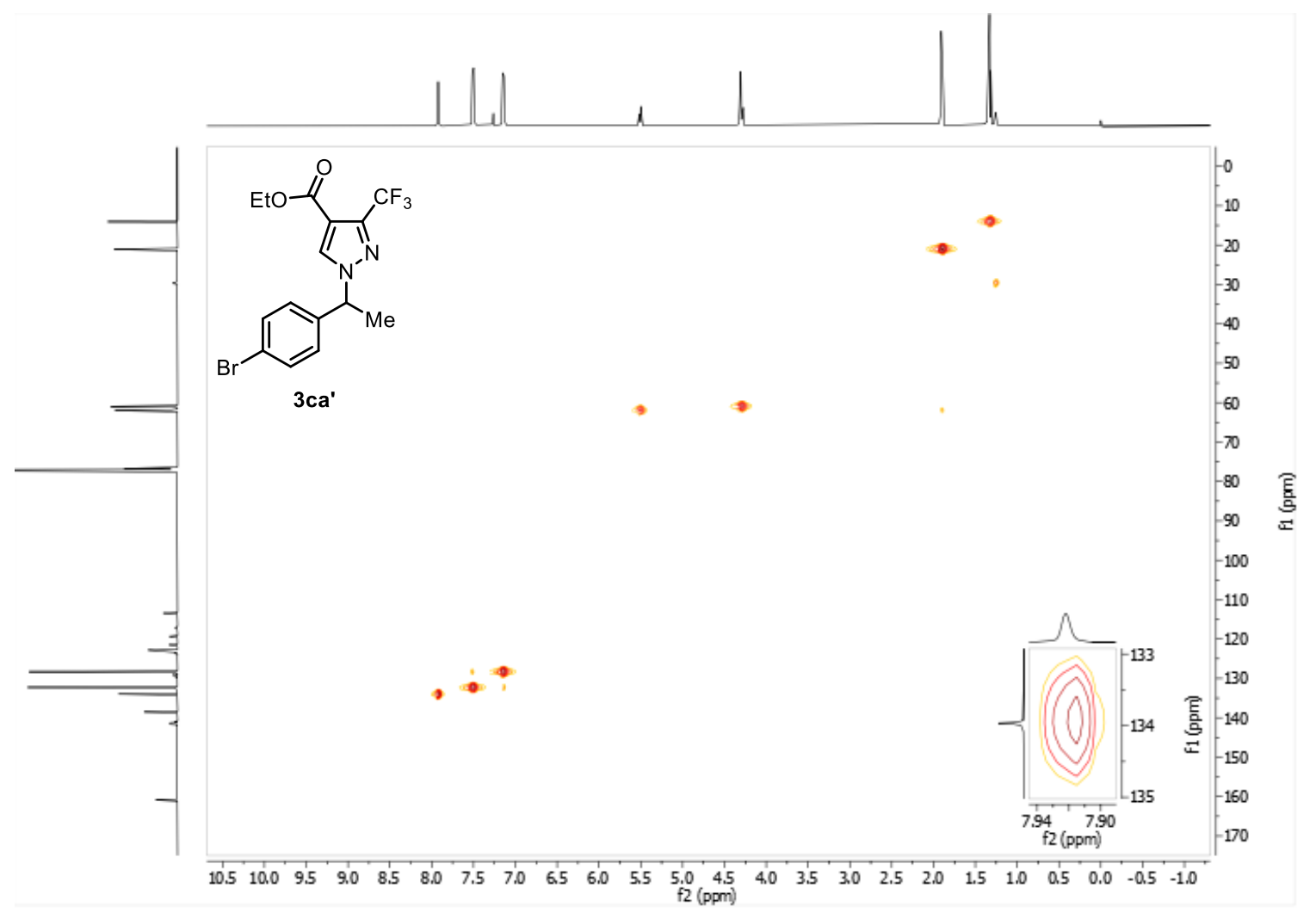


HMBC NMR spectrum of 3ca' in $\mathrm{CDCl}_{3}$ containing $0.03 \%$ (v/v) TMS (500, $126 \mathrm{MHz}$ ).

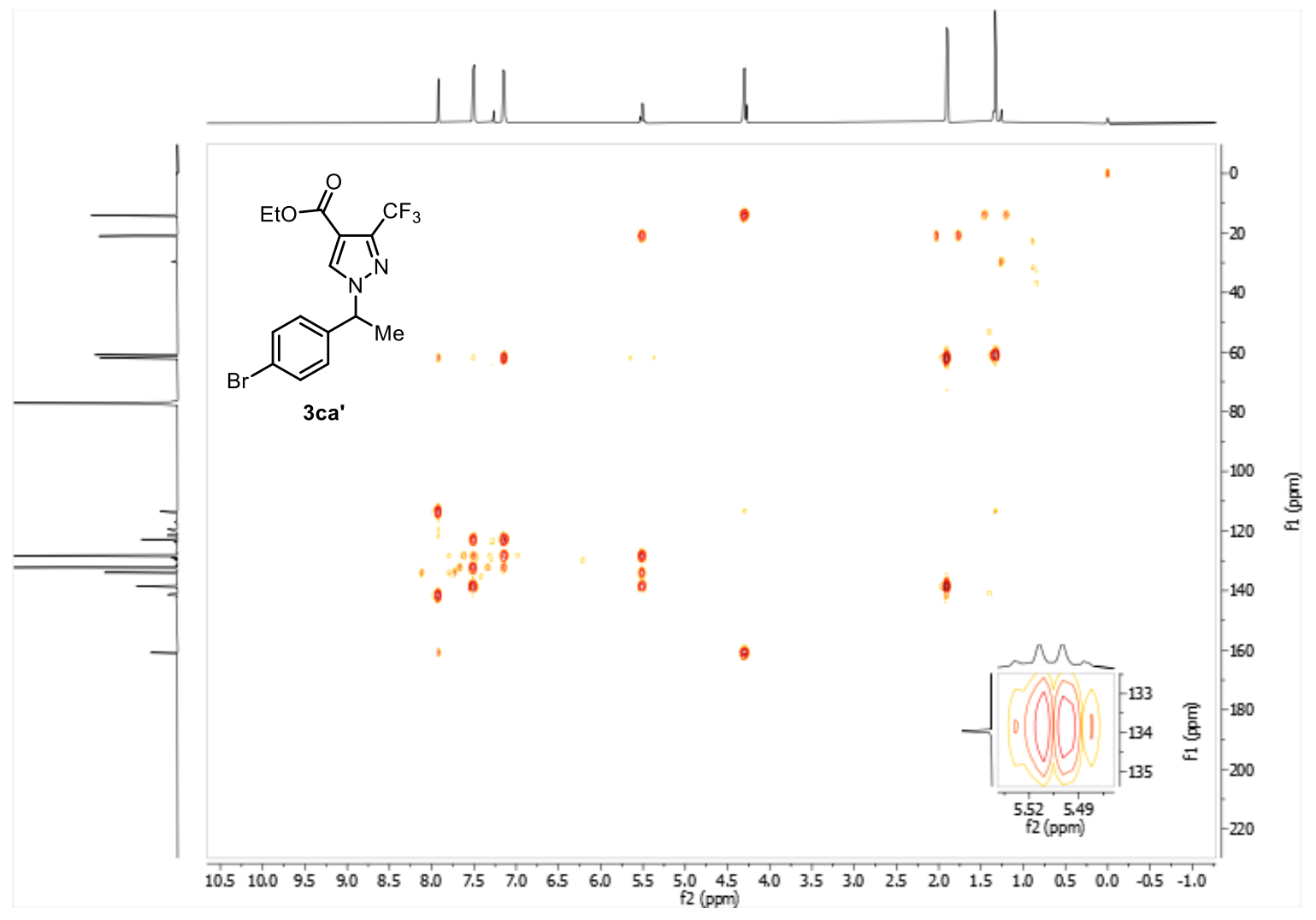

${ }^{1} \mathrm{H}$ NMR spectrum of 3da in $\mathrm{CDCl}_{3}$ containing $0.03 \%$ (v/v) TMS (500 MHz).

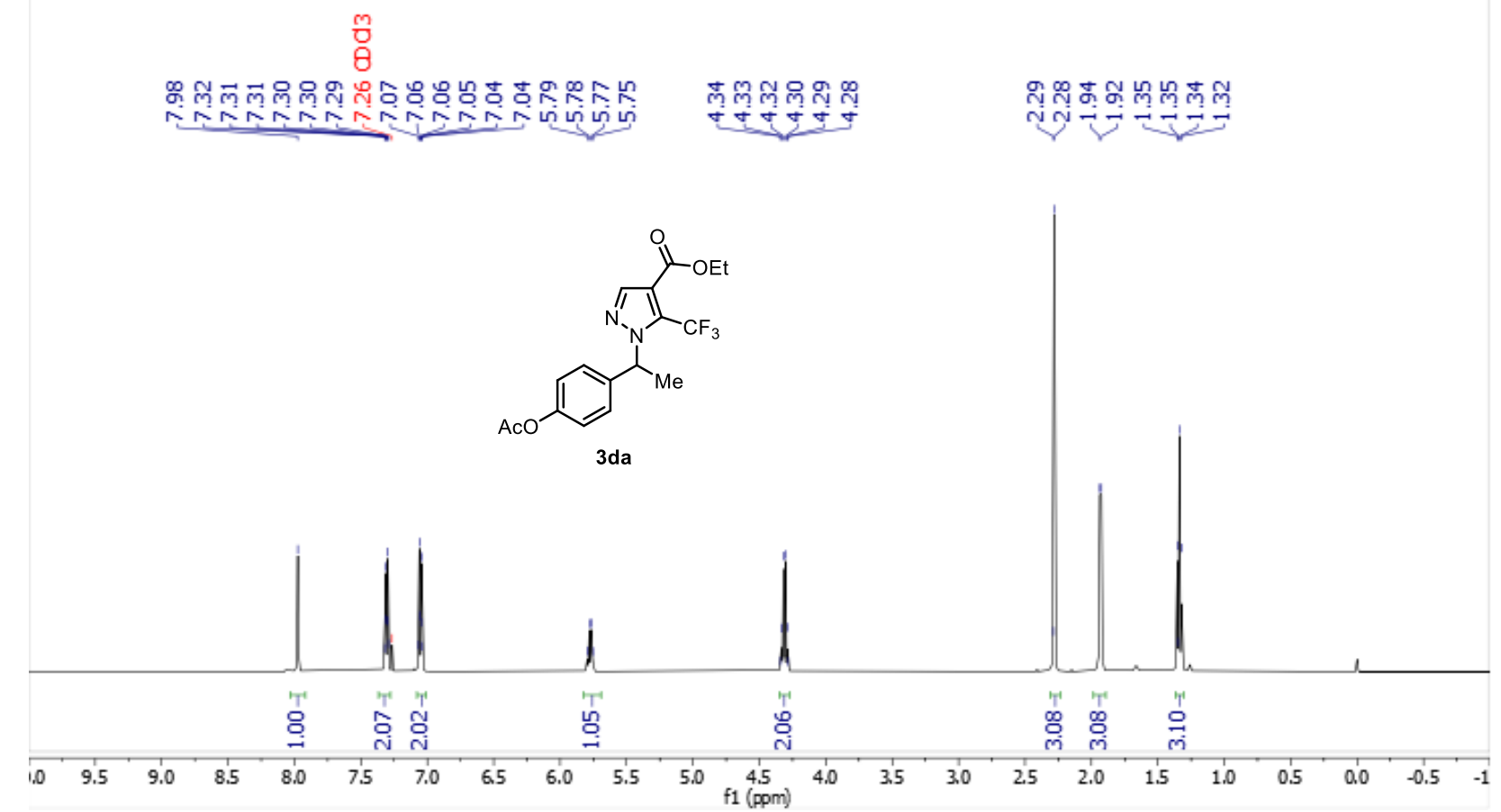


${ }^{13} \mathrm{C}$ NMR spectrum of $\mathbf{3 d a}$ in $\mathrm{CDCl}_{3}$ containing $0.03 \%(\mathrm{v} / \mathrm{v})$ TMS (126 MHz).

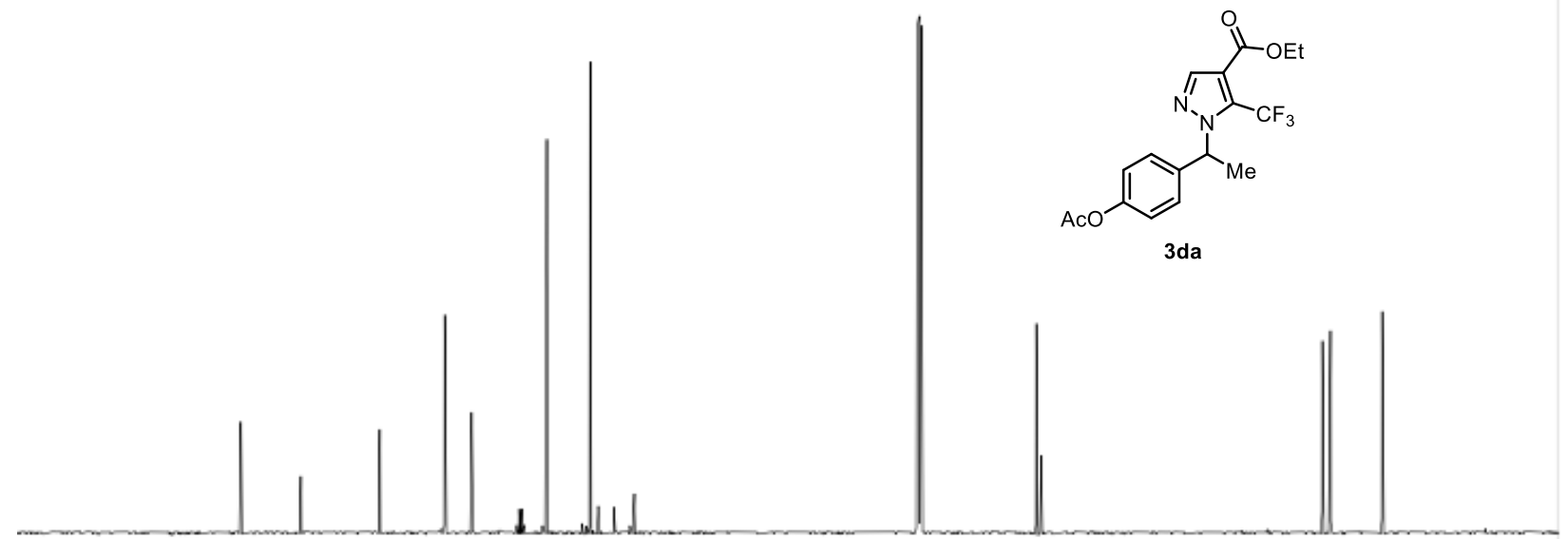

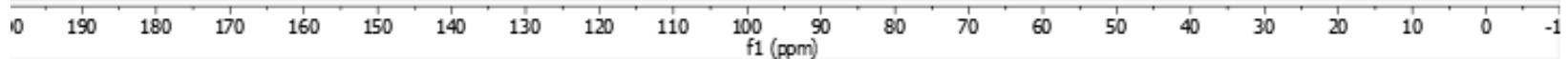

${ }^{19} \mathrm{~F}$ NMR spectrum of $\mathbf{3 d a}$ in $\mathrm{CDCl}_{3}$ containing $0.03 \%$ (v/v) TMS (377 MHz).

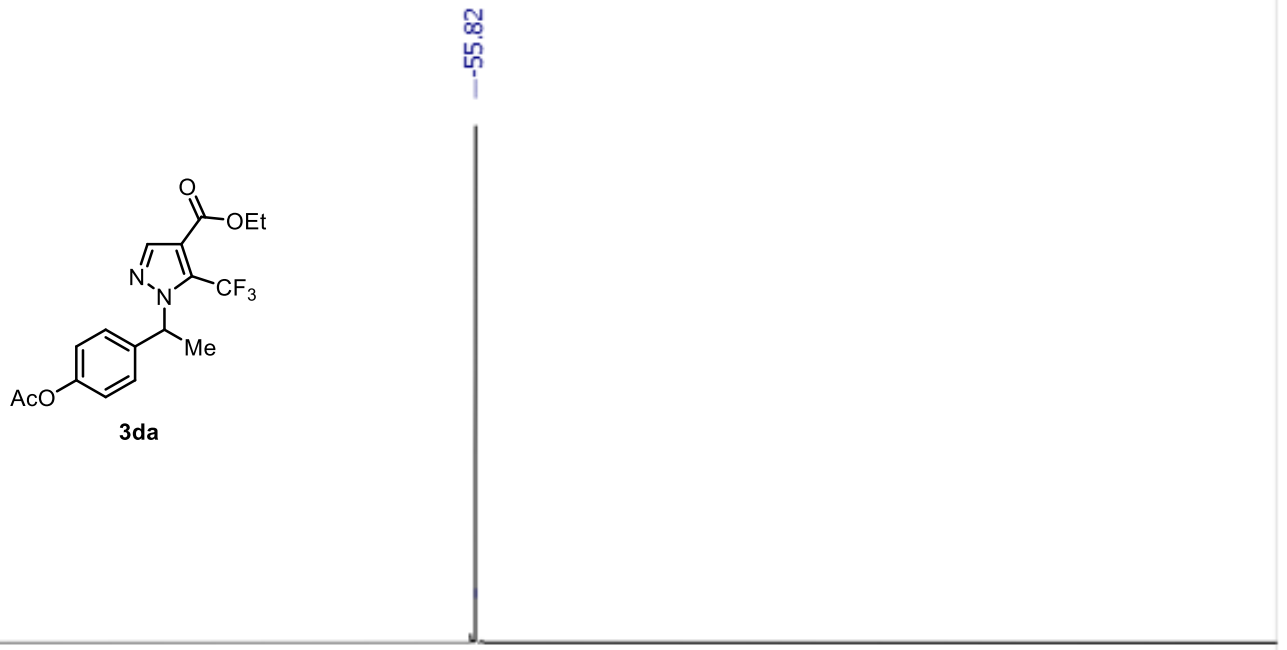

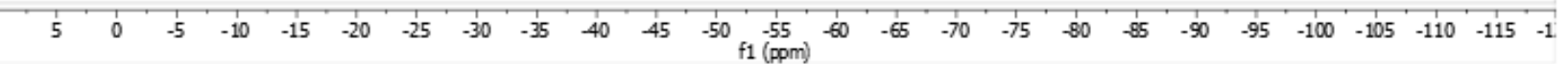


HSQC NMR spectrum of 3da in $\mathrm{CDCl}_{3}$ containing $0.03 \%(\mathrm{v} / \mathrm{v})$ TMS $(500,126 \mathrm{MHz})$.

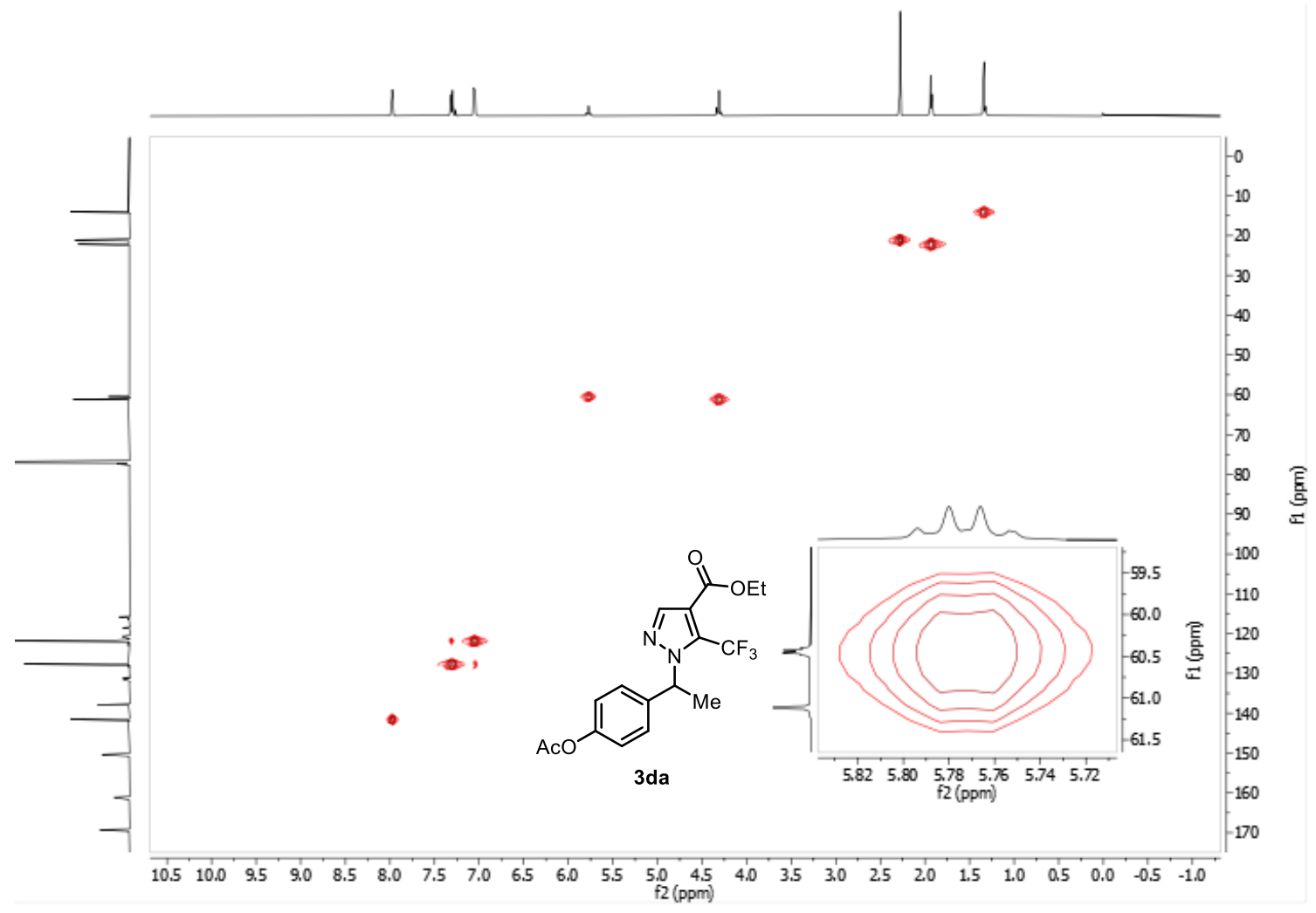

HMBC NMR spectrum of 3da in $\mathrm{CDCl}_{3}$ containing $0.03 \%$ (v/v) TMS $(500,126 \mathrm{MHz}$ ).

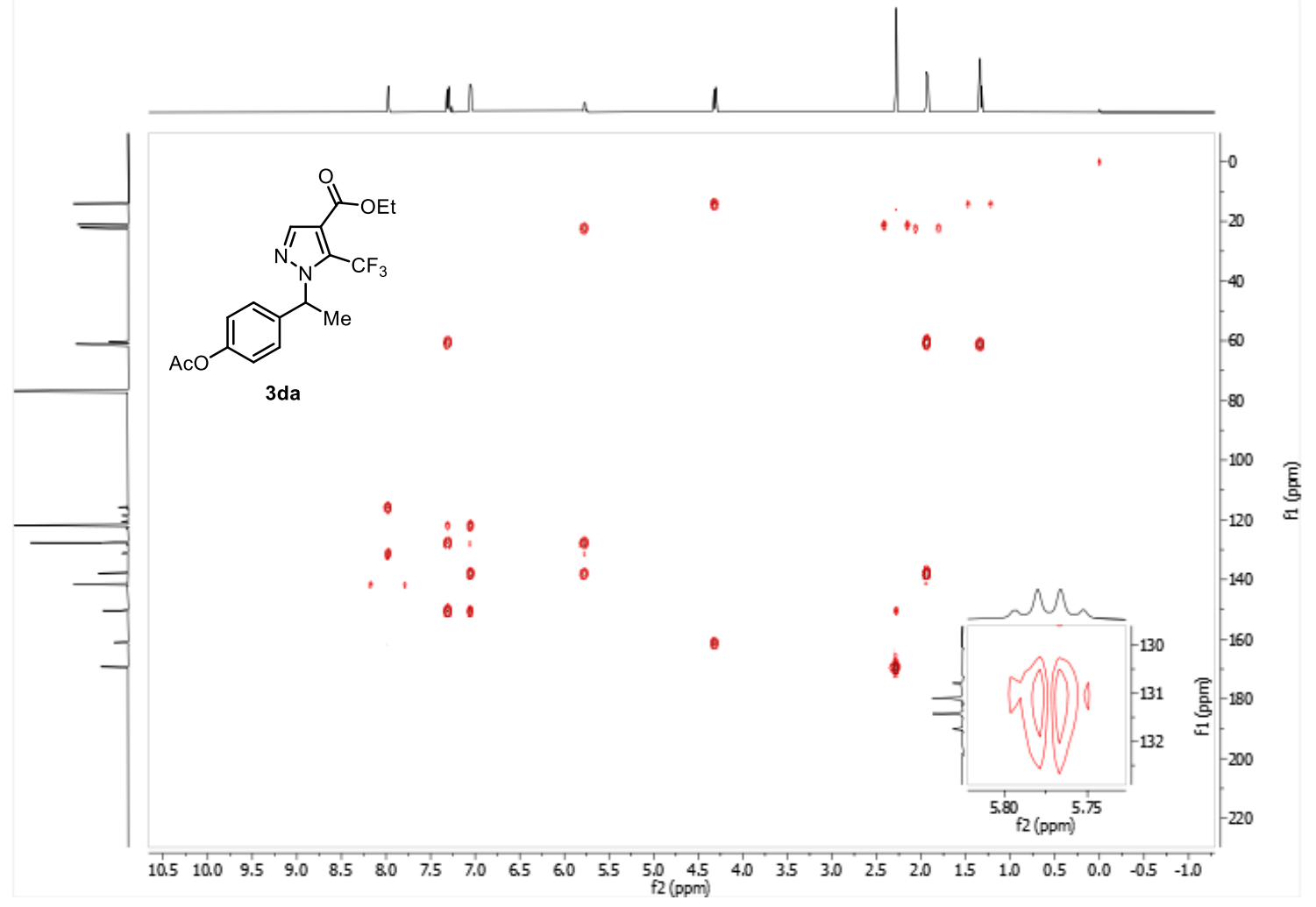


${ }^{1} \mathrm{H}$ NMR spectrum of $\mathbf{3 e a}$ in $\mathrm{CDCl}_{3}$ containing $0.03 \%$ (v/v) TMS (500 MHz).

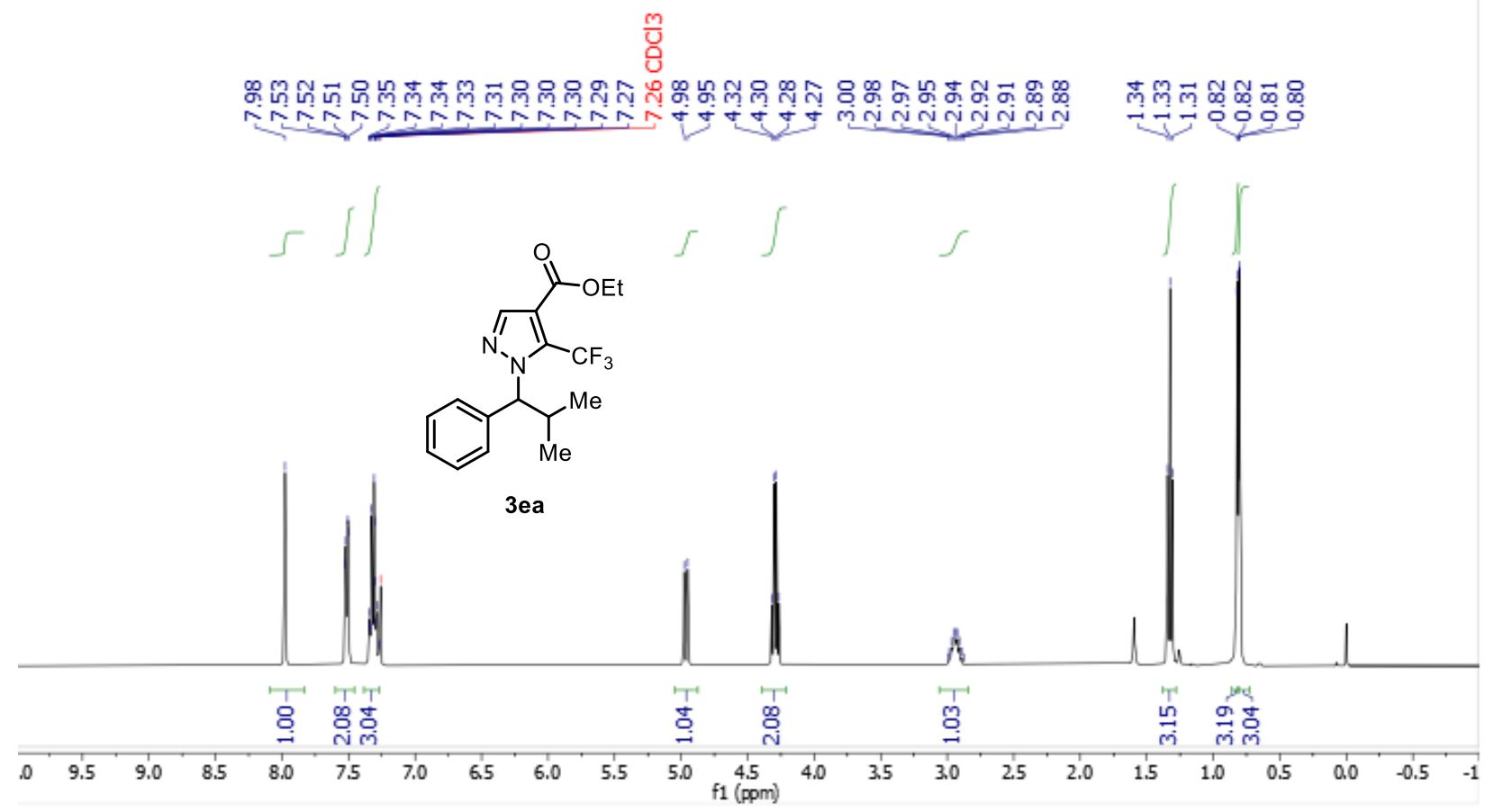

${ }^{13} \mathrm{C}$ NMR spectrum of $\mathbf{3 e a}$ in $\mathrm{CDCl}_{3}$ containing $0.03 \%$ (v/v) TMS (126 MHz).
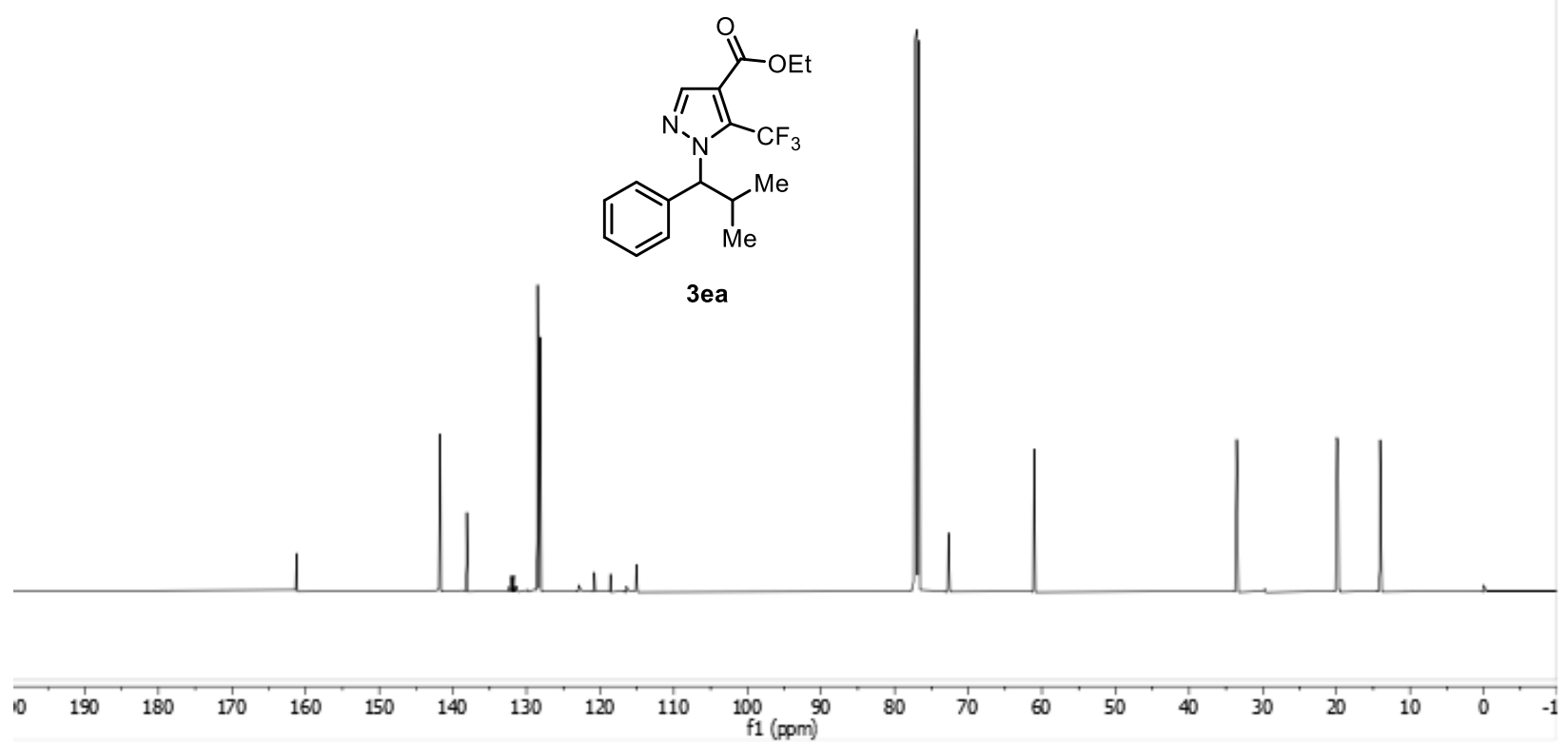
${ }^{19} \mathrm{~F}$ NMR spectrum of 3ea in $\mathrm{CDCl}_{3}$ containing $0.03 \%$ (v/v) TMS (377 MHz).

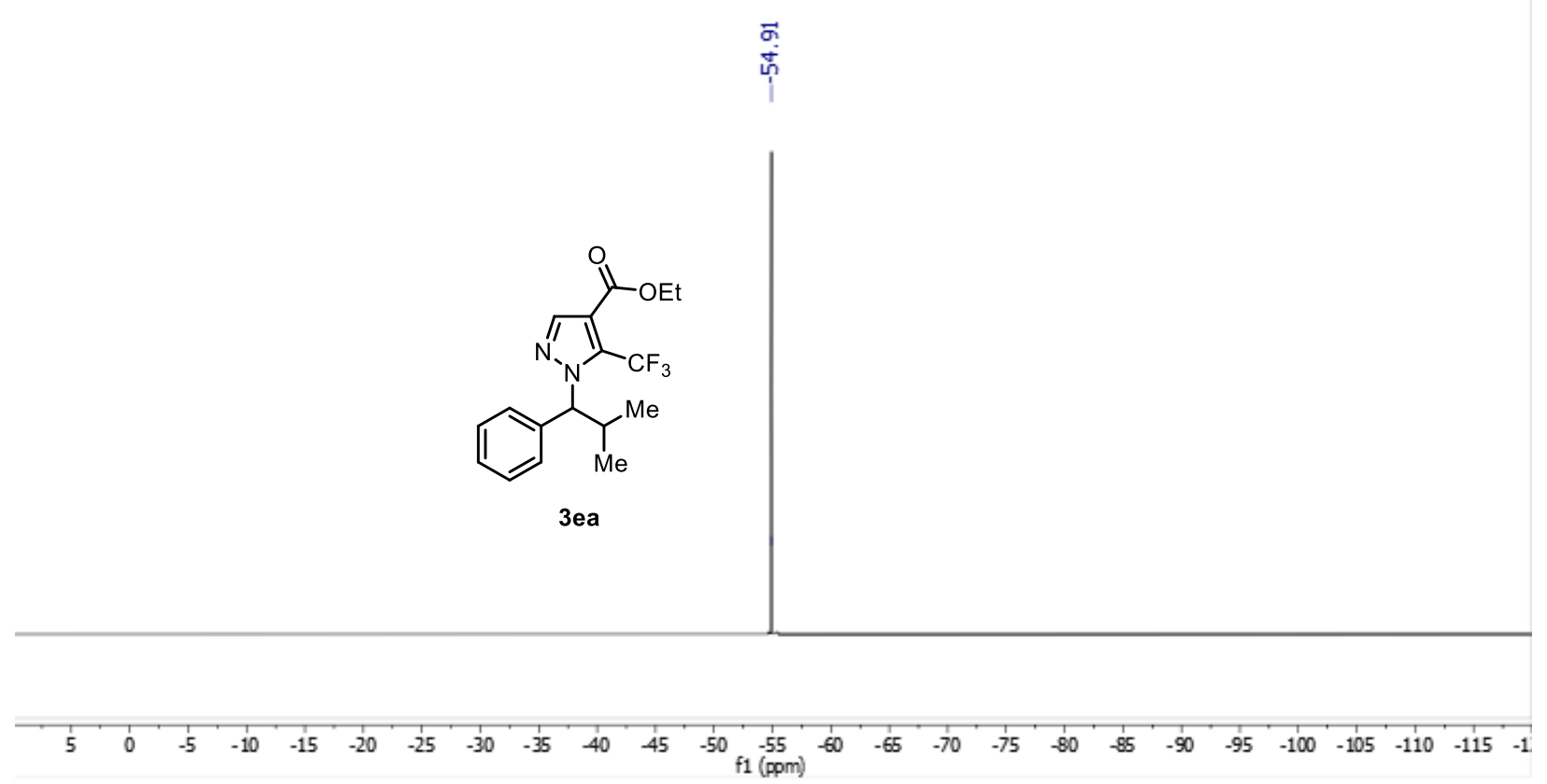

HSQC NMR spectrum of 3ea in $\mathrm{CDCl}_{3}$ containing $0.03 \%$ (v/v) TMS (500, $126 \mathrm{MHz}$ ).

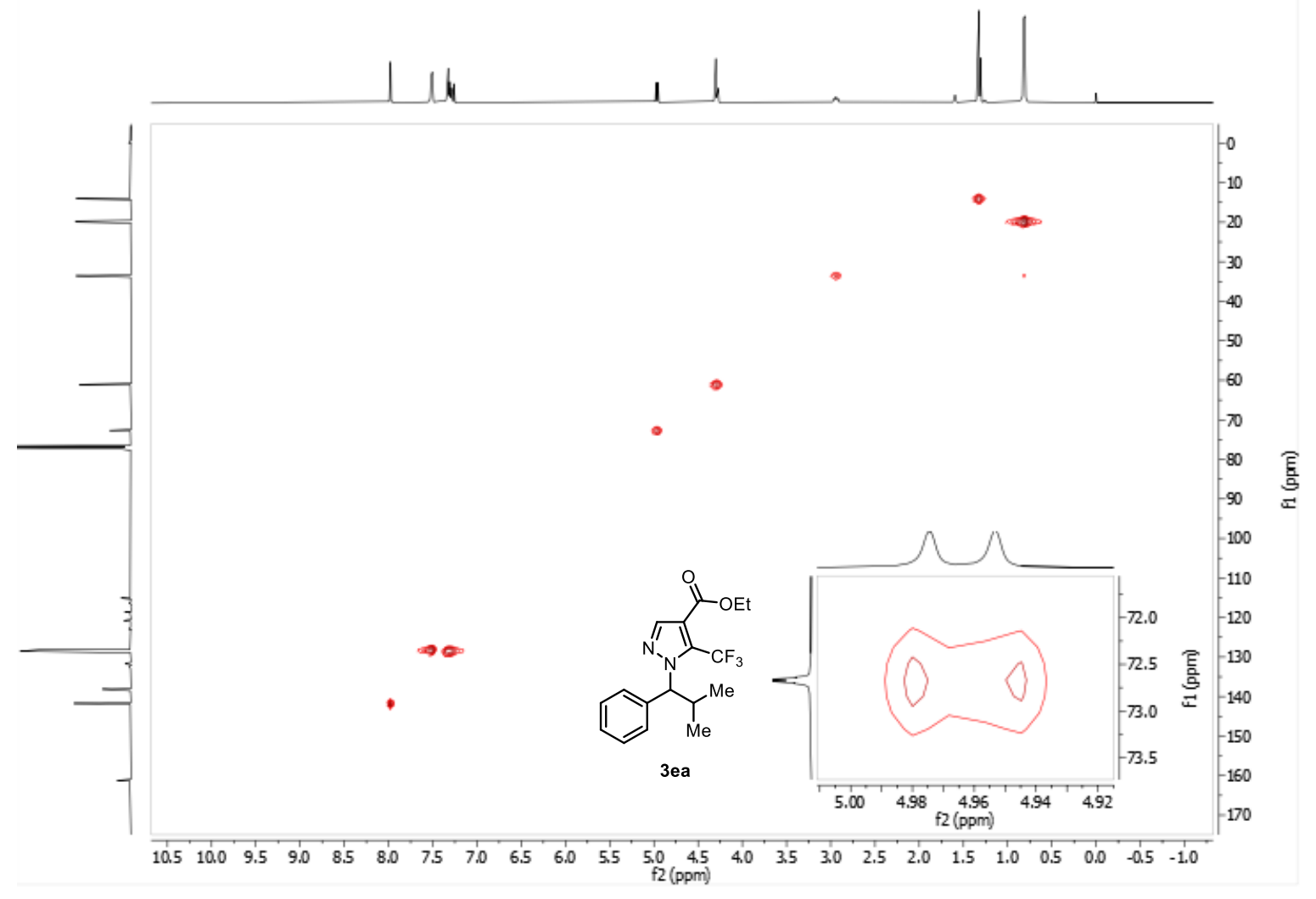


HMBC NMR spectrum of $3 \mathbf{e a}$ in $\mathrm{CDCl}_{3}$ containing $0.03 \%$ (v/v) TMS (500, $126 \mathrm{MHz}$ ).

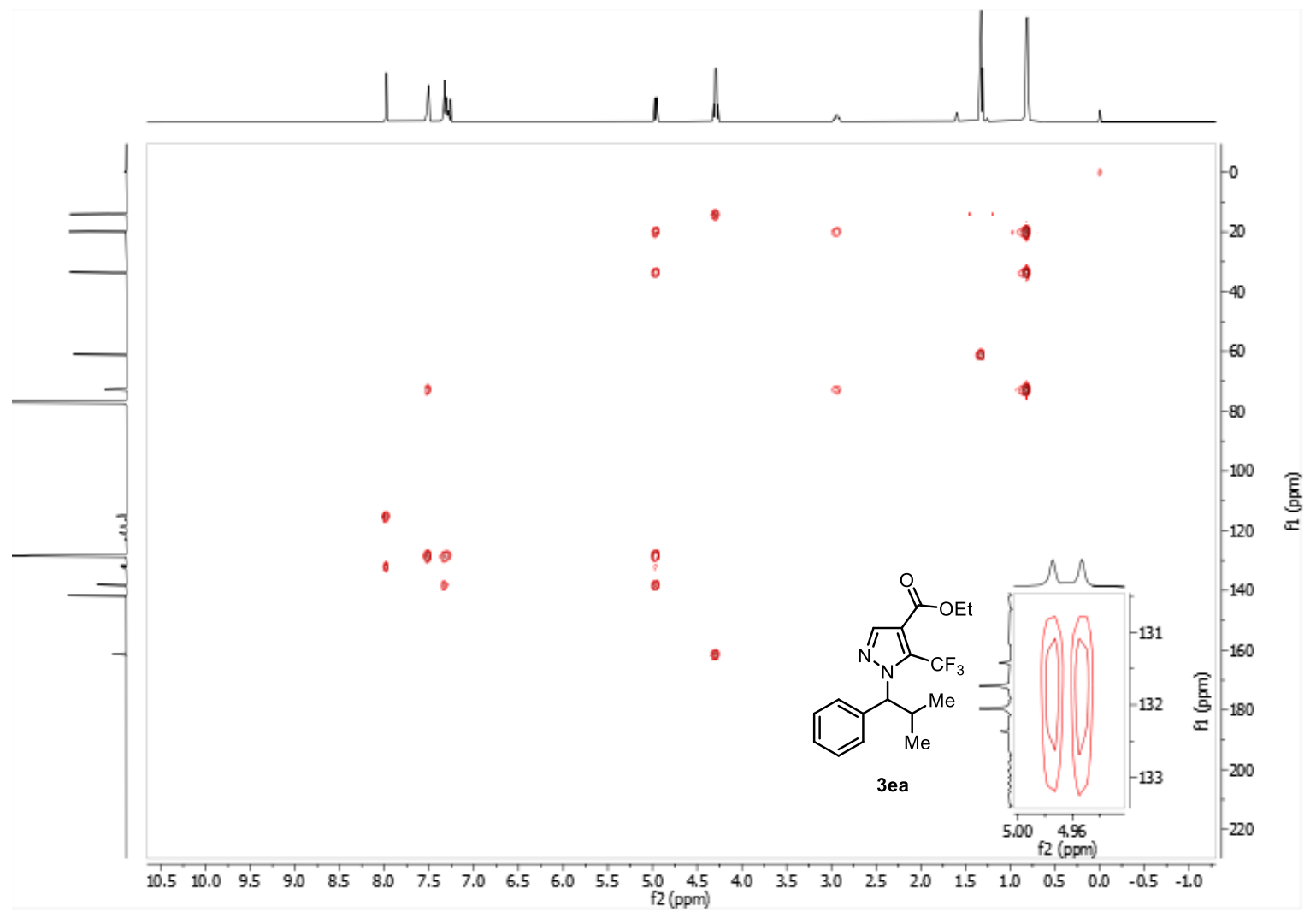

${ }^{1} \mathrm{H}$ NMR spectrum of $\mathbf{3 f a}$ in $\mathrm{CDCl}_{3}$ containing $0.03 \%$ (v/v) TMS (500 MHz).

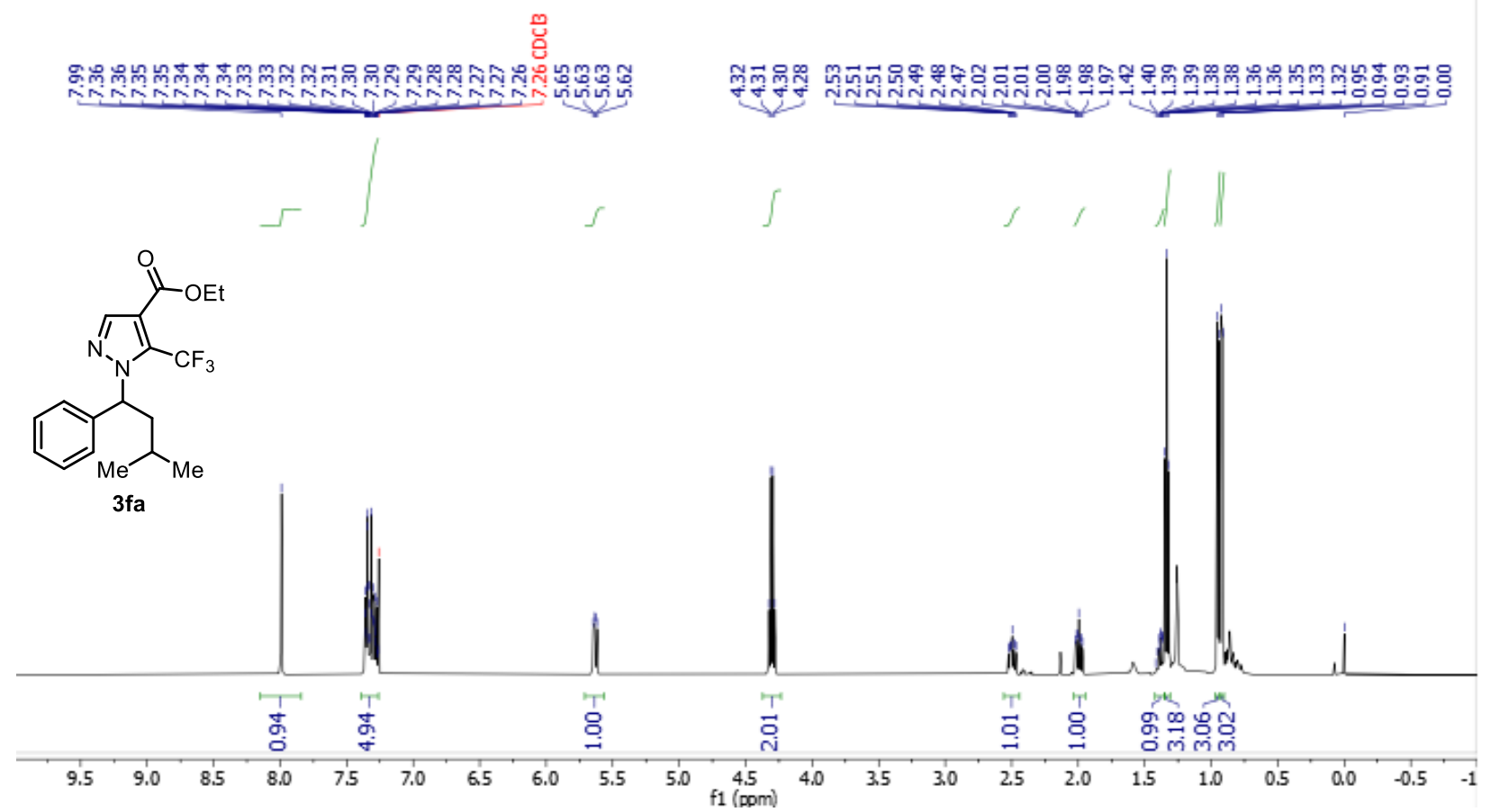


${ }^{13} \mathrm{C}$ NMR spectrum of $\mathbf{3 f a}$ in $\mathrm{CDCl}_{3}$ containing $0.03 \%(\mathrm{v} / \mathrm{v})$ TMS (126 MHz).

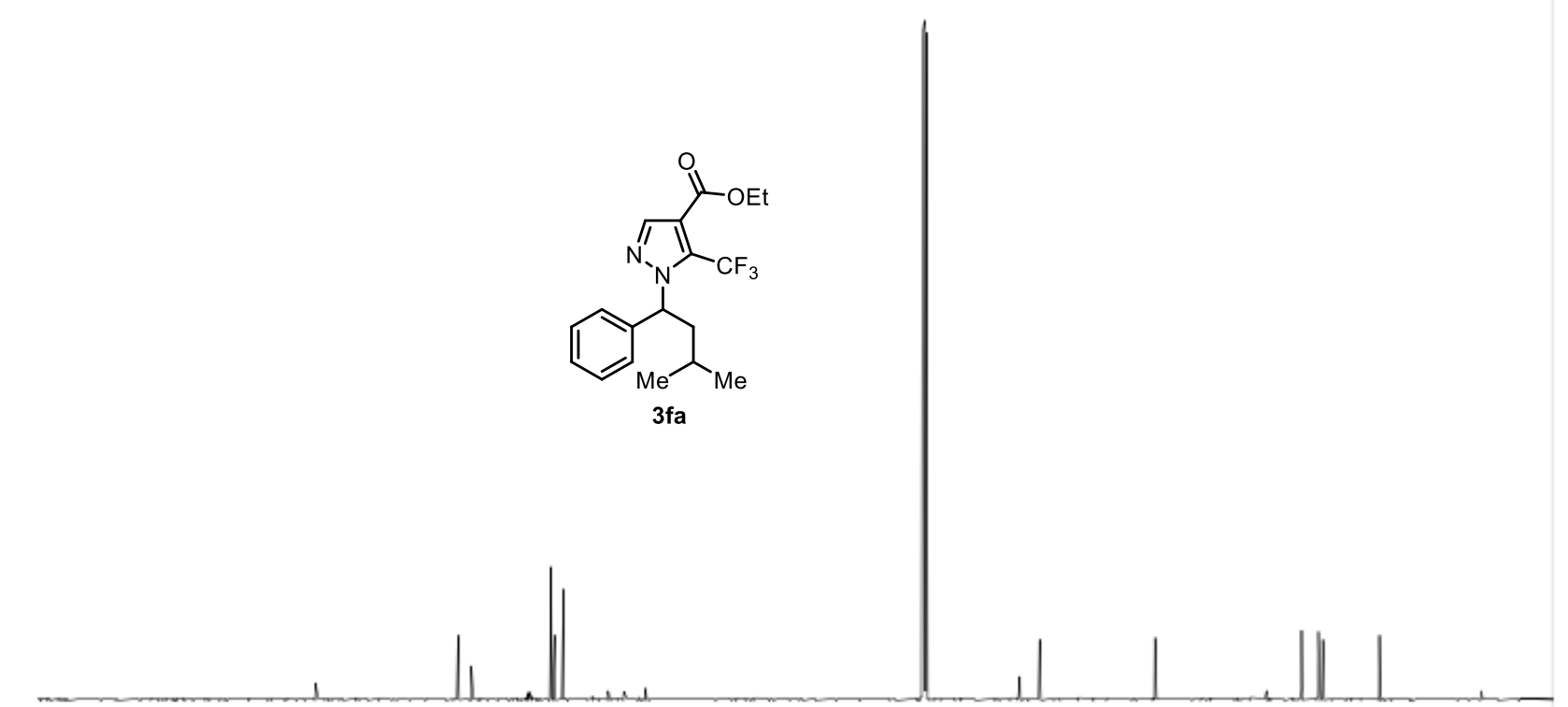

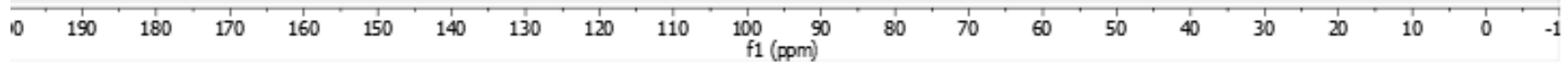

${ }^{19} \mathrm{~F}$ NMR spectrum of $\mathbf{3 f a}$ in $\mathrm{CDCl}_{3}$ containing $0.03 \%$ (v/v) TMS (377 MHz).

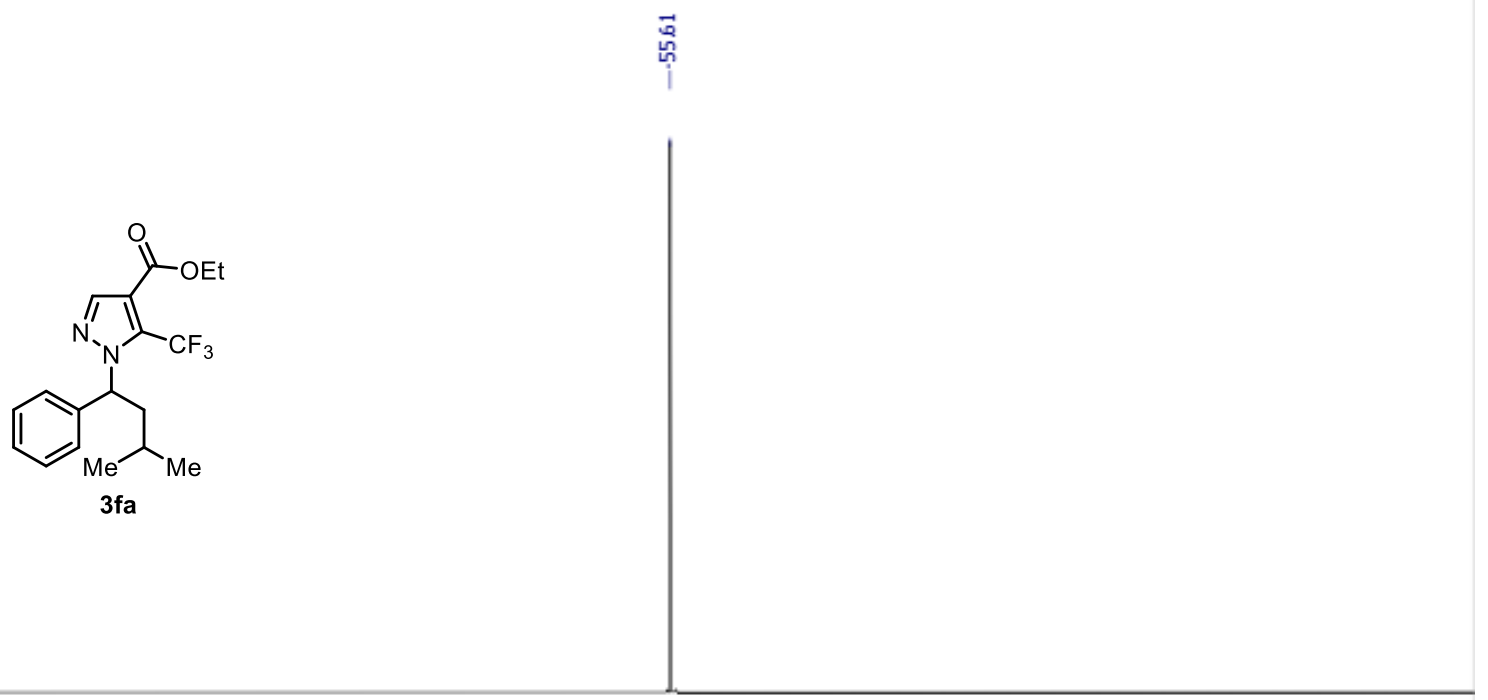

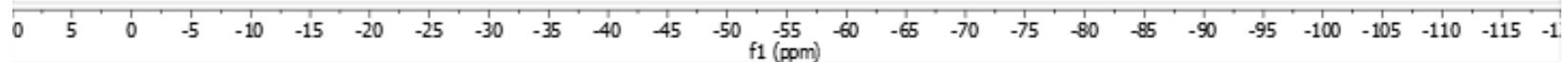


HSQC NMR spectrum of $\mathbf{3 f a}$ in $\mathrm{CDCl}_{3}$ containing $0.03 \%$ (v/v) TMS (500, $126 \mathrm{MHz}$ ).

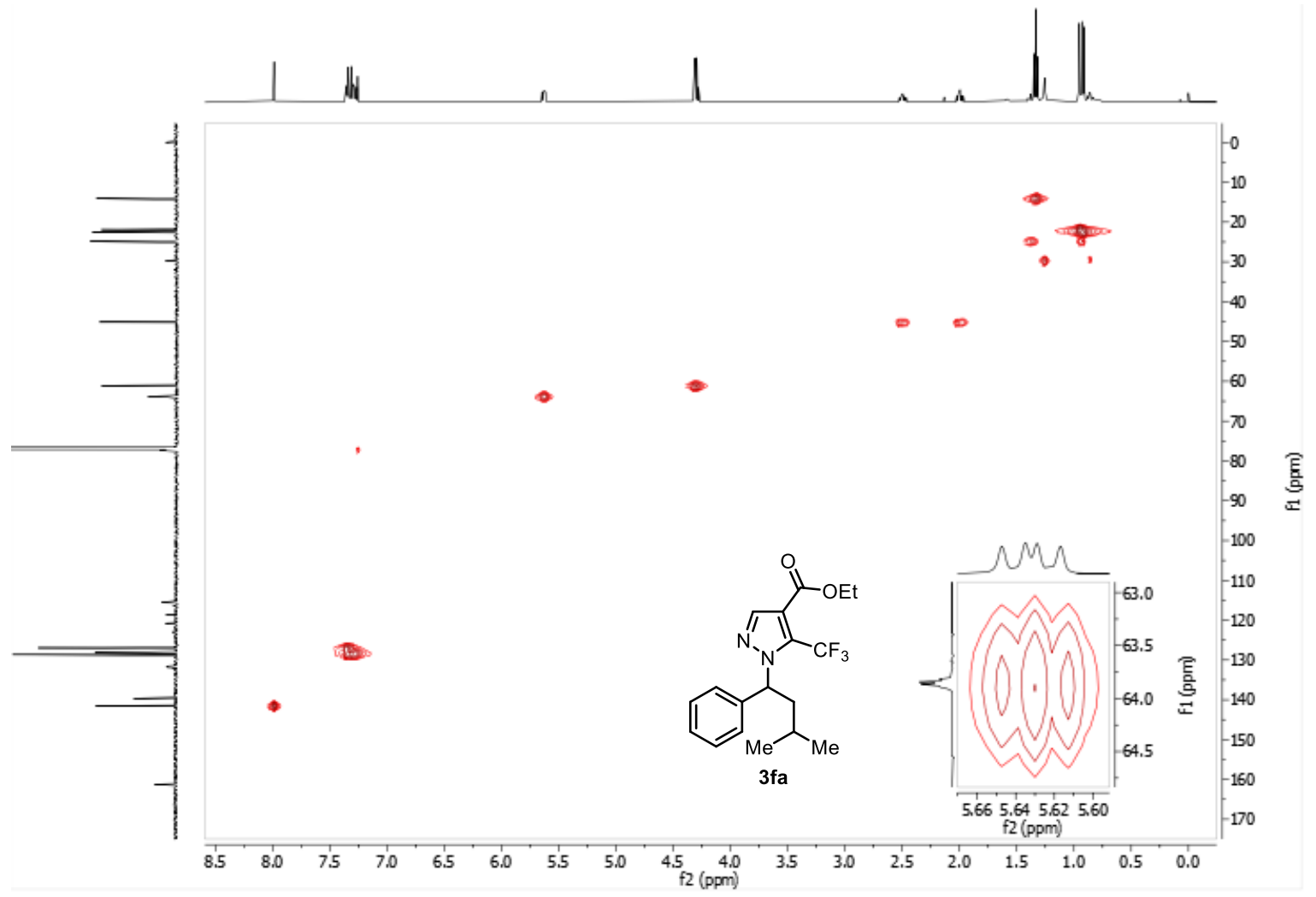

HMBC NMR spectrum of $\mathbf{3 f a}$ in $\mathrm{CDCl}_{3}$ containing $0.03 \%$ (v/v) TMS (500, $126 \mathrm{MHz}$ ).

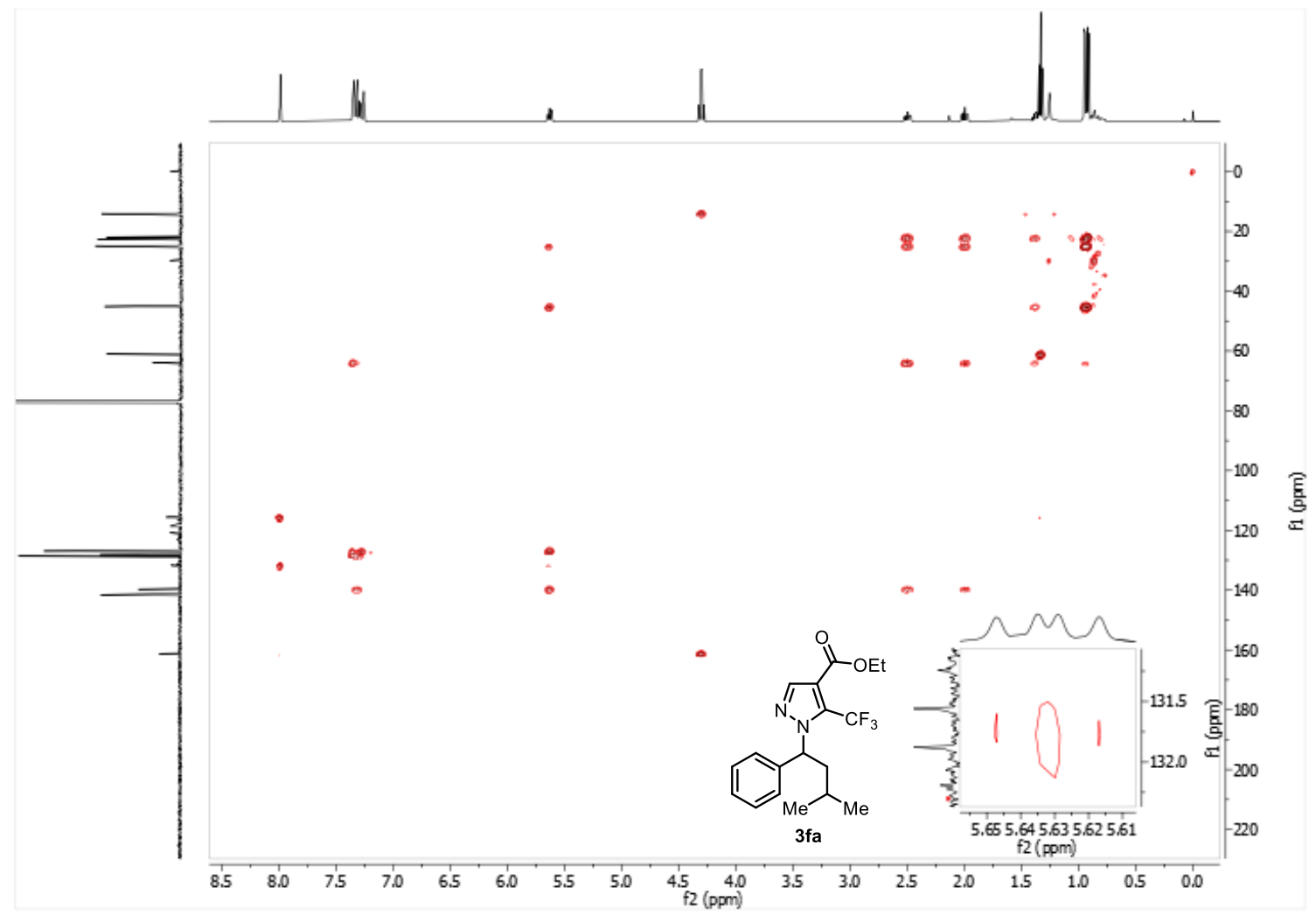


${ }^{1} \mathrm{H}$ NMR spectrum of 3ga-1 in $\mathrm{CDCl}_{3}$ containing $0.03 \%$ (v/v) TMS (500 MHz).
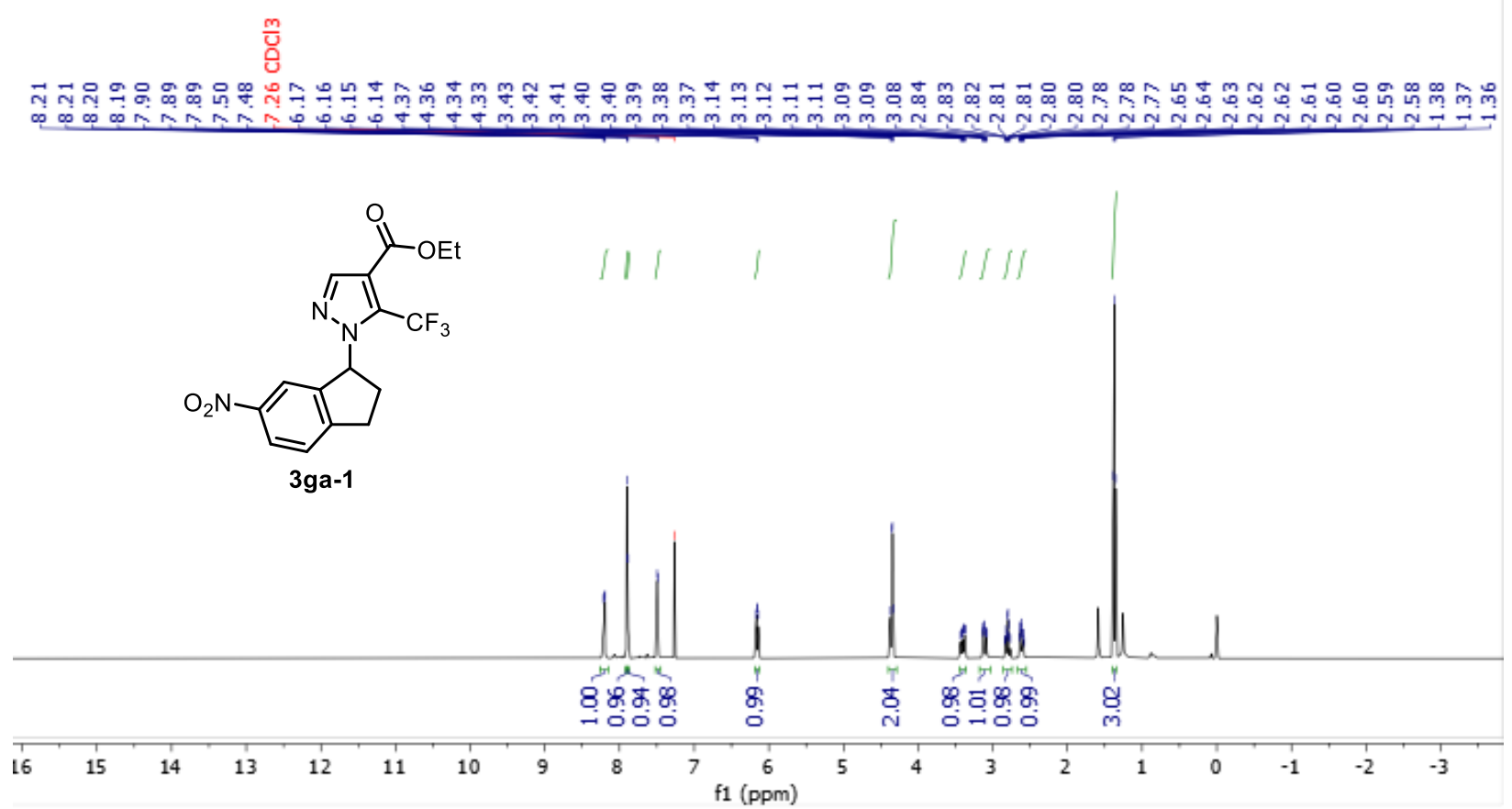

${ }^{13} \mathrm{C}$ NMR spectrum of 3ga-1 in $\mathrm{CDCl}_{3}$ containing $0.03 \%$ (v/v) TMS (126 MHz).
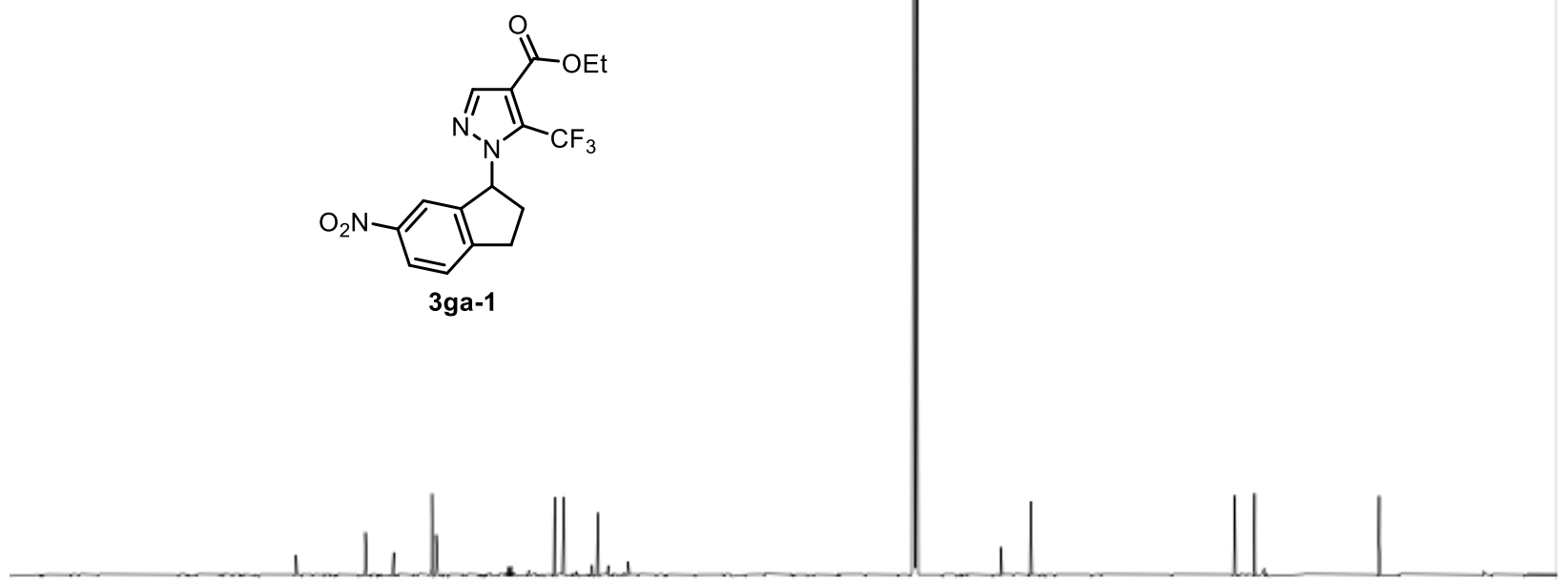

\begin{tabular}{|c|c|c|c|c|c|c|c|c|c|c|c|c|c|c|c|c|c|c|c|c|}
\hline 10 & 190 & 180 & 170 & 160 & 150 & 140 & 130 & 120 & 110 & $\begin{array}{r}100 \\
\mathrm{f} 1\end{array}$ & $\begin{array}{l}90 \\
\text { m) }\end{array}$ & 80 & 70 & 60 & 50 & 40 & 30 & 20 & 10 & 0 \\
\hline
\end{tabular}


${ }^{19} \mathrm{~F}$ NMR spectrum of 3ga-1 in $\mathrm{CDCl}_{3}$ containing $0.03 \%$ (v/v) TMS (377 MHz).
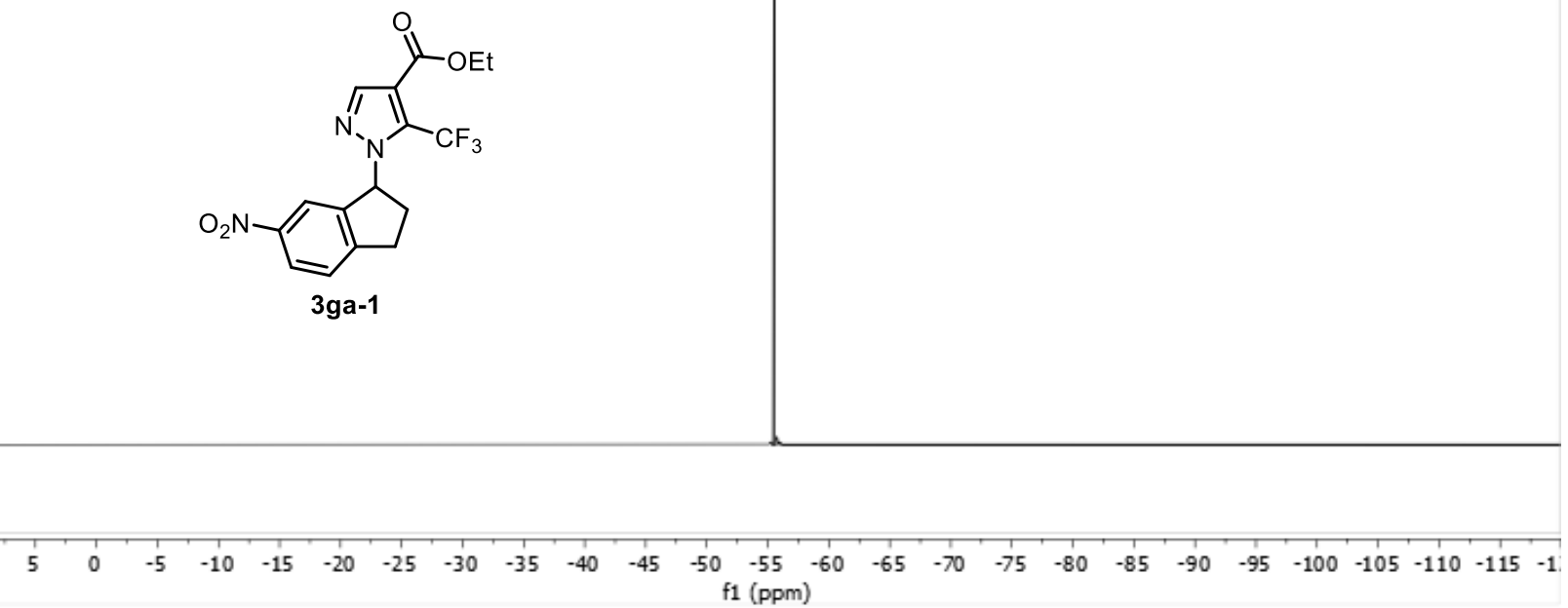

HSQC NMR spectrum of 3ga-1 in $\mathrm{CDCl}_{3}$ containing $0.03 \%$ (v/v) TMS (500, $126 \mathrm{MHz}$ ).

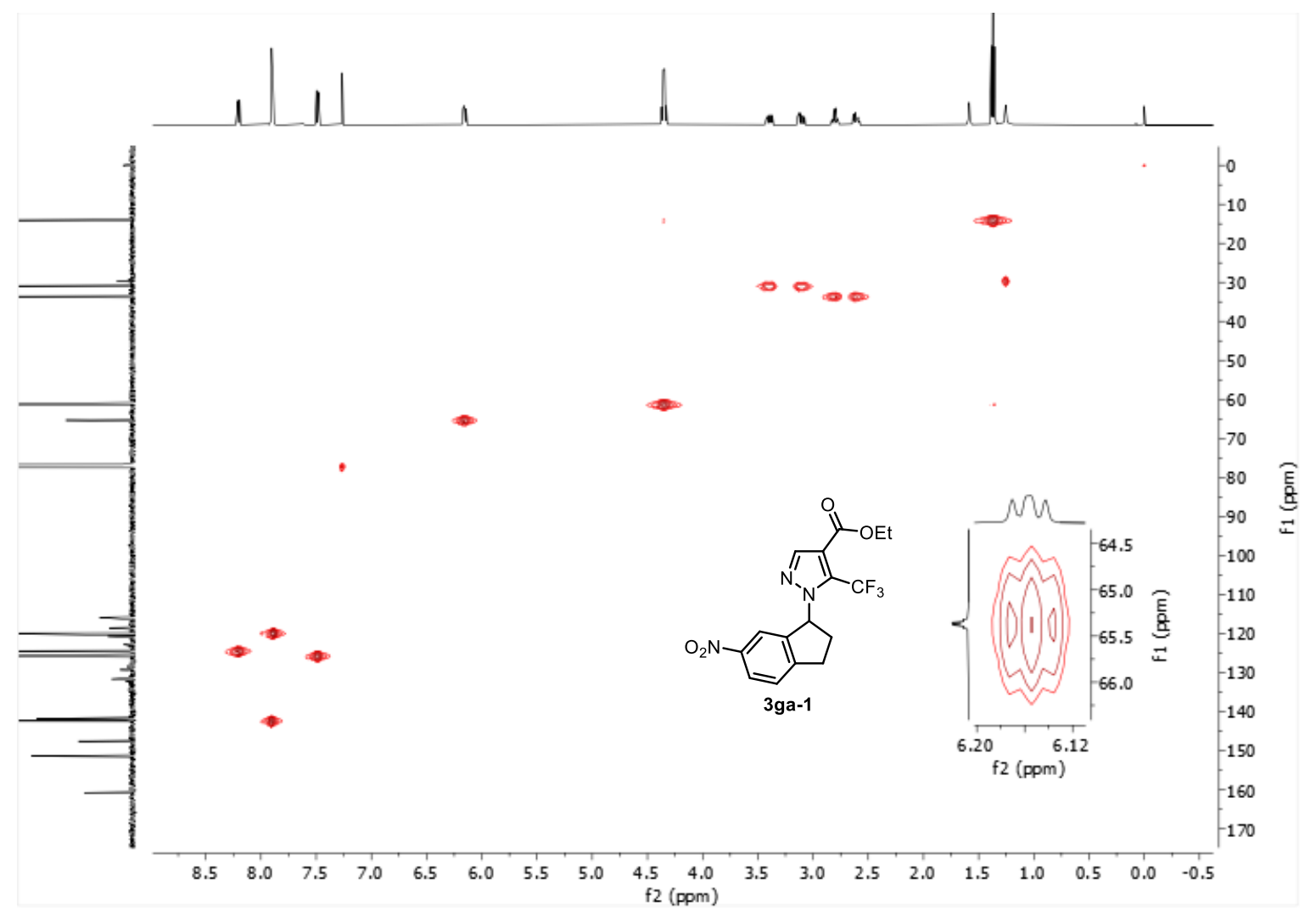


HMBC NMR spectrum of 3ga-1 in $\mathrm{CDCl}_{3}$ containing $0.03 \%$ (v/v) TMS (500, $\left.126 \mathrm{MHz}\right)$.

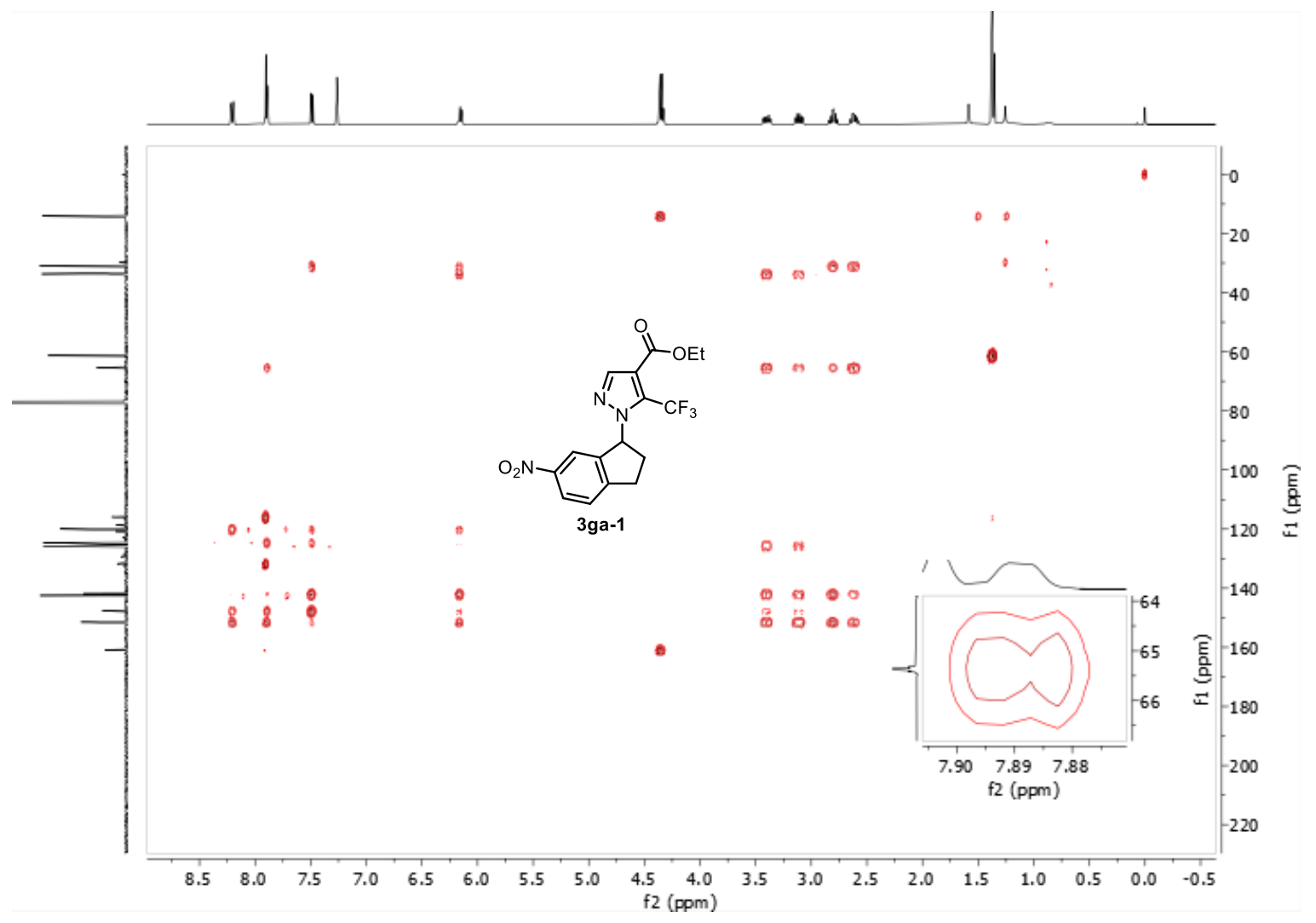

${ }^{1} \mathrm{H}$ NMR spectrum of $\mathbf{3 g a - 2}$ in $\mathrm{CDCl}_{3}$ containing $0.03 \%$ (v/v) TMS (500 MHz).

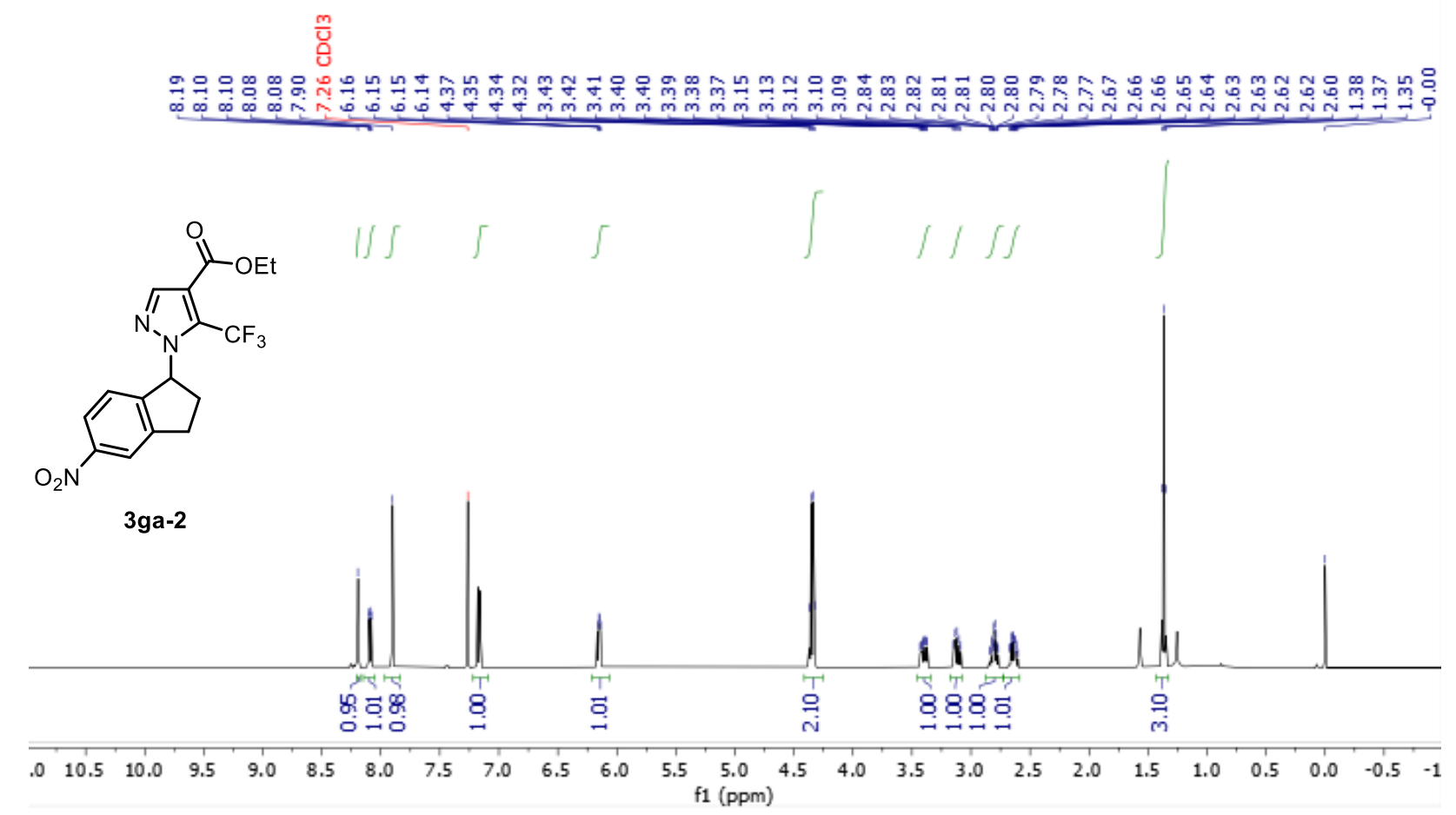


${ }^{13} \mathrm{C}$ NMR spectrum of $\mathbf{3 g a - 2}$ in $\mathrm{CDCl}_{3}$ containing $0.03 \%$ (v/v) TMS (126 MHz).

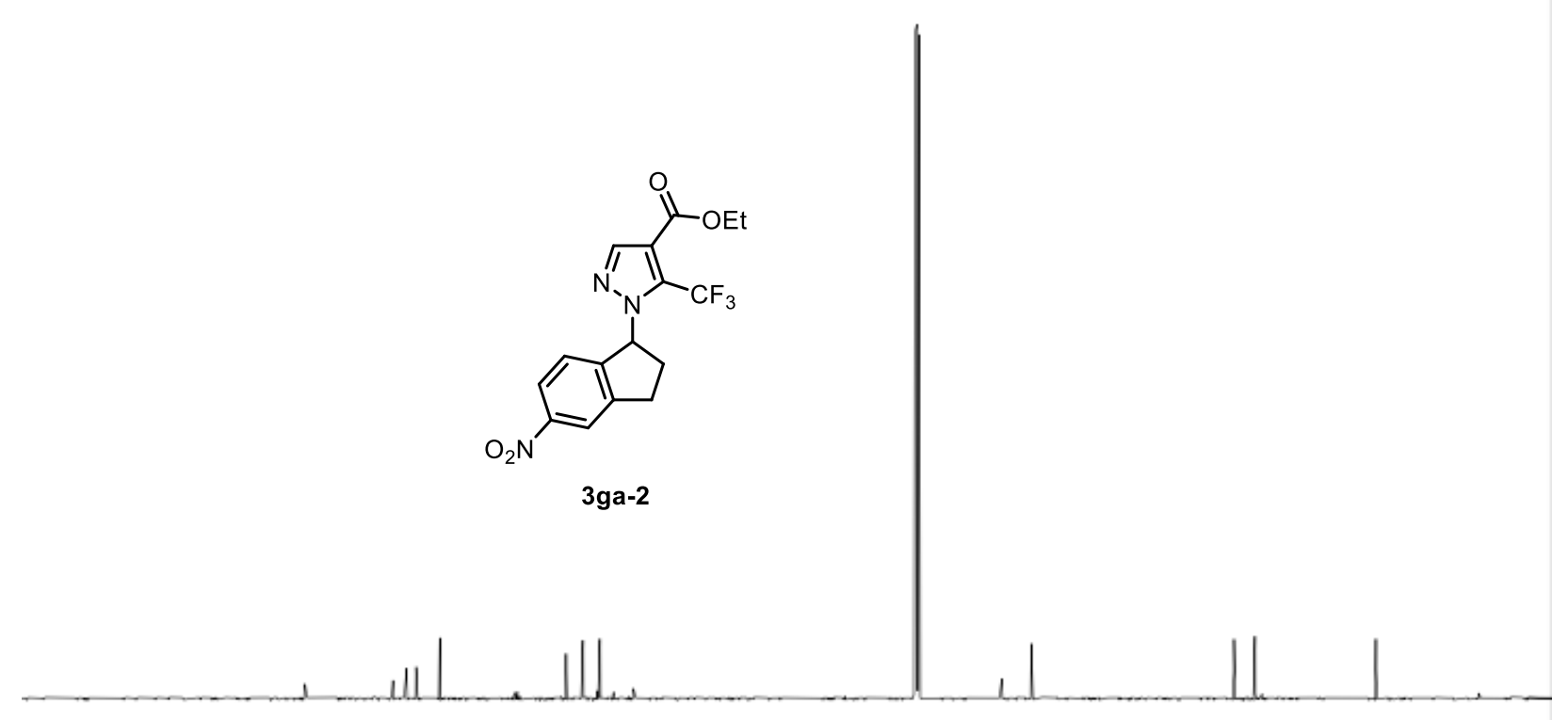

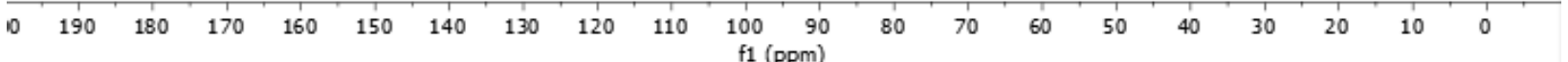

${ }^{19} \mathrm{~F}$ NMR spectrum of $\mathbf{3 g a - 2}$ in $\mathrm{CDCl}_{3}$ containing $0.03 \%(\mathrm{v} / \mathrm{v}) \mathrm{TMS}(377 \mathrm{MHz})$.

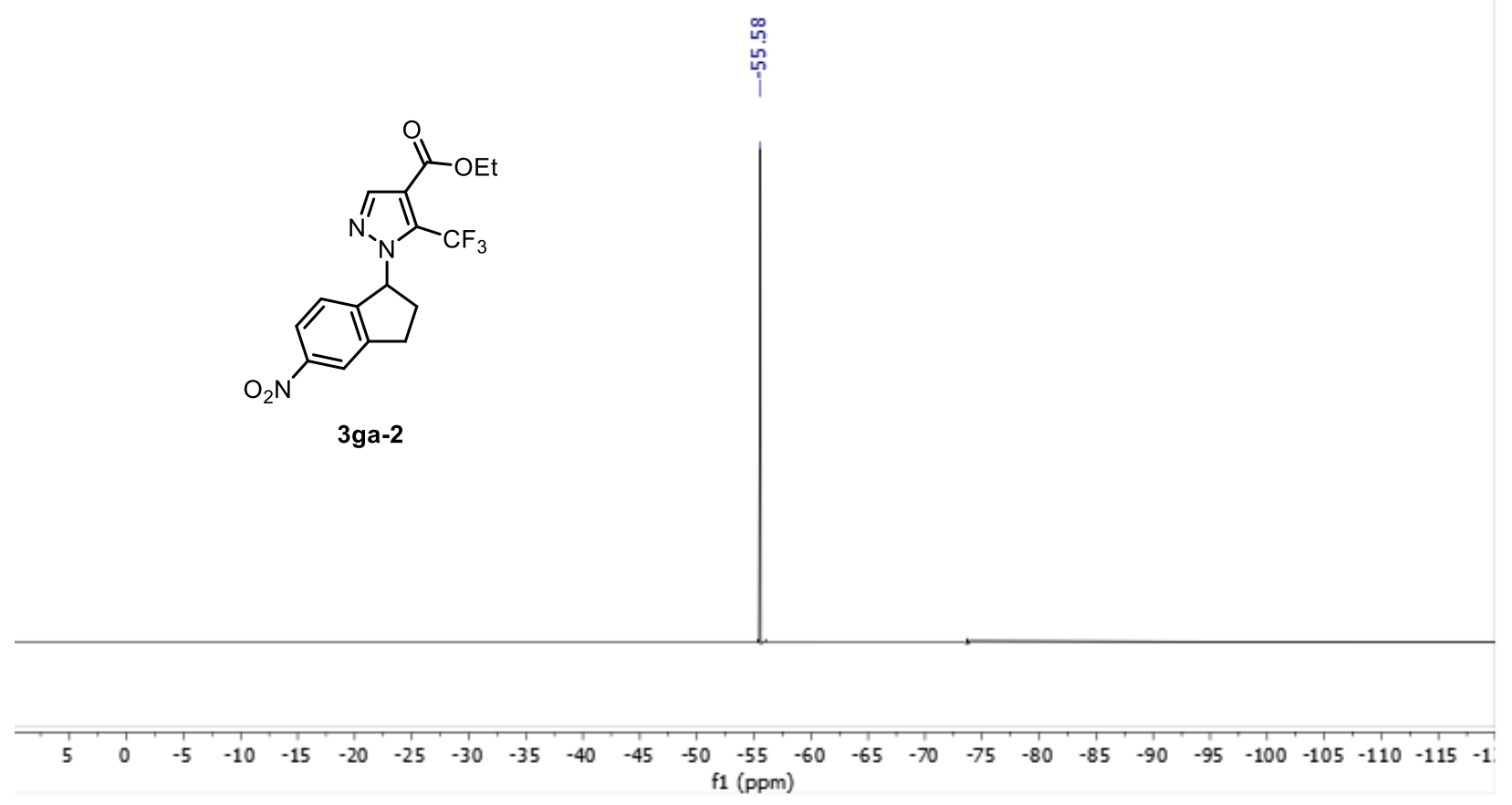


HSQC NMR spectrum of 3ga-2 in $\mathrm{CDCl}_{3}$ containing $0.03 \%$ (v/v) TMS (500, $126 \mathrm{MHz}$ ).

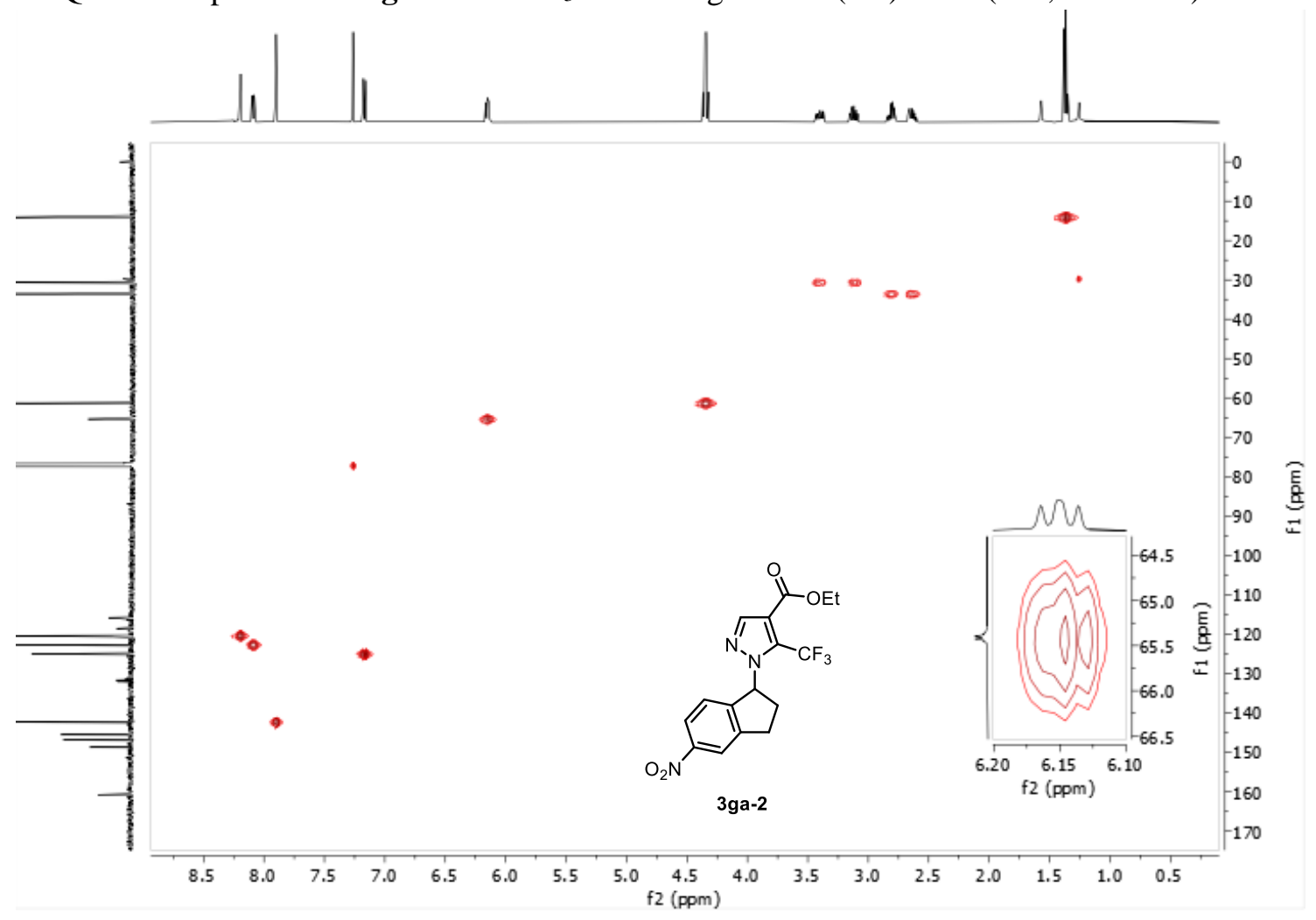

HMBC NMR spectrum of 3ga-2 in $\mathrm{CDCl}_{3}$ containing $0.03 \%(\mathrm{v} / \mathrm{v})$ TMS $(500,126 \mathrm{MHz})$.

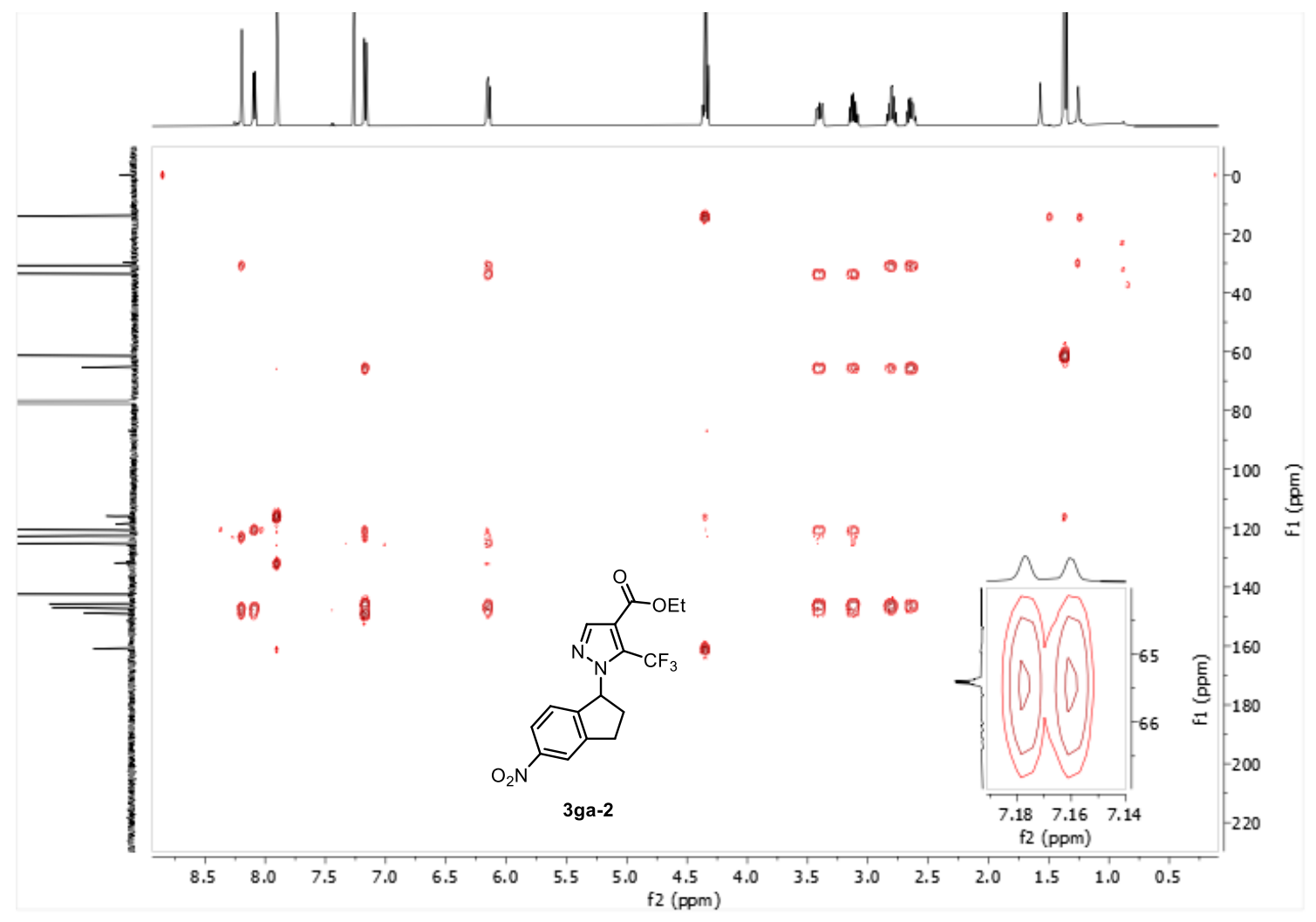


${ }^{1} \mathrm{H}$ NMR spectrum of $\mathbf{3 h a}$ ' in $\mathrm{CDCl}_{3}$ containing $0.03 \%$ (v/v) TMS (500 MHz).

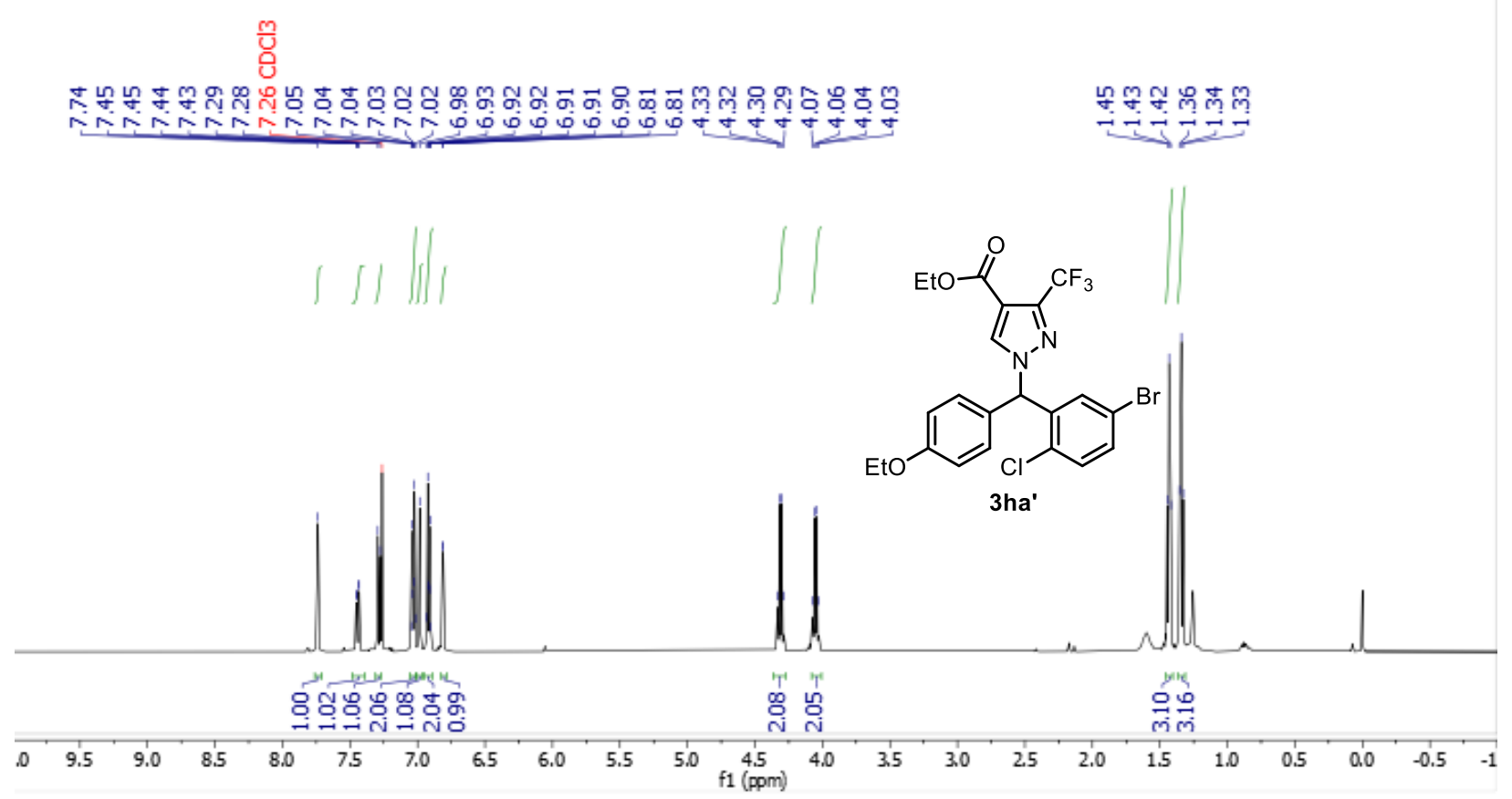

${ }^{13} \mathrm{C}$ NMR spectrum of $\mathbf{3 h a}$ ' in $\mathrm{CDCl}_{3}$ containing $0.03 \%$ (v/v) TMS (126 MHz).

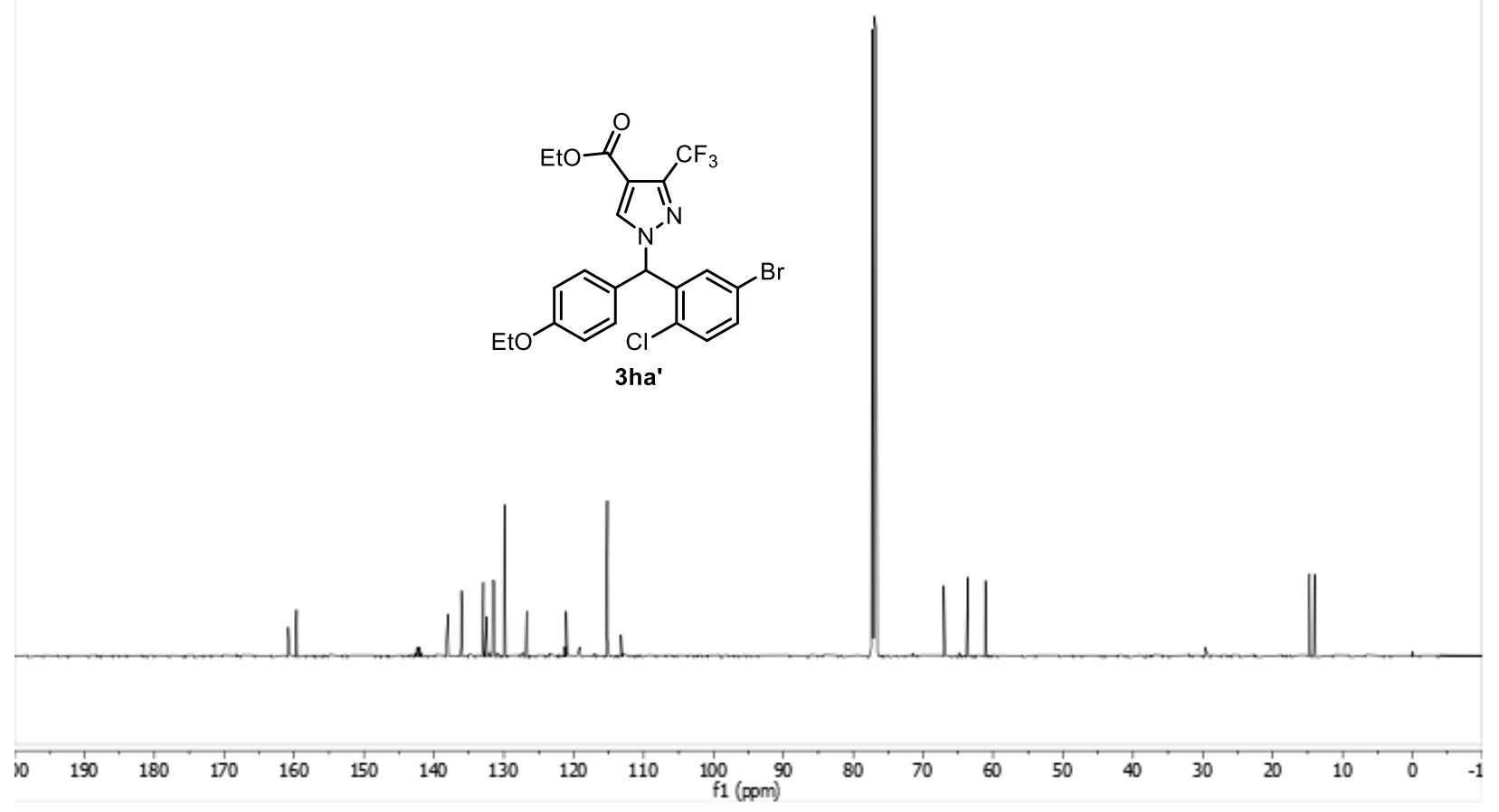


${ }^{19} \mathrm{~F}$ NMR spectrum of $3 \mathbf{h a}$ ' in $\mathrm{CDCl}_{3}$ containing $0.03 \%$ (v/v) TMS (377 MHz).

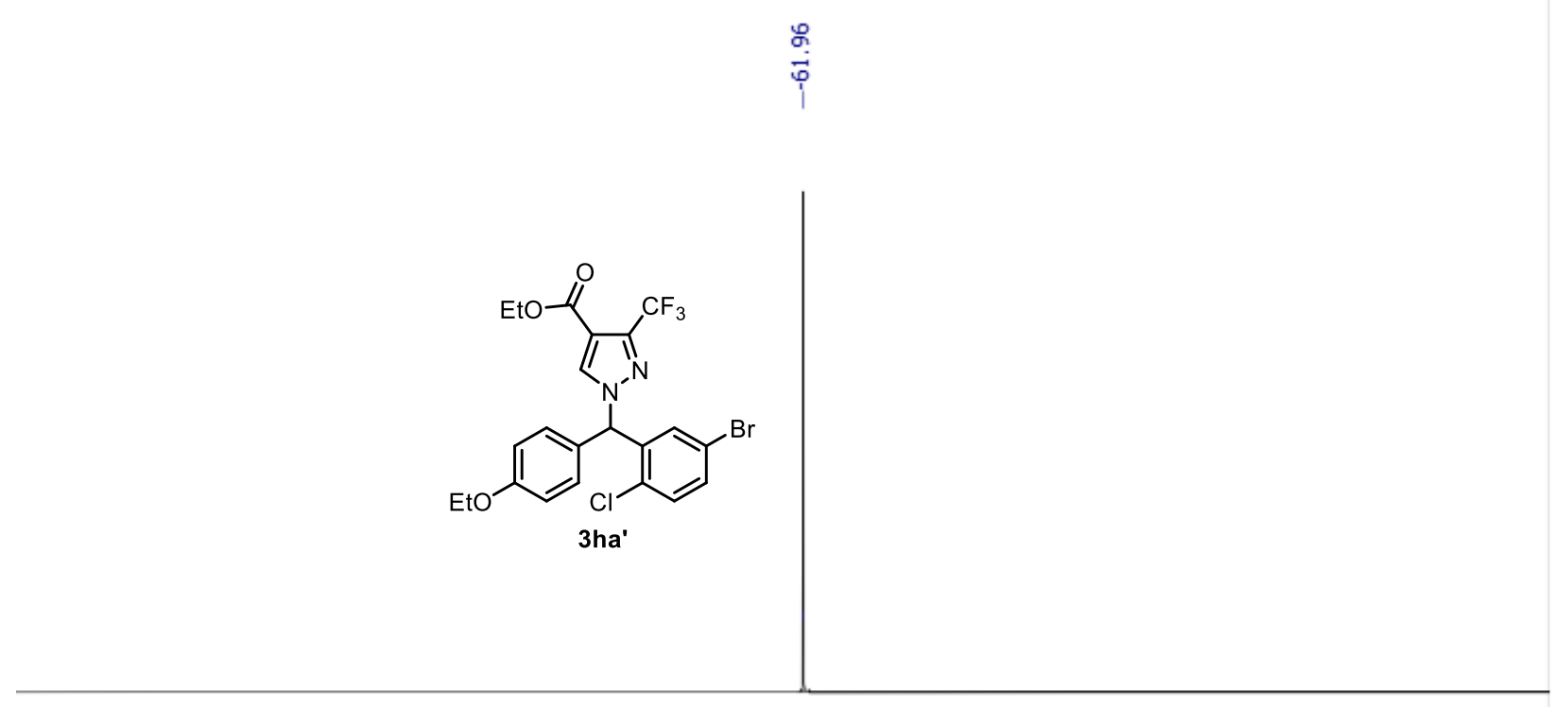

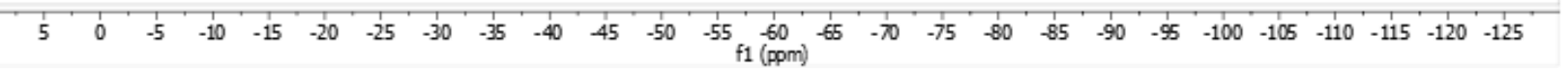

HSQC NMR spectrum of $3 \mathbf{h a}^{\prime}$ in $\mathrm{CDCl}_{3}$ containing $0.03 \%$ (v/v) TMS $(500,126 \mathrm{MHz})$.

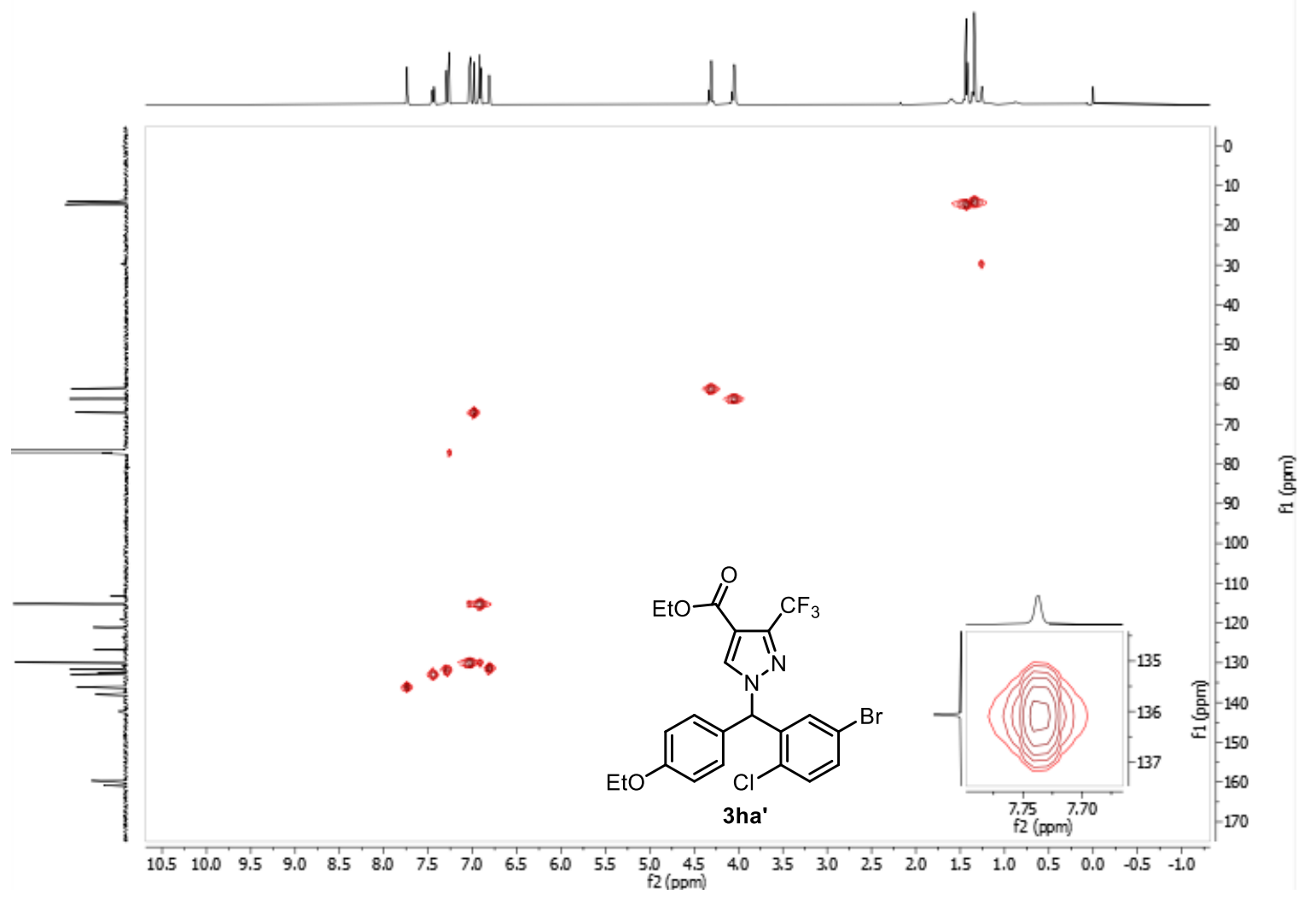


HMBC NMR spectrum of $3 \mathbf{h a}^{\prime}$ in $\mathrm{CDCl}_{3}$ containing $0.03 \%$ (v/v) TMS (500, $126 \mathrm{MHz}$ ).

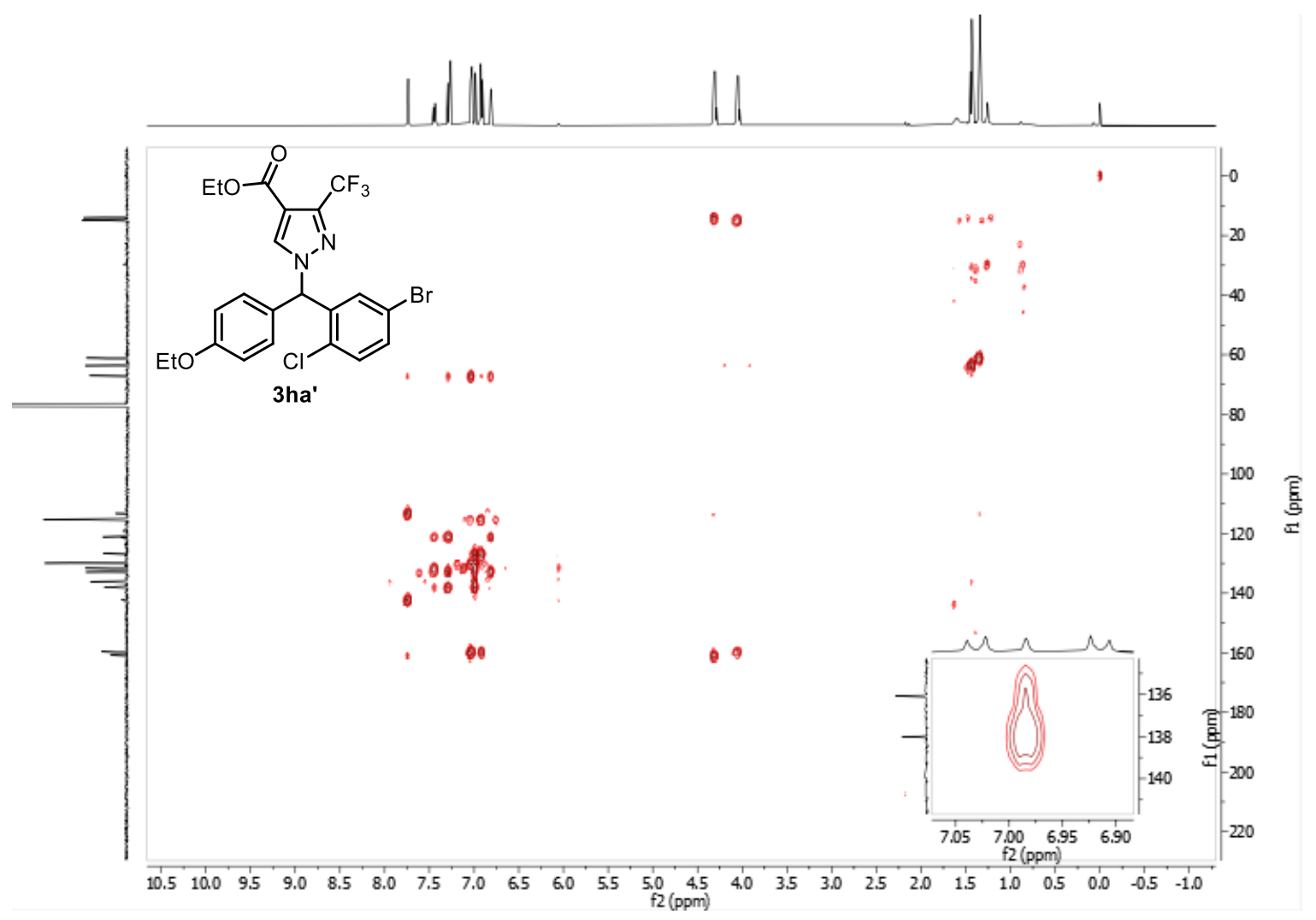

${ }^{1} \mathrm{H}$ NMR spectrum of 3ia' in $\mathrm{CDCl}_{3}$ containing $0.03 \%$ (v/v) TMS (500 MHz).

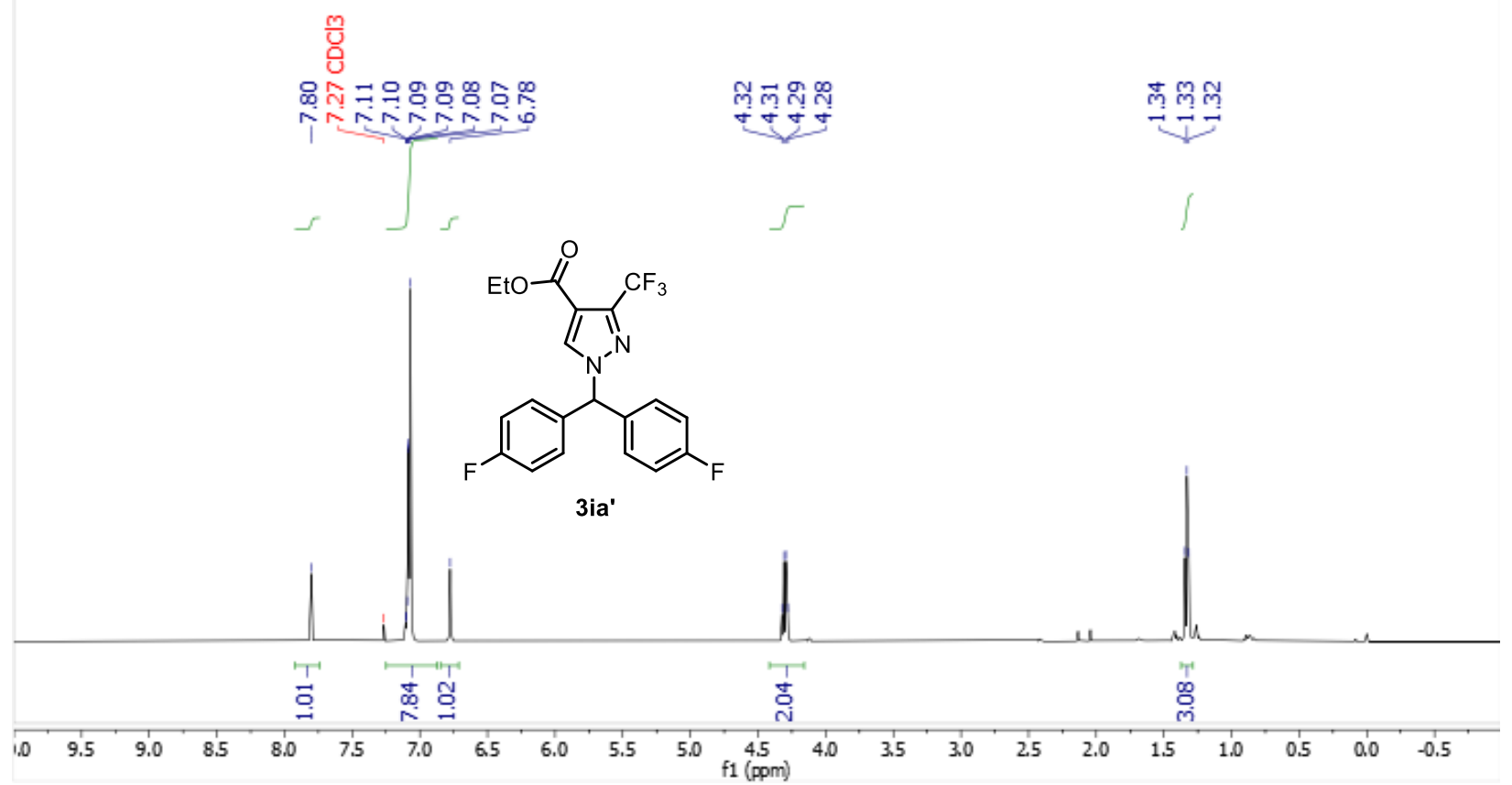


${ }^{13} \mathrm{C}$ NMR spectrum of 3ia' in $\mathrm{CDCl}_{3}$ containing $0.03 \%$ (v/v) TMS (126 MHz).
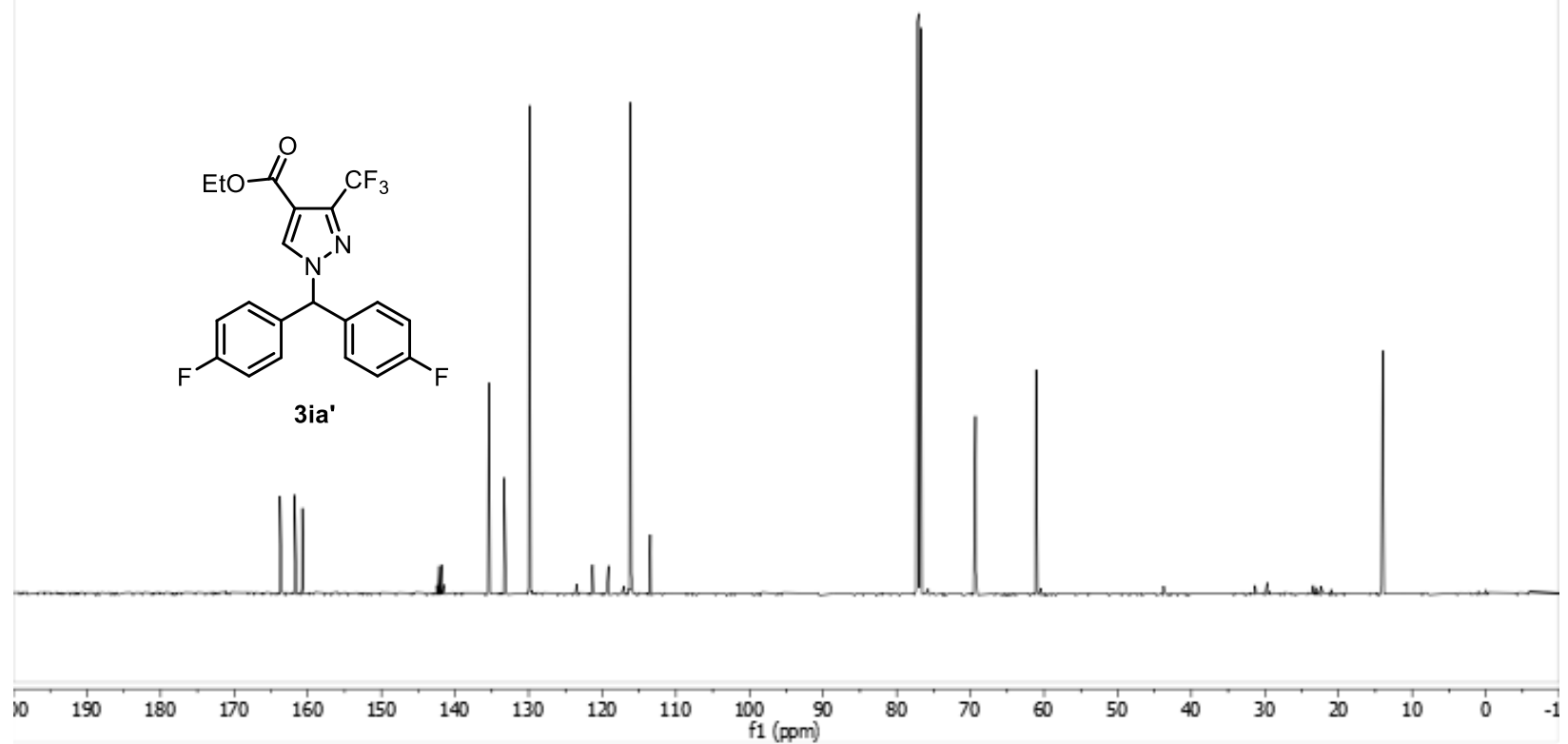

${ }^{19} \mathrm{~F}$ NMR spectrum of 3ia' in $\mathrm{CDCl}_{3}$ containing $0.03 \%$ (v/v) TMS (377 MHz).

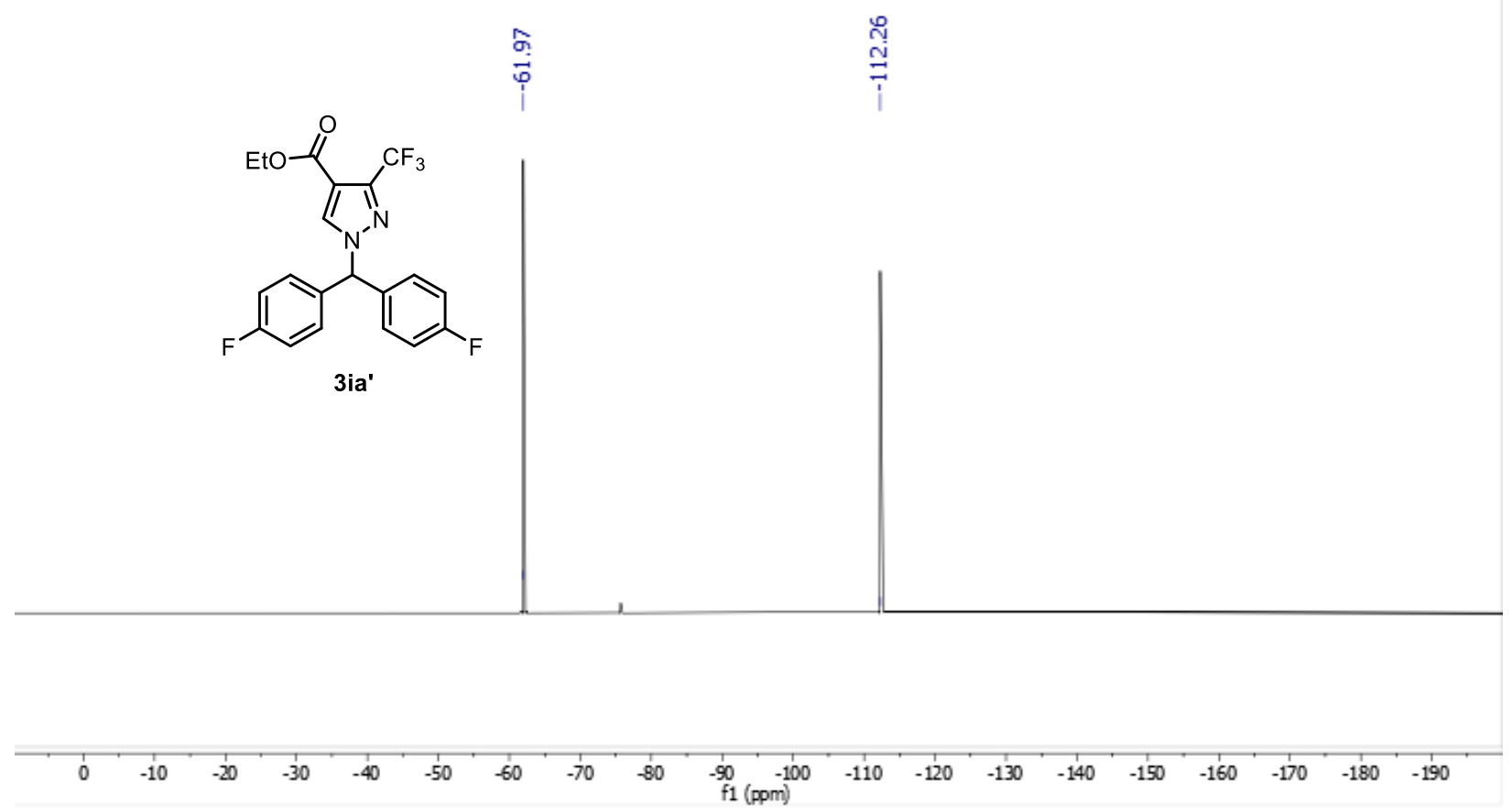


HSQC NMR spectrum of 3ia' in $\mathrm{CDCl}_{3}$ containing $0.03 \%$ (v/v) TMS (500, $126 \mathrm{MHz}$ ).

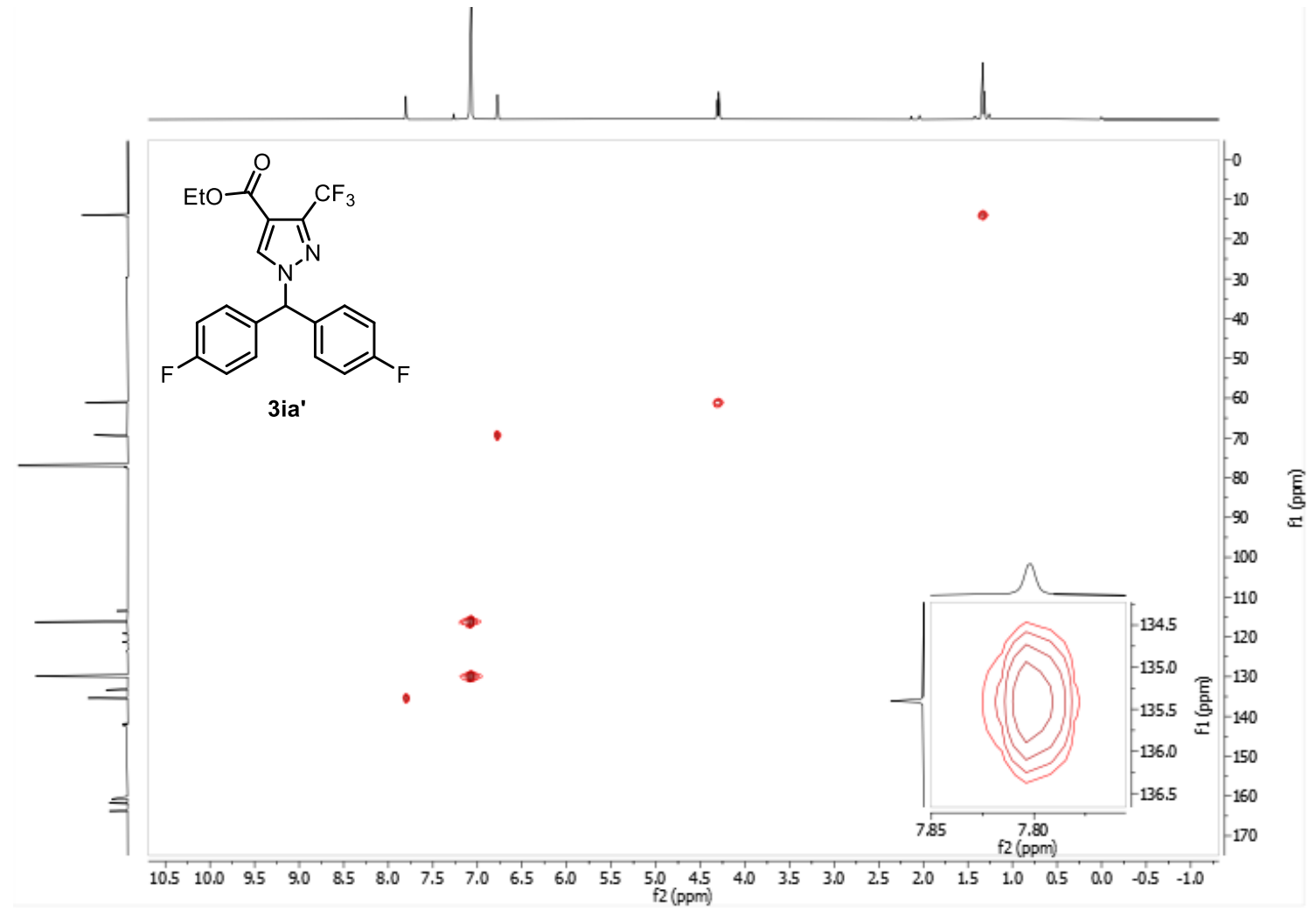

HMBC NMR spectrum of 3ia' in $\mathrm{CDCl}_{3}$ containing $0.03 \%$ (v/v) TMS $(500,126 \mathrm{MHz})$.

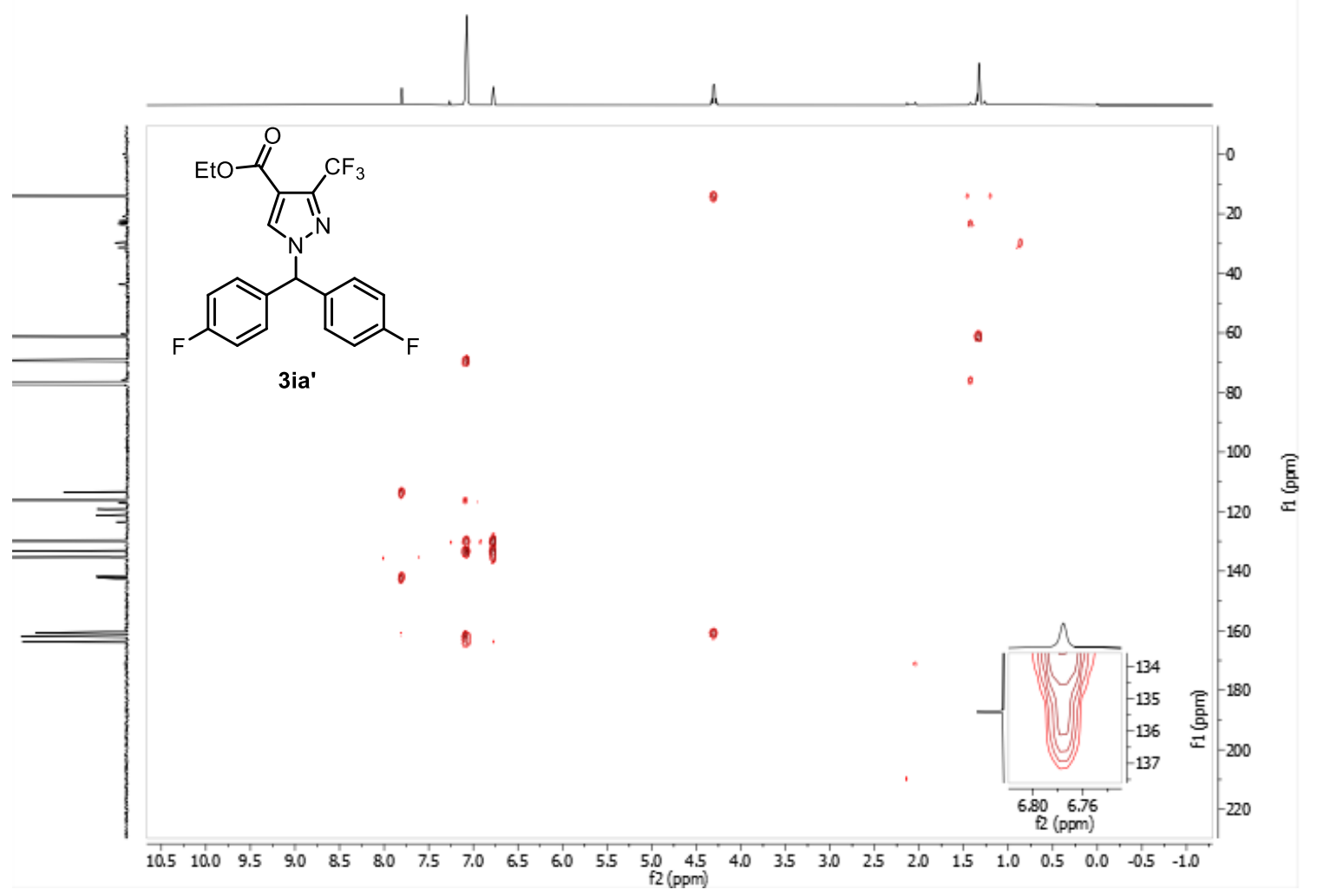


${ }^{1} \mathrm{H}$ NMR spectrum of $\mathbf{3 j a}$ in $\mathrm{CDCl}_{3}$ containing $0.03 \%$ (v/v) TMS (500 MHz).

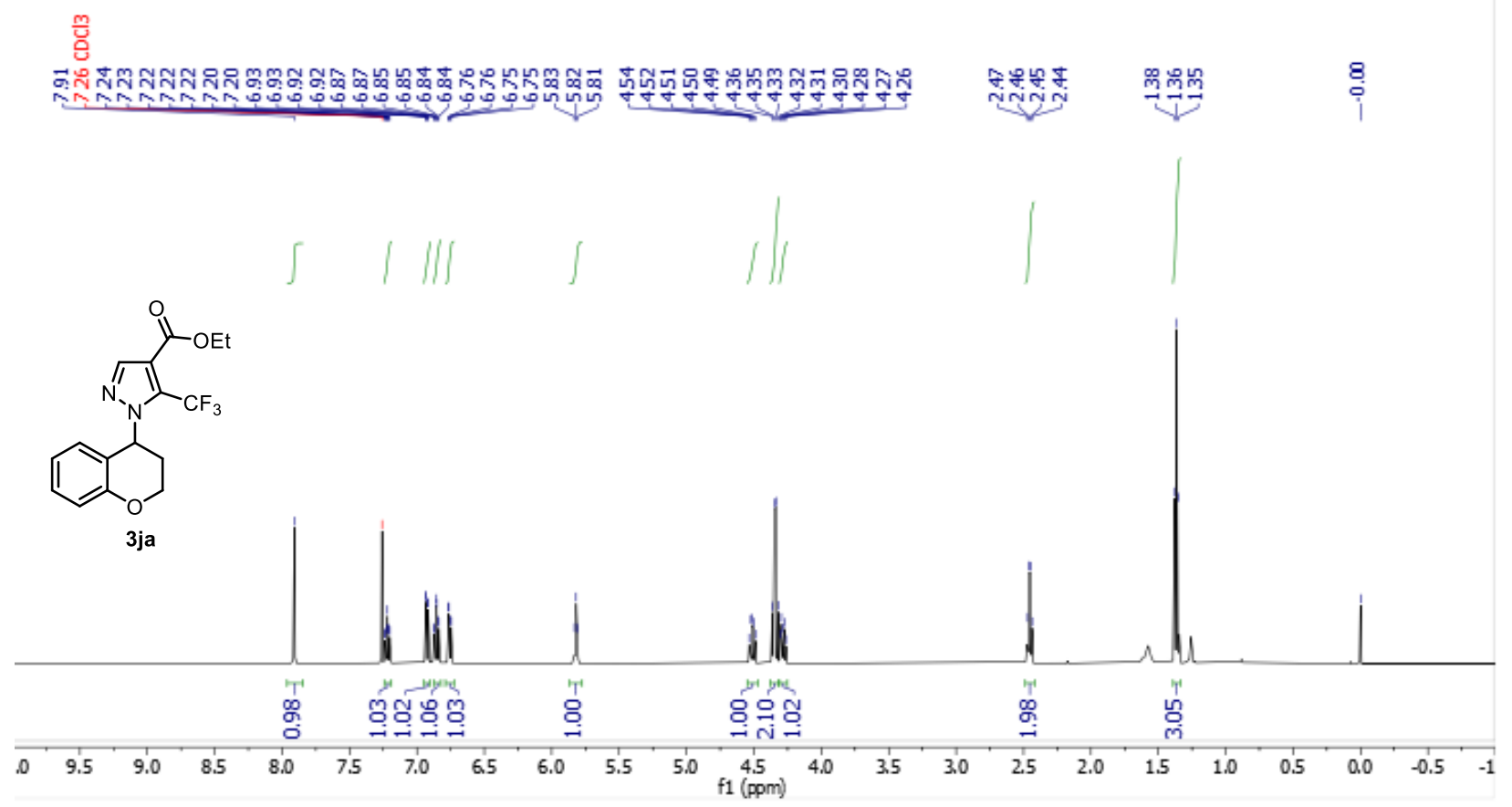

${ }^{13} \mathrm{C}$ NMR spectrum of $\mathbf{3 j a}$ in $\mathrm{CDCl}_{3}$ containing $0.03 \%$ (v/v) TMS (126 MHz).

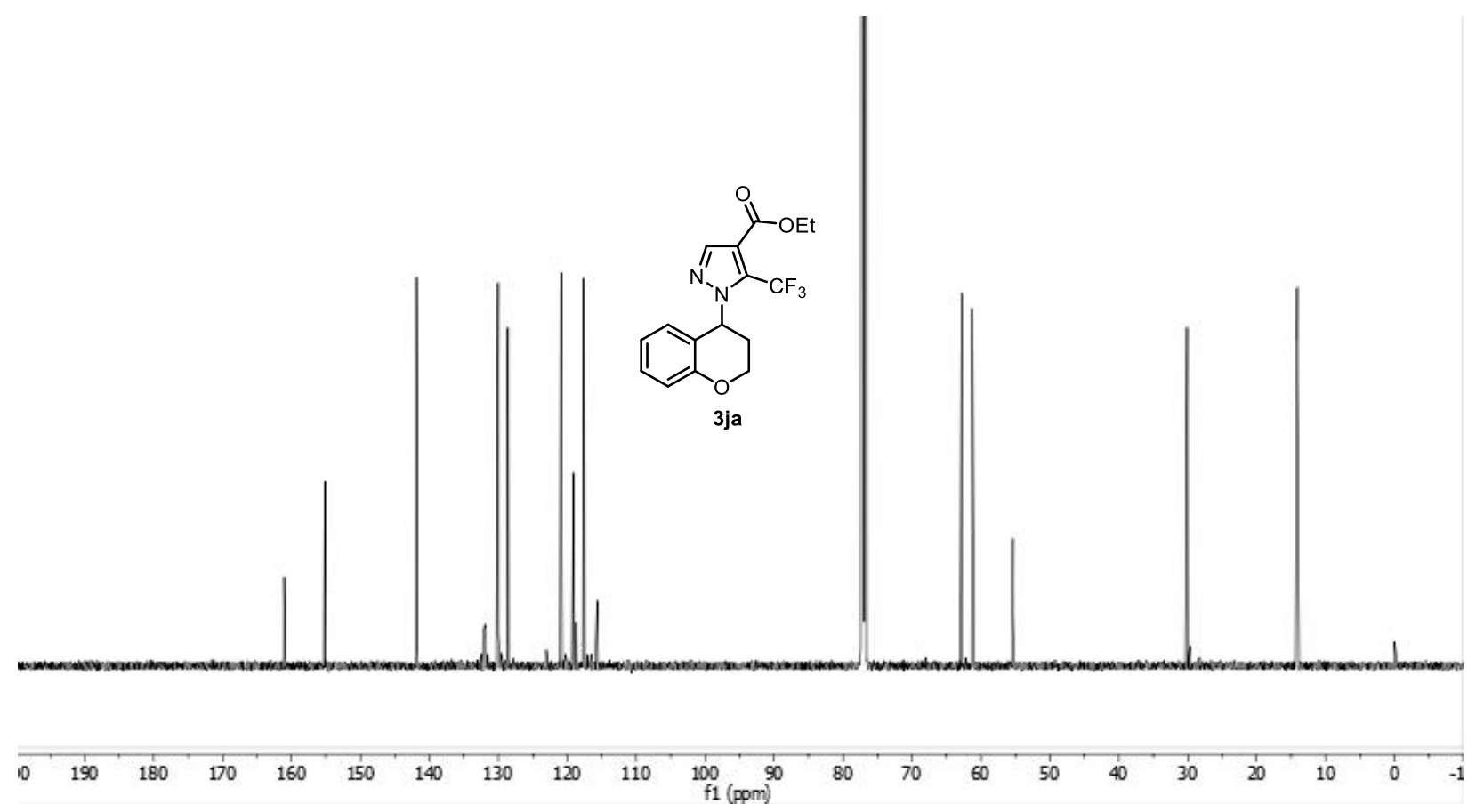


${ }^{19} \mathrm{~F}$ NMR spectrum of $\mathbf{3 j a}$ in $\mathrm{CDCl}_{3}$ containing $0.03 \%$ (v/v) TMS (377 MHz).

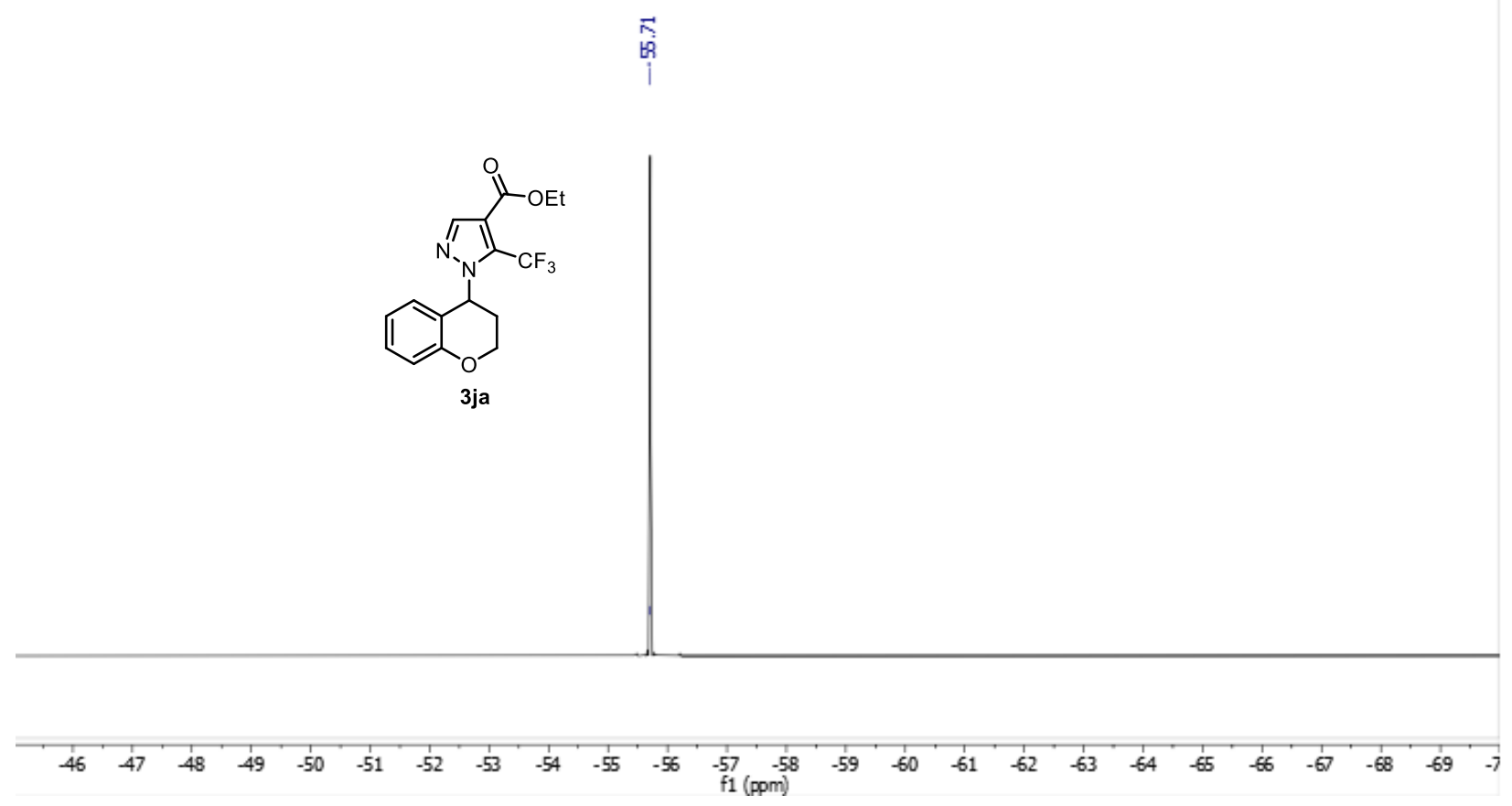

HSQC NMR spectrum of $\mathbf{3 j a}$ in $\mathrm{CDCl}_{3}$ containing $0.03 \%$ (v/v) TMS (500, $126 \mathrm{MHz}$ ).

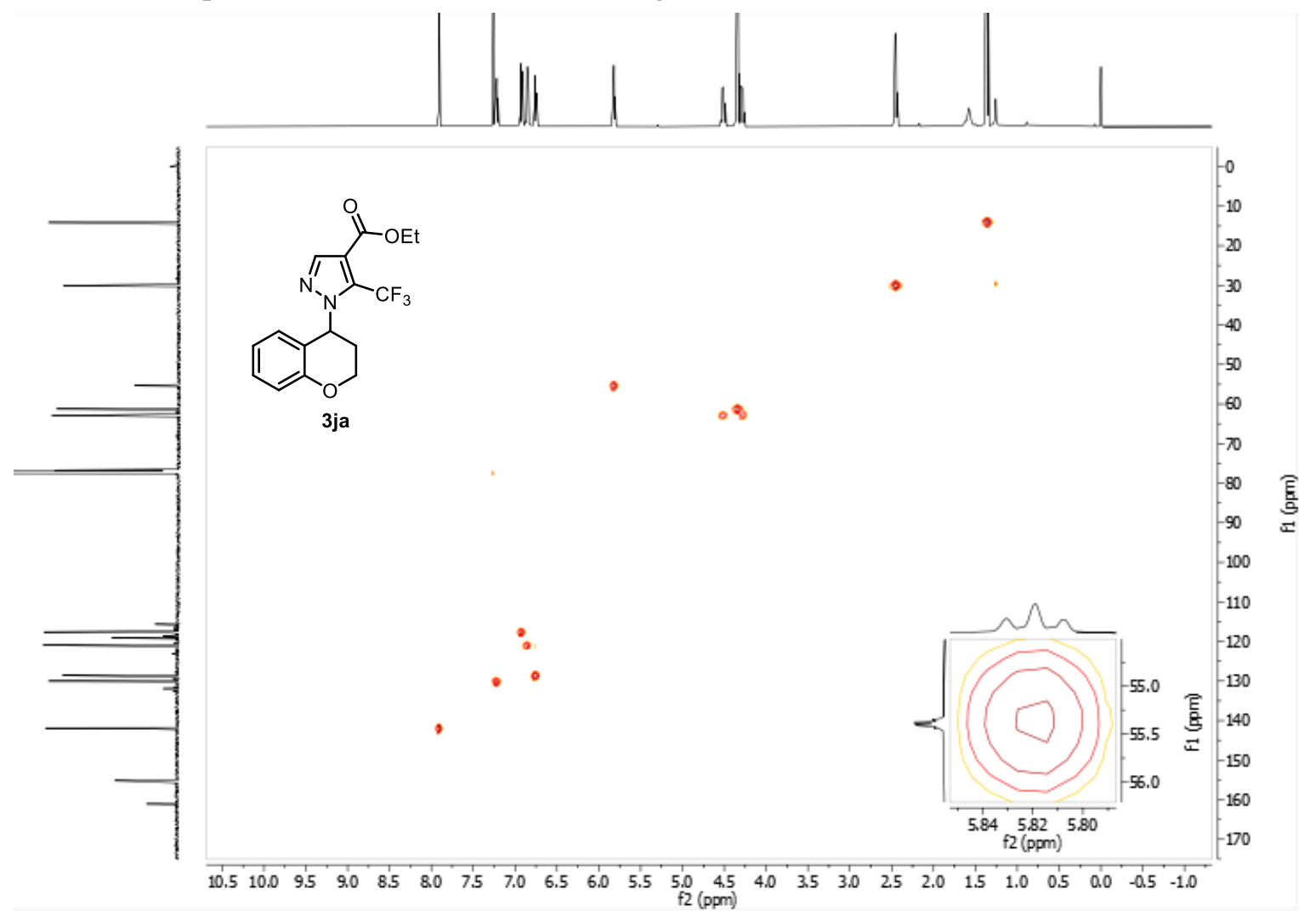


HMBC NMR spectrum of $\mathbf{3 j a}$ in $\mathrm{CDCl}_{3}$ containing $0.03 \%$ (v/v) TMS $(500,126 \mathrm{MHz})$.

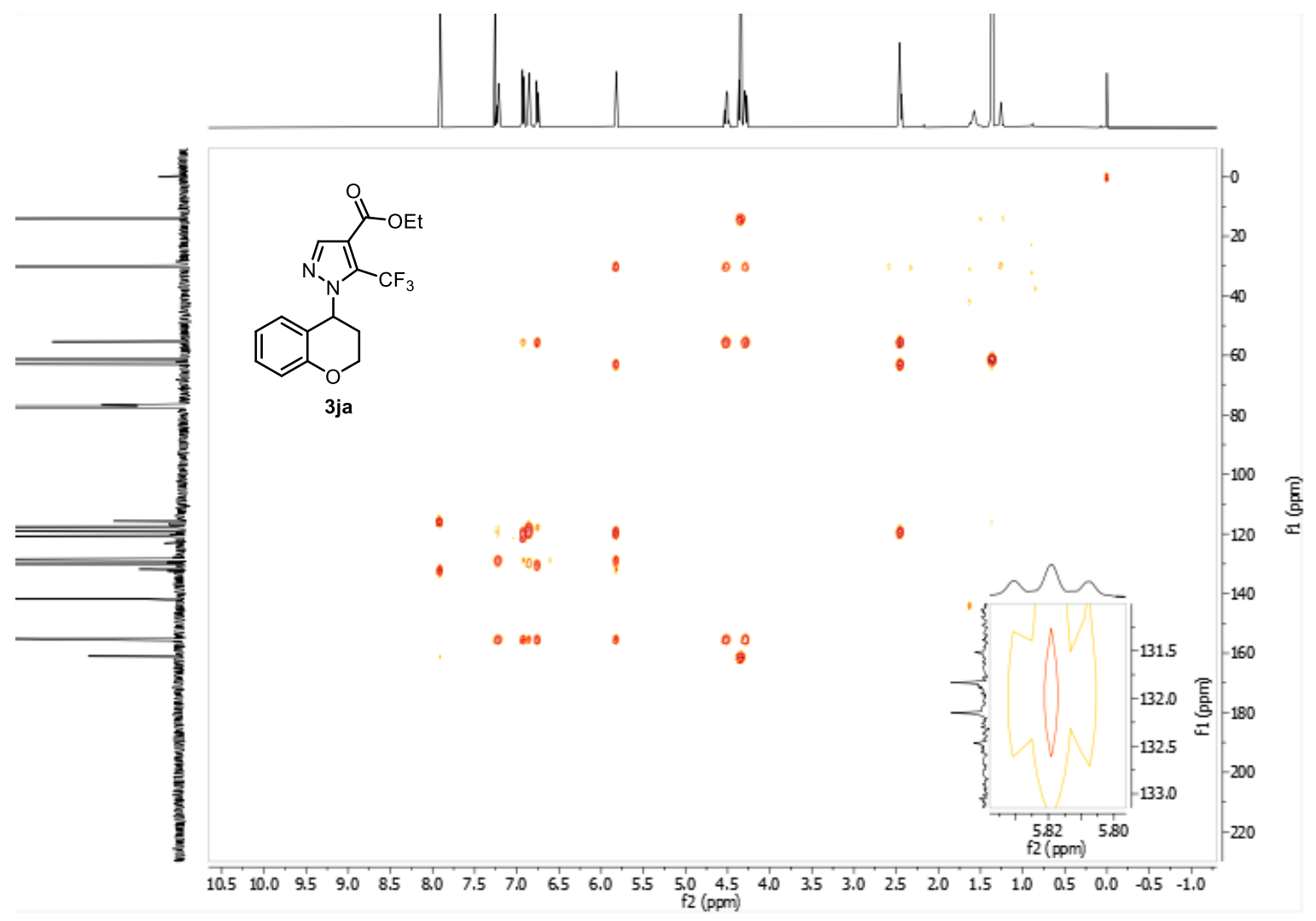

${ }^{1} \mathrm{H}$ NMR spectrum of $\mathbf{3 j a}$ ' in $\mathrm{CDCl}_{3}$ containing $0.03 \%(\mathrm{v} / \mathrm{v})$ TMS (500 MHz).<smiles>CCOC(=O)c1cn(C2CCOc3ccccc32)nc1C(F)(F)F</smiles>
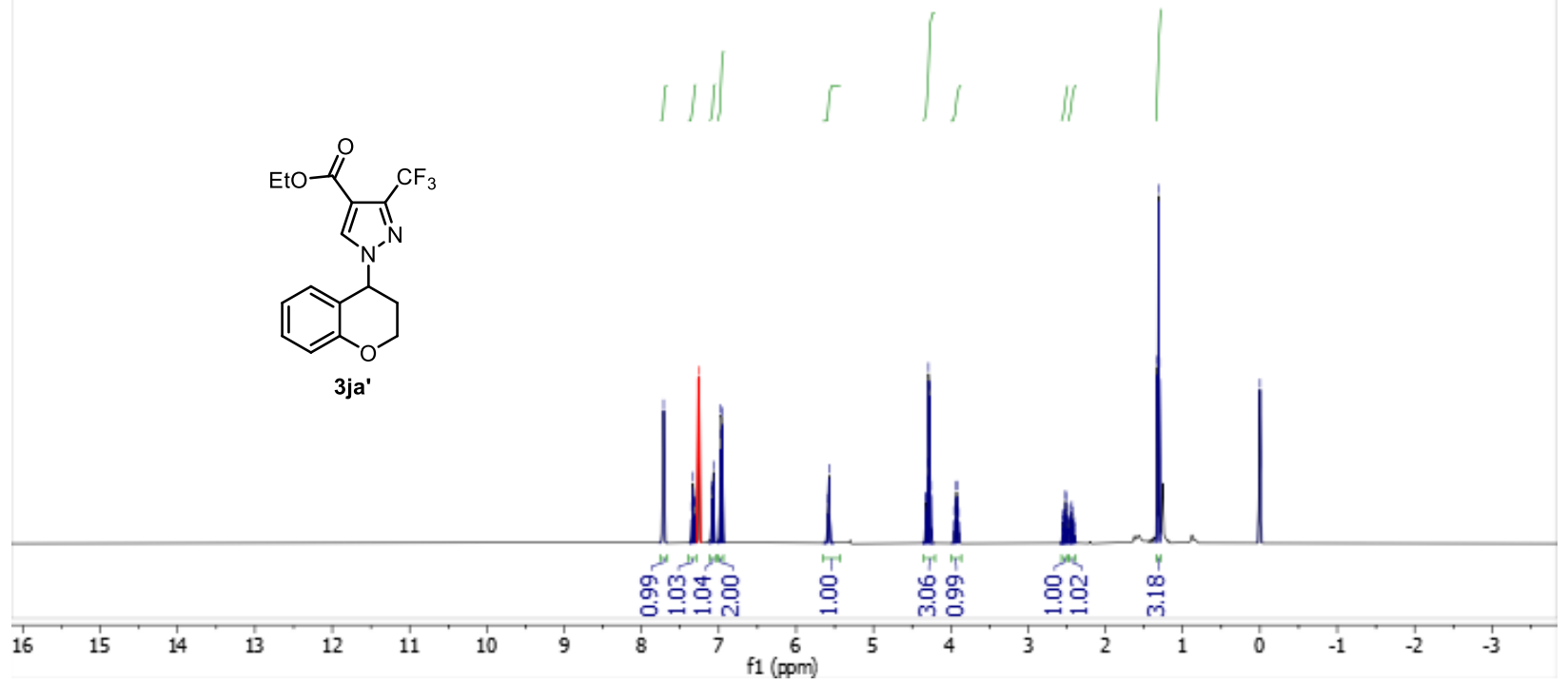
${ }^{13} \mathrm{C}$ NMR spectrum of $\mathbf{3 j a}$ ' in $\mathrm{CDCl}_{3}$ containing $0.03 \%(\mathrm{v} / \mathrm{v}) \mathrm{TMS}(126 \mathrm{MHz})$.
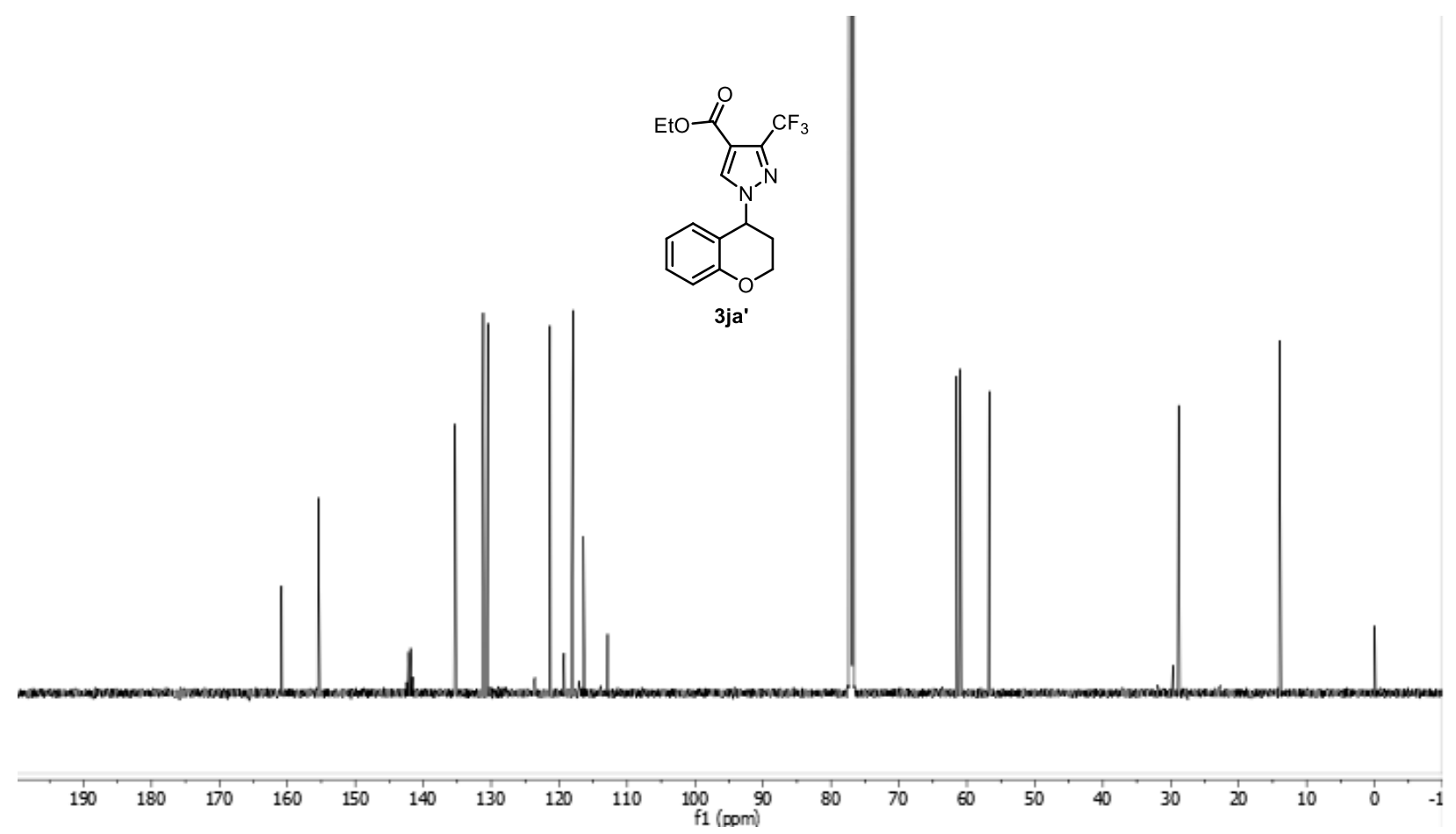

${ }^{19} \mathrm{~F}$ NMR spectrum of $\mathbf{3} \mathbf{j a}$ ' in $\mathrm{CDCl}_{3}$ containing $0.03 \%$ (v/v) TMS (377 MHz).

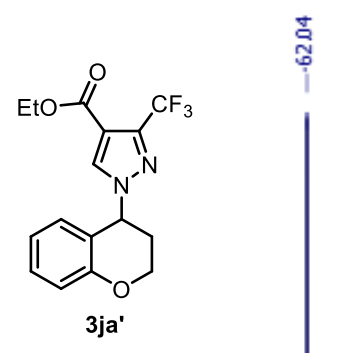

\begin{tabular}{lllllllllllllllllllllllllllllllllllllllllllll}
\hline 0 & 5 & 0 & -5 & -10 & -15 & -20 & -25 & -30 & -35 & -40 & -45 & -50 & -55 & -60 & -65 & -70 & -75 & -80 & -85 & -90 & -95 & -100 & -105 & -110 & -115 & -120 & -125 & -1
\end{tabular} 
HSQC NMR spectrum of $\mathbf{3 j a} \mathbf{a}^{\prime}$ in $\mathrm{CDCl}_{3}$ containing $0.03 \%$ (v/v) TMS (500, $126 \mathrm{MHz}$ ).

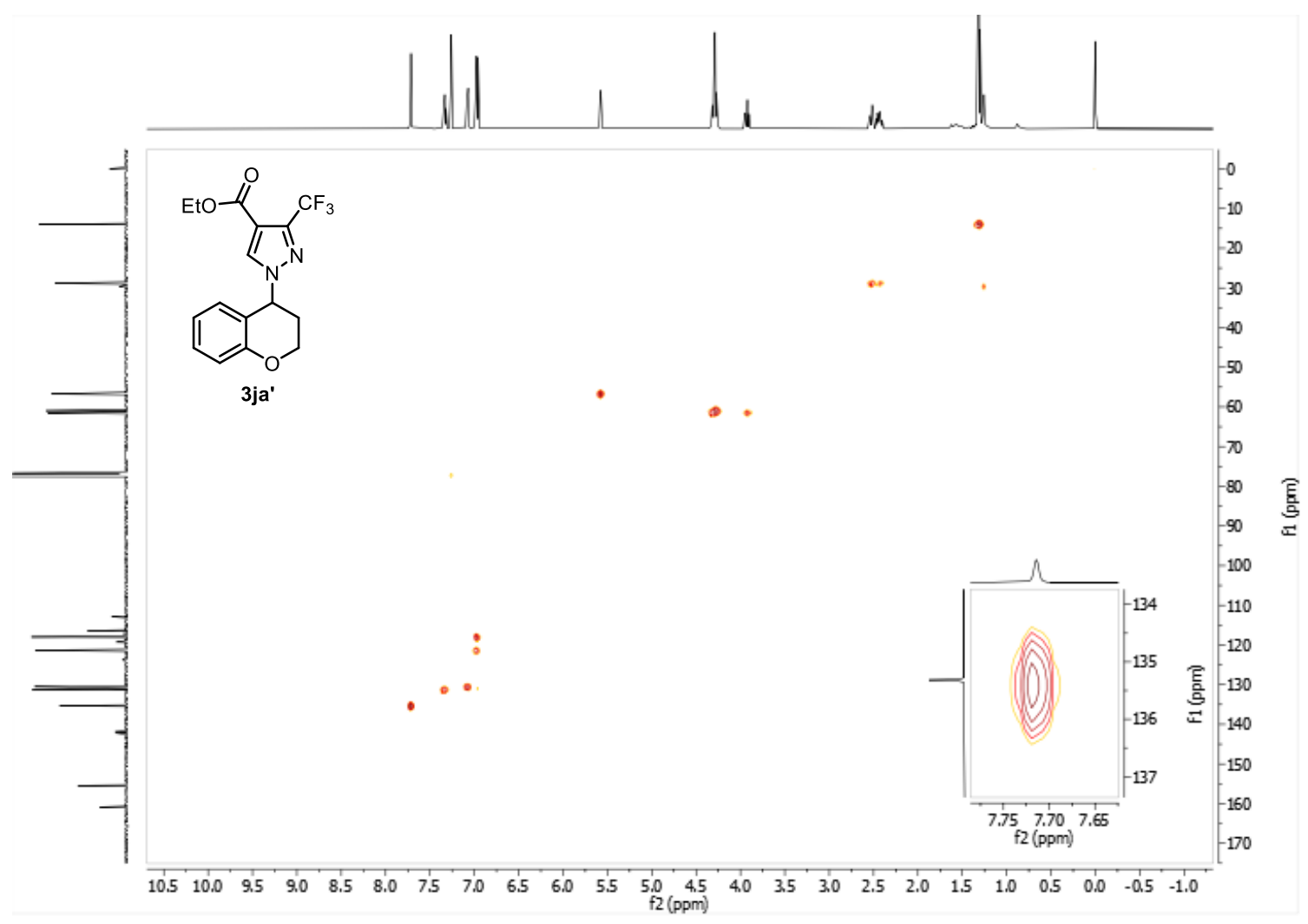

HMBC NMR spectrum of $\mathbf{3 j a} \mathbf{a}^{\prime}$ in $\mathrm{CDCl}_{3}$ containing $0.03 \%$ (v/v) TMS (500, $126 \mathrm{MHz}$ ).

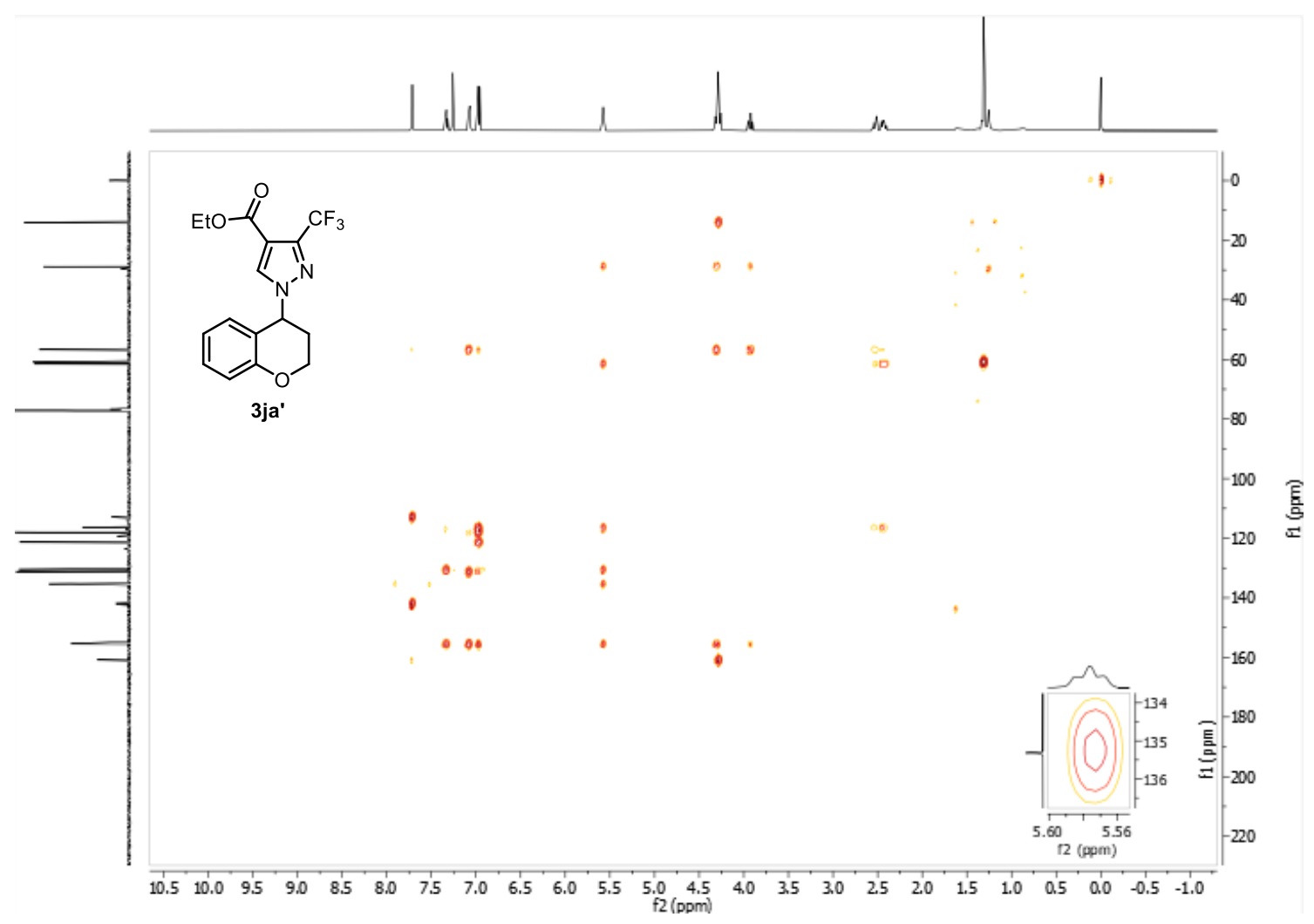


${ }^{1} \mathrm{H}$ NMR spectrum of $3 \mathbf{k a}$ in $\mathrm{CDCl}_{3}$ containing $0.03 \%$ (v/v) TMS (500 MHz).

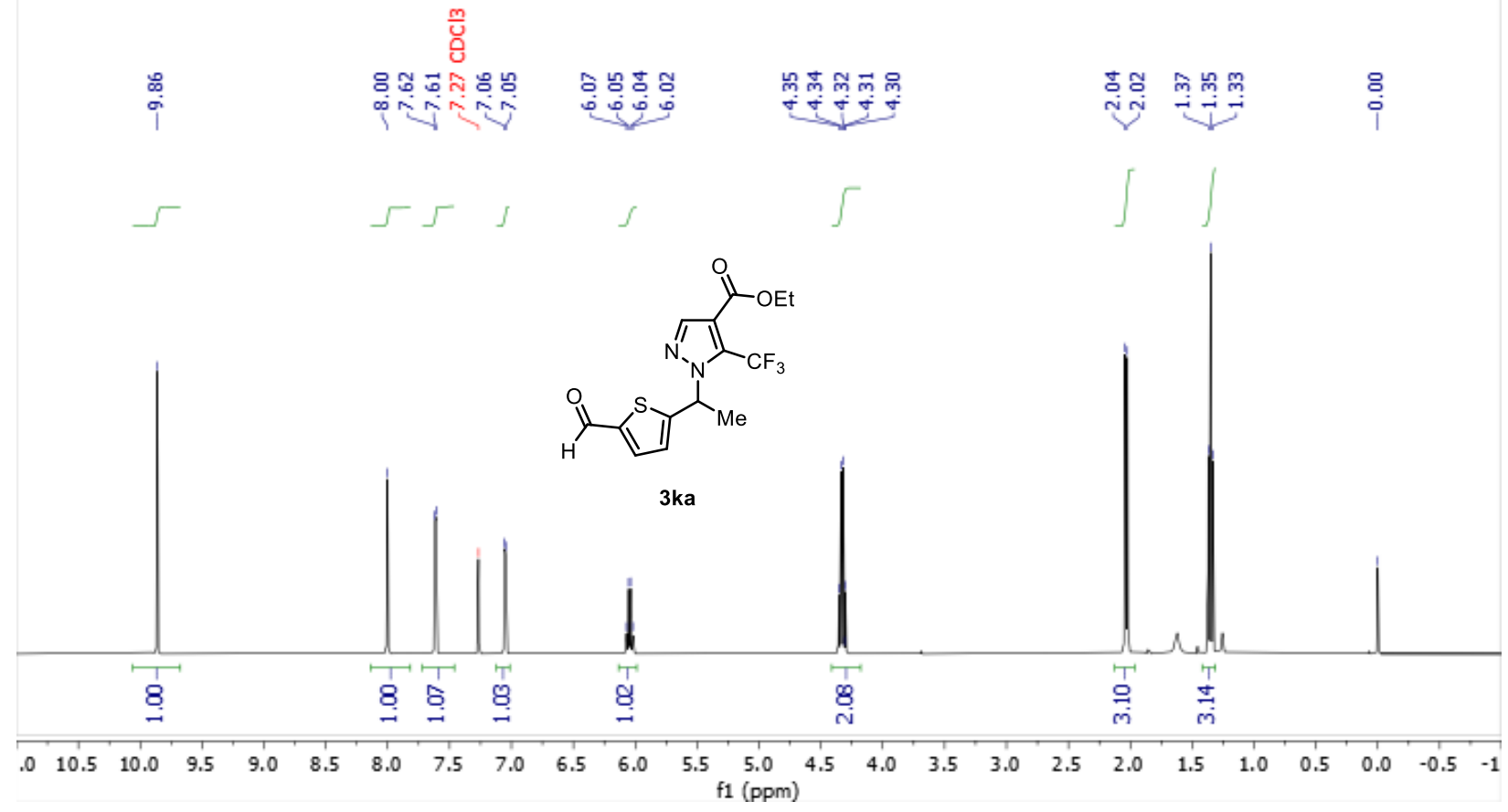

${ }^{13} \mathrm{C}$ NMR spectrum of 3ka in $\mathrm{CDCl}_{3}$ containing $0.03 \%$ (v/v) TMS (126 MHz).

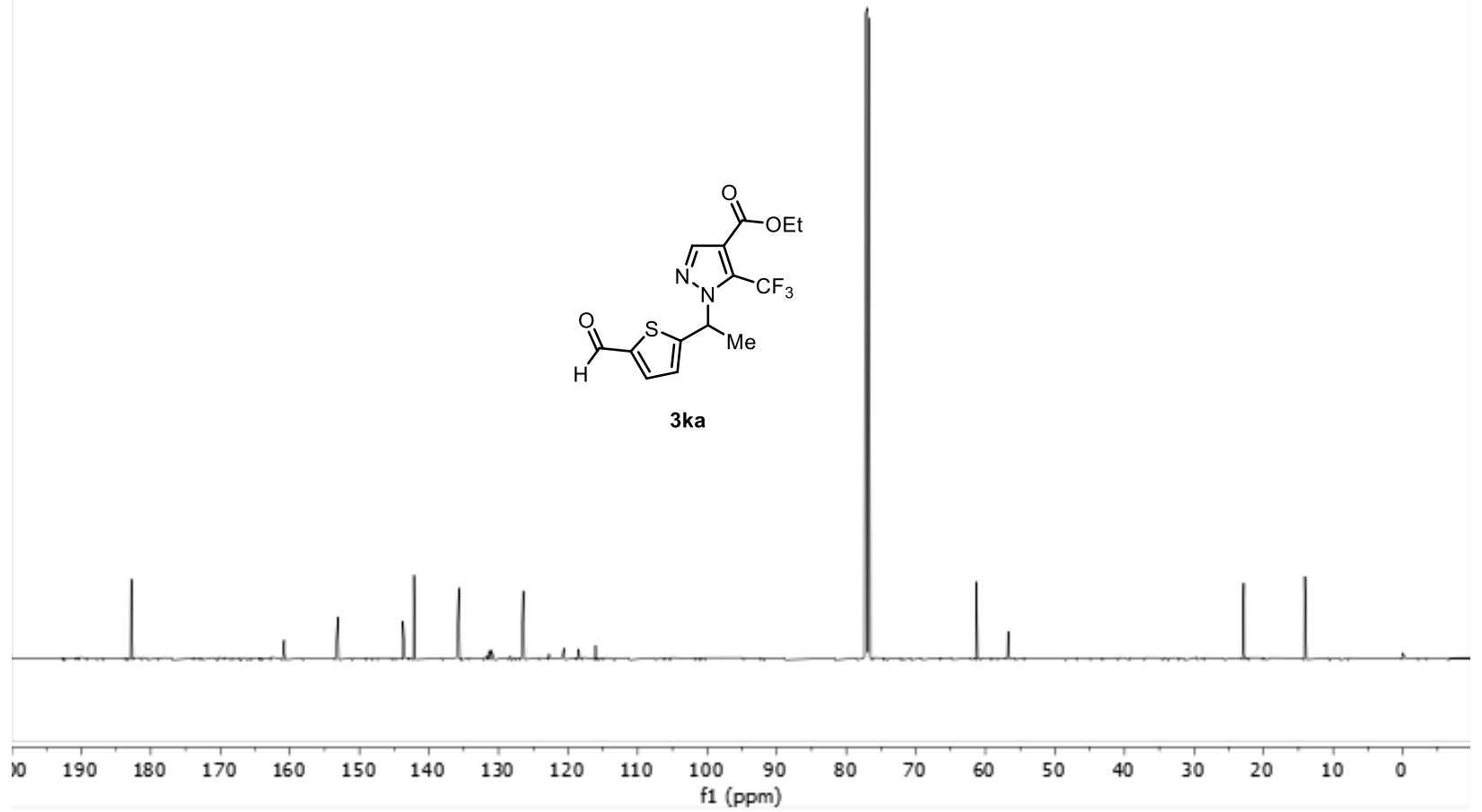


${ }^{19} \mathrm{~F}$ NMR spectrum of $\mathbf{3 k a}$ in $\mathrm{CDCl}_{3}$ containing $0.03 \%$ (v/v) TMS (377 MHz).

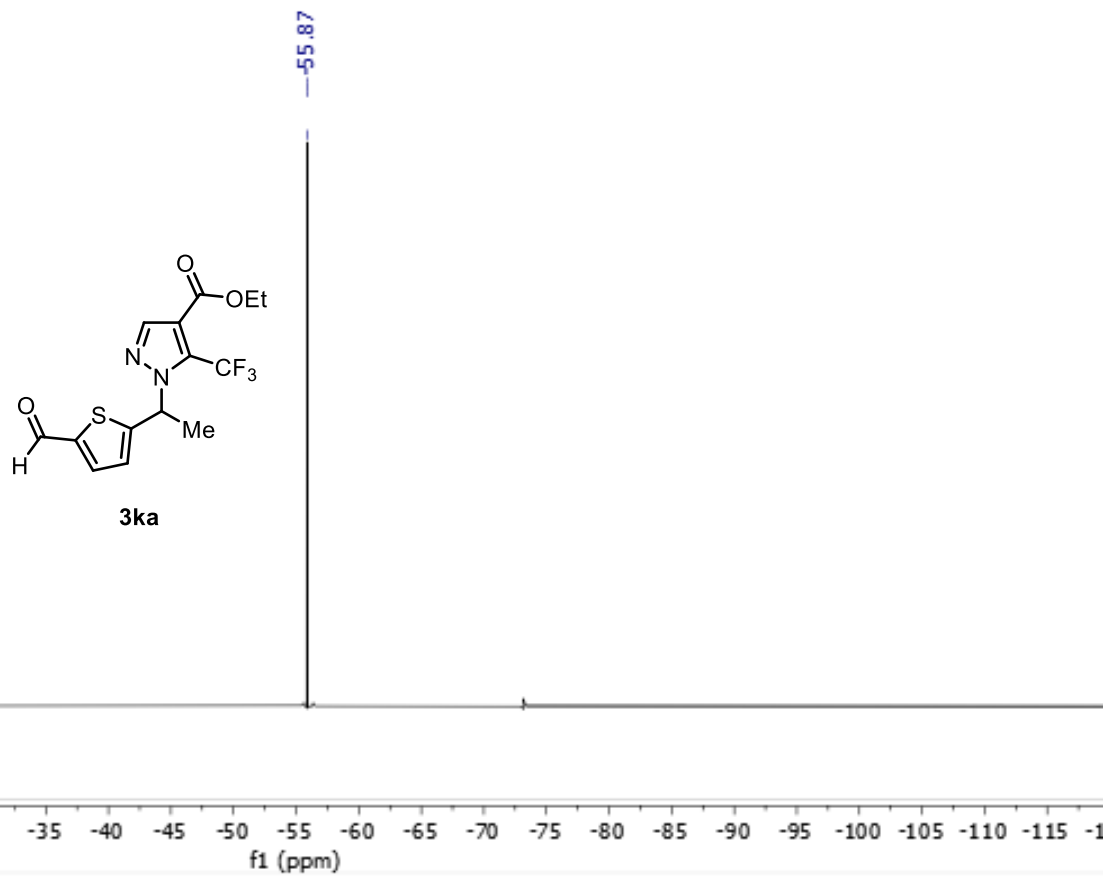

HSQC NMR spectrum of 3ka in $\mathrm{CDCl}_{3}$ containing $0.03 \%$ (v/v) TMS $(500,126 \mathrm{MHz})$.

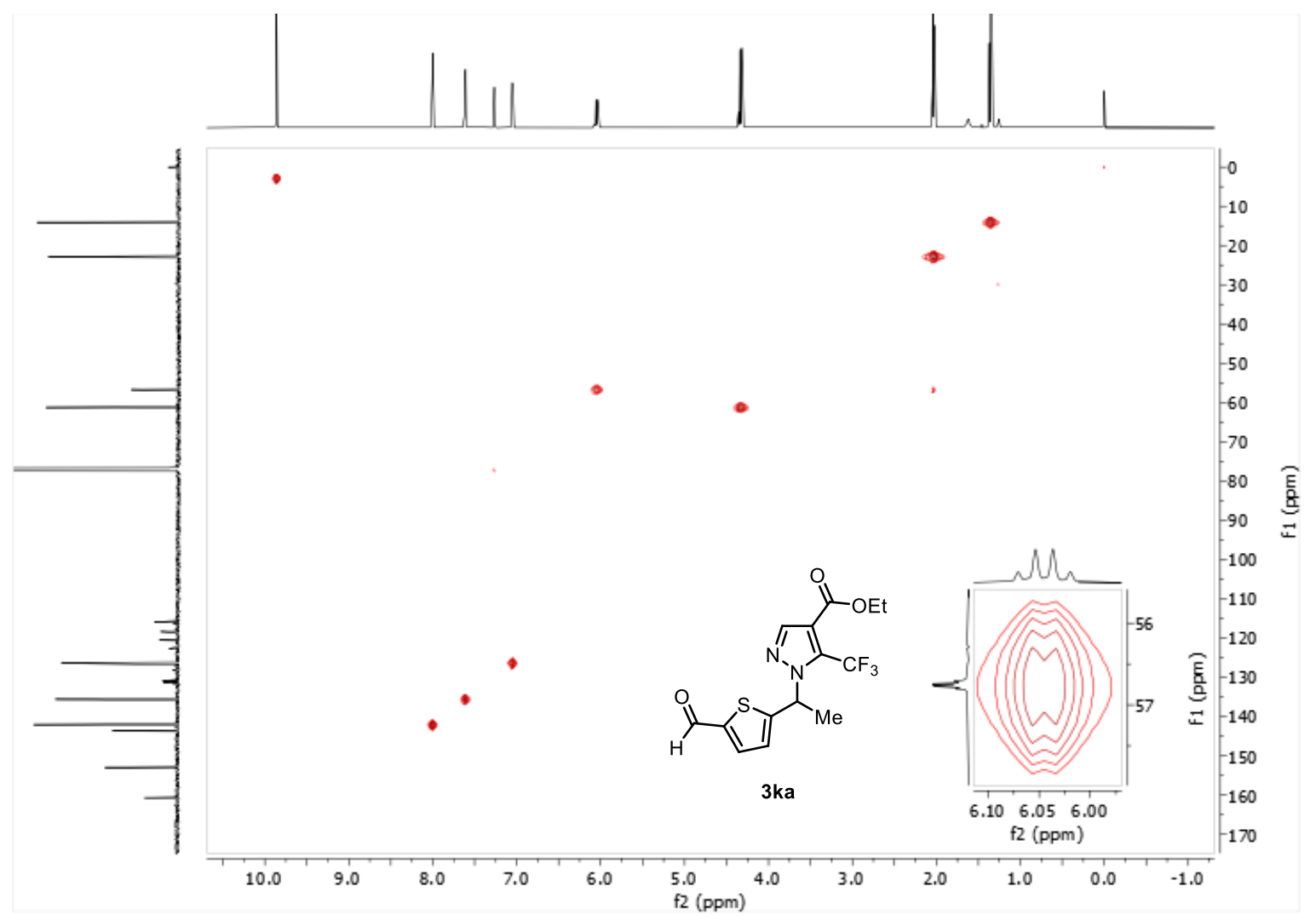


HMBC NMR spectrum of 3ka in $\mathrm{CDCl}_{3}$ containing $0.03 \%$ (v/v) TMS $(500,126 \mathrm{MHz})$.

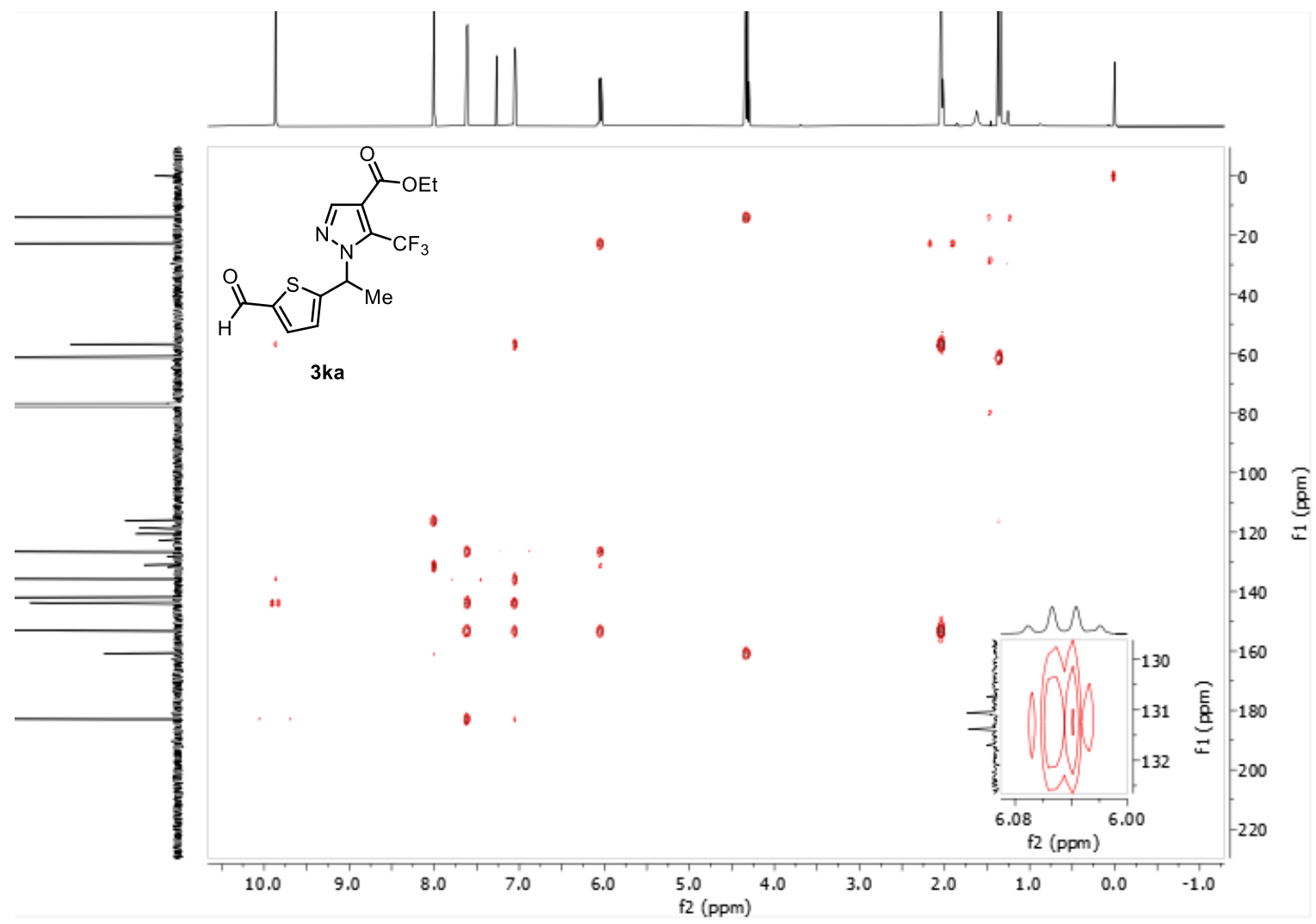

${ }^{1} \mathrm{H}$ NMR spectrum of $\mathbf{3 k a}$ ' in $\mathrm{CDCl}_{3}$ containing $0.03 \%$ (v/v) TMS (500 MHz).

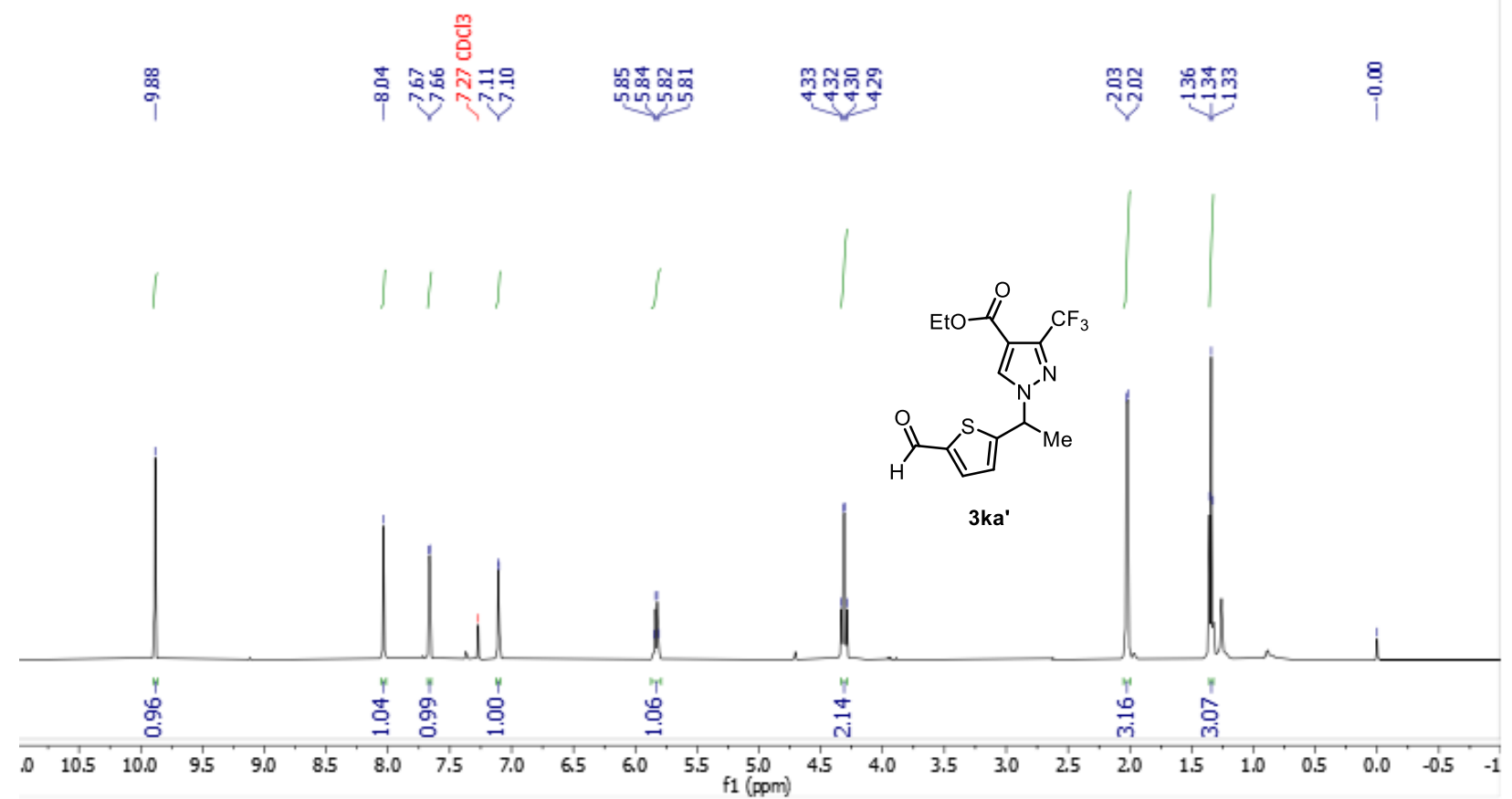


${ }^{13} \mathrm{C}$ NMR spectrum of 3ka' in $\mathrm{CDCl}_{3}$ containing $0.03 \%$ (v/v) TMS (126 MHz).
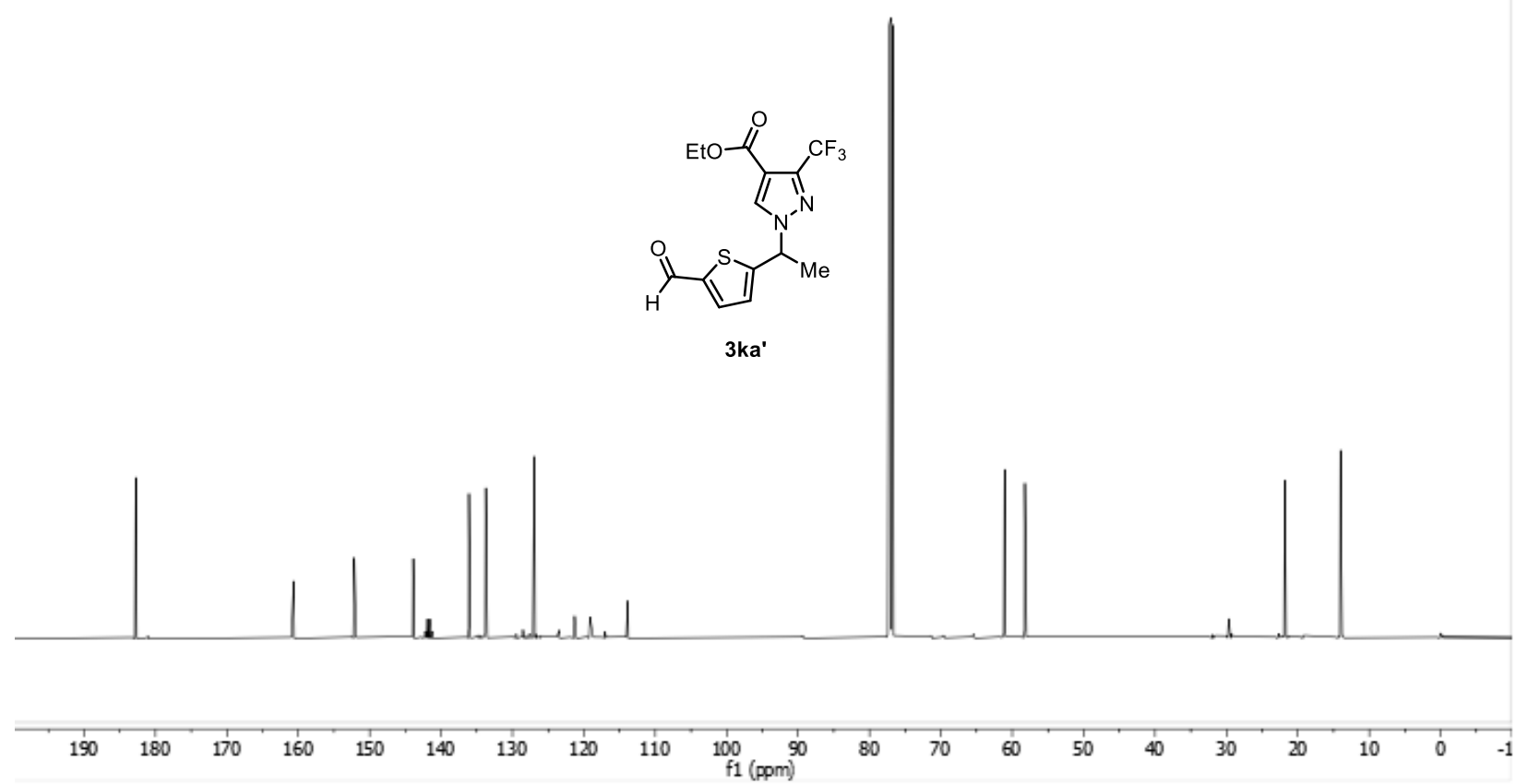

${ }^{19} \mathrm{~F}$ NMR spectrum of $3 \mathbf{k a}$ ' in $\mathrm{CDCl}_{3}$ containing $0.03 \%$ (v/v) TMS (377 MHz).

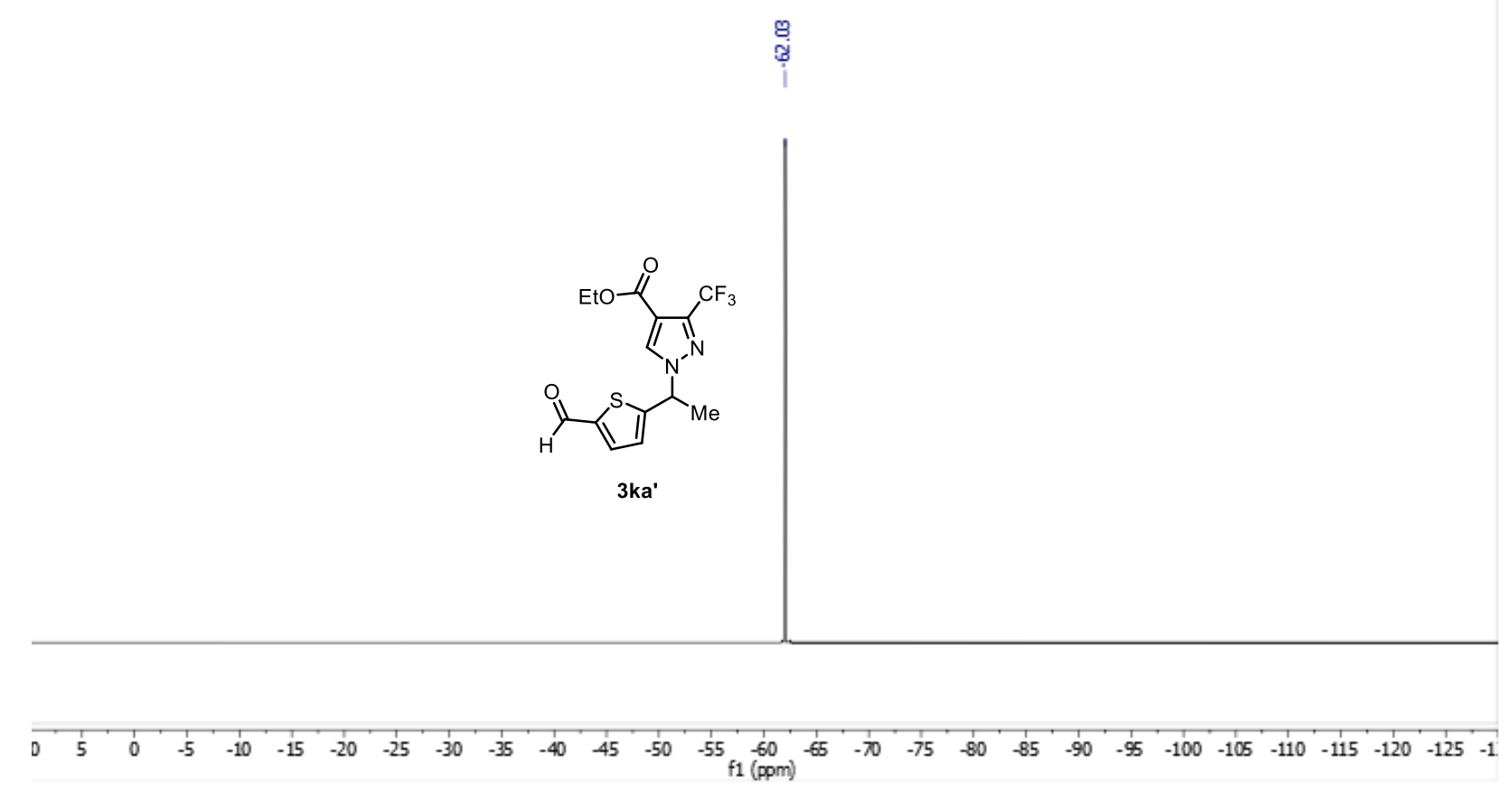


HSQC NMR spectrum of 3ka' in $\mathrm{CDCl}_{3}$ containing $0.03 \%$ (v/v) TMS (500, $126 \mathrm{MHz}$ ).

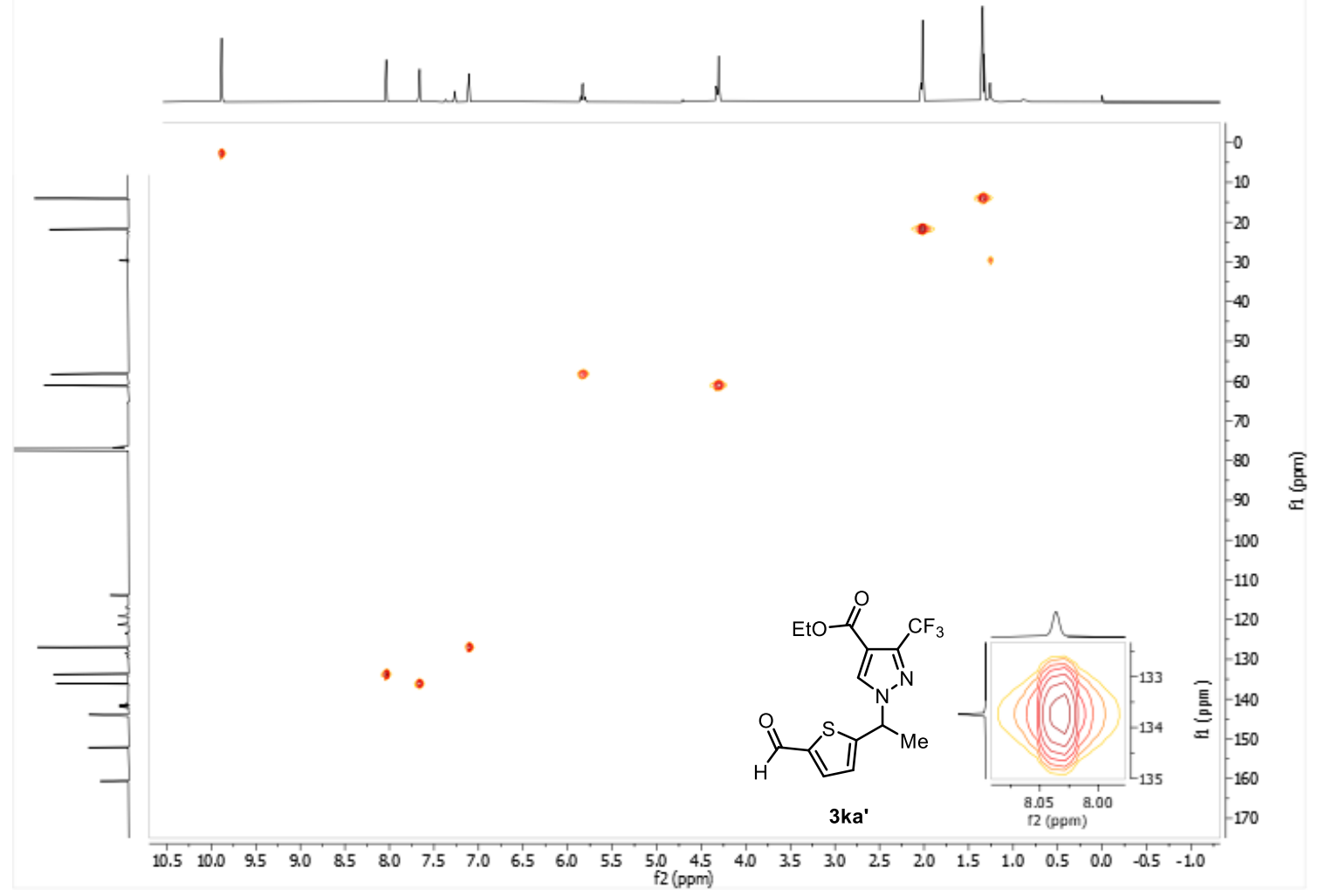

HMBC NMR spectrum of 3ka' in $\mathrm{CDCl}_{3}$ containing $0.03 \%$ (v/v) TMS (500, $126 \mathrm{MHz}$ ).

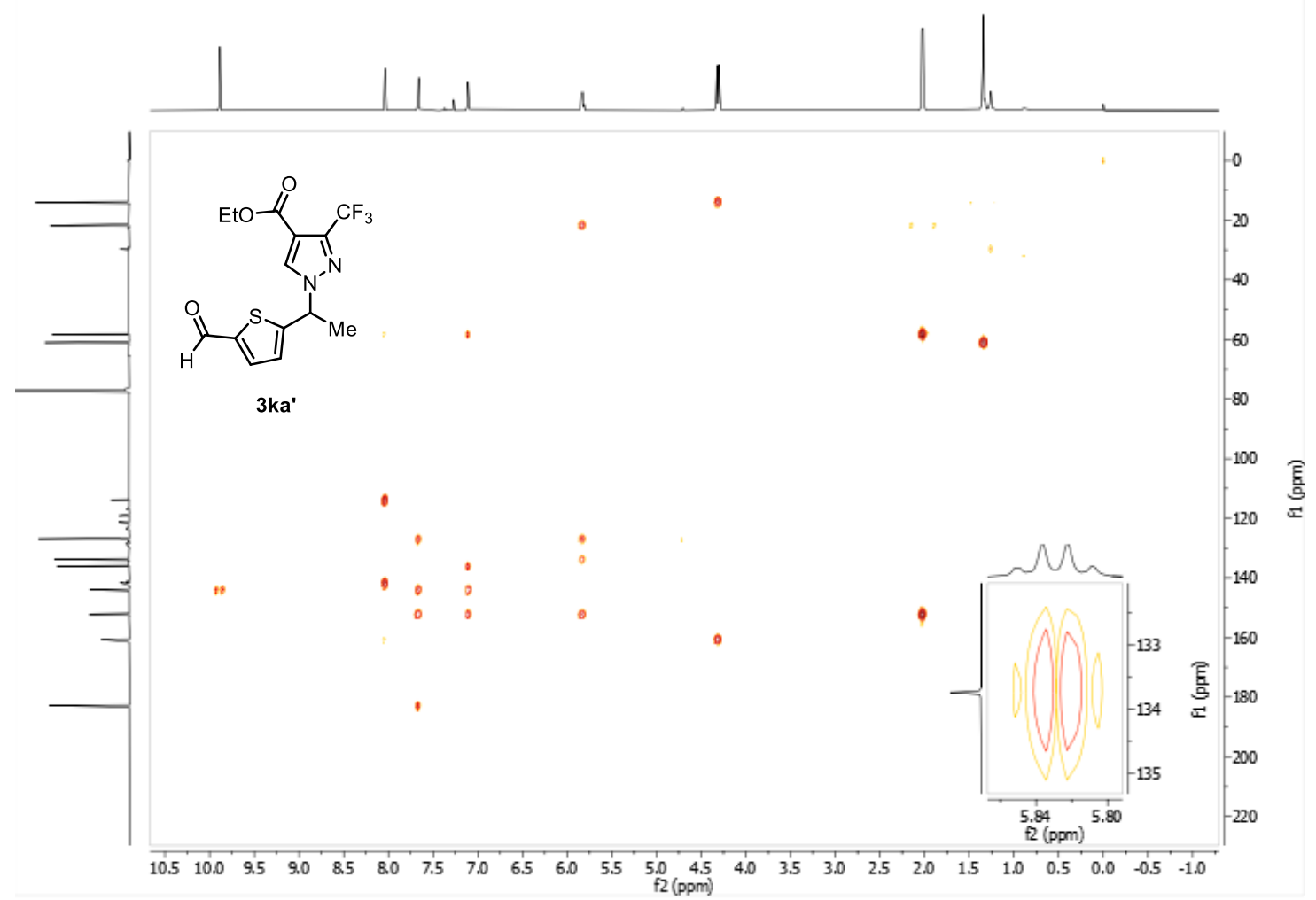


${ }^{1} \mathrm{H}$ NMR spectrum of $3 \mathbf{l a}$ in $\mathrm{CDCl}_{3}$ containing $0.03 \%$ (v/v) TMS (500 MHz).

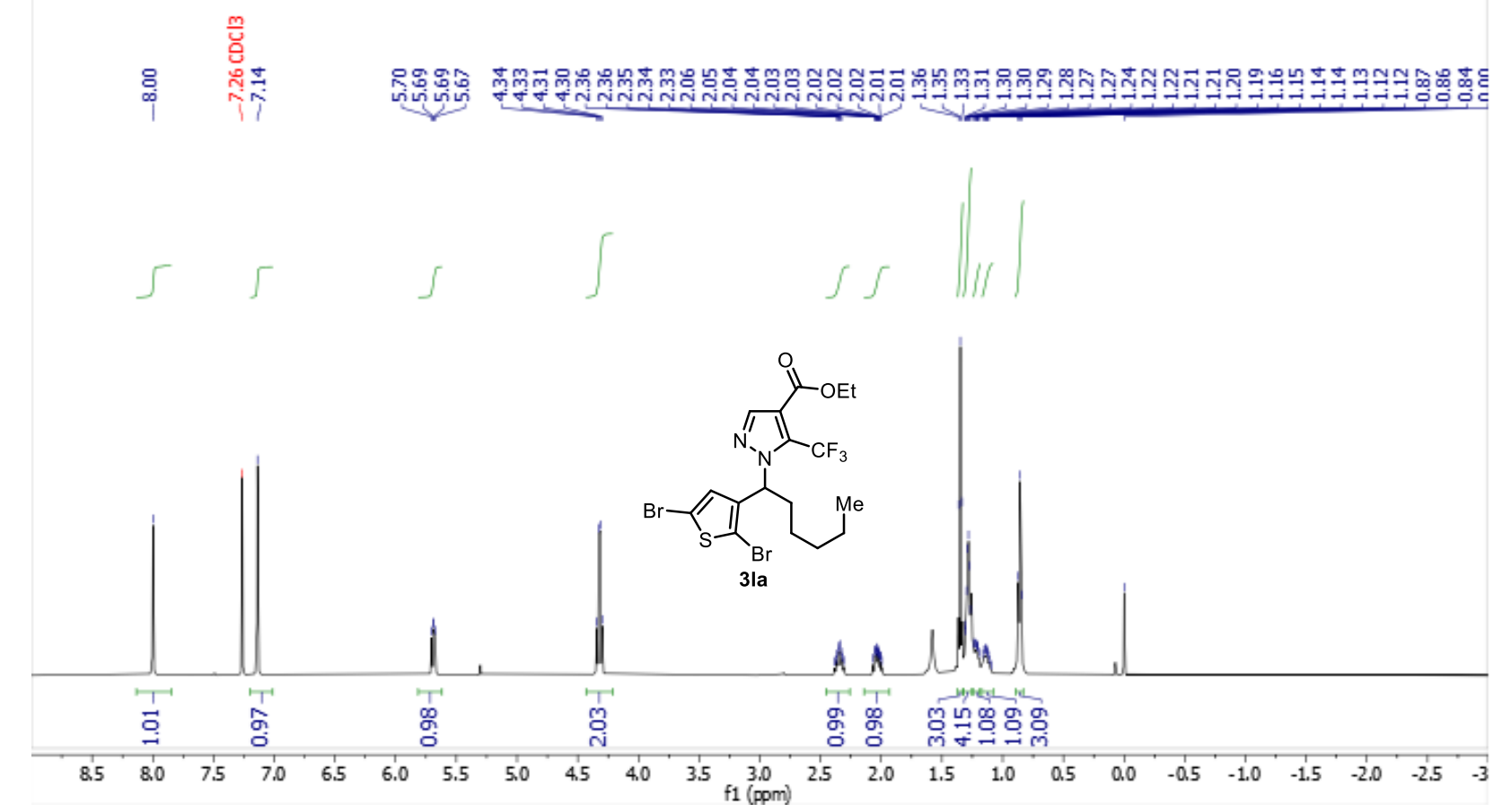

${ }^{13} \mathrm{C}$ NMR spectrum of 3la in $\mathrm{CDCl}_{3}$ containing $0.03 \%$ (v/v) TMS (126 MHz).

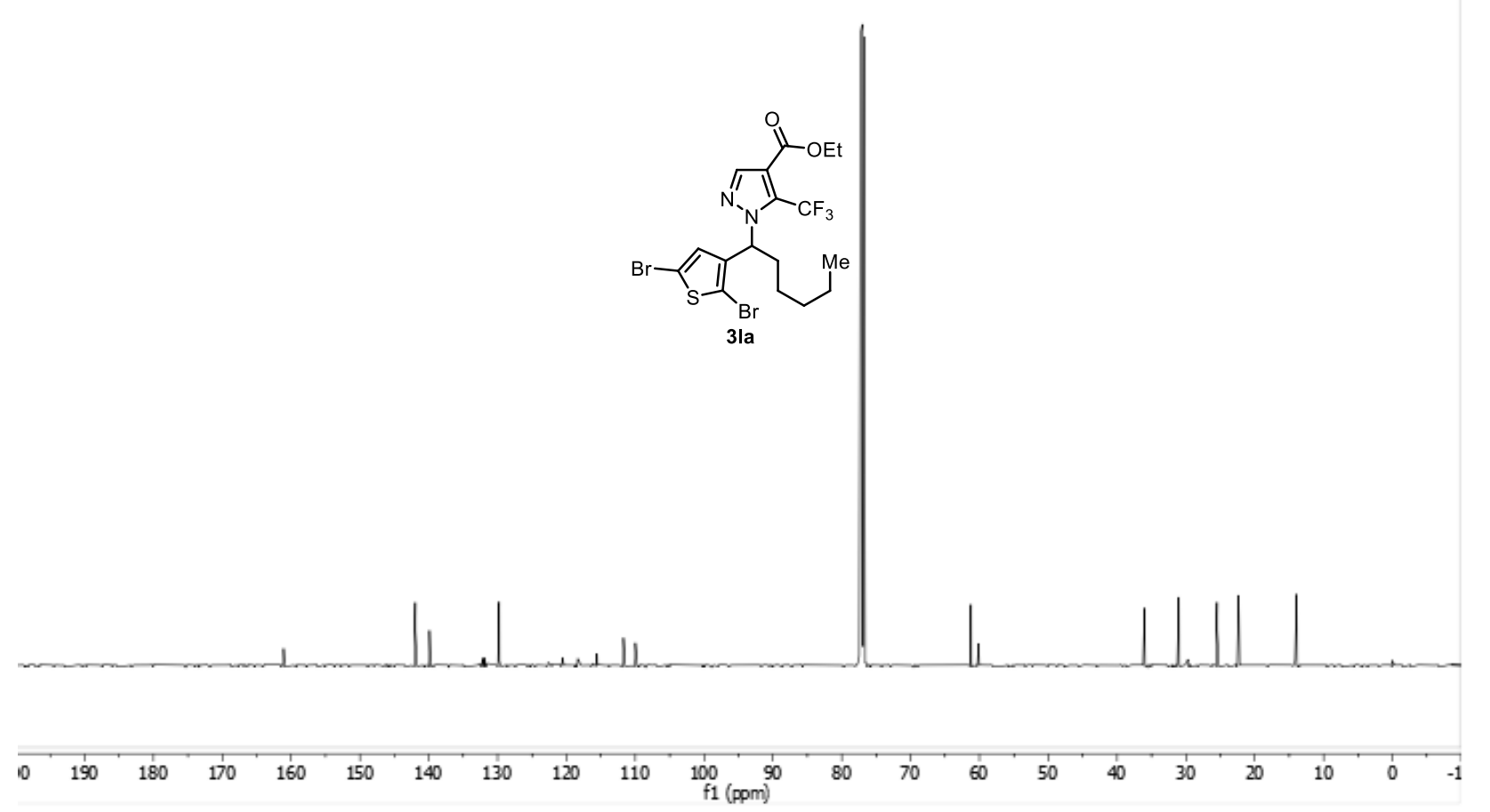


${ }^{19} \mathrm{~F}$ NMR spectrum of 3 la in $\mathrm{CDCl}_{3}$ containing $0.03 \%$ (v/v) TMS (377 MHz).

落

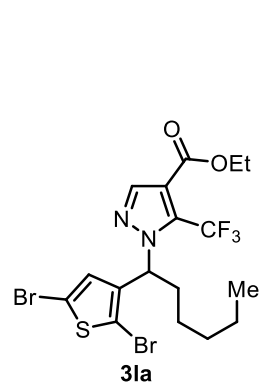

\begin{tabular}{llllllllllllllllllllllllllllll}
\hline 0 & 5 & 0 & -5 & -10 & -15 & -20 & -25 & -10 & -35 & -40 & -45 & -50 & -55 & -60 & -65 & -70 & -75 & -80 & -85 & -90 & -95 & -100 & -105 & -110 & -115 & -1
\end{tabular}

HSQC NMR spectrum of 3la in $\mathrm{CDCl}_{3}$ containing $0.03 \%(\mathrm{v} / \mathrm{v})$ TMS (500, $\left.126 \mathrm{MHz}\right)$.

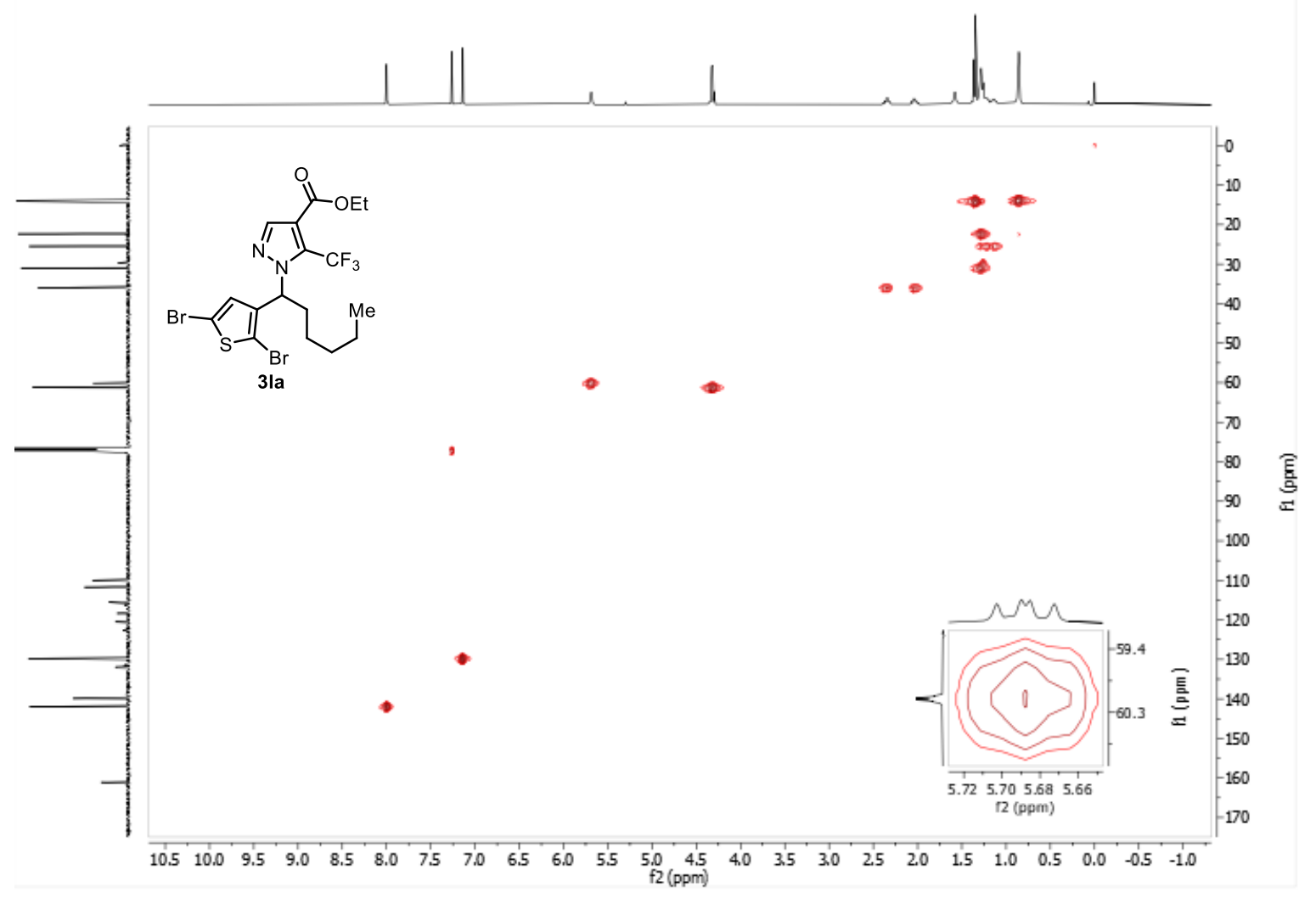


HMBC NMR spectrum of 3la in $\mathrm{CDCl}_{3}$ containing $0.03 \%$ (v/v) TMS (500, $\left.126 \mathrm{MHz}\right)$.

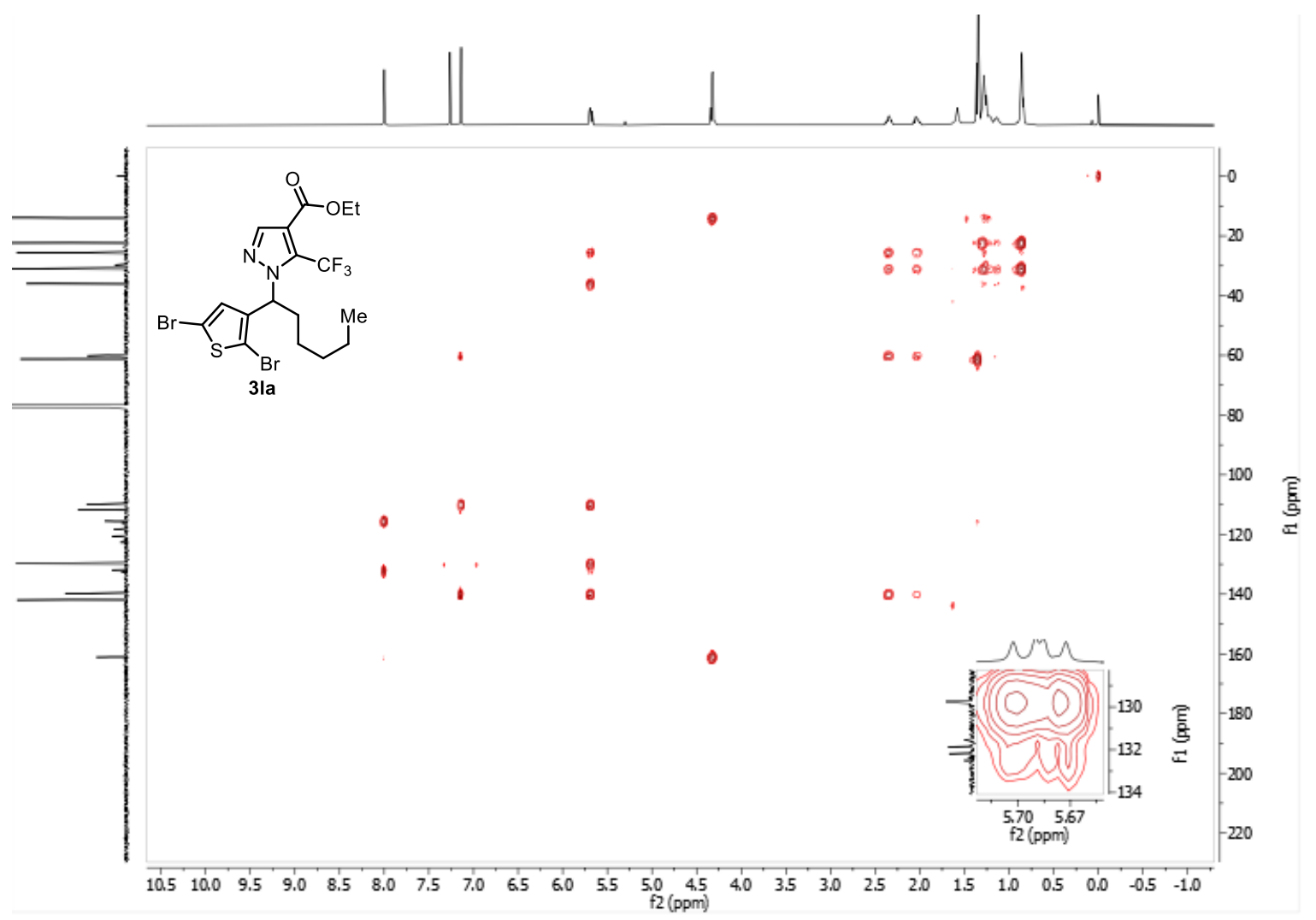

${ }^{1} \mathrm{H}$ NMR spectrum of 3ma-1 in $\mathrm{CDCl}_{3}$ containing $0.03 \%$ (v/v) TMS (500 MHz).

$\stackrel{m}{\mathrm{~g}}$

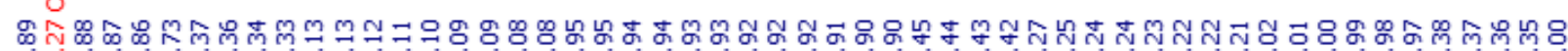
लि ம
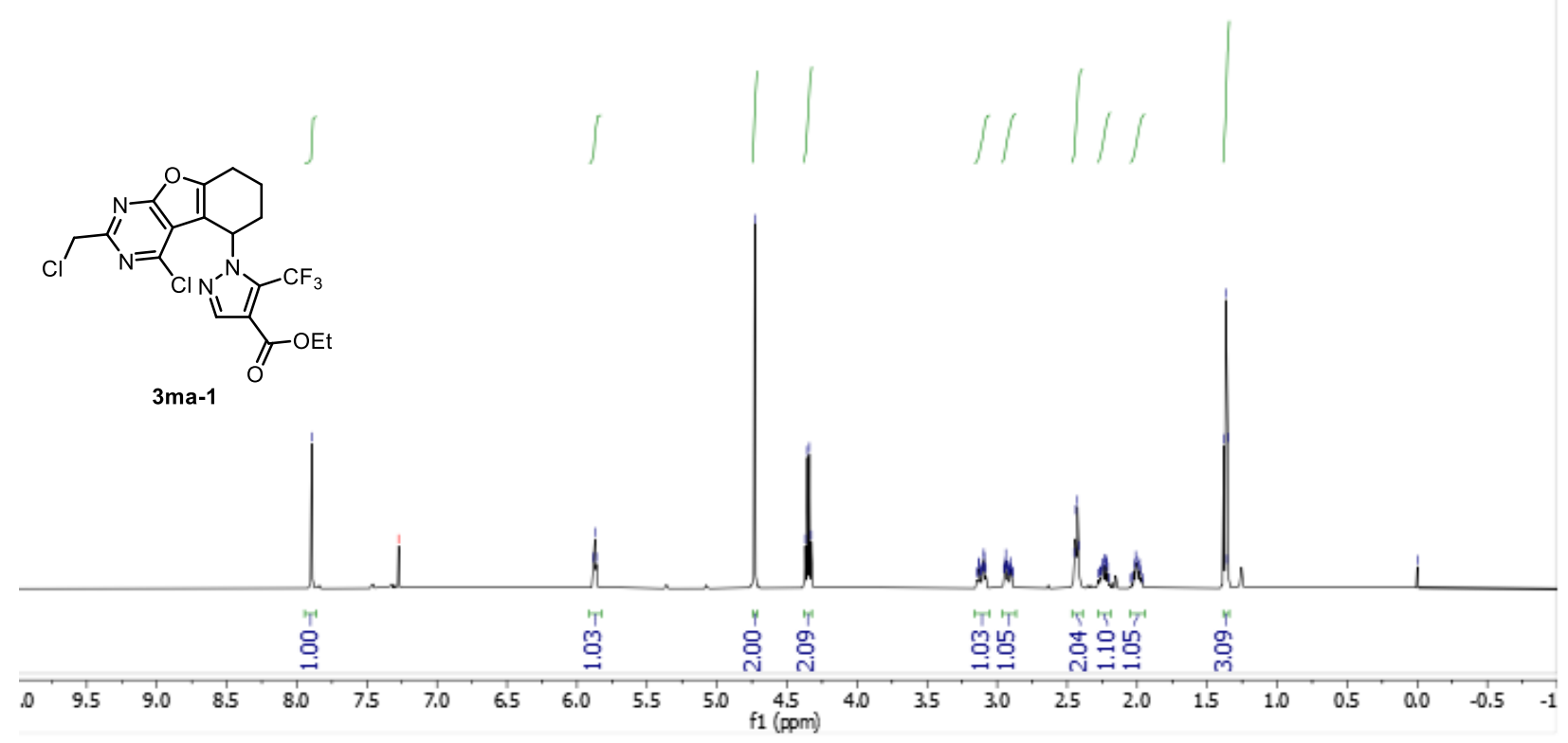
${ }^{13} \mathrm{C}$ NMR spectrum of 3ma-1 in $\mathrm{CDCl}_{3}$ containing $0.03 \%$ (v/v) TMS (126 MHz).
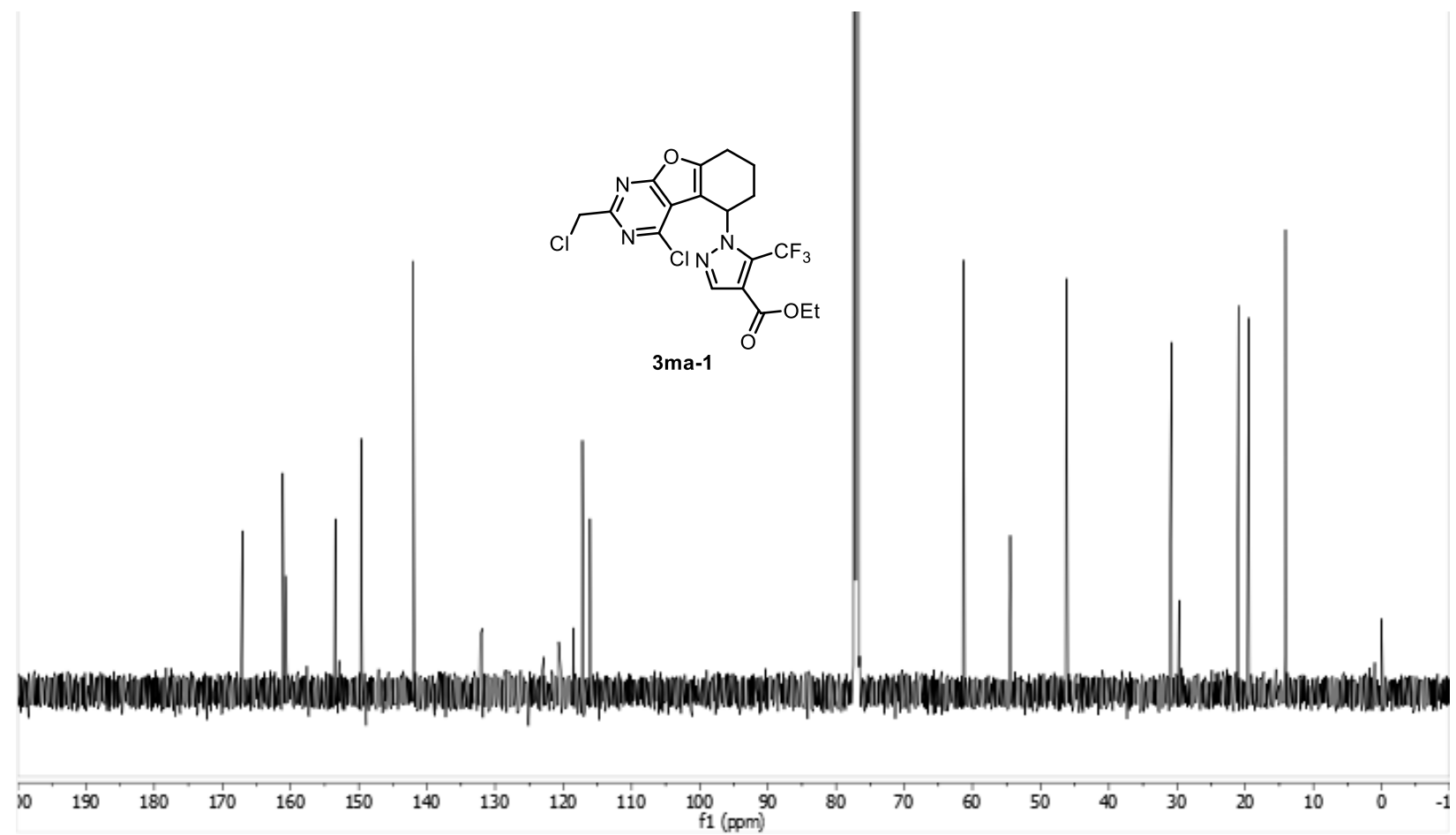

${ }^{19} \mathrm{~F}$ NMR spectrum of 3ma-1 in $\mathrm{CDCl}_{3}$ containing $0.03 \%$ (v/v) TMS (377 MHz).

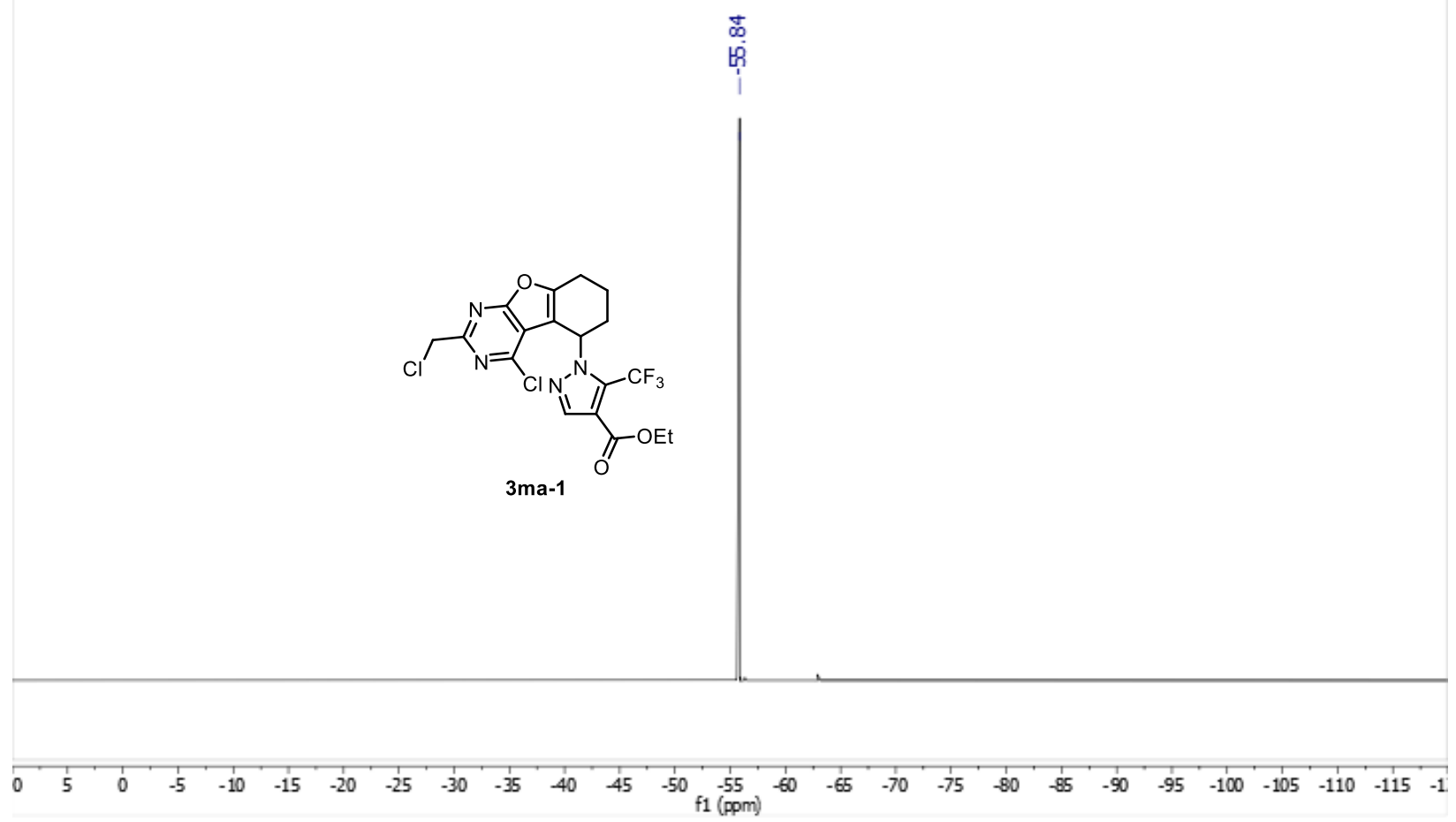


HSQC NMR spectrum of 3ma-1 in $\mathrm{CDCl}_{3}$ containing $0.03 \%$ (v/v) TMS (500, $\left.126 \mathrm{MHz}\right)$.

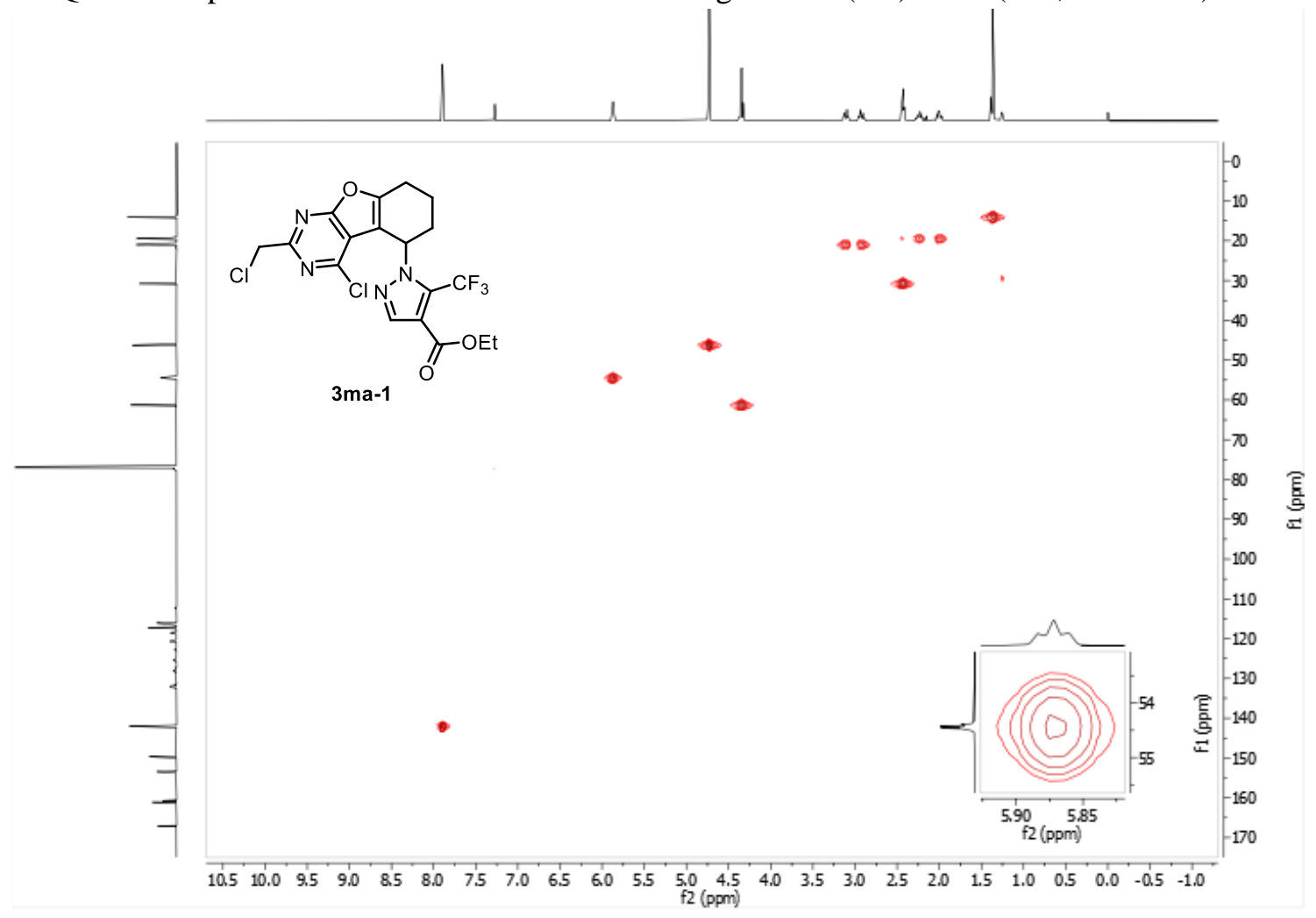

HMBC NMR spectrum of 3ma-1 in $\mathrm{CDCl}_{3}$ containing $0.03 \%$ (v/v) TMS (500, $126 \mathrm{MHz}$ ).

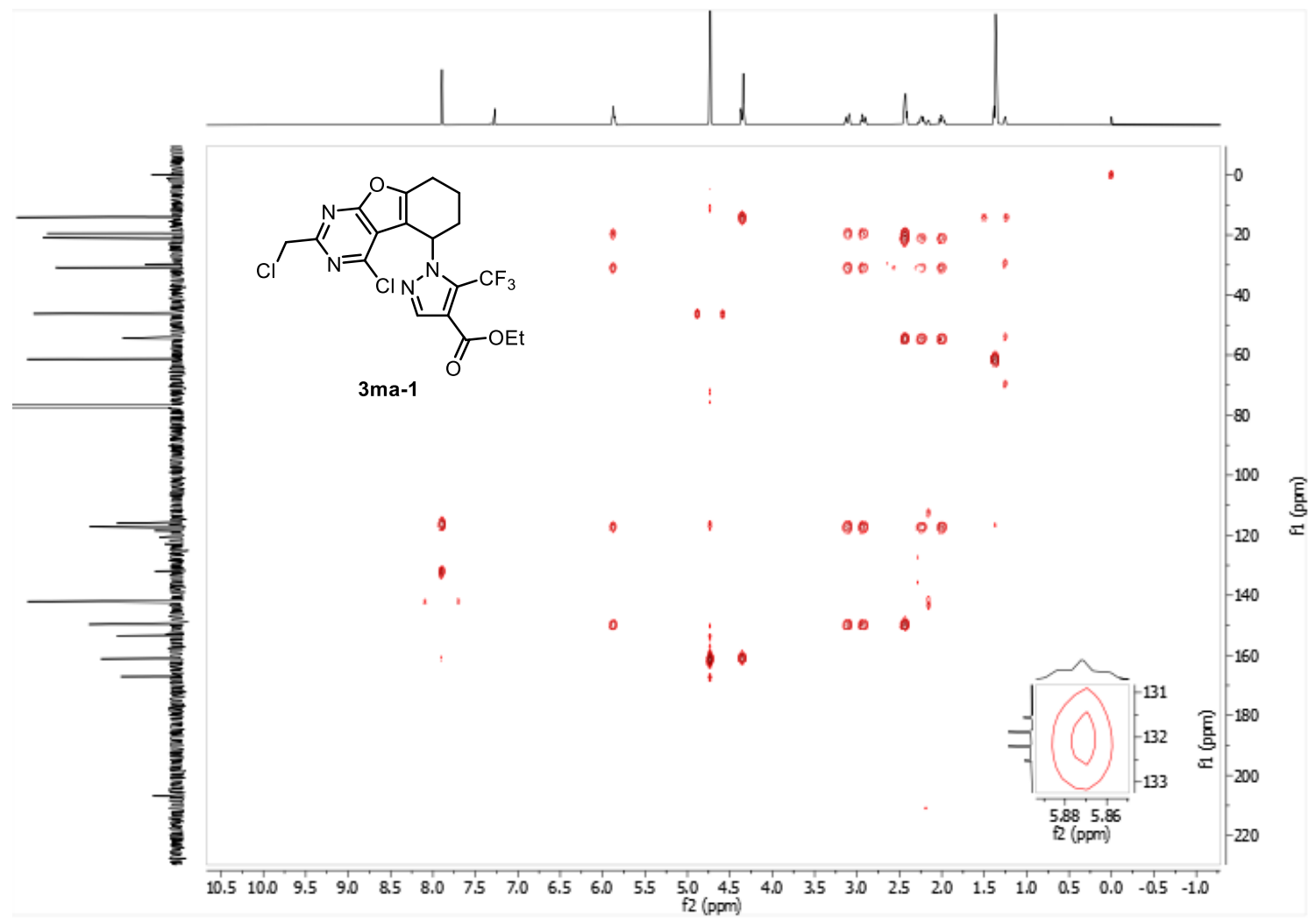


${ }^{1} \mathrm{H}$ NMR spectrum of $\mathbf{3 m a - 2}$ in $\mathrm{CDCl}_{3}$ containing $0.03 \%$ (v/v) TMS (500 MHz).
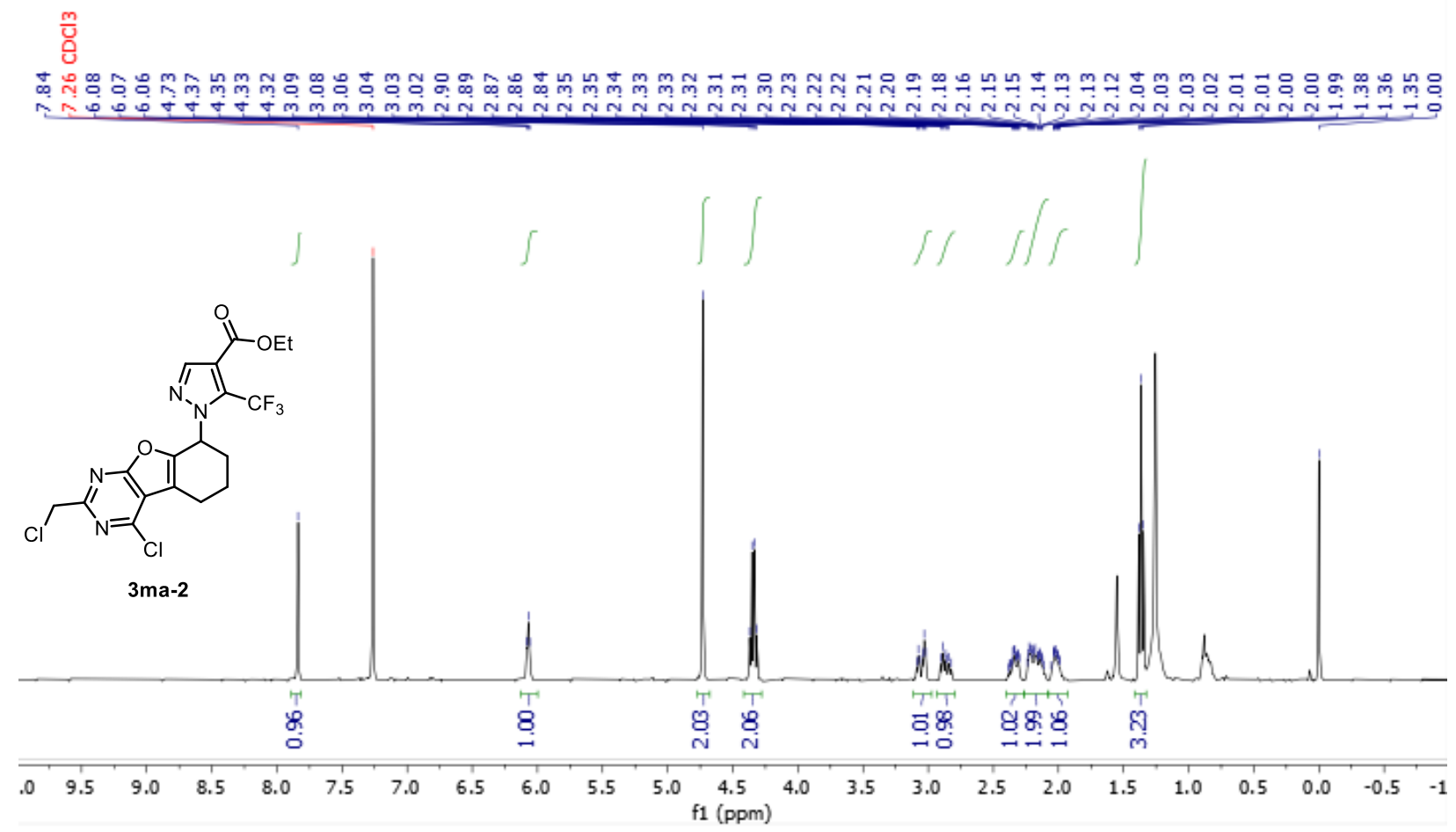

${ }^{13} \mathrm{C}$ NMR spectrum of 3ma-2 in $\mathrm{CDCl}_{3}$ containing $0.03 \%(\mathrm{v} / \mathrm{v}) \mathrm{TMS}(126 \mathrm{MHz})$.

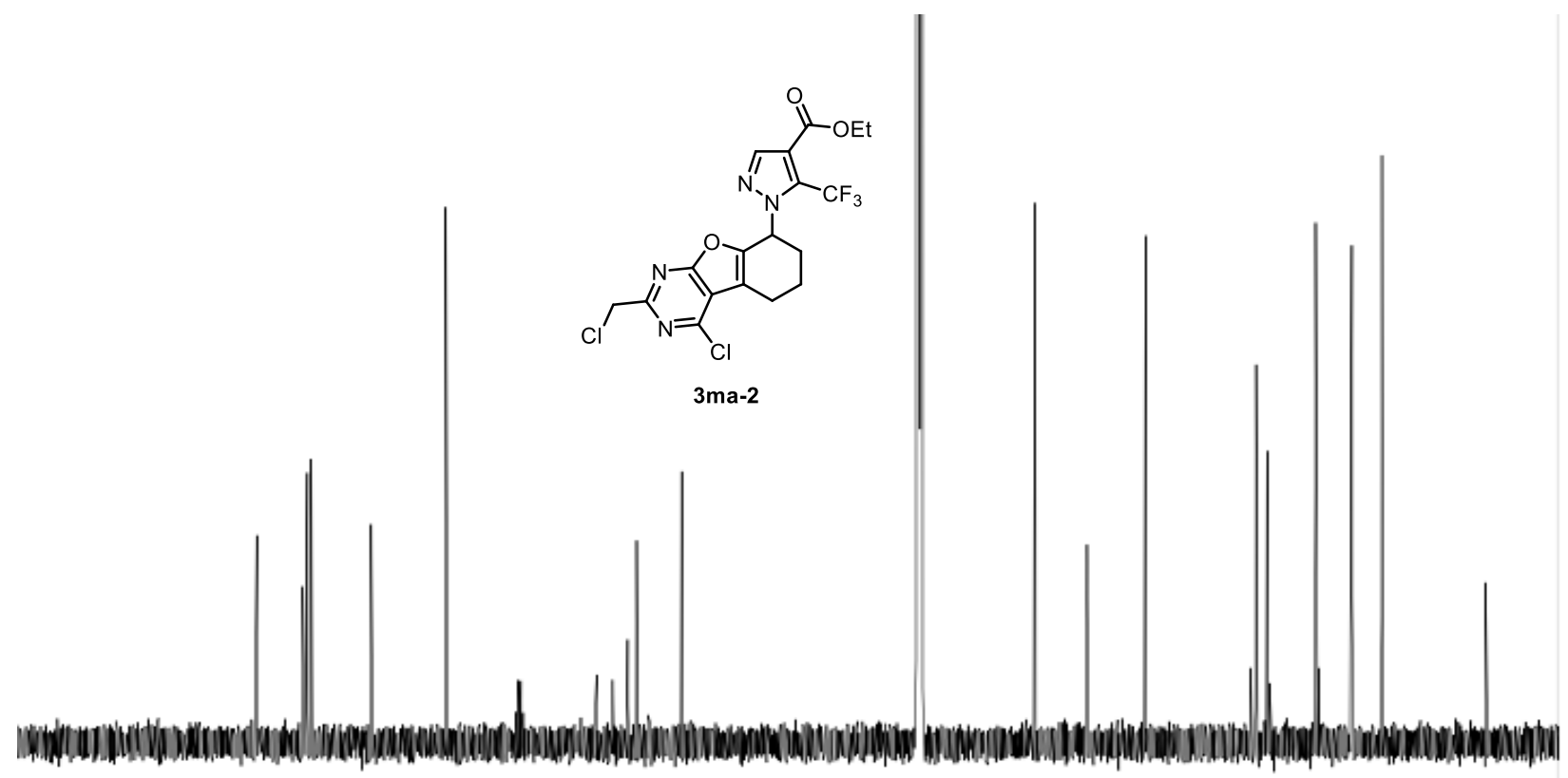

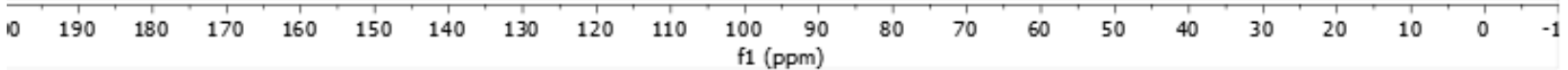


${ }^{19} \mathrm{~F}$ NMR spectrum of $\mathbf{3 m a - 2}$ in $\mathrm{CDCl}_{3}$ containing $0.03 \%$ (v/v) TMS (377 MHz).

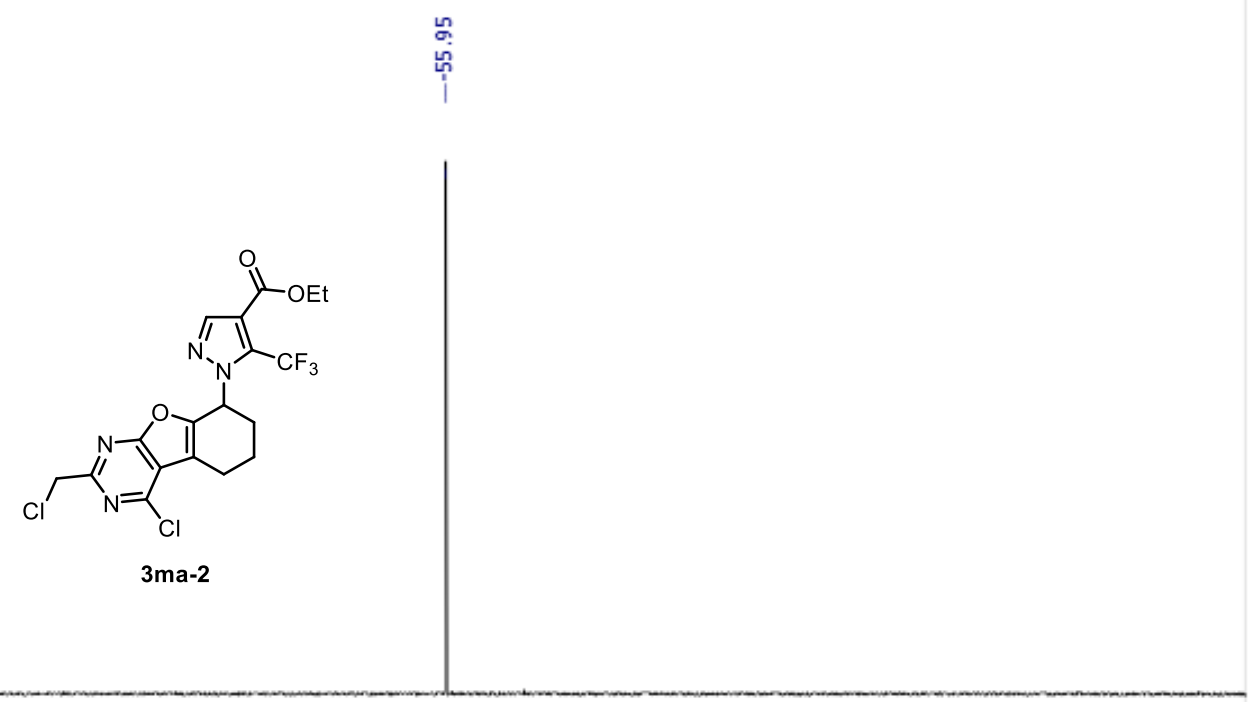

\begin{tabular}{rlllllllllllllllllllllllllllllll}
\hline 5 & 0 & -5 & -10 & -15 & -20 & -25 & -30 & -35 & -40 & -45 & -50 & -55 & -60 & -65 & -70 & -75 & -80 & -85 & -90 & -95 & -100 & -105 & -110 & -115 & -1
\end{tabular}

HSQC NMR spectrum of 3ma-2 in $\mathrm{CDCl}_{3}$ containing $0.03 \%(\mathrm{v} / \mathrm{v})$ TMS $(500,126 \mathrm{MHz})$.

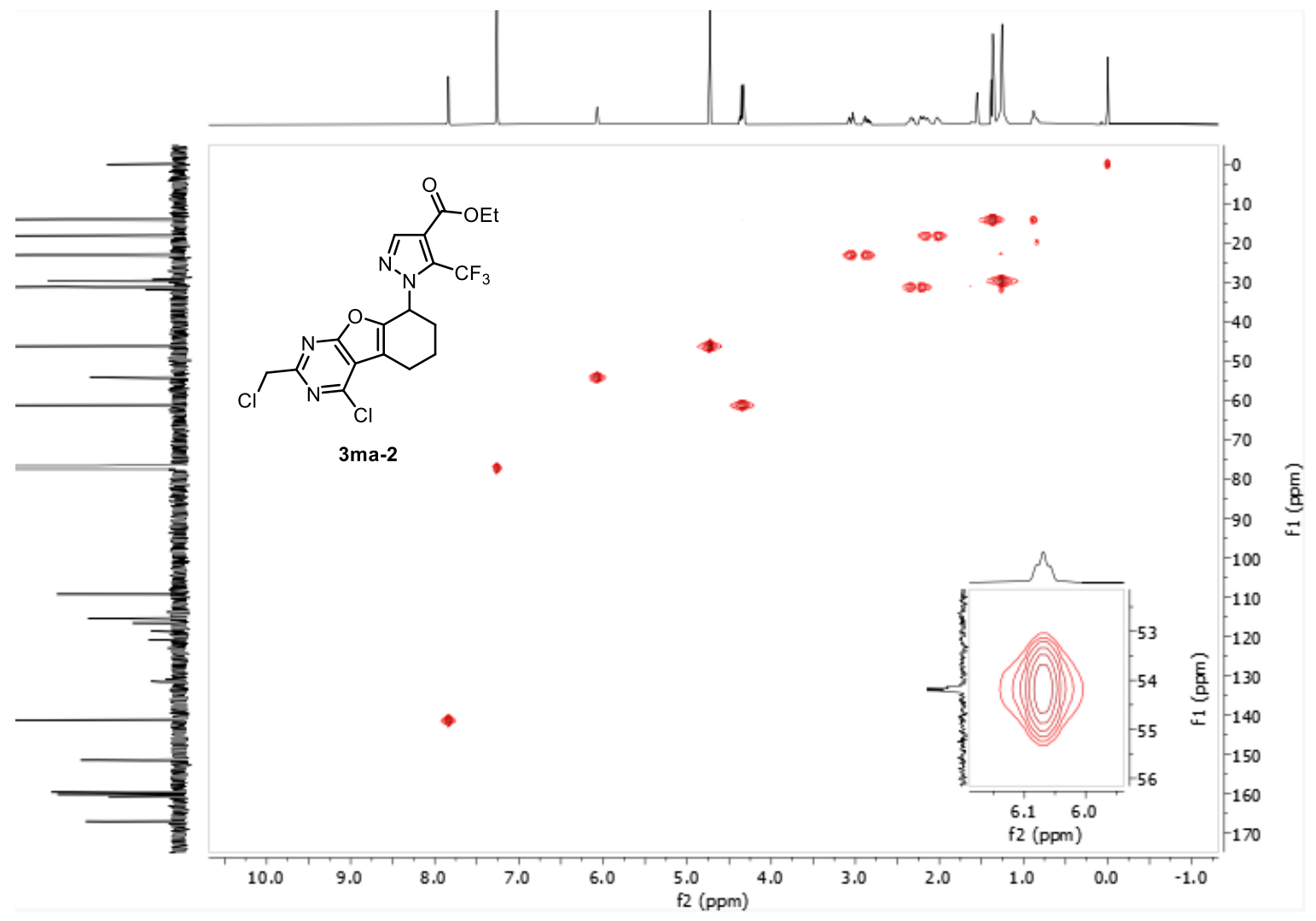


HMBC NMR spectrum of 3ma-2 in $\mathrm{CDCl}_{3}$ containing $0.03 \%(\mathrm{v} / \mathrm{v})$ TMS (500, $\left.126 \mathrm{MHz}\right)$.

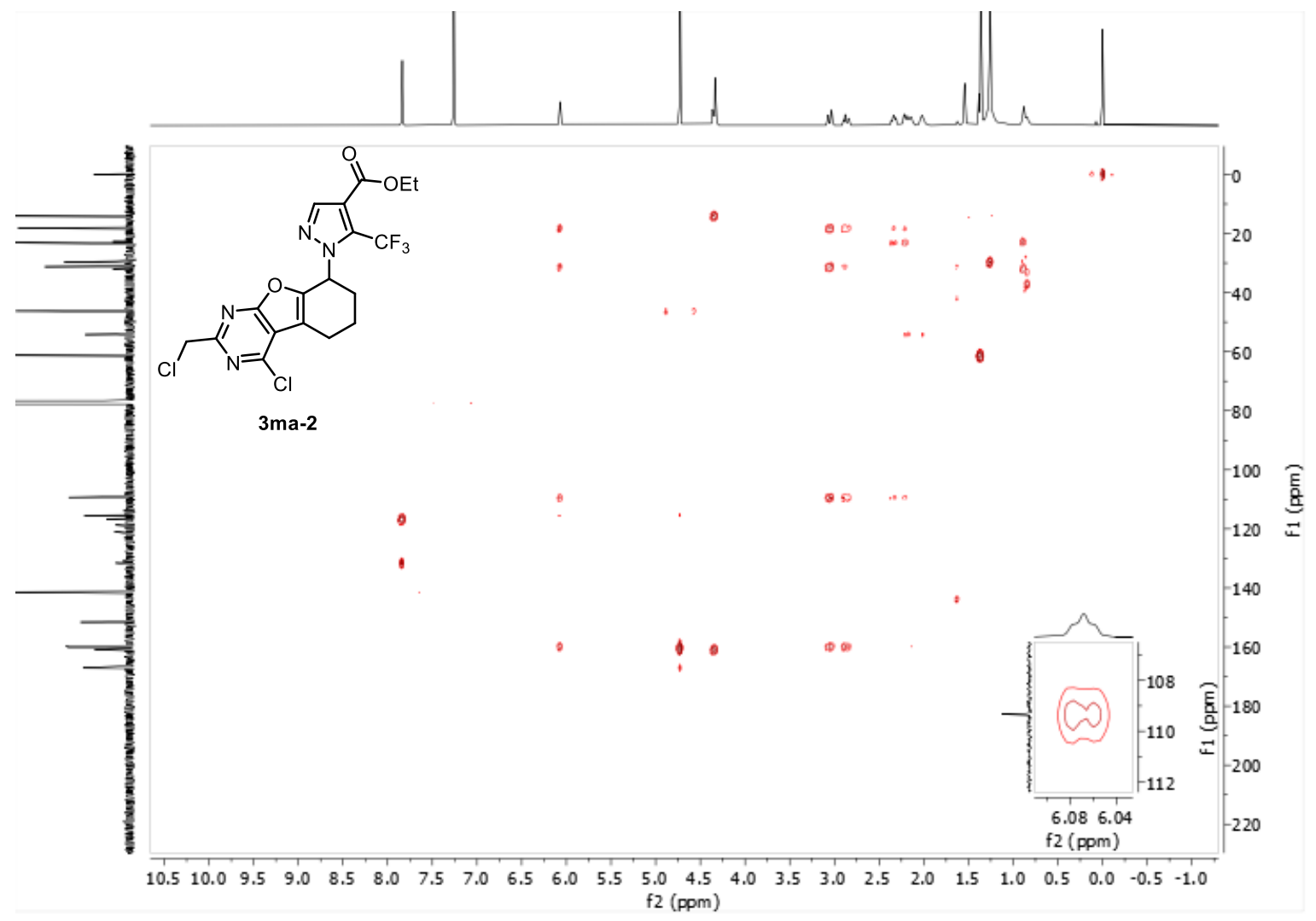

${ }^{1} \mathrm{H}$ NMR spectrum of $\mathbf{3 m a}-\mathbf{1}$ in $\mathrm{CDCl}_{3}$ containing $0.03 \%$ (v/v) TMS (500 MHz).

$\stackrel{n}{8}$

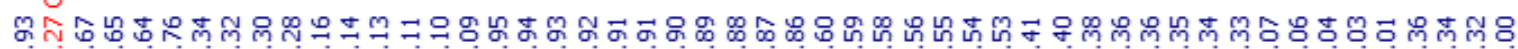

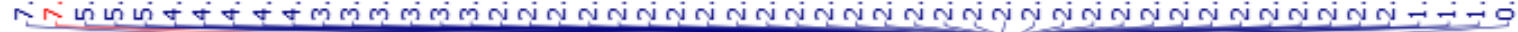

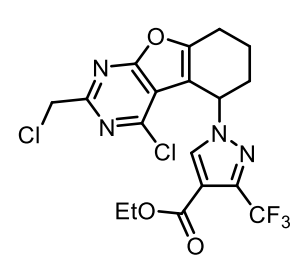

$3 m a '-1$

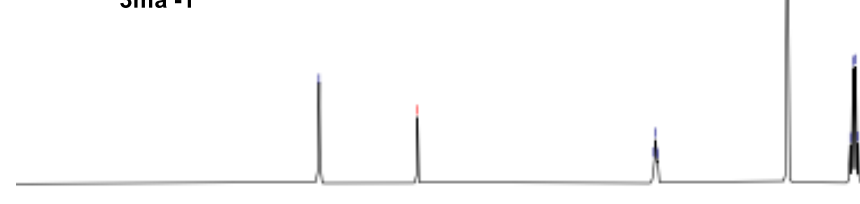

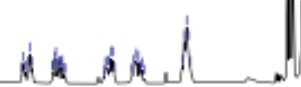

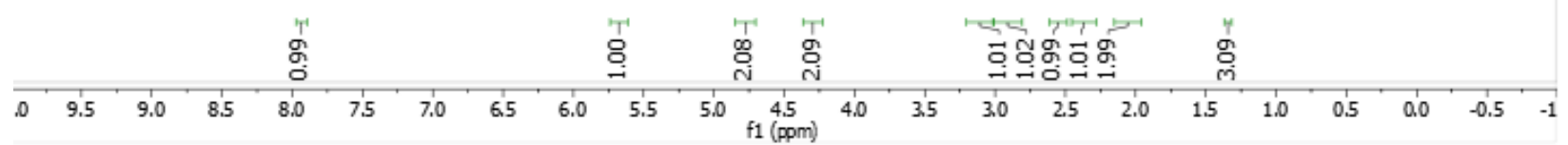


${ }^{13} \mathrm{C}$ NMR spectrum of $\mathbf{3 m a}-\mathbf{1}$ in $\mathrm{CDCl}_{3}$ containing $0.03 \%$ (v/v) TMS (126 MHz).

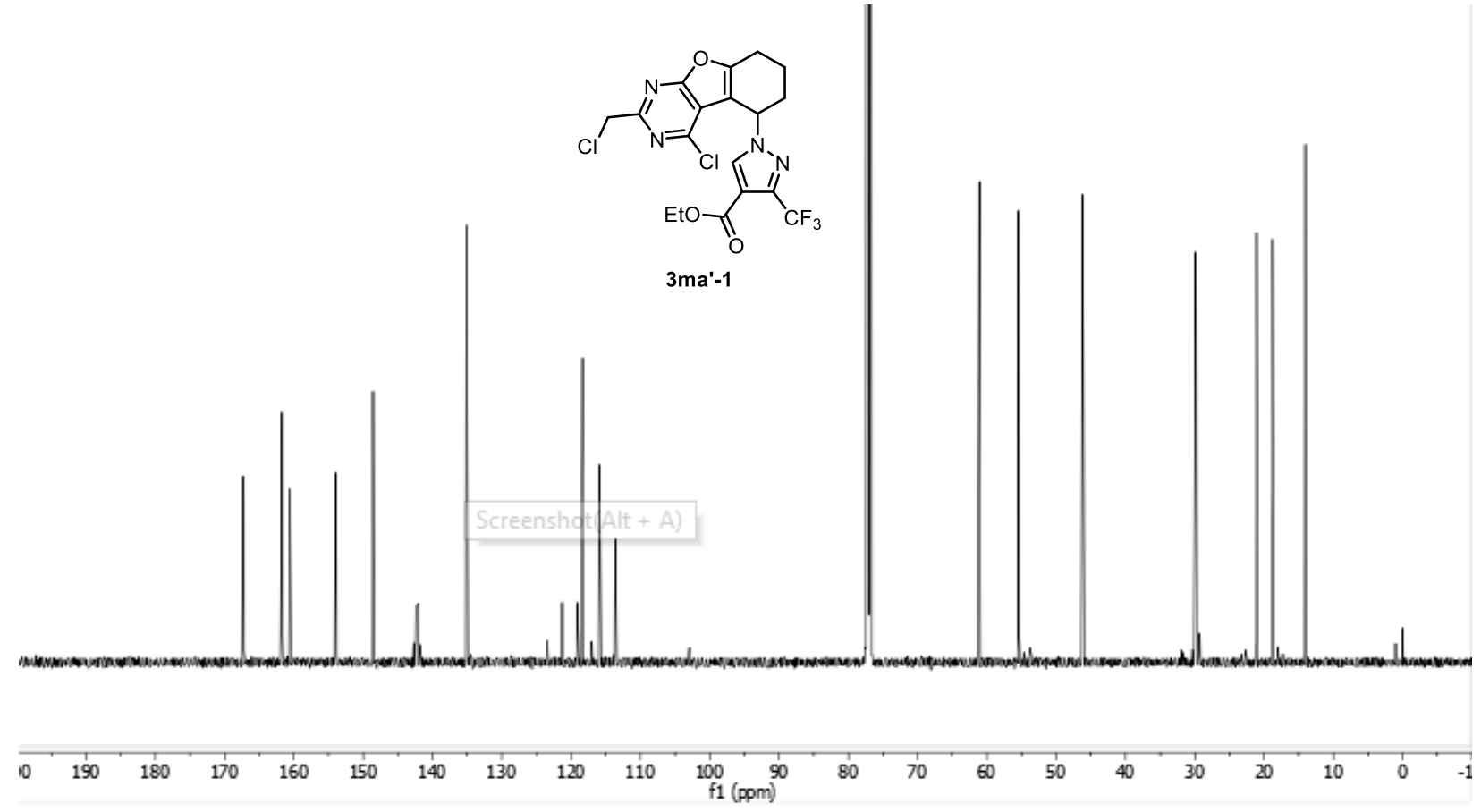

${ }^{19} \mathrm{~F}$ NMR spectrum of $\mathbf{3 m a}-\mathbf{1}$ in $\mathrm{CDCl}_{3}$ containing $0.03 \%$ (v/v) TMS (377 MHz).

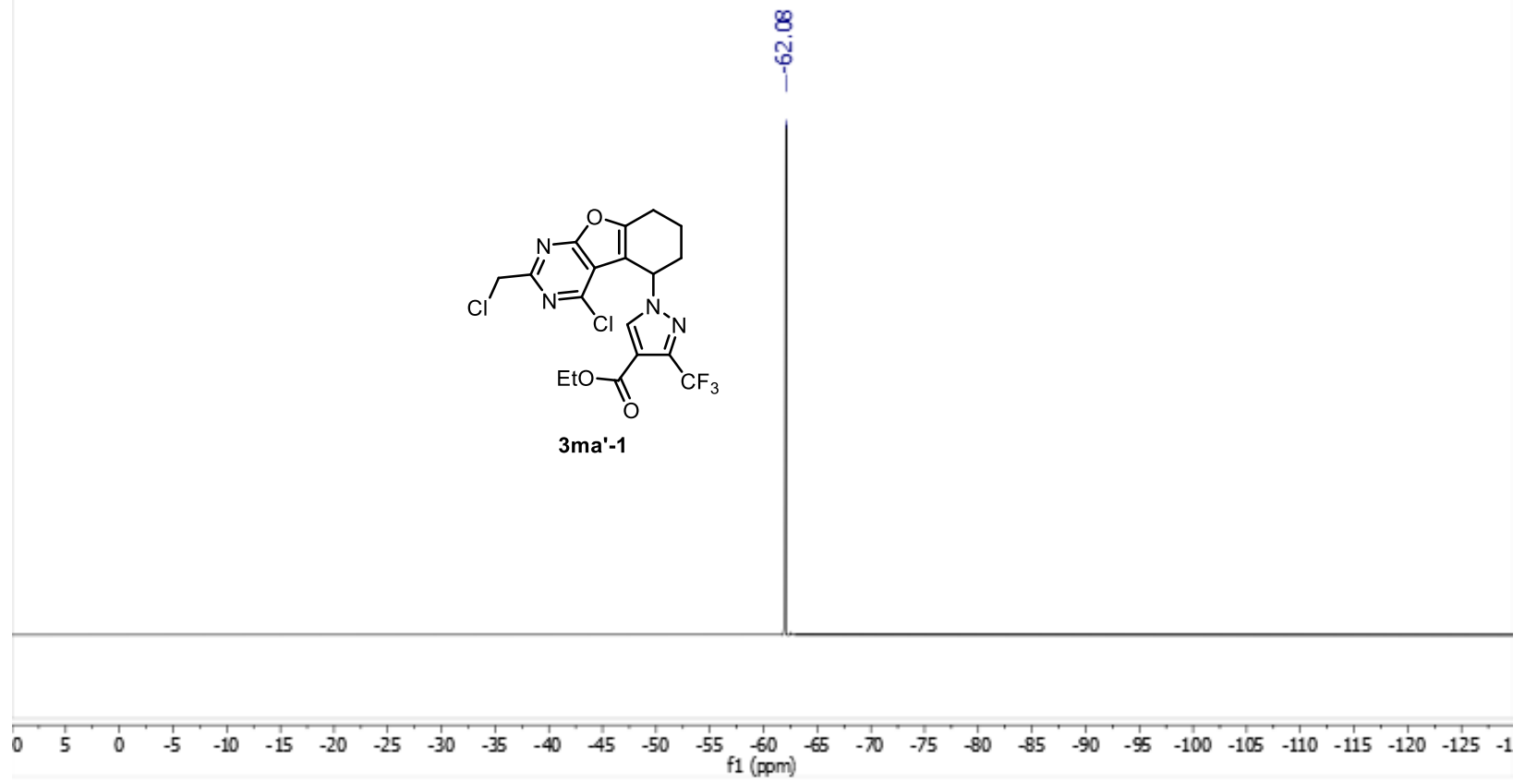


HSQC NMR spectrum of 3ma'-1 in $\mathrm{CDCl}_{3}$ containing $0.03 \%(\mathrm{v} / \mathrm{v})$ TMS (500, $\left.126 \mathrm{MHz}\right)$.

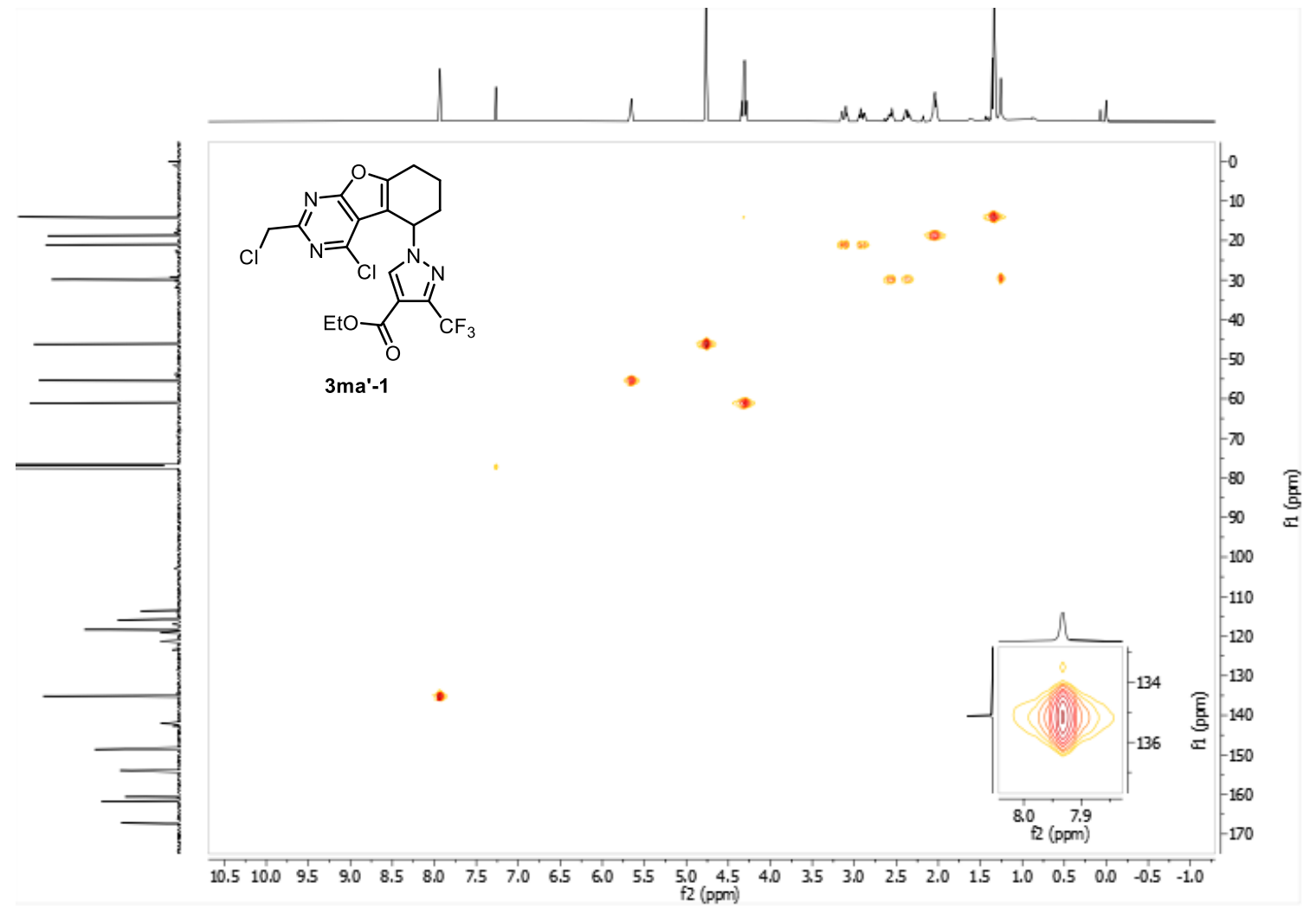

HMBC NMR spectrum of 3ma-1 in $\mathrm{CDCl}_{3}$ containing $0.03 \%(\mathrm{v} / \mathrm{v})$ TMS (500, $\left.126 \mathrm{MHz}\right)$.

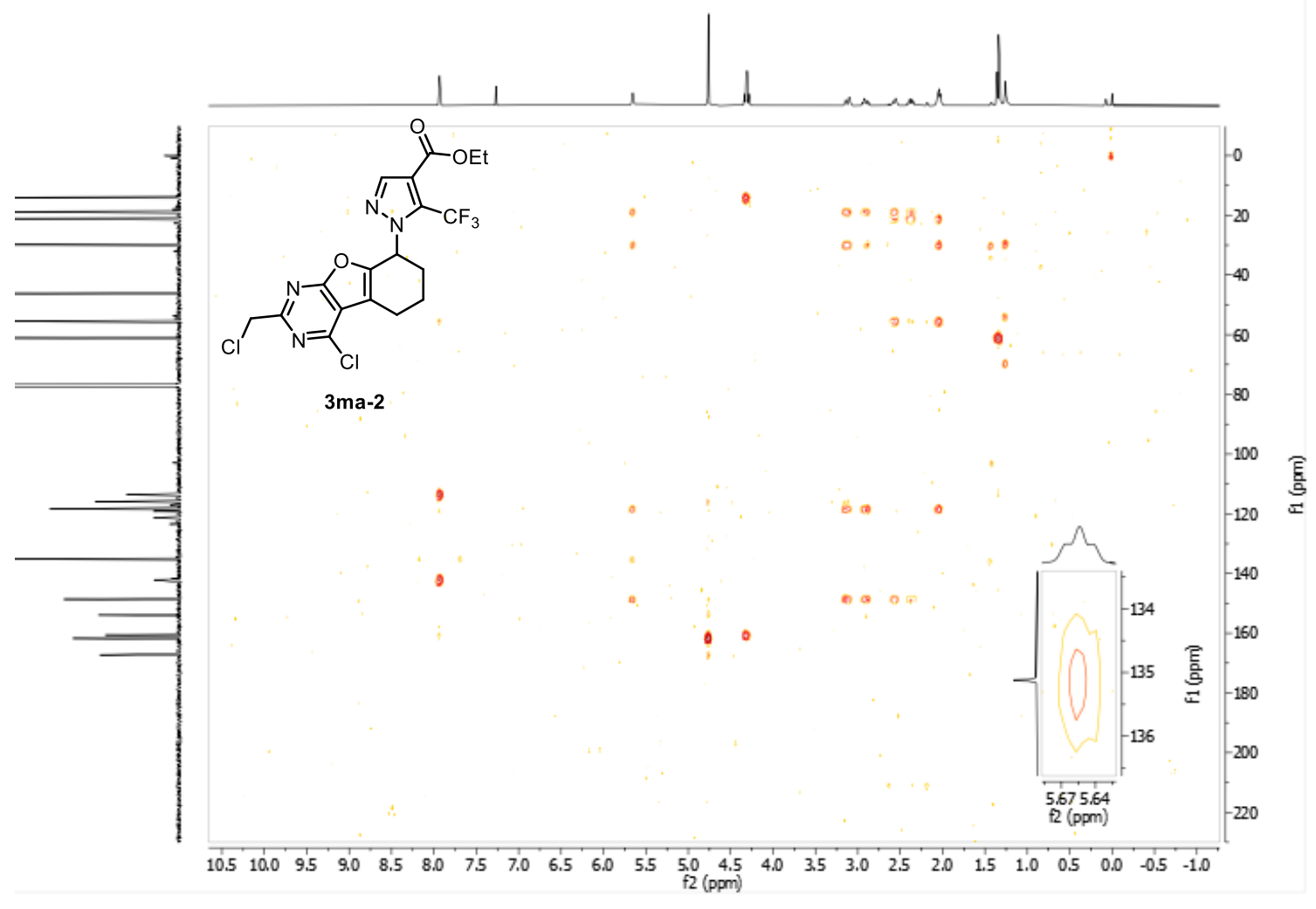


${ }^{1} \mathrm{H}$ NMR spectrum of 3nb-1 in $\mathrm{CDCl}_{3}$ containing $0.03 \%$ (v/v) TMS (500 MHz).
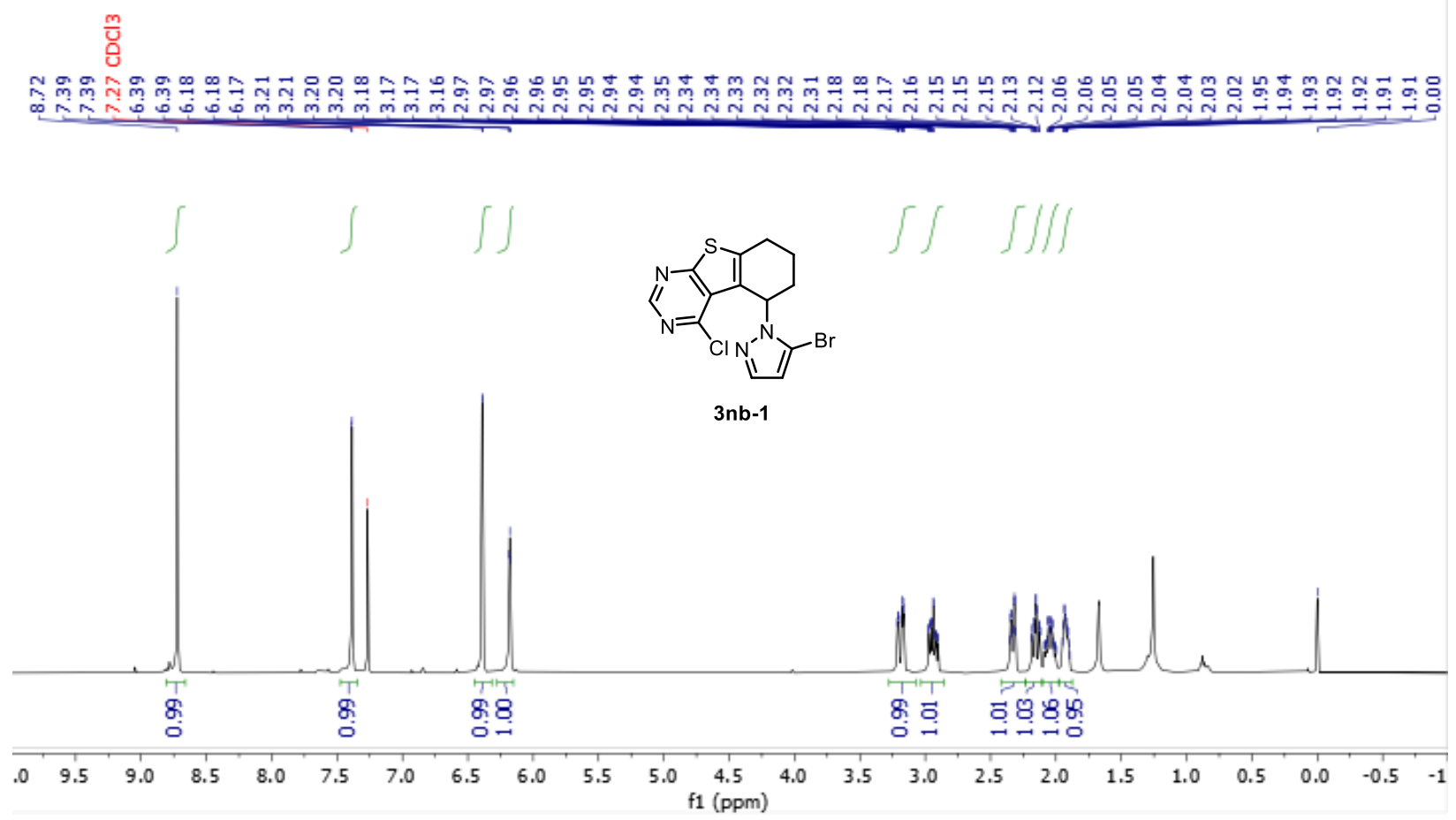

${ }^{13} \mathrm{C}$ NMR spectrum of 3nb-1 in $\mathrm{CDCl}_{3}$ containing $0.03 \%$ (v/v) TMS (126 MHz).
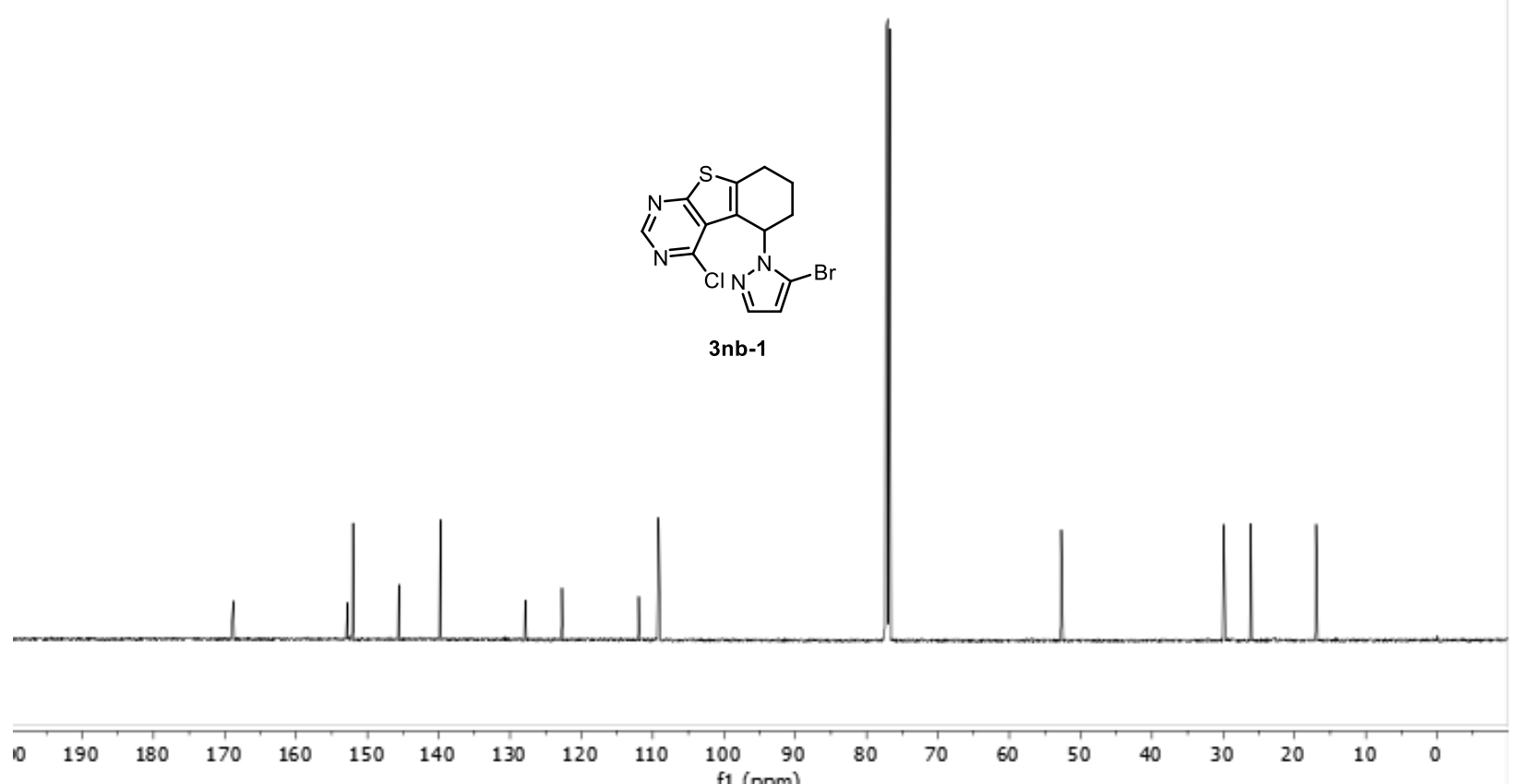
HSQC NMR spectrum of 3nb-1 in $\mathrm{CDCl}_{3}$ containing $0.03 \%$ (v/v) TMS (500, $126 \mathrm{MHz}$ ).

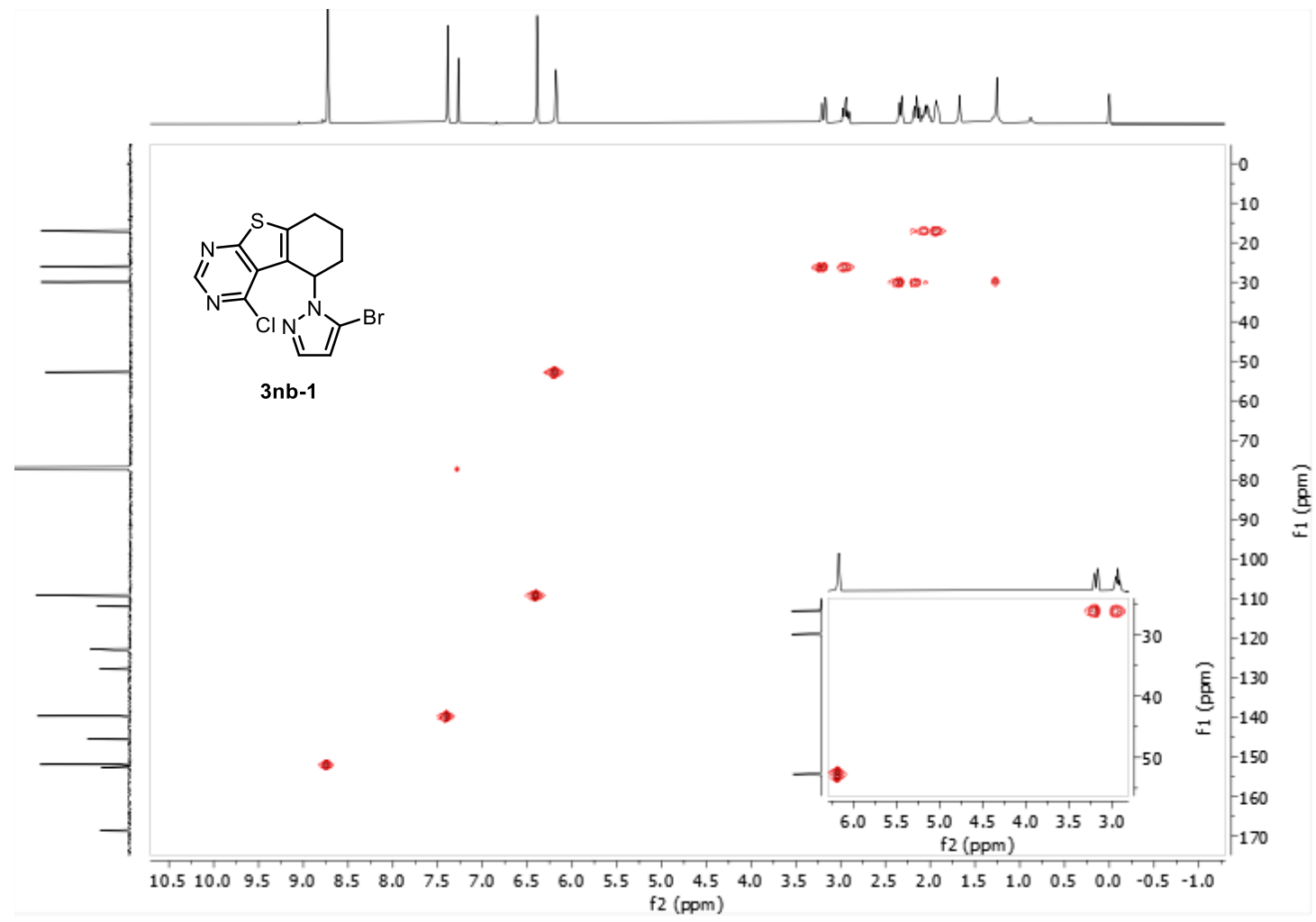

HMBC NMR spectrum of 3nb-1 in $\mathrm{CDCl}_{3}$ containing $0.03 \%$ (v/v) TMS (500, $\left.126 \mathrm{MHz}\right)$.

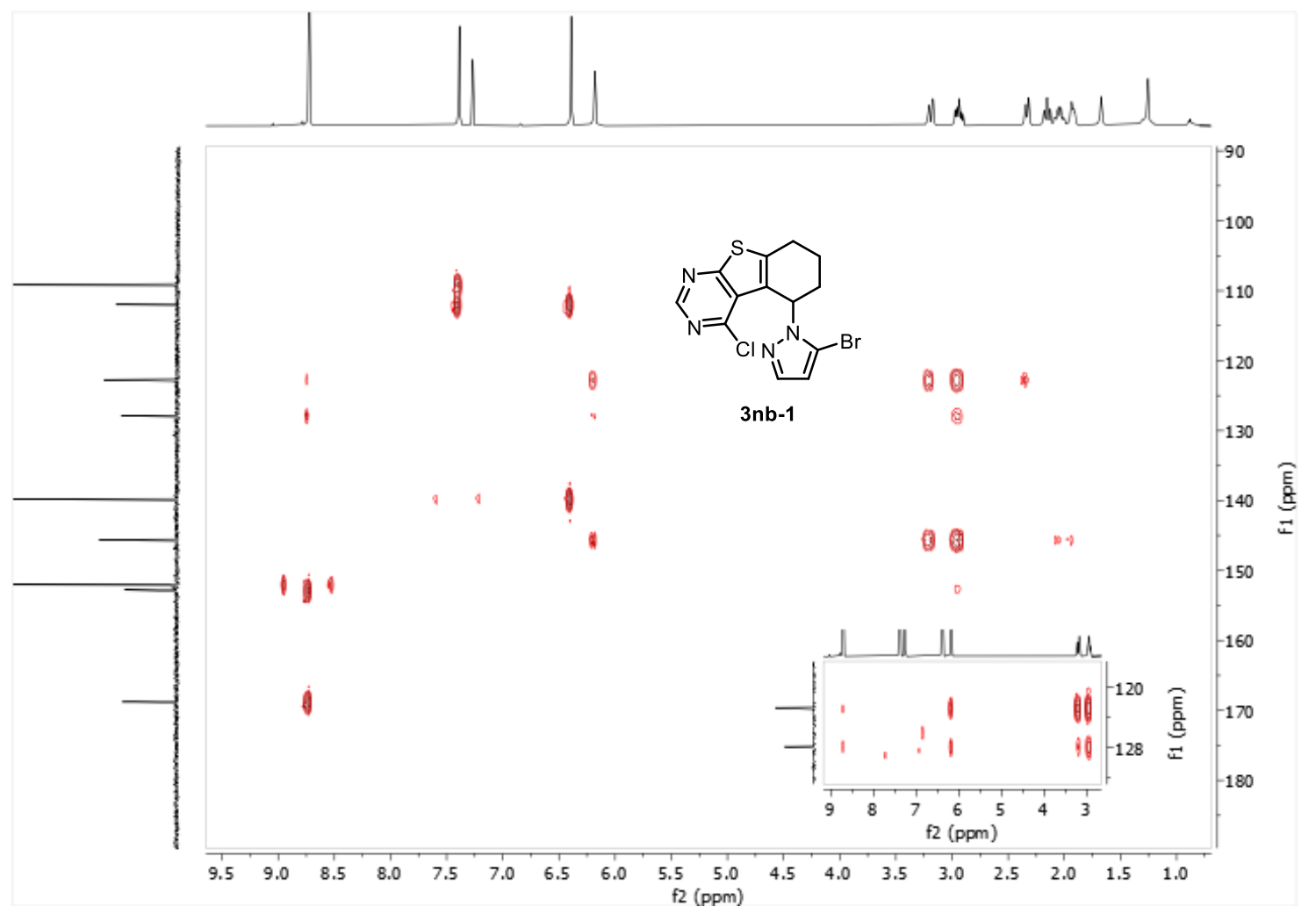


${ }^{1} \mathrm{H}$ NMR spectrum of $\mathbf{3 n b - 2}$ in $\mathrm{CDCl}_{3}$ containing $0.03 \%$ (v/v) TMS (500 MHz).

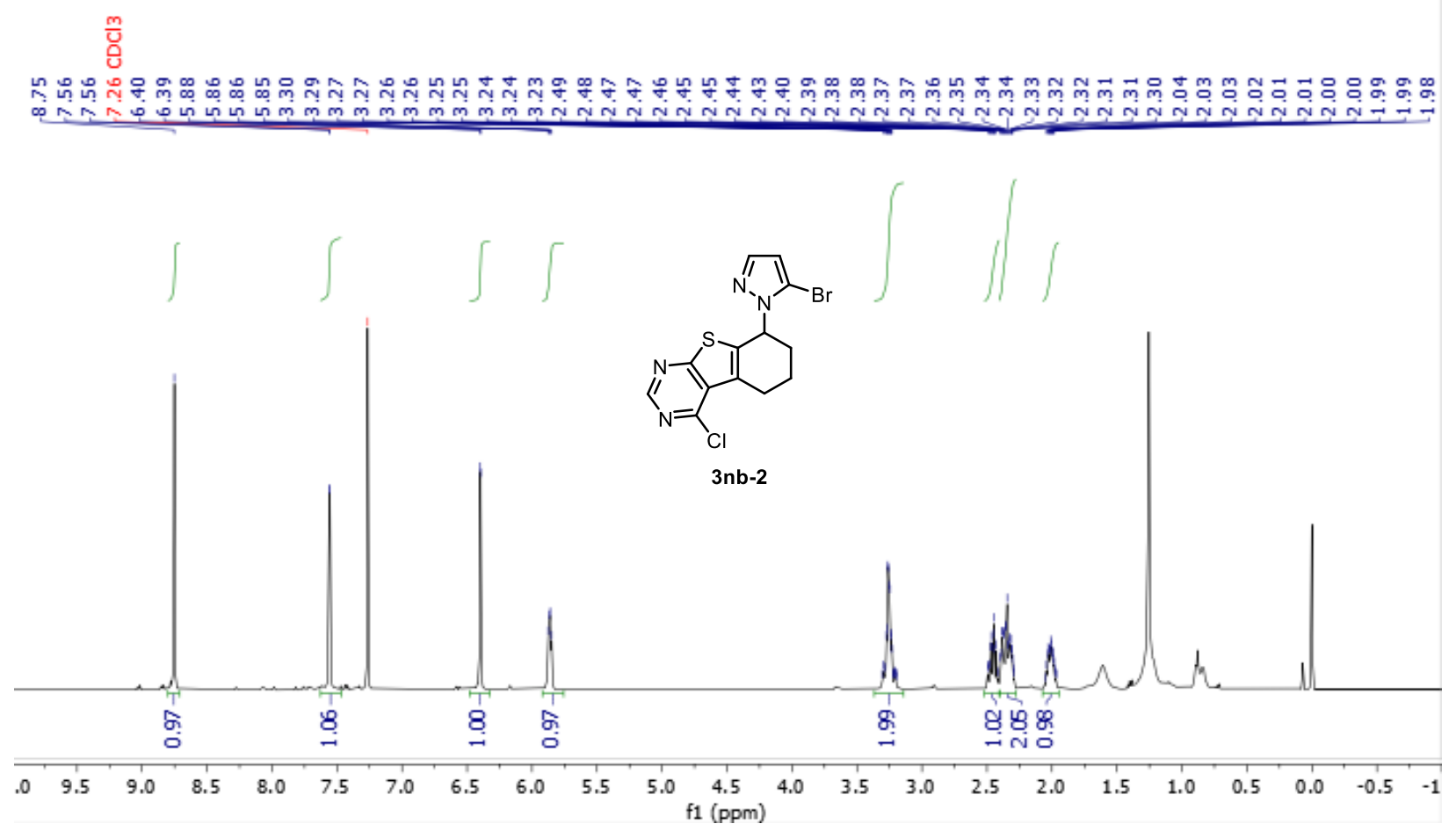

${ }^{13} \mathrm{C}$ NMR spectrum of 3nb-2 in $\mathrm{CDCl}_{3}$ containing $0.03 \%$ (v/v) TMS (126 MHz).

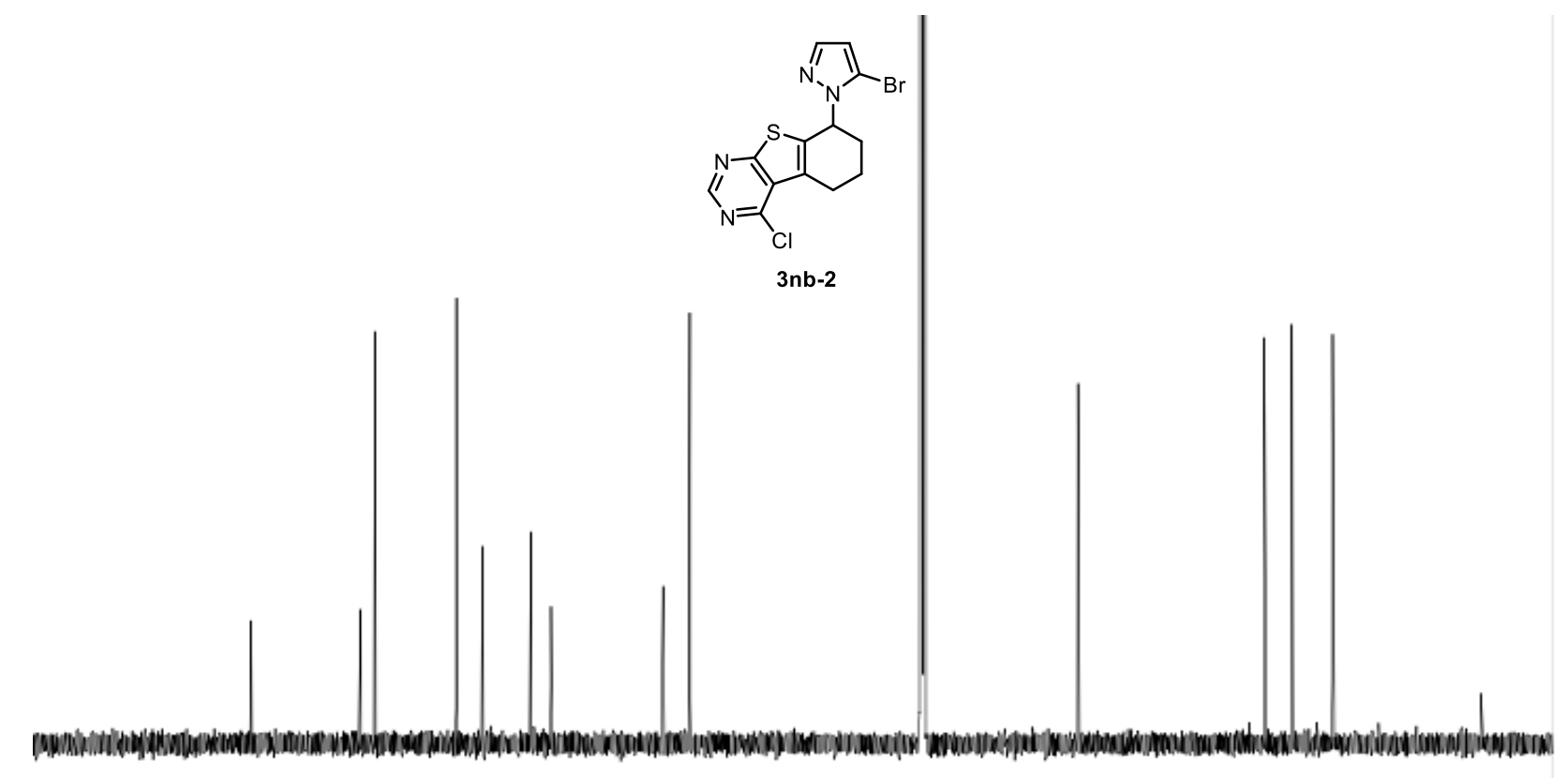

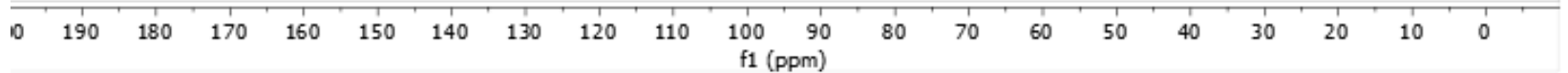


HSQC NMR spectrum of 3nb-2 in $\mathrm{CDCl}_{3}$ containing $0.03 \%(\mathrm{v} / \mathrm{v})$ TMS (500, $126 \mathrm{MHz}$ ).

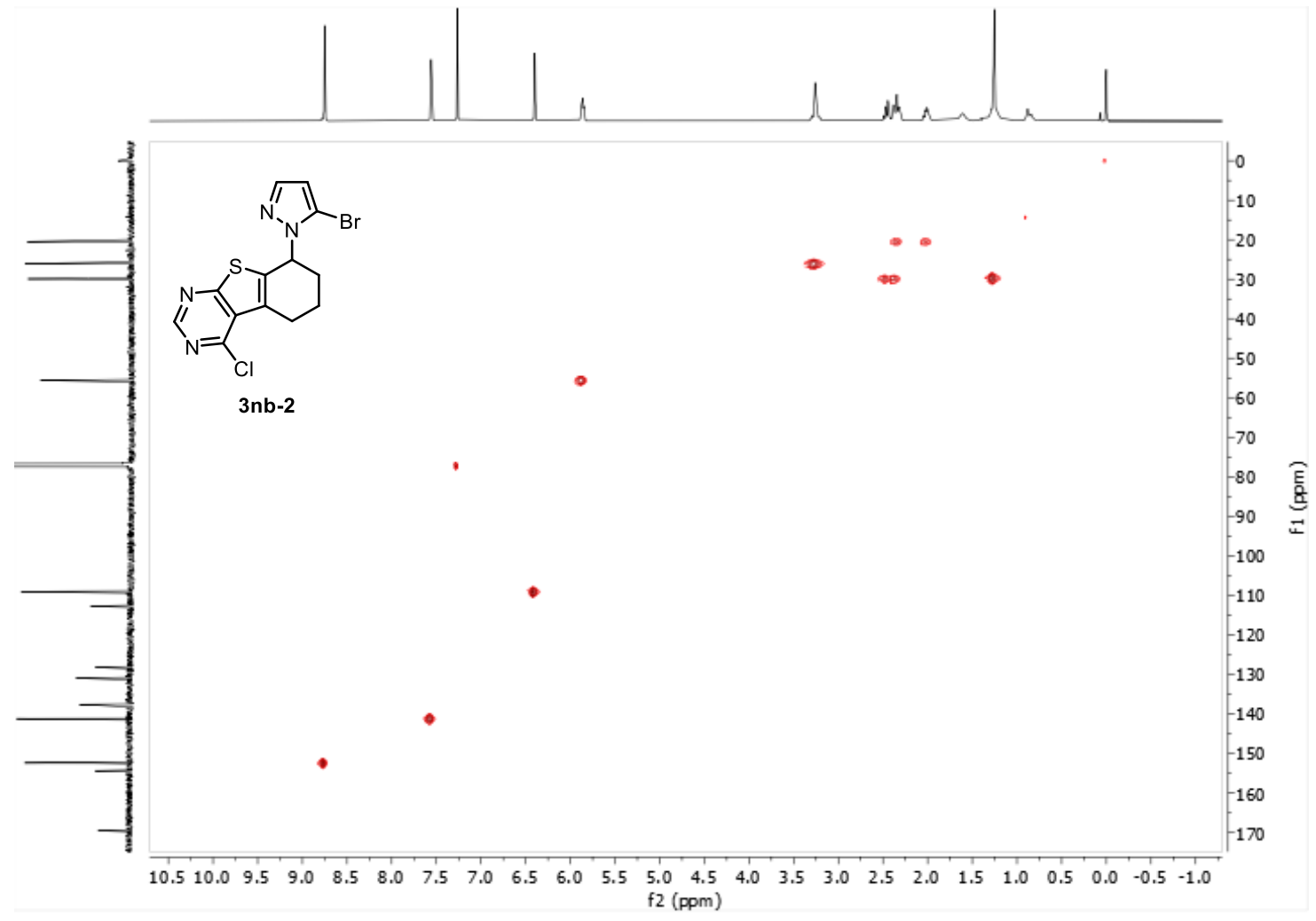

HMBC NMR spectrum of 3nb-2 in $\mathrm{CDCl}_{3}$ containing $0.03 \%$ (v/v) TMS (500, $\left.126 \mathrm{MHz}\right)$.

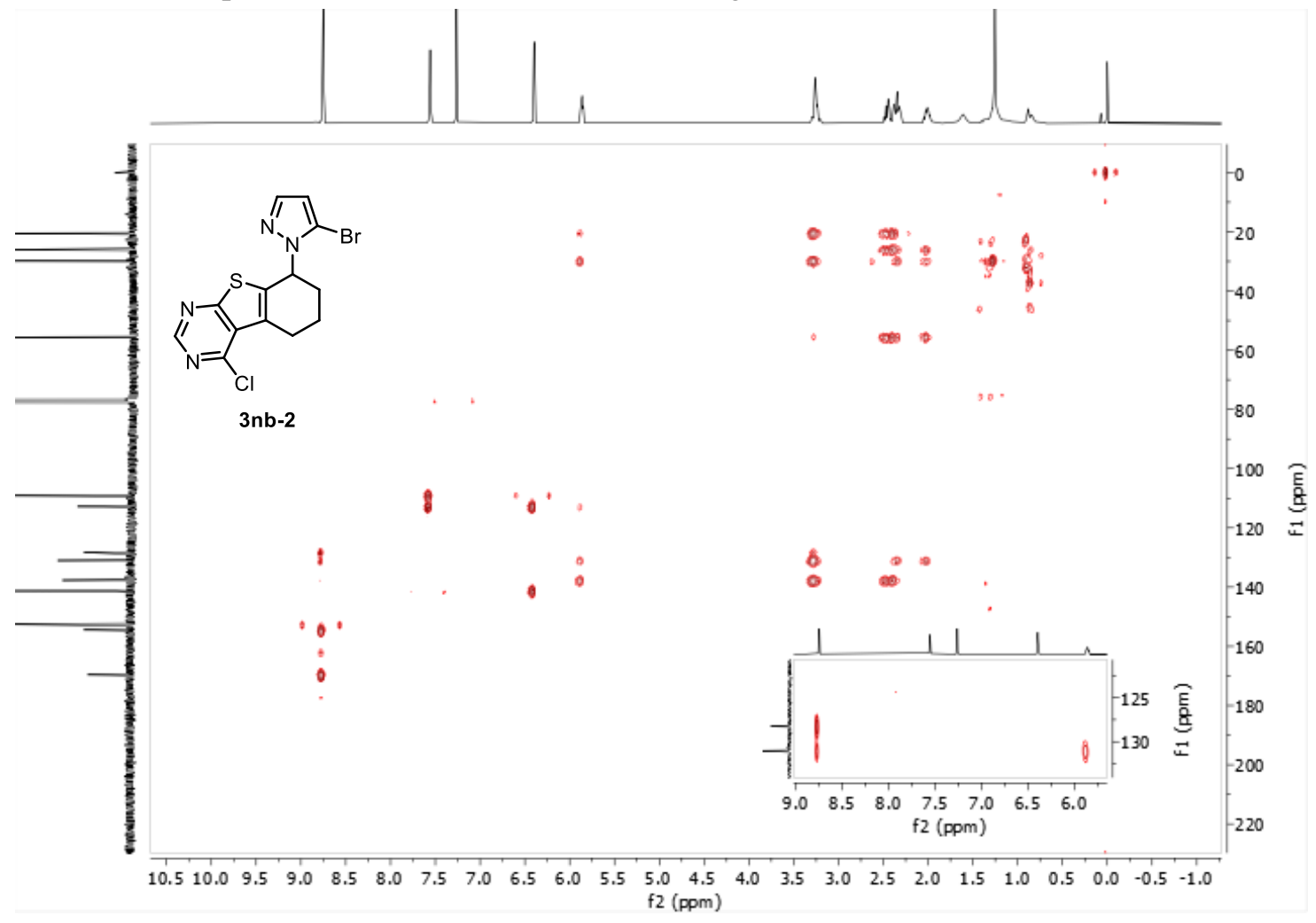


${ }^{1} \mathrm{H}$ NMR spectrum of 3oa-1 in $\mathrm{CDCl}_{3}$ containing $0.03 \%$ (v/v) TMS (500 MHz).

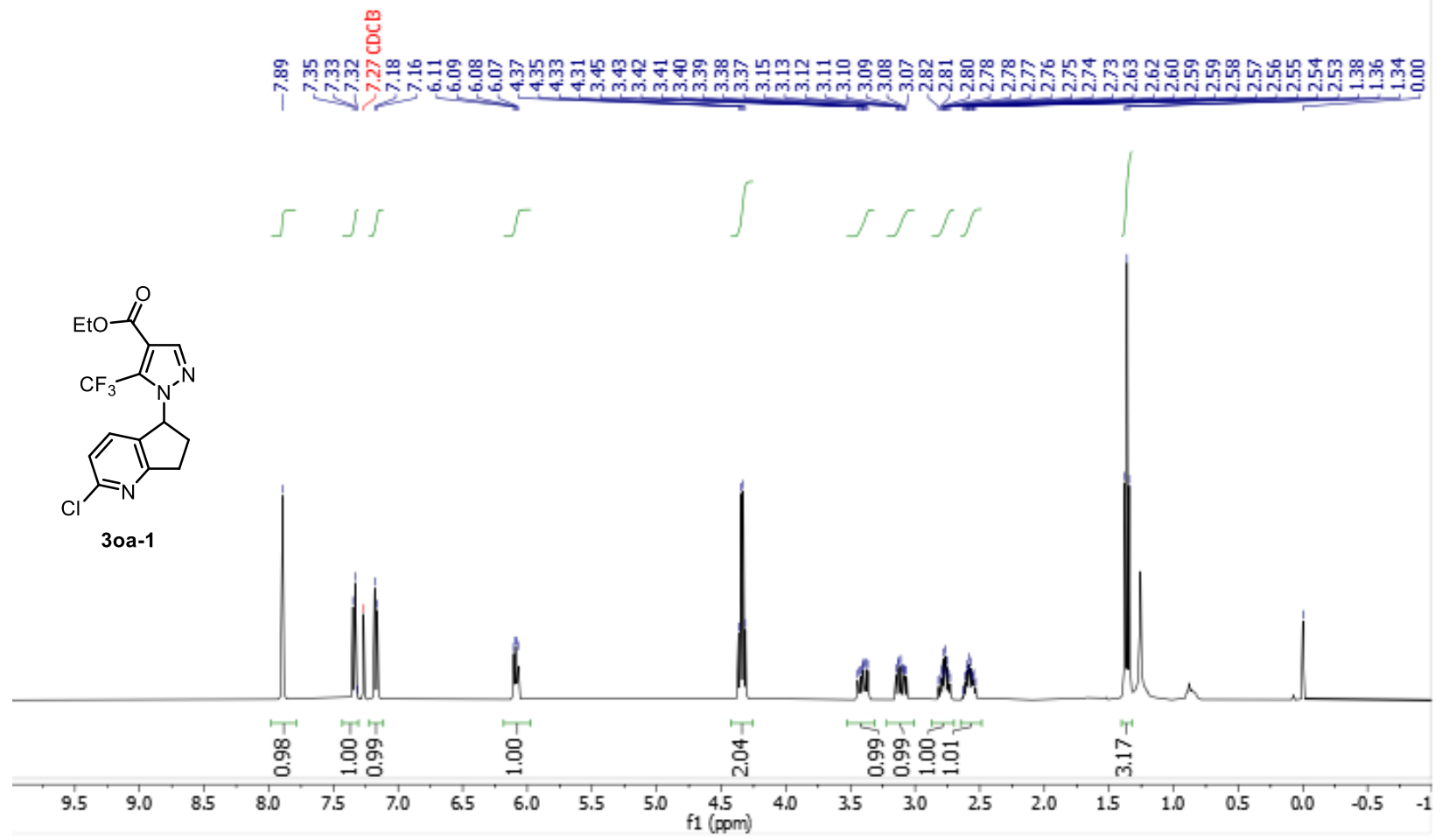

${ }^{13} \mathrm{C}$ NMR spectrum of 3oa-1 in $\mathrm{CDCl}_{3}$ containing $0.03 \%$ (v/v) TMS (126 MHz).

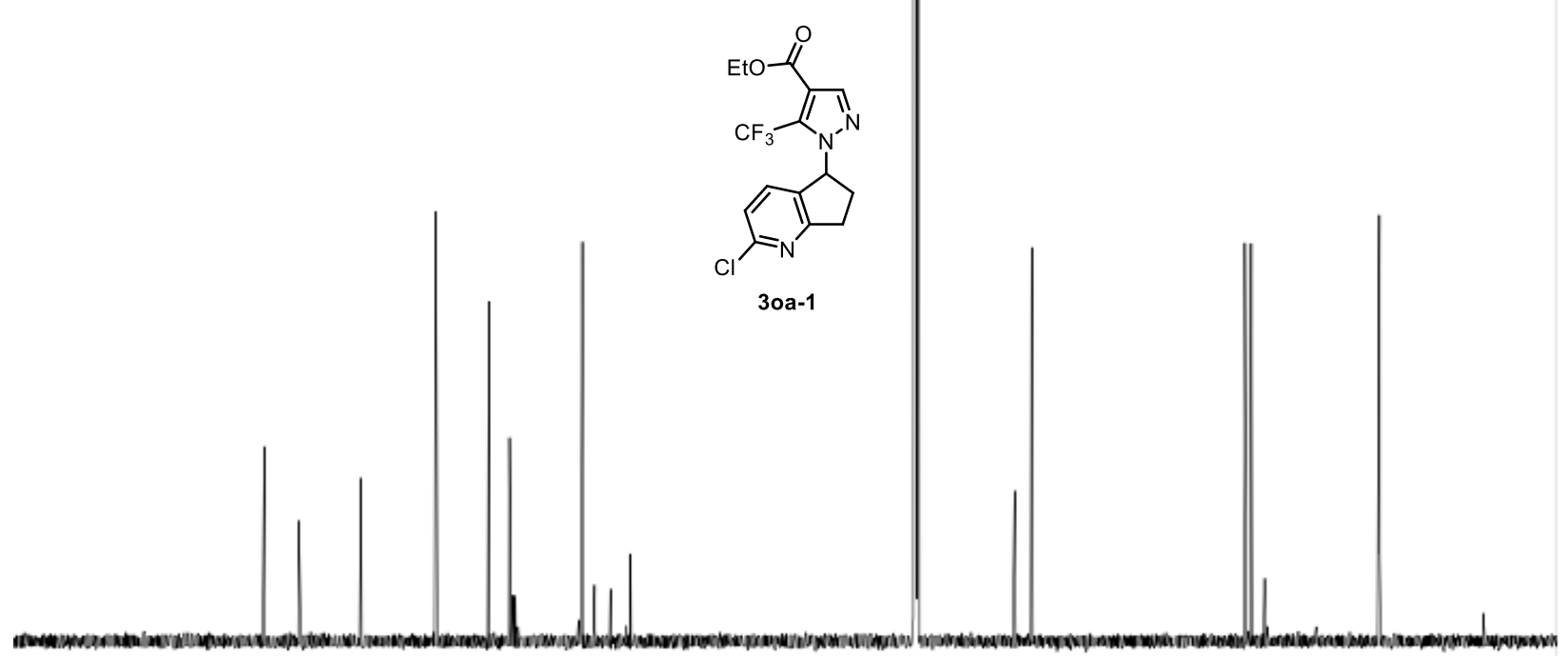


${ }^{19} \mathrm{~F}$ NMR spectrum of 3oa-1 in $\mathrm{CDCl}_{3}$ containing $0.03 \%$ (v/v) TMS (377 MHz).
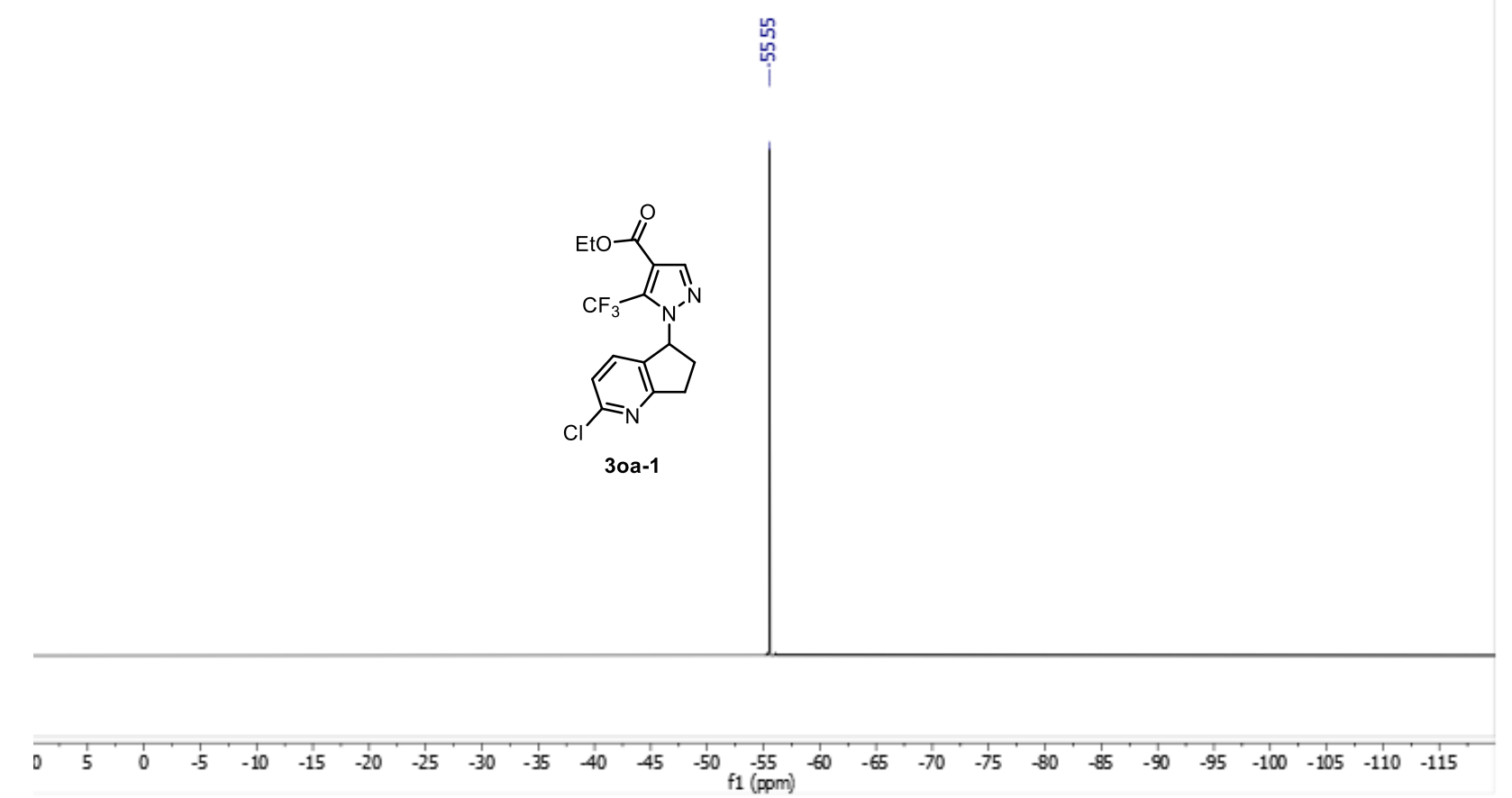

HSQC NMR spectrum of 3oa-1 in $\mathrm{CDCl}_{3}$ containing $0.03 \%$ (v/v) TMS (500, $126 \mathrm{MHz}$ ).

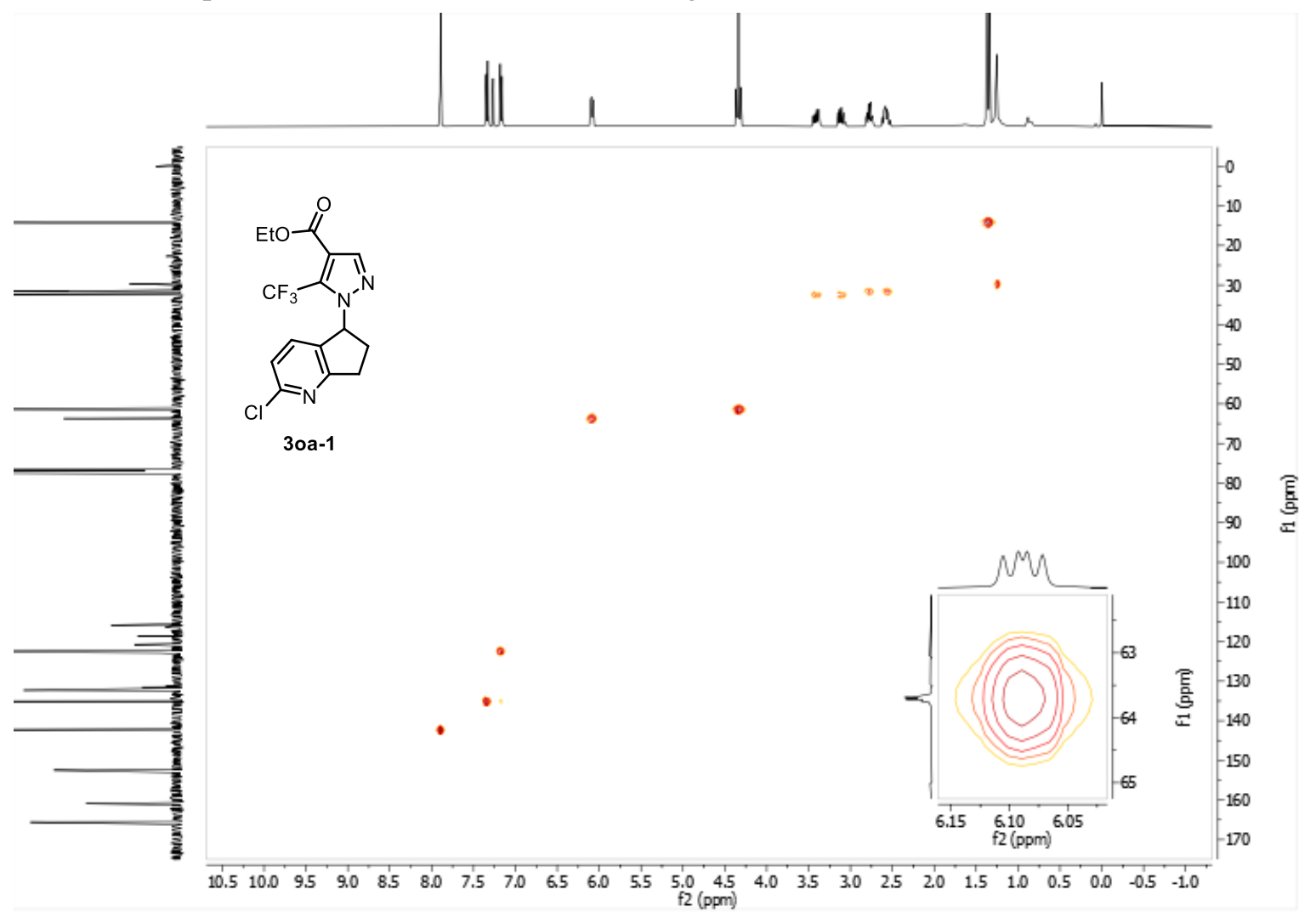


HMBC NMR spectrum of 3oa-1 in $\mathrm{CDCl}_{3}$ containing $0.03 \%(\mathrm{v} / \mathrm{v})$ TMS $(500,126 \mathrm{MHz})$.

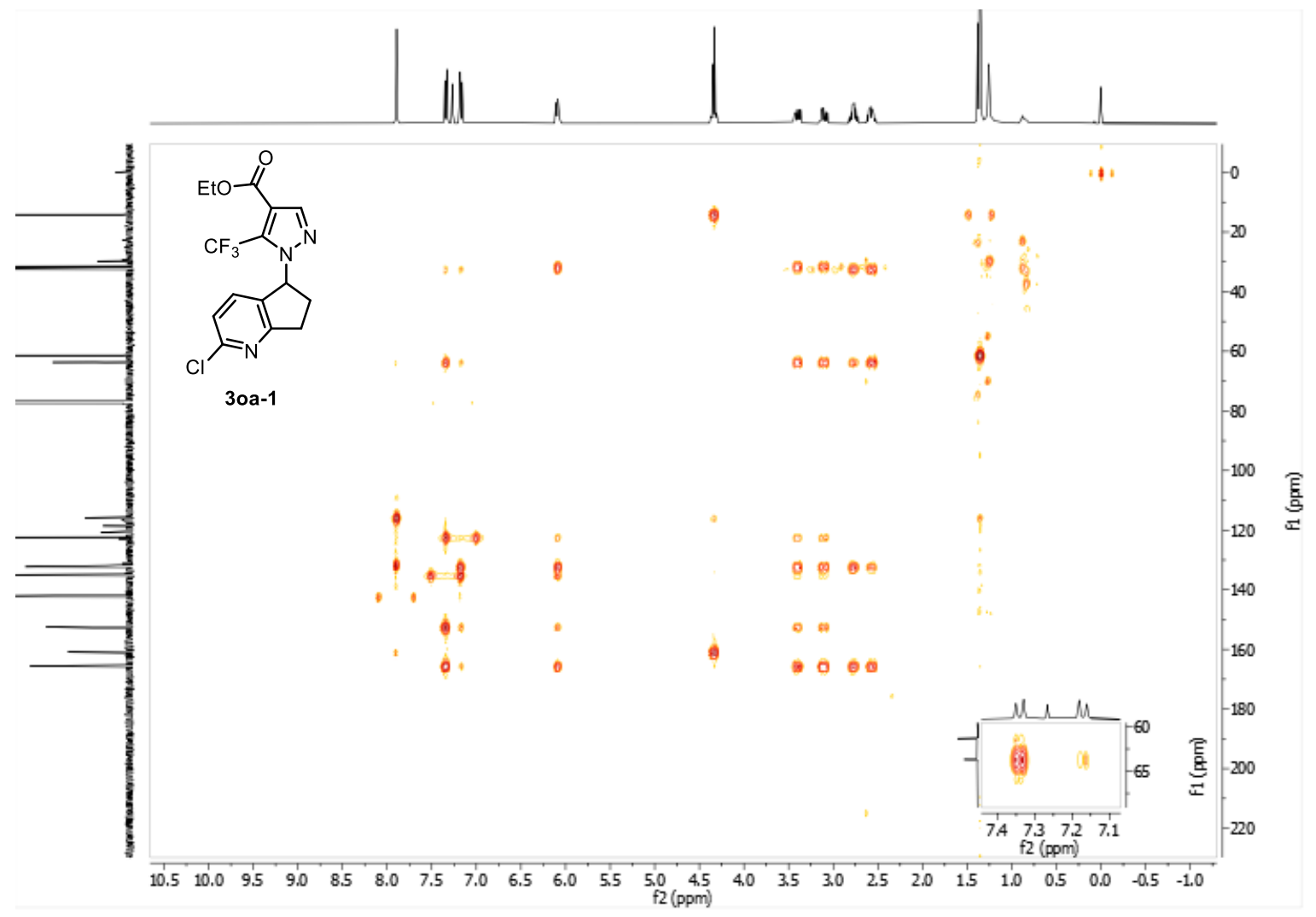

${ }^{1} \mathrm{H}$ NMR spectrum of $\mathbf{3 o a}-2$ in $\mathrm{CDCl}_{3}$ containing $0.03 \%$ (v/v) TMS (500 MHz).

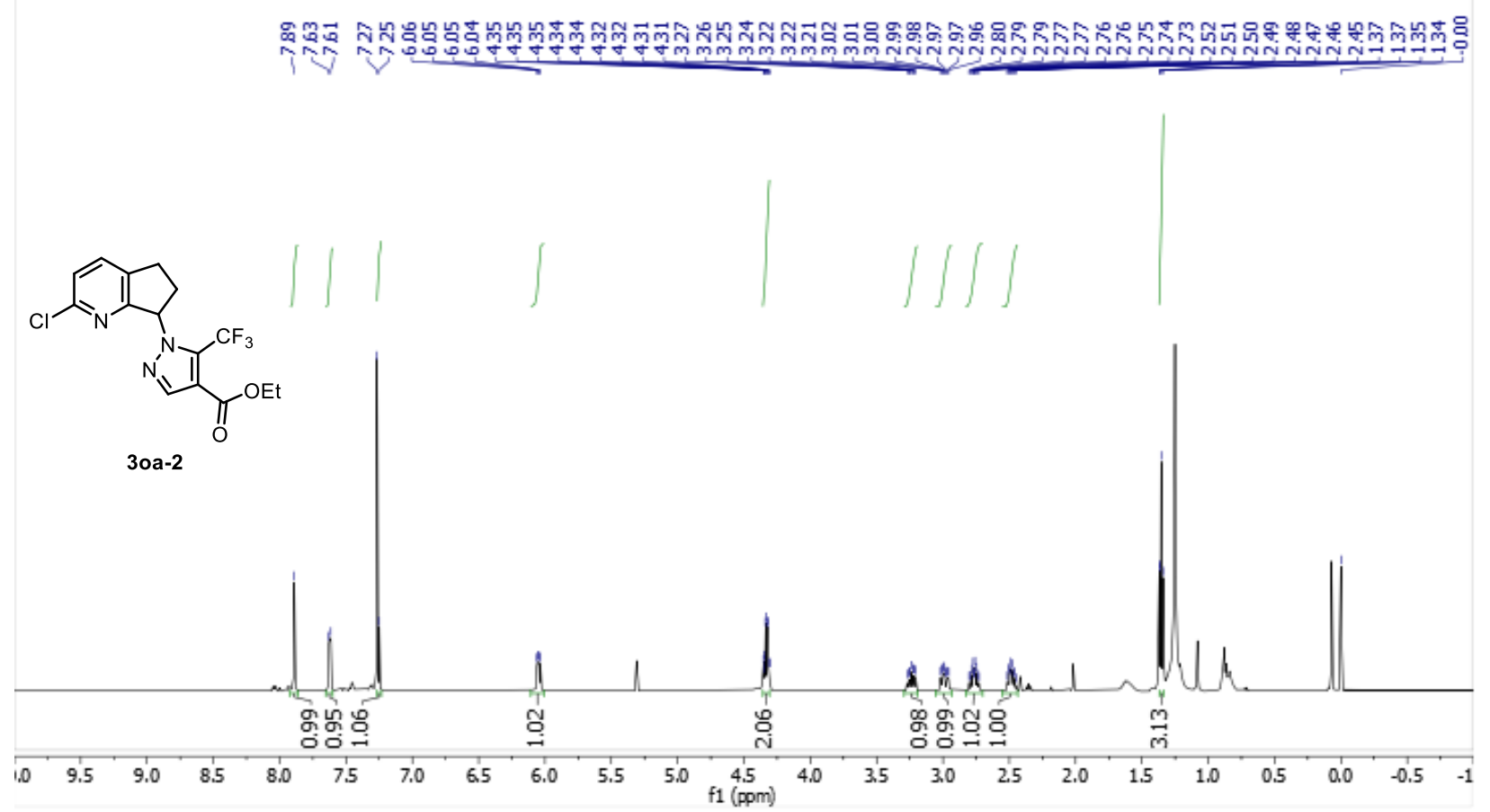


${ }^{13} \mathrm{C}$ NMR spectrum of 3oa-2 in $\mathrm{CDCl}_{3}$ containing $0.03 \%(\mathrm{v} / \mathrm{v})$ TMS (126 MHz).

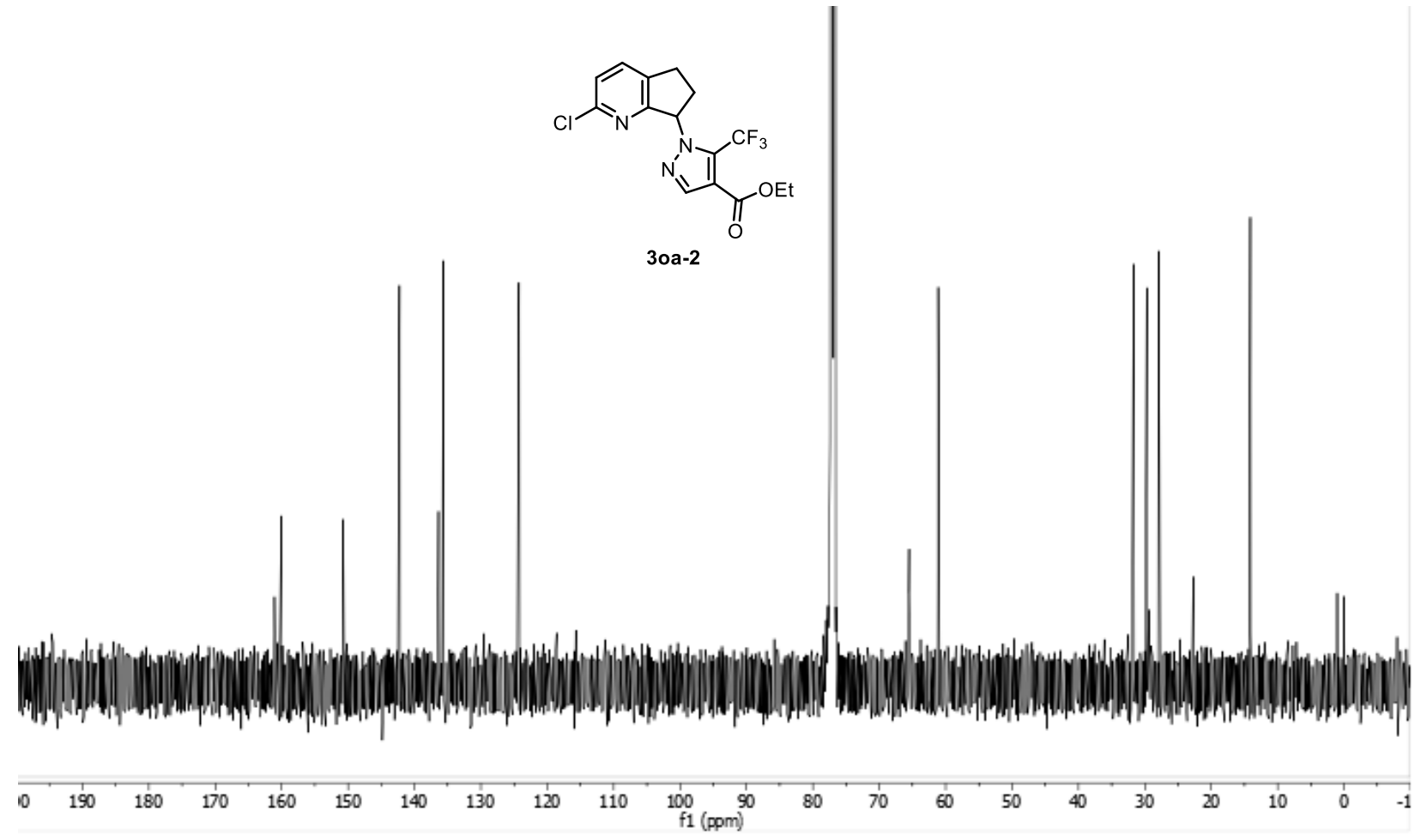

${ }^{19} \mathrm{~F}$ NMR spectrum of $\mathbf{3 o a}-2$ in $\mathrm{CDCl}_{3}$ containing $0.03 \%$ (v/v) TMS (377 MHz).

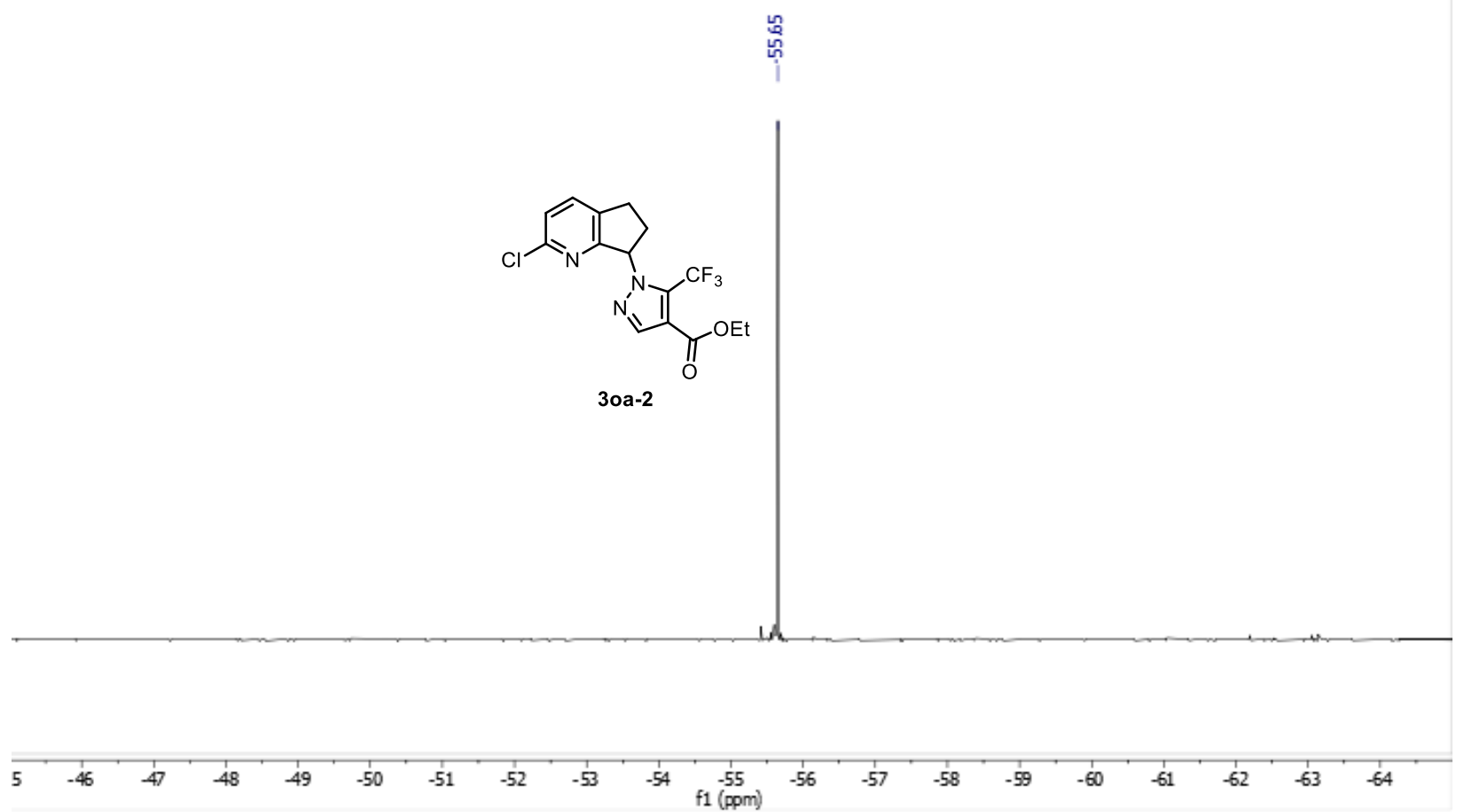


HSQC NMR spectrum of 3oa-2 in $\mathrm{CDCl}_{3}$ containing $0.03 \%$ (v/v) TMS (500, $126 \mathrm{MHz}$ ).

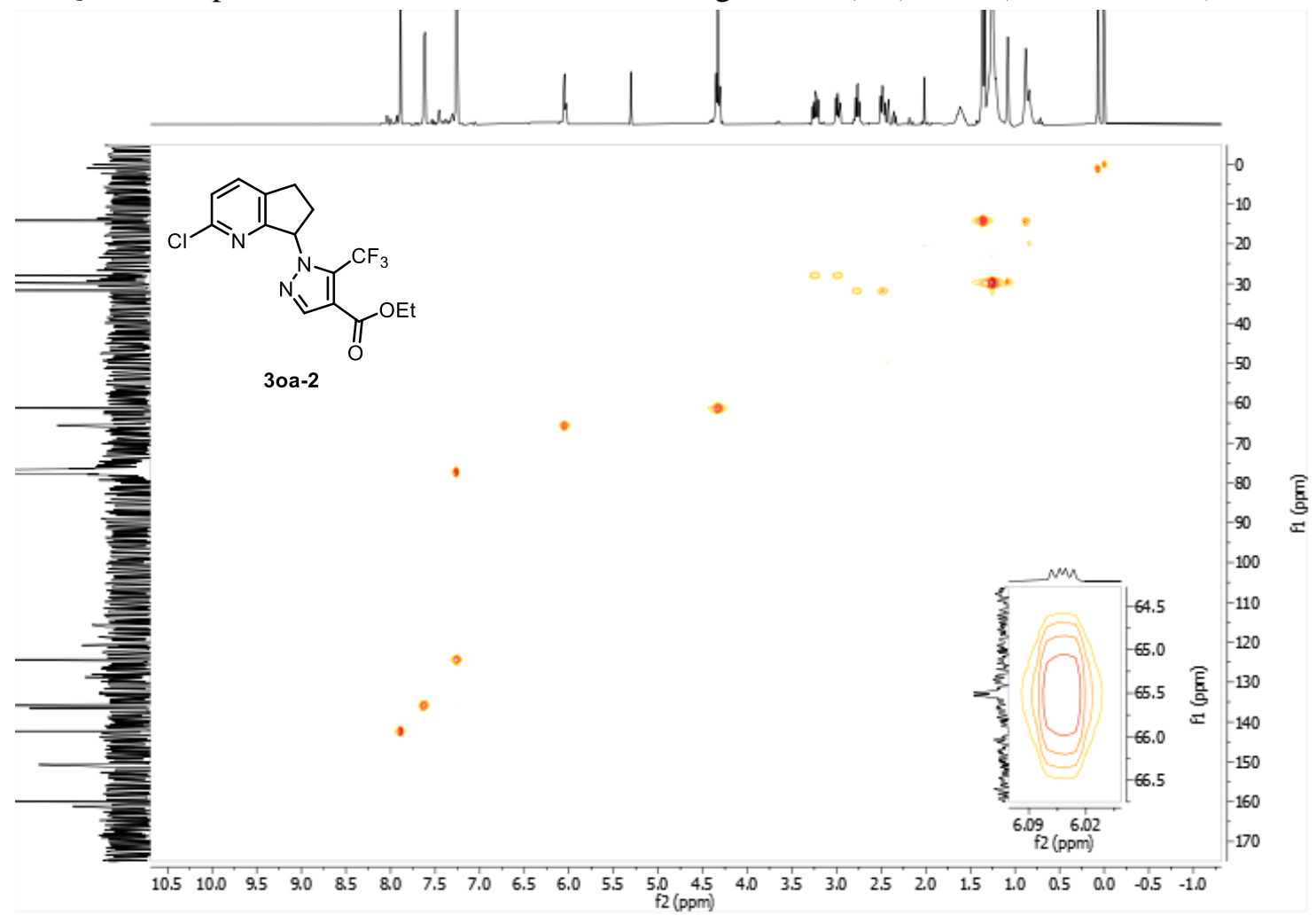

HMBC NMR spectrum of 3oa-2 in $\mathrm{CDCl}_{3}$ containing $0.03 \%$ (v/v) TMS (500, $\left.126 \mathrm{MHz}\right)$.

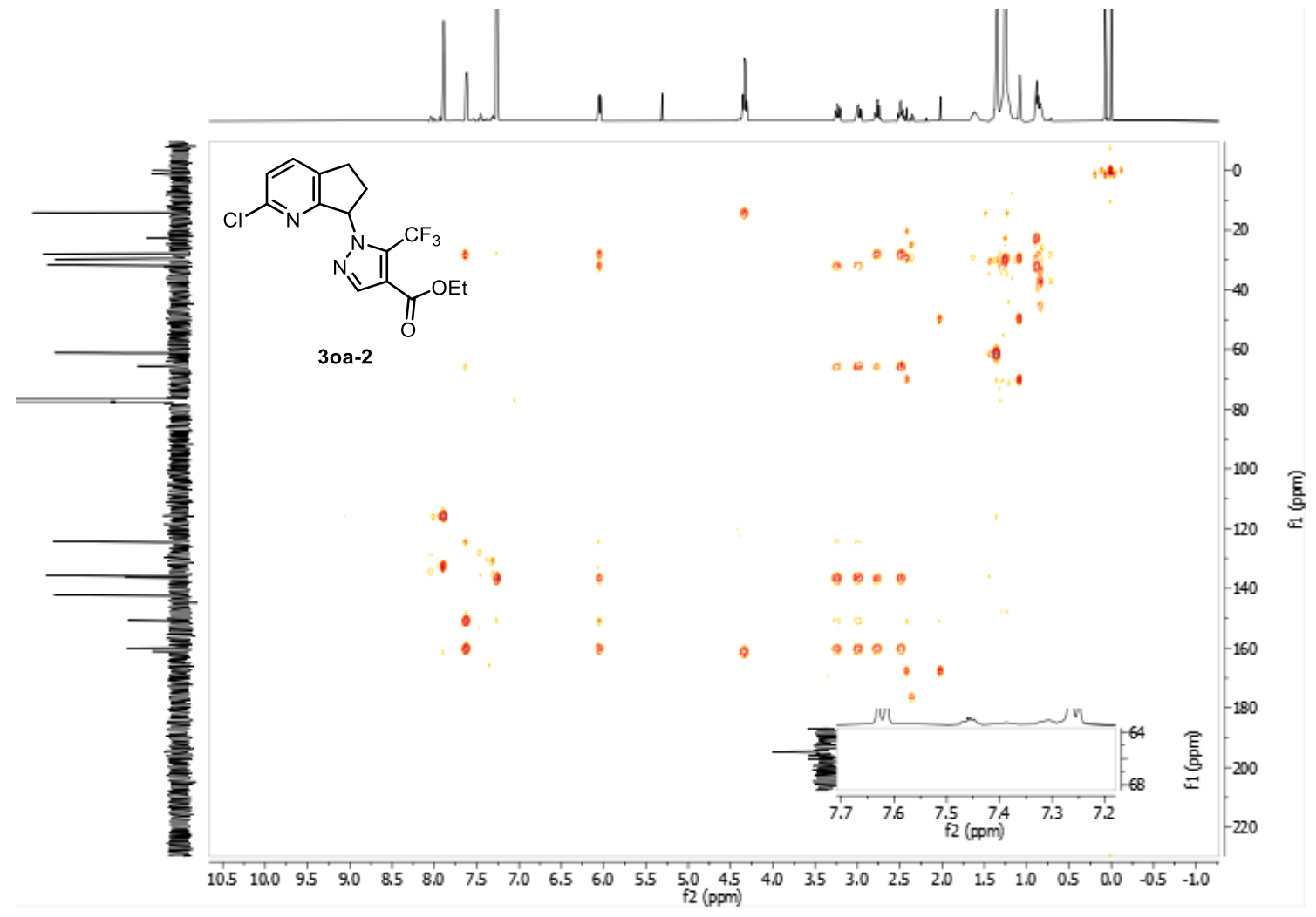


${ }^{1} \mathrm{H}$ NMR spectrum of $\mathbf{3 p a}$ ' in $\mathrm{CDCl}_{3}$ containing $0.03 \%$ (v/v) TMS (500 MHz).

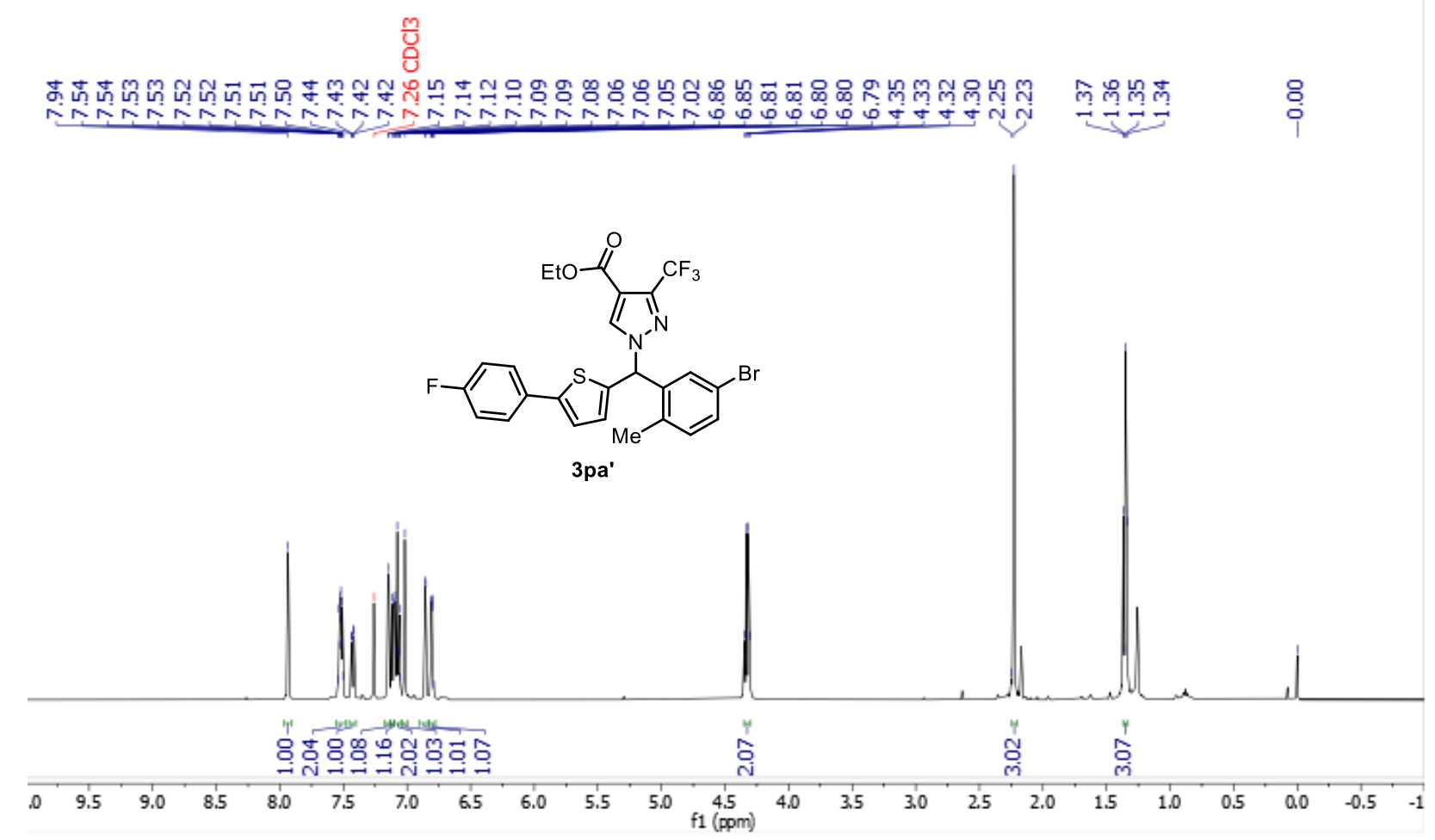

${ }^{13} \mathrm{C}$ NMR spectrum of 3pa' in $\mathrm{CDCl}_{3}$ containing $0.03 \%$ (v/v) TMS (126 MHz).

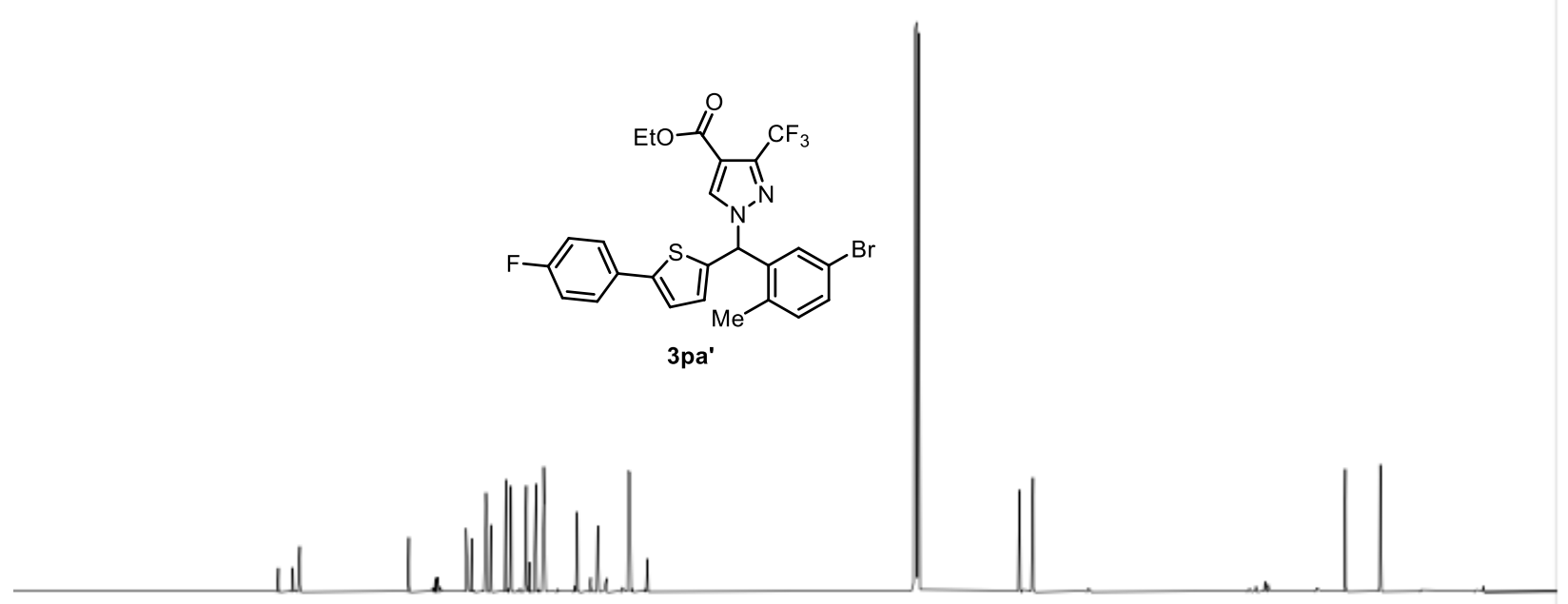


${ }^{19} \mathrm{~F}$ NMR spectrum of $\mathbf{3 p a}$ in $\mathrm{CDCl}_{3}$ containing $0.03 \%$ (v/v) TMS (377 MHz).

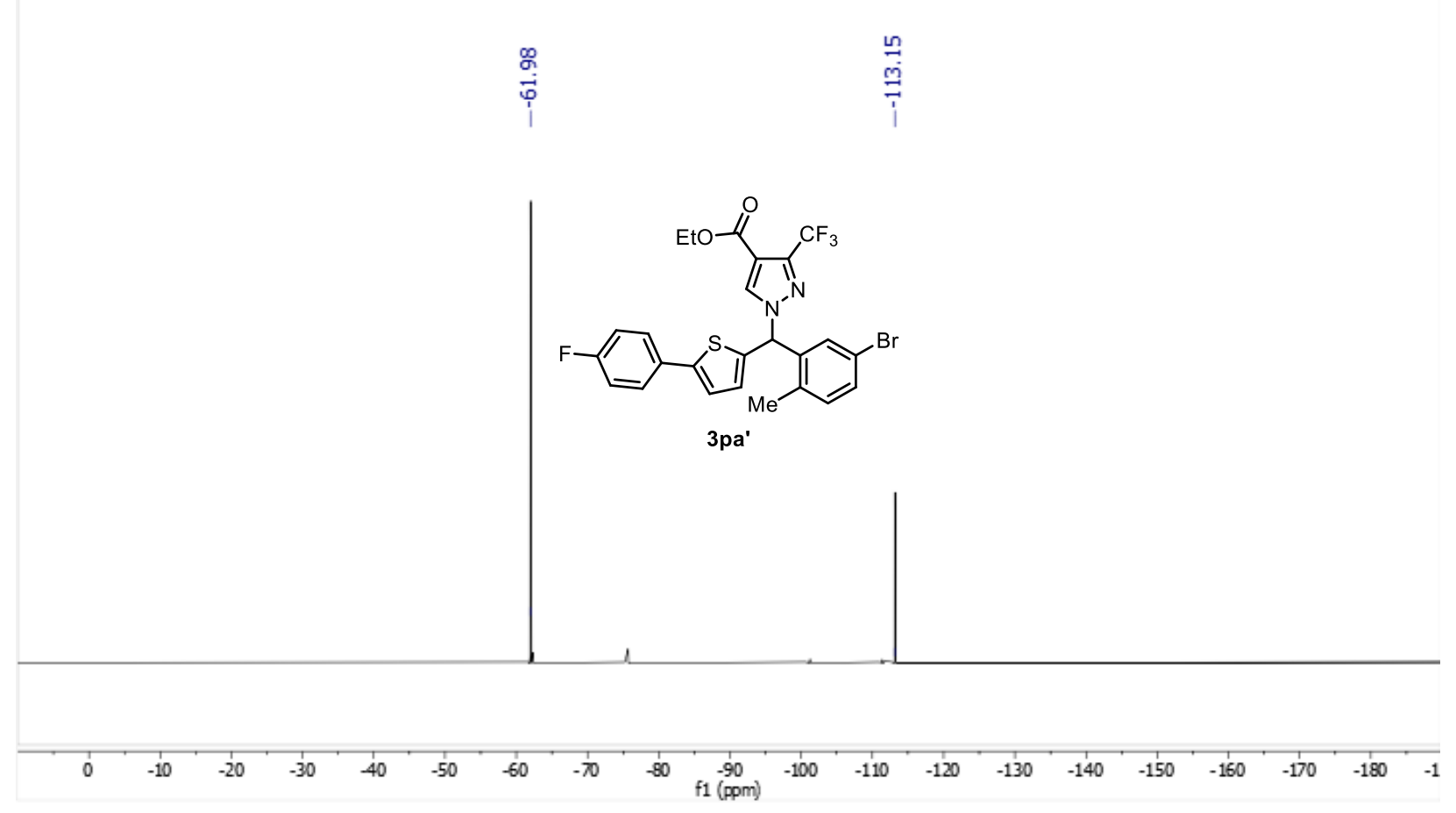

HSQC NMR spectrum of 3pa' in $\mathrm{CDCl}_{3}$ containing $0.03 \%$ (v/v) TMS (500, $126 \mathrm{MHz}$ ).

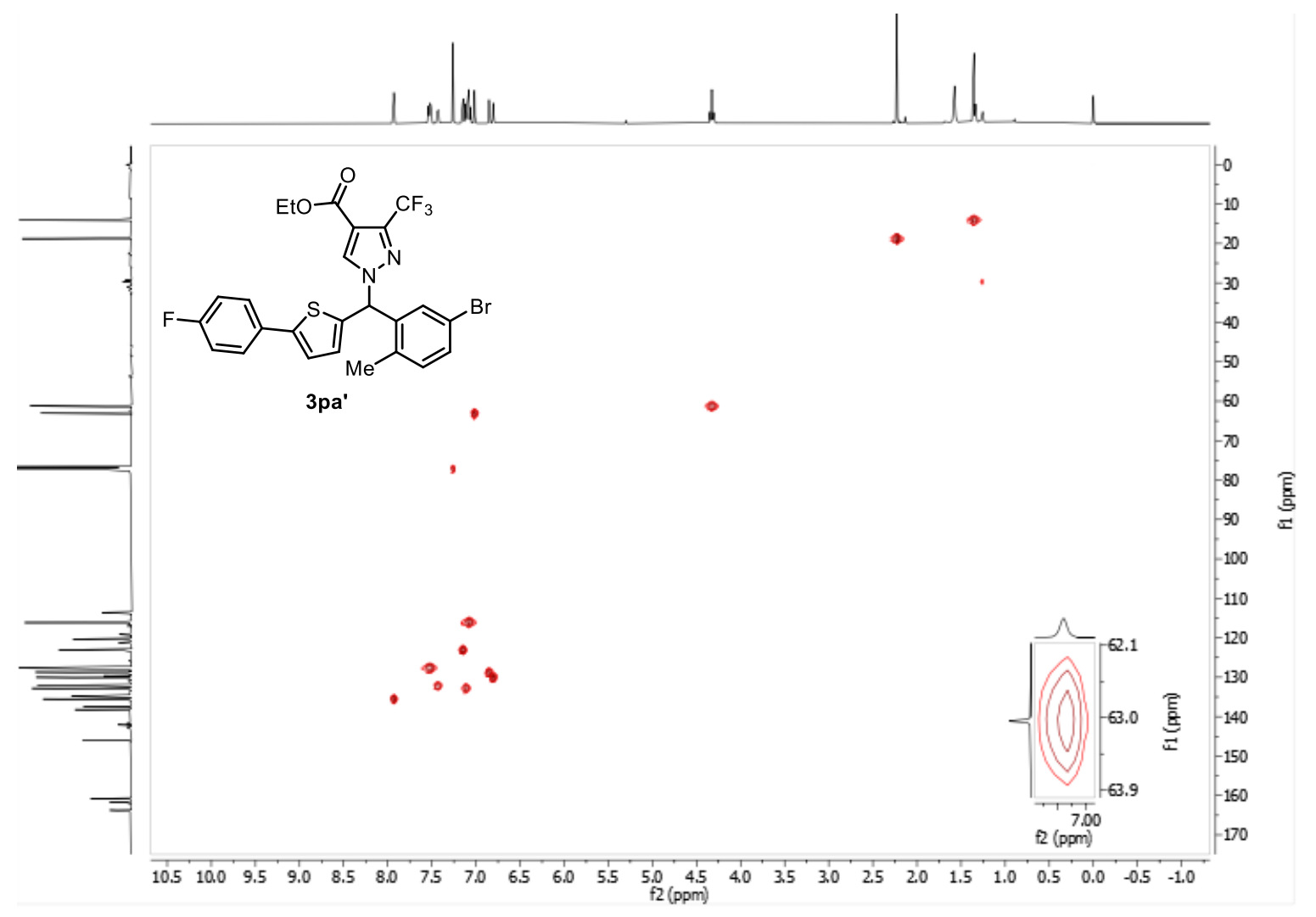


HMBC NMR spectrum of 3pa' in $\mathrm{CDCl}_{3}$ containing $0.03 \%$ (v/v) TMS (500, $126 \mathrm{MHz}$ ).

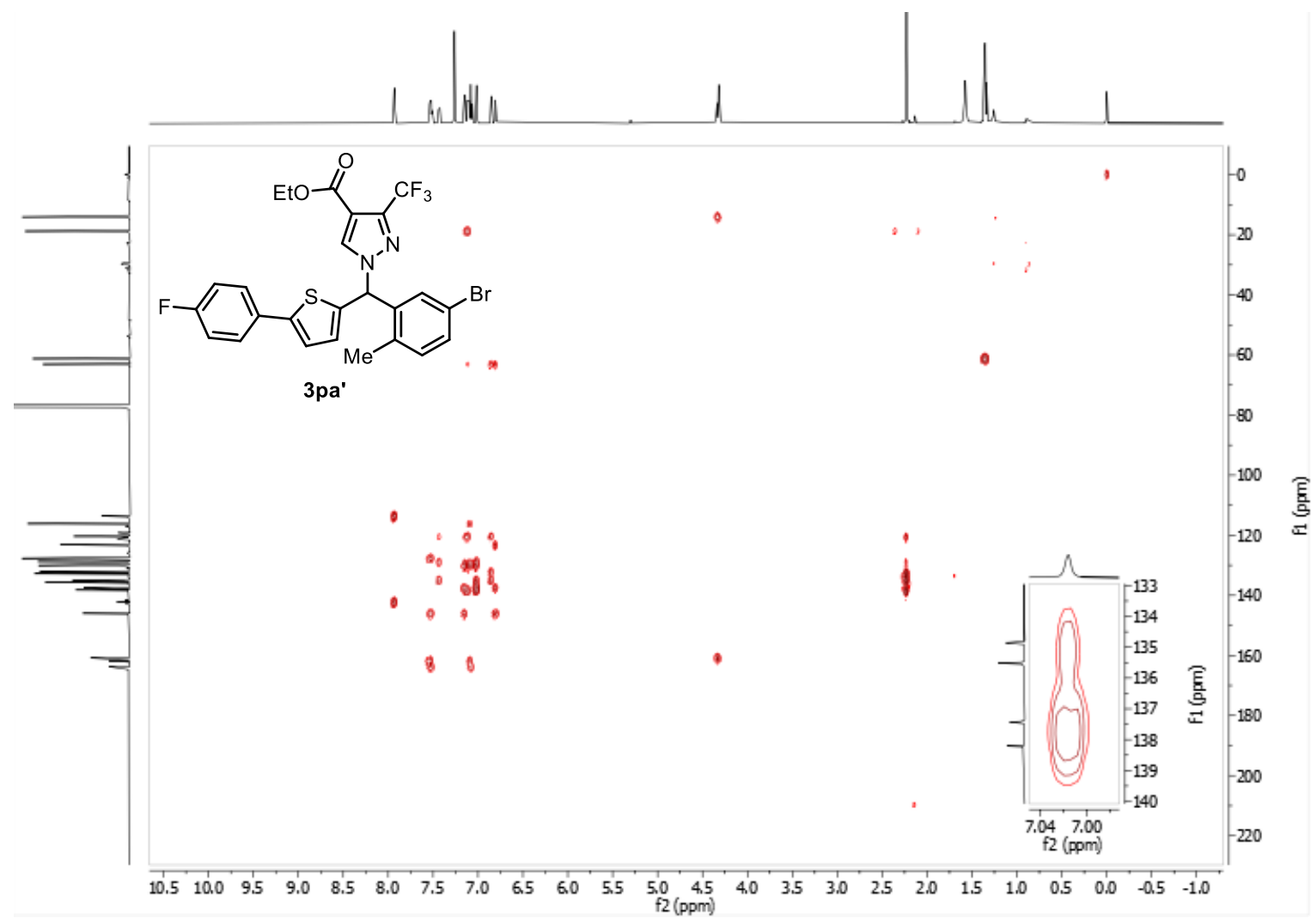

${ }^{1} \mathrm{H}$ NMR spectrum of 3qa in $\mathrm{CDCl}_{3}$ containing $0.03 \%$ (v/v) TMS (500 MHz).

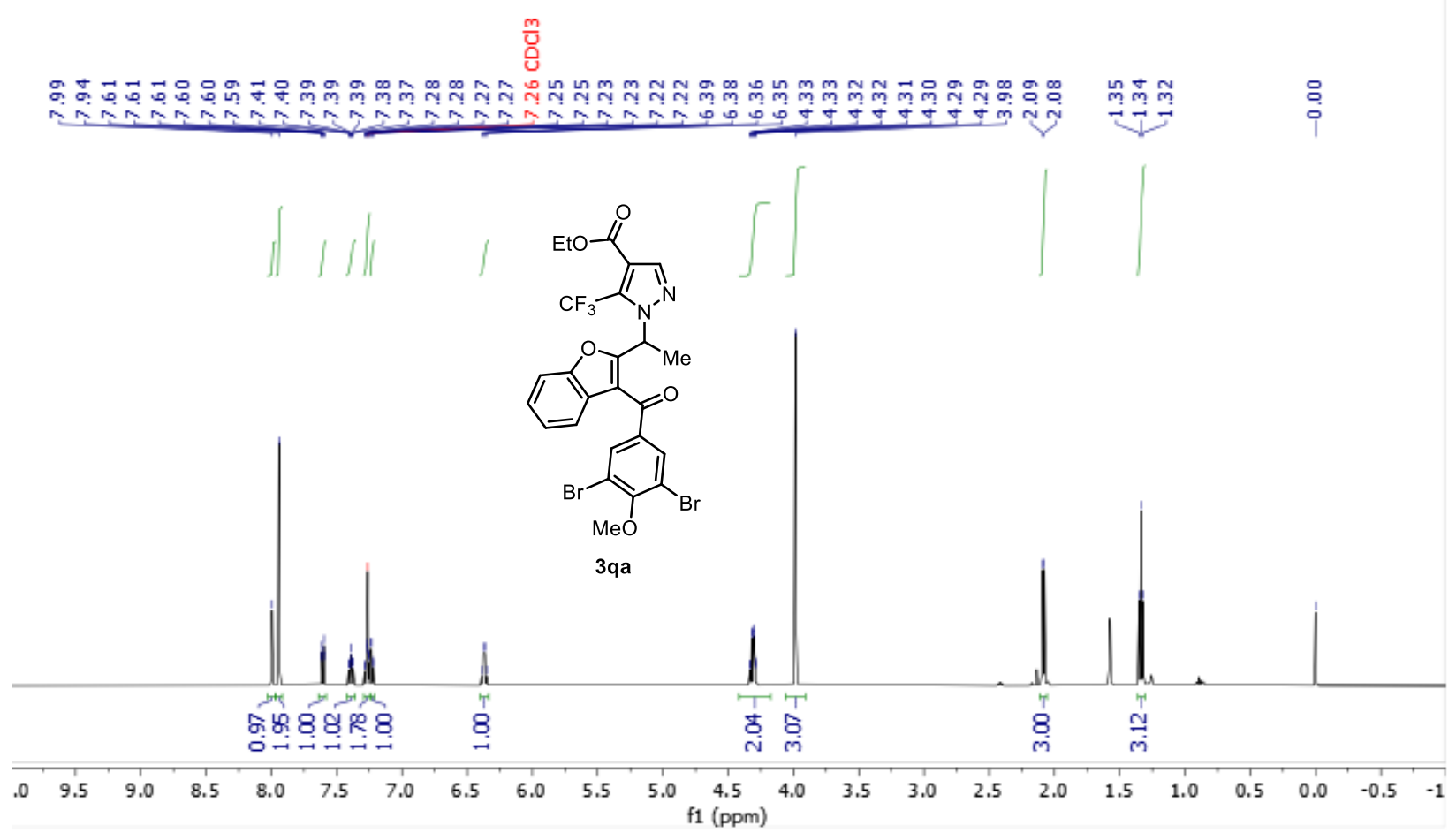


${ }^{13} \mathrm{C}$ NMR spectrum of $\mathbf{3 q a}$ in $\mathrm{CDCl}_{3}$ containing $0.03 \%$ (v/v) TMS (126 MHz).
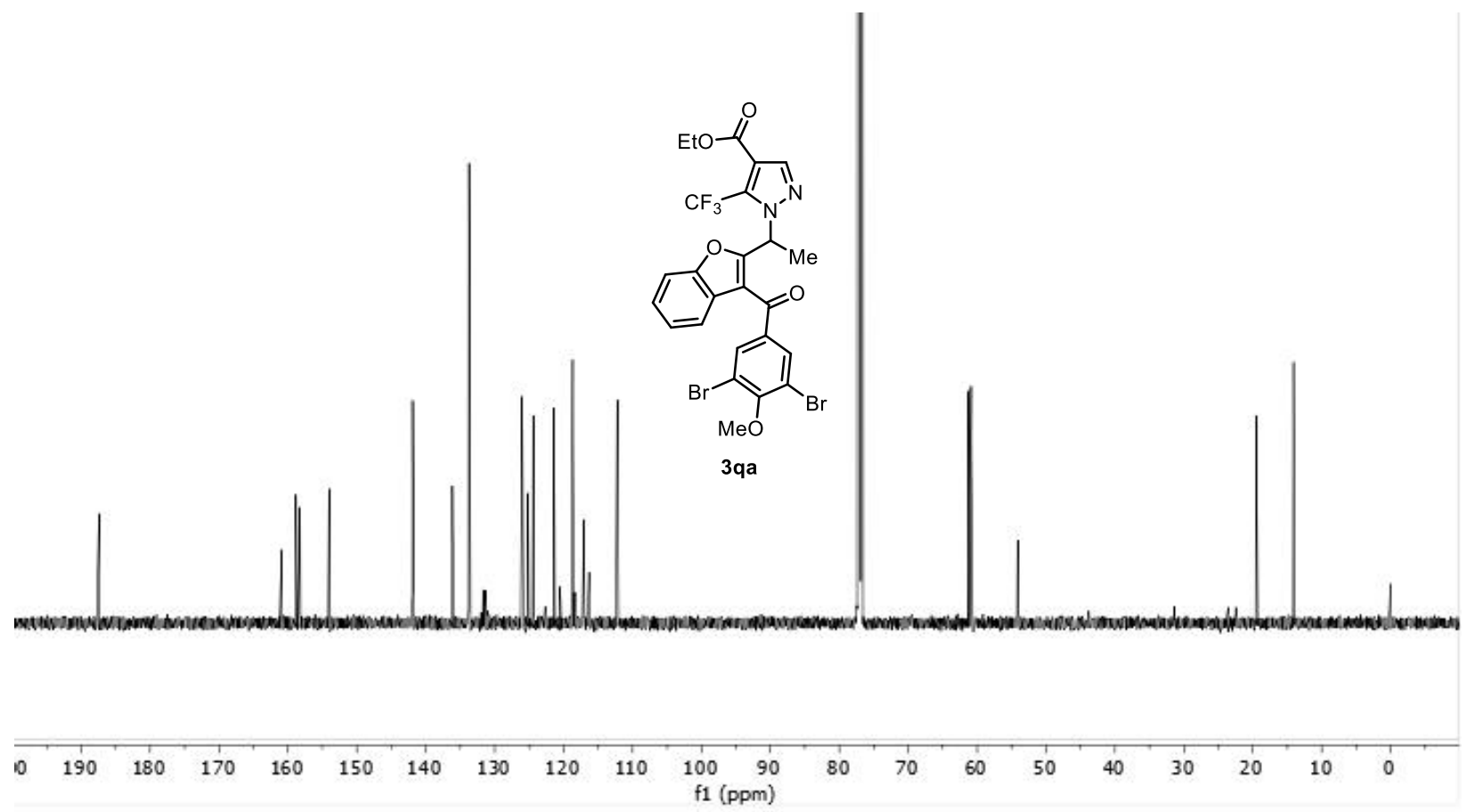

${ }^{19} \mathrm{~F}$ NMR spectrum of $\mathbf{3 q a}$ in $\mathrm{CDCl}_{3}$ containing $0.03 \%(\mathrm{v} / \mathrm{v})$ TMS $(377 \mathrm{MHz})$.

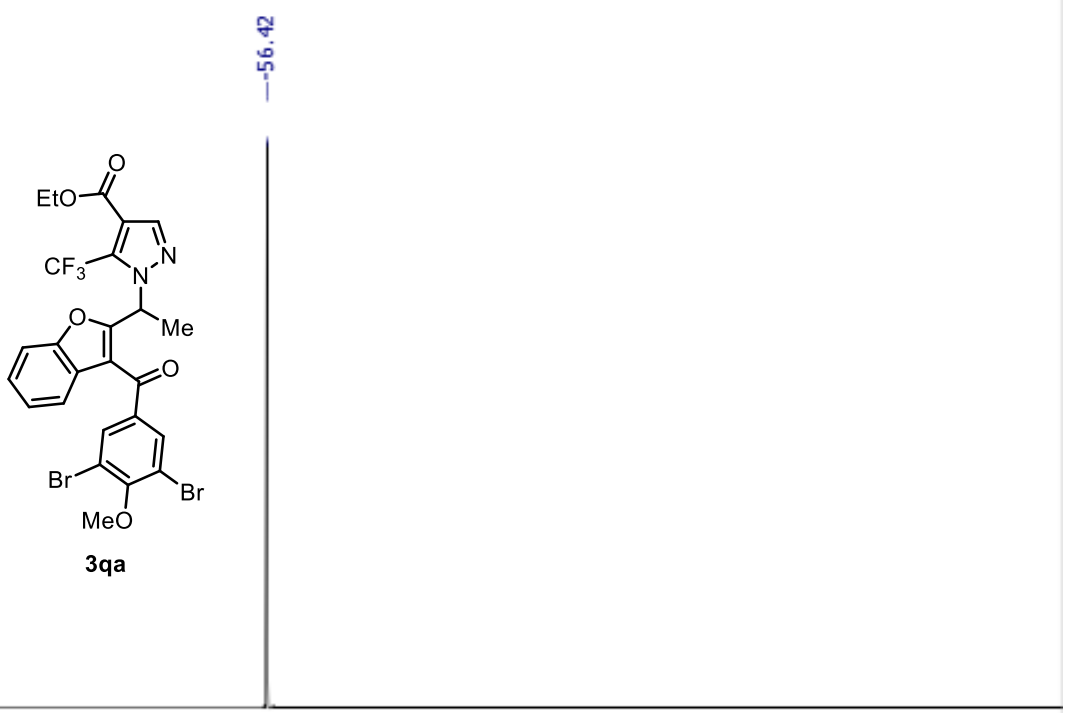

$\begin{array}{lllllllllllllllllllllllllllllllllll}5 & 0 & -5 & -10 & -15 & -20 & -25 & -30 & -35 & -40 & -45 & -50 & -55 & -60 & -65 & -70 & -75 & -80 & -85 & -90 & -95 & -100 & -105 & -110 & -115 & -1\end{array}$


${ }^{1} \mathrm{H}$ NMR spectrum of $\mathbf{3 q a}$ ' in $\mathrm{CDCl}_{3}$ containing $0.03 \%$ (v/v) TMS (500 MHz).

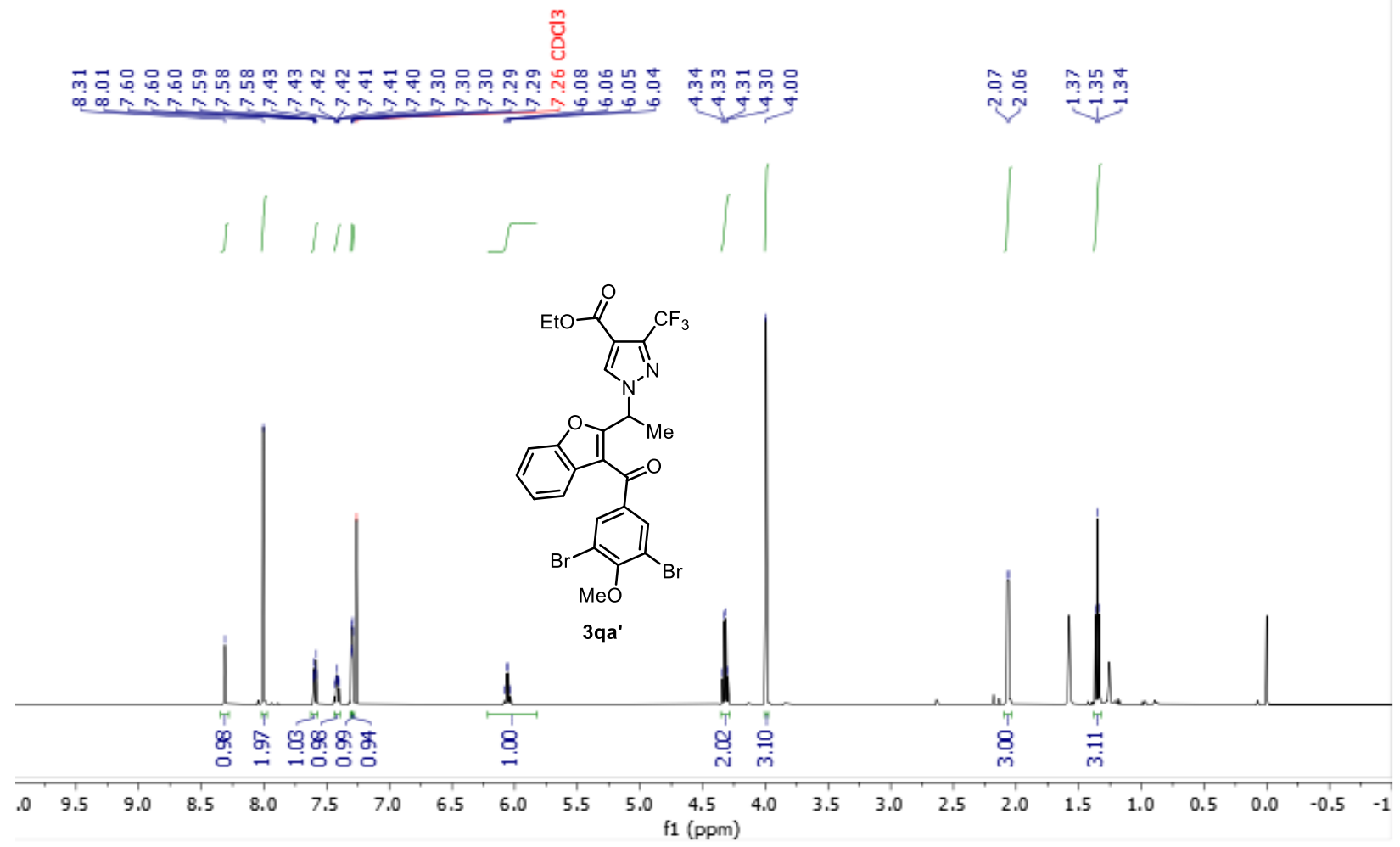

${ }^{13} \mathrm{C}$ NMR spectrum of $\mathbf{3 q a}$ ' in $\mathrm{CDCl}_{3}$ containing $0.03 \%$ (v/v) TMS (126 MHz).
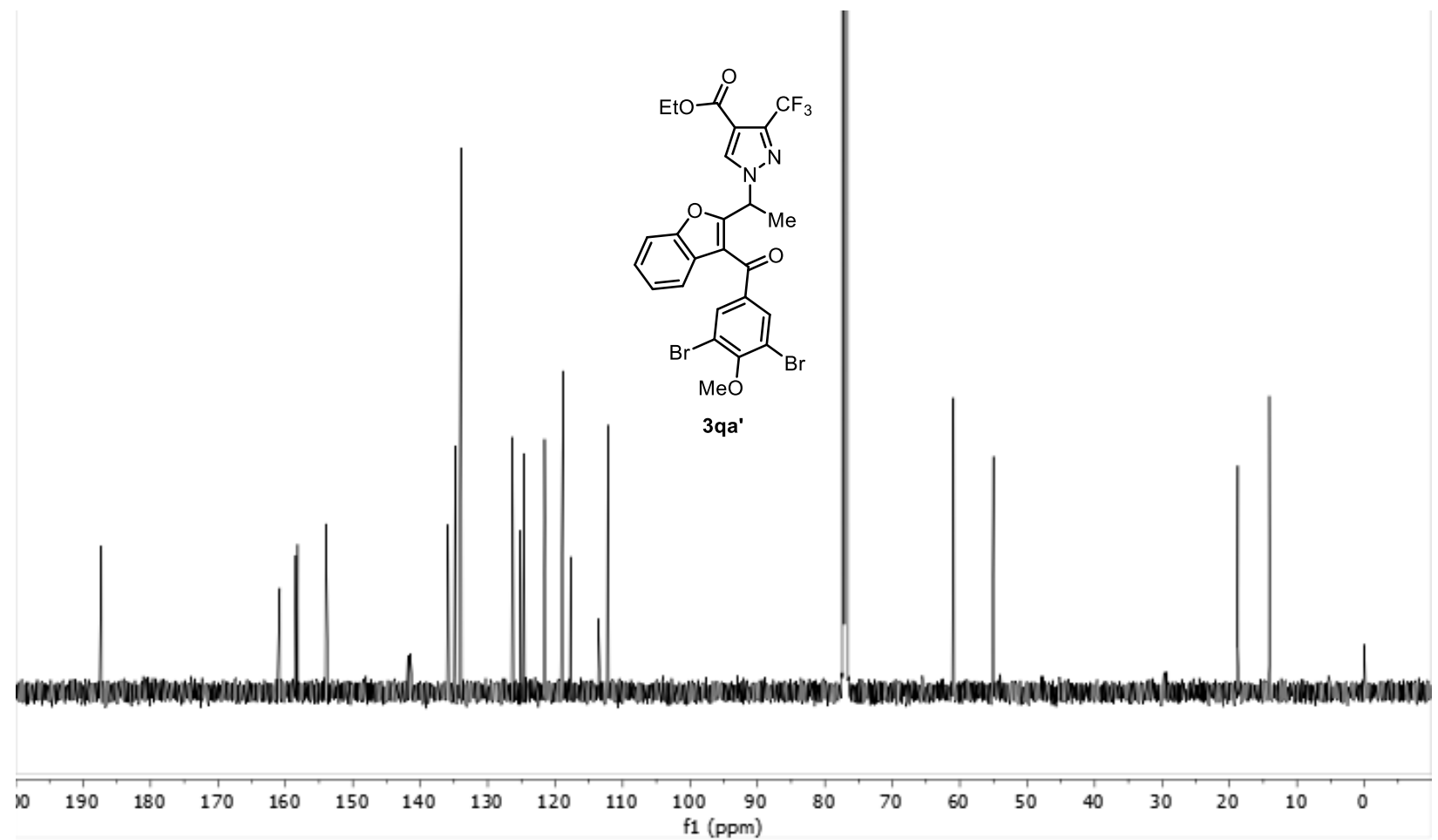
${ }^{19} \mathrm{~F}$ NMR spectrum of 3qa' in $\mathrm{CDCl}_{3}$ containing $0.03 \%$ (v/v) TMS (377 MHz).

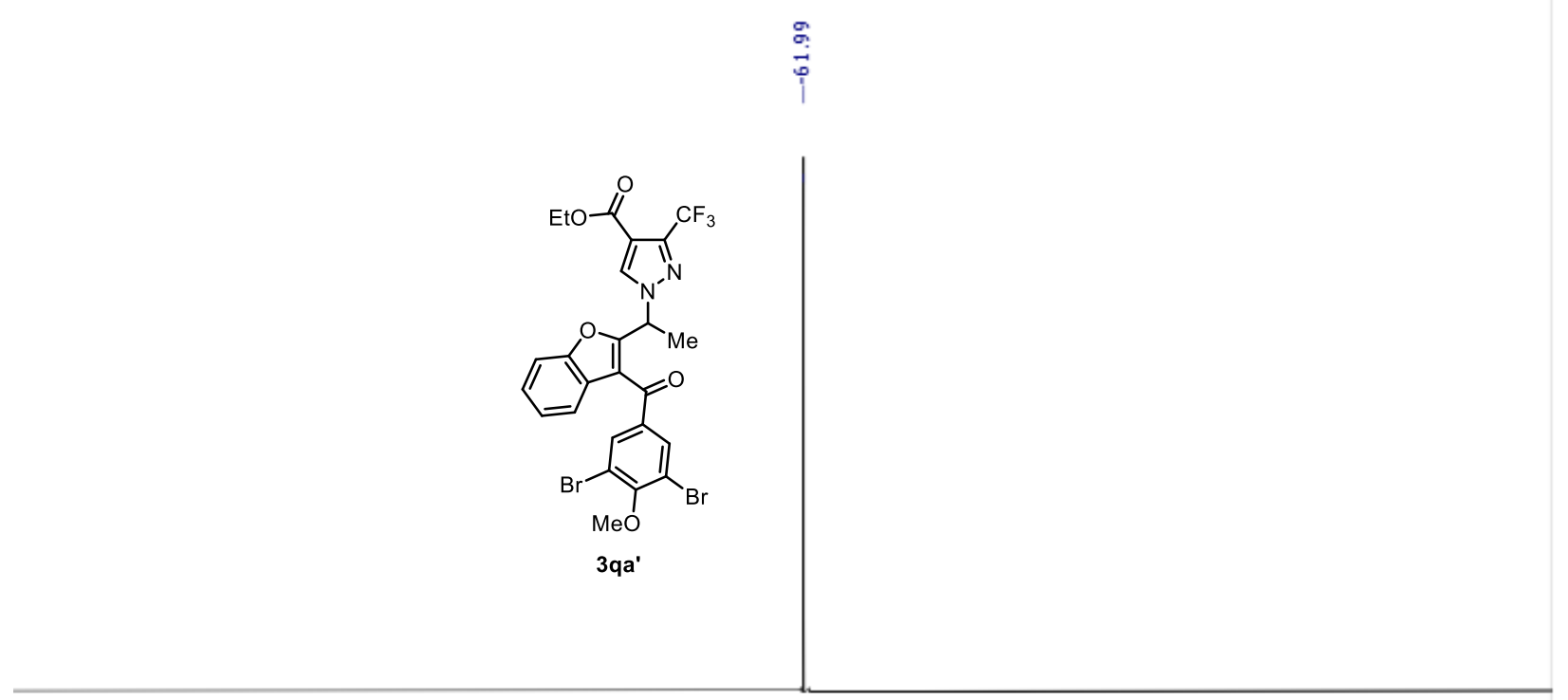

$\begin{array}{rllllllllllllllllllllllllllll}5 & 0 & -5 & -10 & -15 & -20 & -25 & -30 & -35 & -40 & -45 & -50 & -55 & -60 & -65 & -70 & -75 & -80 & -85 & -90 & -95 & -100 & -105 & -110 & -115 & -120 & -125\end{array}$

HSQC NMR spectrum of $\mathbf{3 q a} \mathbf{a}^{\prime}$ in $\mathrm{CDCl}_{3}$ containing $0.03 \%$ (v/v) TMS $(500,126 \mathrm{MHz})$.

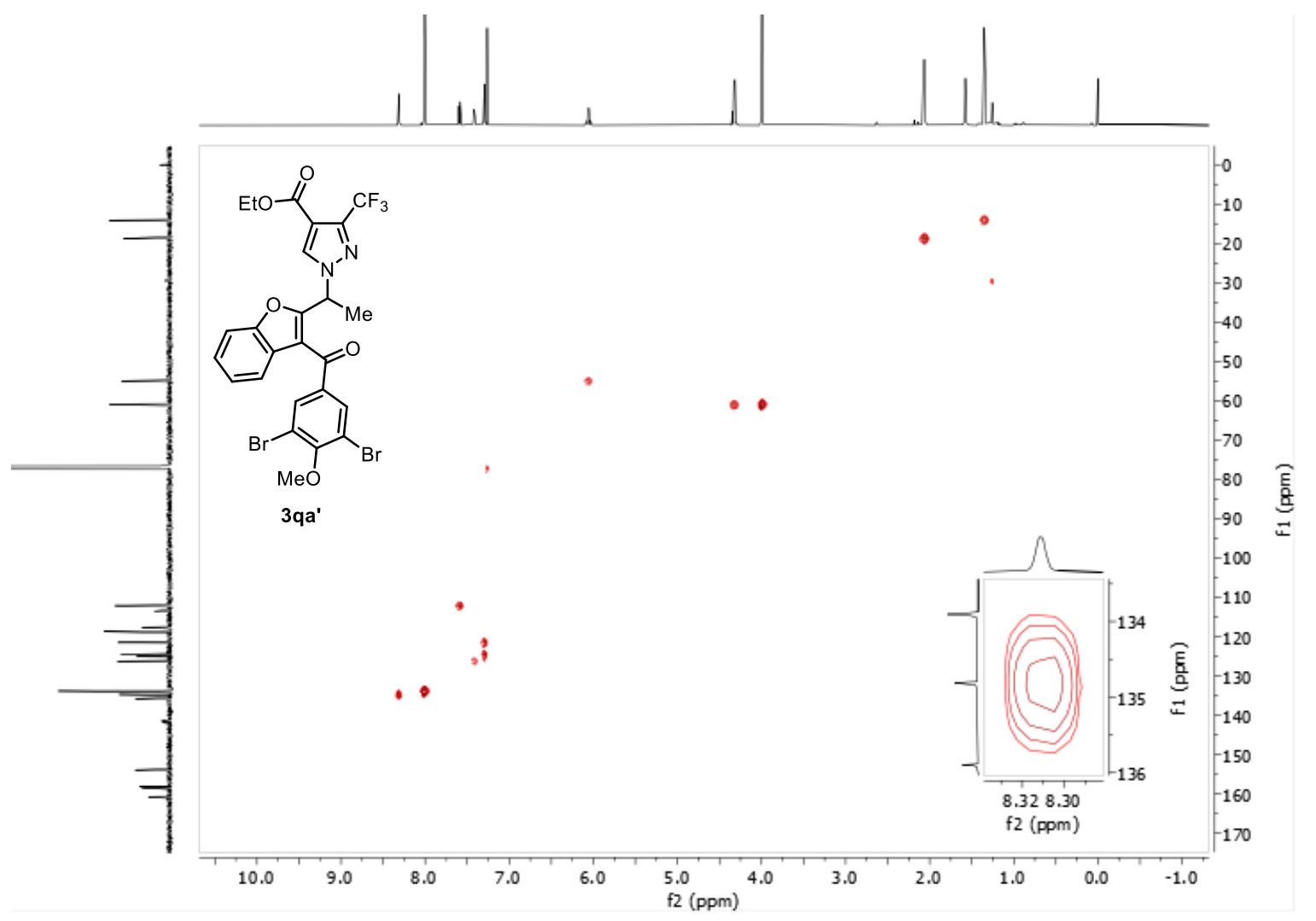


HMBC NMR spectrum of 3qa' in $\mathrm{CDCl}_{3}$ containing $0.03 \%$ (v/v) TMS (500, $126 \mathrm{MHz}$ ).

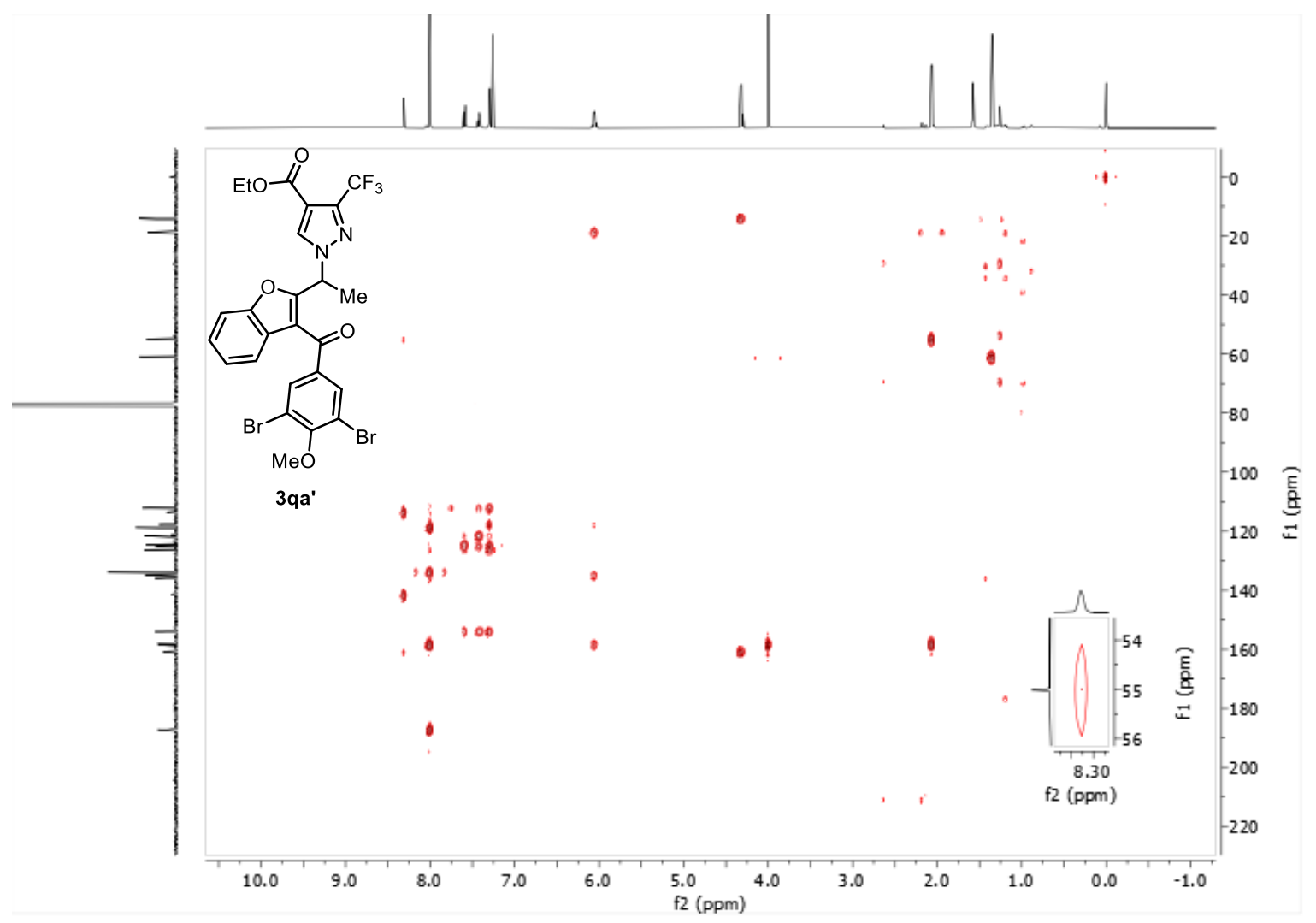

${ }^{1} \mathrm{H}$ NMR spectrum of $3 \mathbf{r b}$ in $\mathrm{CDCl}_{3}$ containing $0.03 \%$ (v/v) TMS (500 MHz).

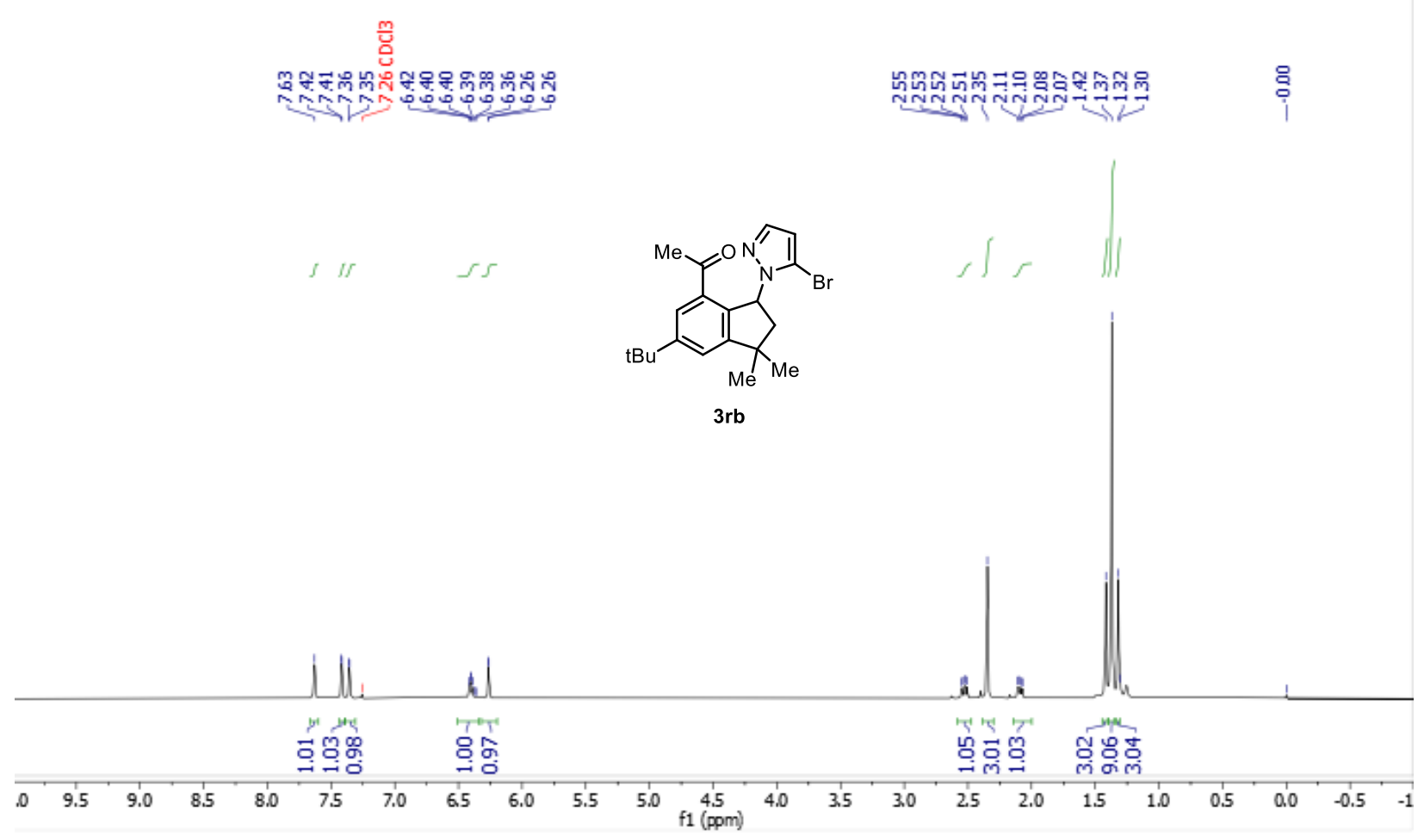


${ }^{13} \mathrm{C}$ NMR spectrum of $\mathbf{3 r b}$ in $\mathrm{CDCl}_{3}$ containing $0.03 \%$ (v/v) TMS (126 MHz).
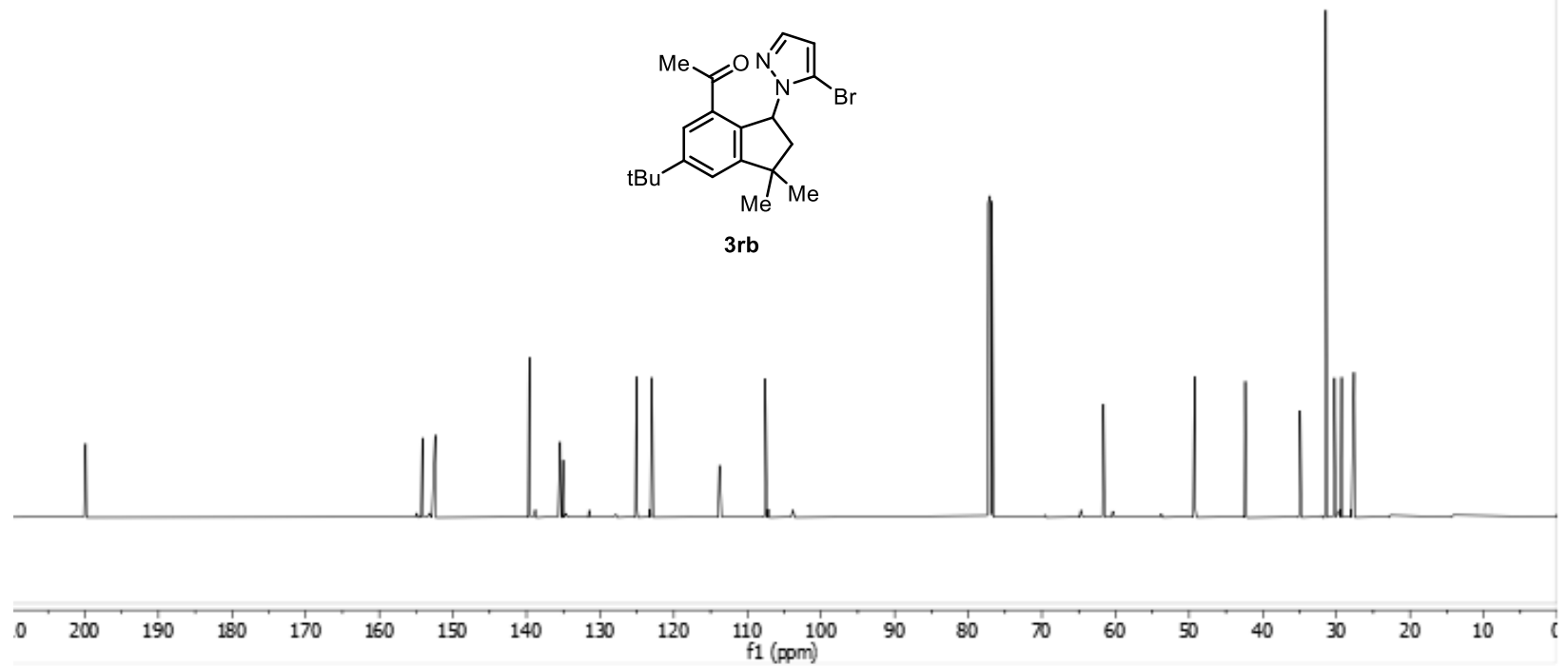

HSQC NMR spectrum of $3 \mathbf{r b}$ in $\mathrm{CDCl}_{3}$ containing $0.03 \%$ (v/v) TMS (500, $126 \mathrm{MHz}$ ).

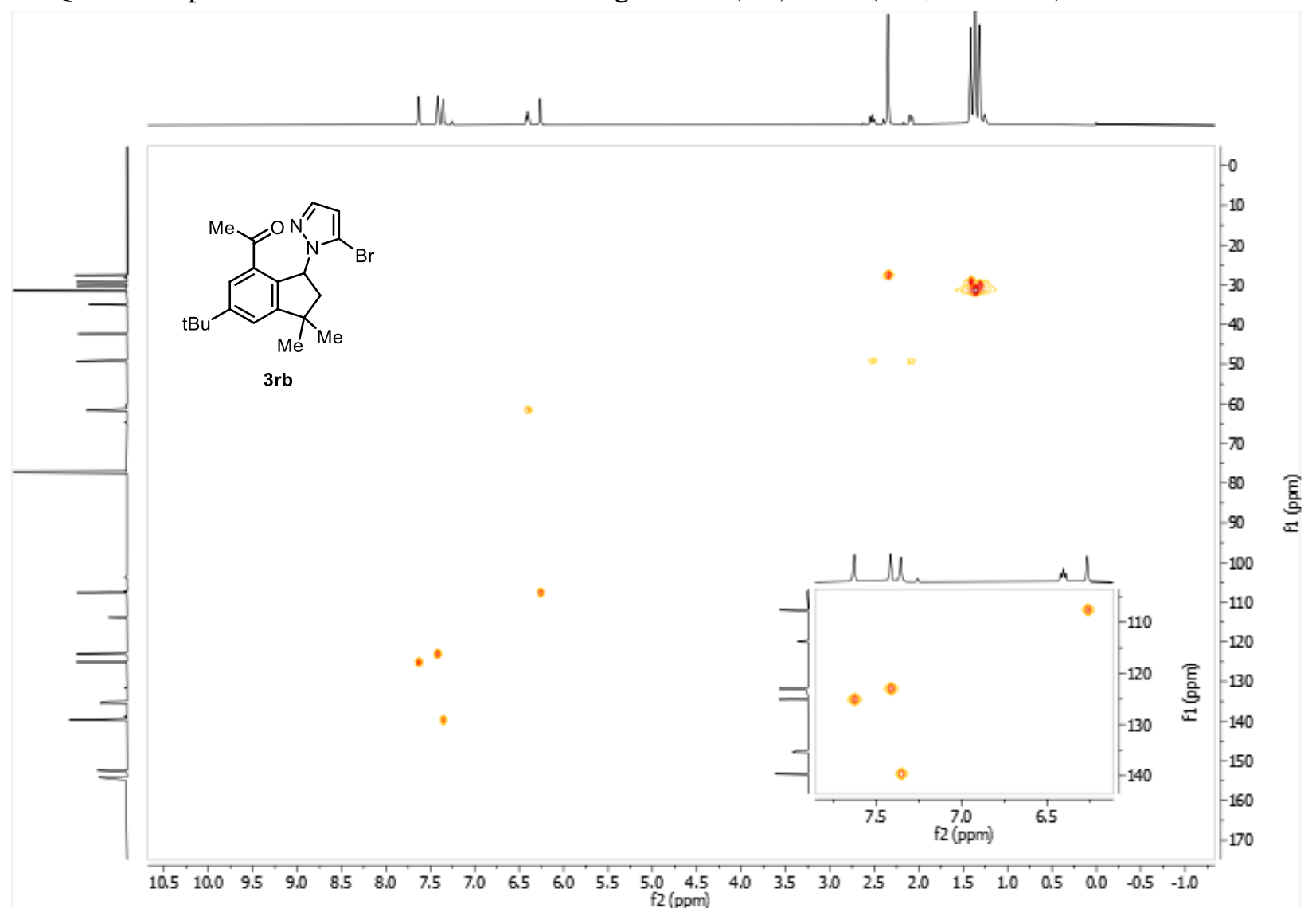


$\mathrm{HMBC}$ NMR spectrum of $\mathbf{3 r b}$ in $\mathrm{CDCl}_{3}$ containing $0.03 \%$ (v/v) TMS (500, $126 \mathrm{MHz}$ ).

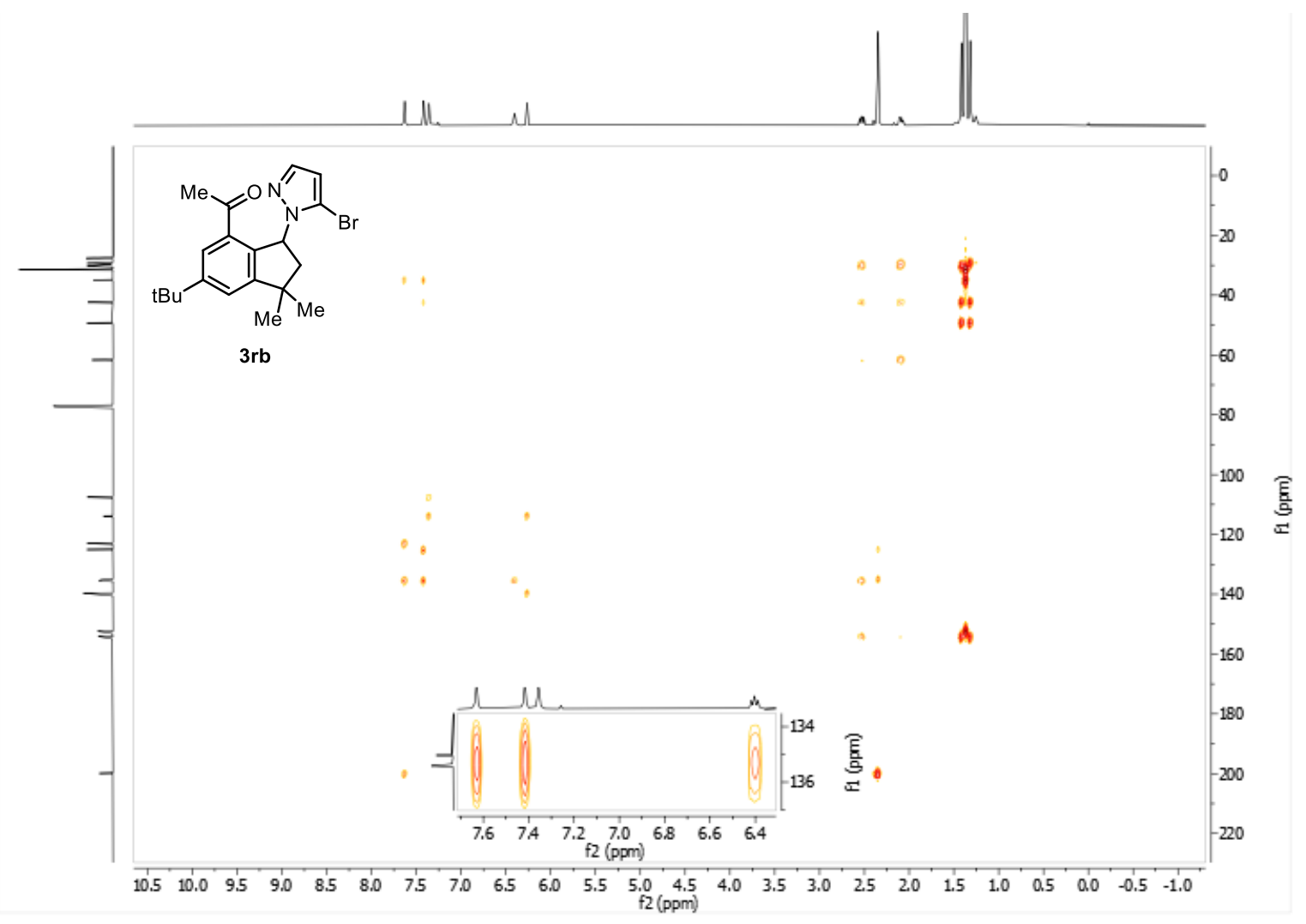

${ }^{1} \mathrm{H}$ NMR spectrum of $3 \mathbf{r b}$ in $\mathrm{CDCl}_{3}$ containing $0.03 \%$ (v/v) TMS (500 MHz).

$\stackrel{8}{8}$

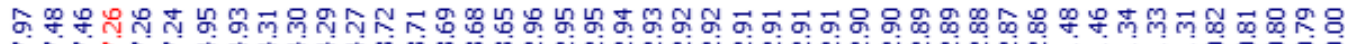

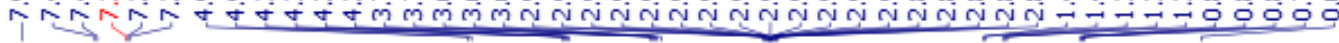

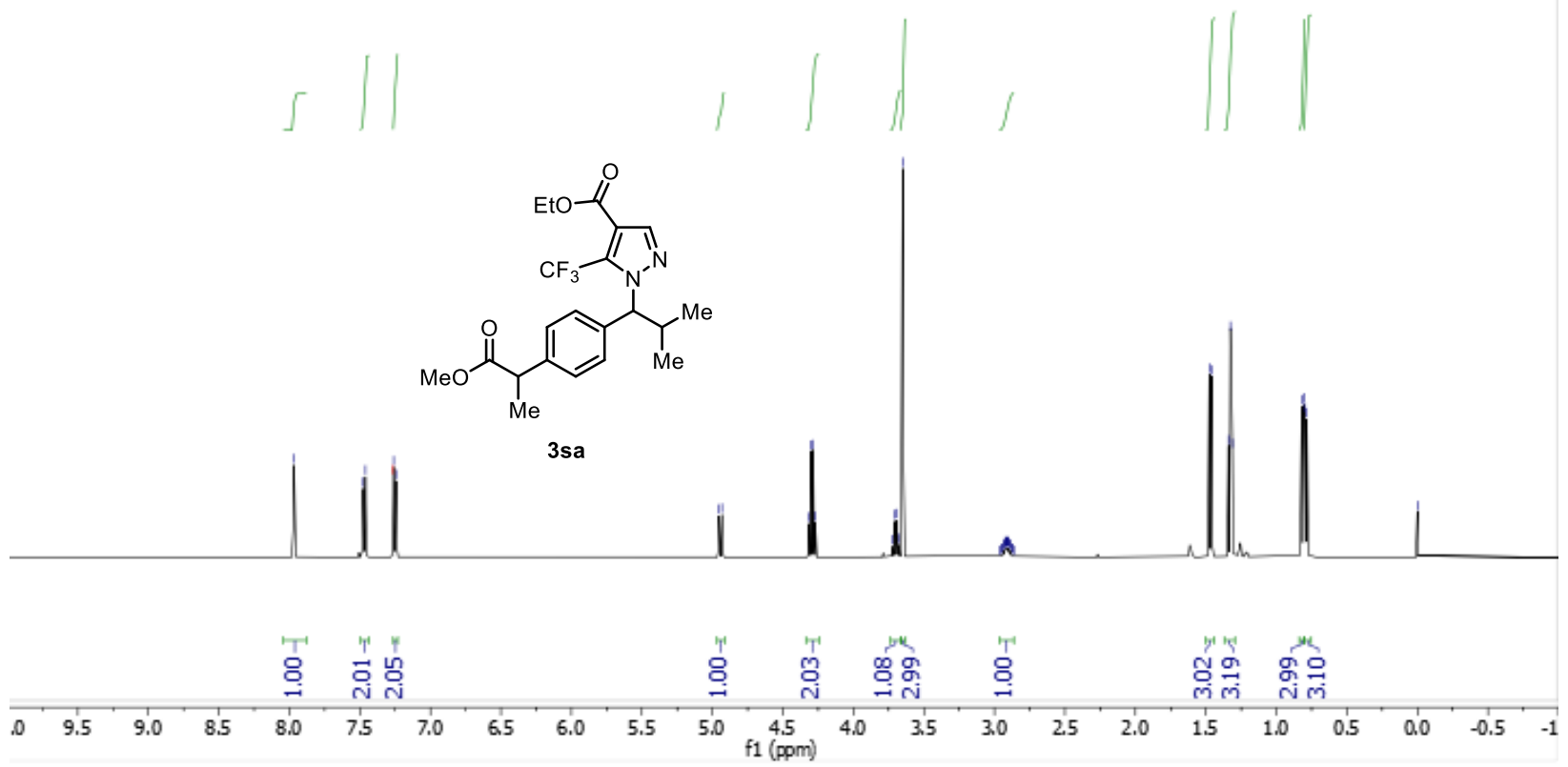


${ }^{13} \mathrm{C}$ NMR spectrum of $3 \mathbf{r b}$ in $\mathrm{CDCl}_{3}$ containing $0.03 \%$ (v/v) TMS (126 MHz).

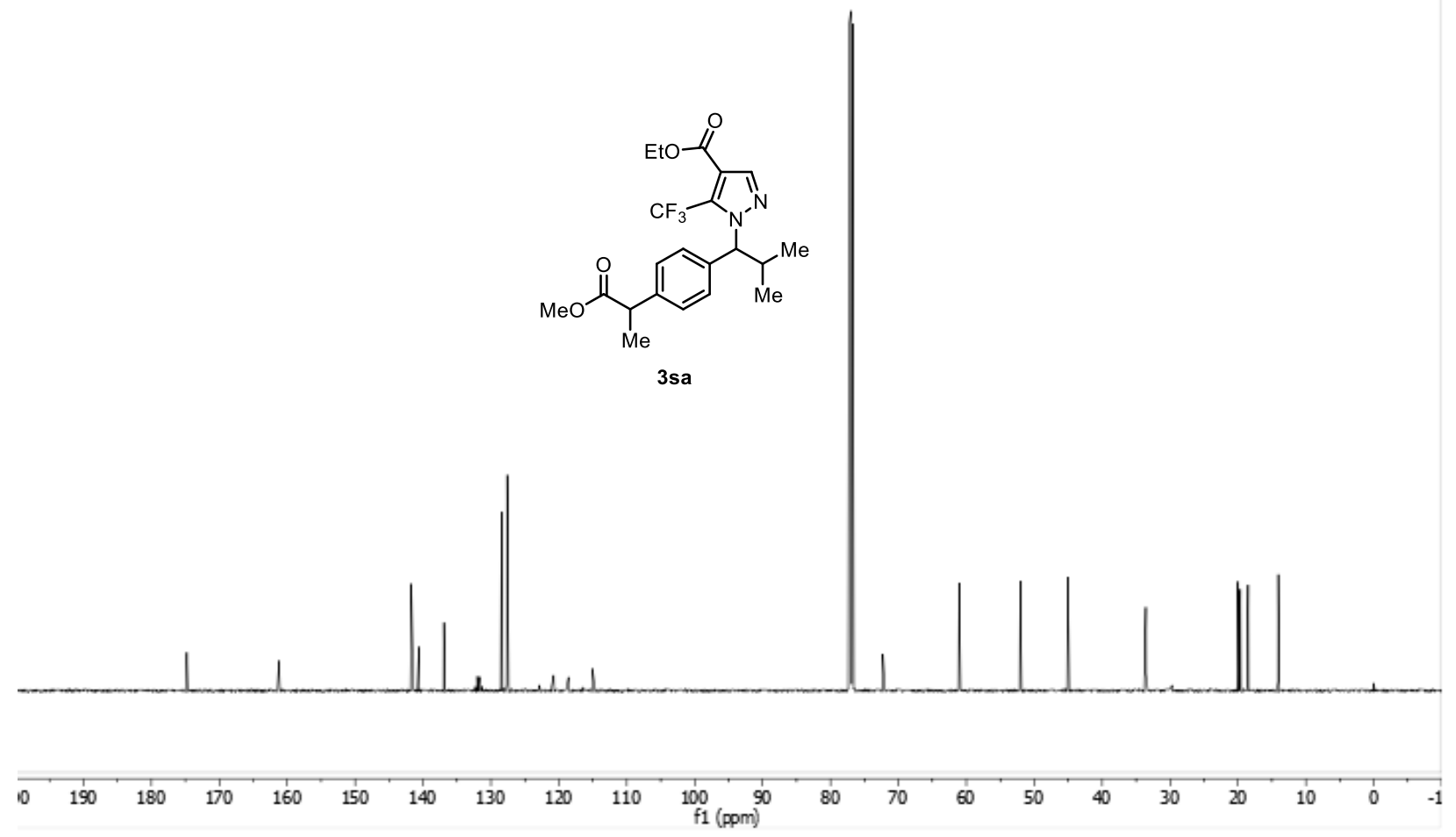

${ }^{19} \mathrm{~F}$ NMR spectrum of $\mathbf{3 r b}$ in $\mathrm{CDCl}_{3}$ containing $0.03 \%$ (v/v) TMS (377 MHz).

$$
\stackrel{\text { 爱 }}{i}
$$

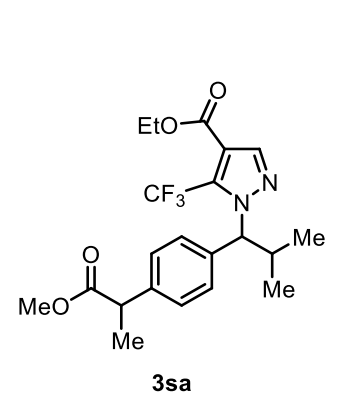


HSQC NMR spectrum of 3rb in $\mathrm{CDCl}_{3}$ containing $0.03 \%$ (v/v) TMS (500, $126 \mathrm{MHz}$ ).

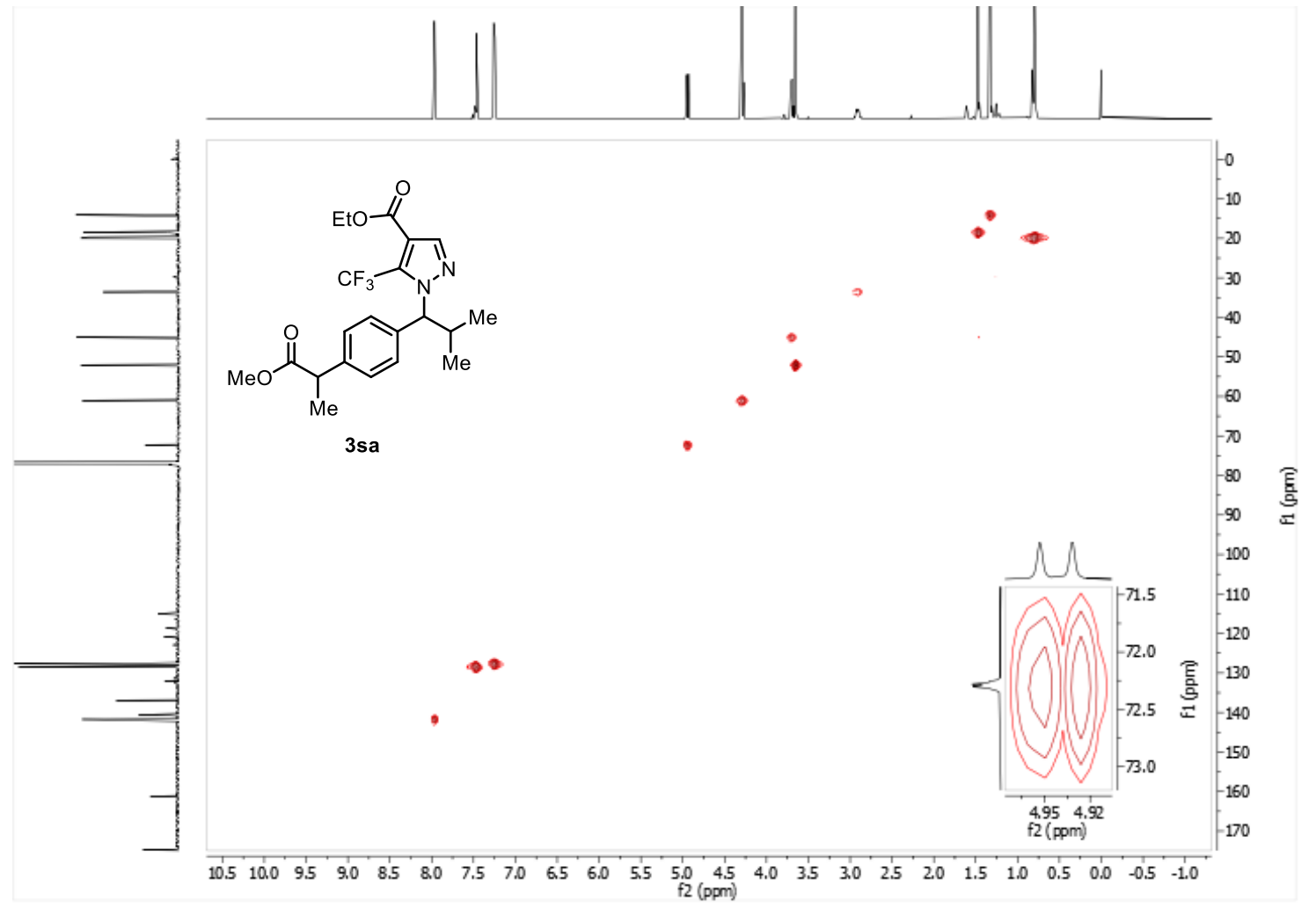

HMBC NMR spectrum of $3 \mathbf{r b}$ in $\mathrm{CDCl}_{3}$ containing $0.03 \%$ (v/v) TMS (500, $126 \mathrm{MHz}$ ).

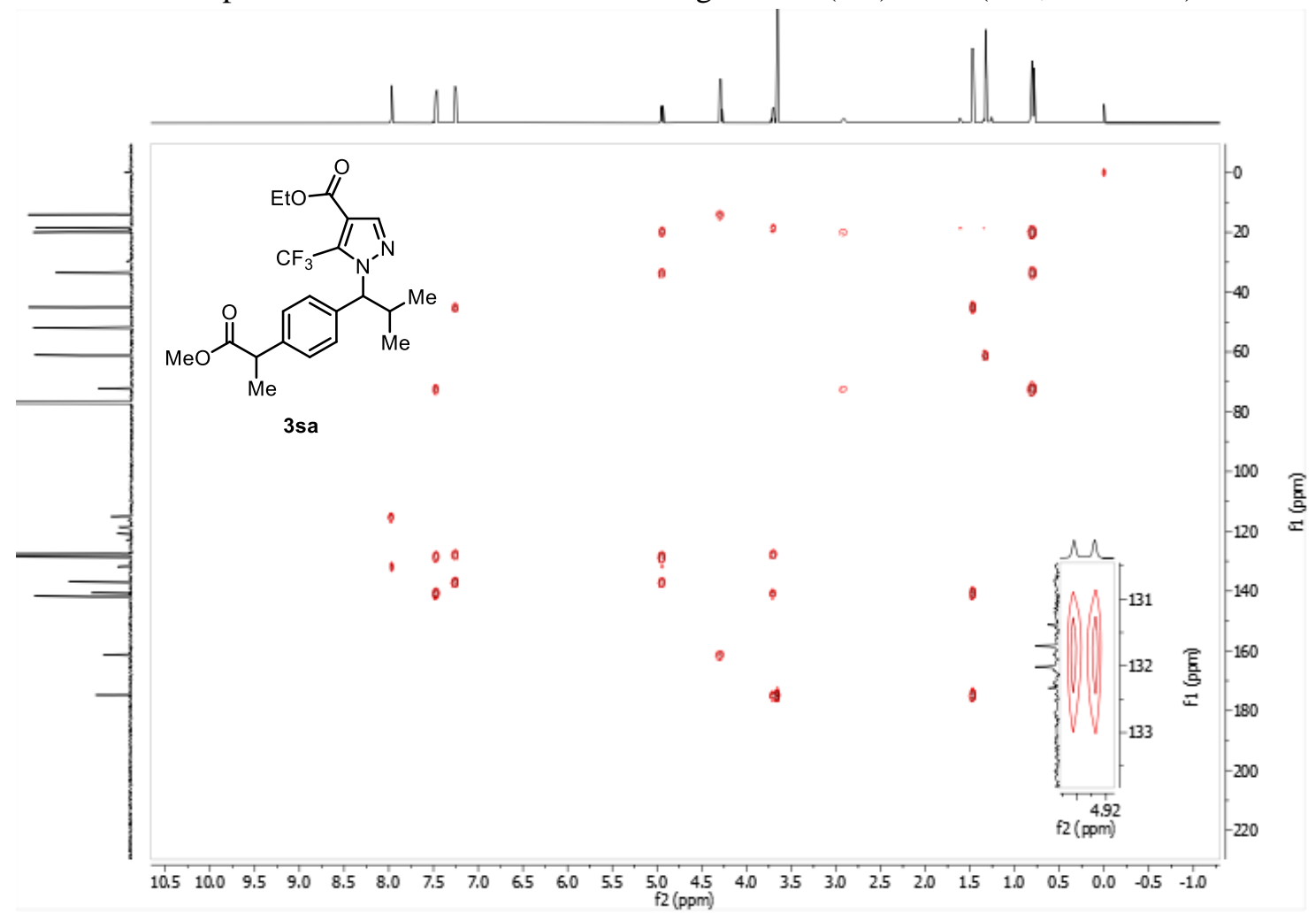


${ }^{1} \mathrm{H}$ NMR spectrum of $\mathbf{3 t b}-\mathbf{1}$ in $\mathrm{CDCl}_{3}$ containing $0.03 \%$ (v/v) TMS (500 MHz).

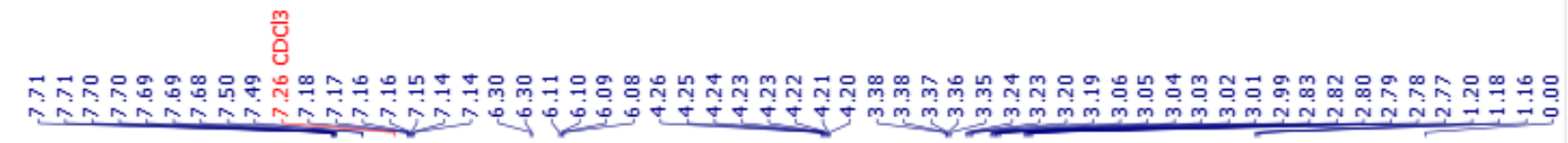<smiles>COC(=O)c1nn(-c2ccc(F)cc2)c2c1CCC2n1ccc(Br)n1</smiles>
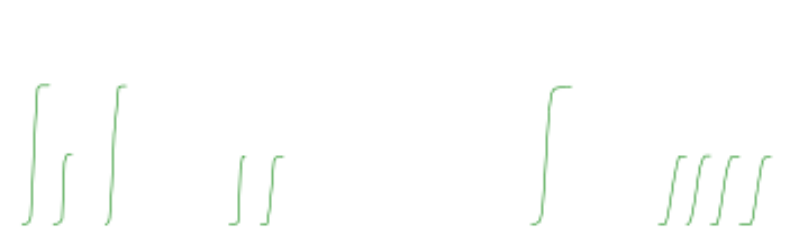

3tb-1

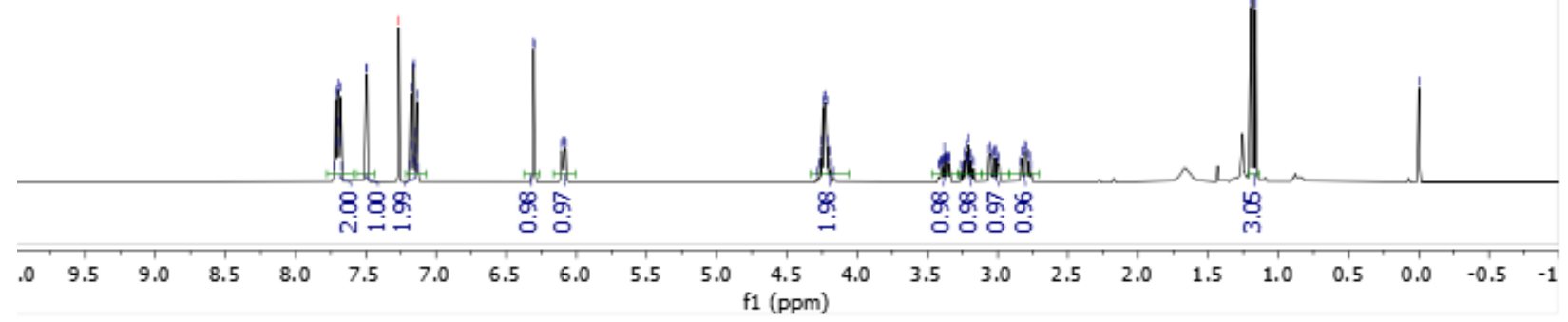

${ }^{13} \mathrm{C}$ NMR spectrum of 3tb-1 in $\mathrm{CDCl}_{3}$ containing $0.03 \%$ (v/v) TMS (126 MHz).

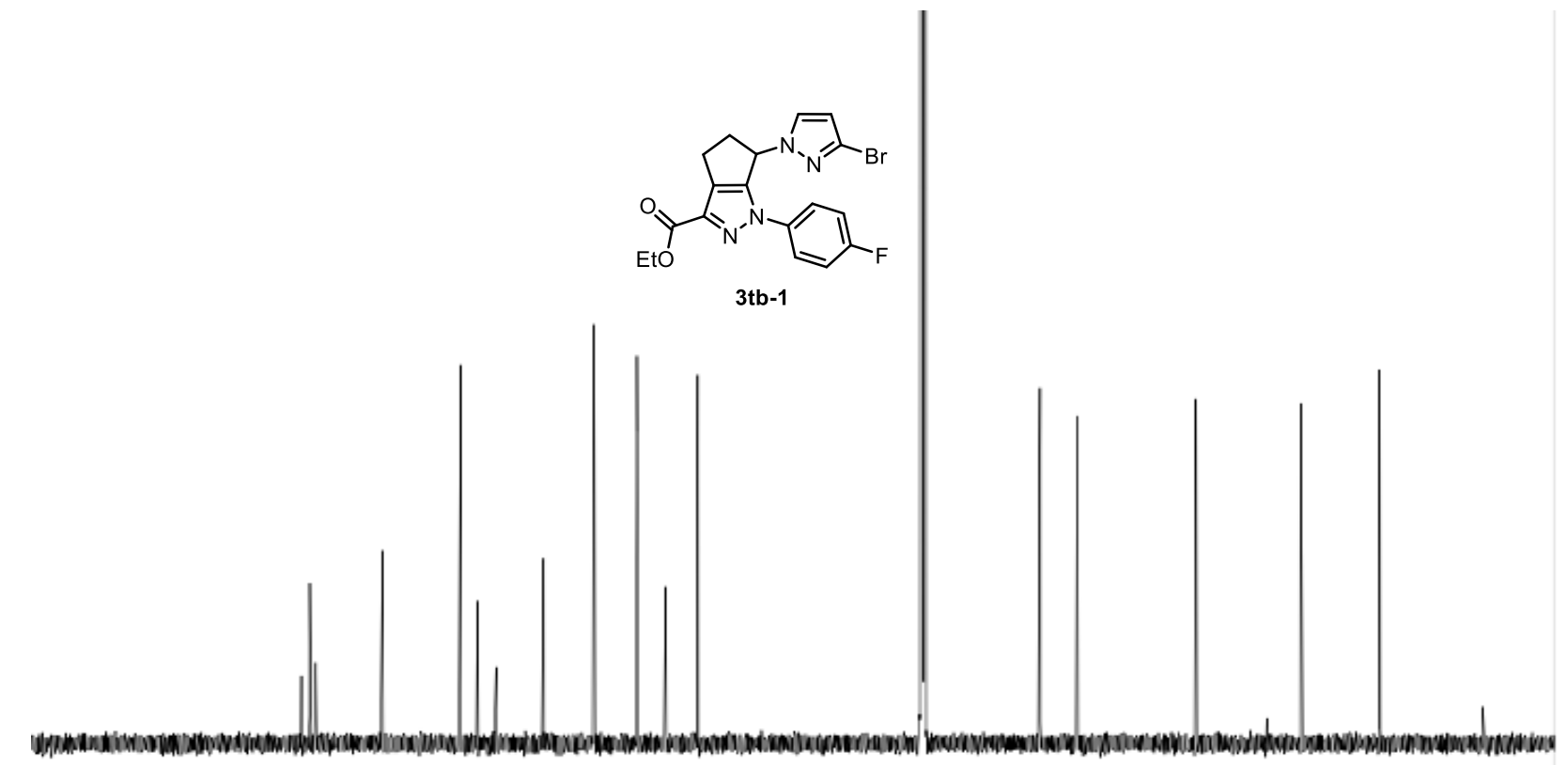

190

180

170

150

140130

120110

10090 
${ }^{19} \mathrm{~F}$ NMR spectrum of 3tb-1 in $\mathrm{CDCl}_{3}$ containing $0.03 \%(\mathrm{v} / \mathrm{v})$ TMS (377 MHz).

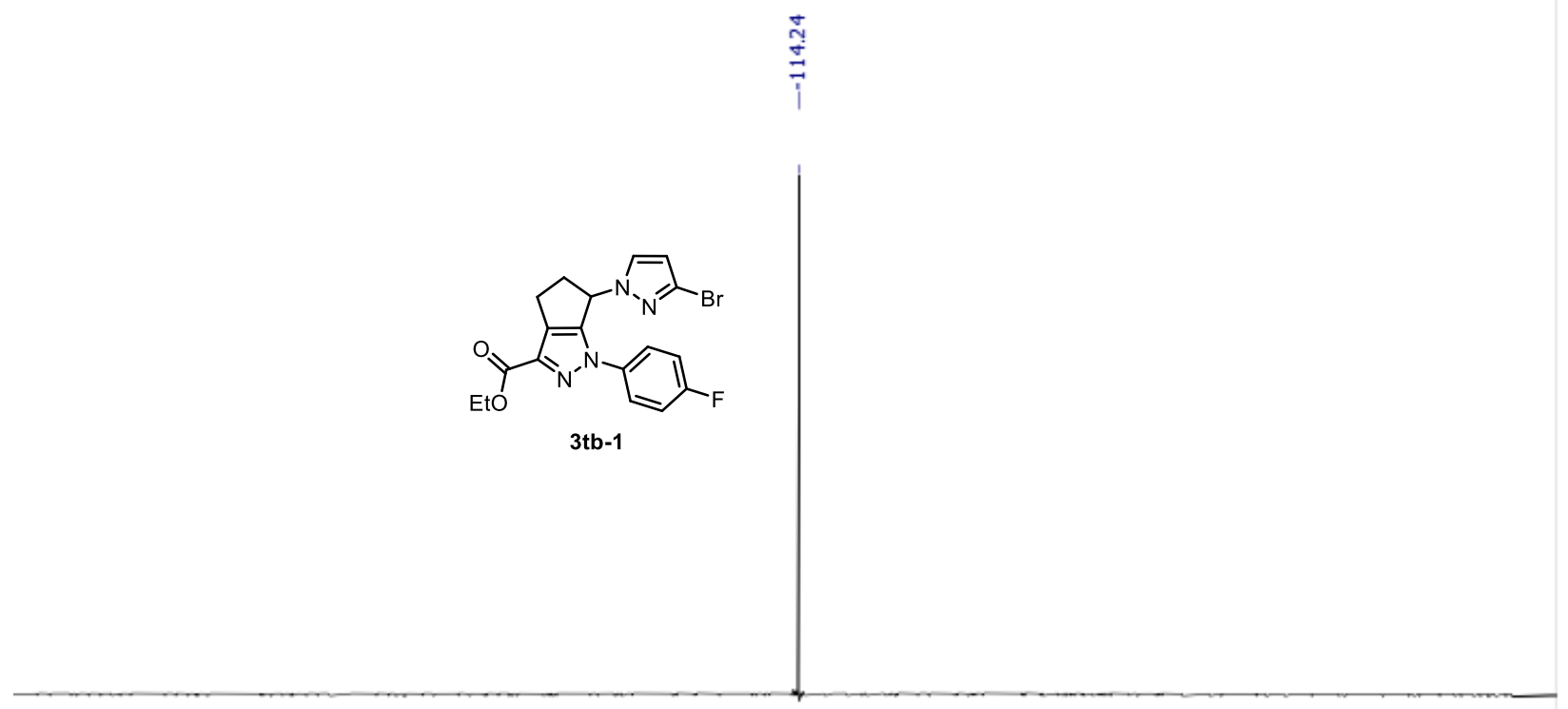

\begin{tabular}{llllllllllllll|l|l|l|l|l|l|}
\hline-30 & -40 & -50 & -60 & -70 & -80 & -90 & -100 & -110 & -120 & -130 & -140 & -150 & -160 & -170 & -180 & -190 & $f 1(\mathrm{ppm})$
\end{tabular}

HSQC NMR spectrum of 3tb-1 in $\mathrm{CDCl}_{3}$ containing $0.03 \%$ (v/v) TMS (500, $126 \mathrm{MHz}$ ).

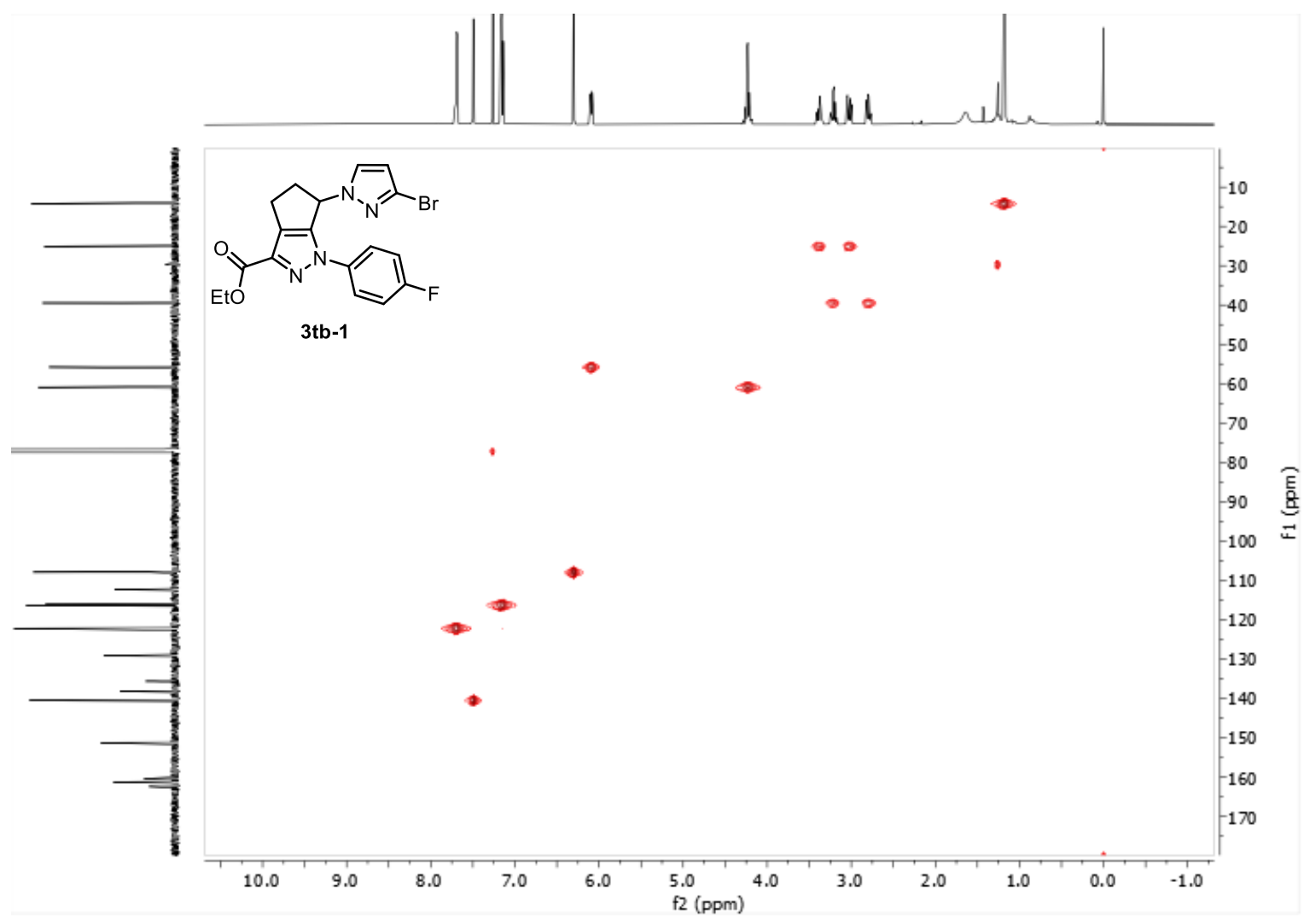


HMBC NMR spectrum of 3tb-1 in $\mathrm{CDCl}_{3}$ containing $0.03 \%$ (v/v) TMS (500, $126 \mathrm{MHz}$ ).

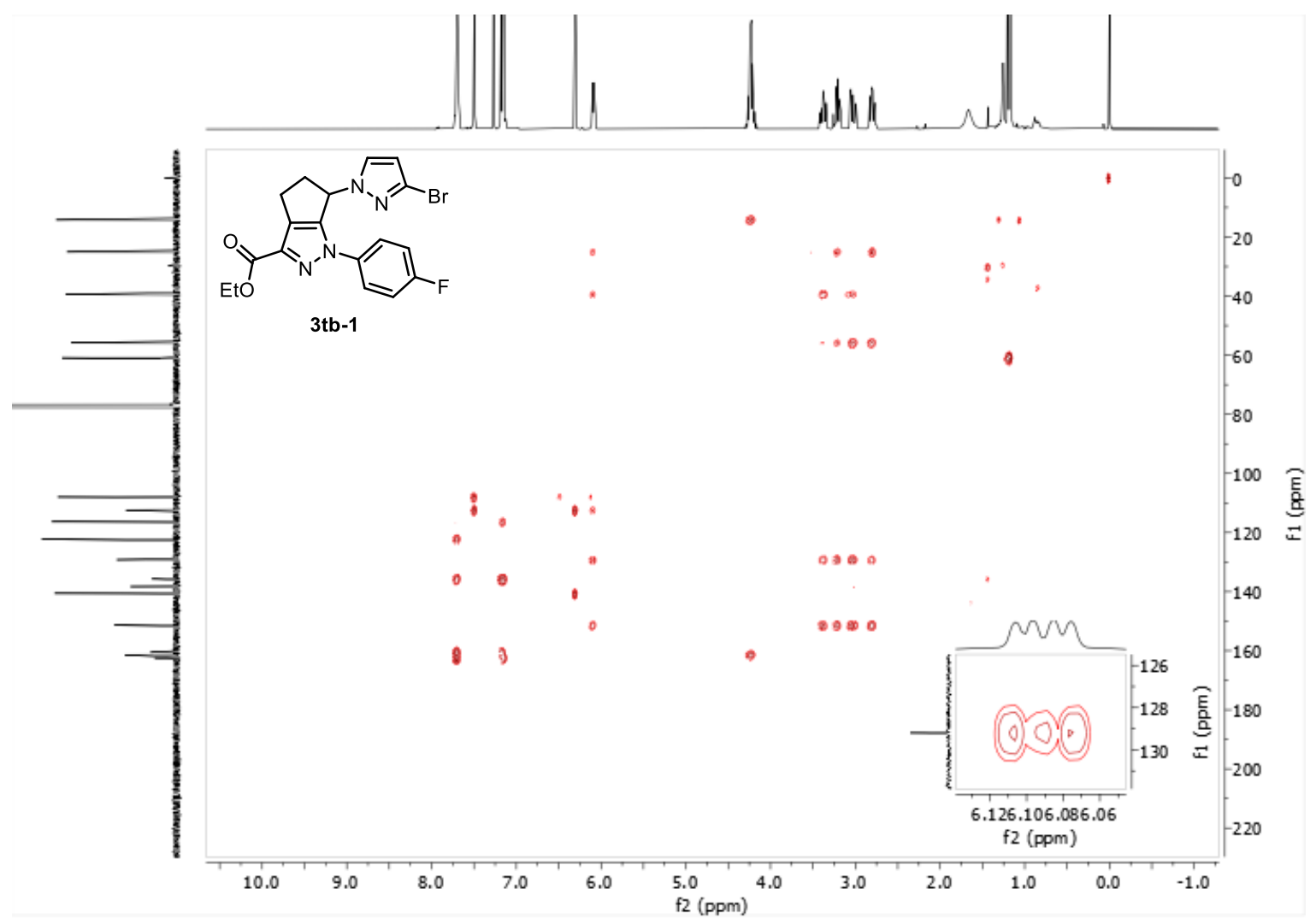

NOESY NMR spectrum of 3tb-1 in $\mathrm{CDCl}_{3}$ containing $0.03 \%$ (v/v) TMS (500, $126 \mathrm{MHz}$ ).

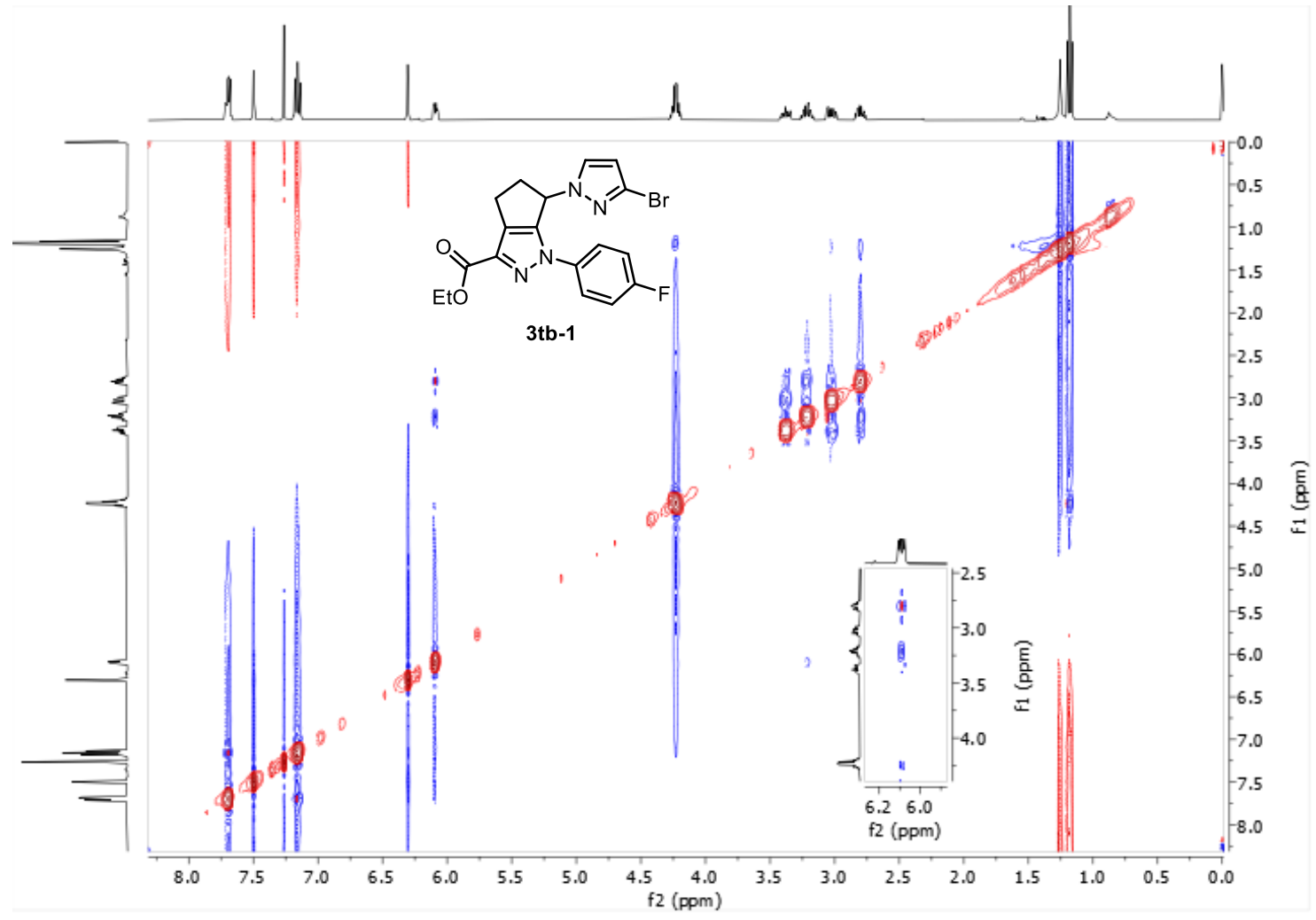


${ }^{1} \mathrm{H}$ NMR spectrum of $\mathbf{3 t b}-2$ in $\mathrm{CDCl}_{3}$ containing $0.03 \%$ (v/v) TMS (500 MHz).

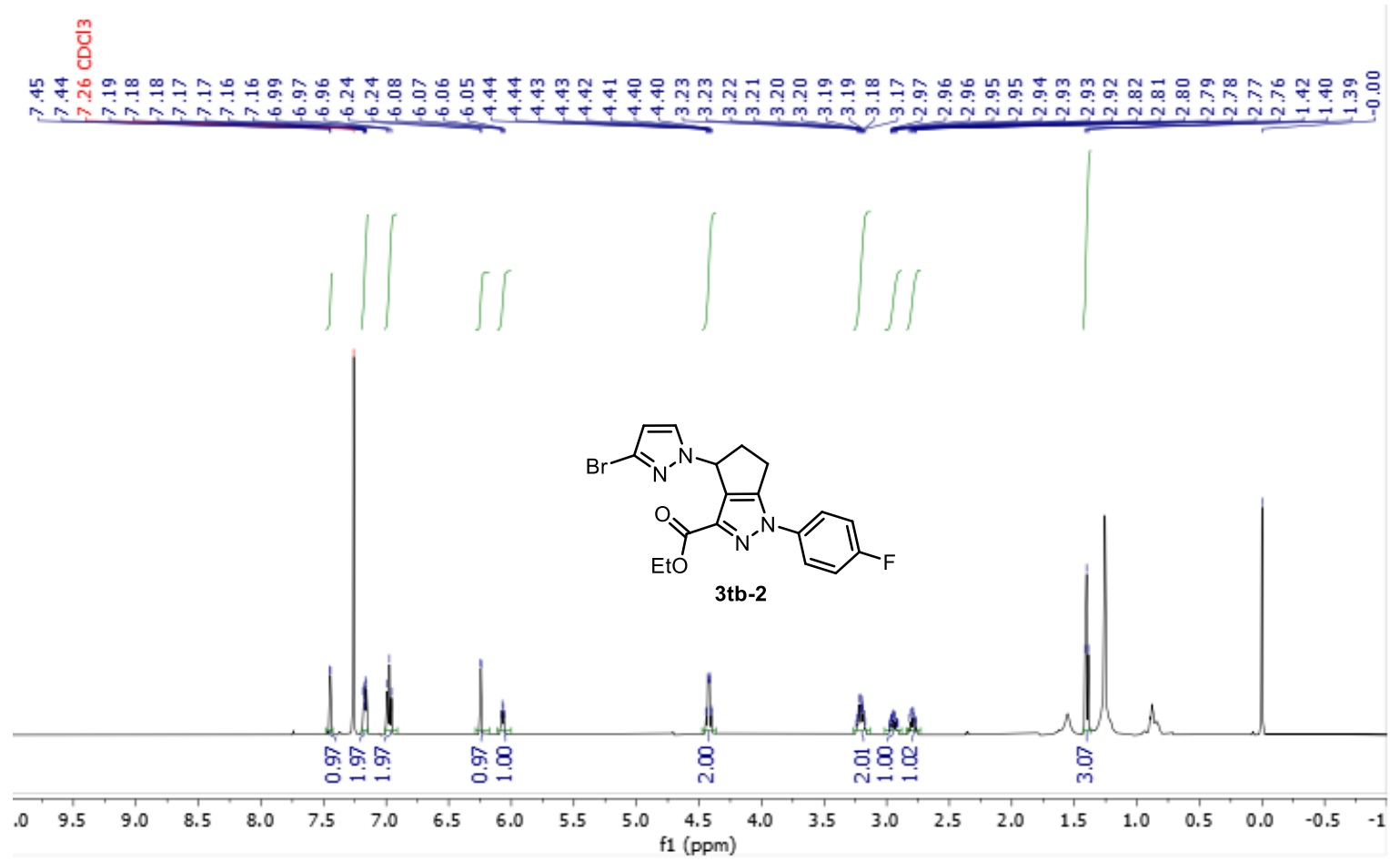

${ }^{13} \mathrm{C}$ NMR spectrum of 3tb-2 in $\mathrm{CDCl}_{3}$ containing $0.03 \%$ (v/v) TMS (126 MHz).

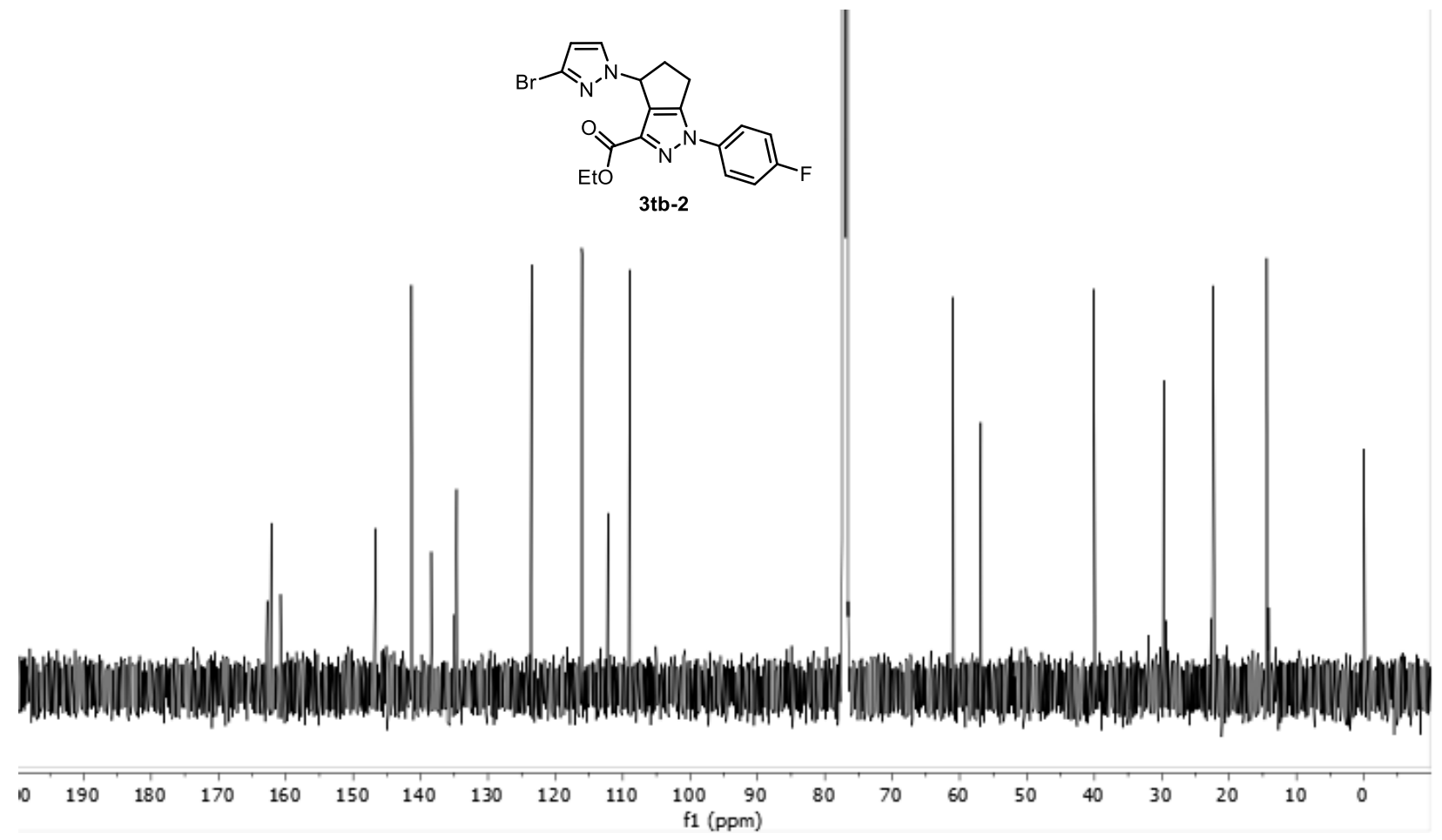


${ }^{19} \mathrm{~F}$ NMR spectrum of 3tb-2 in $\mathrm{CDCl}_{3}$ containing $0.03 \%(\mathrm{v} / \mathrm{v})$ TMS (377 MHz).

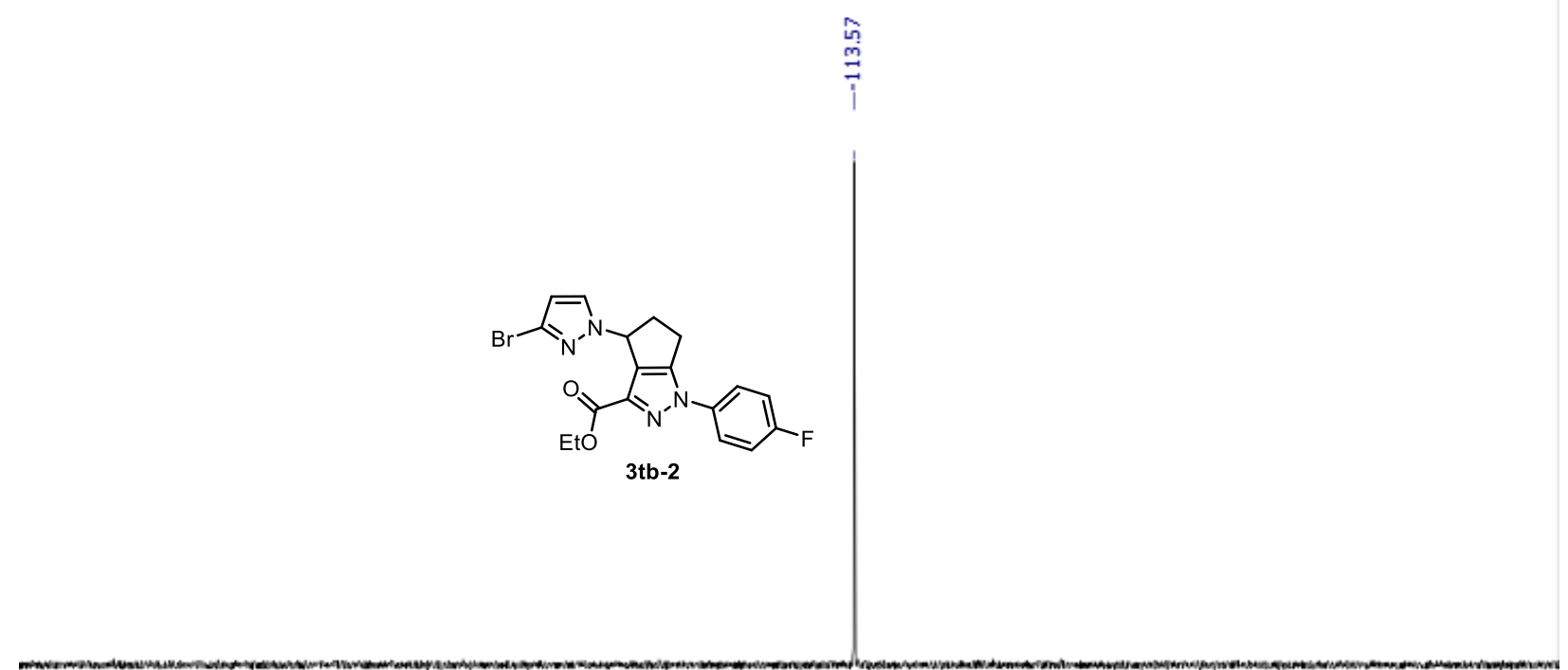

$\begin{array}{llllllllllllllllllllllllll}-101 & -102 & -103 & -104 & -105 & -106 & -107 & -108 & -109 & -110 & -111 & -112 & -113 & -114 & -115 & -116 & -117 & -118 & -119 & -120 & -121 & -122 & -123 & -124 & -1\end{array}$

HSQC NMR spectrum of 3tb-2 in $\mathrm{CDCl}_{3}$ containing $0.03 \%$ (v/v) TMS (500, $\left.126 \mathrm{MHz}\right)$.

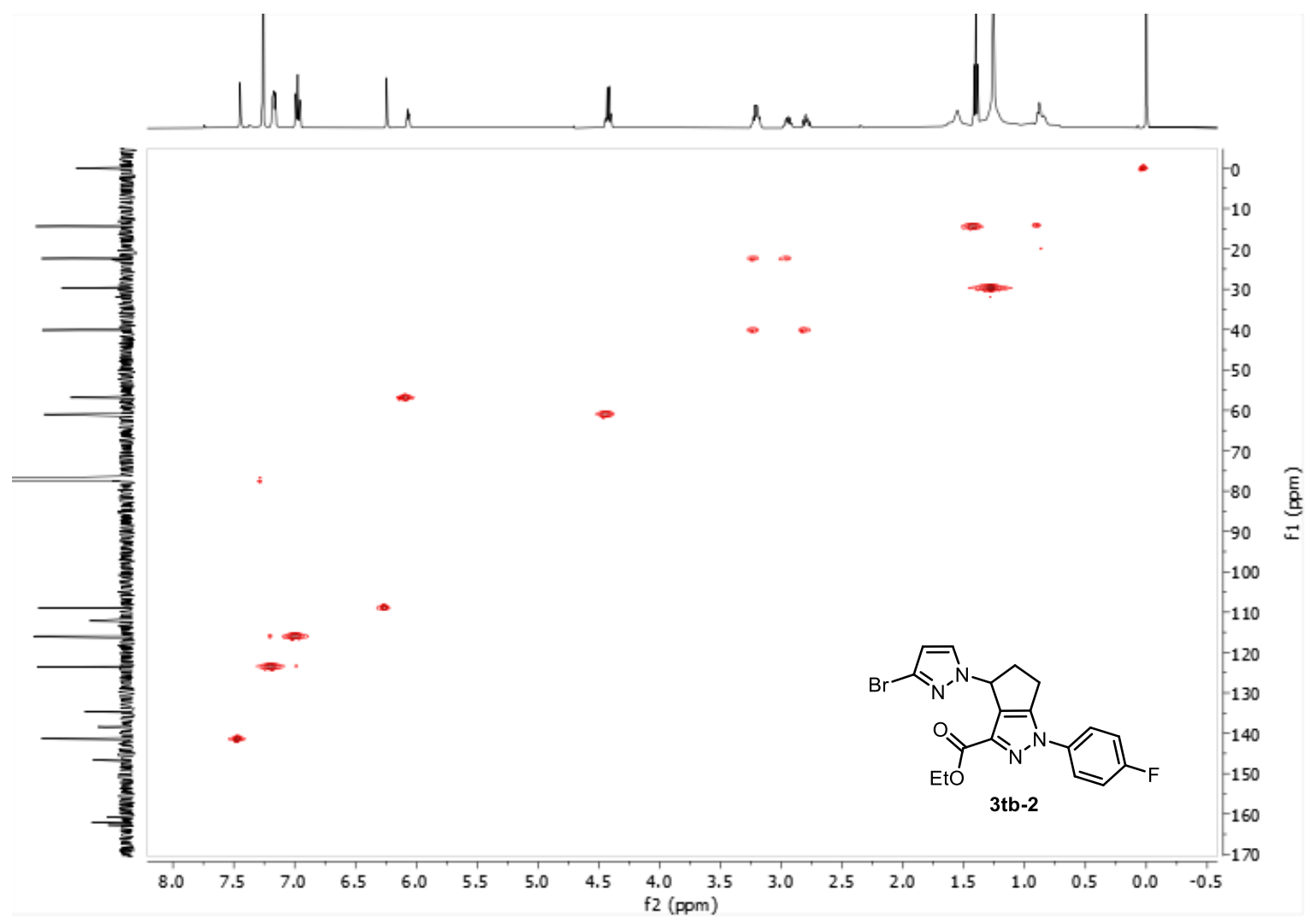


HMBC NMR spectrum of 3tb-2 in $\mathrm{CDCl}_{3}$ containing $0.03 \%$ (v/v) TMS (500, $126 \mathrm{MHz}$ ).

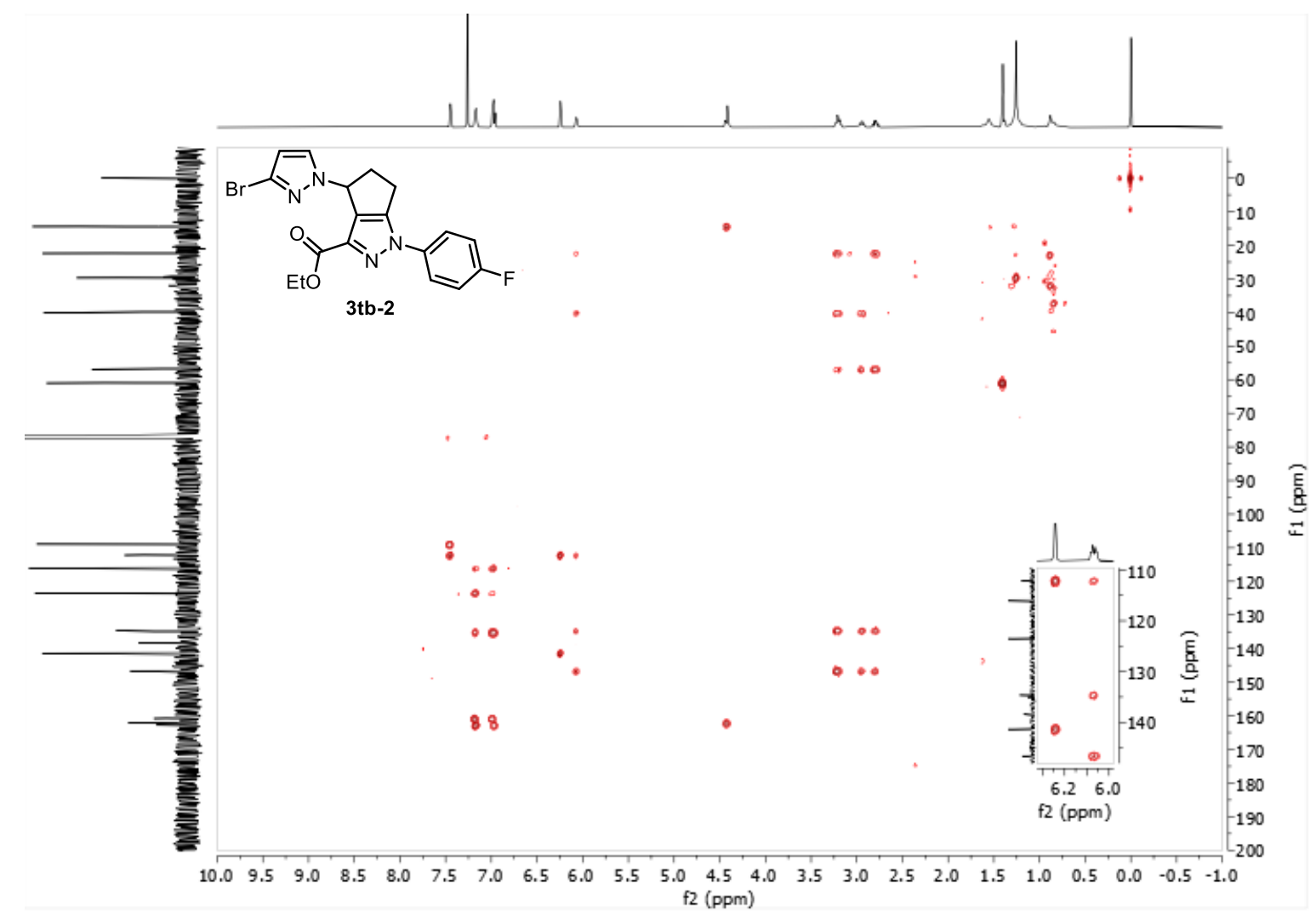

${ }^{1} \mathrm{H}$ NMR spectrum of 3 ua in $\mathrm{CDCl}_{3}$ containing $0.03 \%$ (v/v) TMS (500 MHz).

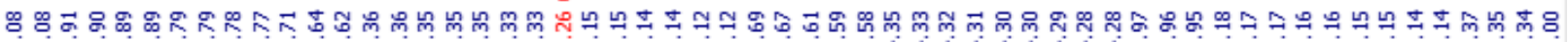

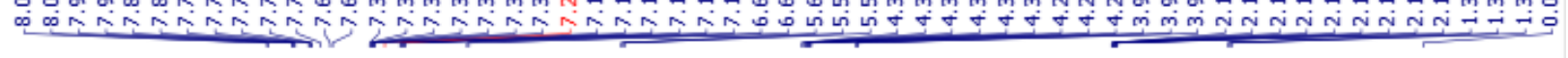

S127 
${ }^{13} \mathrm{C}$ NMR spectrum of 3ua in $\mathrm{CDCl}_{3}$ containing $0.03 \%$ (v/v) TMS (126 MHz).
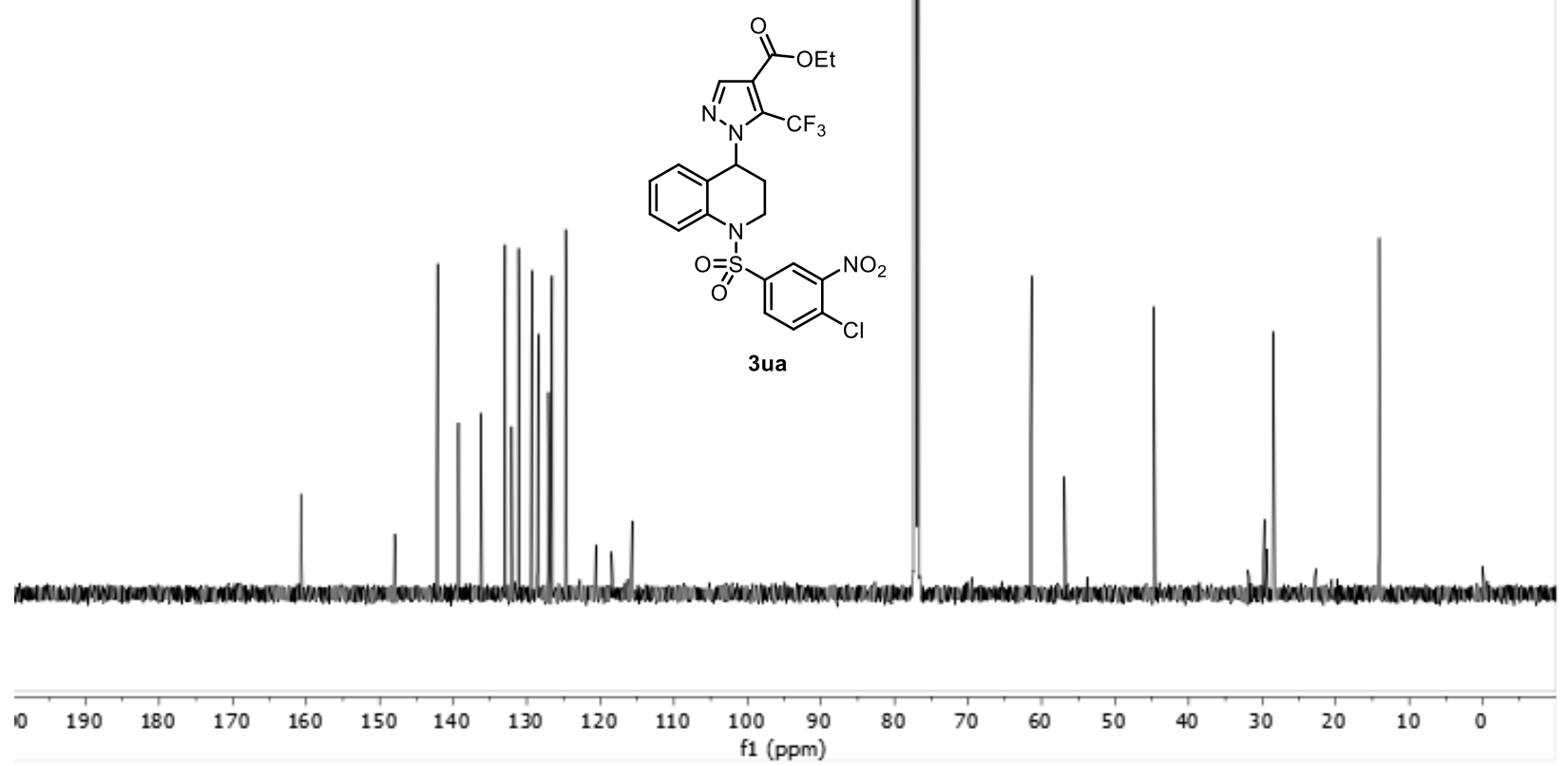

${ }^{19} \mathrm{~F}$ NMR spectrum of 3ua in $\mathrm{CDCl}_{3}$ containing $0.03 \%$ (v/v) TMS (377 MHz).

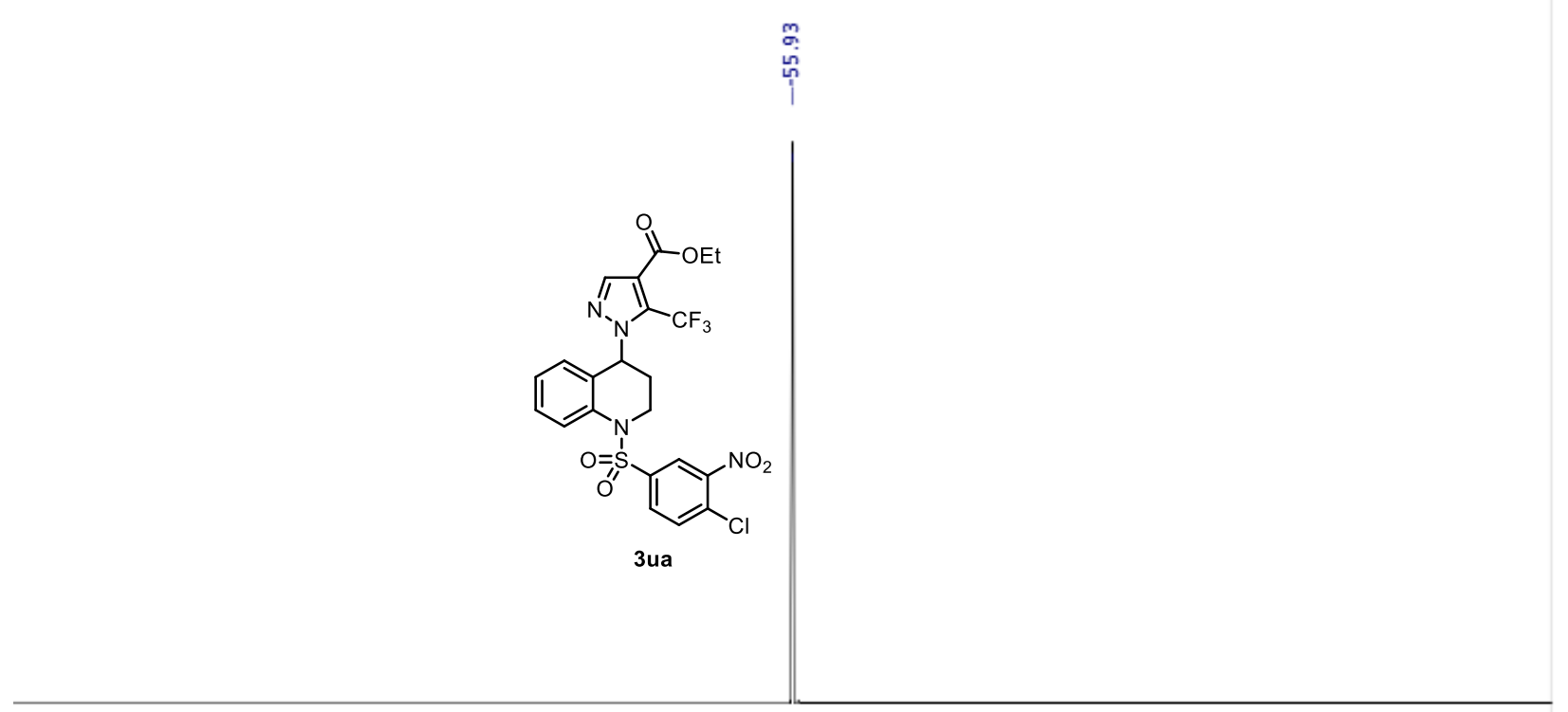

\footnotetext{
$\begin{array}{llllllllllllllllllllllllllll}5 & 0 & -5 & -10 & -15 & -20 & -25 & -30 & -35 & -40 & -45 & -50 & -55 & -60 & -65 & -70 & -75 & -80 & -85 & -90 & -95 & -100 & -105 & -110 & -115 & -1\end{array}$
} 
HSQC NMR spectrum of 3ua in $\mathrm{CDCl}_{3}$ containing $0.03 \%$ (v/v) TMS $(500,126 \mathrm{MHz})$.

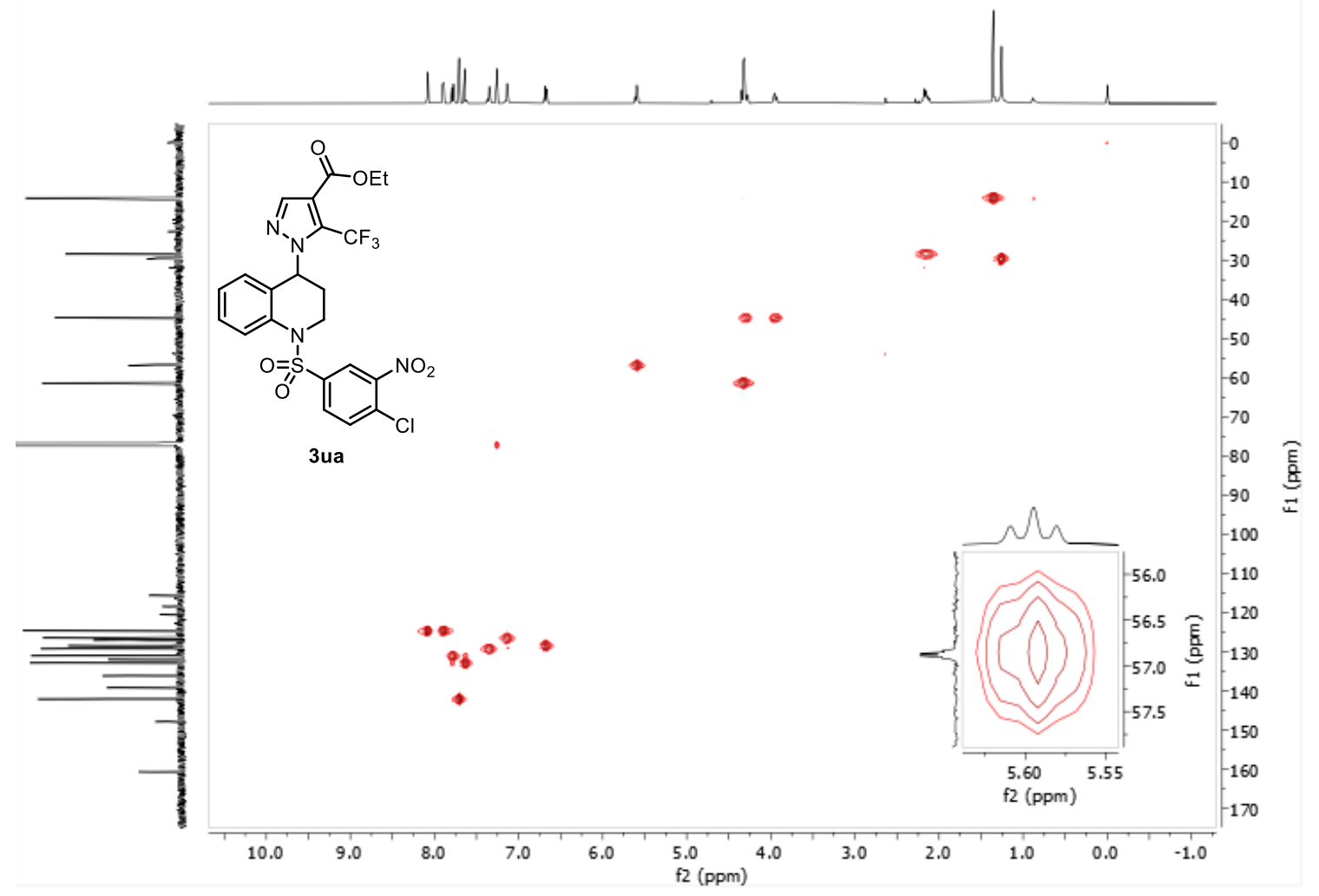

HMBC NMR spectrum of 3ua in $\mathrm{CDCl}_{3}$ containing $0.03 \%$ (v/v) TMS $(500,126 \mathrm{MHz}$ ).

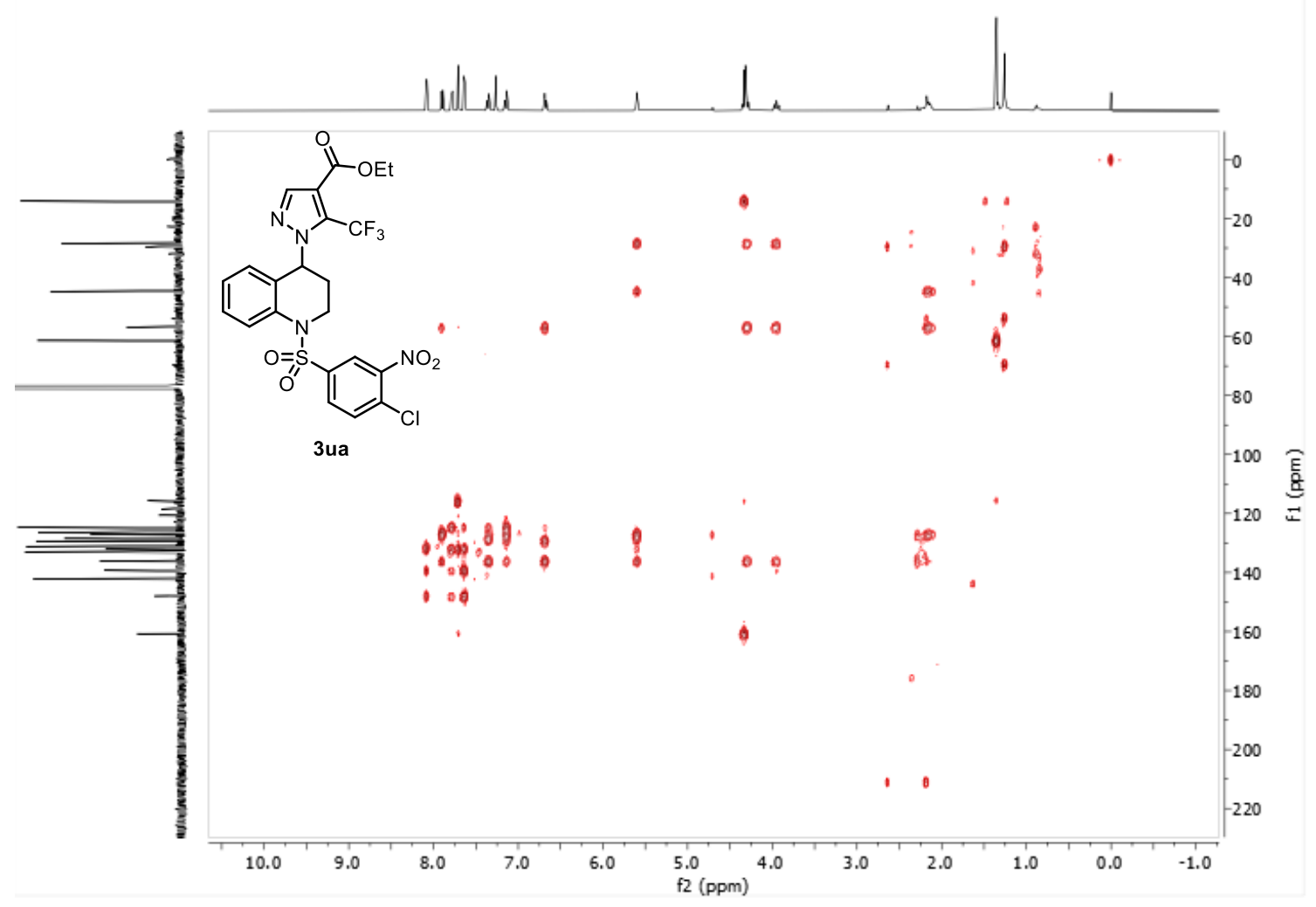


${ }^{1} \mathrm{H}$ NMR spectrum of $3 \mathrm{vc}$ in $\mathrm{CDCl}_{3}$ containing $0.03 \%$ (v/v) TMS (500 MHz).

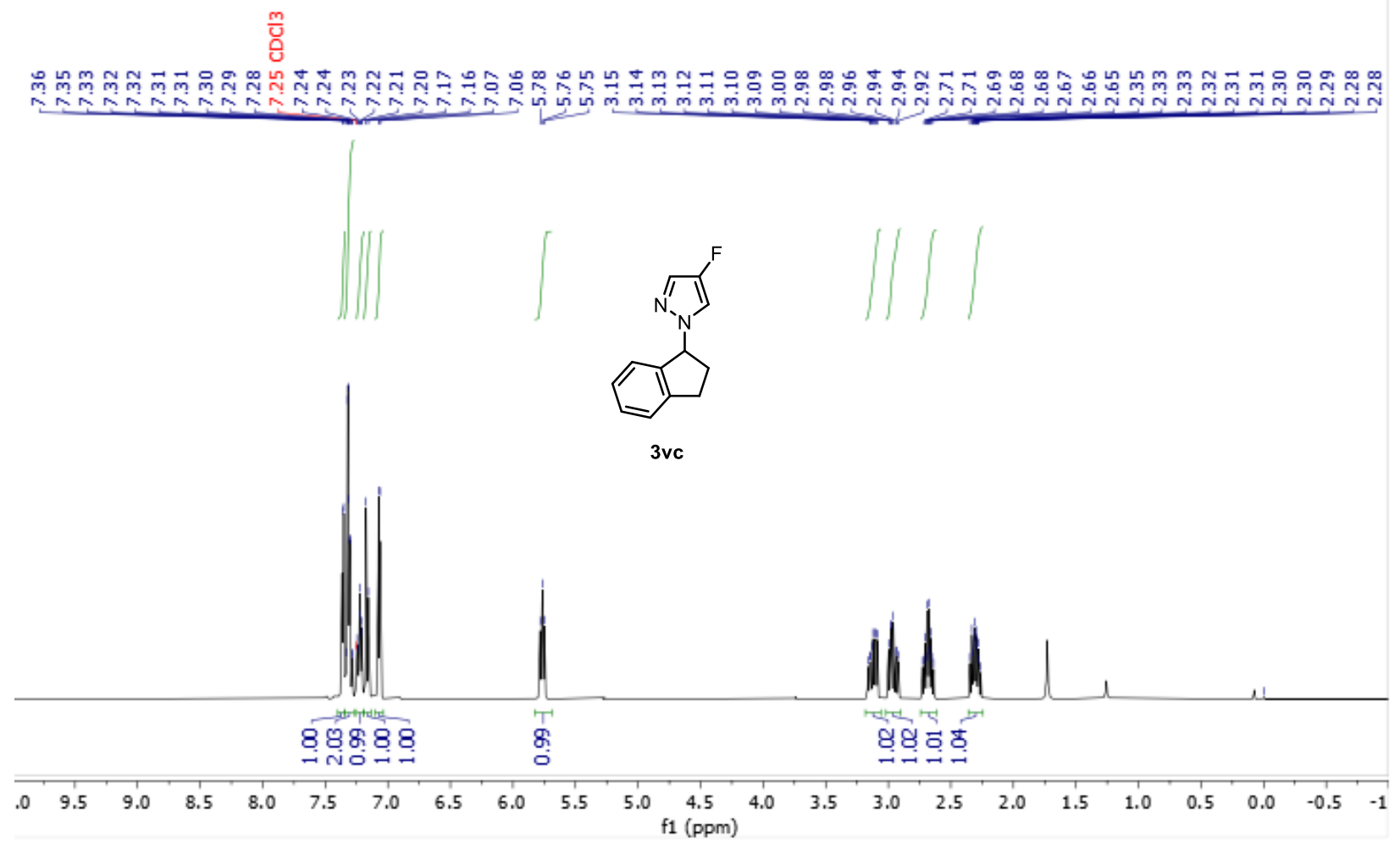

${ }^{13} \mathrm{C}$ NMR spectrum of $\mathbf{3 v c}$ in $\mathrm{CDCl}_{3}$ containing $0.03 \%$ (v/v) TMS (126 MHz).

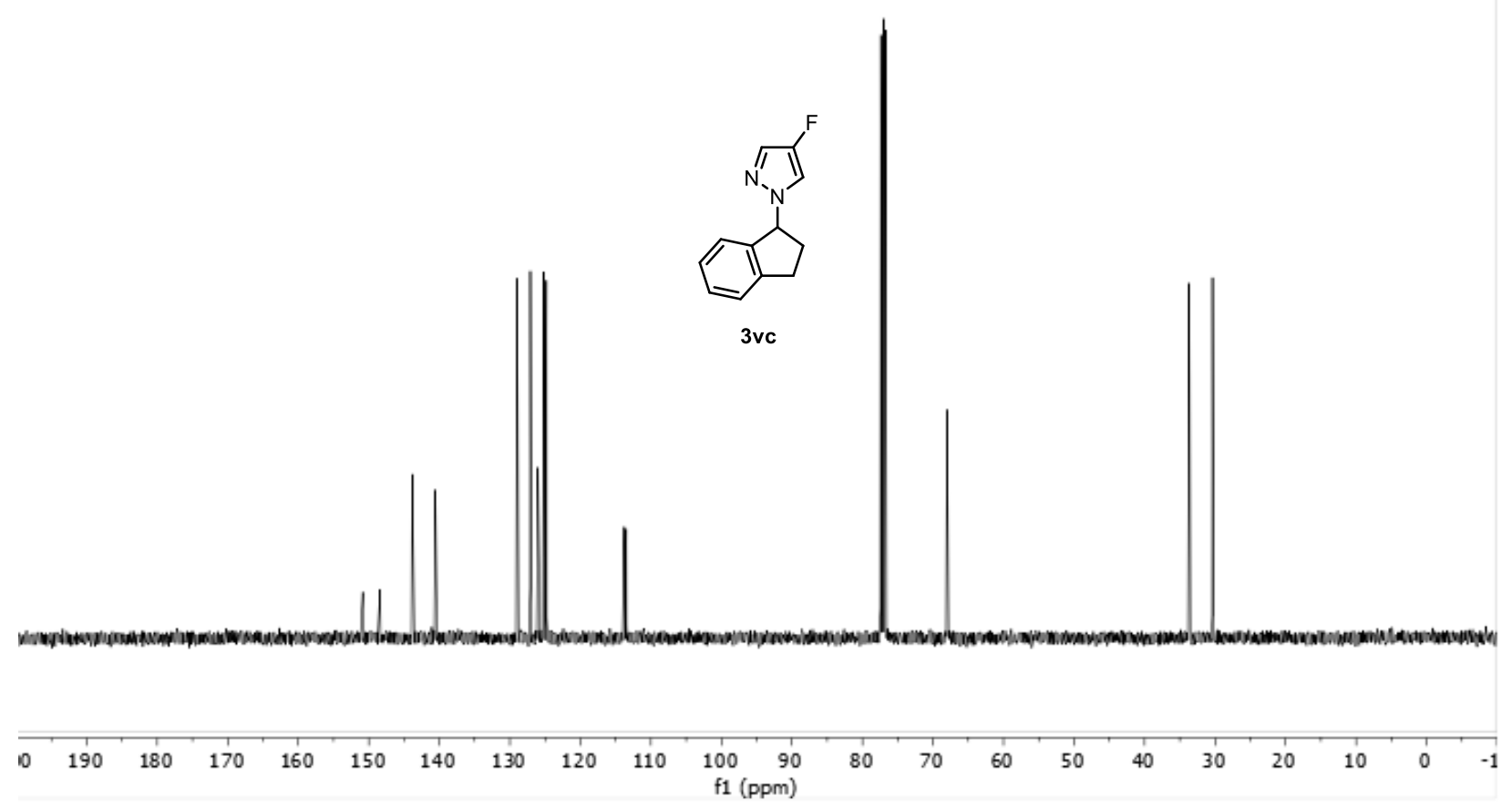


${ }^{19} \mathrm{~F}$ NMR spectrum of $3 \mathbf{v c}$ in $\mathrm{CDCl}_{3}$ containing $0.03 \%$ (v/v) TMS (377 MHz).

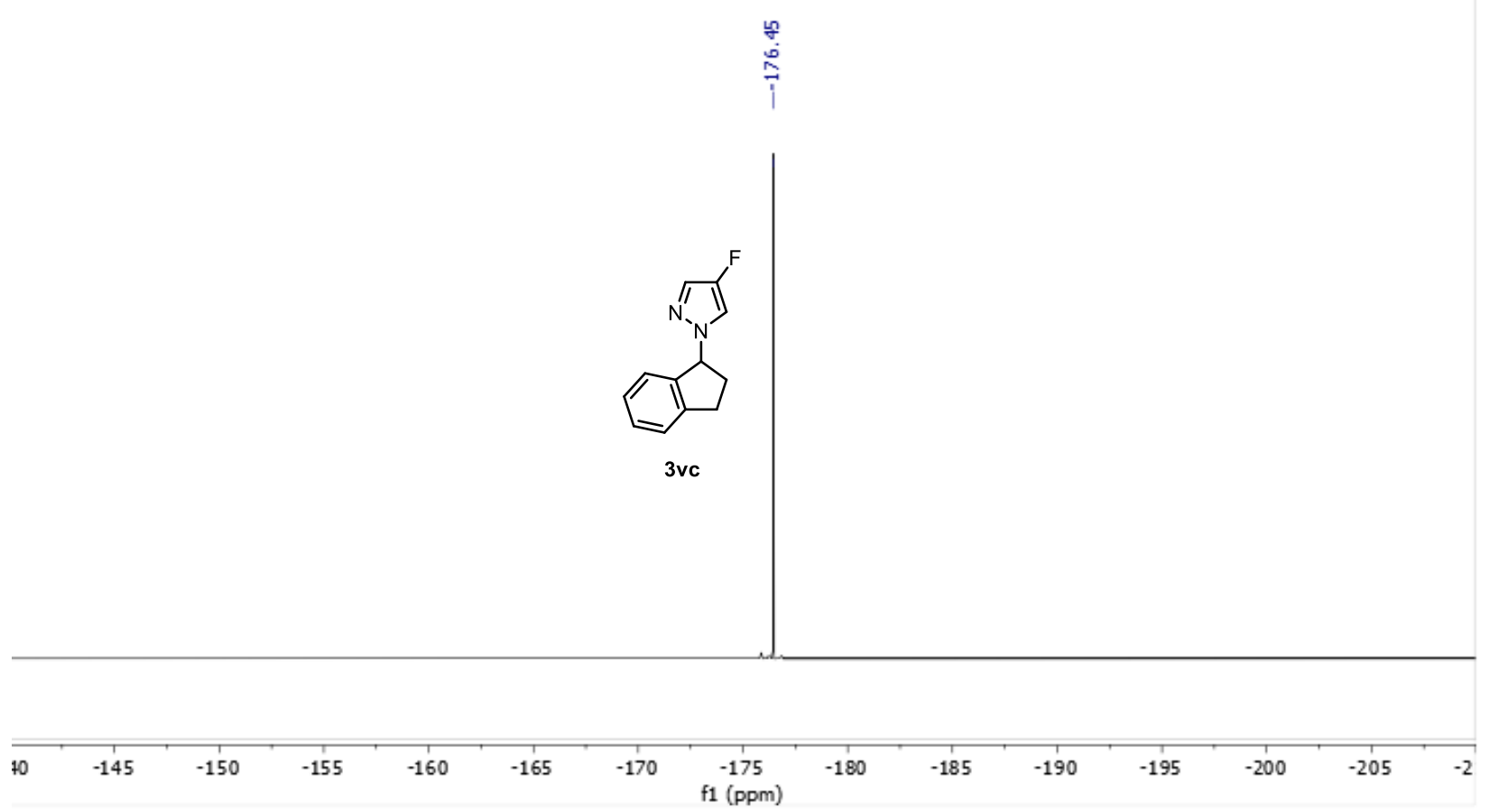

${ }^{1} \mathrm{H}$ NMR spectrum of $\mathbf{3 v d}$ in $\mathrm{CDCl}_{3}$ containing $0.03 \%$ (v/v) TMS (500 MHz).

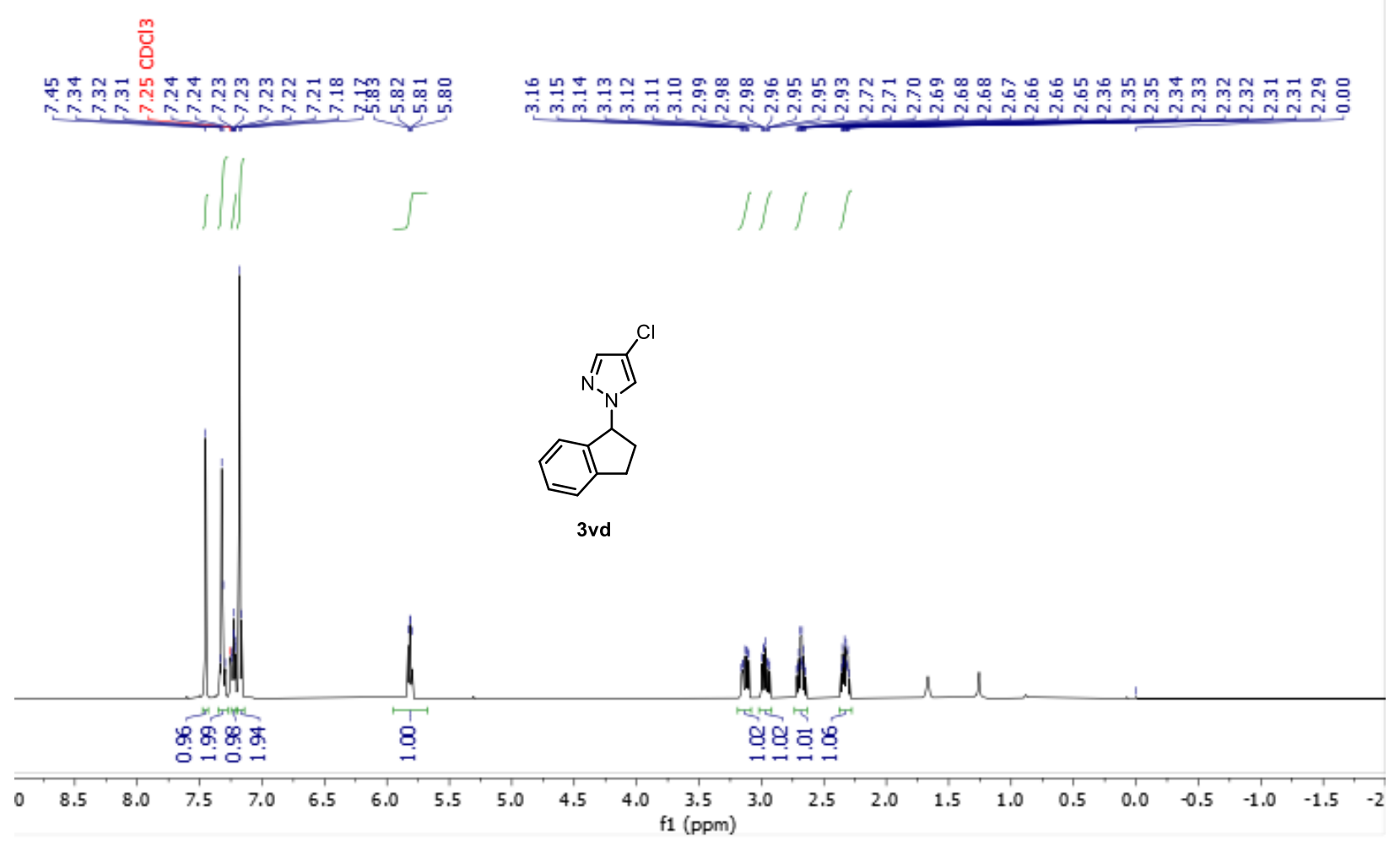


${ }^{13} \mathrm{C}$ NMR spectrum of $\mathbf{3 v d}$ in $\mathrm{CDCl}_{3}$ containing $0.03 \%$ (v/v) TMS (126 MHz).
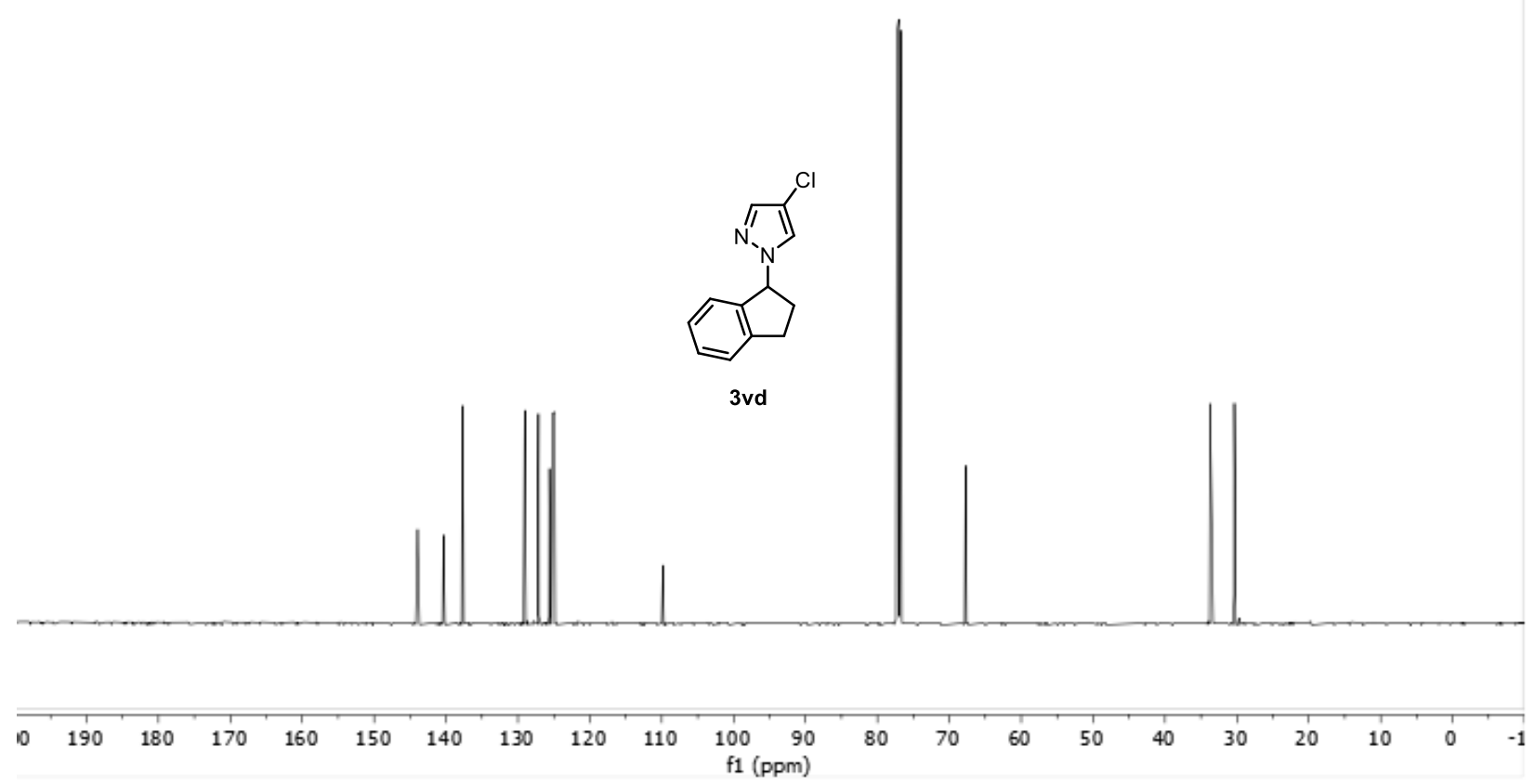

${ }^{1} \mathrm{H}$ NMR spectrum of 3ve in $\mathrm{CDCl}_{3}$ containing $0.03 \%$ (v/v) TMS (500 MHz).

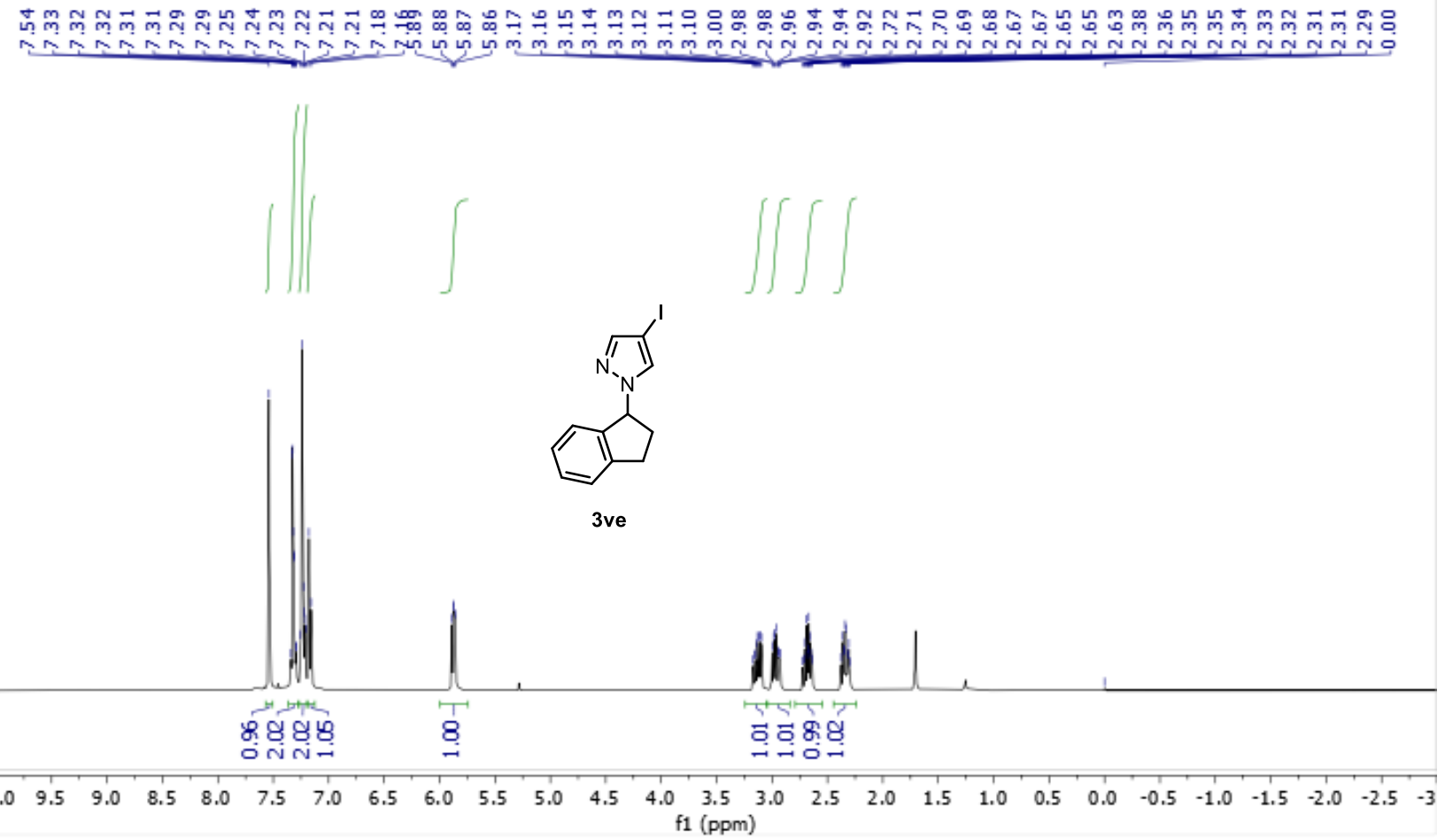


${ }^{13} \mathrm{C}$ NMR spectrum of 3 ve in $\mathrm{CDCl}_{3}$ containing $0.03 \%$ (v/v) TMS (126 MHz).

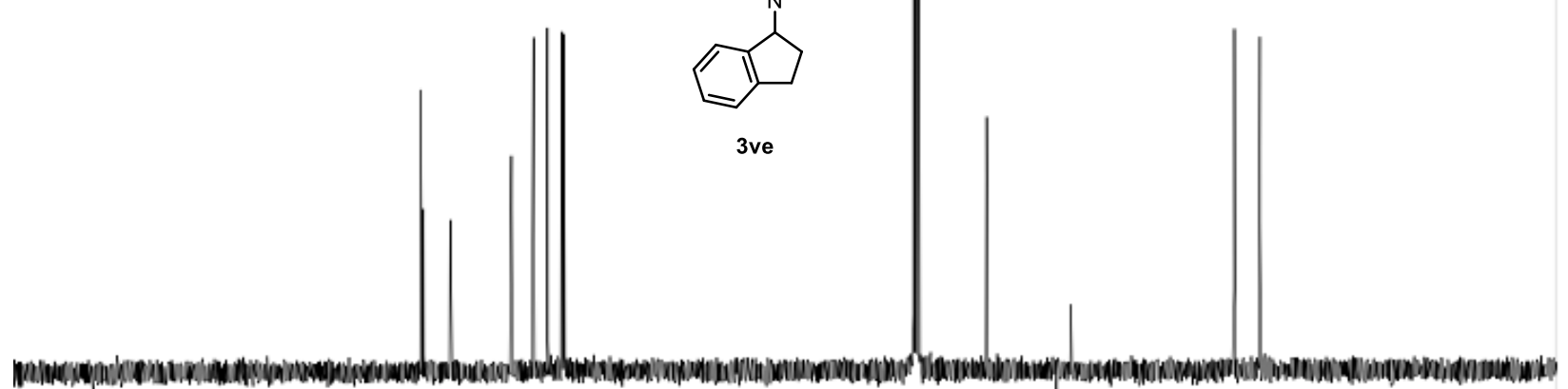

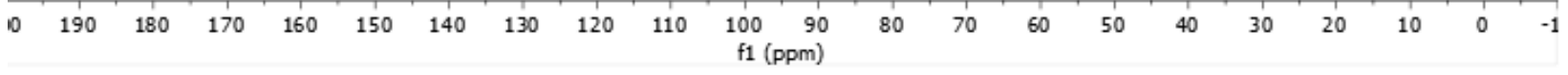

${ }^{1} \mathrm{H}$ NMR spectrum of $\mathbf{3 v f}$ in $\mathrm{CDCl}_{3}$ containing $0.03 \%$ (v/v) TMS (500 MHz).

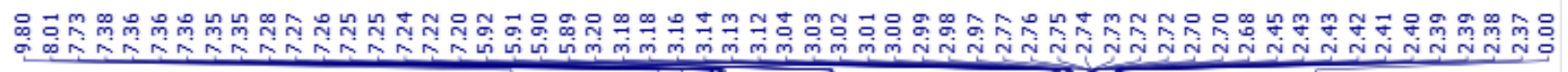

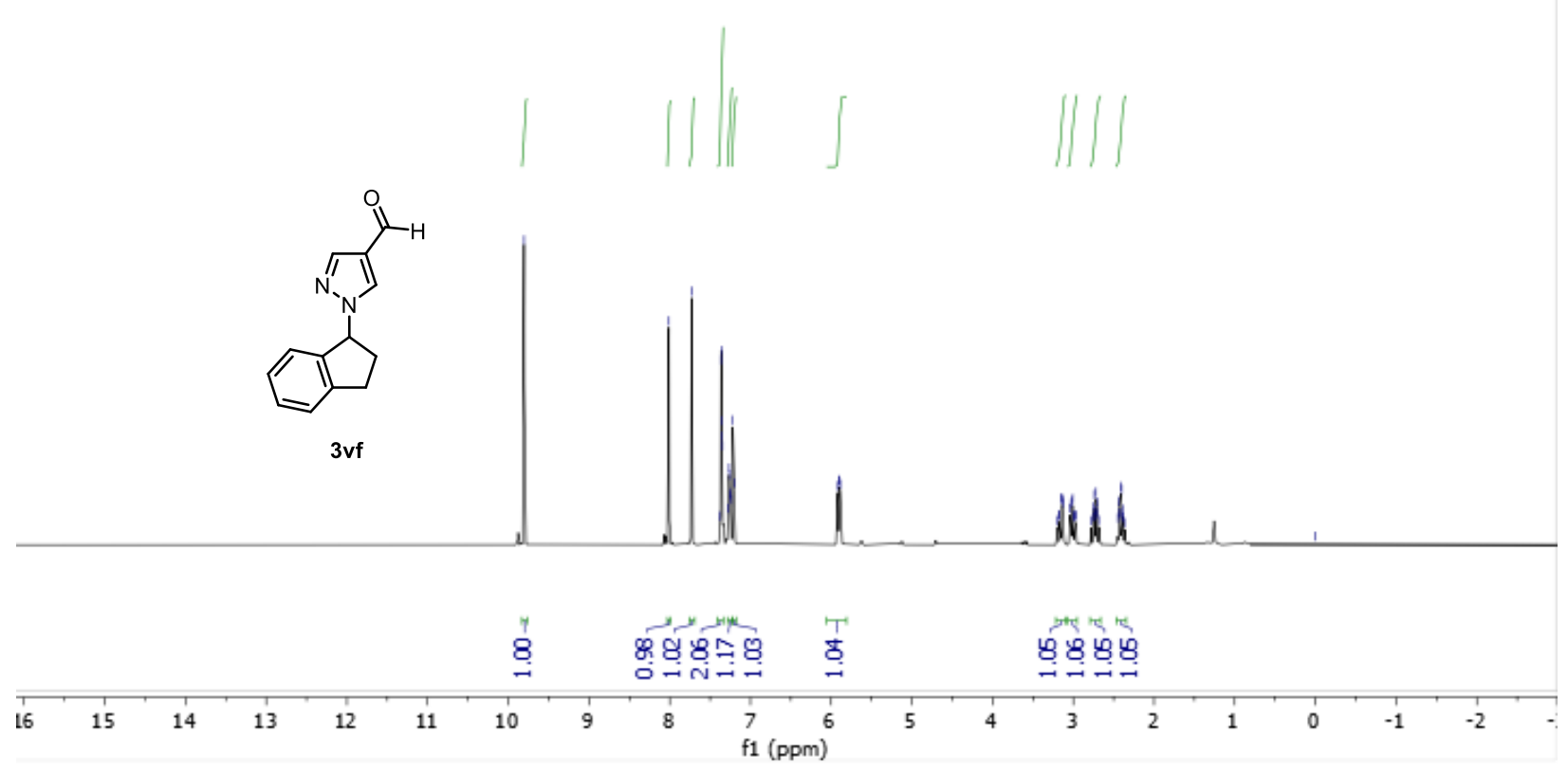


${ }^{13} \mathrm{C}$ NMR spectrum of $\mathbf{3 v f}$ in $\mathrm{CDCl}_{3}$ containing $0.03 \%$ (v/v) TMS (126 MHz).

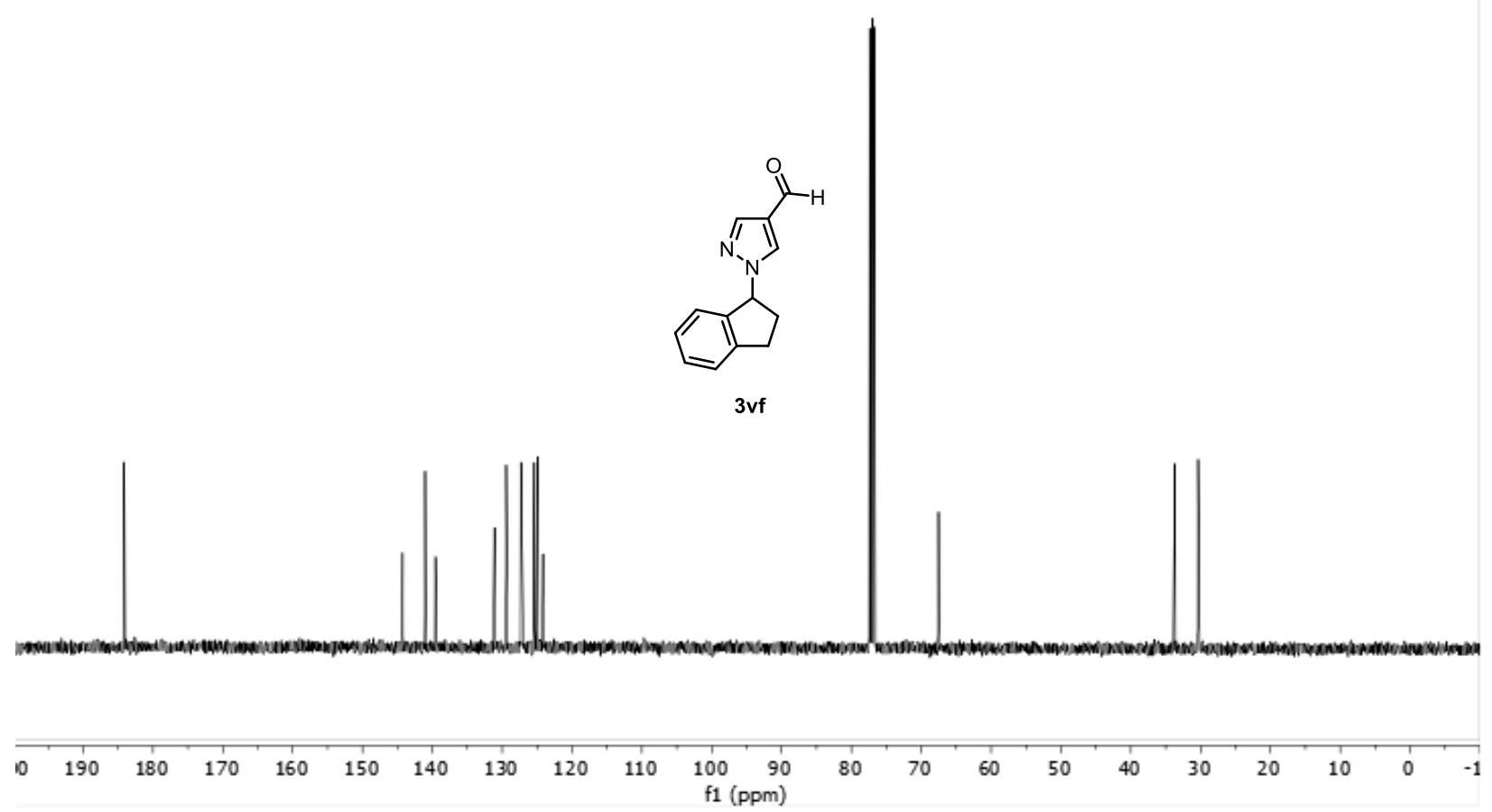

${ }^{1} \mathrm{H}$ NMR spectrum of $\mathbf{3 v g}$ in $\mathrm{CDCl}_{3}$ containing $0.03 \%$ (v/v) TMS (500 MHz).

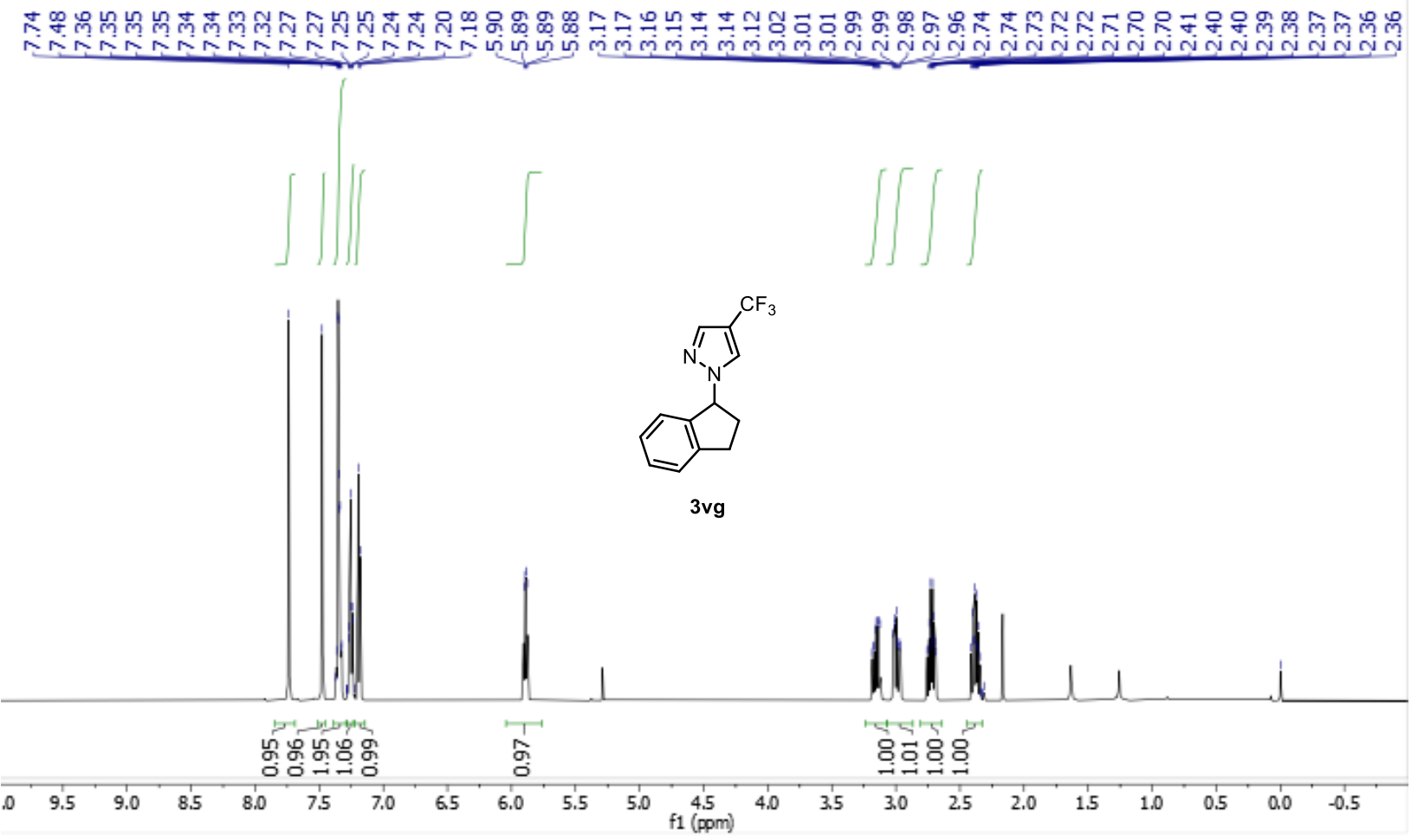


${ }^{13} \mathrm{C}$ NMR spectrum of $\mathbf{3 v g}$ in $\mathrm{CDCl}_{3}$ containing $0.03 \%$ (v/v) TMS (126 MHz).

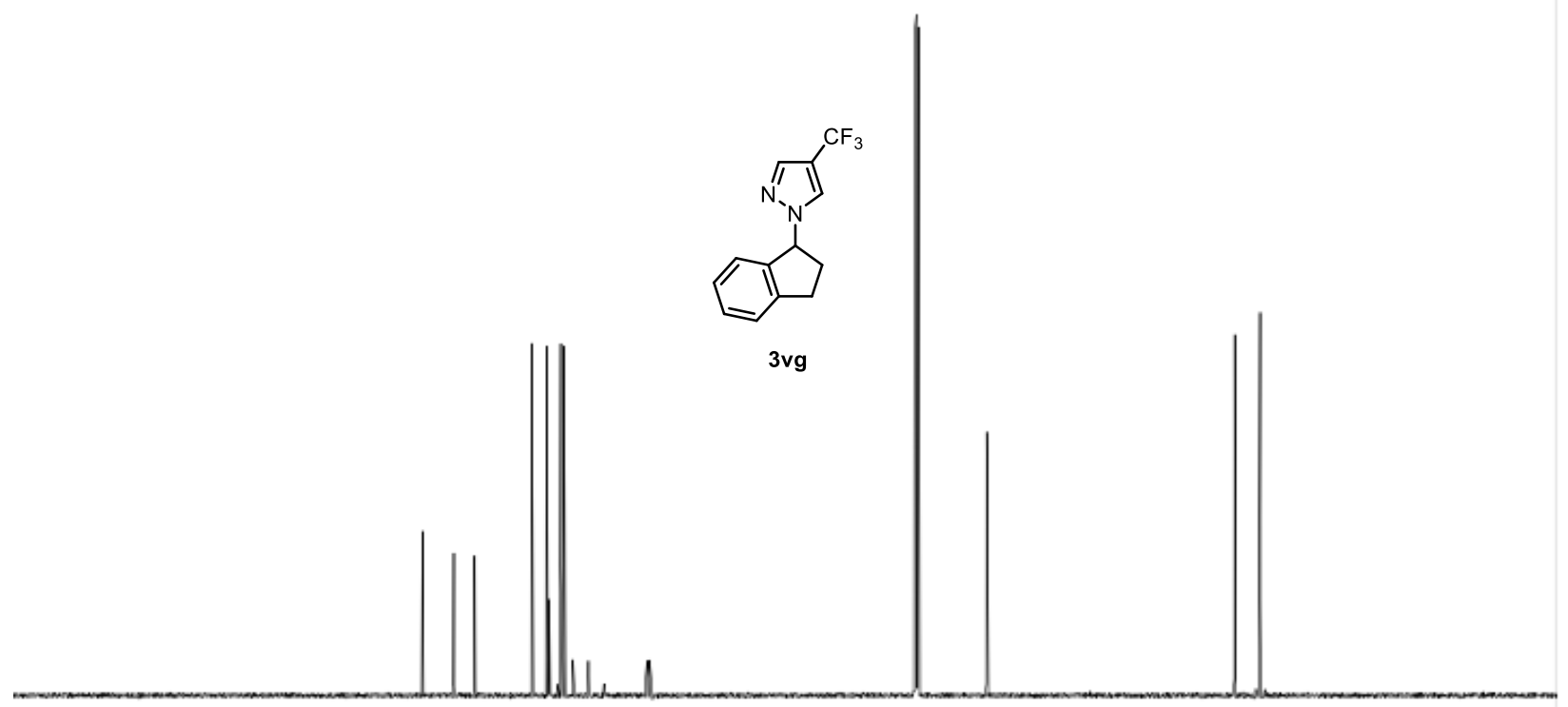

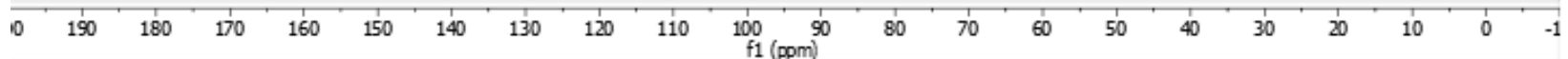

${ }^{19} \mathrm{~F}$ NMR spectrum of $\mathbf{3 v g}$ in $\mathrm{CDCl}_{3}$ containing $0.03 \%$ (v/v) TMS (377 MHz).

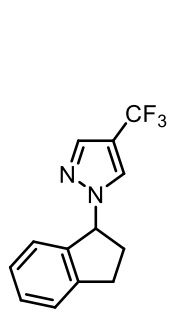

$3 \mathrm{vg}$

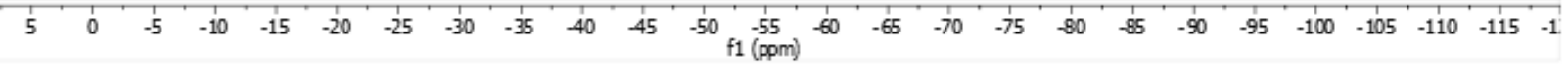


${ }^{1} \mathrm{H}$ NMR spectrum of $\mathbf{3 v h}$ in $\mathrm{CDCl}_{3}$ containing $0.03 \%$ (v/v) TMS (500 MHz).

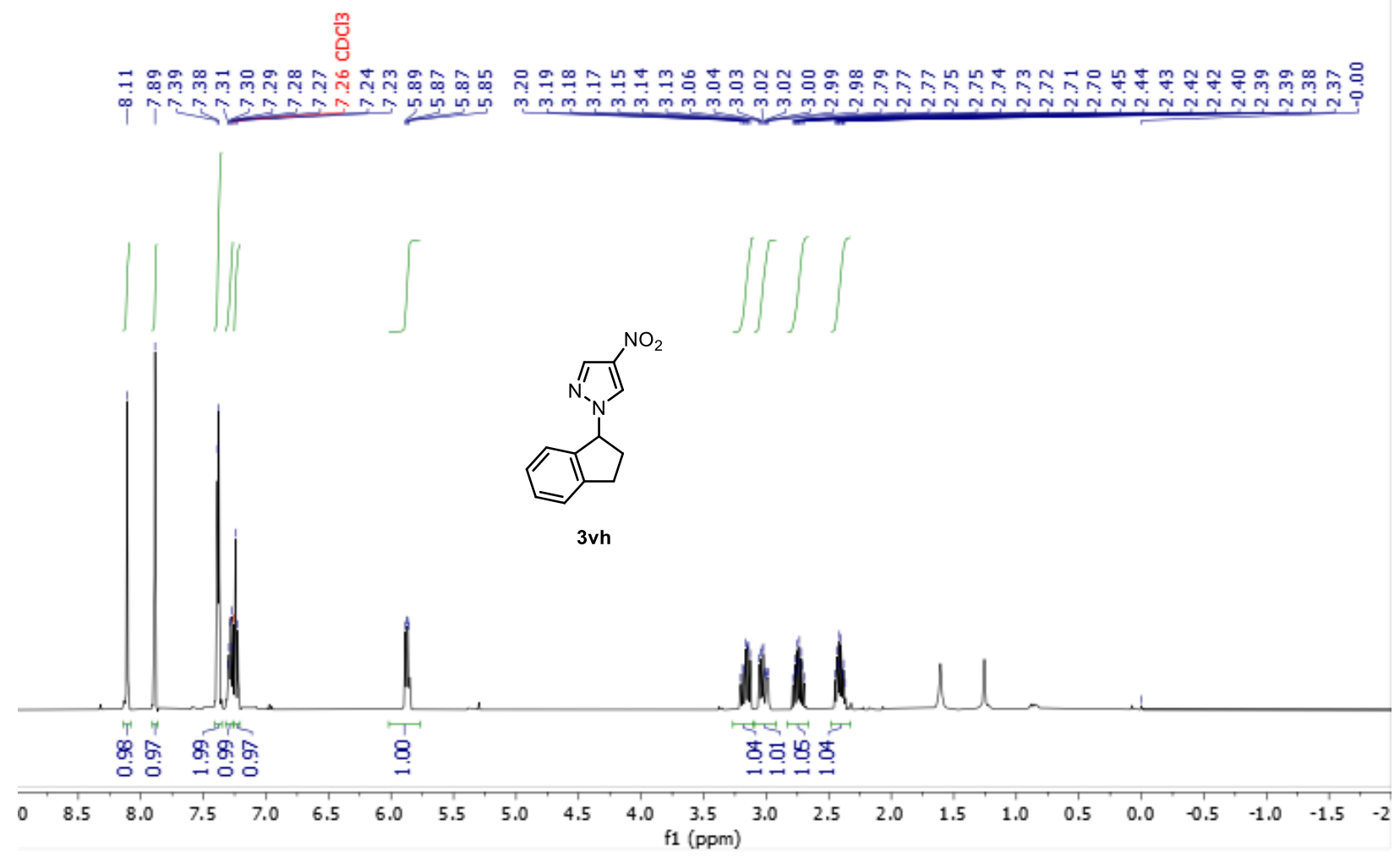

${ }^{13} \mathrm{C}$ NMR spectrum of $\mathbf{3 v h}$ in $\mathrm{CDCl}_{3}$ containing $0.03 \%$ (v/v) TMS (126 MHz).

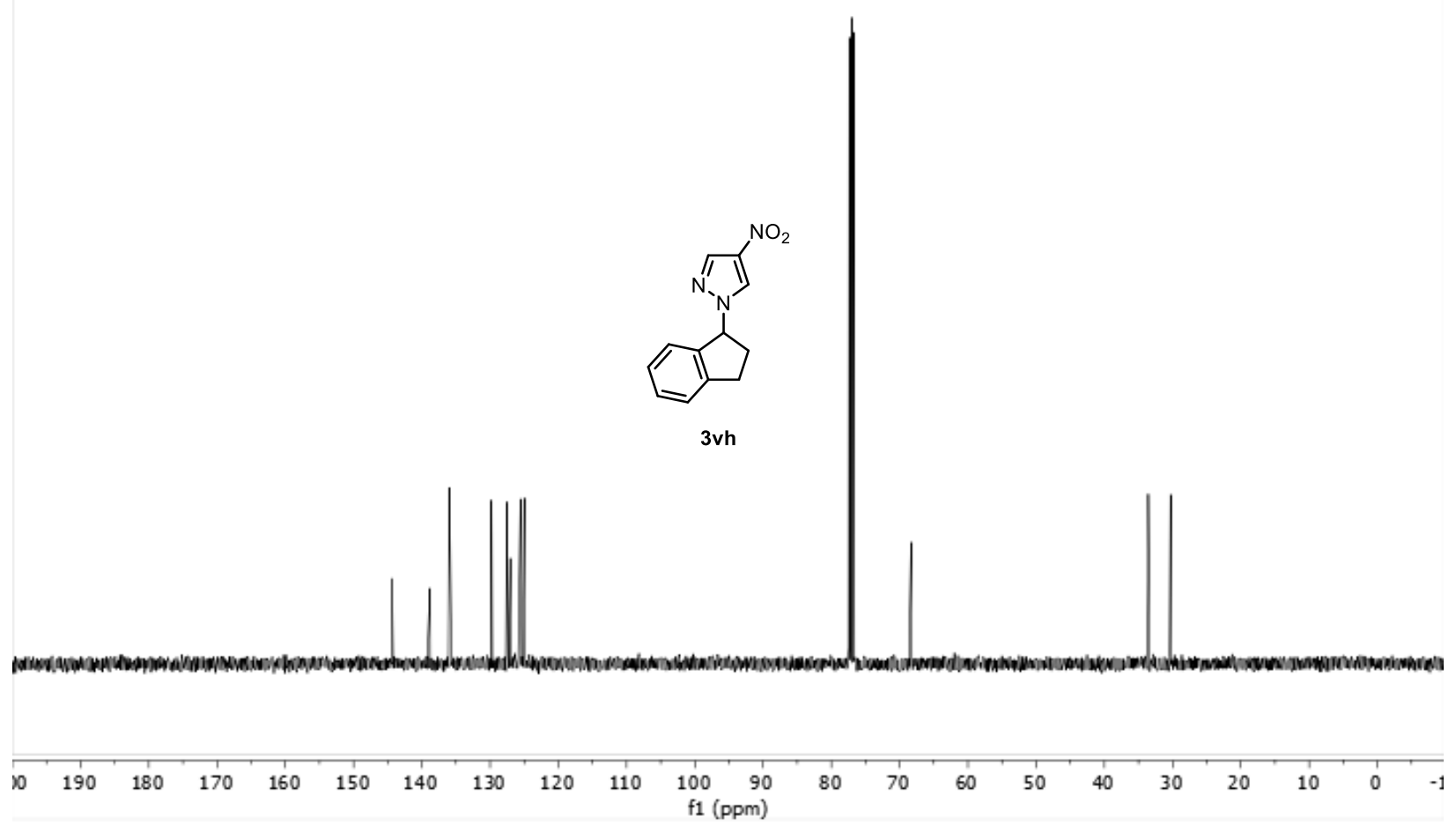


${ }^{1} \mathrm{H}$ NMR spectrum of $\mathbf{3 v c}$ in $\mathrm{CDCl}_{3}$ containing $0.03 \%$ (v/v) TMS (500 MHz).

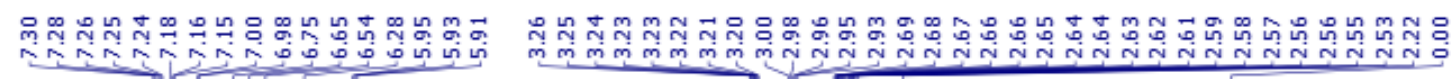
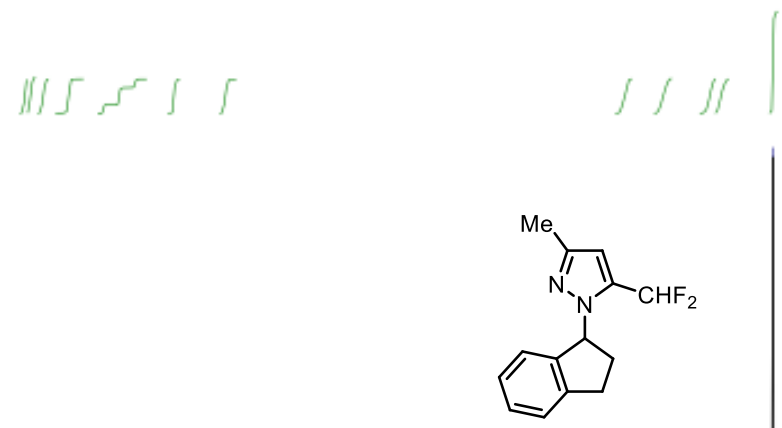

$3 \mathbf{v i}$

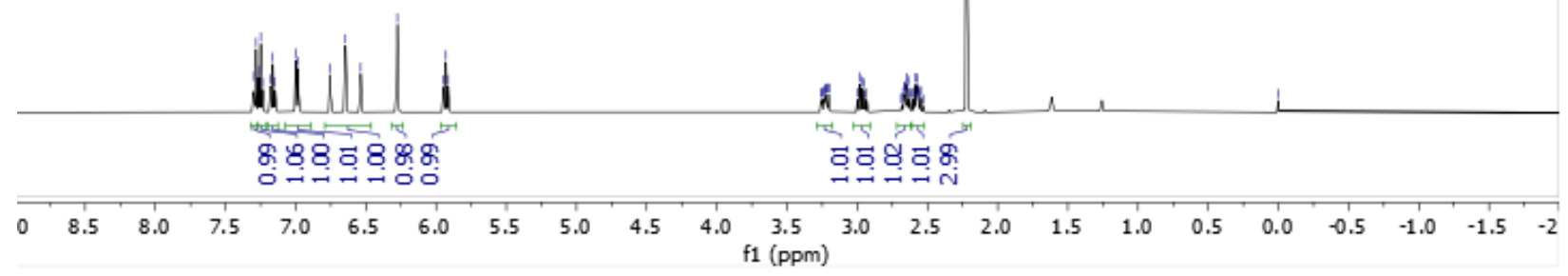

${ }^{13} \mathrm{C}$ NMR spectrum of $\mathbf{3 v c}$ in $\mathrm{CDCl}_{3}$ containing $0.03 \%$ (v/v) TMS (126 MHz).

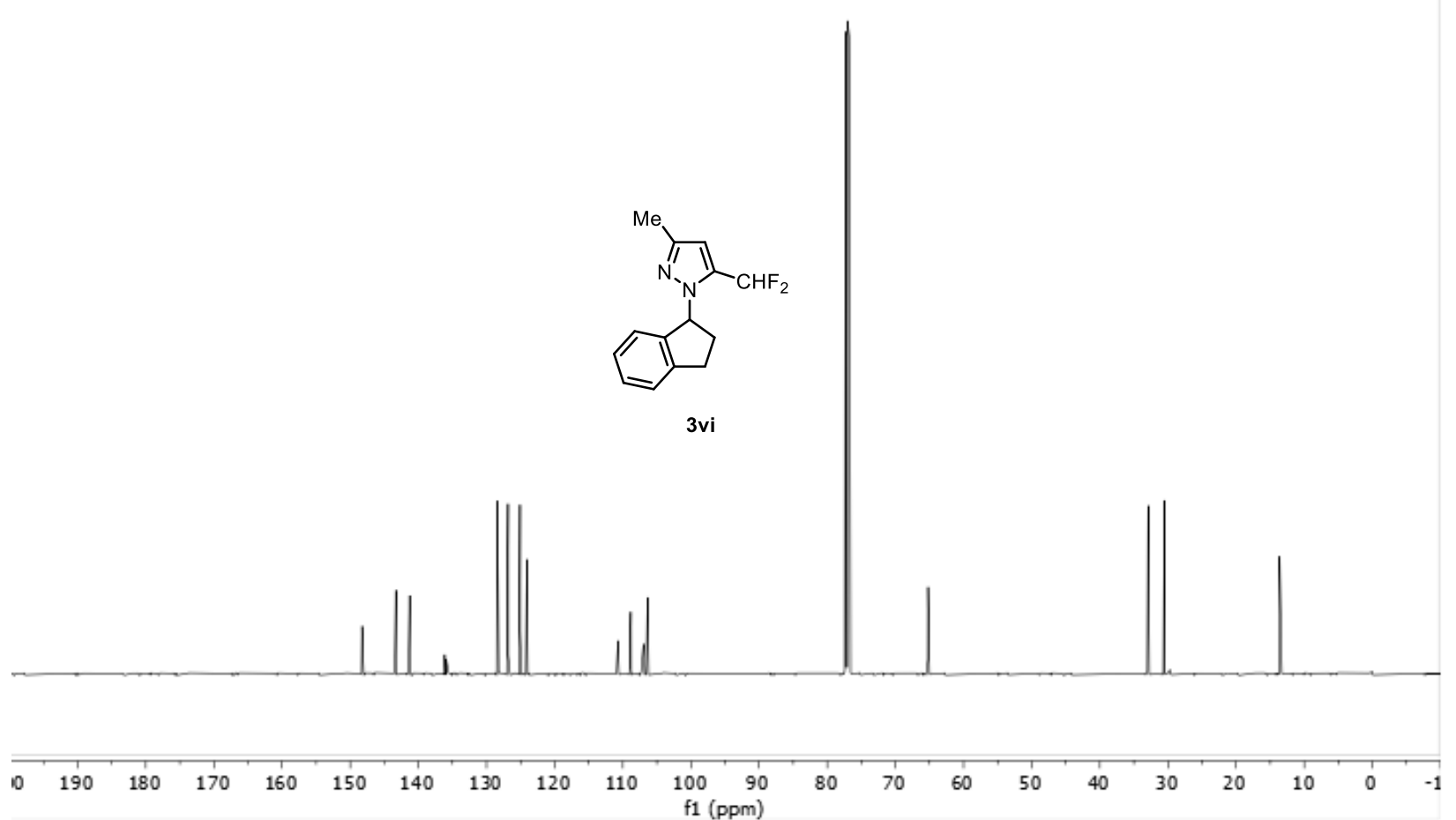


${ }^{19} \mathrm{~F}$ NMR spectrum of $3 \mathbf{v c}$ in $\mathrm{CDCl}_{3}$ containing $0.03 \%$ (v/v) TMS (377 MHz).

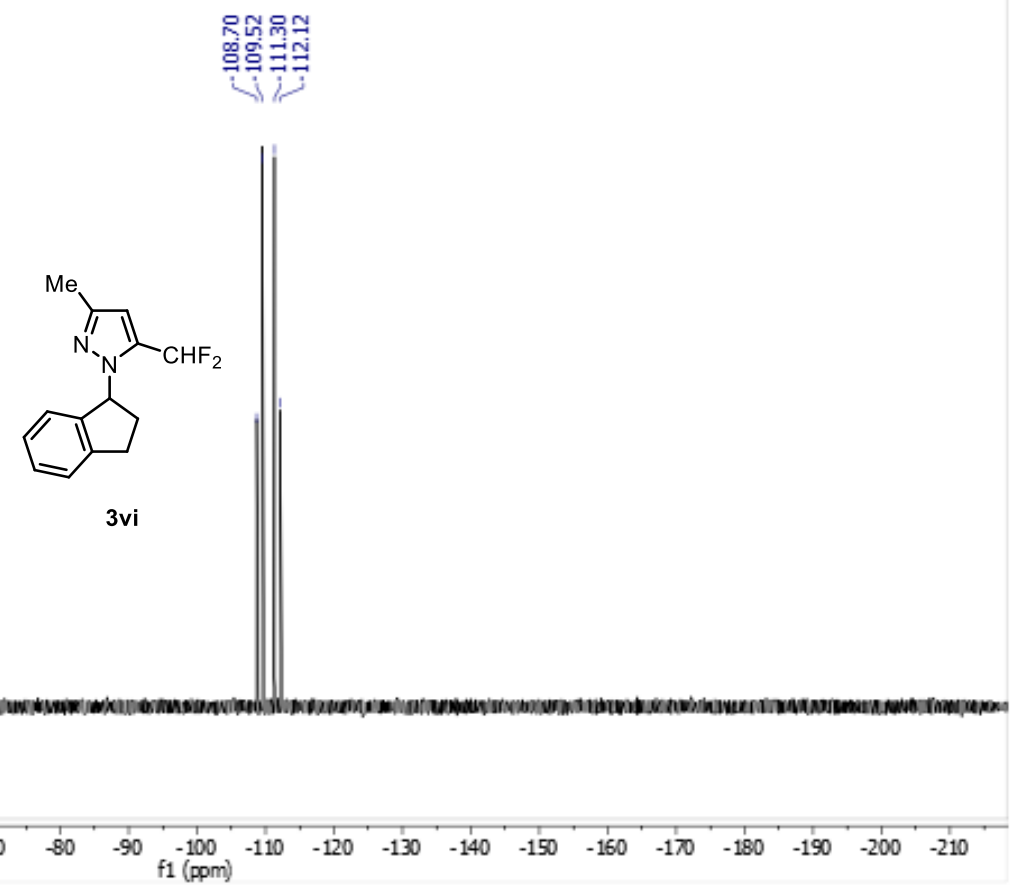

HSQC NMR spectrum of 3ve in $\mathrm{CDCl}_{3}$ containing $0.03 \%$ (v/v) TMS (500, $126 \mathrm{MHz}$ ).

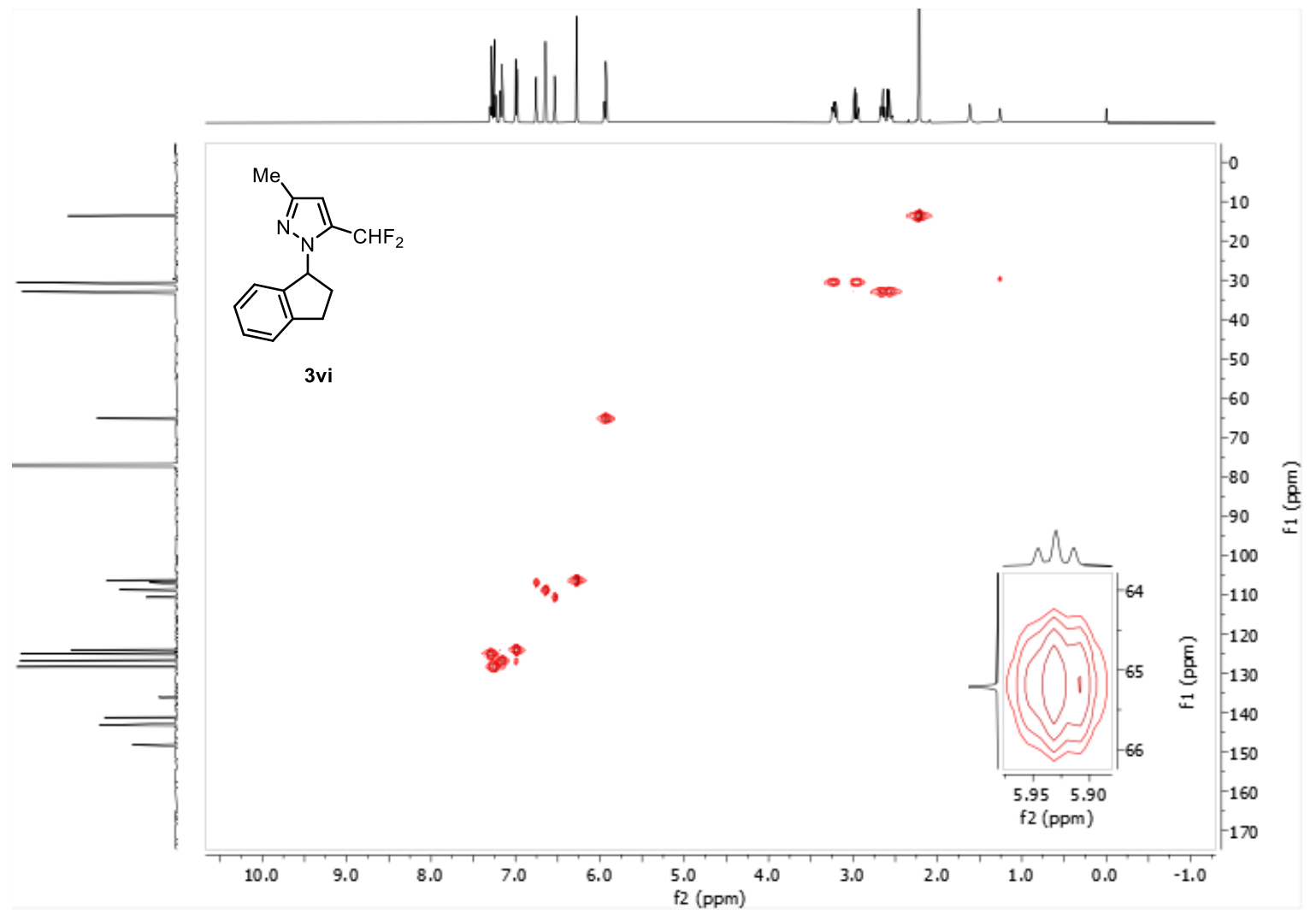


HMBC NMR spectrum of $3 \mathrm{vc}$ in $\mathrm{CDCl}_{3}$ containing $0.03 \%$ (v/v) TMS (500, $126 \mathrm{MHz}$ ).

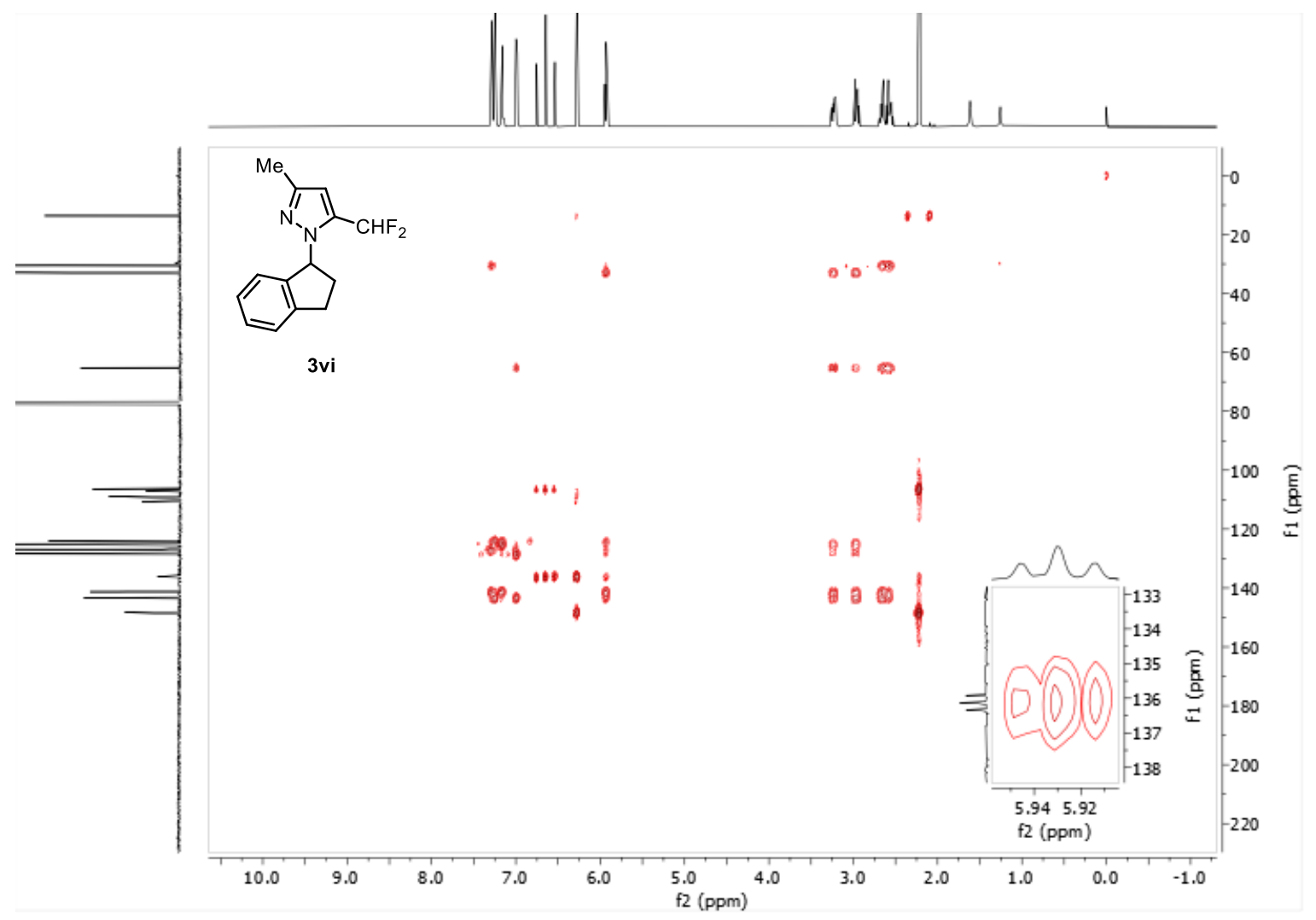

${ }^{1} \mathrm{H}$ NMR spectrum of $\mathbf{3 v j}$ in $\mathrm{CDCl}_{3}$ containing $0.03 \%$ (v/v) TMS (500 MHz).

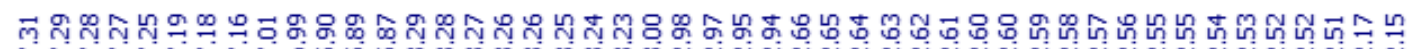

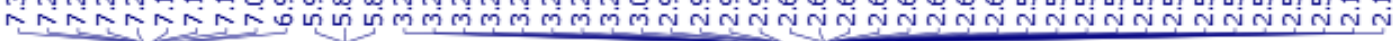
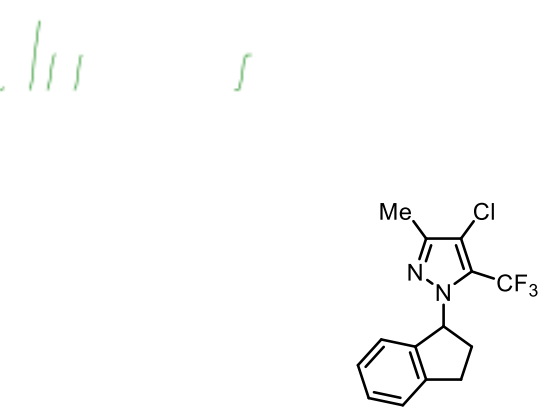

$3 \mathbf{j}$

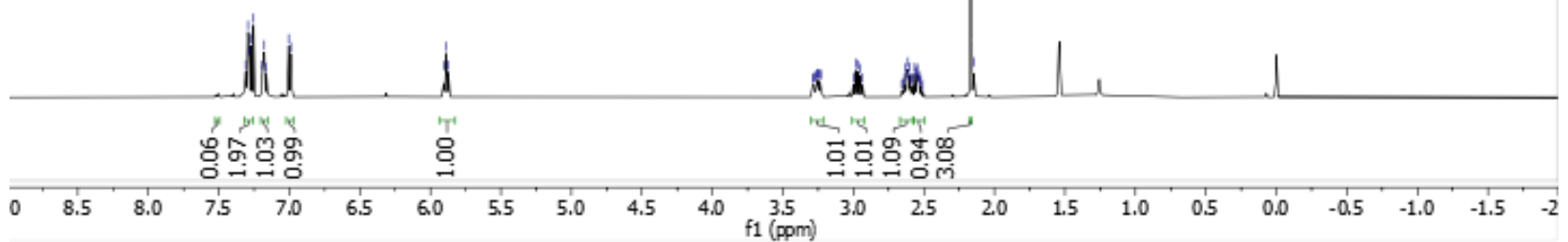


${ }^{13} \mathrm{C}$ NMR spectrum of $\mathbf{3 v j}$ in $\mathrm{CDCl}_{3}$ containing $0.03 \%$ (v/v) TMS (126 MHz).
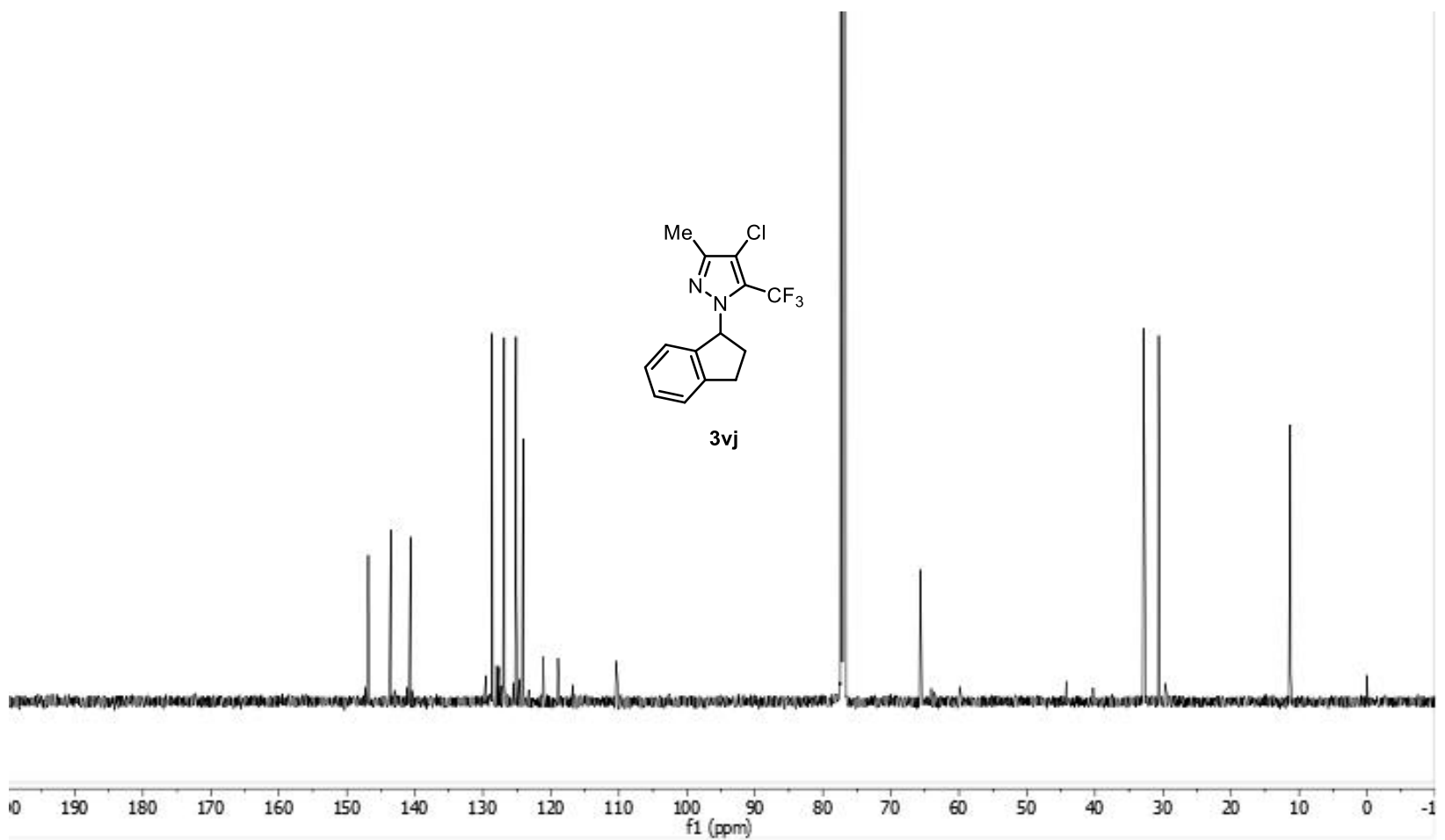

${ }^{19} \mathrm{~F}$ NMR spectrum of $\mathbf{3 v j}$ in $\mathrm{CDCl}_{3}$ containing $0.03 \%$ (v/v) TMS (377 MHz).

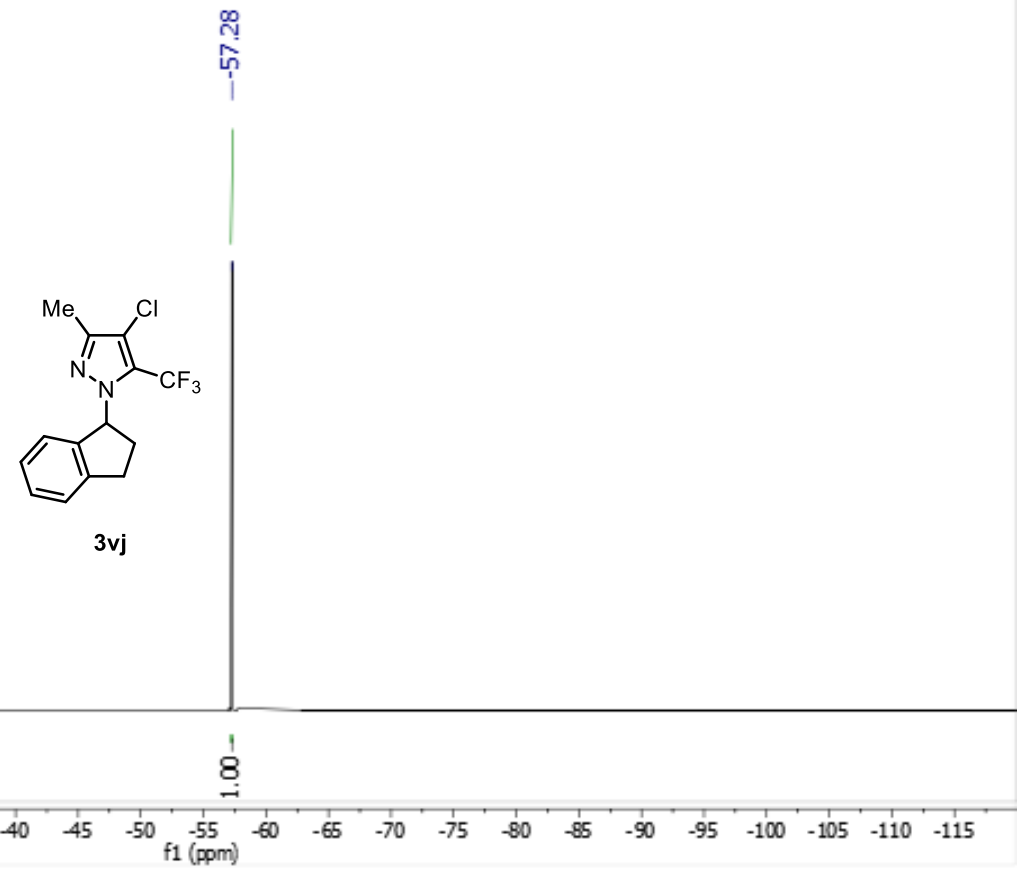


HSQC NMR spectrum of $\mathbf{3 v j}$ in $\mathrm{CDCl}_{3}$ containing $0.03 \%$ (v/v) TMS (500, $126 \mathrm{MHz}$ ).

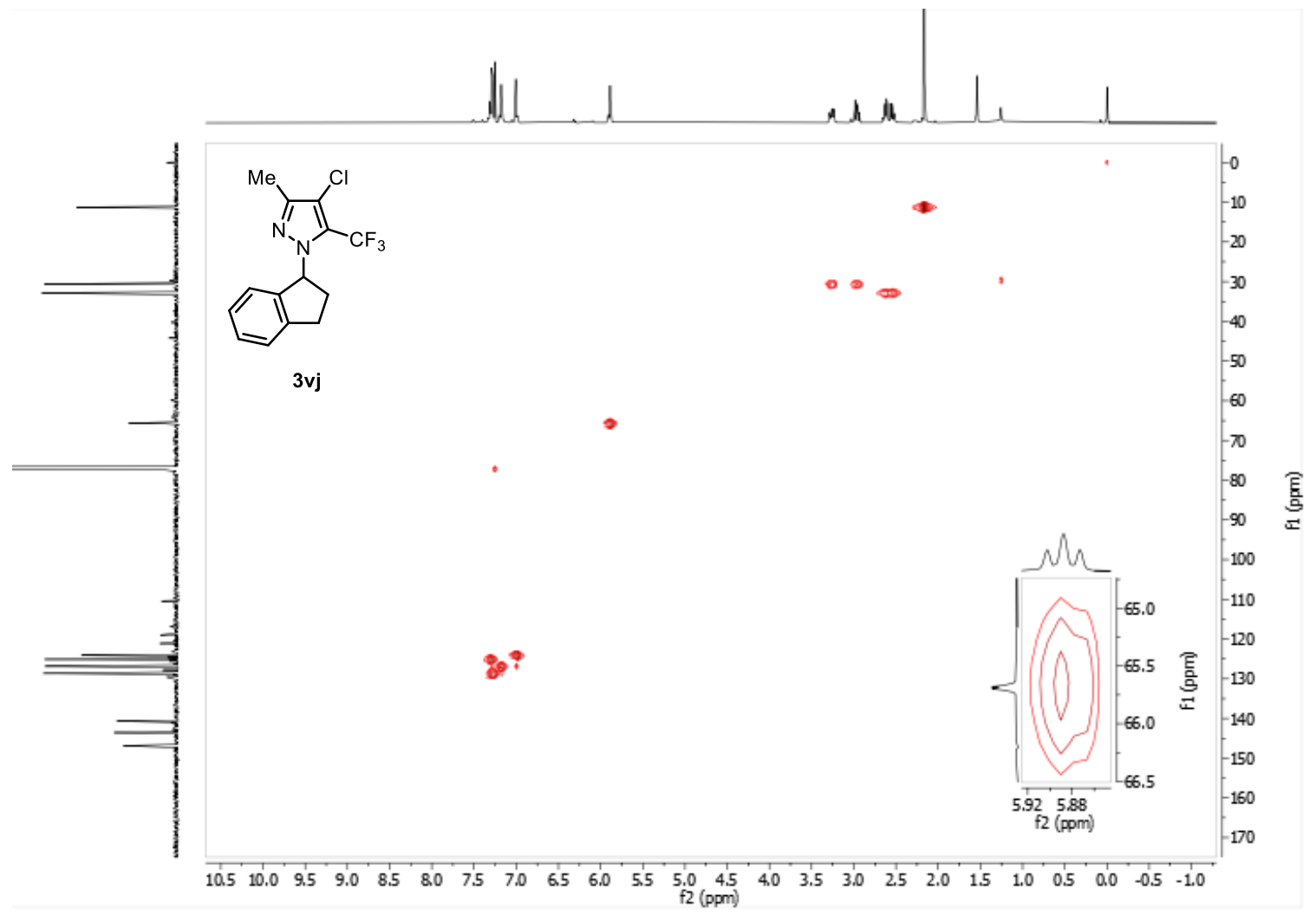

HMBC NMR spectrum of $\mathbf{3 v j}$ in $\mathrm{CDCl}_{3}$ containing $0.03 \%$ (v/v) TMS (500, $126 \mathrm{MHz}$ ).

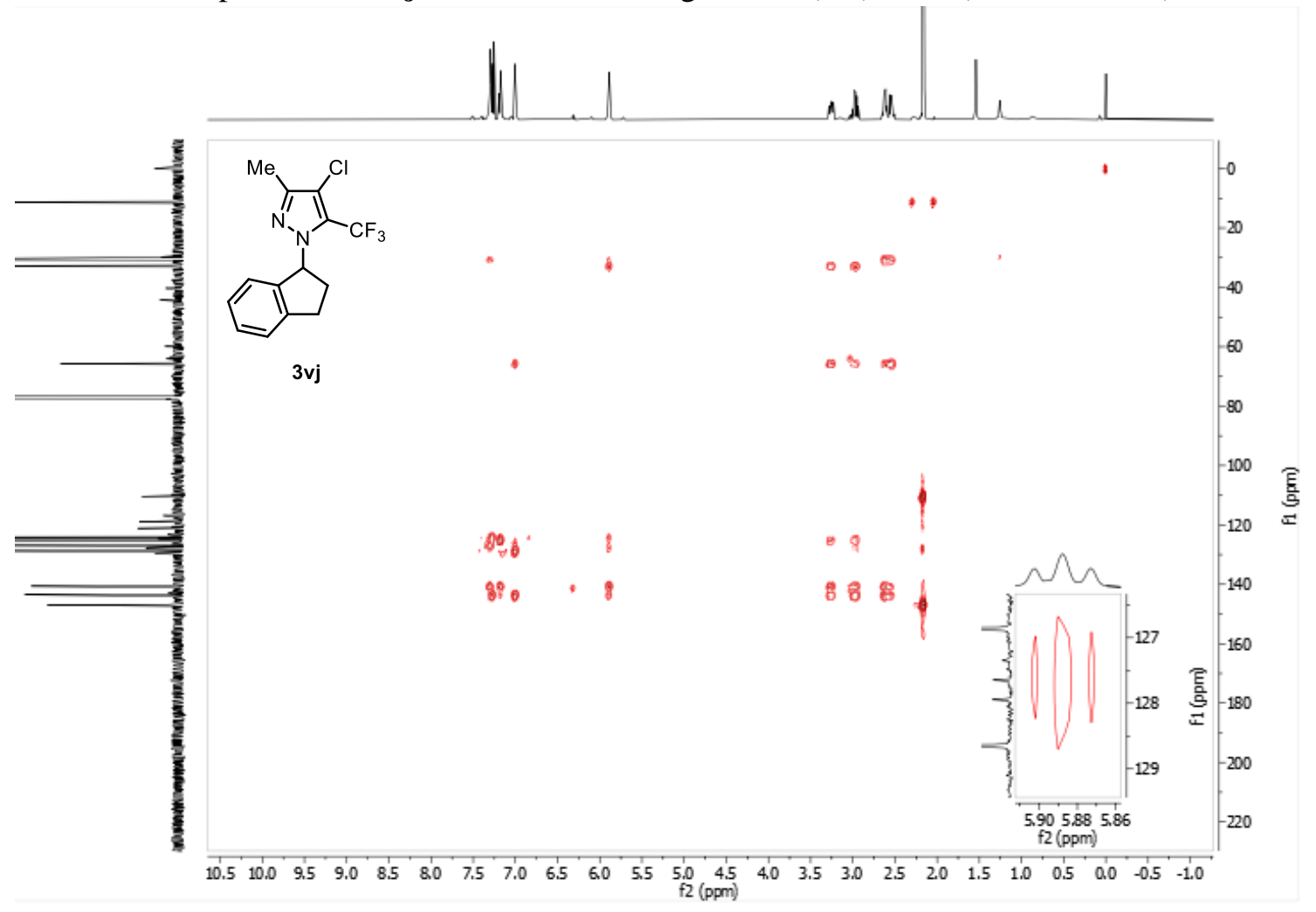


${ }^{1} \mathrm{H}$ NMR spectrum of $\mathbf{3 v j}$ ' in $\mathrm{CDCl}_{3}$ containing $0.03 \%$ (v/v) TMS (500 MHz).

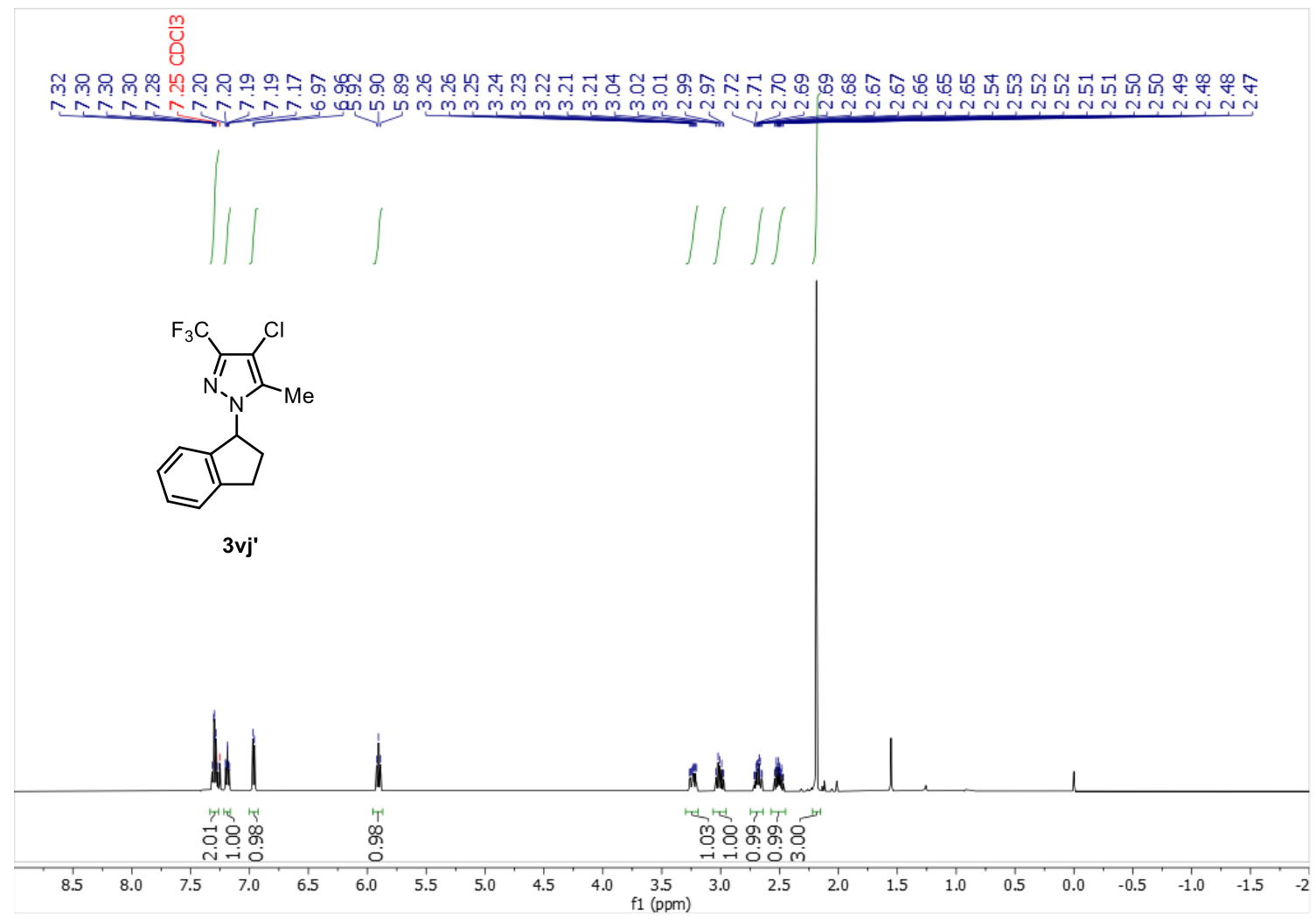

${ }^{13} \mathrm{C}$ NMR spectrum of $\mathbf{3 v j}$ ' in $\mathrm{CDCl}_{3}$ containing $0.03 \%$ (v/v) TMS (126 MHz).

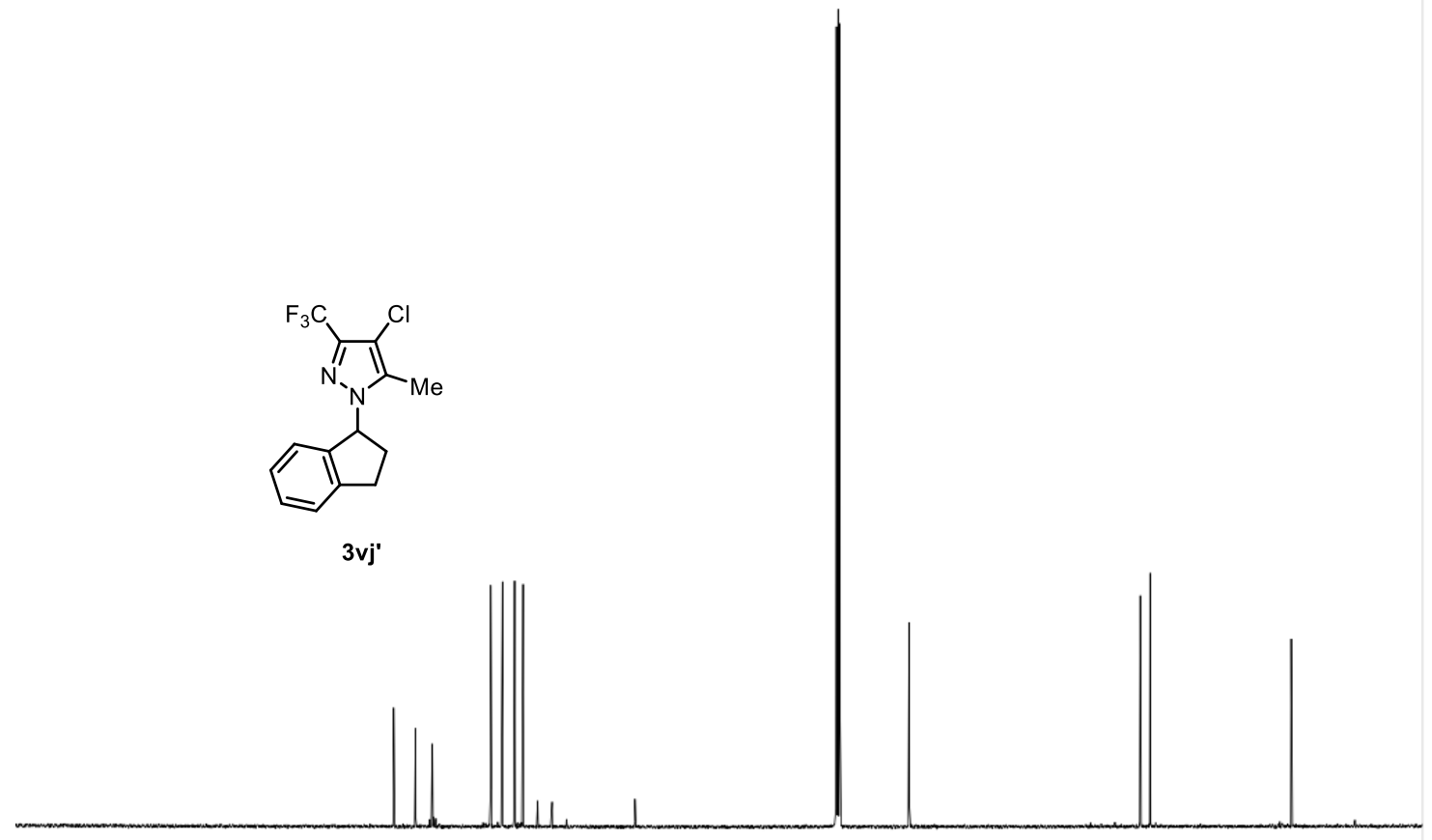

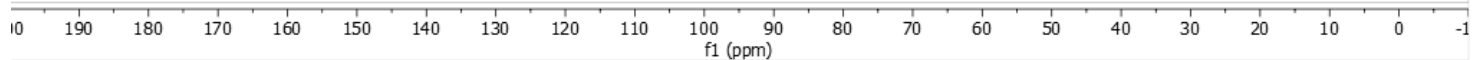


${ }^{19} \mathrm{~F}$ NMR spectrum of $\mathbf{3} \mathbf{v j}$ ' in $\mathrm{CDCl}_{3}$ containing $0.03 \%$ (v/v) TMS (377 MHz).

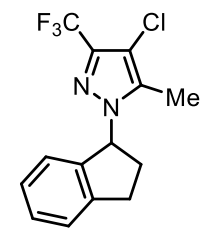

$3 \mathbf{v j}$

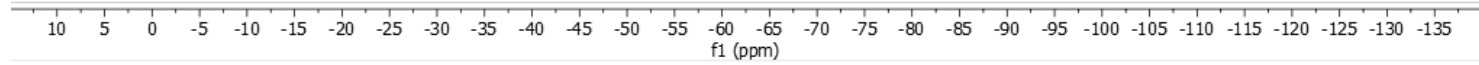

HSQC NMR spectrum of 3vj' in $\mathrm{CDCl}_{3}$ containing $0.03 \%$ (v/v) TMS (500, $126 \mathrm{MHz}$ ).

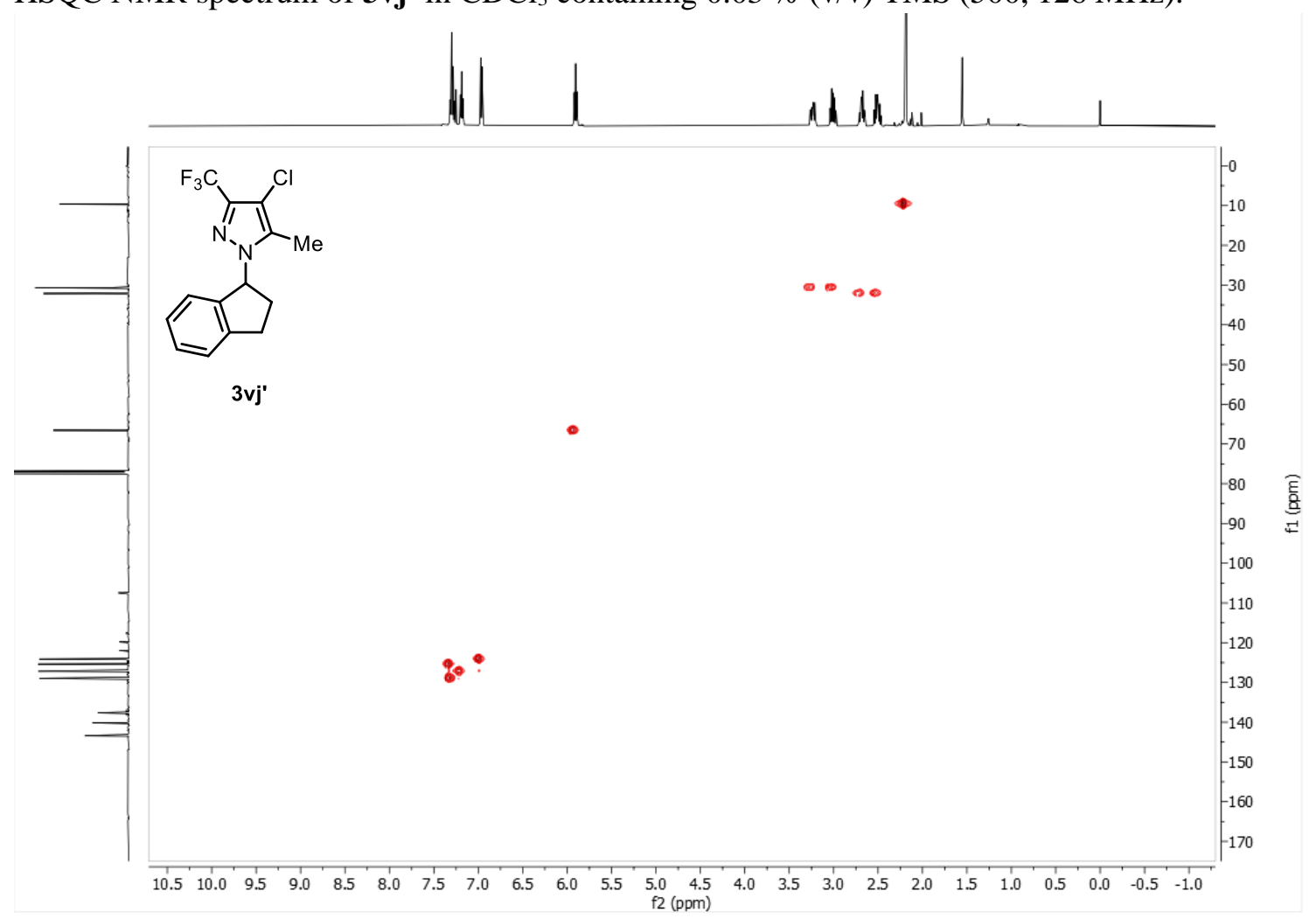


$\mathrm{HMBC}$ NMR spectrum of $\mathbf{3 v j}$ in $\mathrm{CDCl}_{3}$ containing $0.03 \%$ (v/v) TMS (500, $126 \mathrm{MHz}$ ).

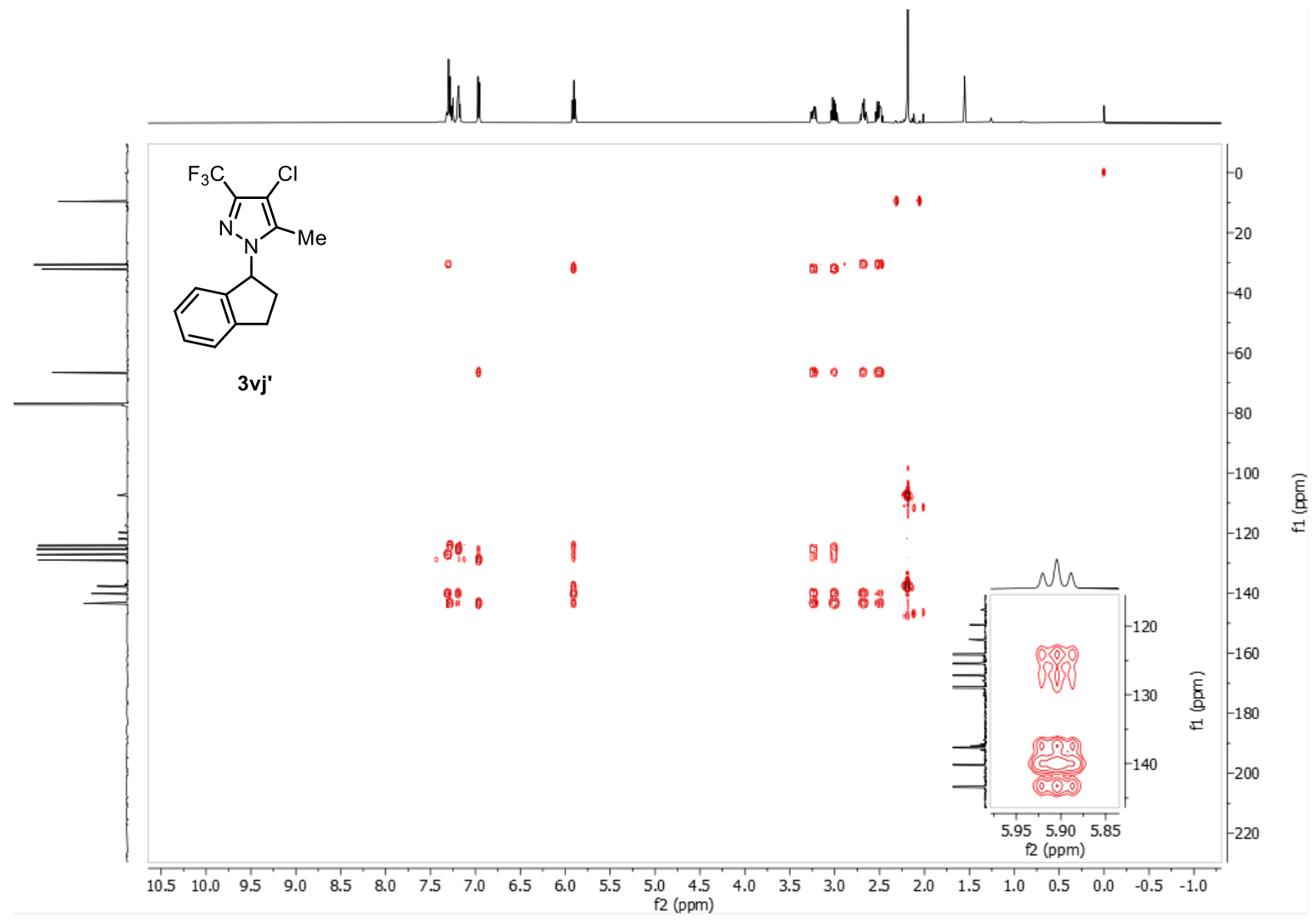

${ }^{1} \mathrm{H}$ NMR spectrum of $\mathbf{3 v k}$ in $\mathrm{CDCl}_{3}$ containing $0.03 \%$ (v/v) TMS (500 MHz).

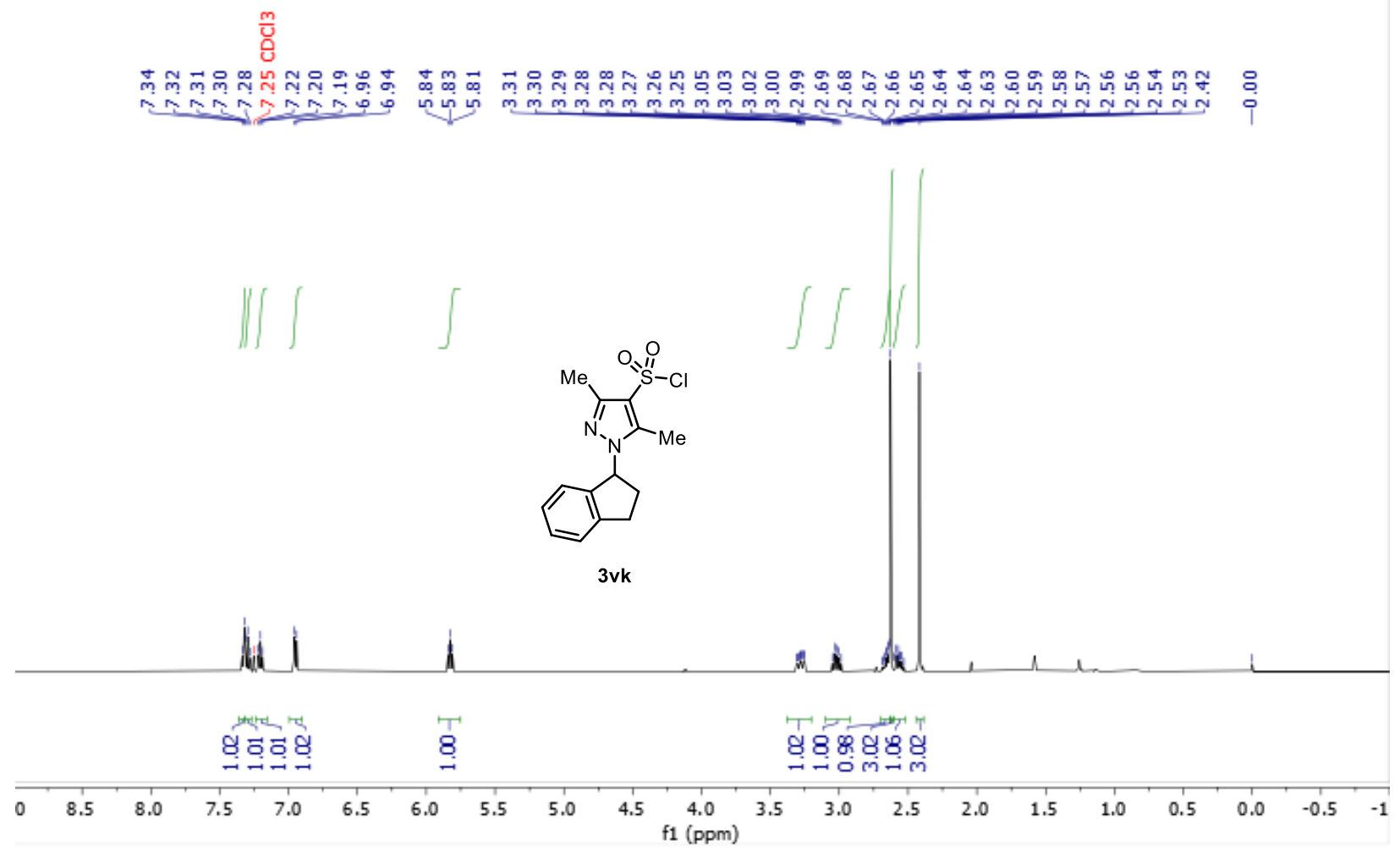


${ }^{13} \mathrm{C}$ NMR spectrum of $\mathbf{3 v k}$ in $\mathrm{CDCl}_{3}$ containing $0.03 \%$ (v/v) TMS (126 MHz).

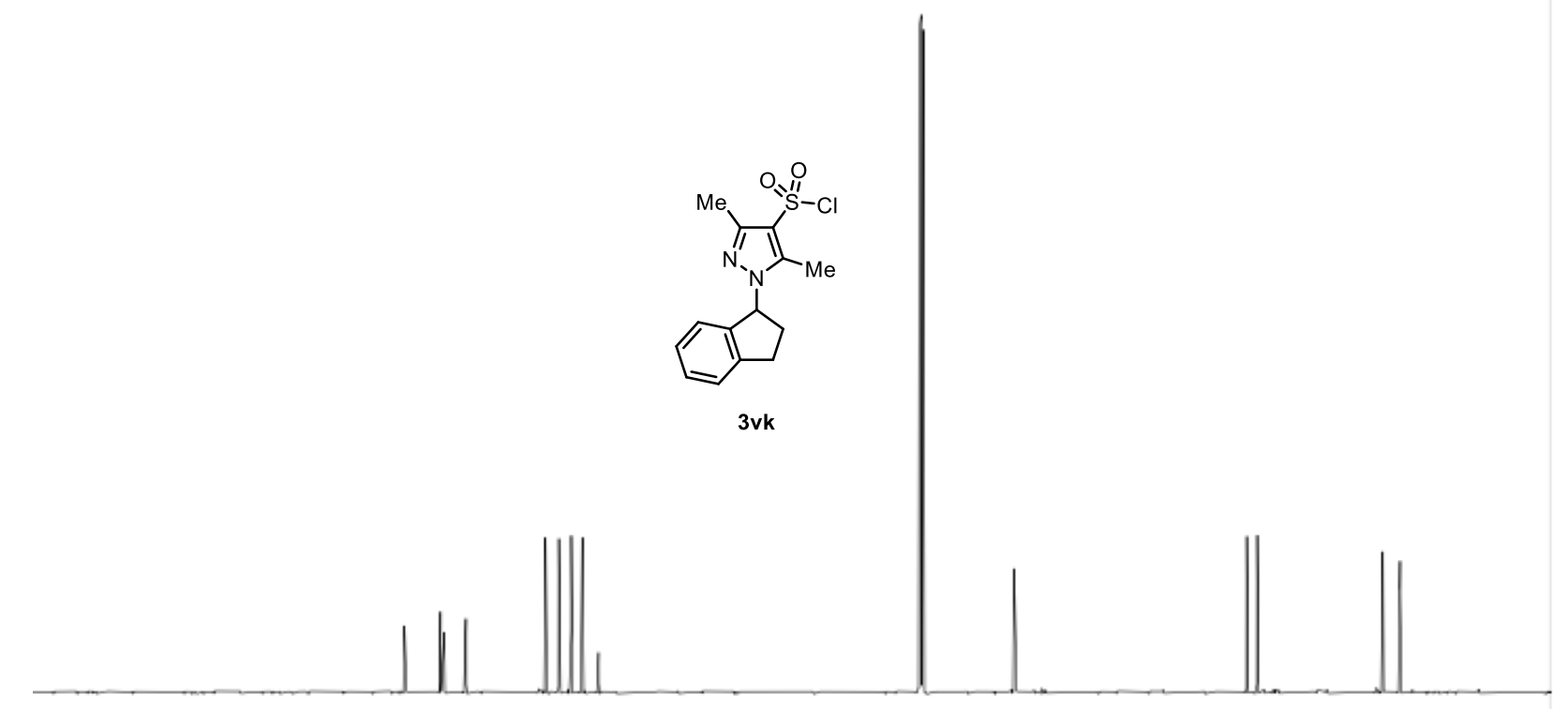

\begin{tabular}{|c|c|c|c|c|c|c|c|c|c|c|c|c|c|c|c|c|c|c|c|c|c|}
\hline 10 & 190 & 180 & 170 & 160 & 150 & 140 & 130 & 120 & 110 & $\begin{array}{r}100 \\
\mathrm{f} 1\end{array}$ & $\begin{array}{r}90 \\
\mathrm{pm})\end{array}$ & 80 & 70 & 60 & 50 & 40 & 30 & 20 & 10 & 0 & -1 \\
\hline
\end{tabular}

${ }^{1} \mathrm{H}$ NMR spectrum of $\mathbf{3 v l}$ in $\mathrm{CDCl}_{3}$ containing $0.03 \%$ (v/v) TMS (500 MHz).

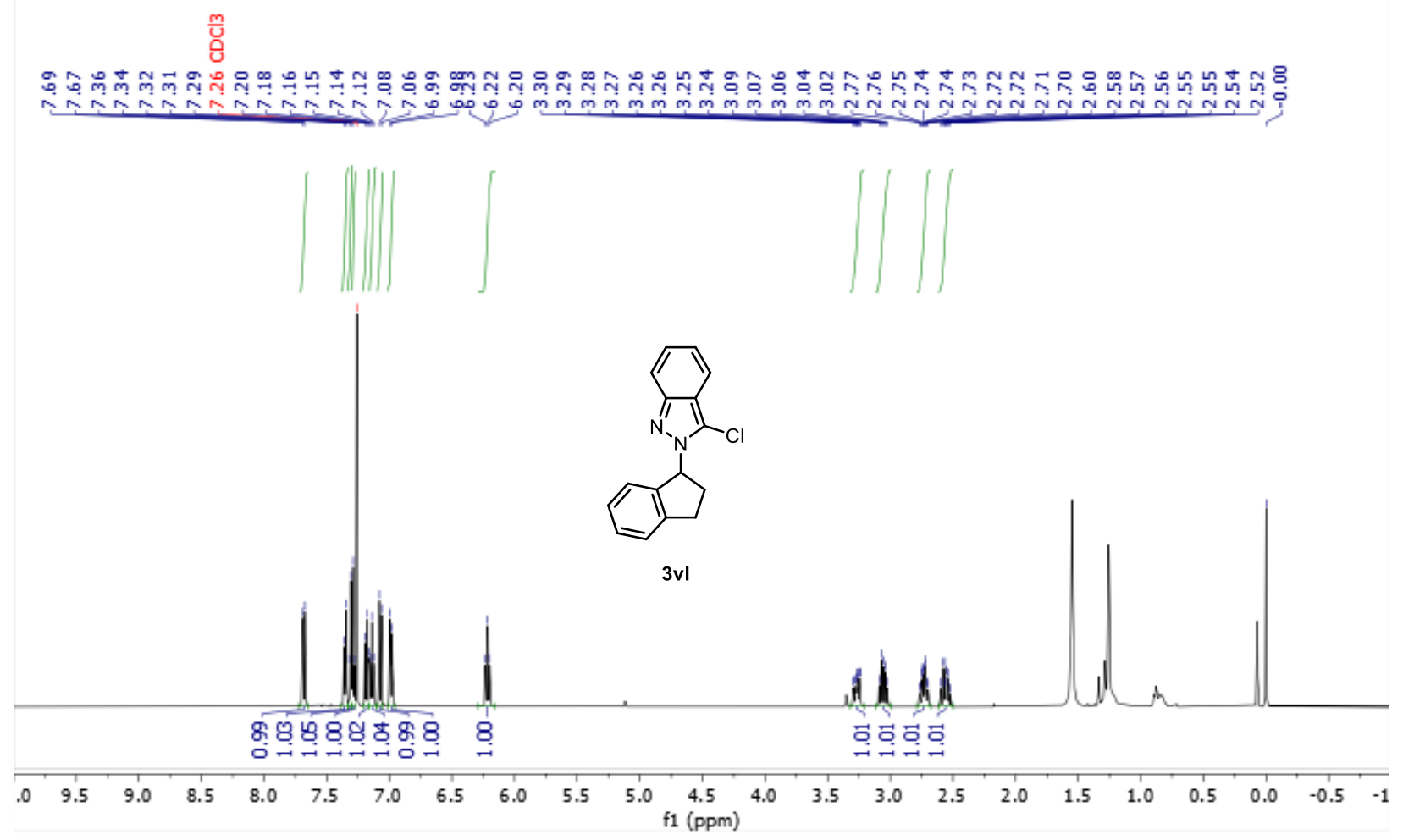


${ }^{13} \mathrm{C}$ NMR spectrum of $\mathbf{3 v l}$ in $\mathrm{CDCl}_{3}$ containing $0.03 \%$ (v/v) TMS (126 MHz).

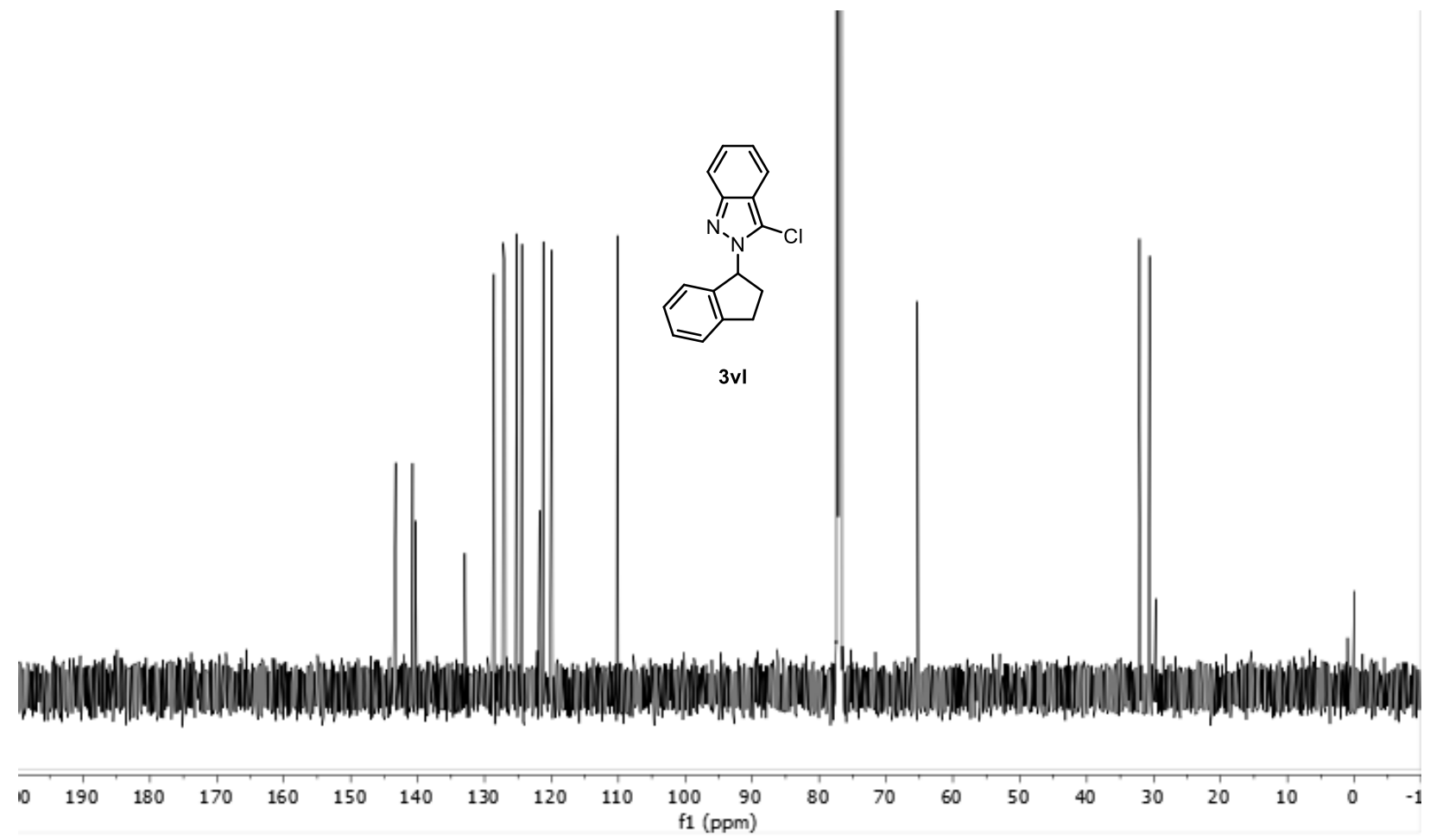

HSQC NMR spectrum of $3 \mathrm{vl}$ in $\mathrm{CDCl}_{3}$ containing $0.03 \%$ (v/v) TMS $(500,126 \mathrm{MHz})$.

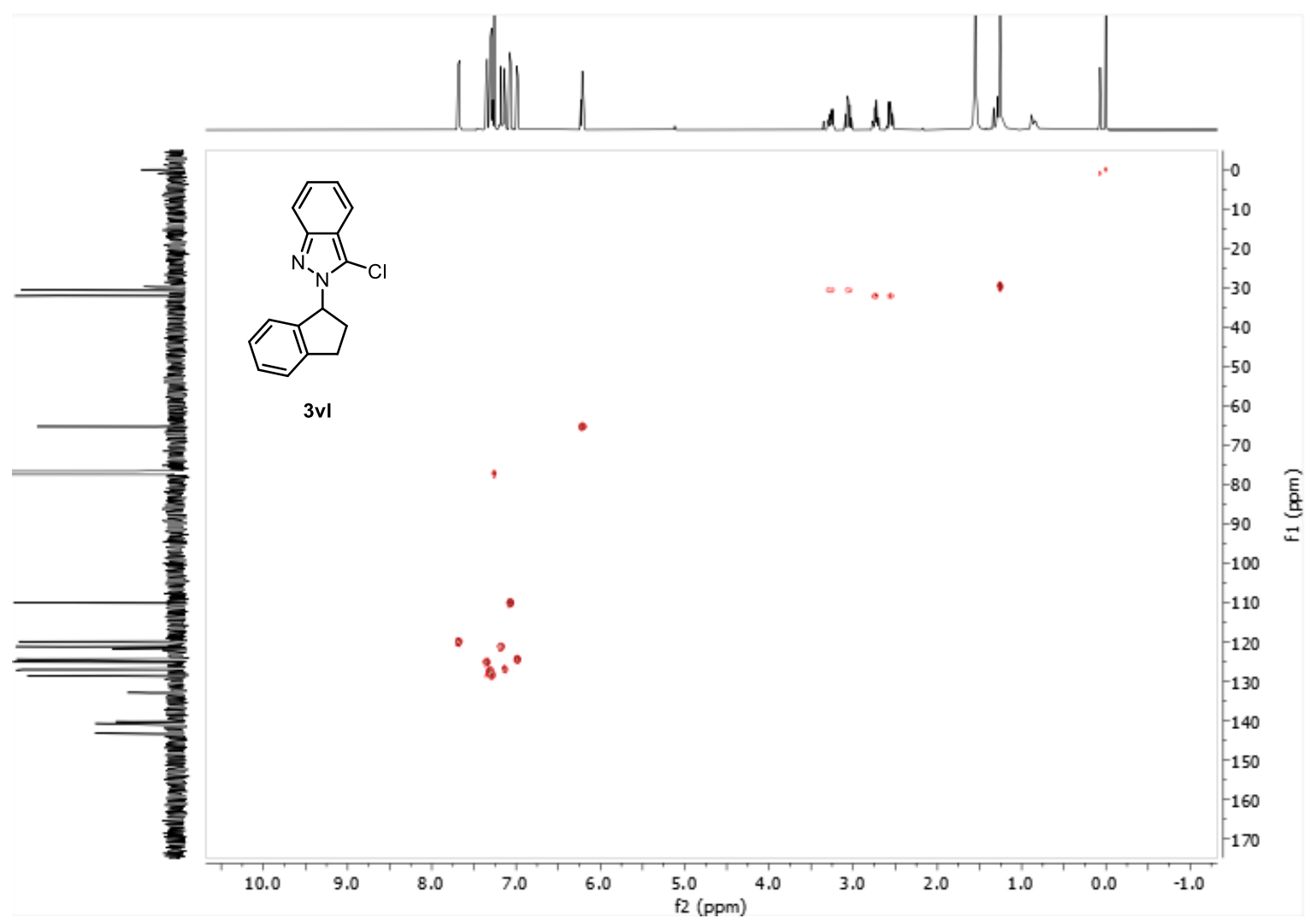


HMBC NMR spectrum of $\mathbf{3 v l}$ in $\mathrm{CDCl}_{3}$ containing $0.03 \%$ (v/v) TMS $(500,126 \mathrm{MHz}$ ).

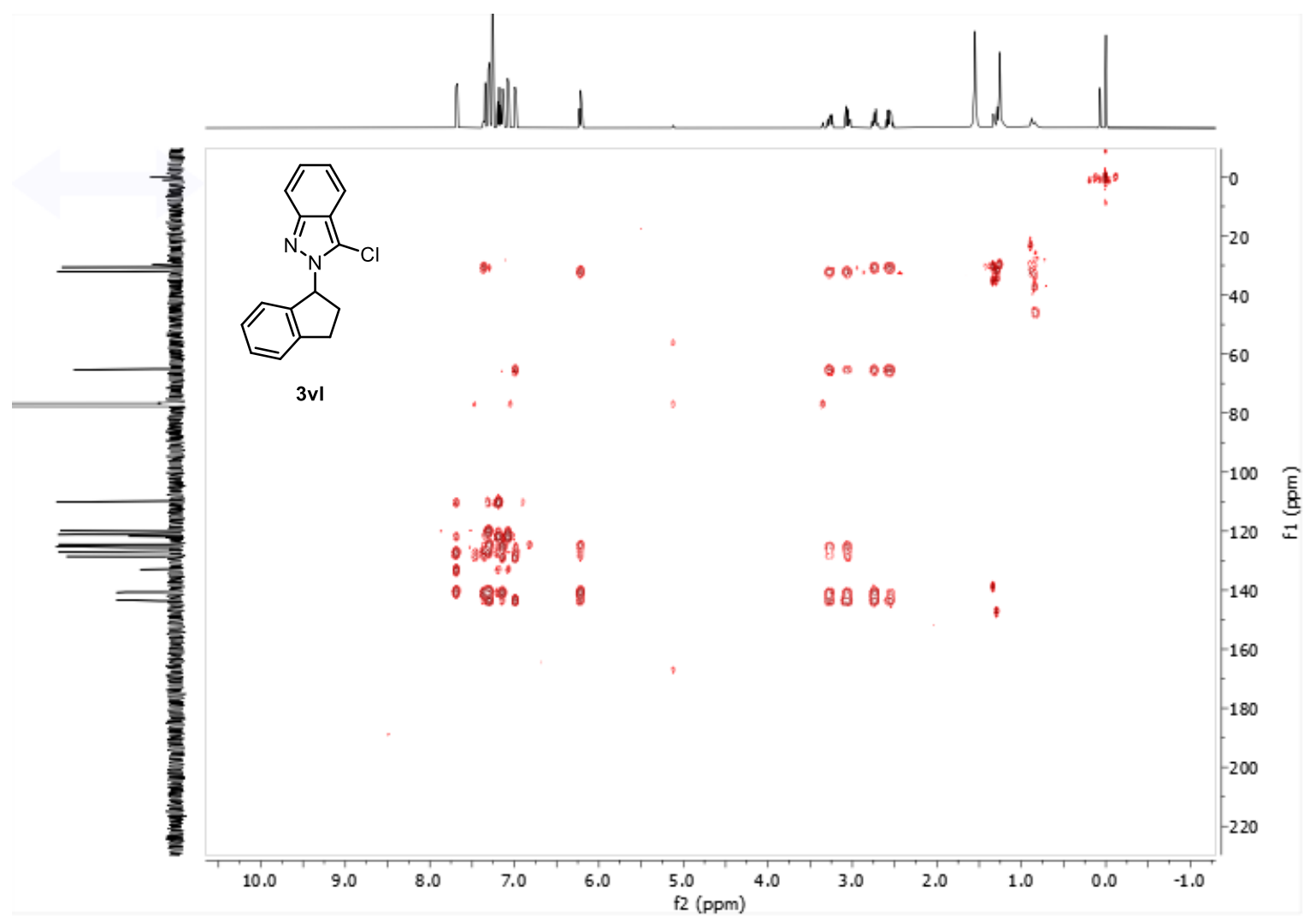

${ }^{1} \mathrm{H}$ NMR spectrum of $\mathbf{3} \mathbf{v m}$ in $\mathrm{CDCl}_{3}$ containing $0.03 \%$ (v/v) TMS (500 MHz).

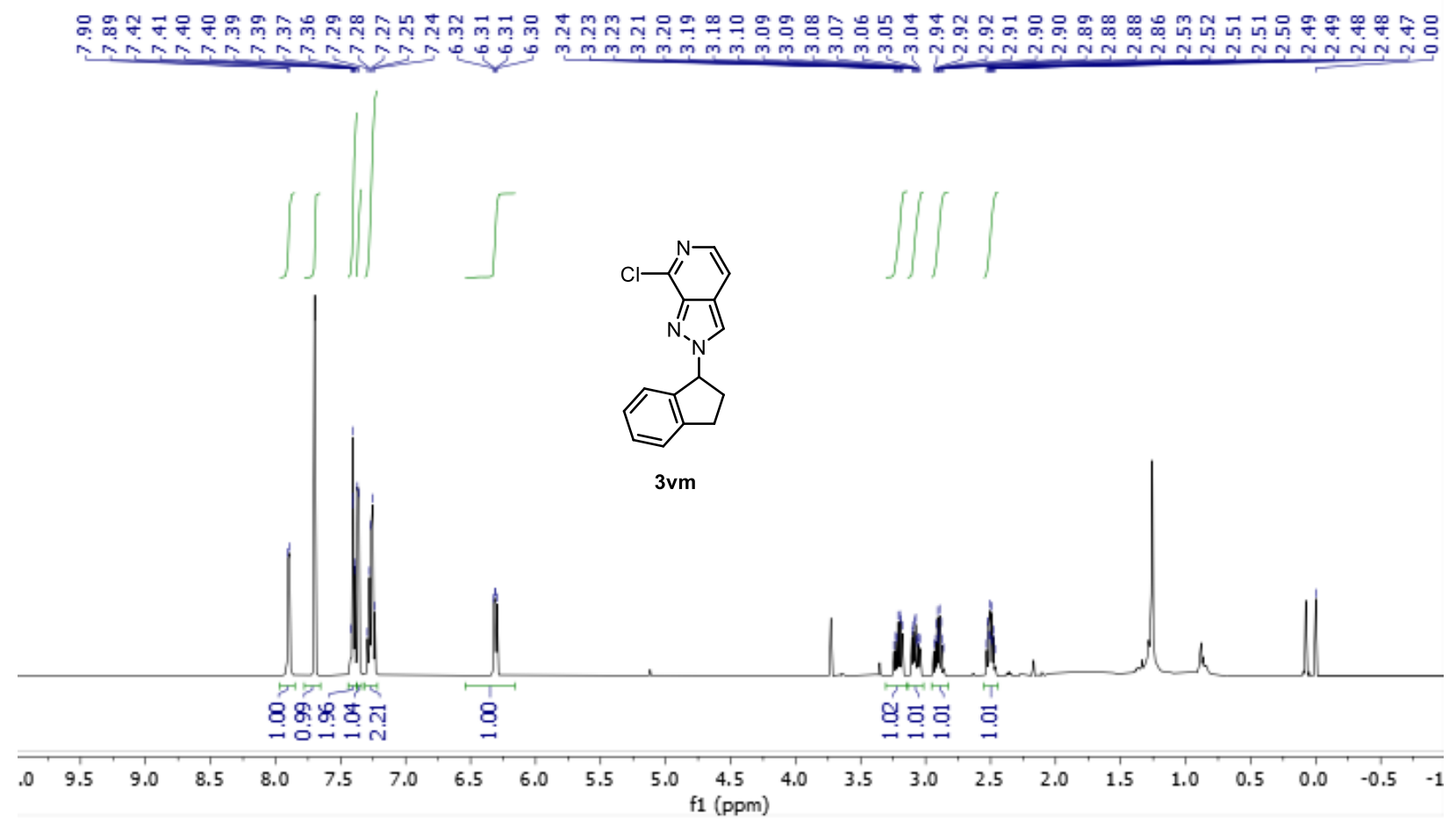


${ }^{13} \mathrm{C}$ NMR spectrum of $\mathbf{3 v m}$ in $\mathrm{CDCl}_{3}$ containing $0.03 \%$ (v/v) TMS (126 MHz).
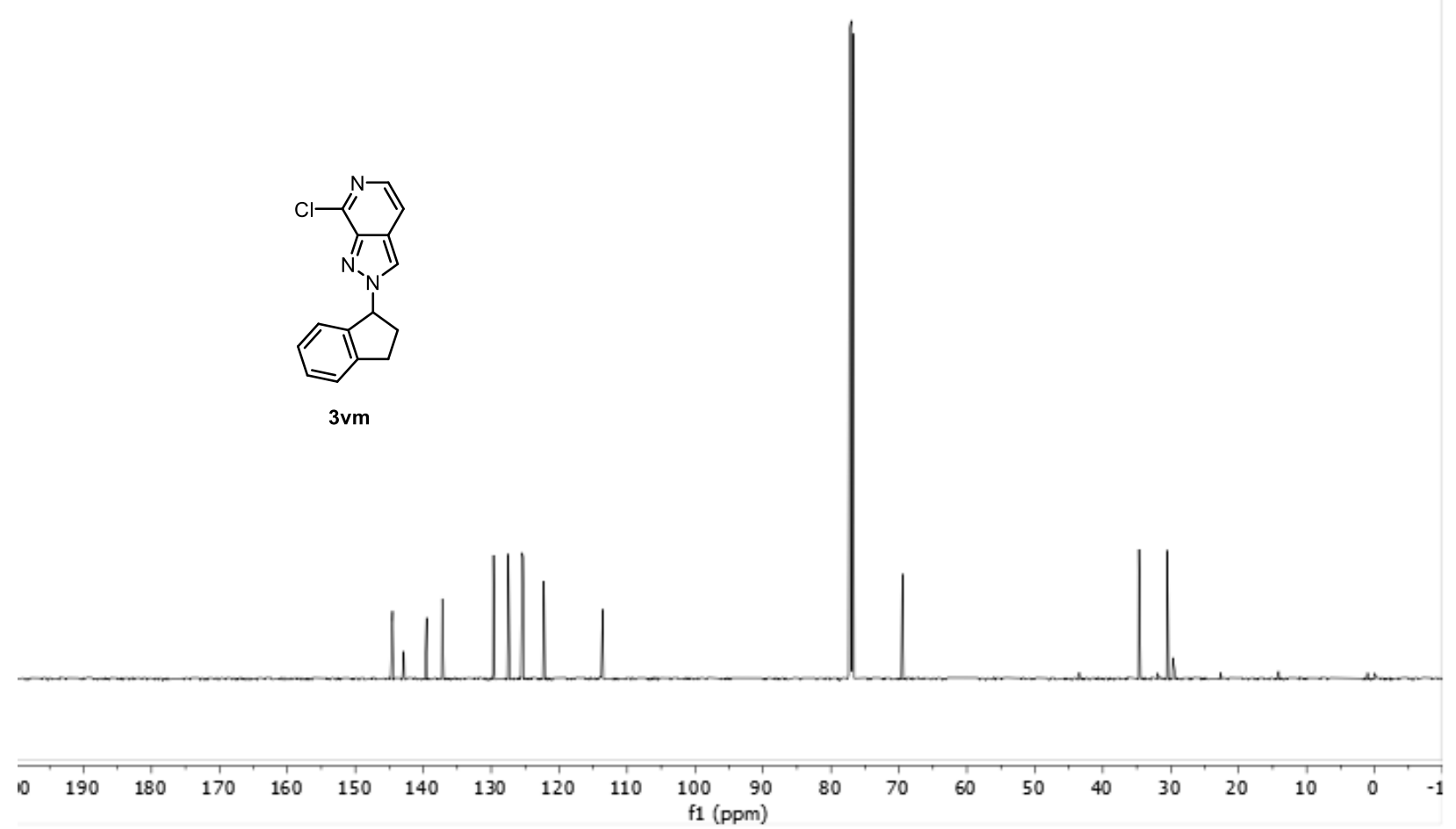

HSQC NMR spectrum of $3 \mathbf{v m}$ in $\mathrm{CDCl}_{3}$ containing $0.03 \%$ (v/v) TMS (500, $126 \mathrm{MHz}$ ).

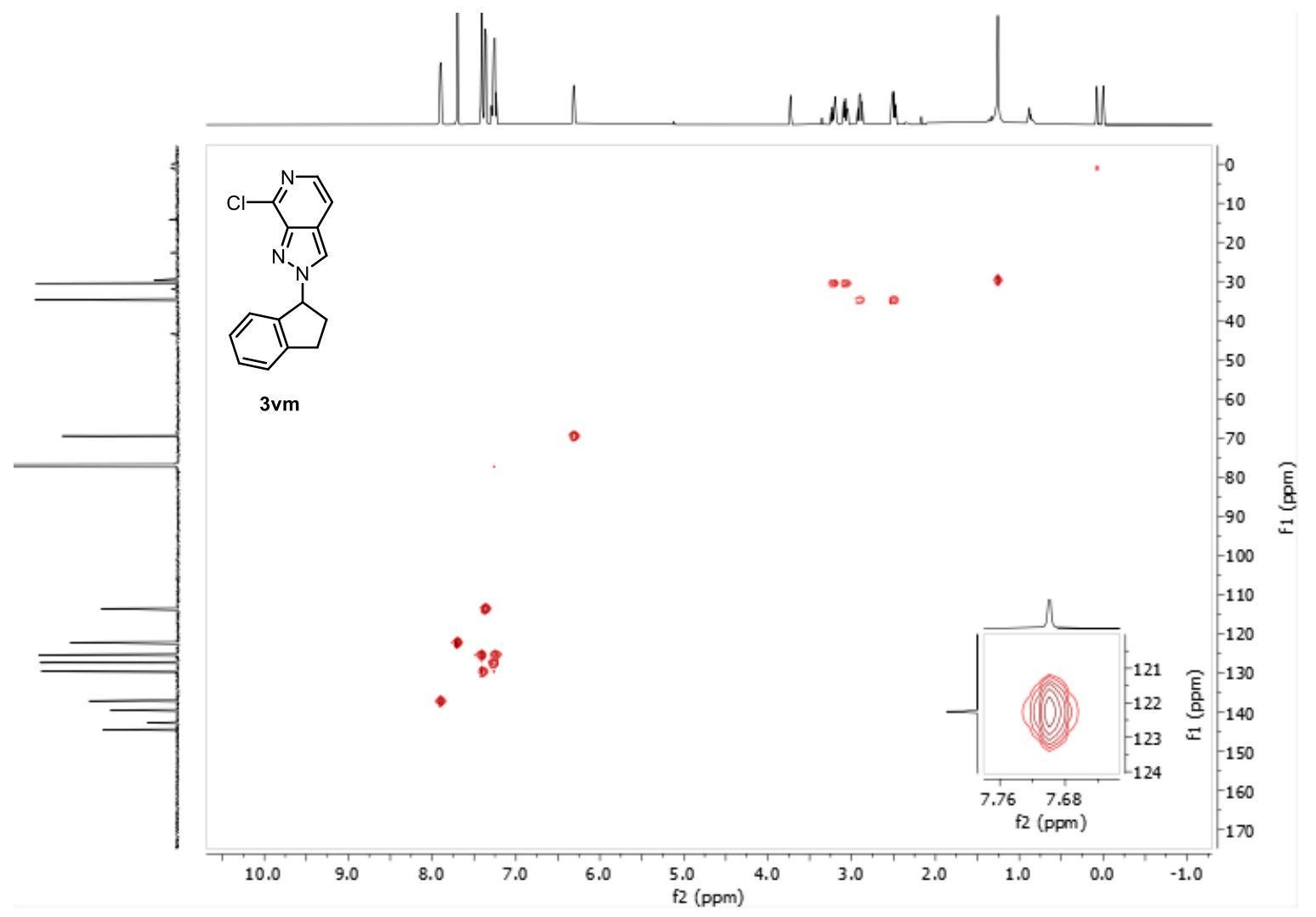


HMBC NMR spectrum of $\mathbf{3 v m}$ in $\mathrm{CDCl}_{3}$ containing $0.03 \%$ (v/v) TMS (500, $126 \mathrm{MHz}$ ).

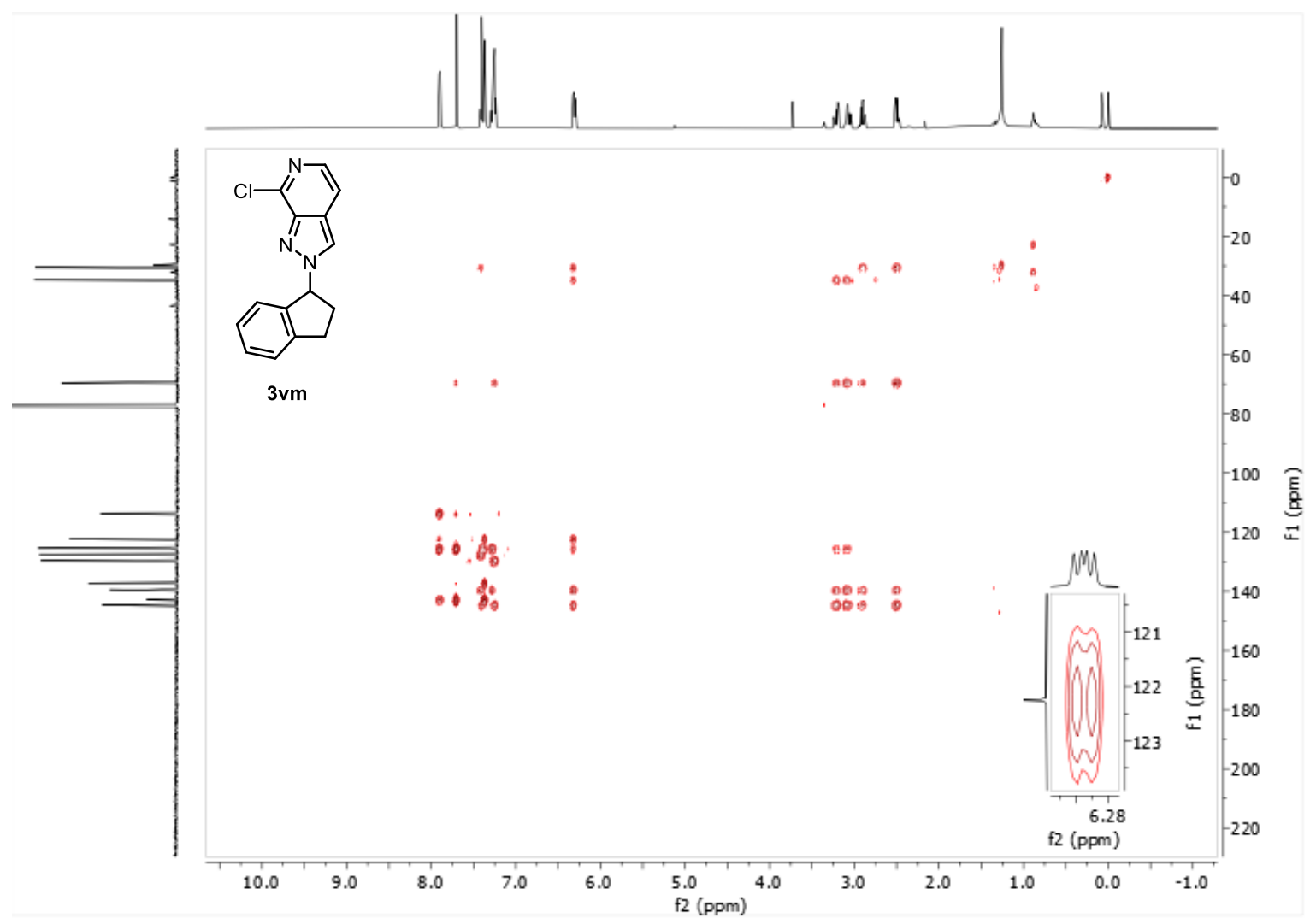

${ }^{1} \mathrm{H}$ NMR spectrum of $3 \mathrm{vn}$ in $\mathrm{CDCl}_{3}$ containing $0.03 \%$ (v/v) TMS (500 MHz).

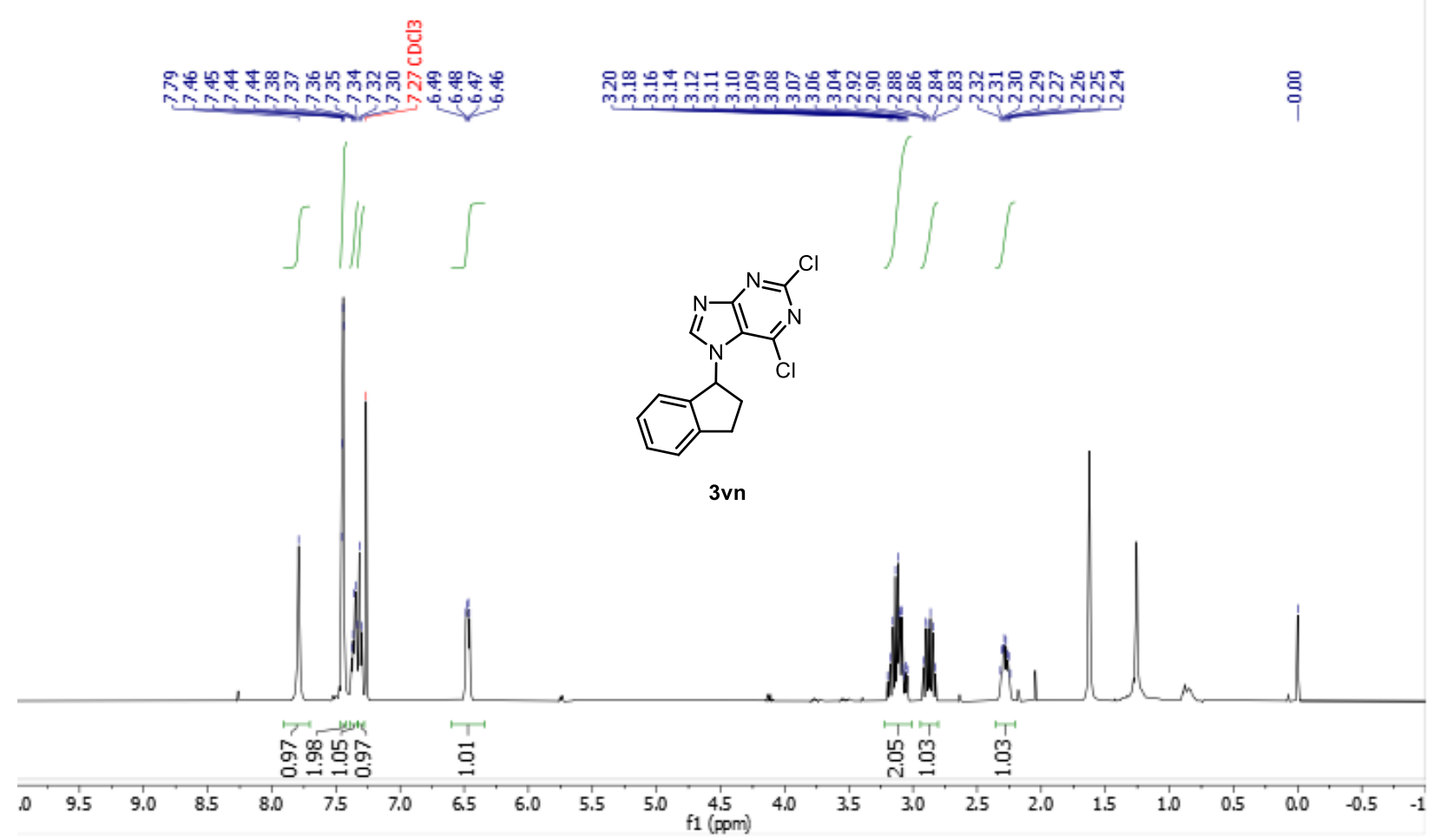


${ }^{13} \mathrm{C}$ NMR spectrum of $\mathbf{3 v n}$ in $\mathrm{CDCl}_{3}$ containing $0.03 \%$ (v/v) TMS (126 MHz).

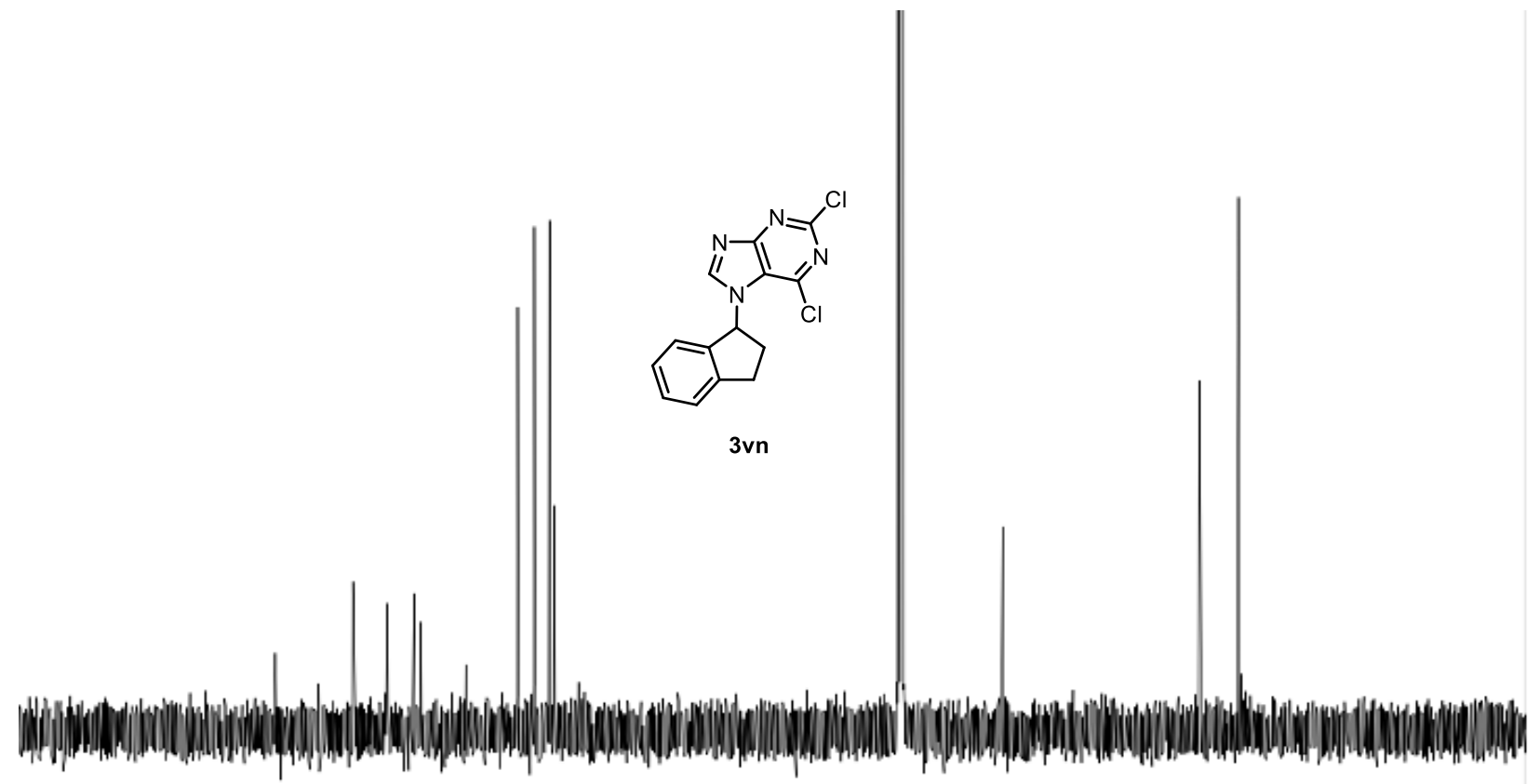

$\begin{array}{lllllllllllllllllllllllllllllll}10 & 190 & 180 & 170 & 160 & 150 & 140 & 130 & 120 & 110 & 100 & 90 & 80 & 70 & 60 & 50 & 40 & 30 & 20 & 10 & 0 & -1\end{array}$

${ }^{1} \mathrm{H}$ NMR spectrum of $\mathbf{3 v n}$ ' in $\mathrm{CDCl}_{3}$ containing $0.03 \%$ (v/v) TMS (500 MHz).

$\stackrel{m}{\mathrm{O}}$

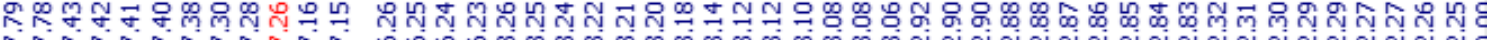

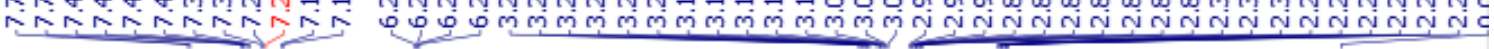

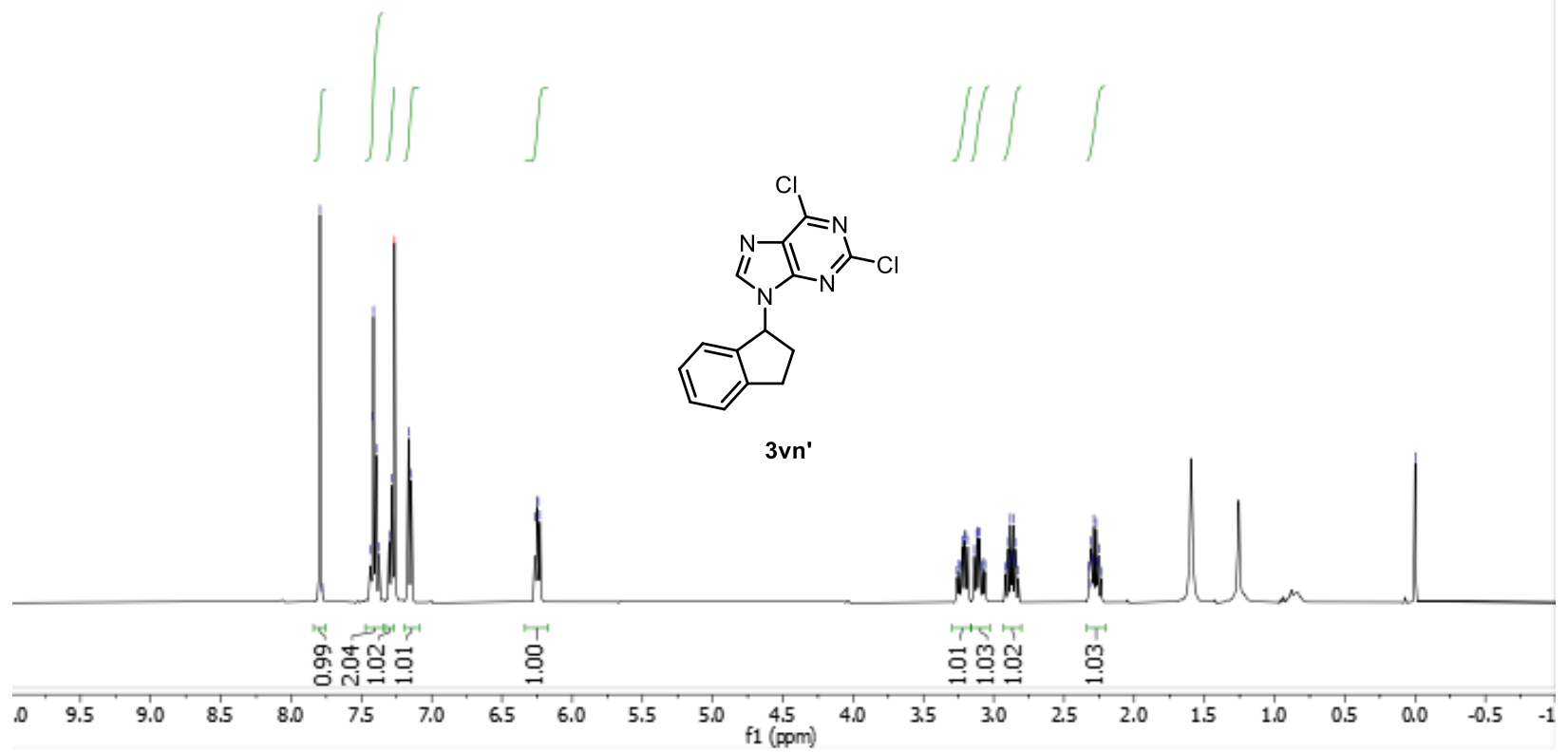


${ }^{13} \mathrm{C}$ NMR spectrum of 3vn' in $\mathrm{CDCl}_{3}$ containing $0.03 \%$ (v/v) TMS (126 MHz).

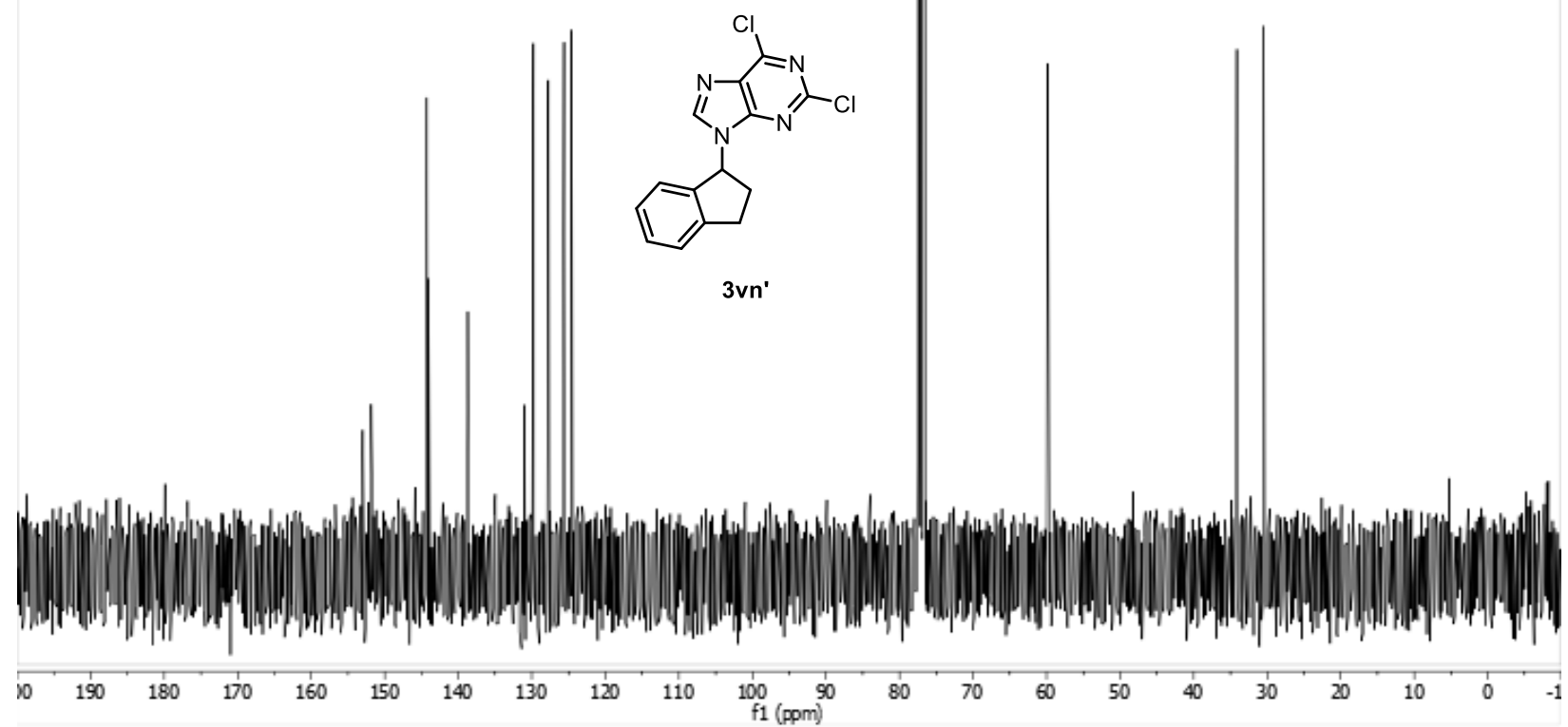

HSQC NMR spectrum of 3vn' in $\mathrm{CDCl}_{3}$ containing $0.03 \%$ (v/v) TMS (500, $126 \mathrm{MHz}$ ).

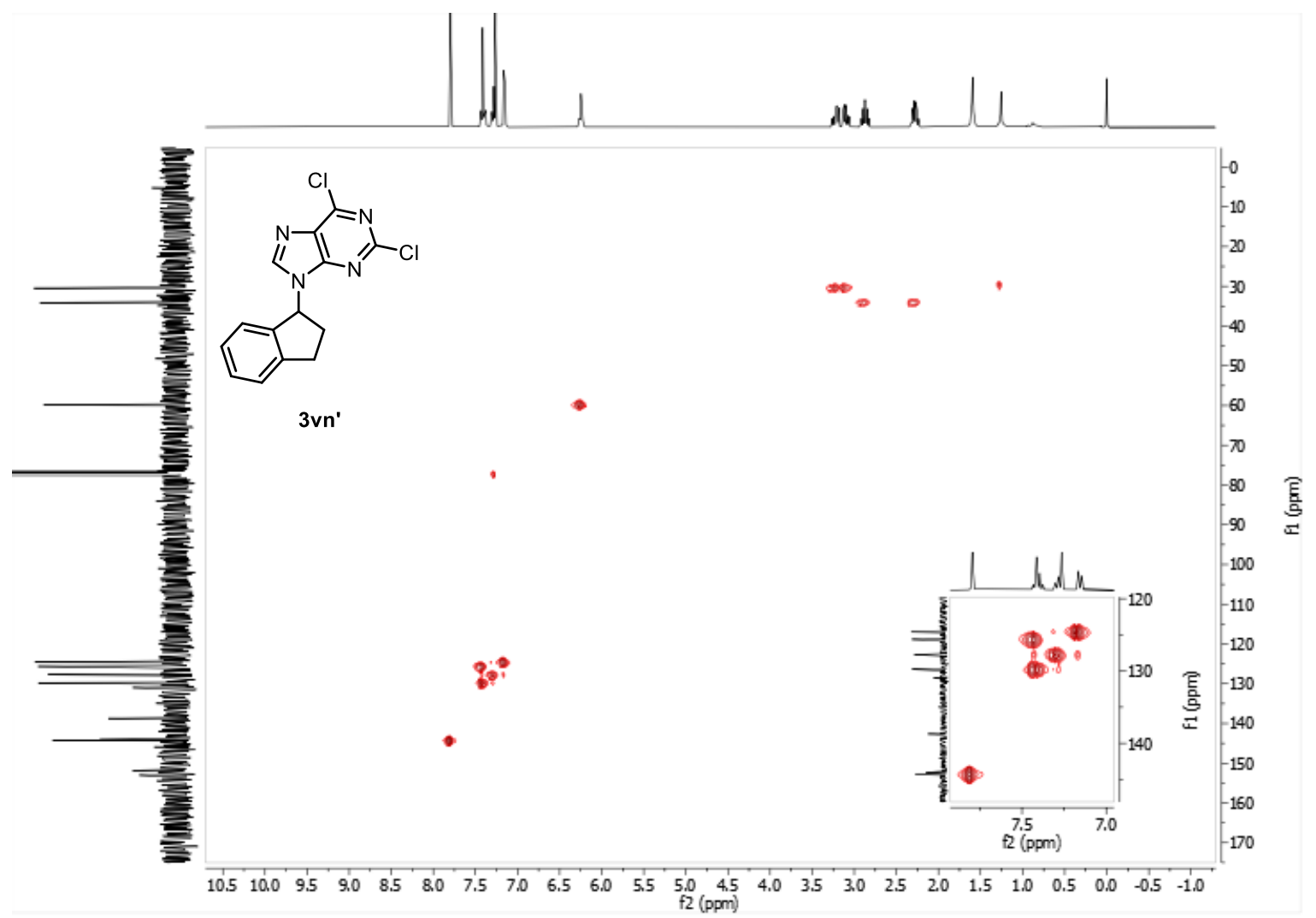


HMBC NMR spectrum of 3vn' in $\mathrm{CDCl}_{3}$ containing $0.03 \%$ (v/v) TMS (500, $126 \mathrm{MHz}$ ).

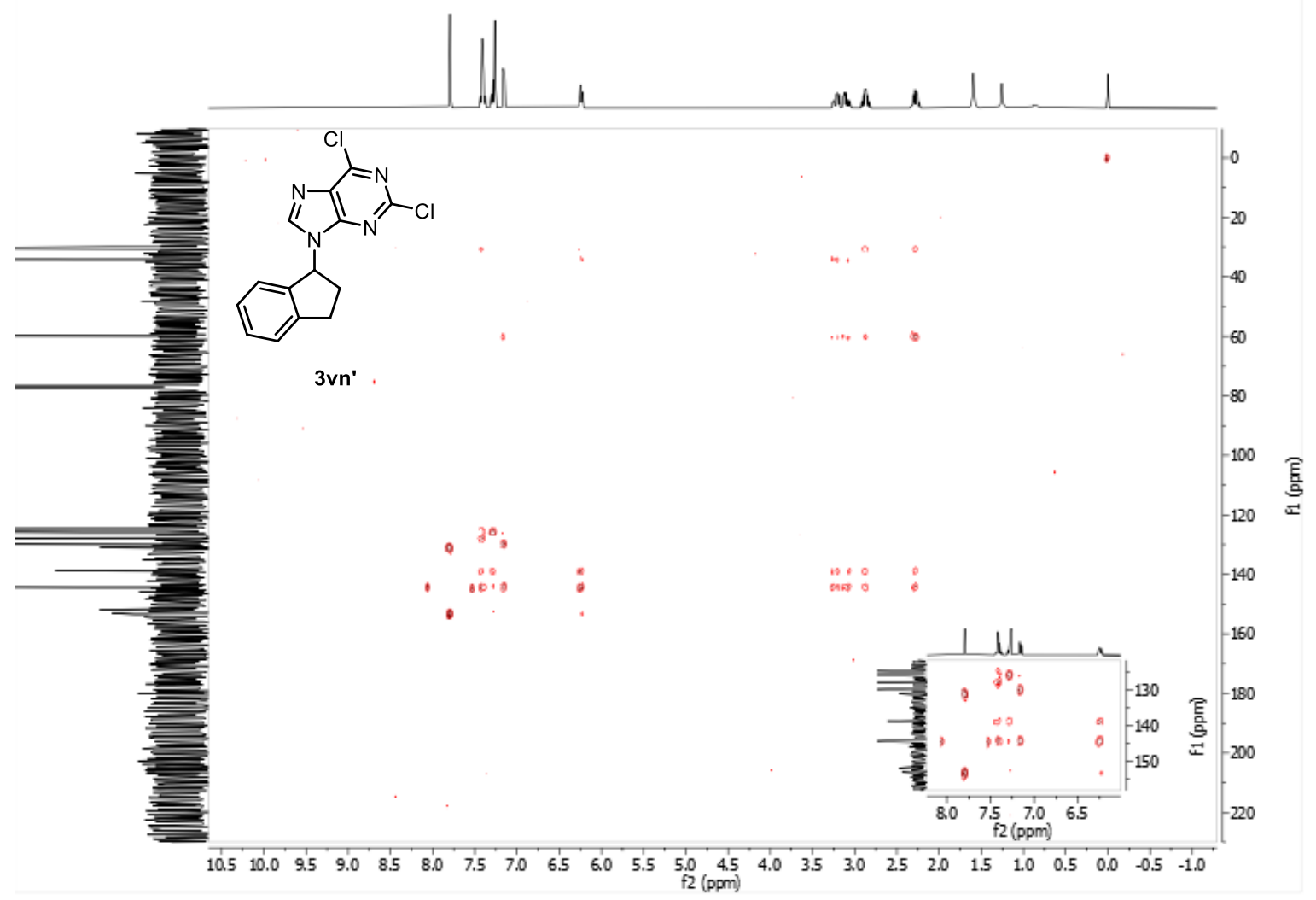

${ }^{1} \mathrm{H}$ NMR spectrum of 3vo in $\mathrm{CDCl}_{3}$ containing $0.03 \%$ (v/v) TMS (500 MHz).

$\stackrel{8}{\mathrm{~g}}$

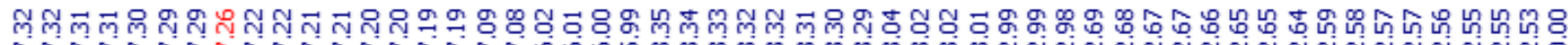

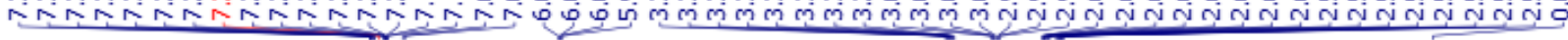

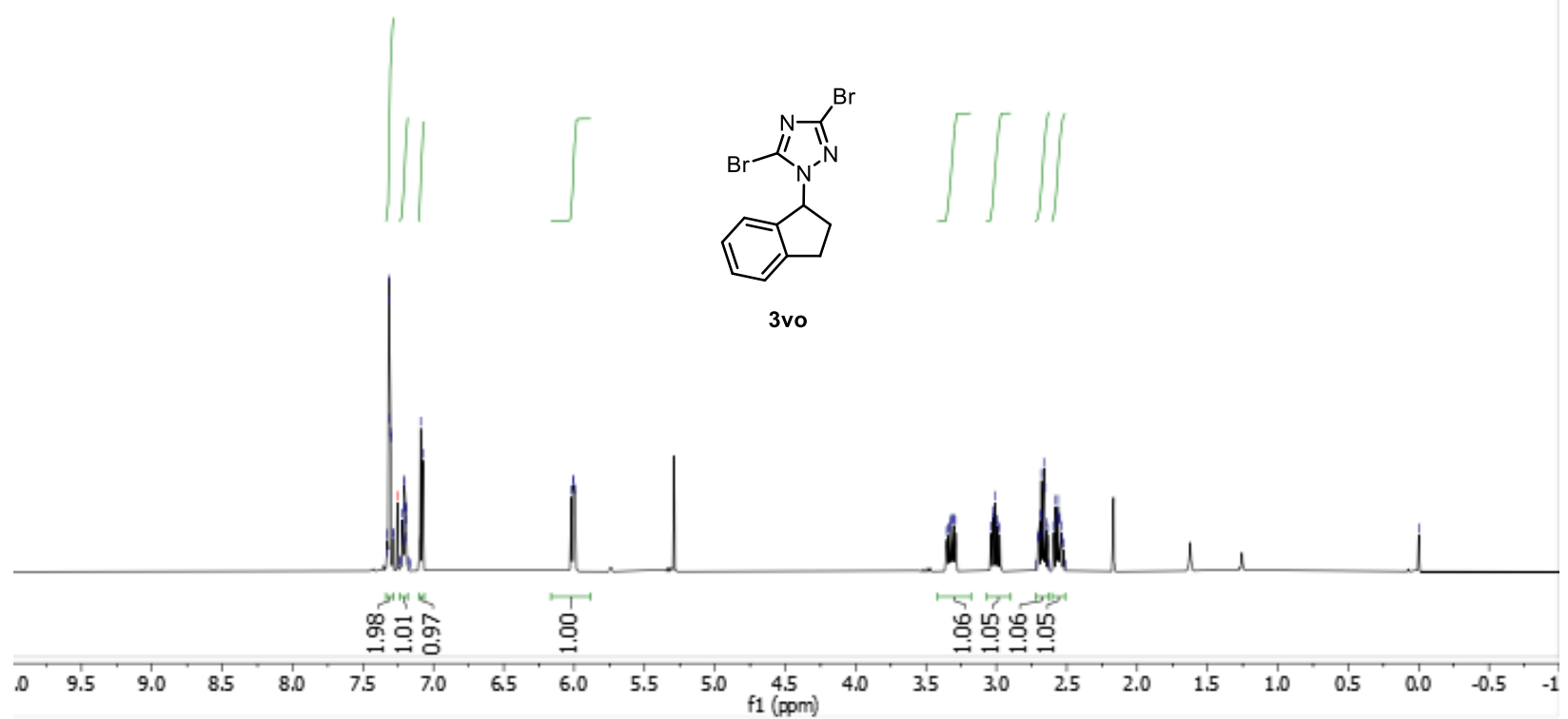


${ }^{13} \mathrm{C}$ NMR spectrum of 3 vo in $\mathrm{CDCl}_{3}$ containing $0.03 \%$ (v/v) TMS (126 MHz).
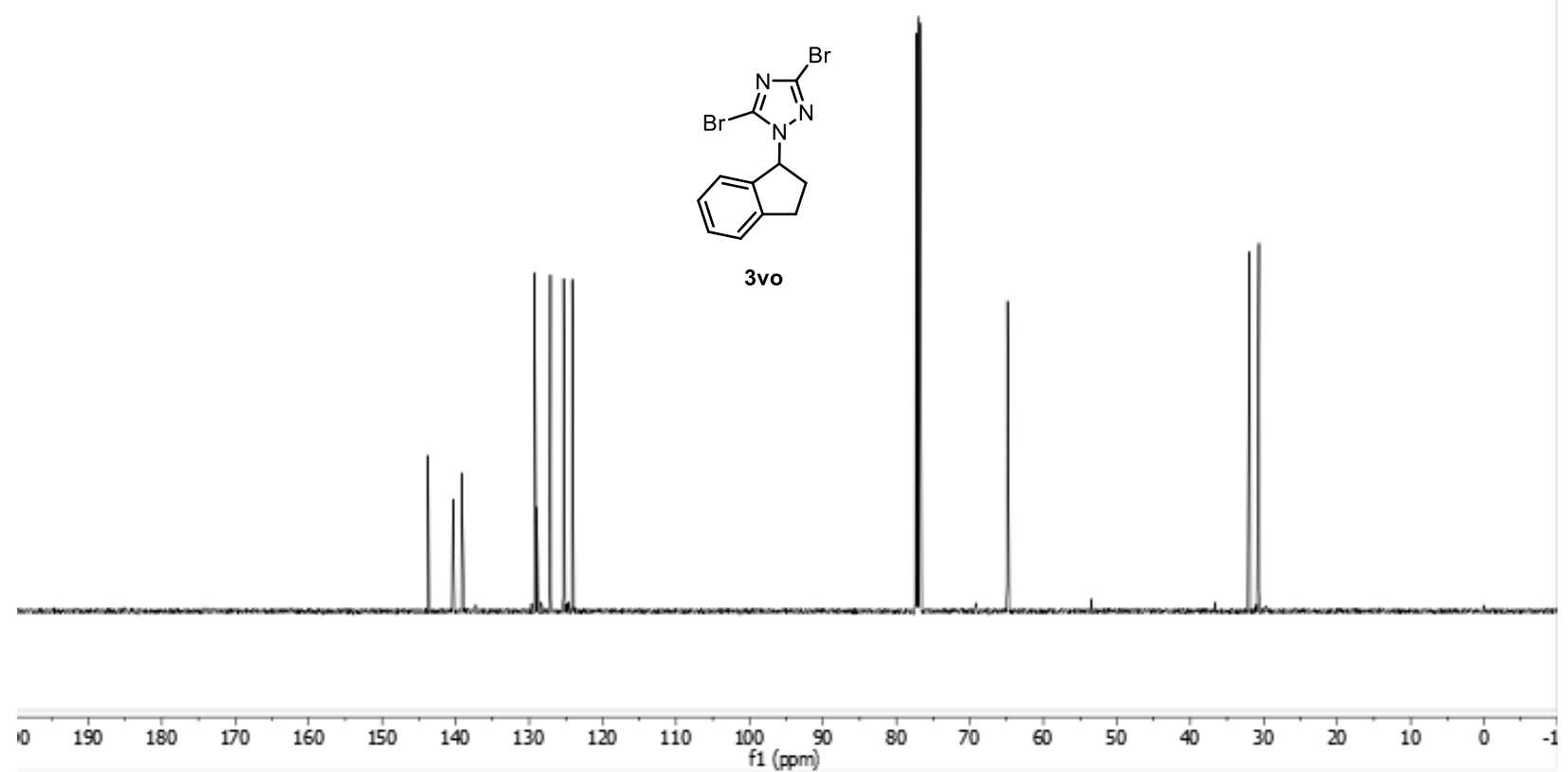

${ }^{1} \mathrm{H}$ NMR spectrum of $\mathbf{3 v p}$ in $\mathrm{CDCl}_{3}$ containing $0.03 \%$ (v/v) TMS (500 MHz).

ํํํ

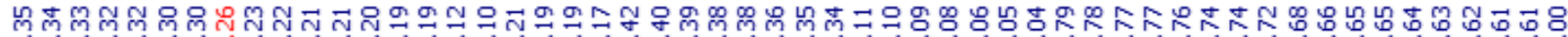

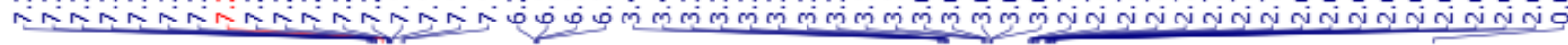

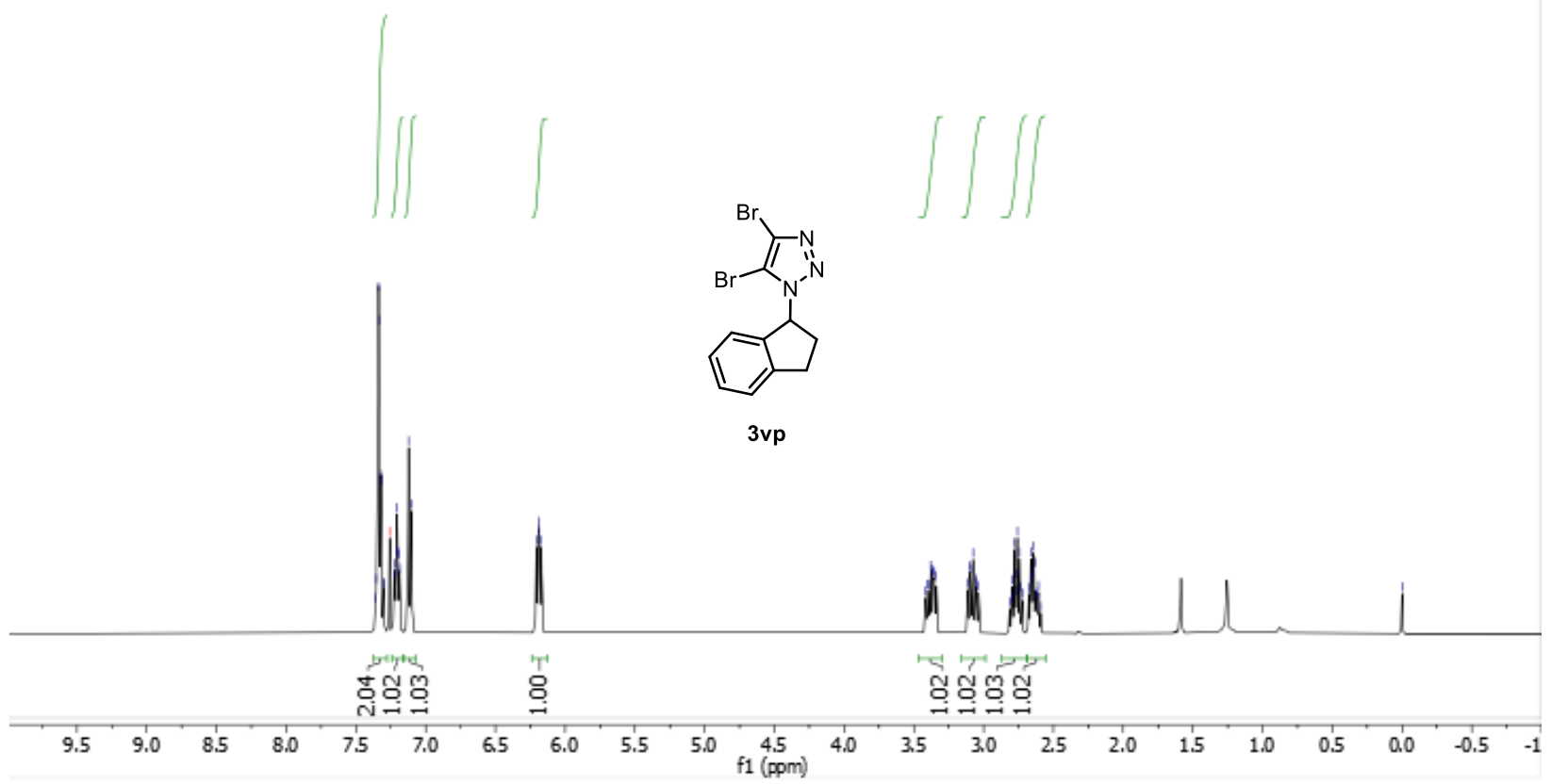


${ }^{13} \mathrm{C}$ NMR spectrum of $\mathbf{3 v p}$ in $\mathrm{CDCl}_{3}$ containing $0.03 \%$ (v/v) TMS (126 MHz).
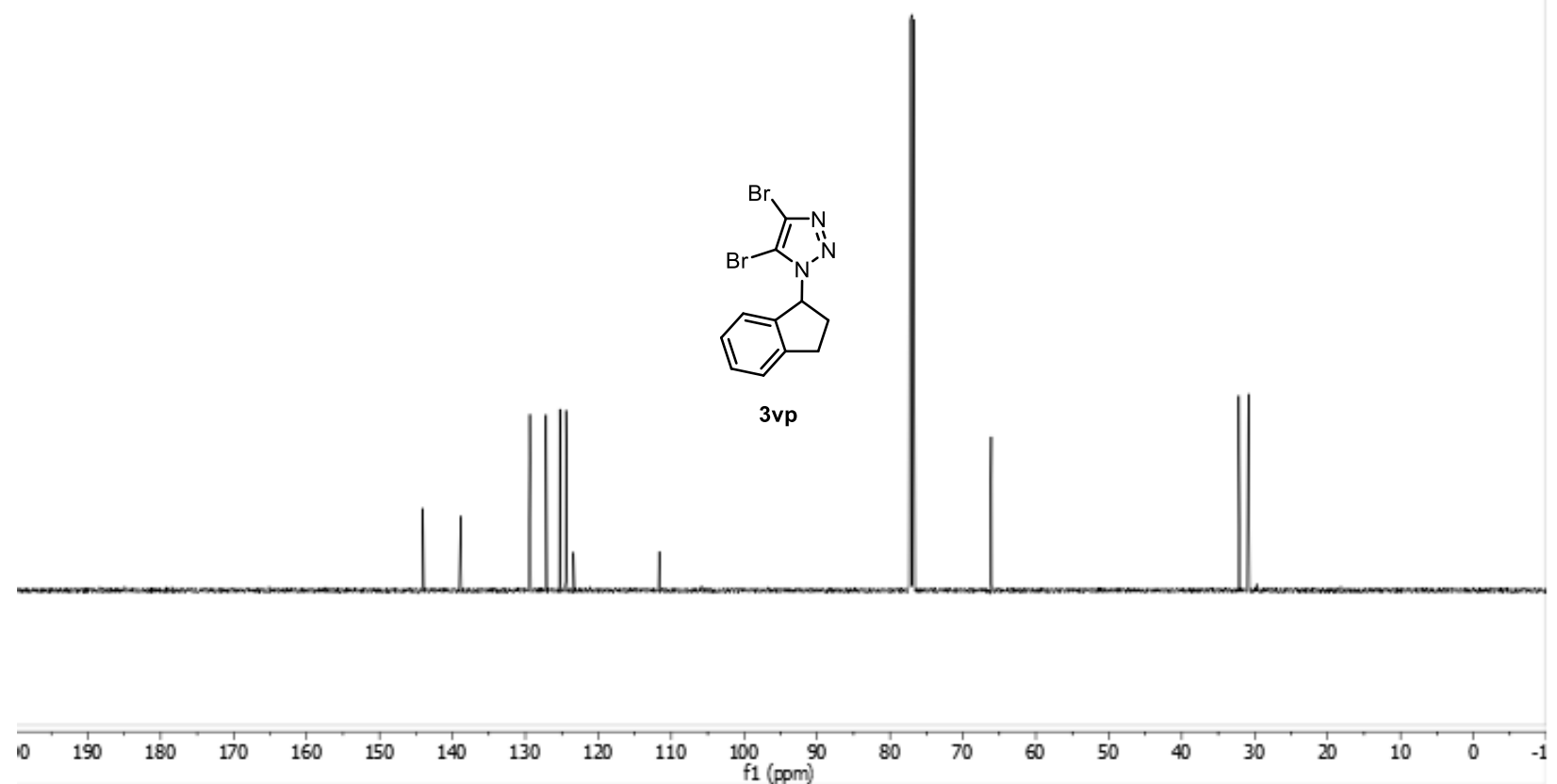

${ }^{1} \mathrm{H}$ NMR spectrum of 3vp' in $\mathrm{CDCl}_{3}$ containing $0.03 \%$ (v/v) TMS (500 MHz).

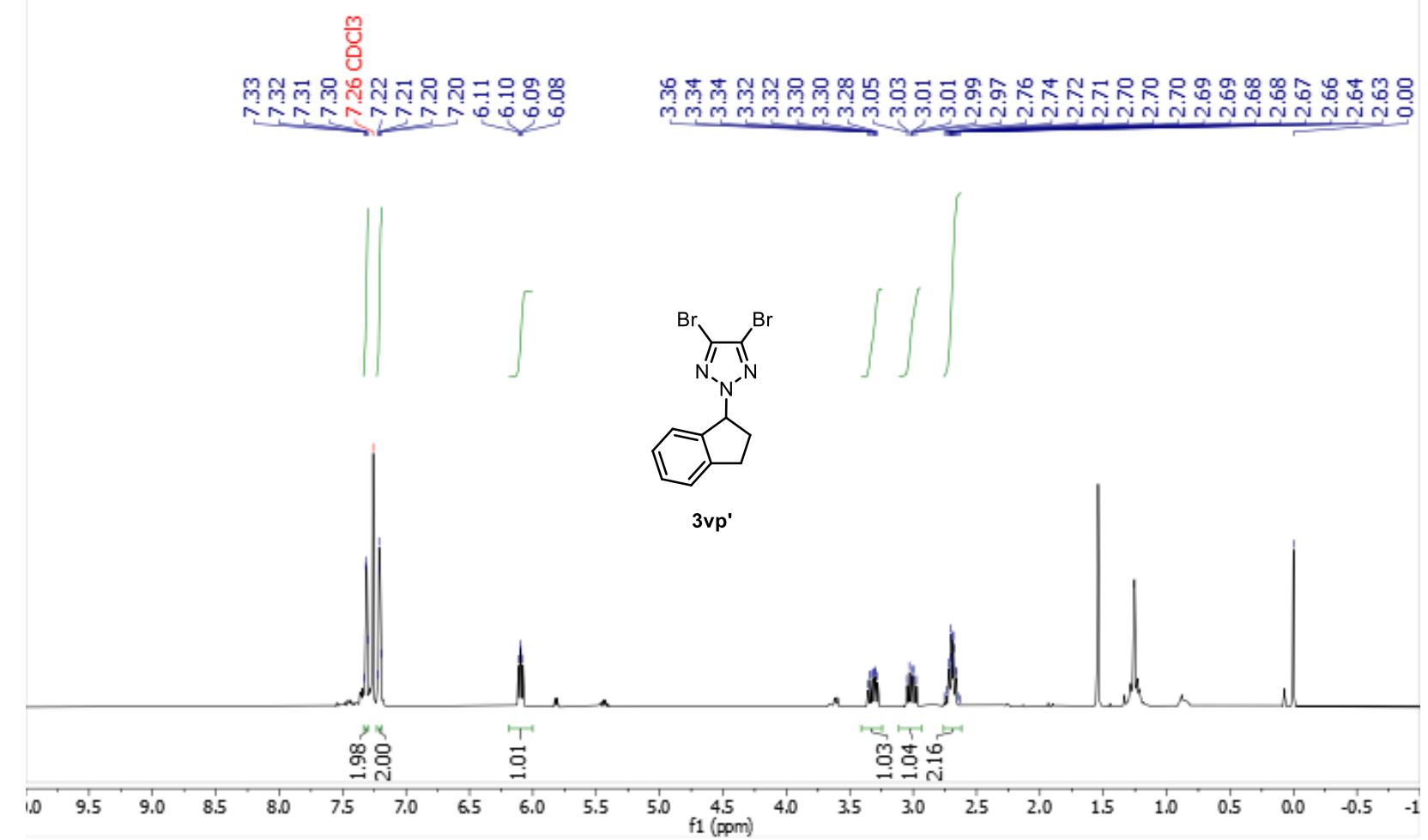


${ }^{13} \mathrm{C}$ NMR spectrum of 3vp' in $\mathrm{CDCl}_{3}$ containing $0.03 \%$ (v/v) TMS (126 MHz).

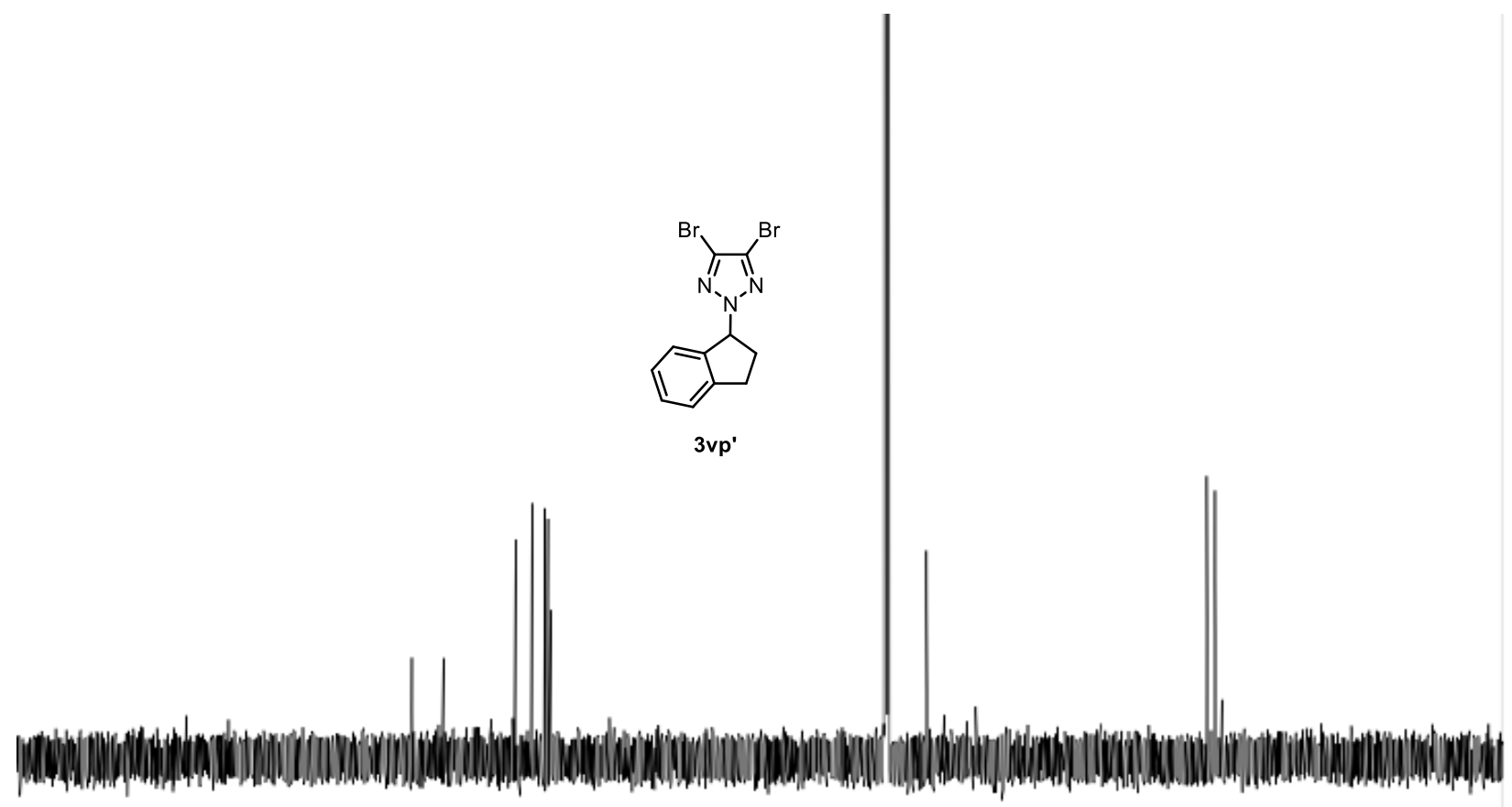

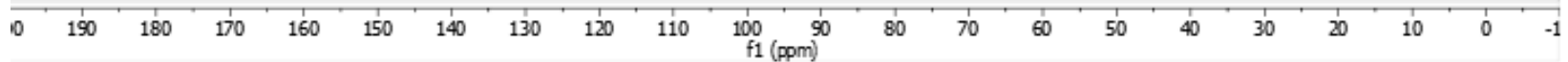

${ }^{1} \mathrm{H}$ NMR spectrum of $\mathbf{3 v q}$ in $\mathrm{CDCl}_{3}$ containing $0.03 \%$ (v/v) TMS (500 MHz).

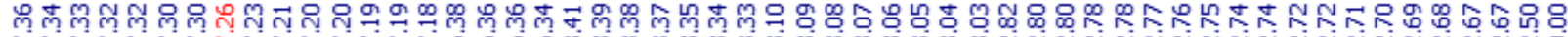

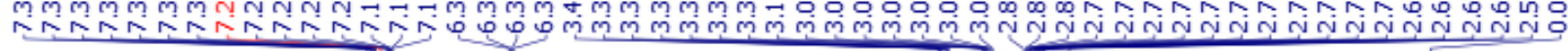
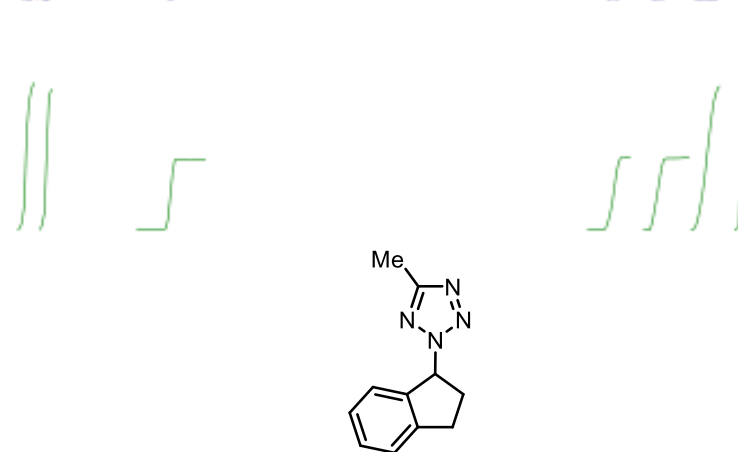

$3 \mathrm{vq}$

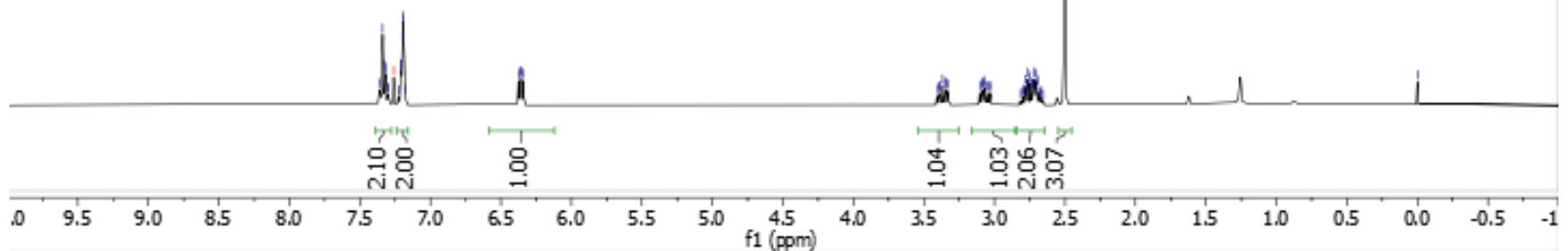


${ }^{13} \mathrm{C}$ NMR spectrum of $\mathbf{3 v q}$ in $\mathrm{CDCl}_{3}$ containing $0.03 \%$ (v/v) TMS (126 MHz).

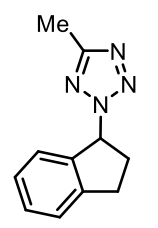

$3 v q$
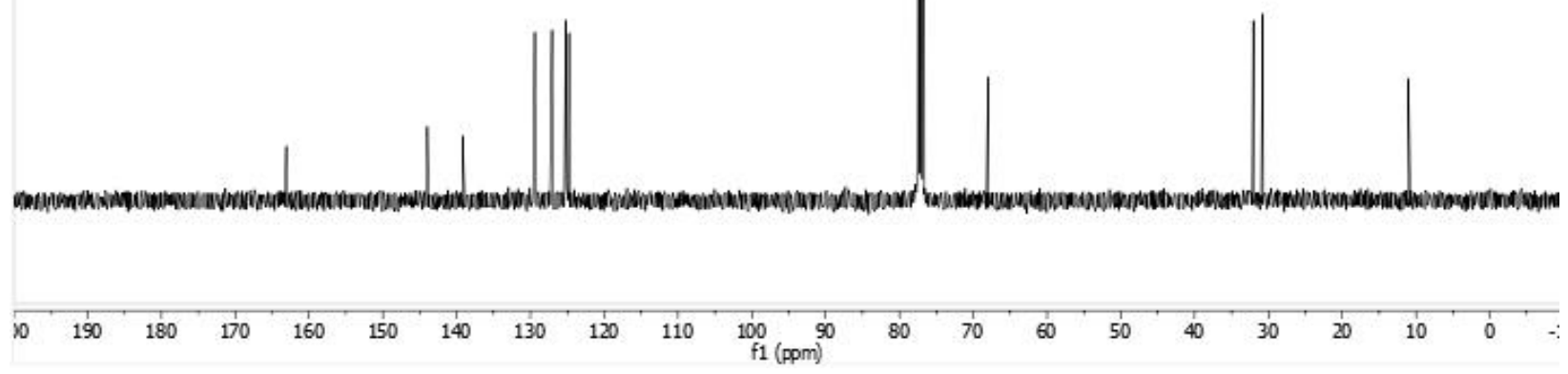

HSQC NMR spectrum of $\mathbf{3 v q}$ in $\mathrm{CDCl}_{3}$ containing $0.03 \%$ (v/v) TMS (500, $126 \mathrm{MHz}$ ).

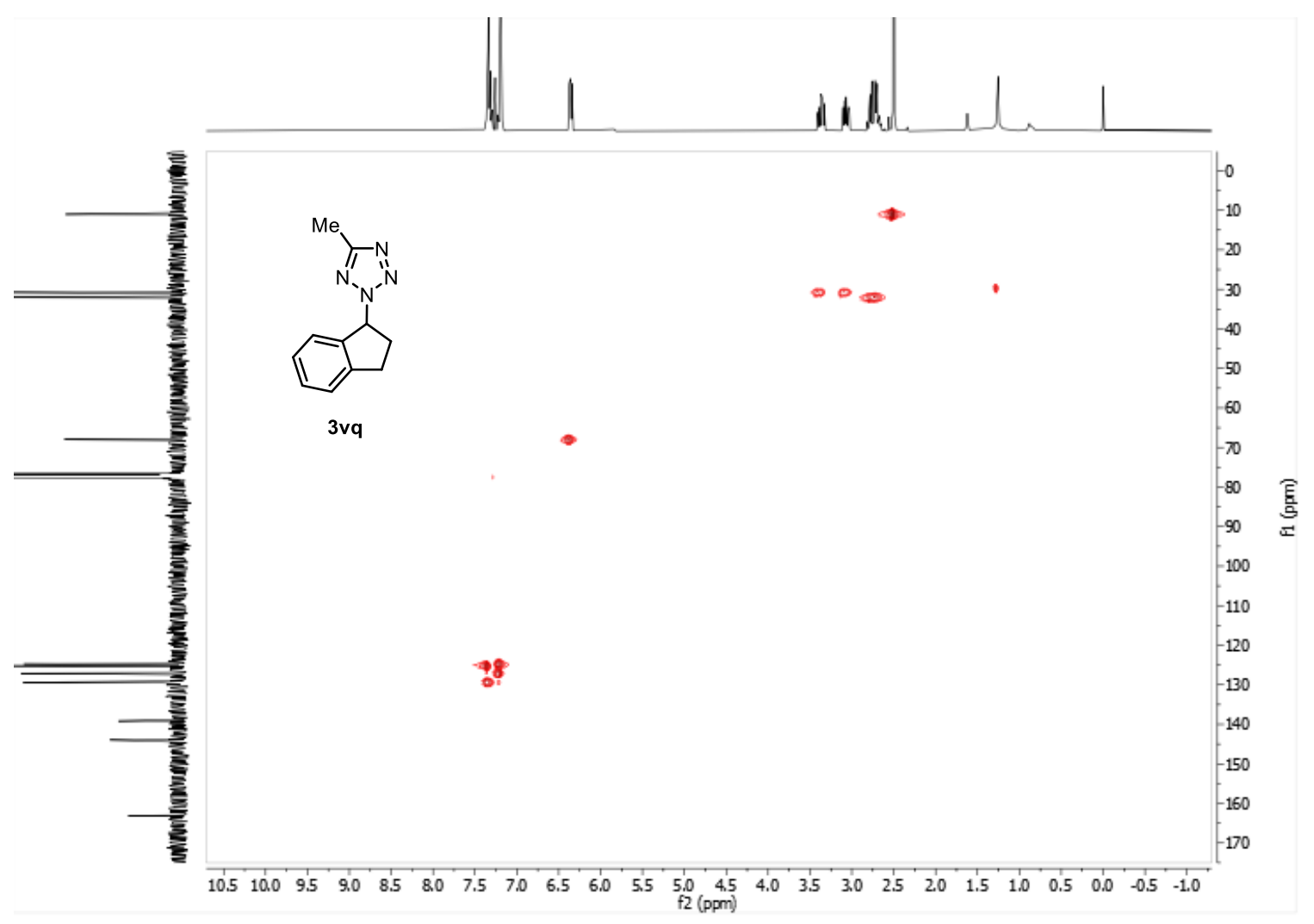


HMBC NMR spectrum of $3 \mathbf{v q}$ in $\mathrm{CDCl}_{3}$ containing $0.03 \%$ (v/v) TMS (500, $126 \mathrm{MHz}$ ).

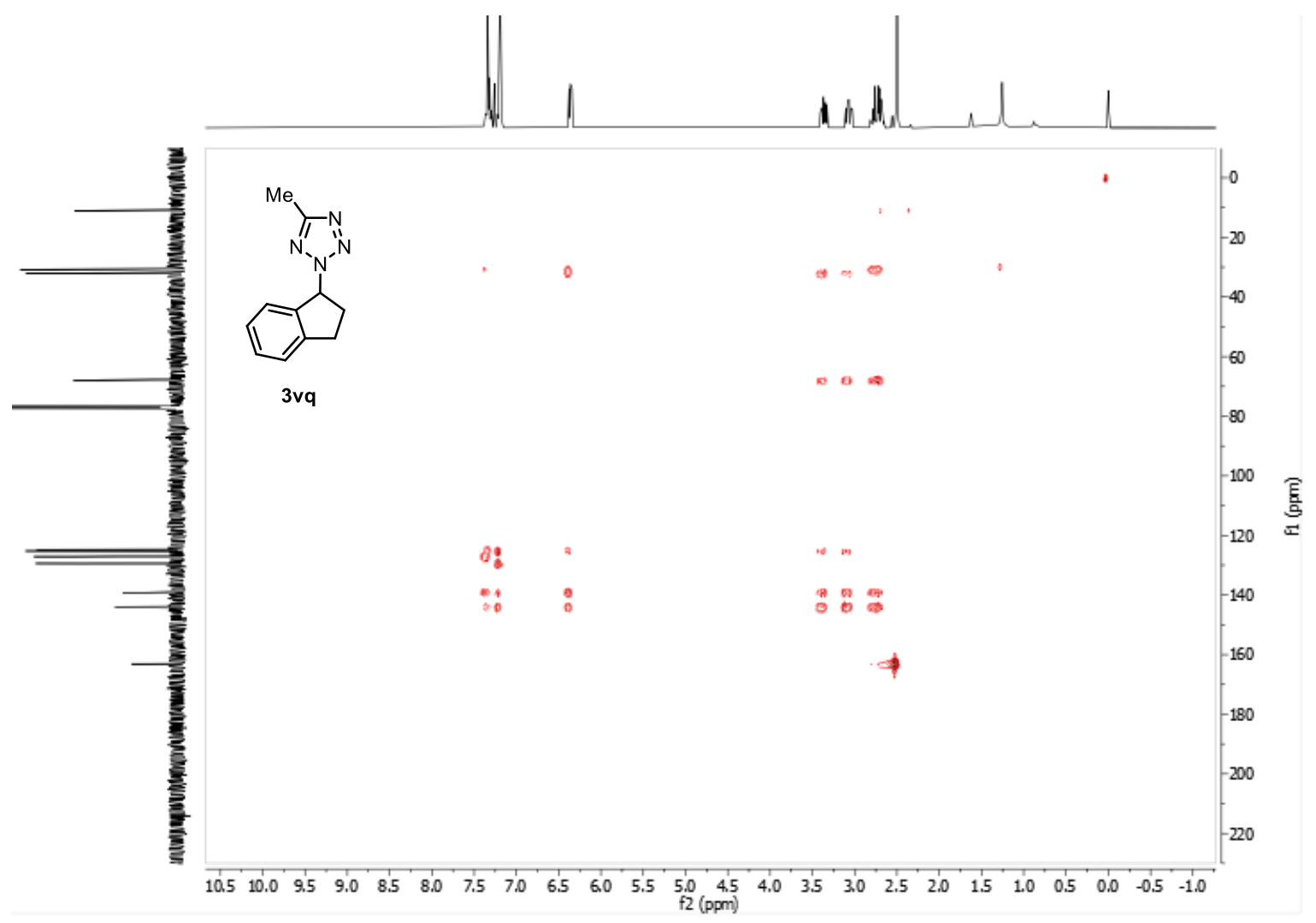

${ }^{1} \mathrm{H}$ NMR spectrum of $\mathbf{3 v q}$ ' in $\mathrm{CDCl}_{3}$ containing $0.03 \%$ (v/v) TMS (500 MHz).

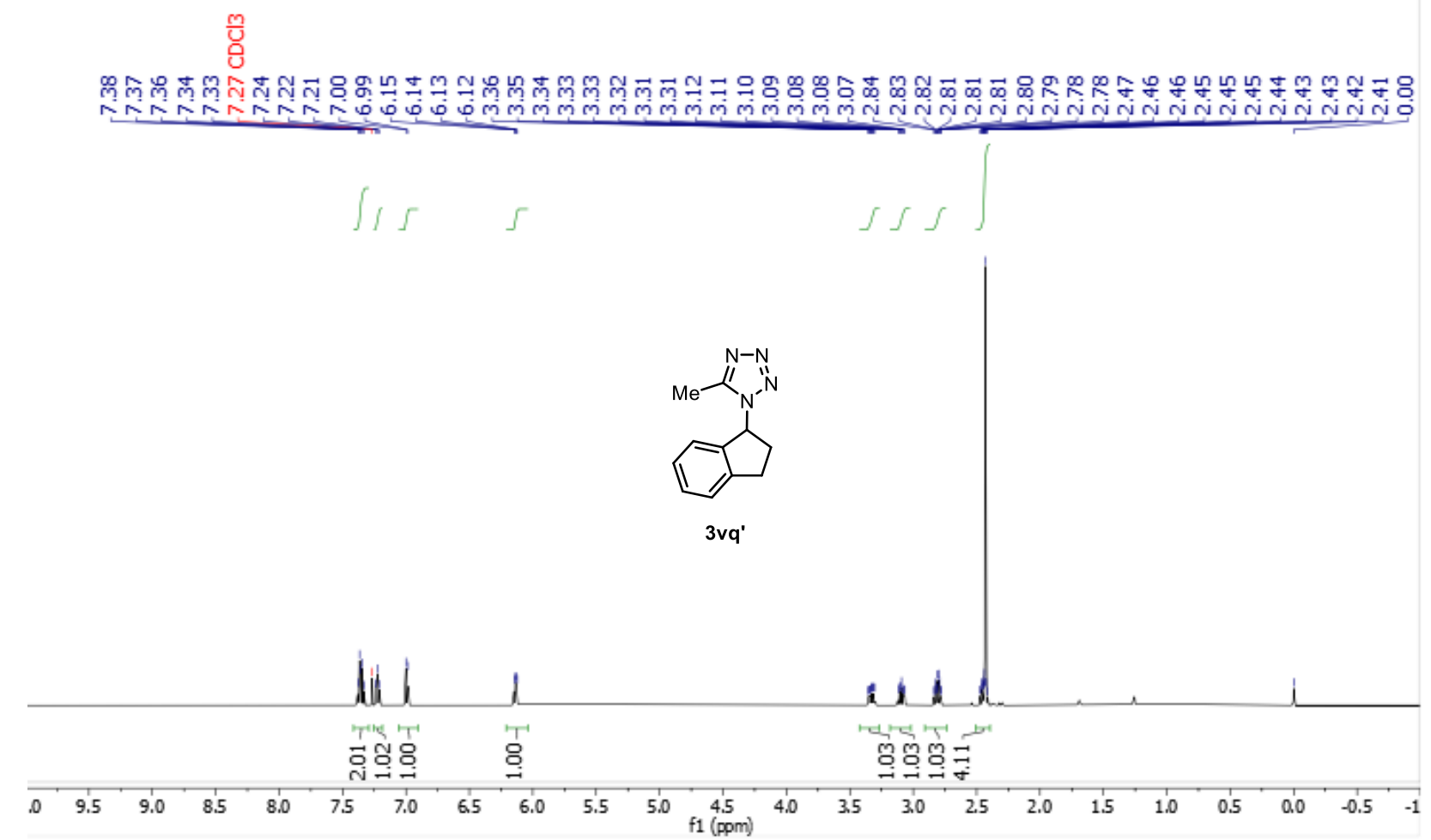


${ }^{13} \mathrm{C}$ NMR spectrum of $\mathbf{3 v q}$ ' in $\mathrm{CDCl}_{3}$ containing $0.03 \%$ (v/v) TMS (126 MHz).
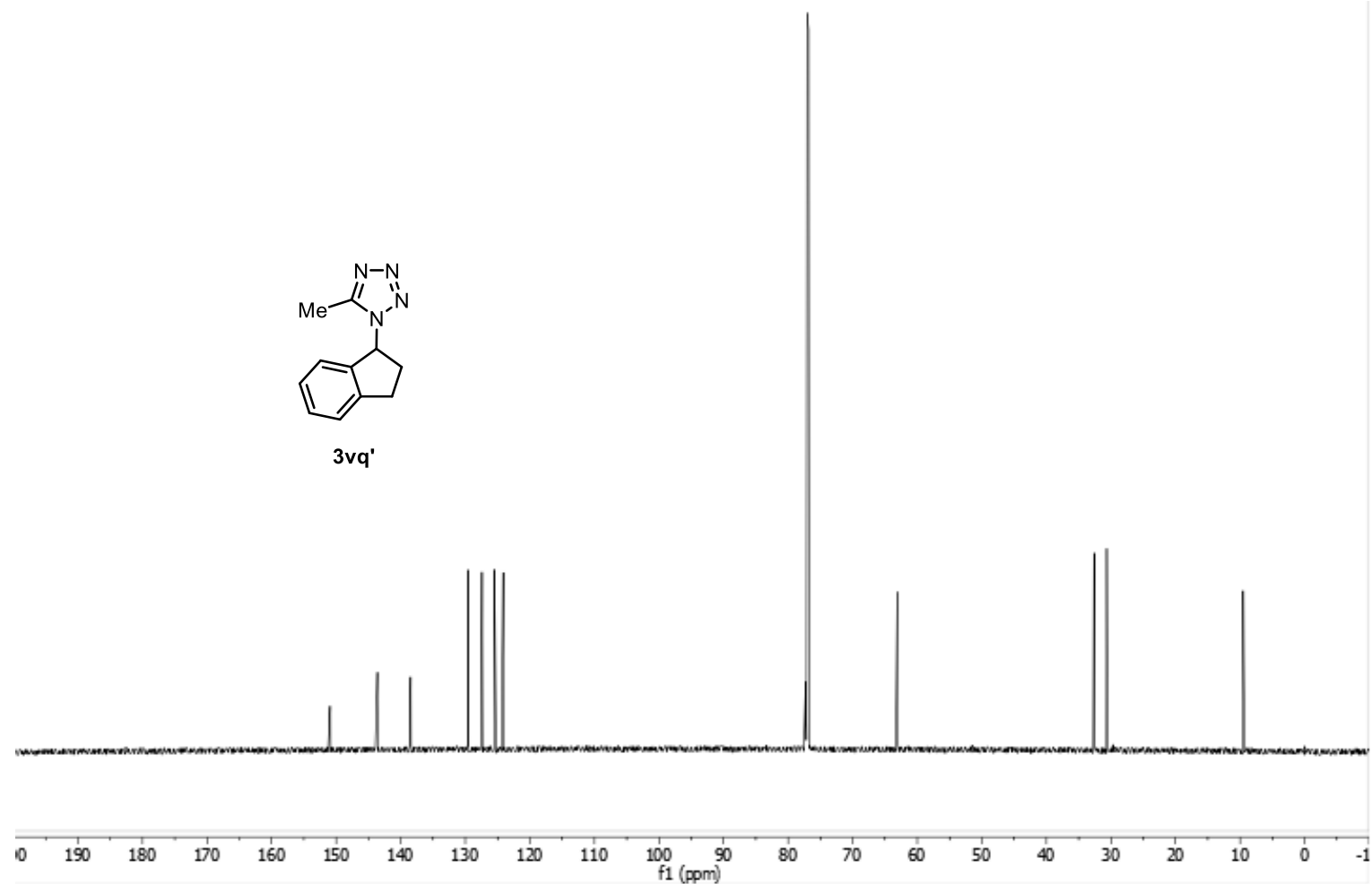

HSQC NMR spectrum of 3vq' in $\mathrm{CDCl}_{3}$ containing $0.03 \%$ (v/v) TMS $(500,126 \mathrm{MHz})$.

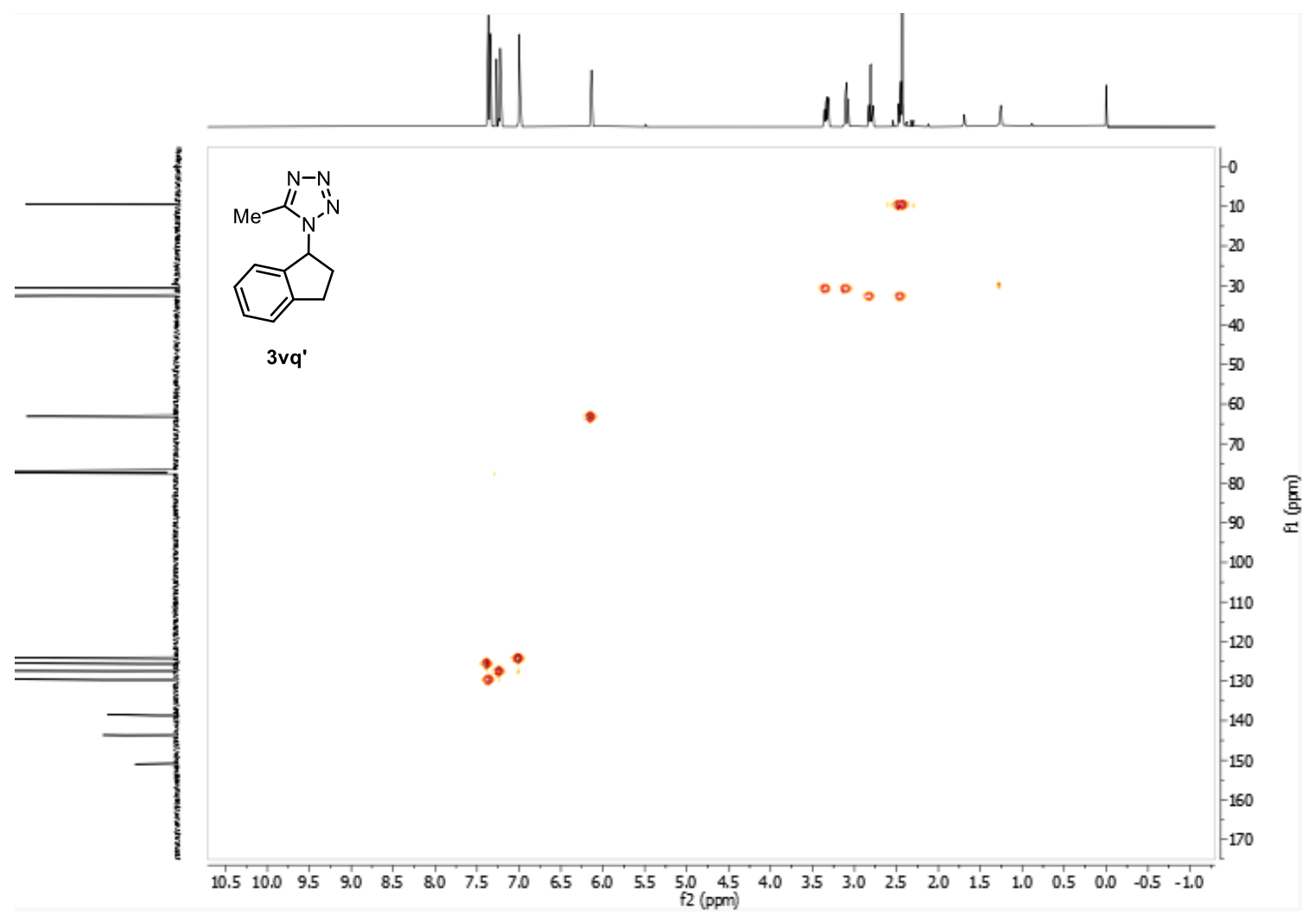


HMBC NMR spectrum of 3vq' in $\mathrm{CDCl}_{3}$ containing $0.03 \%$ (v/v) TMS (500, $126 \mathrm{MHz}$ ).

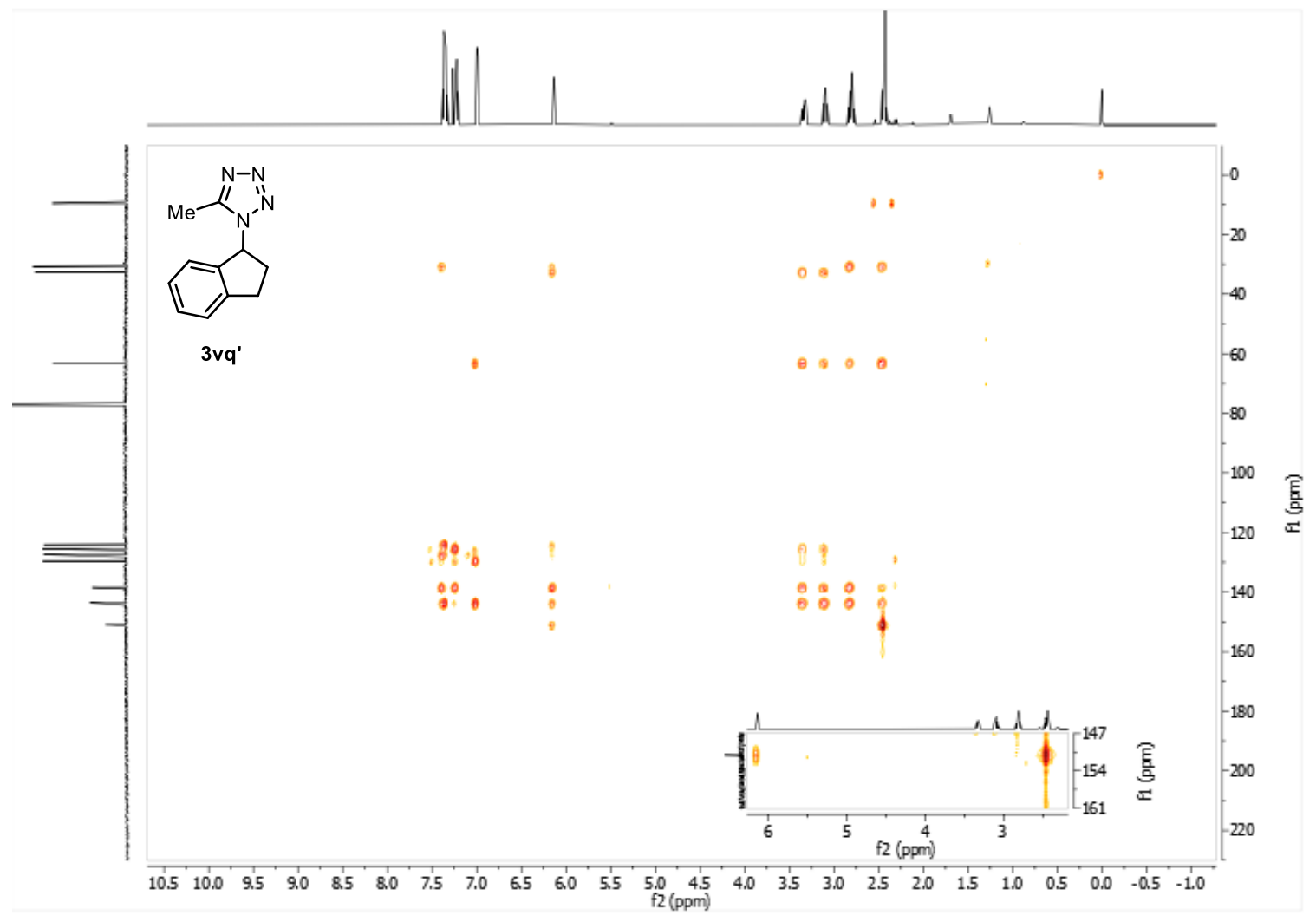

${ }^{1} \mathrm{H}$ NMR spectrum of $3 \mathbf{v r}$ in $\mathrm{CDCl}_{3}$ containing $0.03 \%$ (v/v) TMS (500 MHz).

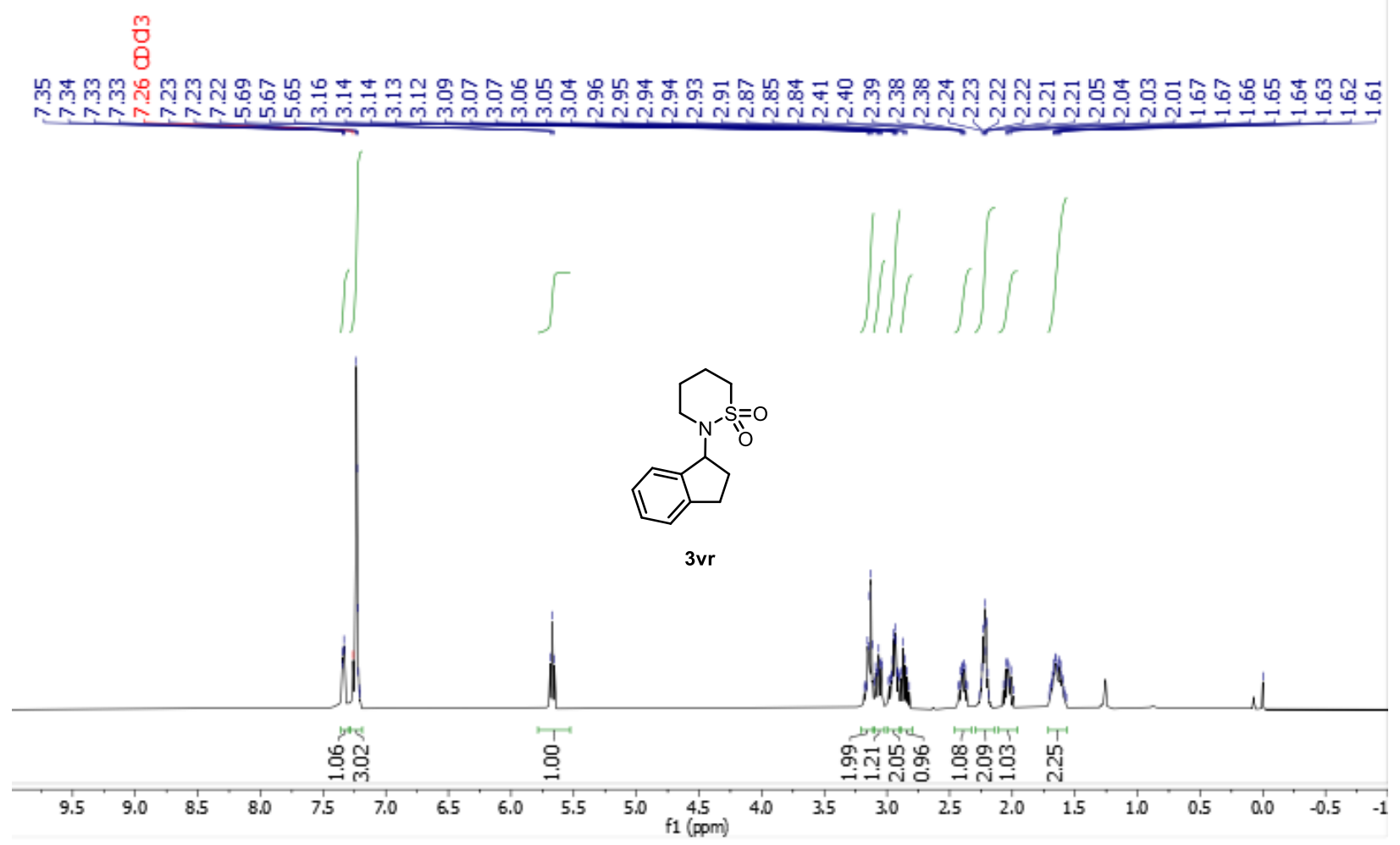


${ }^{13} \mathrm{C}$ NMR spectrum of $\mathbf{3 v r}$ in $\mathrm{CDCl}_{3}$ containing $0.03 \%$ (v/v) TMS (126 MHz).

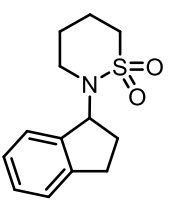

$3 \mathrm{vr}$

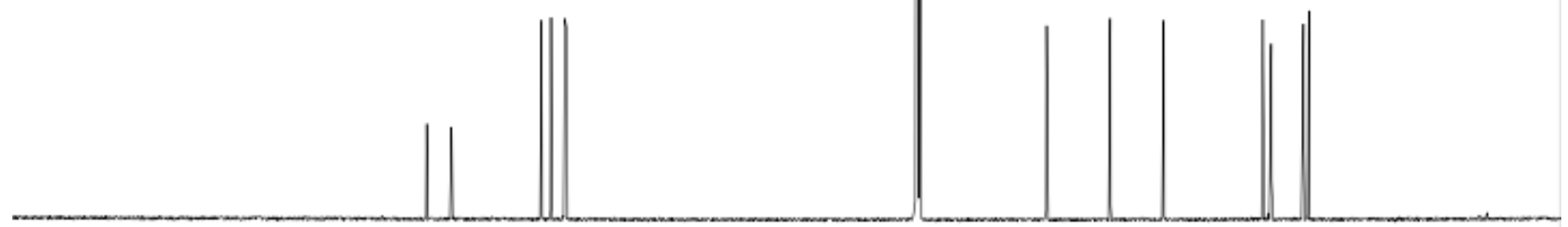

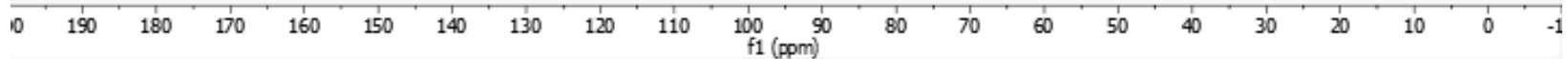

${ }^{1} \mathrm{H}$ NMR spectrum of $\mathbf{3 w h}$ in $\mathrm{CDCl}_{3}$ containing $0.03 \%$ (v/v) TMS (500 MHz).
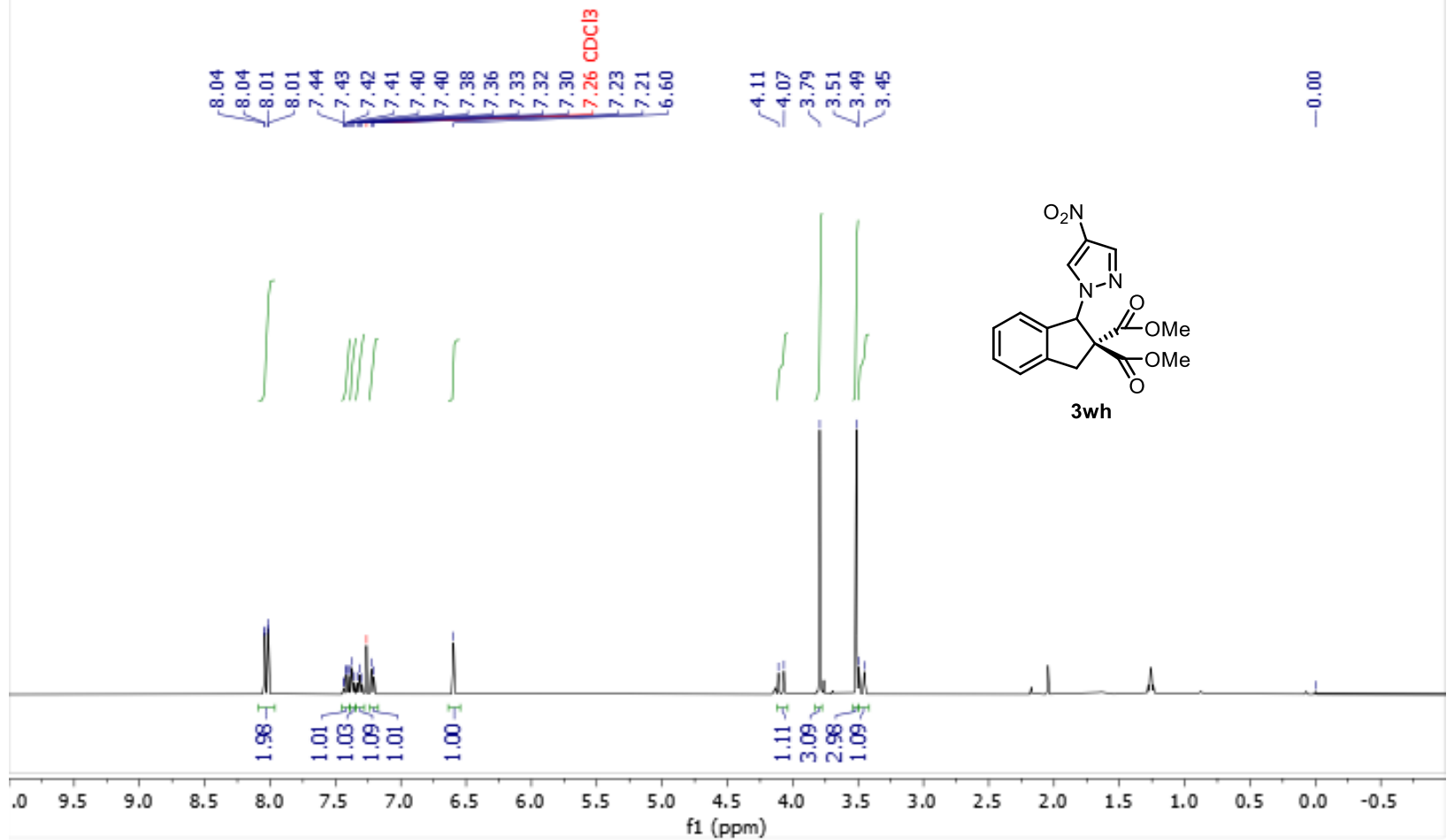
${ }^{13} \mathrm{C}$ NMR spectrum of $3 \mathbf{w h}$ in $\mathrm{CDCl}_{3}$ containing $0.03 \%$ (v/v) TMS (126 MHz).

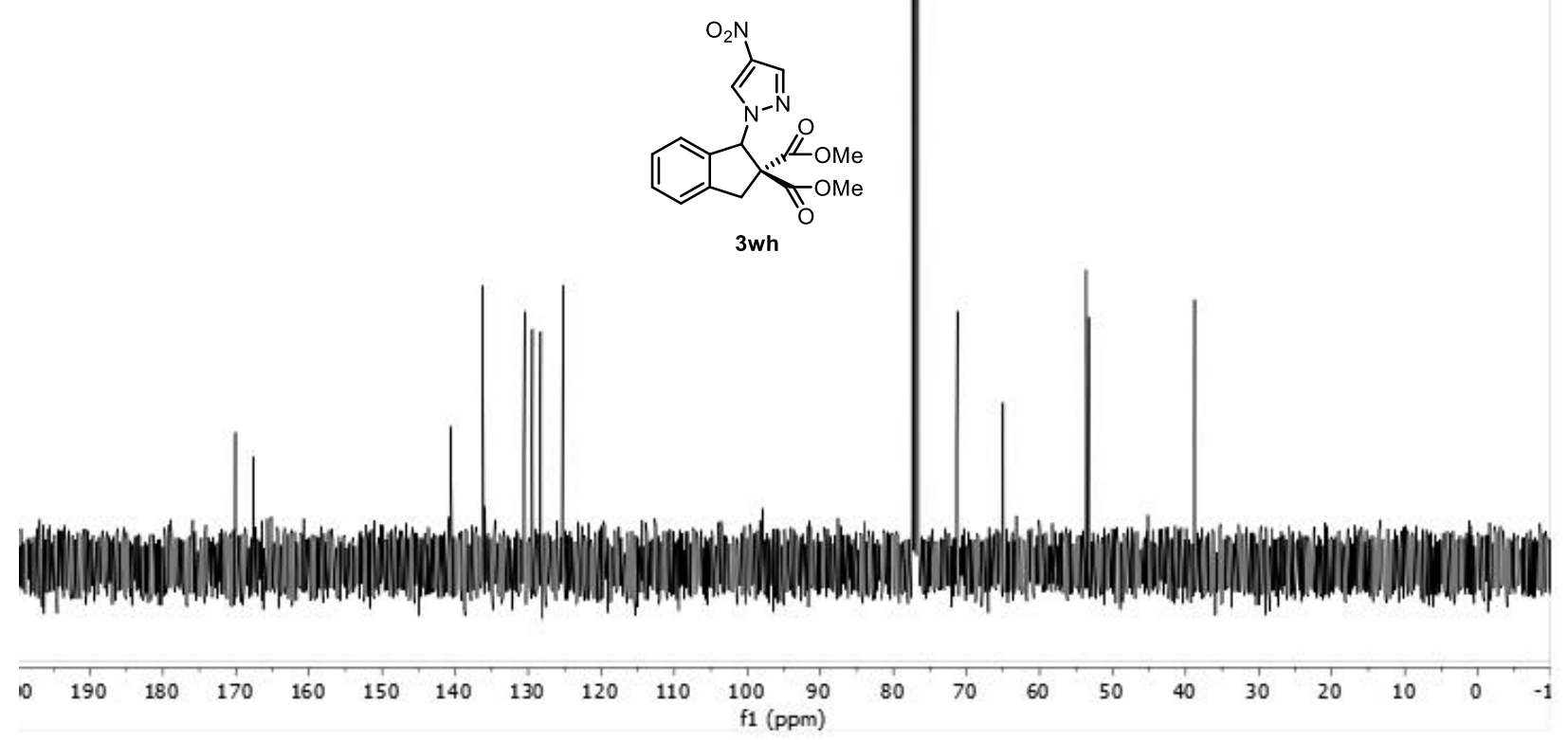

${ }^{1} \mathrm{H}$ NMR spectrum of $\mathbf{3 w a}$ in $\mathrm{CDCl}_{3}$ containing $0.03 \%(\mathrm{v} / \mathrm{v})$ TMS (500 MHz).

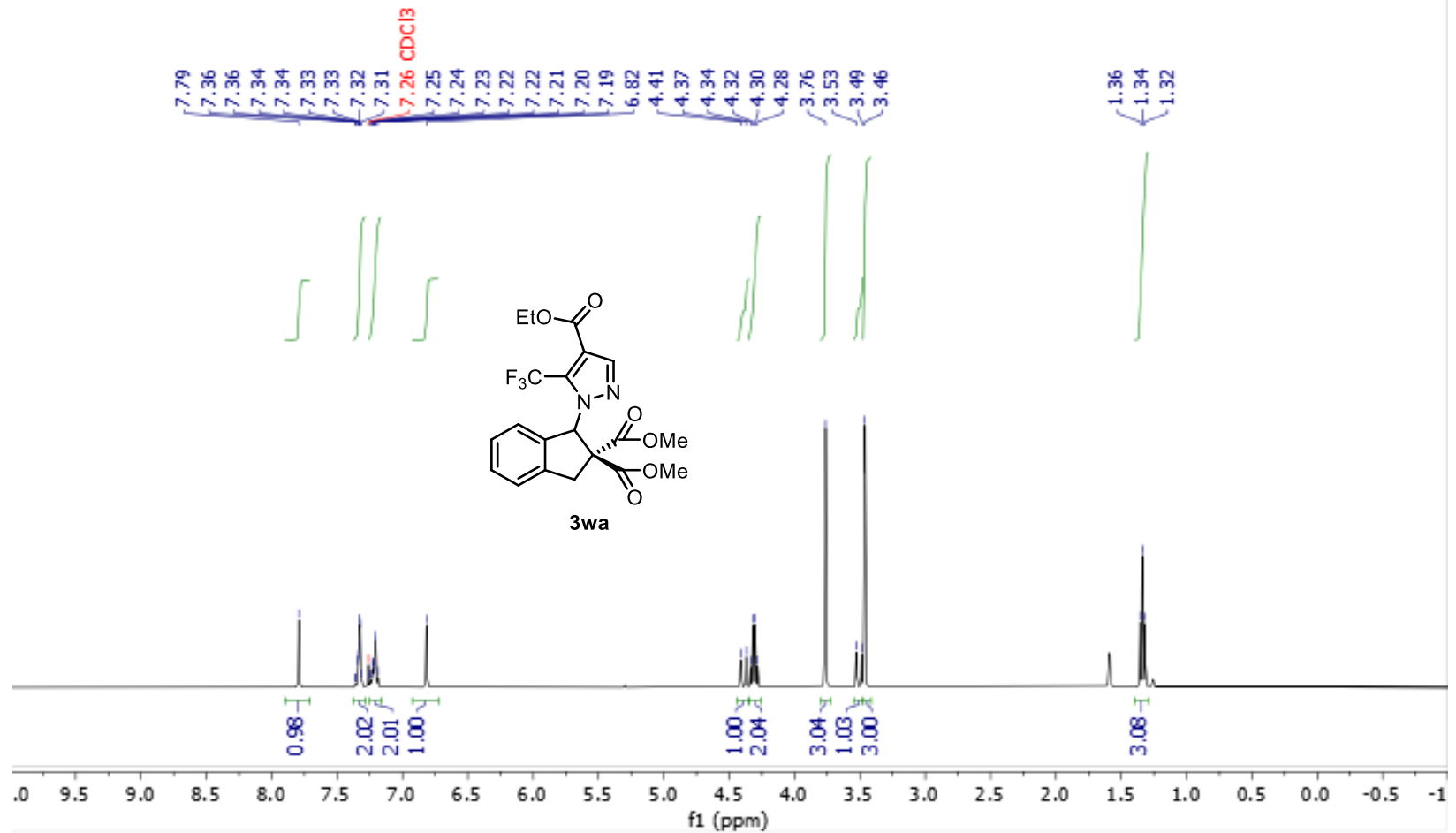


${ }^{13} \mathrm{C}$ NMR spectrum of 3wa in $\mathrm{CDCl}_{3}$ containing $0.03 \%$ (v/v) TMS (126 MHz).
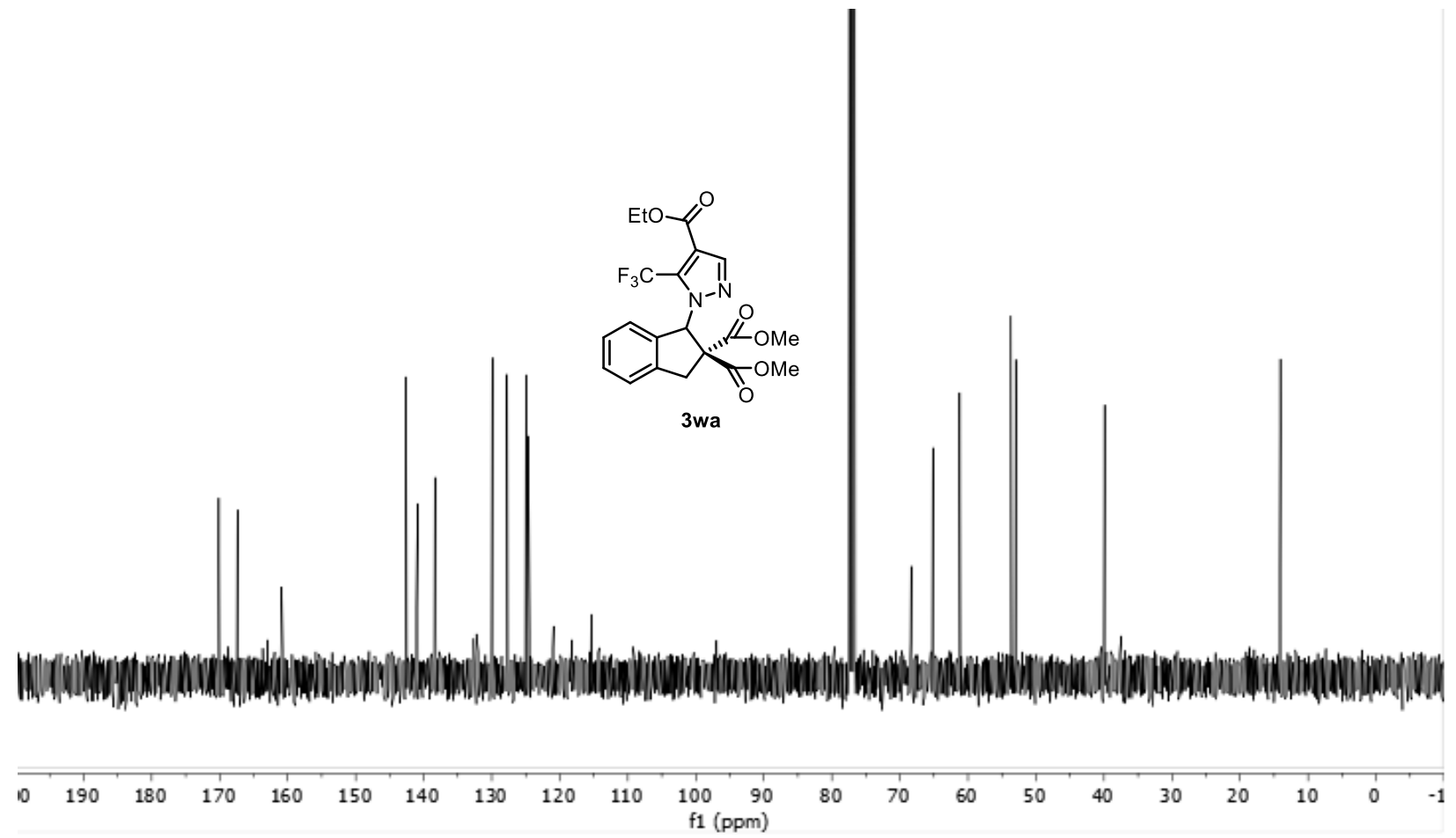

${ }^{19} \mathrm{~F}$ NMR spectrum of 3 wa in $\mathrm{CDCl}_{3}$ containing $0.03 \%$ (v/v) TMS (377 MHz).

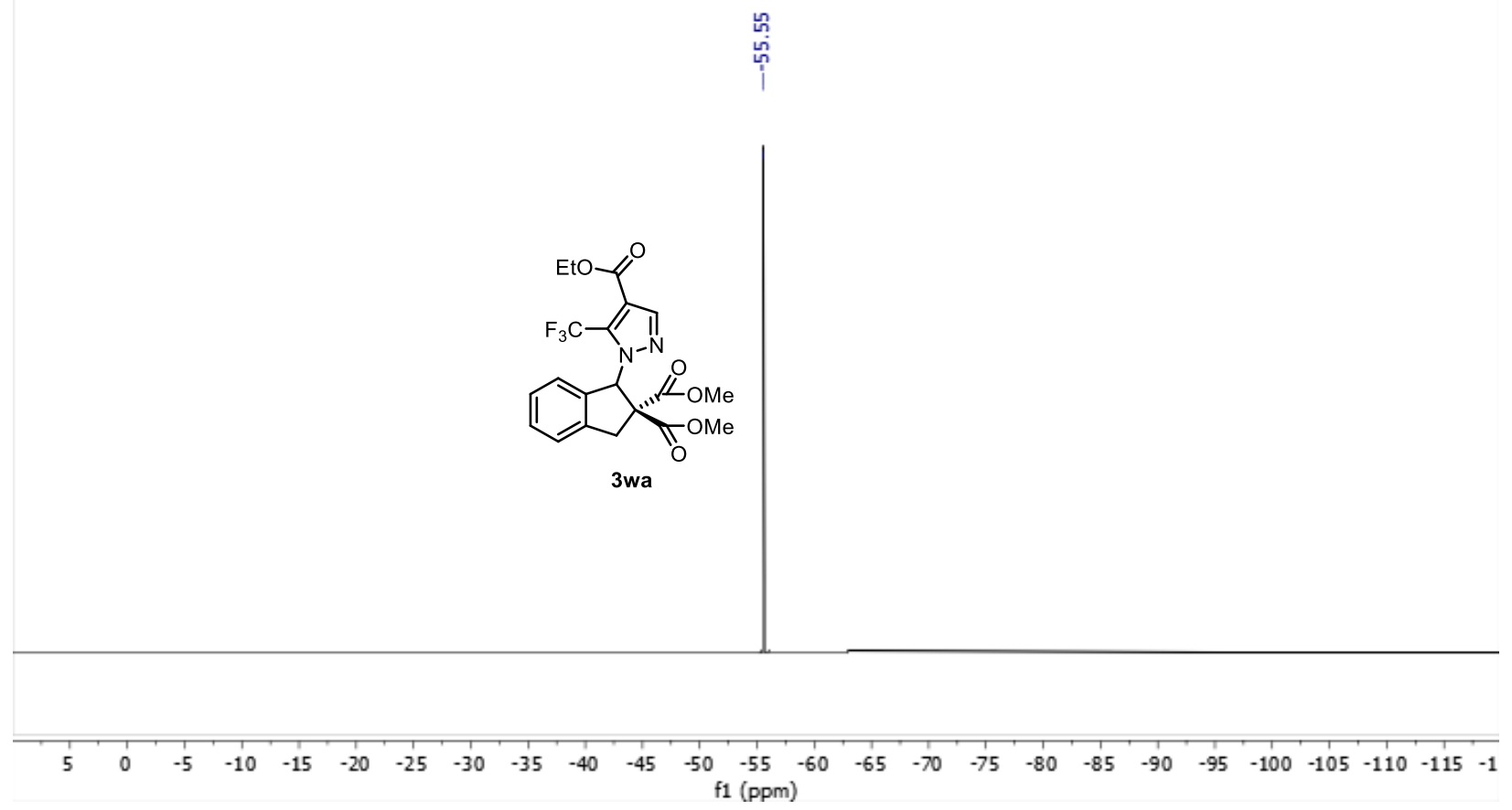


${ }^{1} \mathrm{H}$ NMR spectrum of $\mathbf{3 w a}$ ' in $\mathrm{CDCl}_{3}$ containing $0.03 \%$ (v/v) TMS (500 MHz).

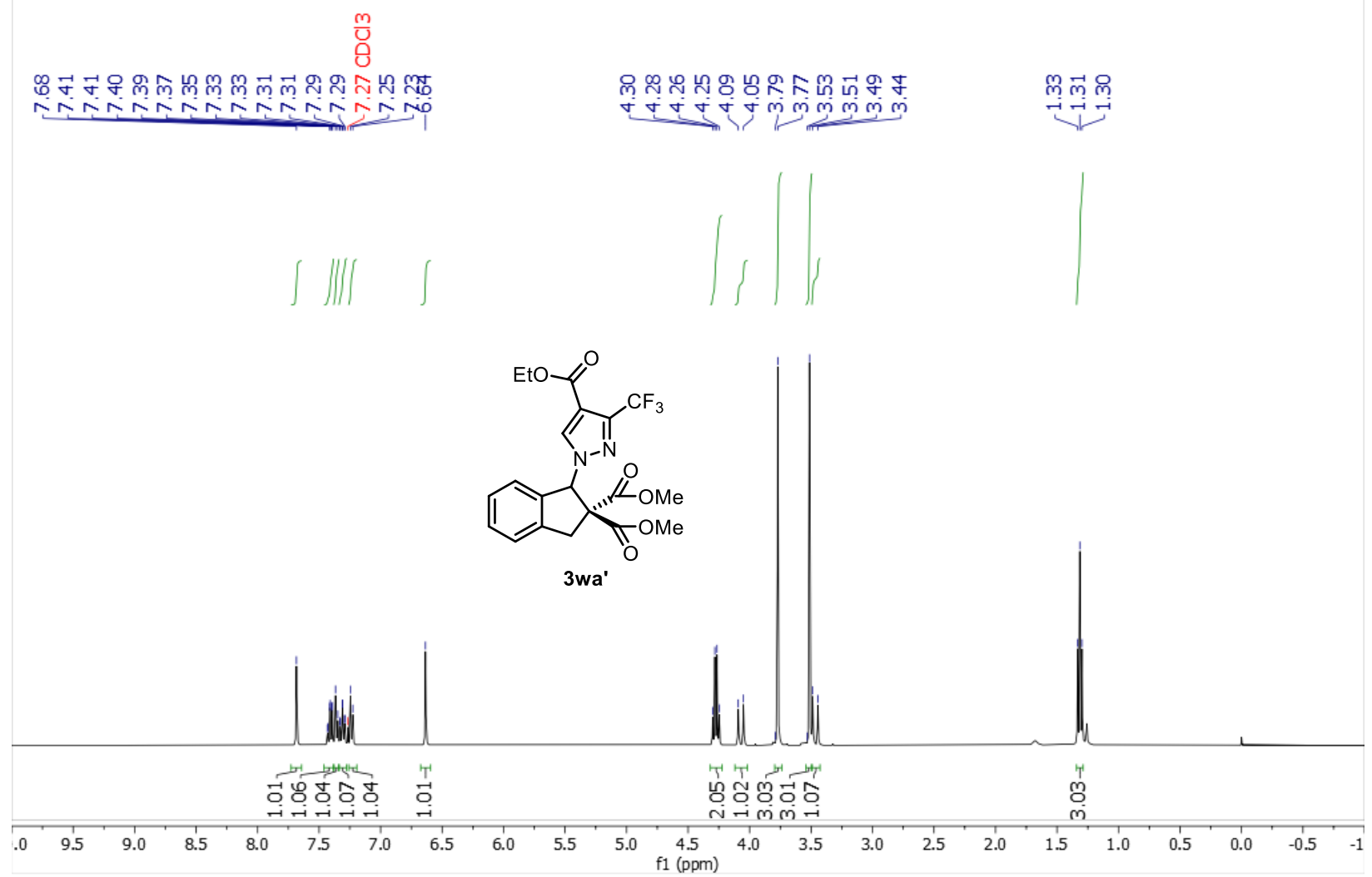

${ }^{13} \mathrm{C}$ NMR spectrum of 3wa' in $\mathrm{CDCl}_{3}$ containing $0.03 \%$ (v/v) TMS (126 MHz).

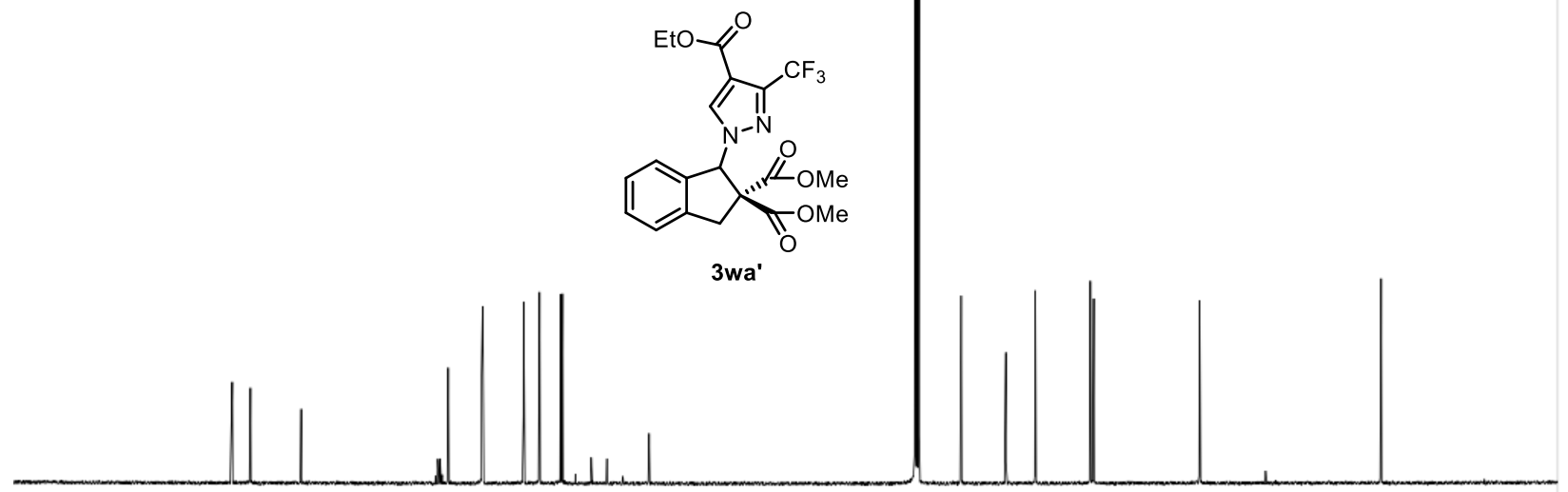


${ }^{19} \mathrm{~F}$ NMR spectrum of $\mathbf{3} \mathbf{w a}^{\prime}$ in $\mathrm{CDCl}_{3}$ containing $0.03 \%$ (v/v) TMS (377 MHz).

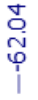

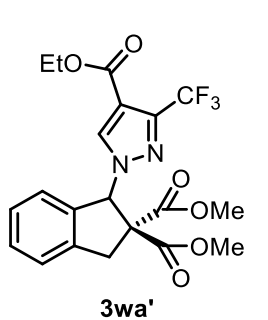

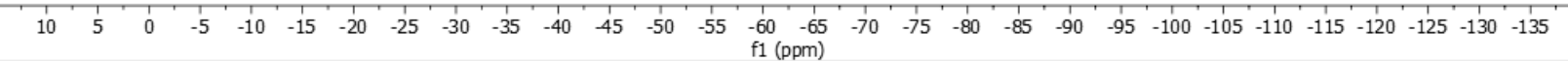

HSQC NMR spectrum of 3wa' in $\mathrm{CDCl}_{3}$ containing $0.03 \%$ (v/v) TMS (500, $126 \mathrm{MHz}$ ).

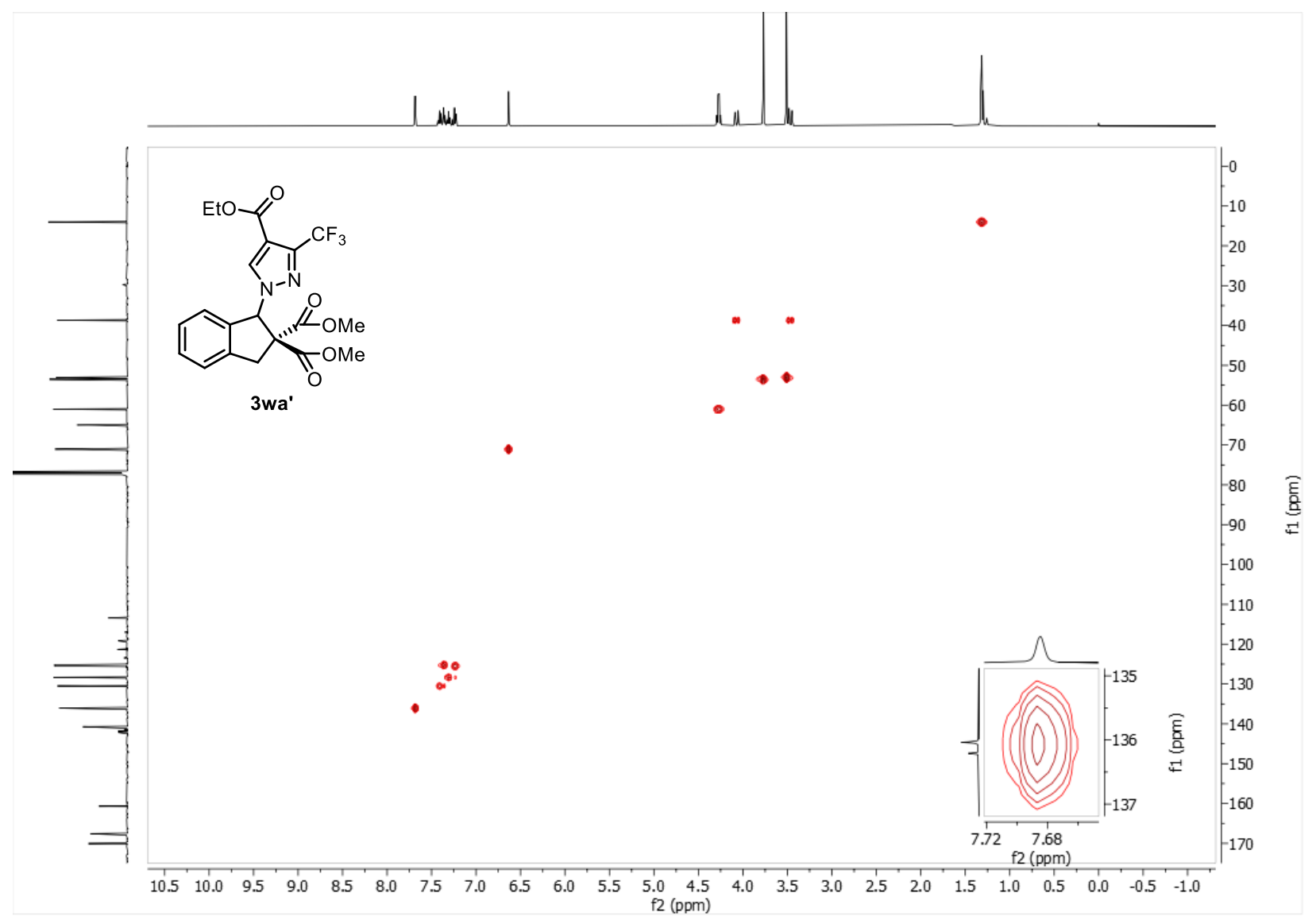


HMBC NMR spectrum of 3wa' in $\mathrm{CDCl}_{3}$ containing $0.03 \%$ (v/v) TMS $(500,126 \mathrm{MHz})$.

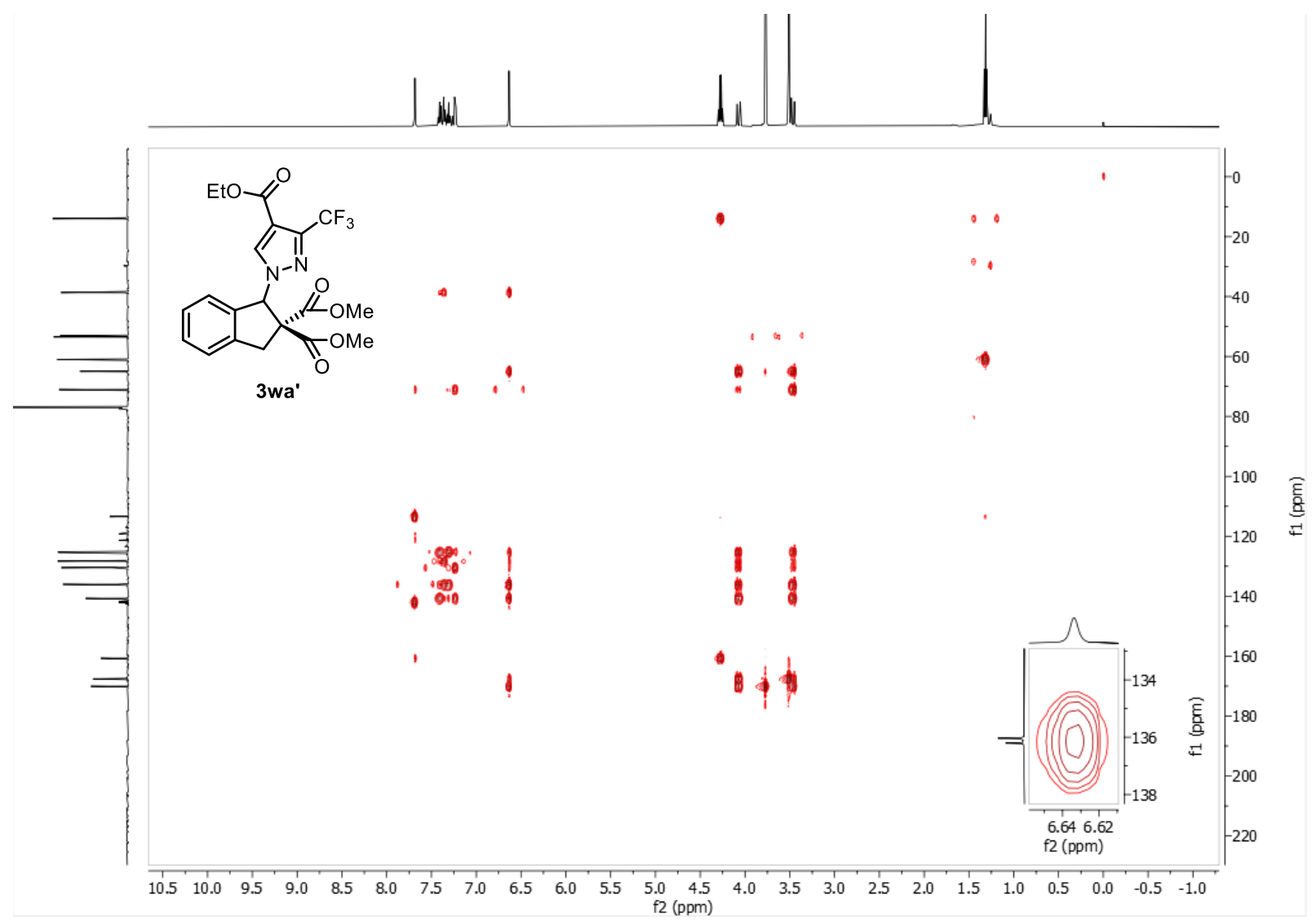

${ }^{1} \mathrm{H}$ NMR spectrum of $3 r f$ in $\mathrm{CDCl}_{3}$ containing $0.03 \%$ (v/v) TMS (500 MHz).

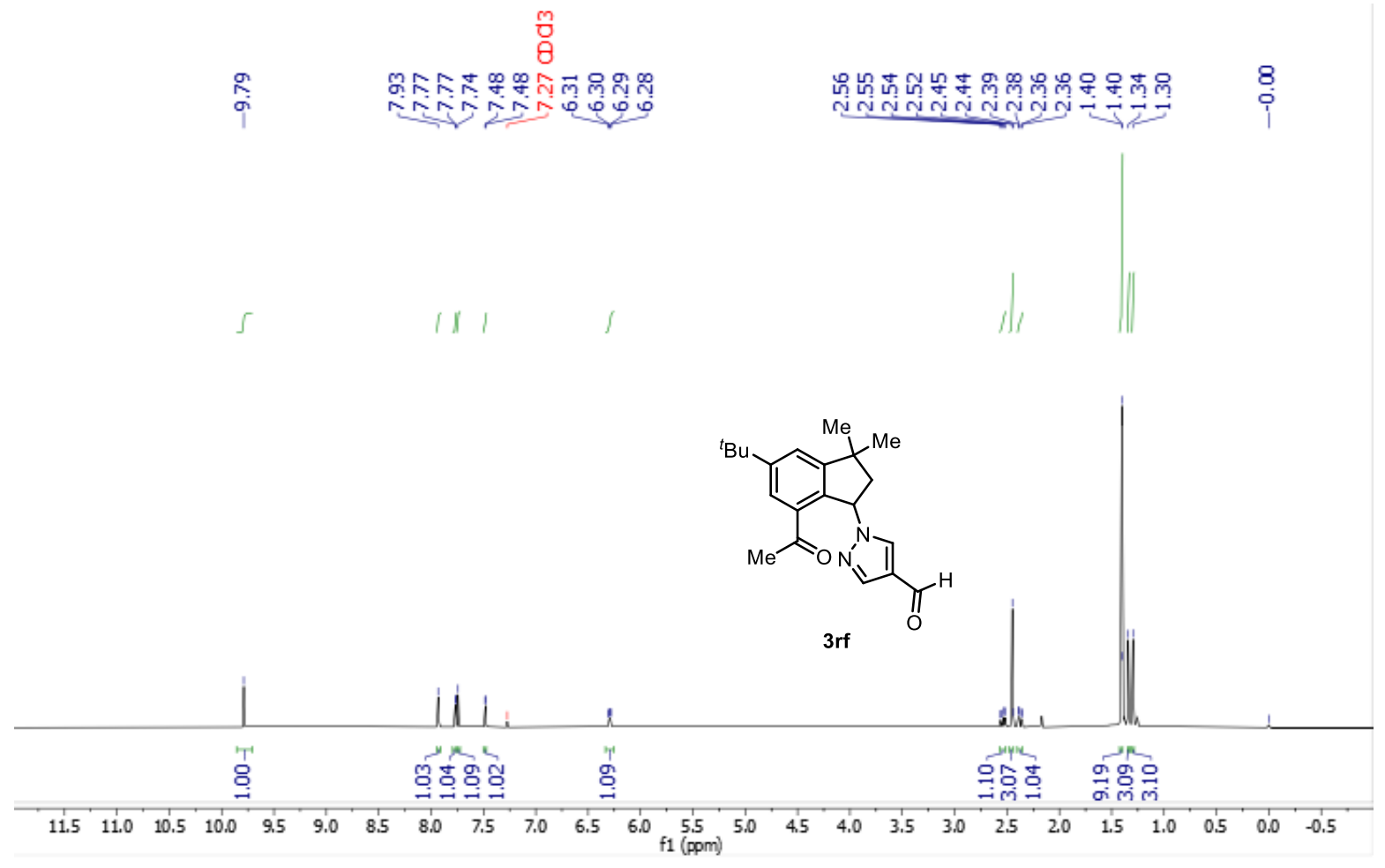


${ }^{13} \mathrm{C}$ NMR spectrum of $3 \mathbf{r f}$ in $\mathrm{CDCl}_{3}$ containing $0.03 \%$ (v/v) TMS (126 MHz).

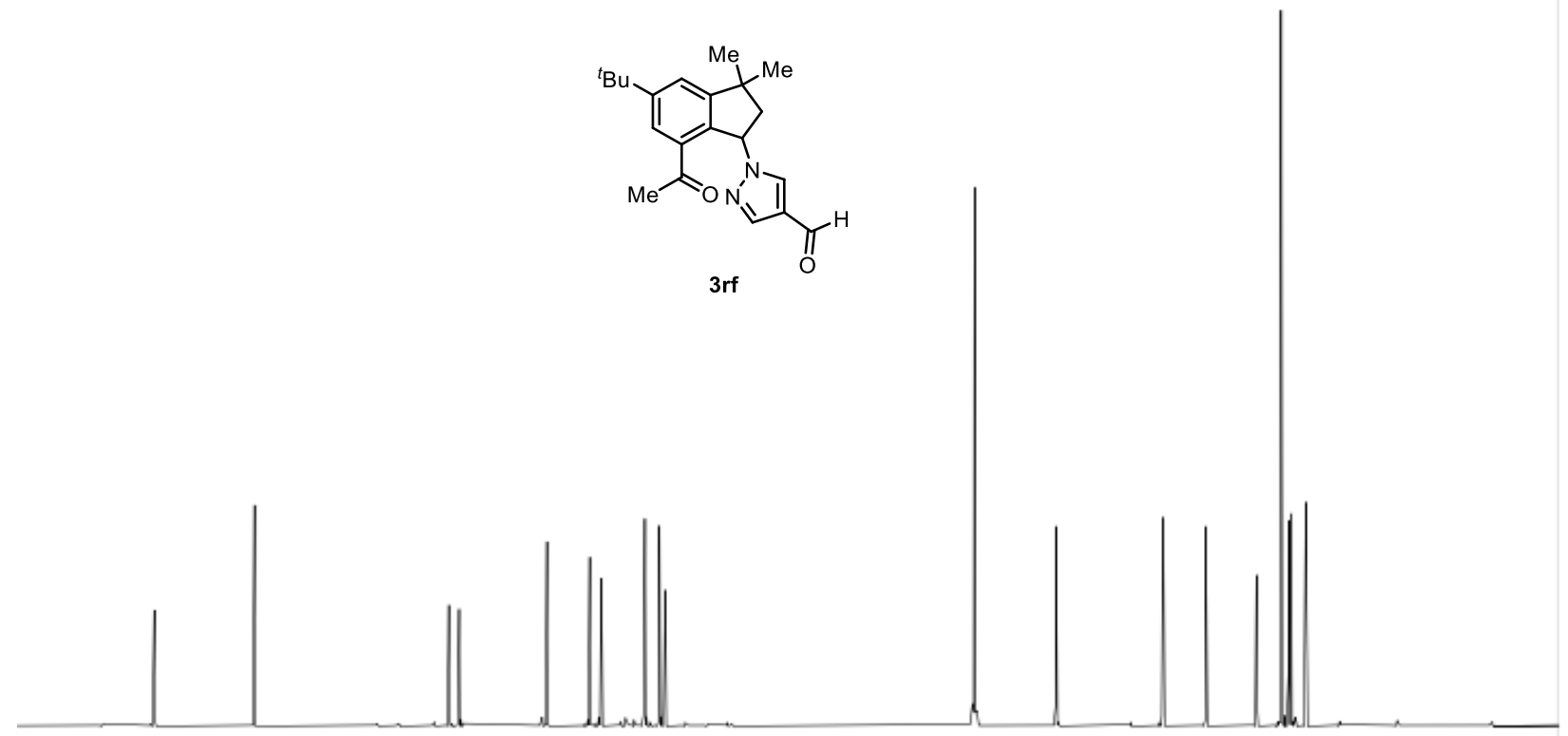

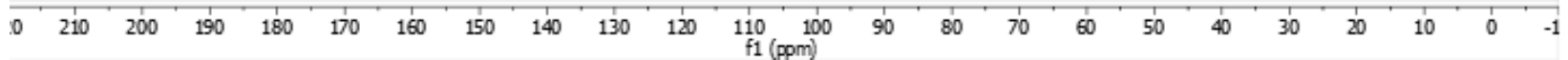

${ }^{1} \mathrm{H}$ NMR spectrum of 3 ri in $\mathrm{CDCl}_{3}$ containing $0.03 \%$ (v/v) TMS (500 MHz).

$\stackrel{m}{8}$

苏苏财内

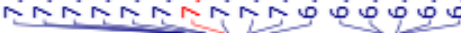

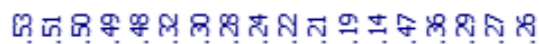

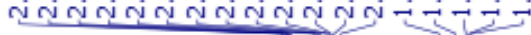

$\stackrel{8}{i}$
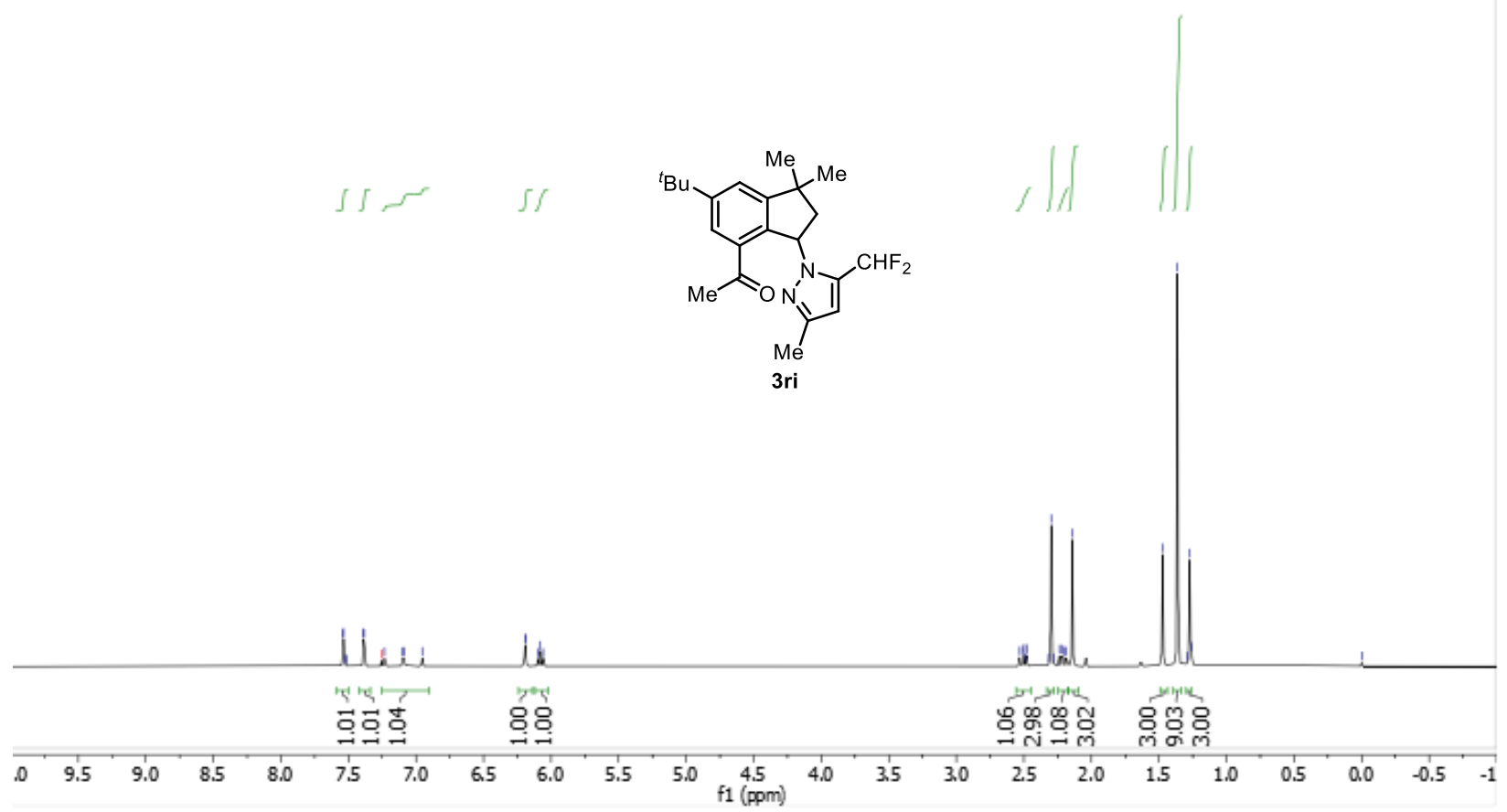
${ }^{13} \mathrm{C}$ NMR spectrum of 3ri in $\mathrm{CDCl}_{3}$ containing $0.03 \%$ (v/v) TMS (126 MHz).

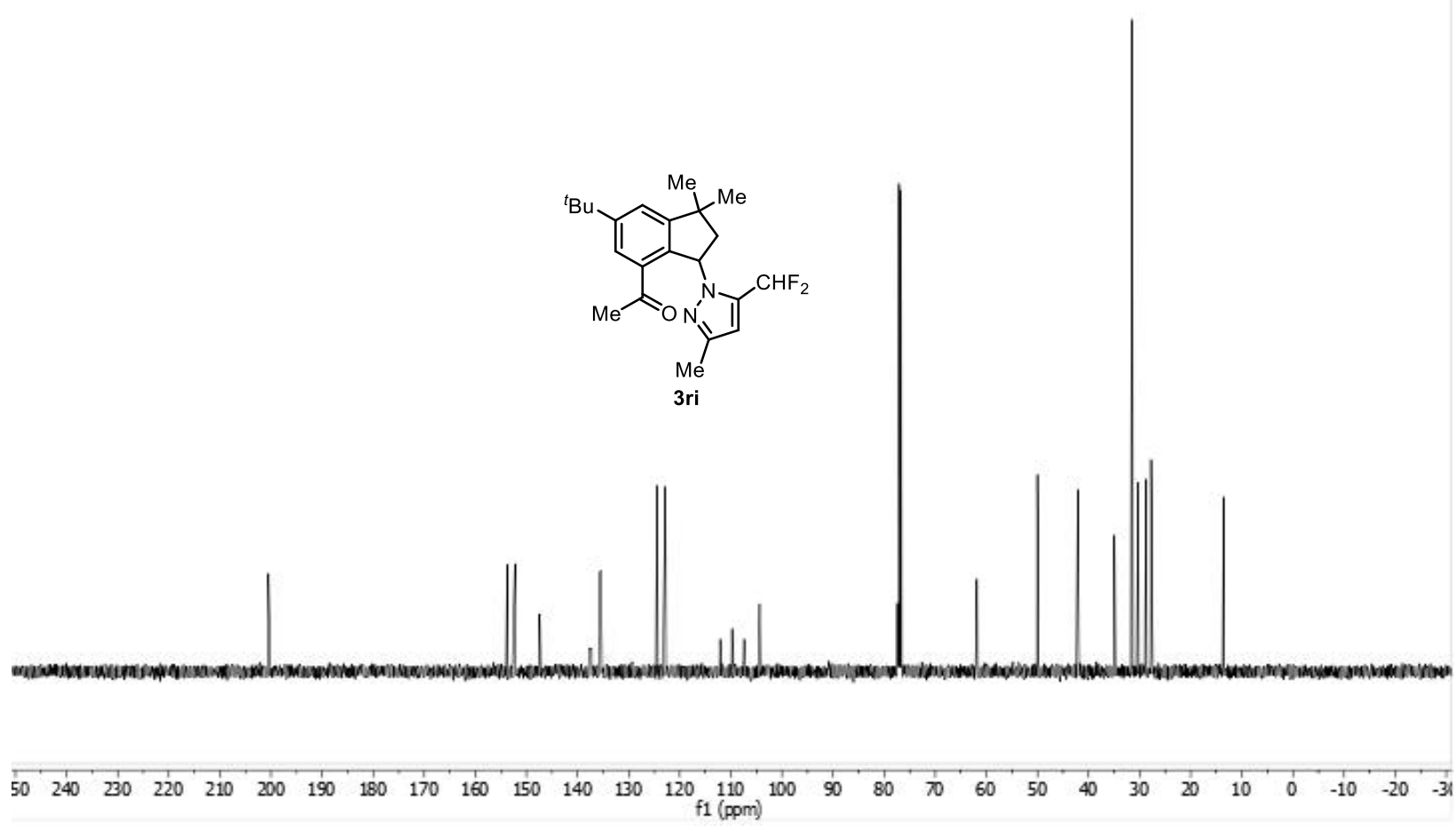

${ }^{19} \mathrm{~F}$ NMR spectrum of 3ri in $\mathrm{CDCl}_{3}$ containing $0.03 \%$ (v/v) TMS (377 MHz).

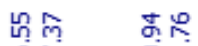

옹

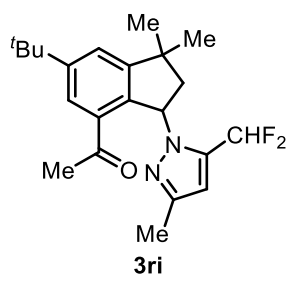

\begin{tabular}{|c|c|c|c|c|c|c|c|c|c|c|c|c|c|c|c|c|}
\hline-30 & -40 & -50 & -60 & -70 & -80 & -90 & -100 & $\begin{array}{c}-110 \\
\mathrm{f} 1 \text { (ppm) }\end{array}$ & -120 & -130 & -140 & -150 & -160 & -170 & -180 & -190 \\
\hline
\end{tabular}


HSQC NMR spectrum of 3wa in $\mathrm{CDCl}_{3}$ containing $0.03 \%$ (v/v) TMS (500, $126 \mathrm{MHz}$ ).

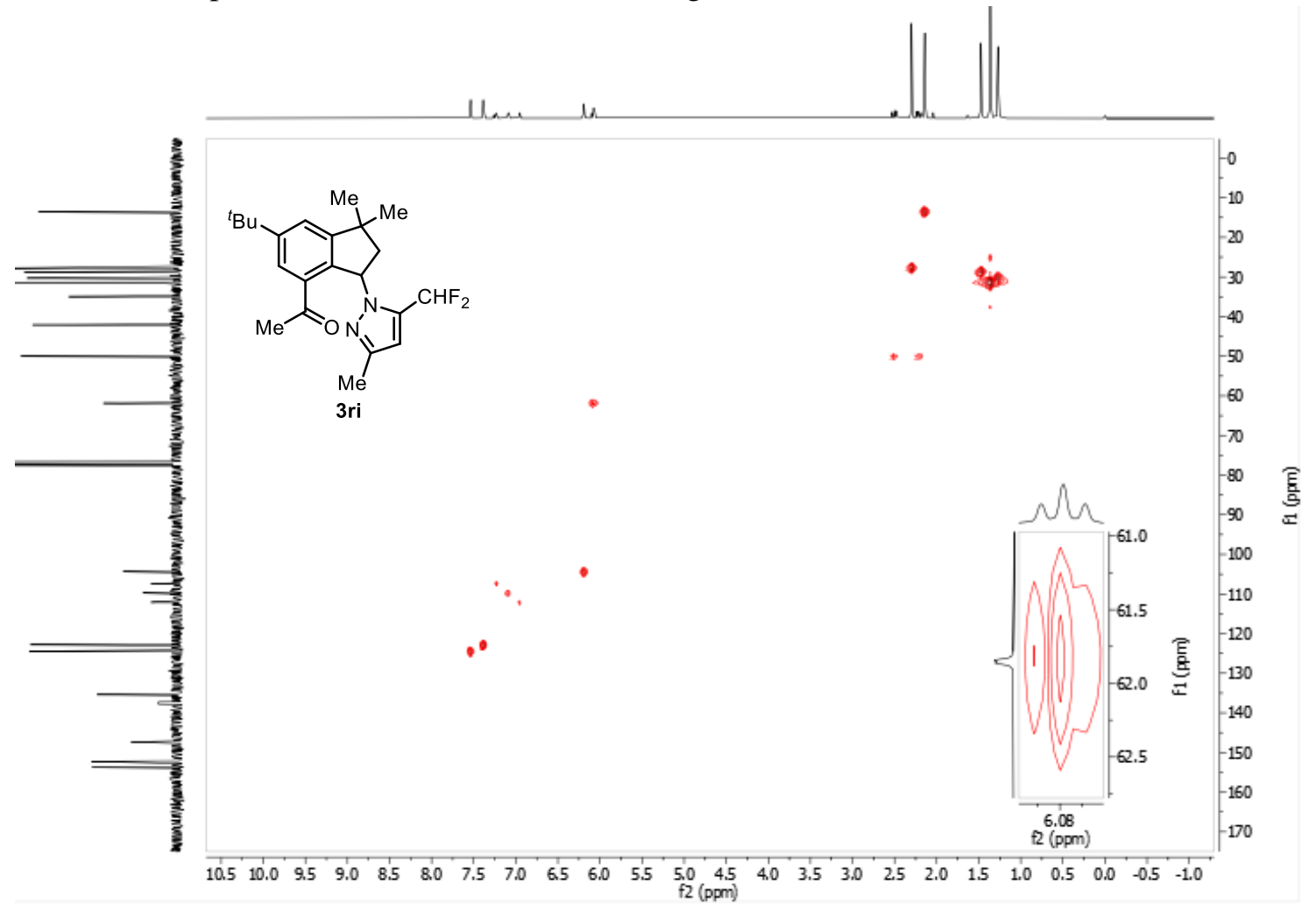

HMBC NMR spectrum of $\mathbf{3 w a}$ in $\mathrm{CDCl}_{3}$ containing $0.03 \%$ (v/v) TMS (500, $126 \mathrm{MHz}$ ).

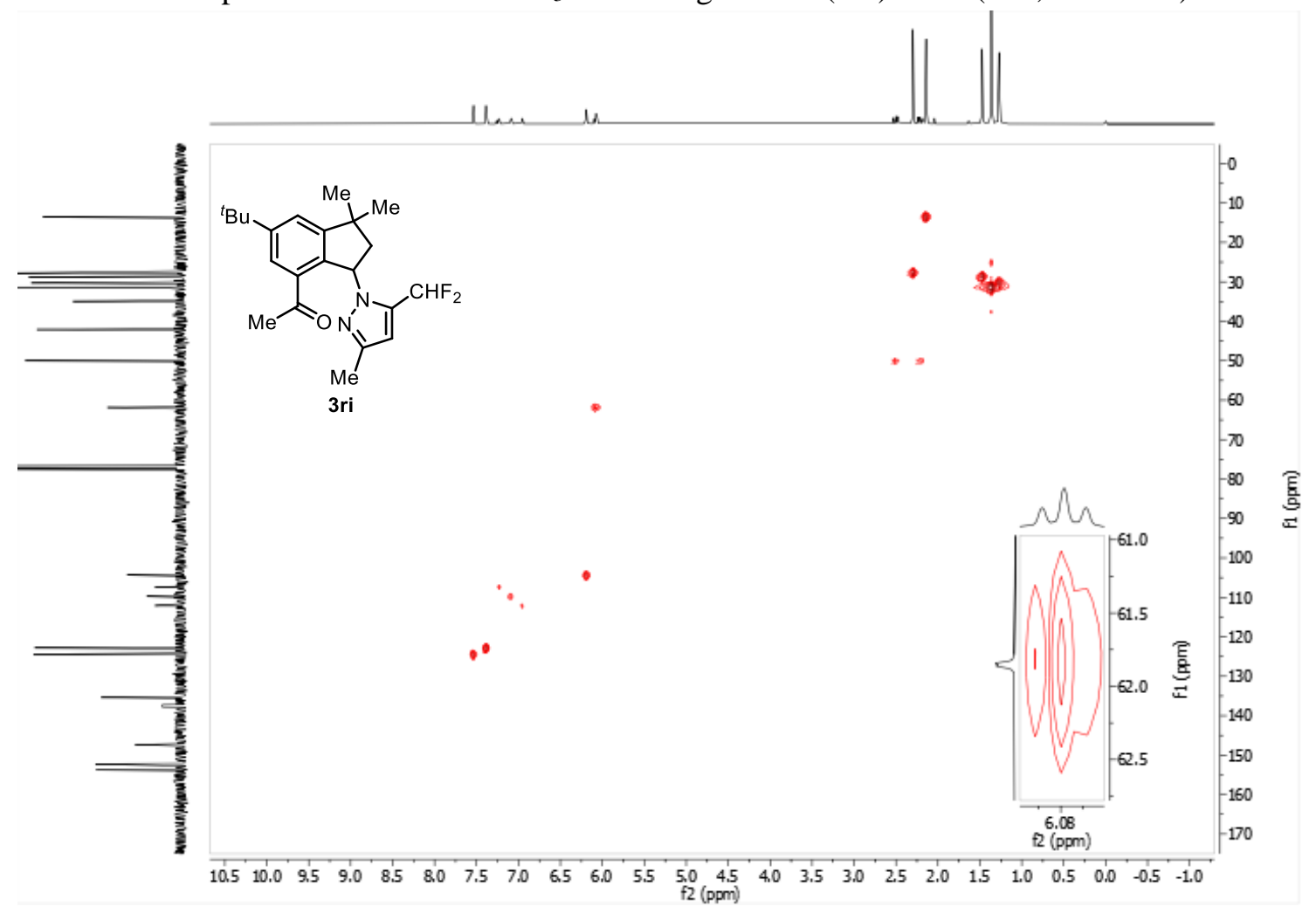


${ }^{1} \mathrm{H}$ NMR spectrum of $3 \mathrm{ti}$ in $\mathrm{CDCl}_{3}$ containing $0.03 \%(\mathrm{v} / \mathrm{v}) \mathrm{TMS}(500 \mathrm{MHz})$.

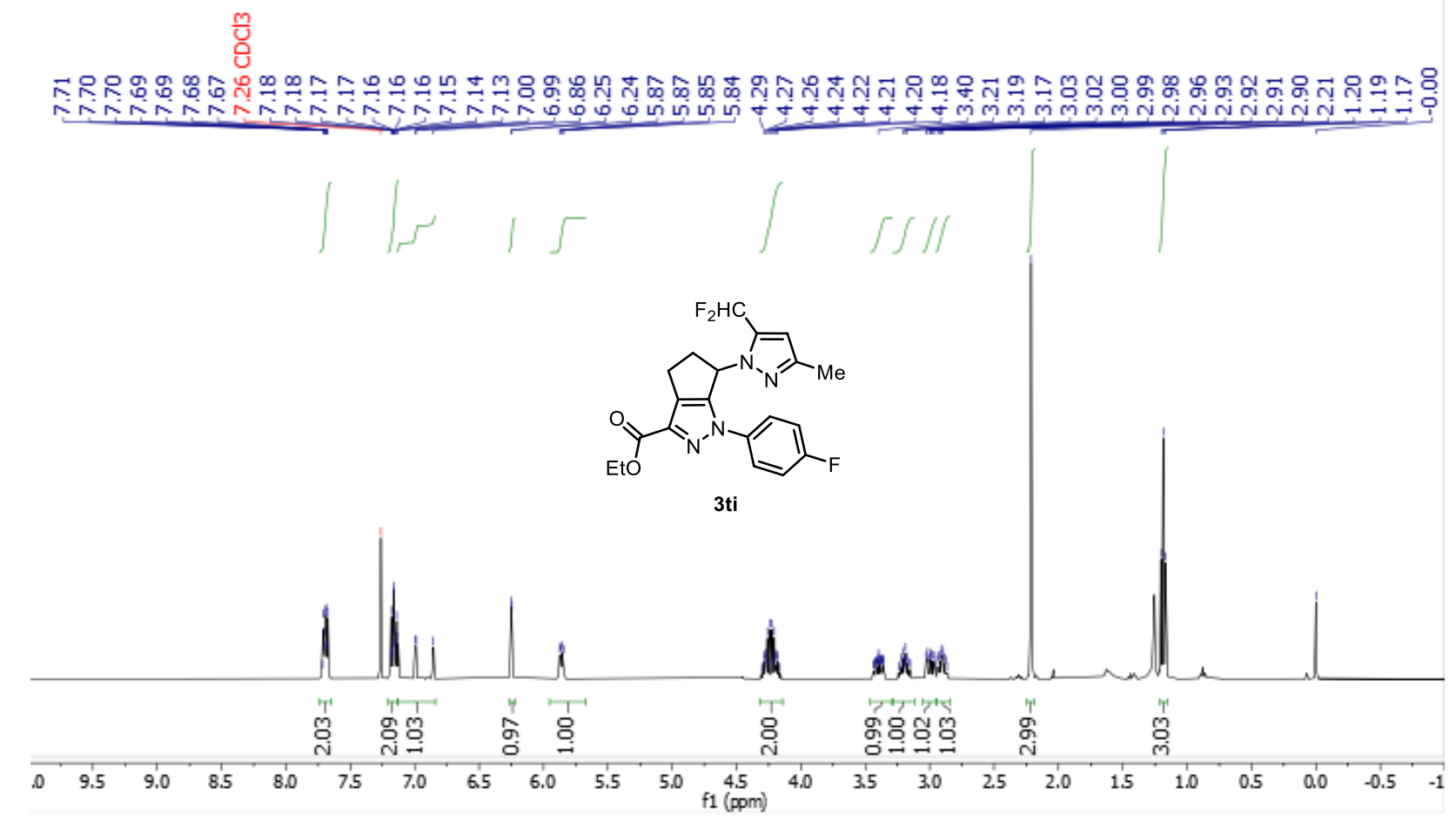

${ }^{13} \mathrm{C}$ NMR spectrum of $3 \mathrm{ti}$ in $\mathrm{CDCl}_{3}$ containing $0.03 \%(\mathrm{v} / \mathrm{v}) \mathrm{TMS}(126 \mathrm{MHz})$.

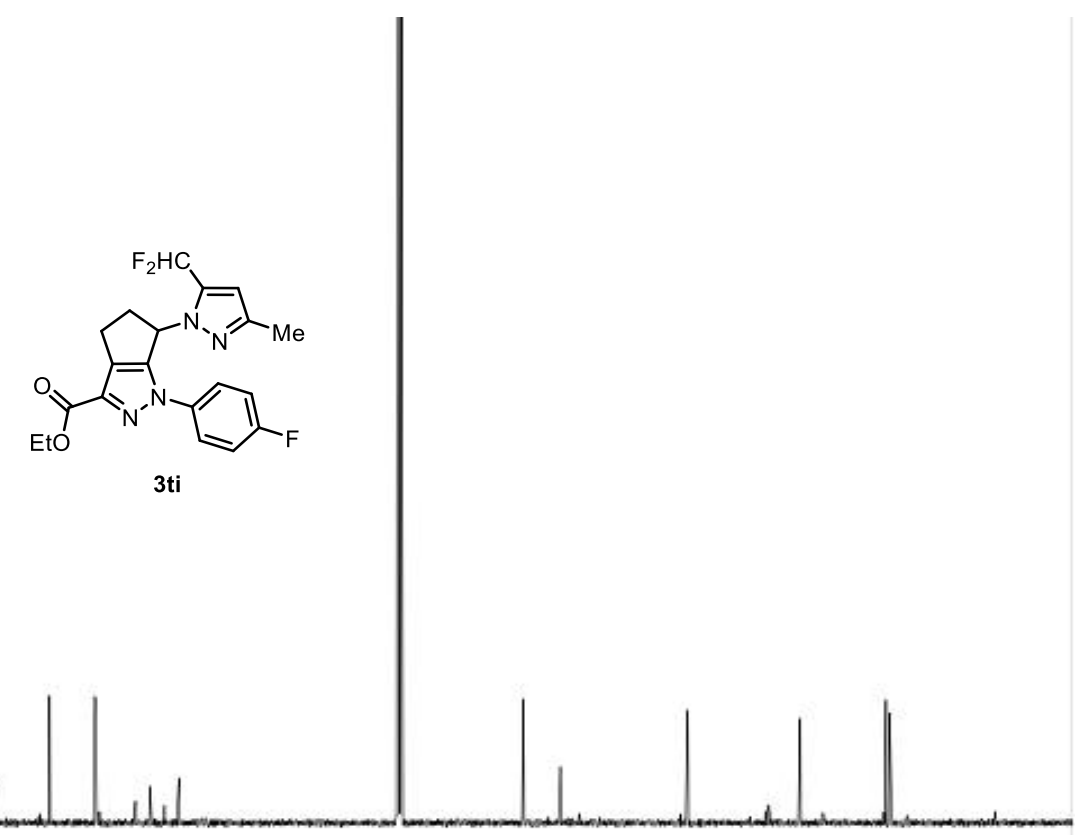

\begin{tabular}{lllllllllllllllllllllllllll}
\hline 0 & 190 & 180 & 170 & 160 & 150 & 140 & 130 & 120 & 110 & 100 & 90 & 80 & 70 & 60 & 50 & 40 & 30 & 20 & 10 & 0 & -1
\end{tabular} 
${ }^{19} \mathrm{~F}$ NMR spectrum of $3 \mathrm{ti}$ in $\mathrm{CDCl}_{3}$ containing $0.03 \%$ (v/v) TMS (377 MHz).
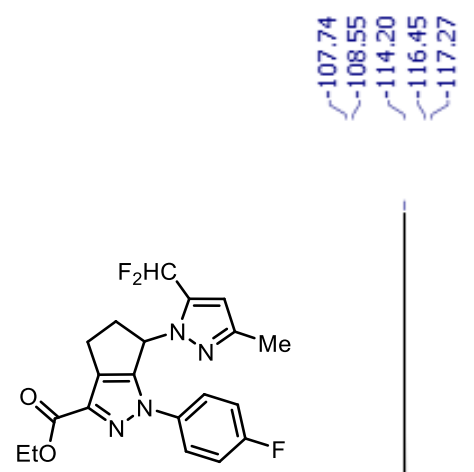

3 ti

\begin{tabular}{|c|c|c|c|c|c|c|c|c|c|c|c|c|c|c|c|c|c|}
\hline-30 & -40 & -50 & -60 & -70 & -80 & -90 & -100 & $\begin{array}{c}-110 \\
\mathrm{f} 1\end{array}$ & -120 & -130 & -140 & -150 & -160 & -170 & -180 & -190 & -2 \\
\hline
\end{tabular}

HSQC NMR spectrum of 3ti in $\mathrm{CDCl}_{3}$ containing $0.03 \%$ (v/v) TMS (500, $126 \mathrm{MHz}$ ).

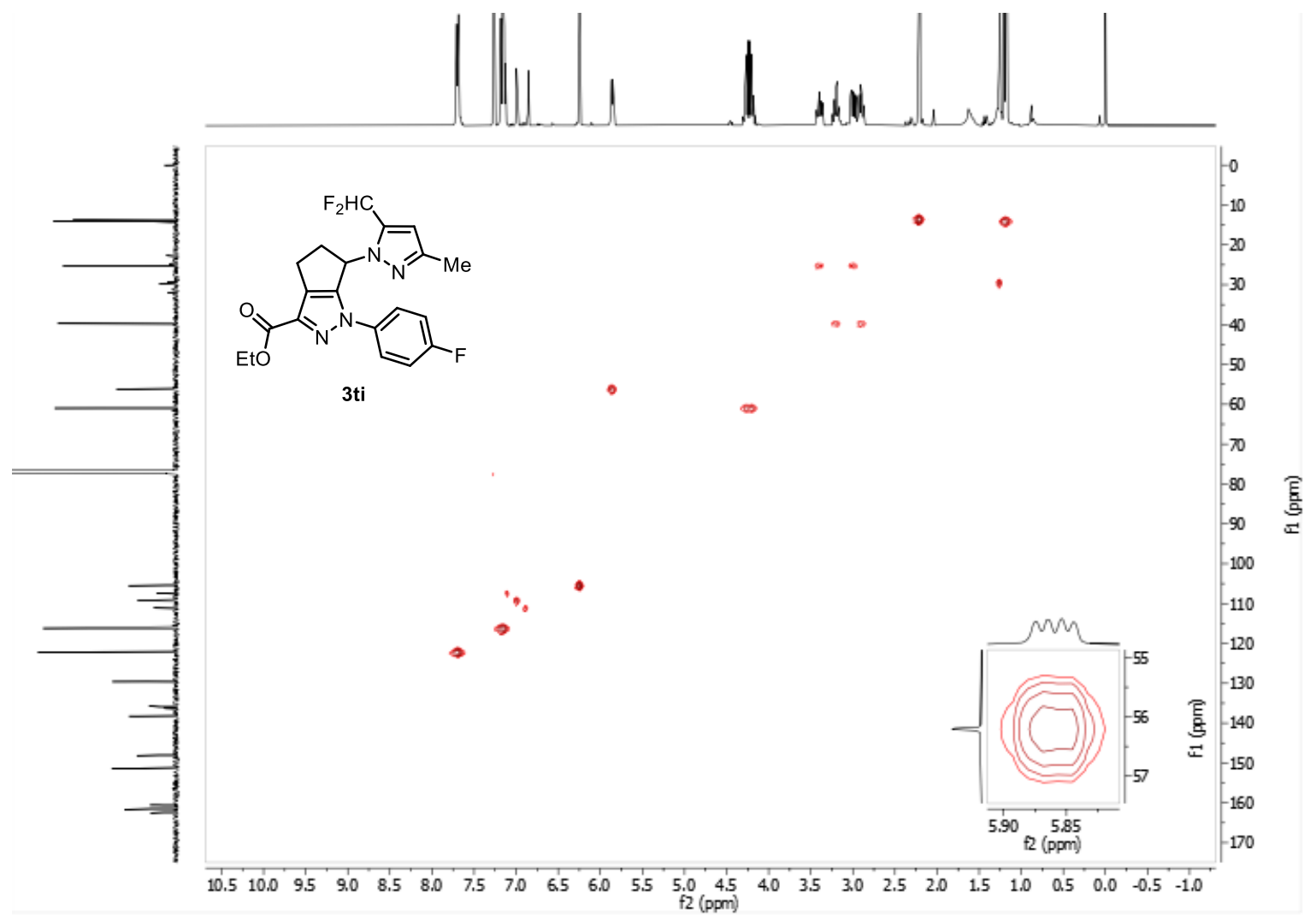


HMBC NMR spectrum of 3ti in $\mathrm{CDCl}_{3}$ containing $0.03 \%$ (v/v) TMS (500, $126 \mathrm{MHz}$ ).

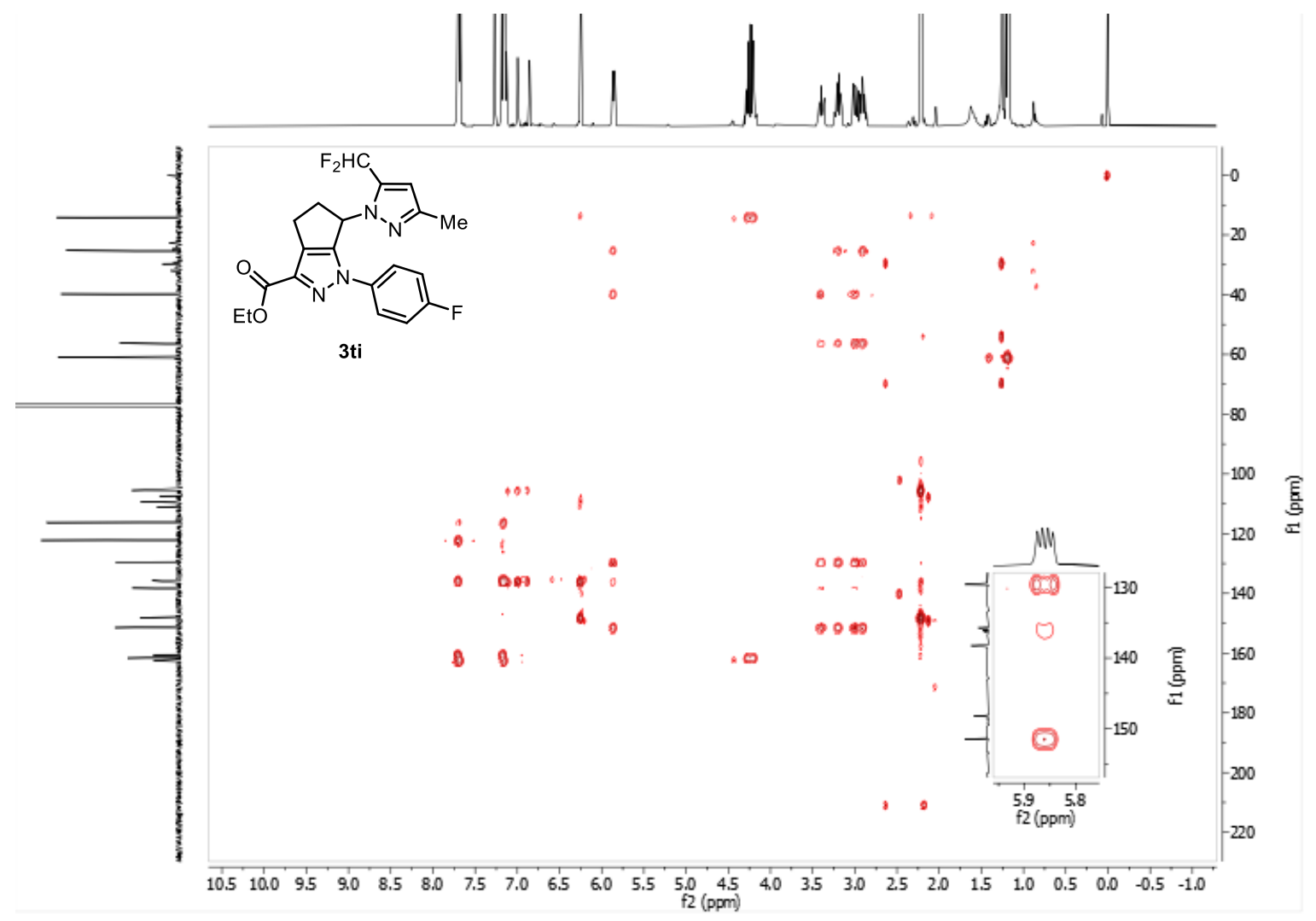

${ }^{1} \mathrm{H}$ NMR spectrum of $\mathbf{3 t s - 1}$ in $\mathrm{CDCl}_{3}$ containing $0.03 \%$ (v/v) TMS (500 MHz).
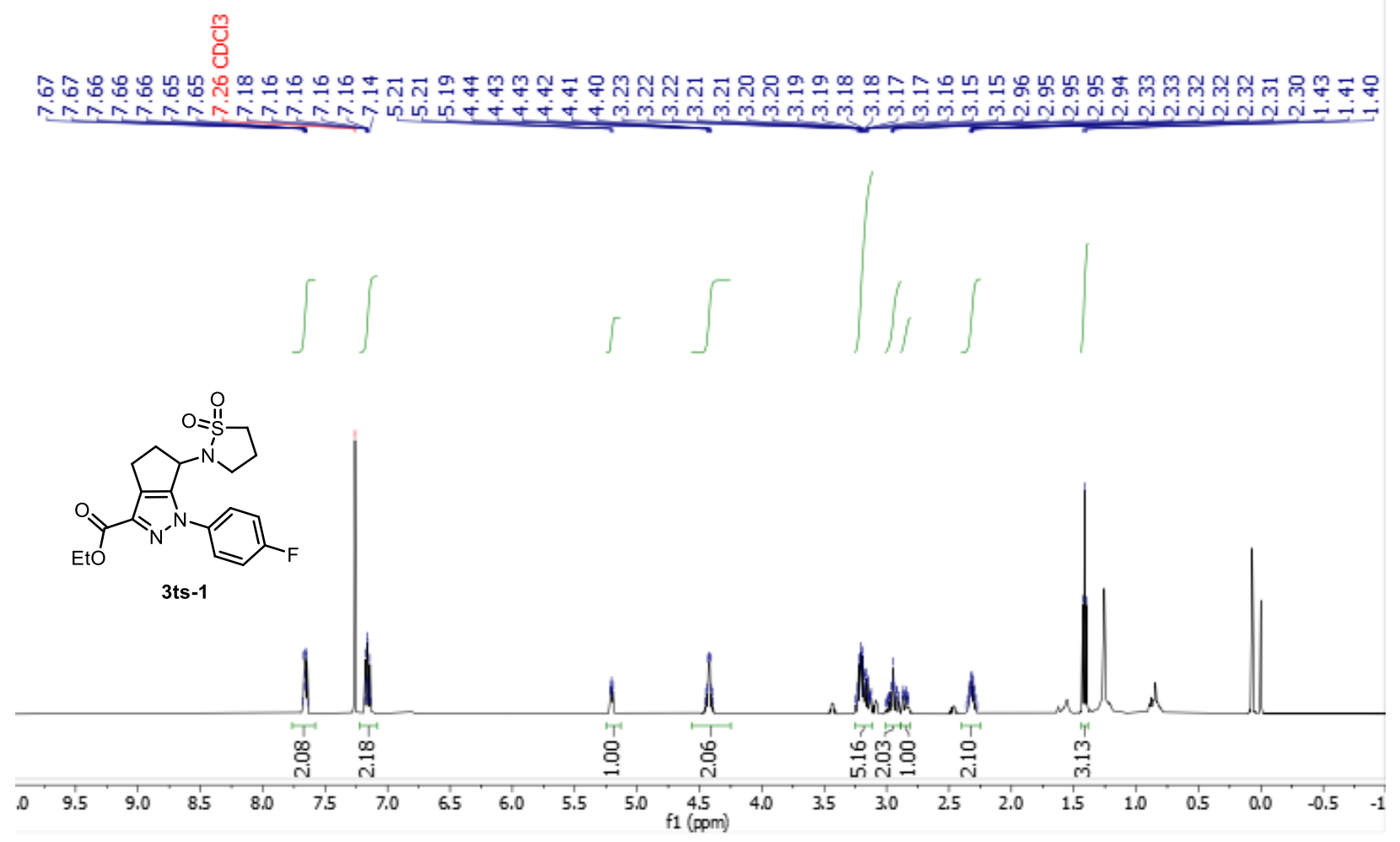
${ }^{13} \mathrm{C}$ NMR spectrum of 3 ts-1 in $\mathrm{CDCl}_{3}$ containing $0.03 \%$ (v/v) TMS (126 MHz).

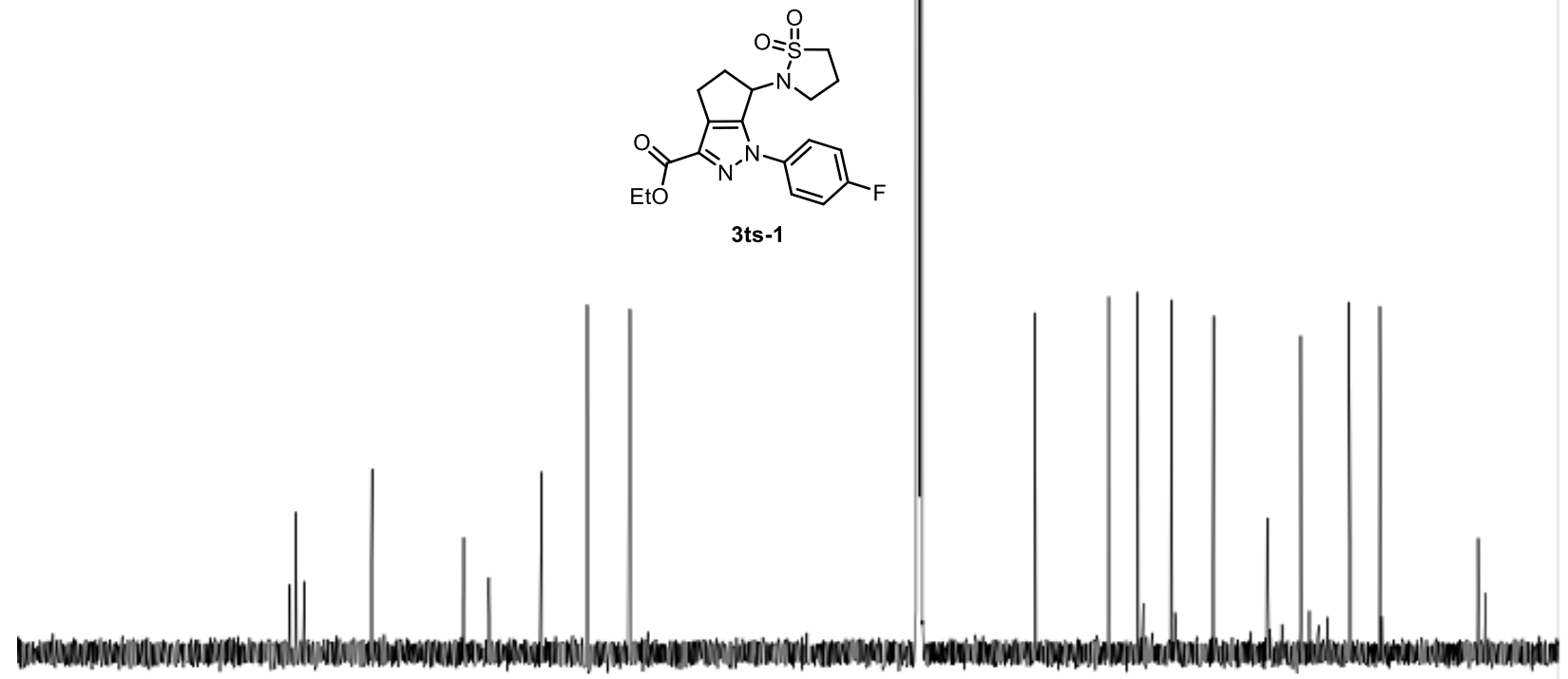

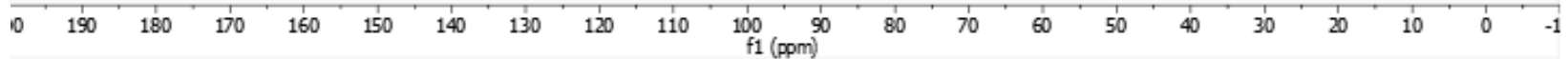

${ }^{19} \mathrm{~F}$ NMR spectrum of $\mathbf{3 t s}-\mathbf{1}$ in $\mathrm{CDCl}_{3}$ containing $0.03 \%$ (v/v) TMS (377 MHz).

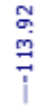<smiles>CCOC(=O)c1nn(-c2ccc(F)cc2)c(N2CCCS2(=O)=O)c1CC</smiles> 
HSQC NMR spectrum of 3ts-1 in $\mathrm{CDCl}_{3}$ containing $0.03 \%$ (v/v) TMS (500, $126 \mathrm{MHz}$ ).

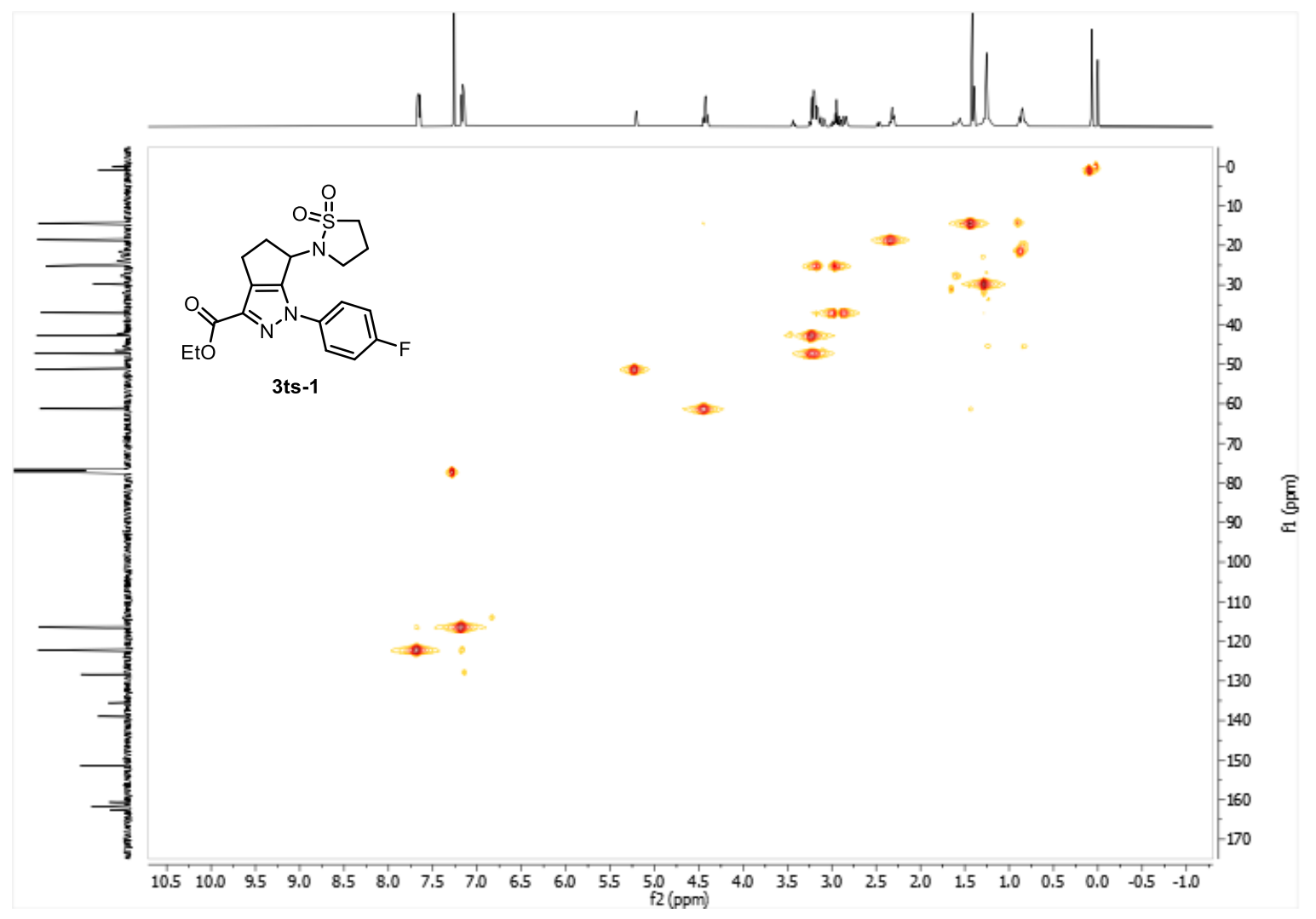

HMBC NMR spectrum of 3ts-1 in $\mathrm{CDCl}_{3}$ containing $0.03 \%$ (v/v) TMS (500, $126 \mathrm{MHz}$ ).

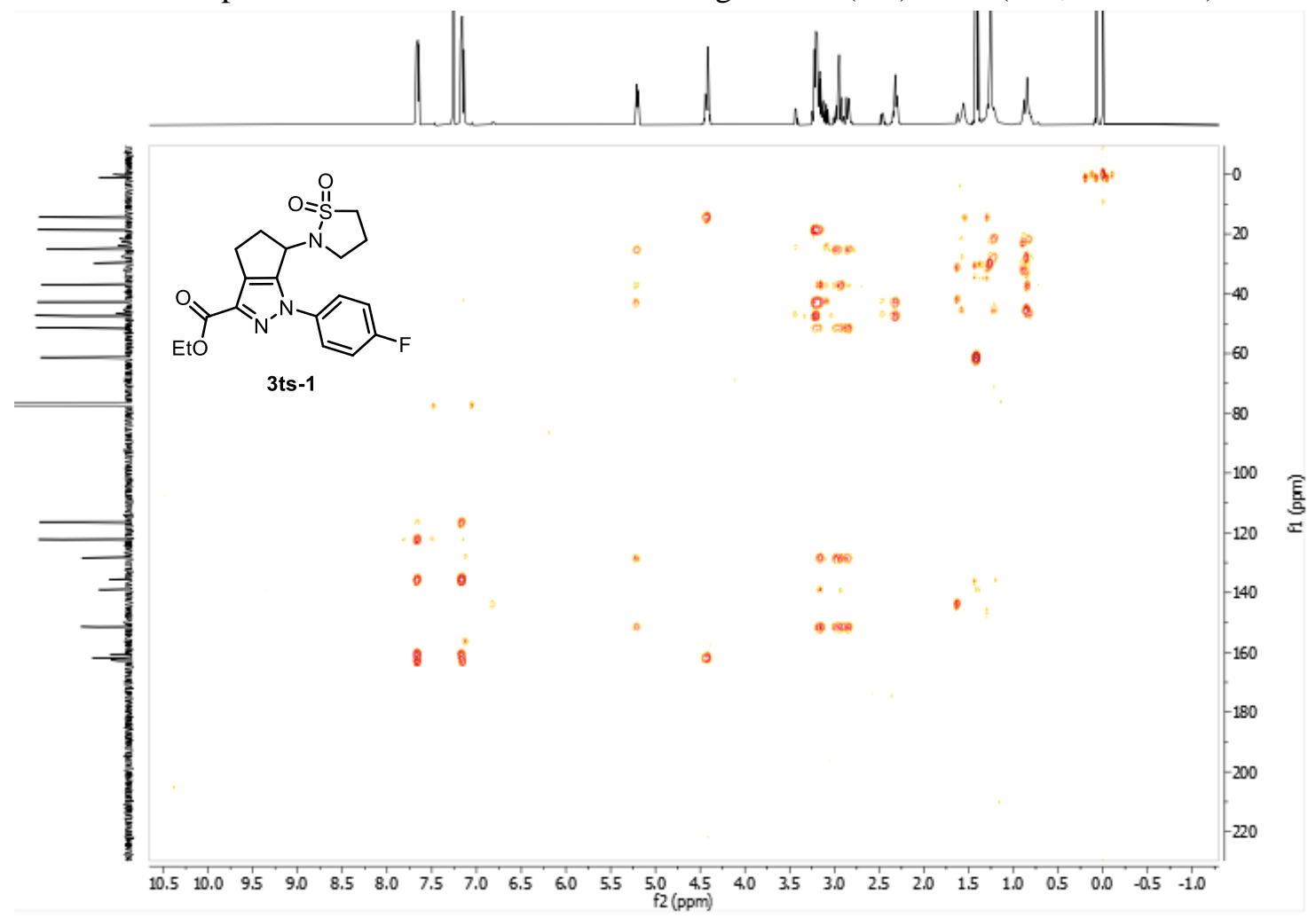


${ }^{1} \mathrm{H}$ NMR spectrum of 3 ts-2 in $\mathrm{CDCl}_{3}$ containing $0.03 \%$ (v/v) TMS (500 MHz).

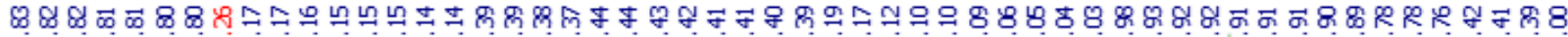

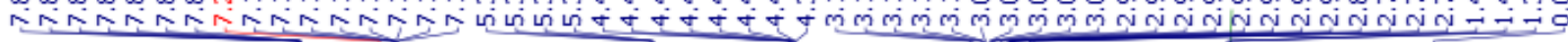

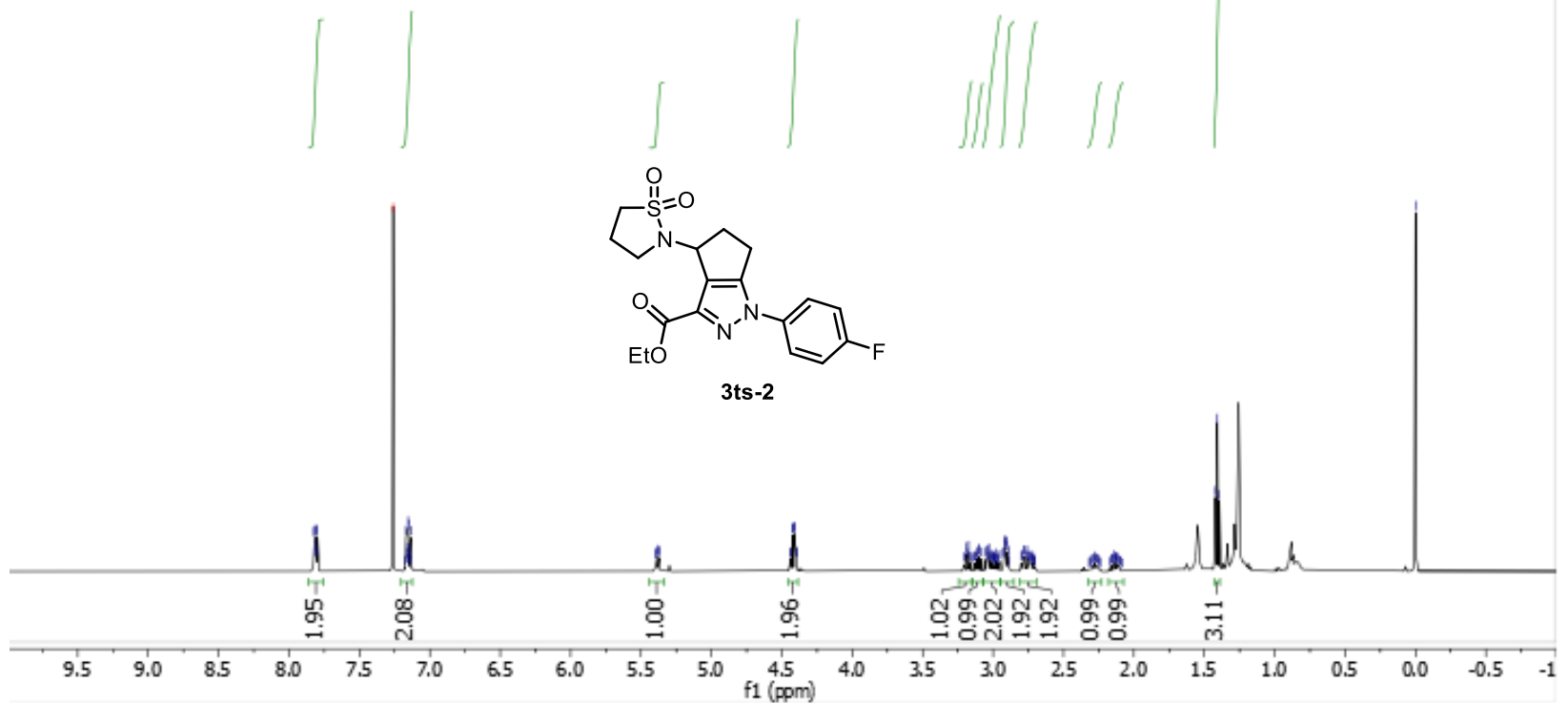

${ }^{13} \mathrm{C}$ NMR spectrum of 3ts-2 in $\mathrm{CDCl}_{3}$ containing $0.03 \%(\mathrm{v} / \mathrm{v})$ TMS $(126 \mathrm{MHz})$.<smiles>CCOC(=O)c1nn(-c2ccc(F)cc2)c2c1CCC2N1CCCS1(=O)=O</smiles>

3ts-2
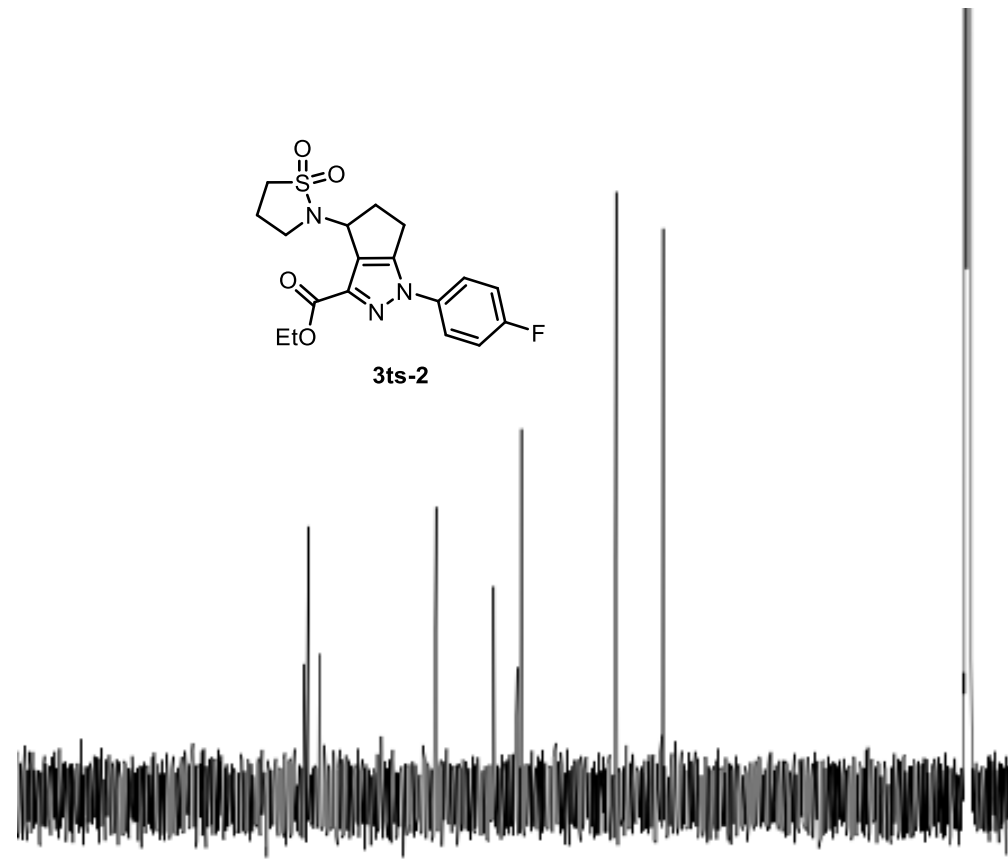

10190

$170 \quad 160$

$150 \quad 140$ f1 (ppm) 
${ }^{19} \mathrm{~F}$ NMR spectrum of $\mathbf{3 t s}-\mathbf{2}$ in $\mathrm{CDCl}_{3}$ containing $0.03 \%$ (v/v) TMS (377 MHz).

$\stackrel{m}{\stackrel{m}{*}}$

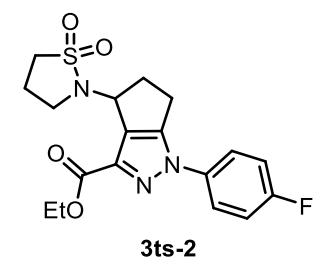

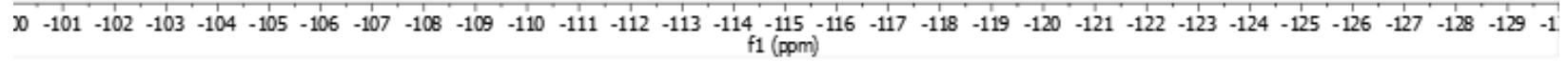
HSQC NMR spectrum of 3ts-2 in $\mathrm{CDCl}_{3}$ containing $0.03 \%$ (v/v) TMS (500, $126 \mathrm{MHz}$ ).

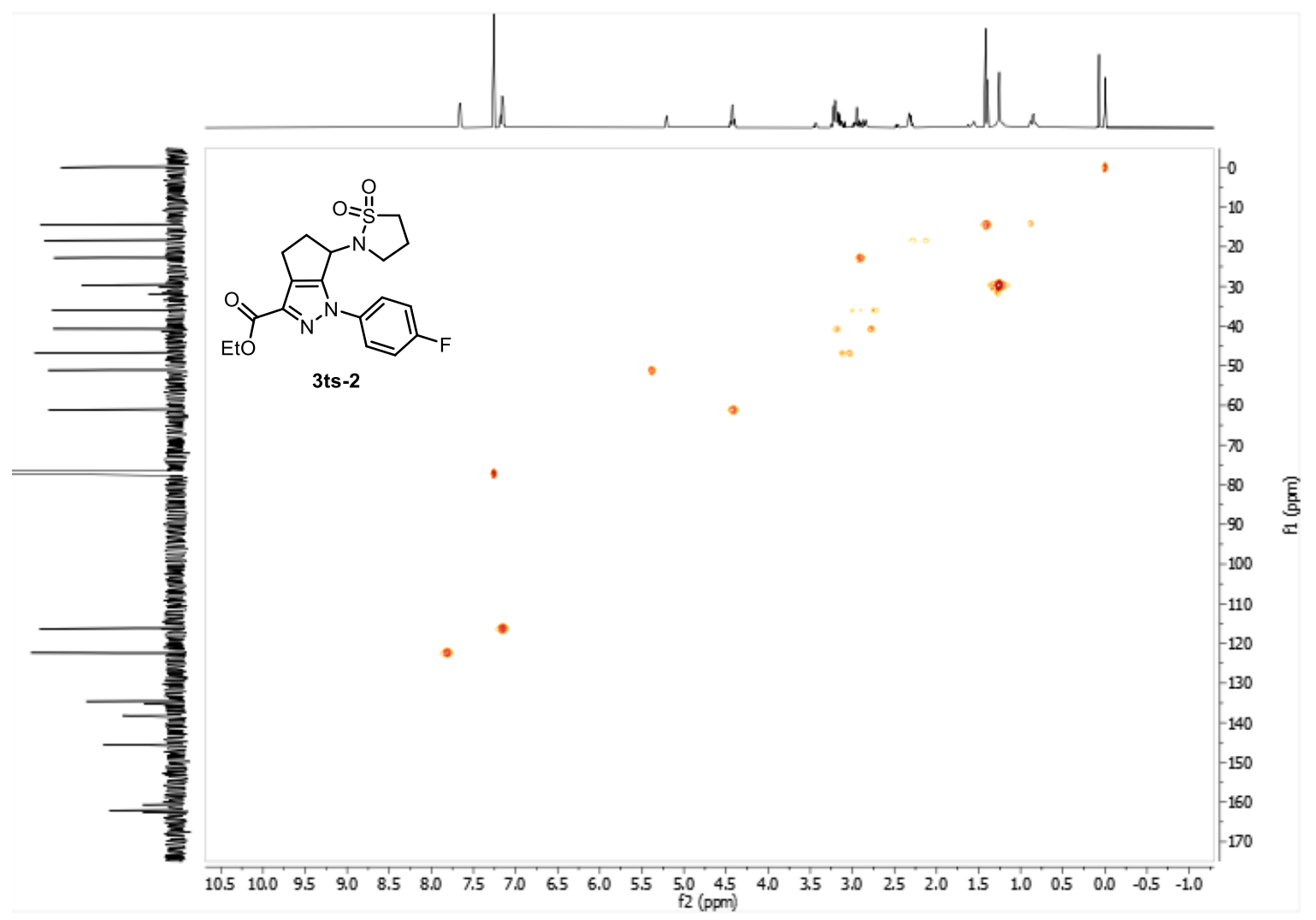


HMBC NMR spectrum of 3ts-2 in $\mathrm{CDCl}_{3}$ containing $0.03 \%$ (v/v) TMS (500, $126 \mathrm{MHz}$ ).

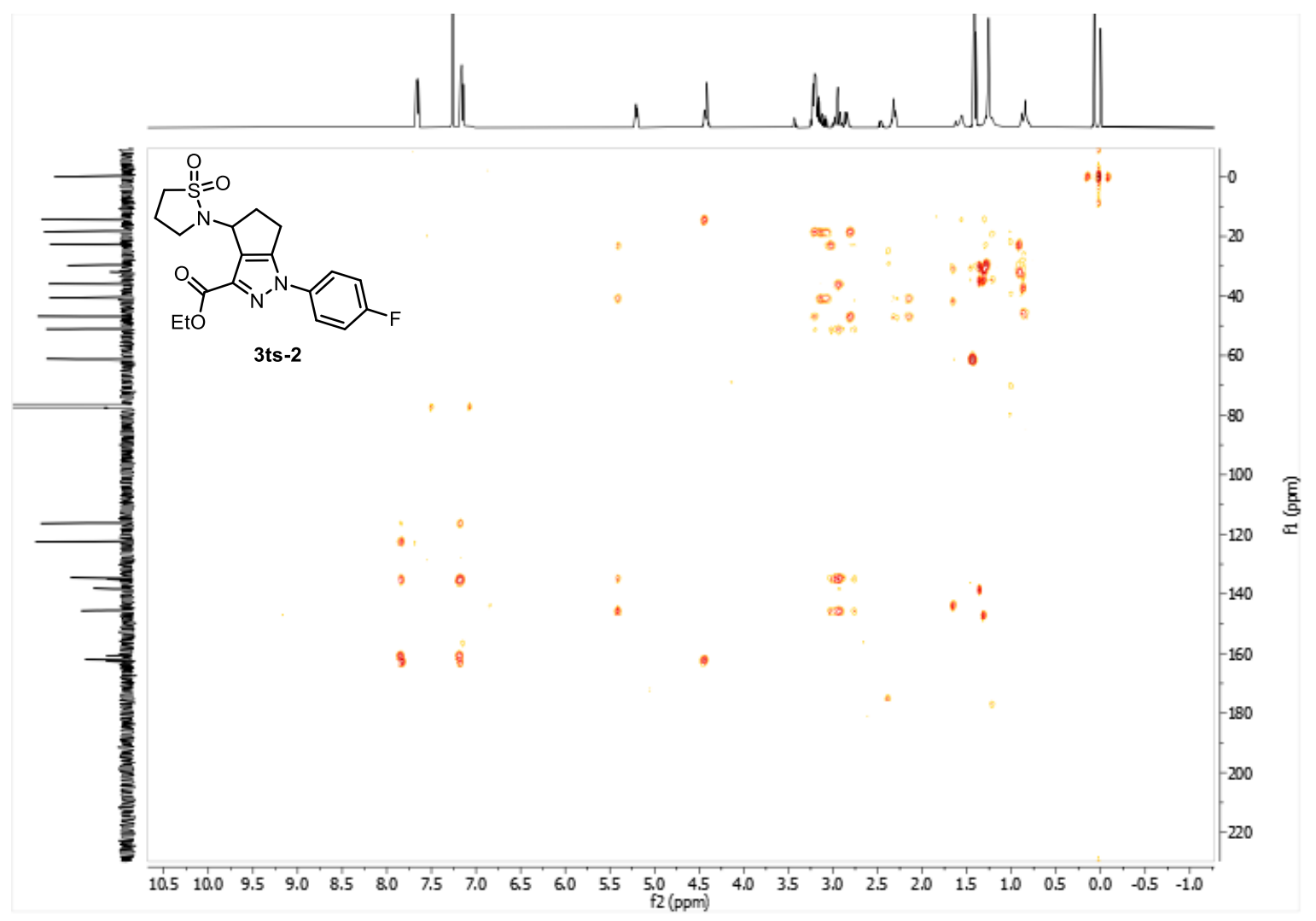

${ }^{1} \mathrm{H}$ NMR spectrum of $\mathbf{3 p p}$ ' in $\mathrm{CDCl}_{3}$ containing $0.03 \%$ (v/v) TMS (500 MHz).

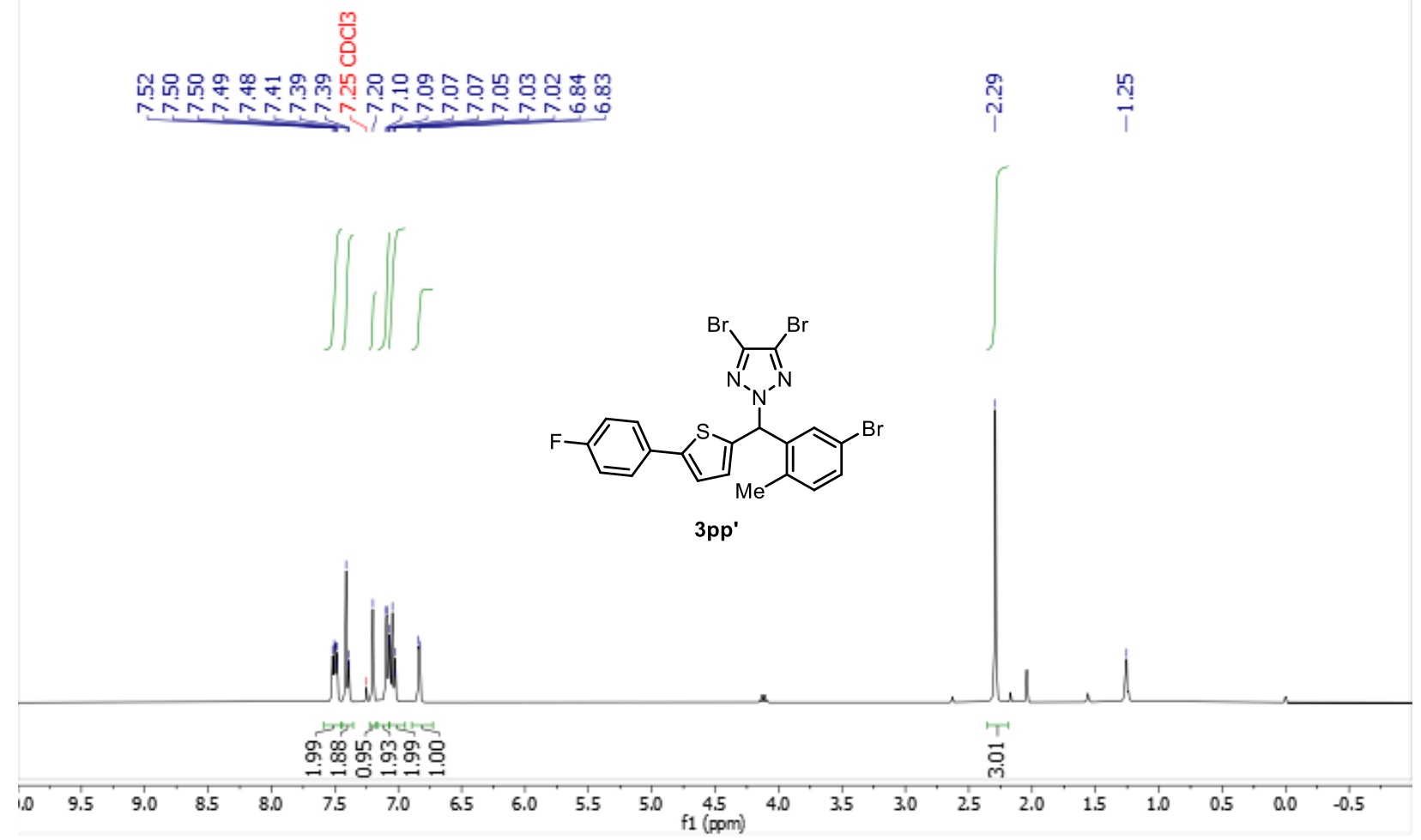


${ }^{13} \mathrm{C}$ NMR spectrum of 3pp' in $\mathrm{CDCl}_{3}$ containing $0.03 \%$ (v/v) TMS (126 MHz).
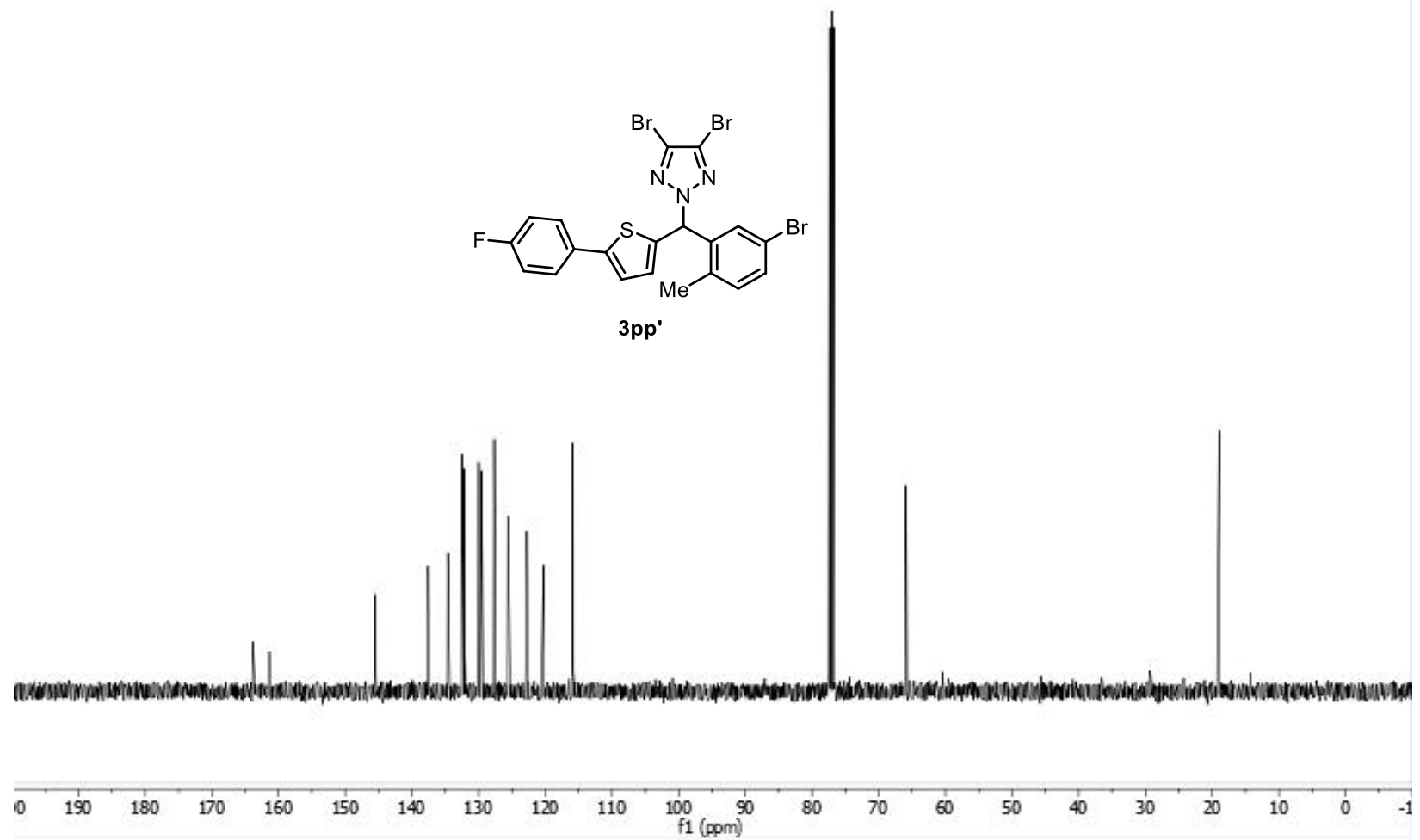

${ }^{19} \mathrm{~F}$ NMR spectrum of 3pp' in $\mathrm{CDCl}_{3}$ containing $0.03 \%$ (v/v) TMS (377 MHz).

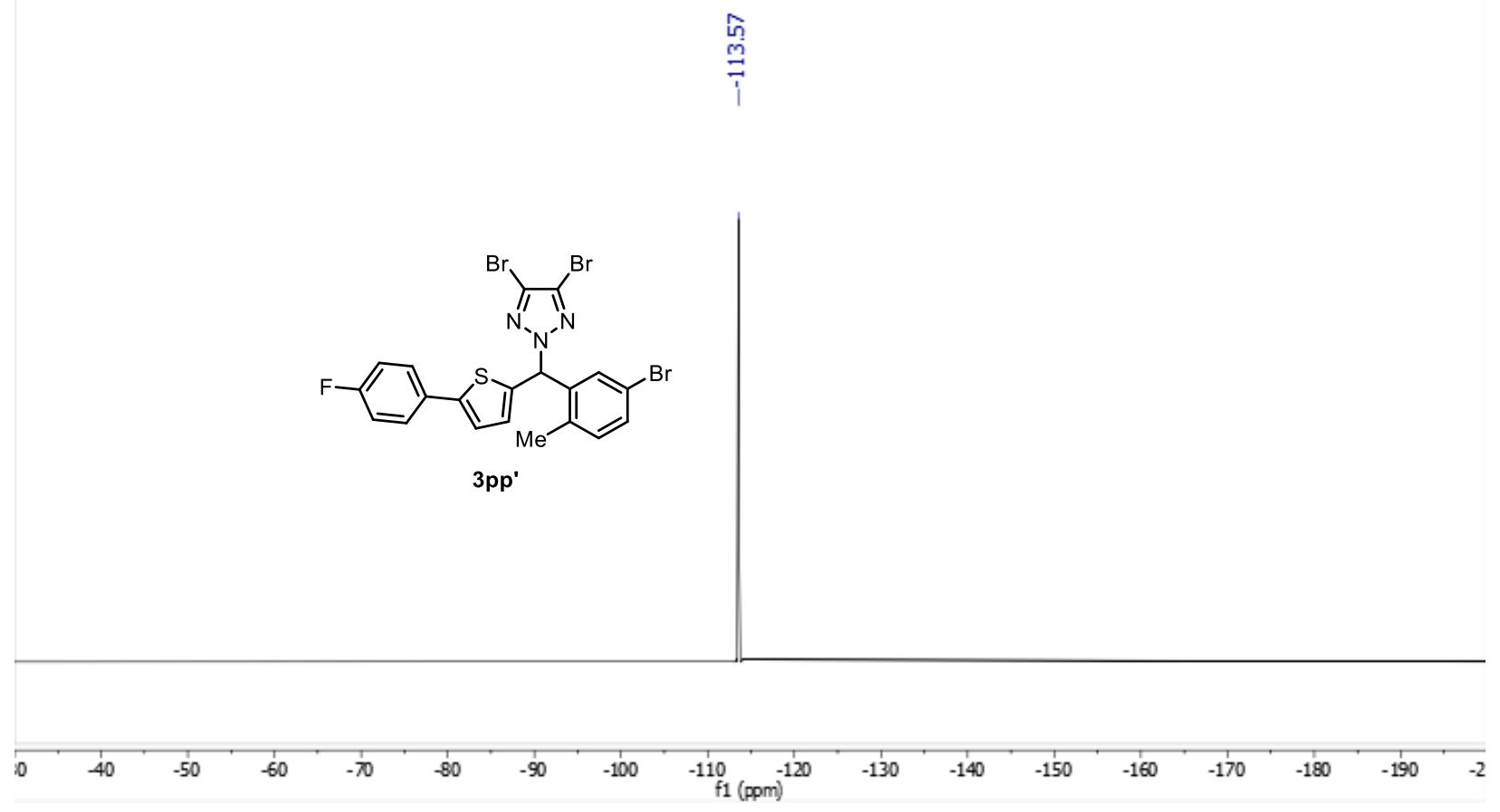


${ }^{1} \mathrm{H}$ NMR spectrum of $\mathbf{3 p s}$ in $\mathrm{CDCl}_{3}$ containing $0.03 \%$ (v/v) TMS (500 MHz).
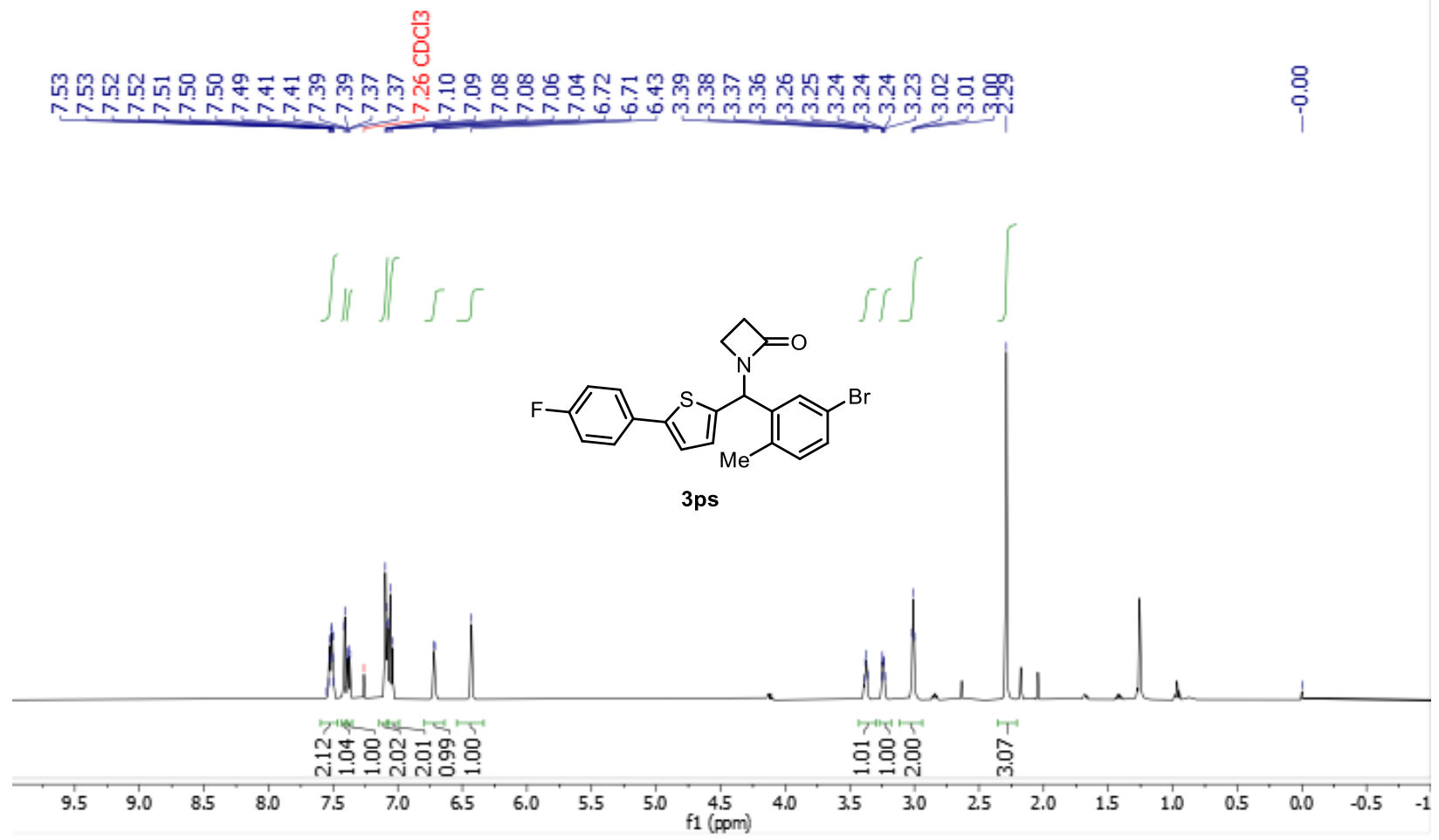

${ }^{13} \mathrm{C}$ NMR spectrum of $\mathbf{3 p s}$ in $\mathrm{CDCl}_{3}$ containing $0.03 \%$ (v/v) TMS (126 MHz).

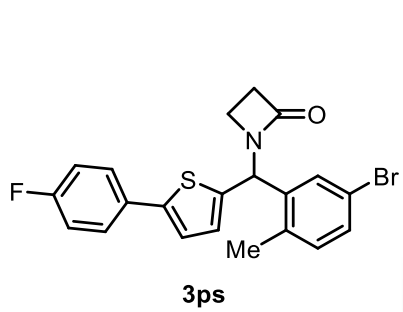

1

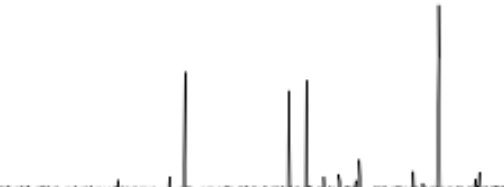


${ }^{19} \mathrm{~F}$ NMR spectrum of $\mathbf{3 p s}$ in $\mathrm{CDCl}_{3}$ containing $0.03 \%$ (v/v) TMS (377 MHz).

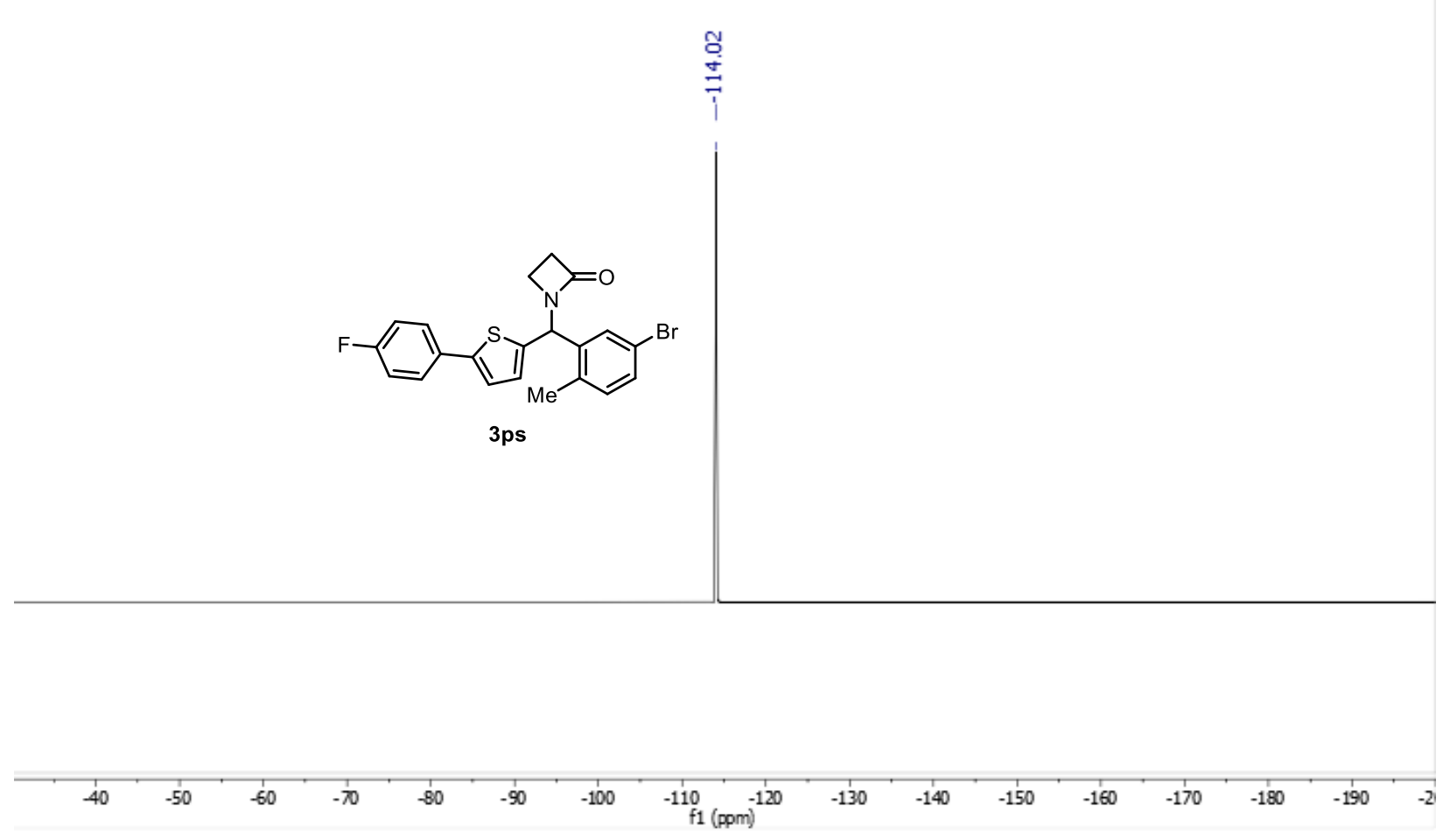

